Beiträge aus der Nordwestdeutschen Forstlichen Versuchsanstalt Band 3

\title{
Ergebnisse angewandter Forschung zur Buche
}

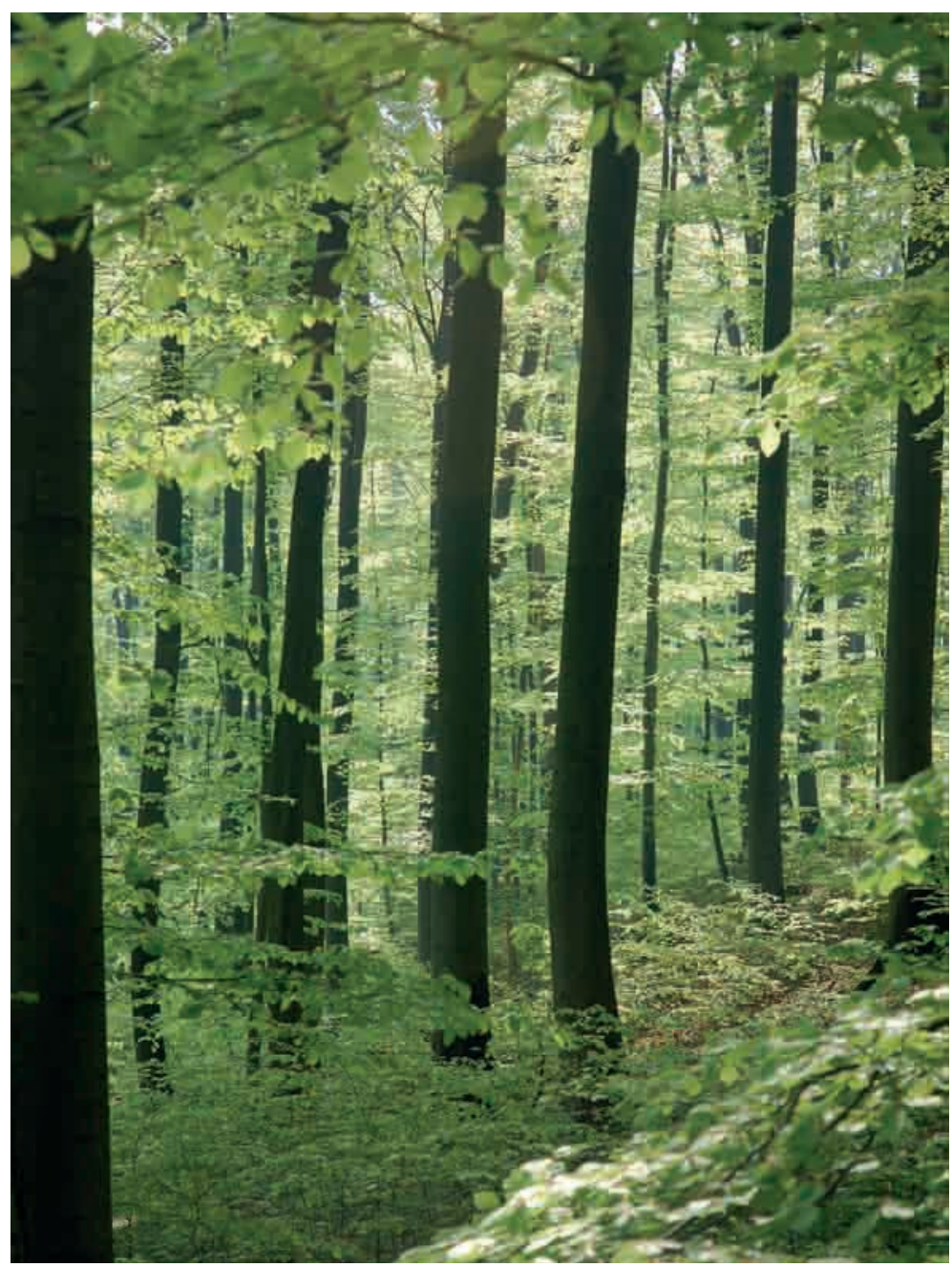



Nordwestdeutsche Forstliche Versuchsanstalt (Hrsg.)

Ergebnisse angewandter Forschung zur Buche

This work is licensed under the Creative Commons License 2.0 "by-nd", allowing you to download, distribute and print the document in a few copies for private or educational use, given that the document stays unchanged and the creator is mentioned. You are not allowed to sell copies of the free version. 
erschienen als Band 3 der Reihe

„Beiträge aus der Nordwestdeutschen Forstlichen Versuchsanstalt“

in den Universitätsdrucken im Universitätsverlag Göttingen 2008 
Nordwestdeutsche

Forstliche Versuchsanstalt (Hrsg.)

\section{Ergebnisse angewandter Forschung zur Buche}

Beiträge aus der

Nordwestdeutschen

Forstlichen Versuchsanstalt

Band 3

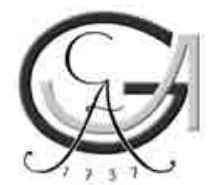

Universitätsverlag Göttingen 2008 


\section{Bibliographische Information der Deutschen Nationalbibliothek}

Die Deutsche Nationalbibliothek verzeichnet diese Publikation in der Deutschen Nationalbibliographie; detaillierte bibliographische Daten sind im Internet über $<$ http://dnb.ddb.de $>$ abrufbar.

Global Forest Decimal Classification: 176.1; 181; 4

\section{Herausgeber}

Nordwestdeutsche Forstliche Versuchsanstalt (NW-FVA)

Grätzelstr. 2, D-37079 Göttingen

Tel.: +49 (0)551-69401-0, Fax: +49 (0)551-69401-160

E-Mail: zentrale@nw-fva.de

www.nw-fva.de

Schriftleitung der Reihe: Prof. Dr. Hermann Spellmann

Redaktion: Inge Kehr, Ulrike Gaertner

Titelfoto: Kalk-Buchenwald bei Göttingen (Dr. Jan Evers)

Dieses Buch ist auch als freie Onlineversion über die Homepage der NW-FVA, des Verlages sowie über den OPAC der Niedersächsischen Staats- und Universitätsbibliothek (http://www.sub.uni-goettingen.de) erreichbar und darf gelesen, heruntergeladen sowie als Privatkopie ausgedruckt werden. Es gelten die Lizenzbestimmungen der Onlineversion. Es ist nicht gestattet, Kopien oder gedruckte Fassungen der freien Onlineversion zu veräußern.

(C) 2008 Universitätsverlag Göttingen http://univerlag.uni-goettingen.de ISBN: 978-3-940344-44-1

ISSN: 1865-6994 


\title{
Vorwort
}

\section{Ergebnisse der angewandten Forschung zur Buche Hintergründe und Zielsetzungen für diesen Buchen-Schwerpunkt-Band}

\begin{abstract}
Alle Waldbesitzarten in Deutschland eint bisher das gemeinsame Leitbild einer nachhaltigen, multifunktionalen Forstwirtschaft. Es wird versucht, auf dem Wege des Kompromisses die vielfältigen Ansprüche an den Wald in unserer dicht besiedelten Kulturlandschaft angemessen zu berücksichtigen. Dazu werden i. d. R. auf der gleichen Fläche Nutz-, Schutz- und Erholungsfunktionen gleichzeitig verfolgt, die Eigentümerinteressen beachtet, die waldbaulichen Ziele und Methoden mit den ökologischen Erfordernissen und den ökonomischen Möglichkeiten in Übereinstimmung gebracht und es wird angestrebt, den nachfolgenden Generationen wenigstens ebensoviel Nutzen aus dem Wald zu sichern, wie er der derzeitigen Generation zur Verfügung steht.
\end{abstract}

Dieses Leitbild wird vom amtlichen Naturschutz und den Umweltverbänden immer stärker angezweifelt, die zunehmend eine Funktionentrennung fordern. Jüngstes Beispiel ist die „Entdeckung“ der Buchenwälder als europäisches Naturerbe (vgl. KNAPP 2007 ${ }^{1}$ im Vorfeld der diesjährigen Vertragsstaatenkonferenz zum Übereinkommen über die biologische Vielfalt (CBD) in Bonn. In seinem Bonner Thesen-Papier zum „Naturerbe Buchenwälder“ fordert das Bundesamt für Naturschutz (BFN 2008²) u. a. Buchenwälder im öffentlichen Besitz großflächig aus der „stofflichen“ Nutzung zu entlassen und in Waldnaturschutzgebieten zu sichern, weil die Buchenwald-Ökosysteme in Deutschland extrem gefährdet seien. Dies steht im deutlichen Widerspruch zu der Tatsache, dass weit über 500.000 ha Buchenwälder aufgrund ihres guten Erhaltungszustandes das Grundgerüst der deutschen FFH-Gebietskulisse im Wald bilden.

Auf der anderen Seite steht die Forderung der Holzwirtschaft nach einer nachhaltigen Rohholzversorgung. Angesichts der Globalisierung der Rohstoff- und Warenmärkte, der Verknappung fossiler Rohstoffe und der zunehmenden Konkurrenz zwischen stofflicher und energetischer Nutzung ist die Rohholznachfrage in den letzten Jahren enorm gestiegen und die Ansprüche an die Holzbereitstellung haben sich wesentlich verändert. Auf den gestiegenen Rohholzbedarf darf die Forstwirtschaft nur unter Wahrung der Nachhaltigkeit reagieren, was eine Über-

\footnotetext{
${ }^{1}$ KNAPP, H. D. (2007): Buchenwälder. Natur und Landschaft 82, 385

2 BfN (Bundesamt für Naturschutz) (2008): Bonner Thesen zum „Naturerbe Buchenwälder“. Download unter http://www.bfn.de/6.html?\&cHash=8affa1489b\&tx_ttnews $\% 5$ Btt_news $\% 5 \mathrm{D}=$ 1115
} 
nutzung der Bestände zur kurzfristigen Überwindung von Versorgungsengpässen ebenso ausschließt wie eine Missachtung der anderen Waldfunktionen.

In diesem Spannungsfeld nationaler und internationaler Forderungen und der forstpolitischen Diskussionen bedarf es einer klaren strategischen Positionierung der Forstwirtschaft zum Naturschutz und zur Holzproduktion im Wald in Abstimmung mit den anderen Funktionen des Waldes. Hierzu sind abgesicherte Erkenntnisse aus der forstlichen Forschung unverzichtbar, um Ziele zu begründen, Risiken zu vermeiden, Umsetzungen effizient zu steuern und die gesellschaftliche Glaubwürdigkeit zu sichern.

Zur Unterstützung einer nachhaltigen, multifunktionalen Buchenwirtschaft wird in diesem Band der Schriftenreihe ein Ausschnitt aus den vielfältigen Ergebnissen der angewandten Forschung an der NW-FVA zur Buche vorgestellt. Die Themen reichen vom Wasser- und Nährstoffhaushalt, über die genetische Vielfalt und deren Nutzung, den biotischen und abiotischen Risiken, den Auswirkungen des Klimawandels, der Strukturdynamik und Artenvielfalt, der waldbaulichen Steuerung bis hin zum Holzaufkommen und der Entwertungsgefahr durch Buchenrotkern. Ergänzt werden diese Entscheidungshilfen durch Beiträge von Kollegen der Forstwissenschaftlichen Fakultät der Universität Göttingen, die sich mit der Verwertung und Verwendung von Buchenholz sowie einer betriebswirtschaftlichen Bewertung von Buchenwäldern befassen.

Der Buchenband soll dazu beitragen, die Diskussion zwischen Forstwirtschaft, Naturschutz, Holzwirtschaft und Gesellschaft anzuregen und zu versachlichen, um tragfähige Lösungen für den Schutz und die Nutzung unserer Buchenwälder auch weiterhin zu sichern.

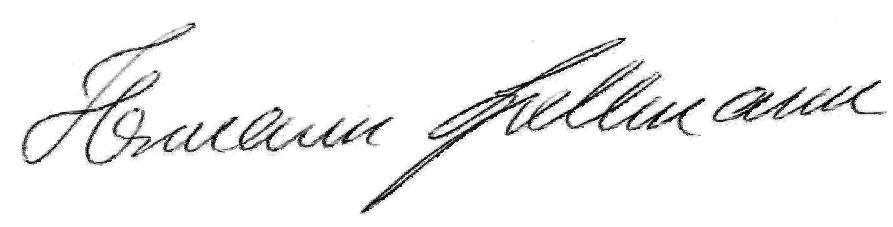

Ltd. FD Prof. Dr. Hermann Spellmann

Leiter der Nordwestdeutschen Forstlichen Versuchsanstalt (NW-FVA) 


\section{Inhaltsverzeichnis}

Vorwort

Inhaltsverzeichnis

Nährstoffhaushalt von Buchenbeständen auf Muschelkalk, Basalt und Buntsandstein

(H. Meesenburg, K. J. Meiwes, H. Fortmann, B. Scheler, J. Eichhorn) 1

1 Einleitung 3

2 Material und Methoden 3

3 Bodenzustand und -veränderungen 6

4 Stoffhaushalt 10

5 Schlussfolgerungen 15

$\begin{array}{ll}\text { Literatur } & 17\end{array}$

Auswirkungen von Bodenschutzkalkungen auf Buchenwälder (Fagus sylvatica L.)

(J. Evers, I. Dammann, A. Noltensmeier, R.-V. Nagel) 21

1 Einleitung 23

2 Material und Methoden $\quad 24$

3 Ergebnisse 30

4 Diskussion 45

$\begin{array}{ll}\text { Literatur } & 48\end{array}$

Genetische Vielfalt nordwestdeutscher Buchenwälder

(A. Janßen, K. Gebhardt, W. Steiner)

1 Einleitung 52

2 Untersuchungen zur genetischen Vielfalt von Buchenbeständen 53

3 Beeinflussungen der genetischen Variation 57

4 Diskussion und Ausblick 64

Literatur $\quad 65$ 
Schutz durch Nutzung forstlicher Genressourcen der Buche (Fagus sylvatica L.) in Nordwestdeutschland

(J. R. G. Kleinschmit, H.-M. Rau, K. Gebhardt) 69

1 Einleitung 71

2 Prüfung von Buchen-Nachkommenschaften 71

3 Herkunftsempfehlungen, verfügbares Vermehrungsgut 74

4 Programm zur Erhaltung forstlicher Genressourcen 76

5 Herkunftssicherung 81

6 Diskussion $\quad 82$

$\begin{array}{ll}\text { Literatur } & 84\end{array}$

Komplexe Erkrankungen an Buche

$\begin{array}{ll}\text { (U. Bressem) } & 87\end{array}$

1 Einleitung 89

2 Beschreibung der drei wichtigsten Schadenstypen 89

3 Umfrage zu Schäden an Buche im Zuständigkeitsbereich der $\begin{array}{ll}\text { NW-FVA } & 97\end{array}$

4 Diskussion und Wertung der Ergebnisse 102

$\begin{array}{ll}\text { Literatur } & 105\end{array}$

Untersuchungen zur Trockenheitstoleranz der Buche am Beispiel des witterungsextremen Jahres 2003

(J. Eichhorn, I. Dammann, E. Schönfelder, M. Albrecht, W. Beck, U. Paar) 109

1 Einleitung 111

2 Resilienzeigenschaften als Bewertungskriterium für die Vitalität der $\begin{array}{ll}\text { Buche } & 114\end{array}$

3 Konzept zur Erfassung der Resilienz von Bäumen und Wäldern 115

4 Übersicht Hauptergebnisse 128

5 Bewertung Resilienzeigenschaften, Vitalität und Risiko der Buche 129

Literatur

131 
Der Klimawandel und seine Auswirkungen auf die Buchenwälder in Deutschland

(J. Sutmöller, H. Spellmann, C. Fiebiger, M. Albert)

135

1 Einleitung

137

2 Klimatische Ansprüche der Buche 138

3 Klimaszenarien 143

4 Folgen des Klimawandels für die Buche 149

5 Fazit 154

Literatur 155

Aspekte der Biodiversität von Buchenwäldern -

Konsequenzen für eine naturnahe Bewirtschaftung

(P. Meyer, Mar. Schmidt)

1 Einleitung 161

2 Verbreitung von Buchenwäldern 163

3 Artenvielfalt 167

4 Strukturvielfalt 176

5 Schlussfolgerungen 184

Literatur 186

Waldumbau mit Buche unter Berücksichtigung ihrer

ökologischen Ansprüche

(H. Rumpf, R. Petersen) 193

1 Einleitung 195

2 Versuche der NW-FVA zum Umbau von Nadelholzreinbeständen mit Buche 197

$\begin{array}{ll}\text { Literatur } & 216\end{array}$

Wachstum, Behandlung und Ertrag von Reinbeständen der

Rotbuche (Fagus sylvatica L.) in Nordwestdeutschland

(R.-V. Nagel, H. Spellmann) 221

1 Einleitung 222

2 Ziele der Buchen-Bestandespflege 224

3 Für die Bestandessteuerung wichtige ökologische Eigenschaften der

Rotbuche 226 
$4 \quad$ Wachstumstrends der Buche in Nordwestdeutschland 228

5 Einfluss der Bestandesbehandlung auf Struktur, Qualitätsentwicklung und Ertrag von Buchenbeständen

6 Diskussion 255

Literatur

Methodische Ansätze und Ergebnisse zur Quantifizierung des Buchen-Rotkerns in Hessen

(Mat. Schmidt, S. Nowack, R. Riebeling)

1 Einleitung 268

2 Datengrundlage 269

3 Methodik 274

4 Ergebnisse 276

5 Diskussion 283

$\begin{array}{ll}\text { Literatur } & 288\end{array}$

Das mittelfristige Buchenholzaufkommen in Niedersachsen und Deutschland

(J. Hansen, J. Nagel, Mat. Schmidt, H. Spellmann) 291

1 Einführung 293

2 Methodik der Szenariosimulation 294

3 Ausgangszustand, Zuwachs und Nutzungen in der Inventurperiode $1987-2002 \quad 297$

4 Buchenholzpotenziale Niedersachsen 299

5 Buchenholzpotenziale Deutschland 302

$\begin{array}{ll}6 & \text { Regionale Validierung und Kalibrierung der } \\ \text { Durchmesserzuwachsprognose } & 307\end{array}$

$\begin{array}{ll}\text { Literatur } & 309\end{array}$

Verwertung und Verwendung von Buchenholz

(F. Hapla, H. Militz) 311

1 Einleitung 312

2 Rundholzsortierung 313

3 Holzeigenschaften 314

$4 \quad$ Furnierausbeute von rotkernigen Buchen in Zielstärke 317 
5 Schnittholzausbeute von rotkernigen Buchen in Zielstärke 319

6 Innovative Holzprodukte 319

7 Verwendungsbereiche 321

8 Fazit 323

$\begin{array}{ll}\text { Literatur } & 324\end{array}$

Betriebswirtschaftliche Bewertung von Buchenwäldern

(B. Möhring, G. Leefken, C. Gutsche) 327

1 Einleitung 329

2 Reinerträge im Wandel der Zeit 330

3 Risiko und Diversifikation 332

4 Berücksichtigung der betrieblichen Liquidität 335

$5 \quad$ Forstwirtschaftliche Realität: Betriebsvergleichsergebnisse 337

6 Schlussfolgerungen $\quad 340$

Literatur $\quad 341$ 



\title{
Nährstoffhaushalt von Buchenbeständen auf Muschelkalk, Basalt und Buntsandstein
}

\section{Nutrient cycling in beech stands on shell limestone, basalt and new red sandstone}

\author{
Henning Meesenburg, Karl Josef Meiwes, Heike Fortmann, Birte Scheler und \\ Johannes Eichborn
}

\section{Zusammenfassung}

Unterschiede und Gemeinsamkeiten des Nährstoffstatus und der Nährstoffbilanzen von drei Buchenaltbeständen mit unterschiedlichen Standorteigenschaften werden vorgestellt. Die Böden der Bestände Göttinger Wald, Zierenberg und Solling differieren deutlich bezüglich ihres Säure-Base-Status. Der Boden des Bestandes Göttinger Wald ist aus kalkhaltigem Substrat entstanden und befindet sich bis in den Oberboden im Karbonat-Pufferbereich, während am Standort Zierenberg mit Böden aus Basaltverwitterungsschutt im Oberboden bereits eine Versauerung eingesetzt hat. Auf dem Buntsandstein im Solling ist der gesamte durchwurzelte Boden aus Lösslehm dem Aluminium-Pufferbereich zuzuordnen. Dort sind in einer mächtigen Humusauflage erhebliche Nährstoffmengen gespeichert.

Die atmogenen Säureeinträge unterscheiden sich an den drei Standorten nur wenig und sind während der vergangenen Jahrzehnte deutlich reduziert worden. 
Die Stickstoffeinträge haben dagegen nur leicht abgenommen und liegen deutlich über dem Bedarf der Bestände für den Biomassezuwachs. Im Solling waren nur sehr geringe Stickstoffausträge zu verzeichnen. Im Göttinger Wald und insbesondere in Zierenberg traten im Beobachtungszeitraum erhebliche Stickstoffausträge auf, die in Zierenberg einen steigenden Trend aufwiesen und in einer im Mittel negativen Stickstoffbilanz resultierten. Im Solling wurde seit 1990 eine negative Calcium-Bilanz beobachtet. Dies unterstreicht für den Buntsandsteinstandort die Bedeutung der Bodenschutzkalkung und rät zur Vorsicht bei der Nutzung nährstoffreichen Schwachholzes, um übermäßige Nährstoffentzüge zu vermeiden.

Stichworte: Buche, Fagus sylvatica, Stickstoffsättigung, Versauerung, Stoffbilanzen, Bodenveränderung

\begin{abstract}
Differences and similarities in the nutrient status and nutrient budget of three mature beech ecosystems with different site conditions are presented. At the sites, Göttinger Wald, Zierenberg and Solling, the soils differ substantially with respect to their acid/base status. Soils at the Göttinger Wald site have developed from limestone, and the whole profile is at the carbonate buffer range, whereas the soils derived from weathered basalt debris at the Zierenberg site show obvious early signs of acidification in the top layers. At the Solling site the soil in the rooting zone of the new red sandstone is at the aluminium buffer range. At this site a considerable proportion of nutrients is stored in the top organic layer.

The atmospheric deposition of acids is very similar at the three sites, and has been reduced substantially during the last decades. Yet the deposition of nitrogen decreased only slightly, and is much higher than that required for growth. Nitrogen seepage at the Solling site was very low. Nitrogen losses at the Göttinger Wald, and particularly the Zierenberg site are much higher, with an increasing trend during the observation period, resulting in a negative average nitrogen budget at Zierenberg site. At the Solling site, a negative calcium budget has been observed since 1990. This highlights the importance of liming on new red sandstone sites, and the need for cautious utilisation of the nutrient-rich smallwood to avoid unnecessary nutrient withdrawal from these sites.
\end{abstract}

Keywords: beech, Fagus sylvatica, nitrogen saturation, acidification, element budgets, soil changes 


\section{Einleitung}

Unter natürlichen Bedingungen ist die Buche in Mitteleuropa auf einer weiten Amplitude von Standortseigenschaften die konkurrenzstärkste Baumart (LEUSCHNER et al. 2006). Ihre ökologische Spannweite wird auch in bewirtschafteten Beständen weitgehend ausgenutzt. Die Stoffkreisläufe in Buchenwäldern und anderen Waldökosystemen in Mitteleuropa wurden in den letzten Dekaden durch anthropogene Stoffeinträge weitgehend verändert. Während dabei in den $1960 \mathrm{er}$ bis 1990er Jahren die Einträge von Säuren wie Schwefelsäure und Salpetersäure eine besondere Rolle spielten, ist seit etwa den 1980er Jahren die Deposition eutrophierenden Stickstoffs $(\mathrm{N})$ ein eigenständiges Problem. Atmogene N-Einträge werden als wesentliche Triebkraft für ein beschleunigtes Waldwachstum und zunehmende Kohlenstoffsequestrierung, aber auch für Nährstoffimbalanzen, erhöhte Nitratausträge und veränderte Pflanzendiversität angesehen (DISE u. Wright 1995, KAHLE et al. 2005, DE VRIES et al. 2006, SEIDLING u. FISCHER 2008). Diese unter dem Begriff Stickstoffsättigung zusammengefassten Phänomene werden in unterschiedlichen Waldökosystemen der nördlichen Hemisphäre beobachtet (ABER et al. 1989, 1998, BRUMME u. KHANNA 2008). Zukünftig und in Ansätzen schon jetzt zu erkennen werden sich aufgrund des globalen Klimawandels vermutlich Veränderungen des Wasser- und Stoffhaushalts von Buchenwäldern ergeben.

\section{Material und Methoden}

\subsection{Untersuchte Waldökosysteme}

Bei den hier vorgestellten drei Fallstudien Göttinger Wald, Zierenberg und Solling handelt es sich um mehr oder weniger gleichaltrige und reine Buchenaltbestände. Sie befinden sich im südniedersächsischen (Göttinger Wald, Solling) bzw. nordhessischen (Zierenberg) Bergland und repräsentieren typische Buchenwälder auf unterschiedlichem Ausgangssubstrat (s. Tab. 1). Die Untersuchungsflächen unterliegen relativ ähnlichen klimatischen Bedingungen, unterscheiden sich aber beträchtlich bezüglich des Säure-Base-Status der Böden und der Zusammensetzung der Bodenvegetation. Sie sind als Monitoringstandorte in das europäische Level II-Programm des ICP Forests (HAUSSMANN u. LUX 1997) und das niedersächsische Boden-Dauerbeobachtungsprogramm (Göttinger Wald und Solling, MEESENBURG et al. 1997) bzw. die Waldökosystemstudie Hessen (Zierenberg, EICHHORN 1992) eingebunden.

Die Monitoringfläche Göttinger Wald wurde 1980 eingerichtet und liegt nahe dem Trauf der Göttinger Wald-Schichtstufe (MEIWES u. BEESE 1988). Der nahezu reine Buchenbestand enthält eine geringe Beimischung von Esche, Ahorn, Eiche und Ulme. Die dichte und artenreiche Bodenvegetation wird durch Waldmeister, 
Bärlauch und Bingelkraut geprägt. Die Böden sind flachgründig mit Entwicklungstiefen von 20-50 cm und enthalten bis nahe an die Oberfläche Karbonat.

Die Fallstudie Zierenberg wird seit 1989 untersucht und befindet sich $14 \mathrm{~km}$ nordwestlich von Kassel am nordöstlichen Hang des Kleinen Gudenberg, welcher als basaltische Kuppe eine Muschelkalkfläche des nordhessischen Berglands überragt (EICHHORN 1995). Auf der Hauptuntersuchungsfläche bildet basaltischer Schutt (Limburgit) von ungefähr $90 \mathrm{~cm}$ Mächtigkeit das Substrat für die Bodenentwicklung und bedeckt den unterliegenden Muschelkalk. Der Waldbestand besteht fast ausschließlich aus Buche. Die Bodenvegetation wird von Bingelkraut und Brennessel dominiert. Letztere erreicht Bestandeshöhen von mehr als $1 \mathrm{~m}$.

Tabelle 1: $\quad$ Beschreibung der untersuchten Buchenwälder

\begin{tabular}{|c|c|c|c|c|}
\hline & Einheit & Göttinger Wald & Zierenberg & Solling \\
\hline Geogr. Breite & $\left.{ }^{\circ}\right]$ & N513' & $\mathrm{N} 51^{\circ} 22^{\prime}$ & $\mathrm{N} 51^{\circ} 46^{\prime}$ \\
\hline Geogr. Länge & {$\left[{ }^{\circ}\right]$} & $\mathrm{E} 10^{\circ} 03^{\prime}$ & $\mathrm{E} 09^{\circ} 16^{\prime}$ & $\mathrm{E} 09^{\circ} 34^{\prime}$ \\
\hline Höhe & {$[\mathrm{m}]$ ü. NN } & 420 & 450 & 504 \\
\hline Neigung & {$\left[{ }^{\circ}\right]$} & $0-3$ & 15 & $0-3$ \\
\hline Exposition & & west & nordost & süd \\
\hline Bestandesalter & [a] (2008) & $127-140$ & 162 & 170 \\
\hline Bestandesdichte & [Bäume ha-1] & 252 & 132 & 212 \\
\hline Mittl. BHD & {$[\mathrm{cm}]$} & 45,0 & 55 & 46,1 \\
\hline Mittl. Höhe & {$[\mathrm{m}]$} & 33,6 & 36,1 & 29,6 \\
\hline Derbholzvolumen & {$\left[\mathrm{m}^{3} \mathrm{ha}^{-1}\right]$} & 680 & 533 & 529 \\
\hline $\begin{array}{l}\text { Natürl. Waldgesell- } \\
\text { schaft }\end{array}$ & & $\begin{array}{l}\text { Hordelymo- } \\
\text { Fagetum }\end{array}$ & $\begin{array}{l}\text { Hordelymo- } \\
\text { Fagetum }\end{array}$ & Luzulo-Fagetum \\
\hline Ausgangsgestein & & Kalkstein & $\begin{array}{l}\text { Basalt über } \\
\text { Kalkstein }\end{array}$ & $\begin{array}{l}\text { Sandstein mit } \\
\text { Lössauflage }\end{array}$ \\
\hline Bodentyp & & $\begin{array}{l}\text { Rendzina / Terra } \\
\text { fusca }\end{array}$ & Braunerde & $\begin{array}{l}\text { podsolige } \\
\text { Braunerde }\end{array}$ \\
\hline Bodentyp FAO & & $\begin{array}{l}\text { Rendzic Lepto- } \\
\text { sol/Calcaric } \\
\text { Cambisol }\end{array}$ & $\begin{array}{l}\text { Eutric } \\
\text { Cambisol }\end{array}$ & Dystric Cambisol \\
\hline Humusform & & F-Mull & F-Mull & typischer Moder \\
\hline Niederschlag ${ }^{1}$ & {$[\mathrm{~mm}]$} & 709 & 754 & 1193 \\
\hline $\begin{array}{l}\text { Niederschlag } \\
(\text { Mai - Okt. })^{1}\end{array}$ & {$[\mathrm{~mm}]$} & 410 & 406 & 553 \\
\hline Mittl. Temperatur ${ }^{1}$ & {$\left[{ }^{\circ} \mathrm{C}\right]$} & 9,3 & 7,5 & 7,6 \\
\hline $\begin{array}{l}\text { Mittl. Temperatur } \\
(\text { Mai - Okt. })^{1}\end{array}$ & {$\left[{ }^{\circ} \mathrm{C}\right]$} & 14,5 & 12,9 & 12,8 \\
\hline
\end{tabular}

1Periode 1990 - 2002 
Die Daueruntersuchungsfläche Solling befindet sich im Zentrum des SollingPlateaus. Die Hochfläche wird vom Mittleren Buntsandstein gebildet, der von einer Lösslehmauflage von etwa 60-80 cm überdeckt wird. Der Waldbestand wird ausschließlich von Buche gebildet, die aus Naturverjüngung hervorgegangen ist (ELLENBERG et al. 1986). Die spärliche Bodenvegetation wird von wenigen Arten gebildet, von denen Sauerklee und Waldhainsimse die höchsten Deckungsgrade erreichen.

\subsection{Untersuchungsprogramm}

Die Untersuchungsprogramme der drei Fallstudien sind, bedingt durch verschiedene Laufzeiten und organisatorische Einbindungen, unterschiedlich (s. Tab. 2). Die Methodik ist aber durch die Orientierung an Untersuchungsvorschriften des ICP Forests (UN/ECE 2004) bzw. Boden-Dauerbeobachtungsprogramms (BARTH et al. 2002) und verschiedener Qualitätssicherungsschritte bezüglich der hier behandelten Stoffhaushaltskenngrößen vergleichbar.

Tabelle 2: $\quad$ Zusammenstellung der auf den Monitoringflächen durchgefübrten Untersuchungen und deren räumlicher und zeitlicher Auflösung

\begin{tabular}{|c|c|c|}
\hline Untersuchung & $\begin{array}{l}\text { räumliche } \\
\text { Auflösung }\end{array}$ & $\begin{array}{l}\text { zeitliche } \\
\text { Auflösung }\end{array}$ \\
\hline Stoffflussmessungen & & \\
\hline Freilandniederschlag & $3-6^{*}$ & fortlaufend \\
\hline Kronentraufe & $15-20 *$ & fortlaufend \\
\hline Streufall & $12-20^{*}$ & fortlaufend \\
\hline Bodenlösung (3-5 Tiefenstufen) & $3-12 *$ & fortlaufend \\
\hline $\begin{array}{l}\text { Meteorologische Messungen: Lufttemperatur, Bodentemperatur, } \\
\text { Feuchte, Windgeschwindigkeit, Niederschlag, Globalsstrahlung, } \\
\text { Gaskonzentrationen }\left(\mathrm{SO}_{2}, \mathrm{NO}_{2}, \mathrm{NH}_{3}, \mathrm{O}_{3}\right)\end{array}$ & - & fortlaufend \\
\hline Bodenhydrologische Messungen: Matrixpotenzial, Wassergehalt & $3-5^{*}$ & fortlaufend \\
\hline Chemische Bodeninventur & & \\
\hline Nährelemente & $8-24 *$ & $10 \mathrm{a}$ \\
\hline Schwermetalle & $8-24 *$ & $10 \mathrm{a}$ \\
\hline Zuwachsmessungen & $\begin{array}{l}\text { alle Bäume } \\
\text { auf } 0,25 \text { ha }\end{array}$ & $1-5 \mathrm{a}$ \\
\hline Kronenansprache & $\begin{array}{l}\text { Min. } 50 \\
\text { Bäume }\end{array}$ & $1 \mathrm{a}$ \\
\hline Vegetationsaufnahme & $4 \times 100 \mathrm{~m}^{2}$ & $1 \mathrm{a}$ \\
\hline Blatt-Elementgehalte & 6-9 Bäume & $1 \mathrm{a}$ \\
\hline
\end{tabular}

*Proben/Monitoringfläche 
Dargestellt werden hier Ergebnisse von chemischen Bodeninventuren sowie von Stoffflüssen mit den Teilprozessen atmogene Deposition, Sickerwasseraustrag, Freisetzung von Elementen über die Mineralverwitterung sowie Festlegung von Elementen in der Holzbiomasse.

Die atmogene Deposition wurde mittels ständig offener Sammelgefäße auf Freiflächen und unterhalb des Kronendaches kontinuierlich erfasst (MEESENBURG et al. 1997). Zusätzlich wurde der Stammablauf über Manschetten in Sammelgefäßen aufgefangen. Gesamtdepositionsraten wurden über ein Kronenraumbilanzmodell (ULRICH 1994) unter Berücksichtigung von trocken deponierten Stoffen sowie Austauschprozessen im Kronenraum geschätzt.

Die Bodenlösung wurde mittels Saugkerzen bzw. -platten in mehrfacher Wiederholung und mehreren Tiefenstufen gewonnen. Gehalte von Stoffen unterhalb des Hauptwurzelraumes (90-100 cm Bodentiefe) wurden als charakteristisch für den Sickerwasseraustrag angesehen. Die entsprechenden Wasserflüsse wurden mit einem Wasserhaushaltsmodell berechnet (HÖRMANN 1997).

Elementfreisetzungsraten durch die Mineralverwitterung wurden statisch mit dem Modell PROFILE (SVERDRuP u. WARFVINGE 1993) unter Verwendung von gemessenen Mineralgehalten der jeweiligen Böden geschätzt. Ebenso wurde die Elementfestlegung in der Holzbiomasse statisch mittels mittlerer Elementkonzentrationen in verschiedenen Baumkompartimenten und mittleren Zuwachsraten geschätzt (RADEMACHER et al. 2008).

\section{Bodenzustand und -veränderungen}

\subsection{Der Bodenzustand der Monitoringflächen im Vergleich}

Die Böden der Monitoringflächen wurden aus unterschiedlichen Ausgangssubstraten gebildet und decken einen Großteil der in Mitteleuropa in Waldböden vorkommenden Pufferbereiche ab. Im Göttinger Wald haben sich die Böden aus Kalkstein entwickelt, der als nahezu reiner Calcit kaum Magnesium $(\mathrm{Mg})$ enthält. Der Verwitterungsrückstand auf der Monitoringfläche Göttinger Wald ist sehr ton$(58 \%)$ und schluffhaltig $(41 \%)$ und besteht im Wesentlichen aus Quarz $(31 \%)$, Illit (20\%), Montmorillonit (15\%), Glimmern (14\%) und Feldspäten (10\%). Dem hohen Calciumcarbonat-Gehalt entsprechend ist der $\mathrm{pH}$-Wert bis in den Oberboden $(0-10 \mathrm{~cm}: 5,7-6,9)$ relativ hoch und steigt mit zunehmender Bodentiefe an (s. Abb. 1); er liegt damit im Carbonat-Pufferbereich.

In Zierenberg sind die Böden aufgrund des basaltischen Ausgangsgesteins ebenfalls basenreich, aber bis in den Unterboden karbonatfrei. Die Verwitterungsdecke ist außer durch den Basalt auch durch Lössablagerungen geprägt. Letzteres bedingt einen hohen Schluffanteil $(70-80 \%)$ und hohe Quarz- sowie Illitgehalte. 
Hohe Anteile an schnell verwitterbaren Mineralen wie Pyroxen, Hornblende, Olivin und Smektit stammen aus dem Basalt. Die pH-Werte liegen im Oberboden etwas niedriger als im Göttinger Wald und indizieren eine beginnende Versauerung. Der Boden ist dem Silikat-Pufferbereich zuzuordnen.

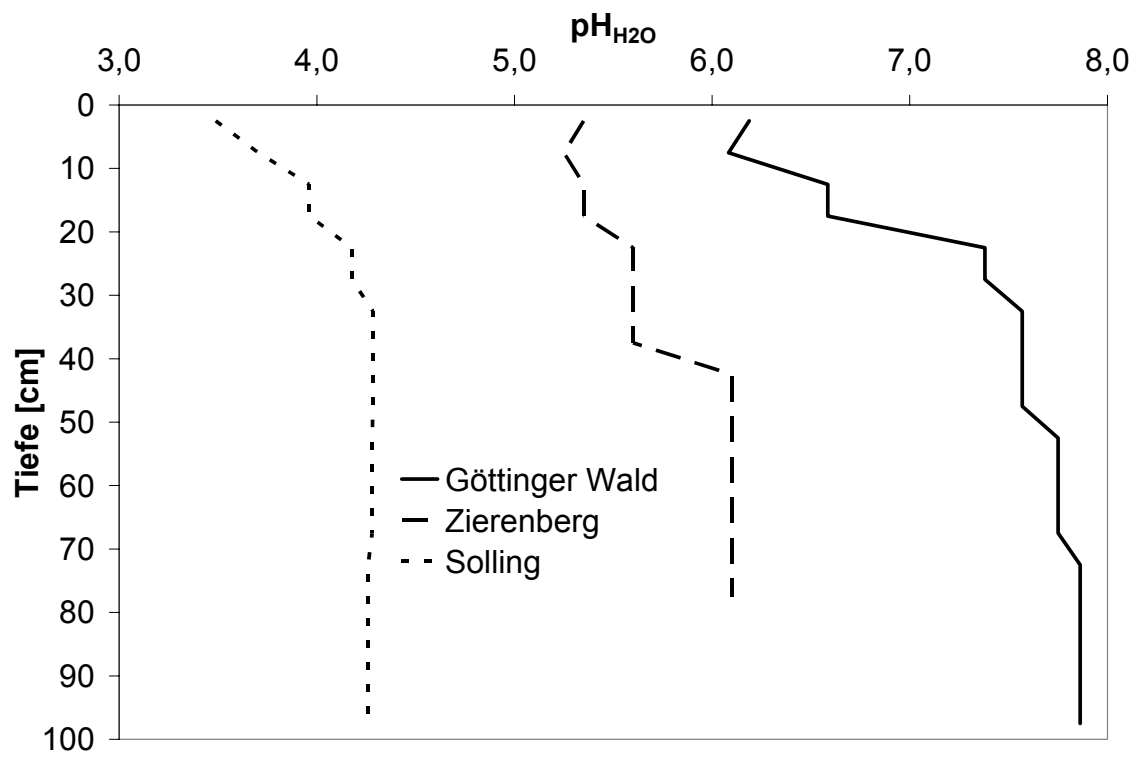

Abbildung 1: Tiefenfunktionen des mittleren $\mathrm{pH}_{\mathrm{H} 2 \mathrm{O}}$-Wertes im Mineralboden der Monitoringflächen

Der Standort Solling ist repräsentativ für Böden im Aluminium-Pufferbereich mit geringer Basensättigung und hohen Gehalten an austauschbarem Aluminium (Al) und Protonen. Die $\mathrm{pH}_{\mathrm{H} 2 \mathrm{O}}$-Werte liegen im Oberboden um 3,4 und steigen im Unterboden auf Werte um 4,3 an. Die Böden sind aus Buntsandsteinverwitterungsmaterial entstanden, welches von einer $60-80 \mathrm{~cm}$ mächtigen Lösslehmauflage überlagert wird. Die Mineralzusammensetzung wird durch Quarz (47-74\%), Feldspäte (10-16\%) und Illite (8-28 \%) geprägt.

Aufgrund der verschiedenen Ausgangssubstrate sind die Nährstoffvorräte auf den Untersuchungsstandorten unterschiedlich verteilt. Im Mineralboden sind die austauschbaren Ca-Vorräte im Göttinger Wald sehr hoch, in Zierenberg hoch und im Solling sehr gering (s. Tab. 3). Die Mg-Vorräte sind in Zierenberg sehr hoch, im Göttinger Wald mittel und im Solling gering. Hinsichtlich Phosphor sind die Standorte Solling und Göttinger Wald mittelmäßig und Zierenberg sehr gut ausgestattet. Die Vorräte an austauschbarem Kalium (K) sind im Göttinger Wald und Zierenberg doppelt so hoch wie im Solling. Die in der organischen Auflage akkumulierten Nährstoffvorräte sind dagegen im Solling aufgrund der Mächtigkeit der Humusauflage hoch. Für Mg und Ca liegen die in der organischen Auflage gespeicherten Vorräte in der gleichen Größenordnung wie die Vorräte austauschbarer 
Kationen im Mineralboden. Im Göttinger Wald und in Zierenberg sind kaum Nährstoffe in der organischen Auflage gespeichert, da die Streu durch die hohe biologische Aktivität schnell umgesetzt und in den Mineralboden inkorporiert wird.

Tabelle 3: $\quad$ Nährstoffvorräte in der organischen Auflage und im Mineralboden $(0-100 \mathrm{~cm})$

\begin{tabular}{|c|c|c|c|}
\hline & $\begin{array}{c}\text { Göttinger Wald } \\
\mathrm{kg} \mathrm{ha} \mathrm{h}^{-1}\end{array}$ & $\begin{array}{c}\text { Zierenberg } \\
\mathrm{kg} \mathrm{ha}^{-1}\end{array}$ & $\begin{array}{l}\text { Solling } \\
\mathrm{kg} \mathrm{ha}^{-1}\end{array}$ \\
\hline $\mathrm{KO}_{\mathrm{lfh}}$ & 71 & 15 & 287 \\
\hline $\mathrm{K}^{*} 0-100 \mathrm{~cm}$ & 1625 & 712 & 545 \\
\hline $\mathrm{KO}_{\mathrm{lfh}}+0-100 \mathrm{~cm}$ & 1696 & 727 & 832 \\
\hline $\mathrm{Mg} \mathrm{O}_{\mathrm{lfh}}$ & 36 & 32 & 89 \\
\hline $\mathrm{Mg}^{*} 0-100 \mathrm{~cm}$ & 507 & 4944 & 91 \\
\hline $\mathrm{Mg} \mathrm{O}_{\text {lfh }}+0-100 \mathrm{~cm}$ & 543 & 4976 & 180 \\
\hline $\mathrm{Ca} \mathrm{O}_{\text {lfh }}$ & 369 & 161 & 210 \\
\hline $\mathrm{Ca}^{*} 0-100 \mathrm{~cm}$ & 57908 & 13469 & 219 \\
\hline $\mathrm{Ca} \mathrm{O}_{\mathrm{lfh}}+0-100 \mathrm{~cm}$ & 58277 & 13630 & 429 \\
\hline $\mathrm{PO}_{\mathrm{lfh}}$ & 10 & 13 & 94 \\
\hline P 0-100 cm & 3886 & 10131 & 3774 \\
\hline $\mathrm{P} \mathrm{O}_{\mathrm{lfh}}+0-100 \mathrm{~cm}$ & 3896 & 10144 & 3868 \\
\hline $\mathrm{N}_{\mathrm{ges}} \mathrm{O}_{\mathrm{lfh}}$ & 200 & 223 & 1890 \\
\hline $\mathrm{N}_{\text {ges }} 0-100 \mathrm{~cm}$ & 16520 & 10570 & 9930 \\
\hline $\mathrm{N}_{\text {ges }} \mathrm{O}_{\text {lfh }}+0-100 \mathrm{~cm}$ & 16720 & 10793 & 11820 \\
\hline
\end{tabular}

* Im Mineralboden werden für Zierenberg und Solling das austauschbare $\mathrm{K}, \mathrm{Mg}$ und $\mathrm{Ca}$ im $1 \mathrm{~N}$ $\mathrm{NH}_{4} \mathrm{Cl}$-Perkolat, für den Göttinger Wald im gepufferten 0,2M-BaCl 2 -Perkolat aufgeführt.

\subsection{Veränderungen der Bodenvorräte auf der Monitoringfläche im Solling}

Die Buchen-Monitoringfläche im Solling wird seit 1966 beobachtet. Während dieser Zeit wurden wiederholt Inventuren der Nährstoffvorräte in der organischen Auflage und im Mineralboden durchgeführt (MEESENBURG et al. 1999, MEIWES et al. 2002). Mittels einfacher linearer Regression wurden Veränderungen der Bodenvorräte von $\mathrm{C}, \mathrm{N}, \mathrm{P}$ und $\mathrm{Ca}$ untersucht.

Der Humusvorrat in der organischen Auflage hat im Zeitraum 1966 - 2001 um durchschnittlich $630 \mathrm{~kg} \mathrm{ha}^{-1} \mathrm{a}^{-1}$ zugenommen (MEIWES et al. 2002). Ein ähnlicher Trend ist für einen benachbarten Fichtenbestand festzustellen. Dabei hat sich die Qualität, d. h. die chemische Zusammensetzung des Humus mit Ausnahme des $\mathrm{C} / \mathrm{P}-\mathrm{Verhältnisses,}$ welches angestiegen ist, kaum verändert. Dies bedeutet wiederum, dass in diesem Zeitraum eine Akkumulation von Auflagehumus stattgefunden 
hat. Für $\mathrm{N}$ und $\mathrm{Ca}$ ergeben sich dabei Akkumulationsraten von $21 \mathrm{bzw}$. 2,5 $\mathrm{kg} \mathrm{ha}^{-1} \mathrm{a}^{-1}$. Nach COVINGTON (1981) sowie BÖTTCHER u. SPRINGOB (2001) ist eine Auflagehumusakkumulation mit zunehmendem Bestandesalter ein natürlicher Vorgang in bewirtschafteten Wäldern. Unterstellt man jedoch für den Buchenbestand im Solling ein vollständiges Fehlen der Humusauflage bei Bestandesbegründung und eine lineare Zunahme des Auflagehumusvorrats bis zum Beginn der Beobachtung 1966, so liegen die Akkumulationsraten im Zeitraum 1966 - 2001 deutlich höher. Daraus lässt sich entweder auf eine Zunahme der Streufallraten oder auf eine Reduktion der Streuabbauraten schließen. Aus der Analyse der gemessenen Streufallmengen ergeben sich weder für den Buchen- noch für den benachbarten Fichtenbestand Anhaltspunkte für eine erhöhte Streuproduktion (KHANNA et al. 2008).

Als Ursache für eine Reduktion der Streumineralisierung kommen ein verändertes Bestandesinnenklima, der hohe Versauerungsgrad der Humusauflage oder erhöhte Schwermetall- bzw. N-Gehalte in Frage (MEIWES et al. 2002). Da sich die Streufallmengen nicht verändert haben, ist davon auszugehen, dass sich der das Bestandesklima beeinflussende Kronenschlussgrad ebenfalls nicht verändert hat. Ein Anstieg der Lufttemperatur und der Niederschlagsmenge würden sich eher positiv auf die Streuzersetzung auswirken.

Eine starke Versauerung der Humusauflage kann zu einem verminderten Streuabbau beitragen. So wurde beispielsweise eine verminderte $\mathrm{CO}_{2}$-Atmung in versauerten Humusauflagen verschiedener Standorte und Baumarten festgestellt (MEIWES et al. 2002).

Ebenfalls können erhöhte Schwermetallgehalte zu einer Hemmung der Mineralisation beitragen. Die Gehalte an Blei und Kupfer liegen in der Humusauflage im Solling teilweise im Bereich oder über den von TYLER (1992) angegebenen Grenzwerten für eine Hemmung der Streuzersetzung. Allerdings haben die Einträge von Blei und Kupfer mit dem Streufall und dem Bestandesniederschlag im Solling seit den 1970er Jahren deutlich abgenommen.

Nach BERG u. MATZNER (1997) führen hohe N-Gehalte der Streu zu einem raschen Abbau der Zellulosebestandteile, während der Ligninabbau eher gehemmt wird. Dies führt dazu, dass ein hoher Anteil schwer abbaubarer Verbindungen im Humus angereichert wird. Im Solling liegen die N-Gehalte der Streu in einem Bereich, der nach BERG (2000) einen hohen Anteil schwer abbaubarer Verbindungen erwarten lässt.

Welche der genannten Hypothesen, hoher Versauerungsgrad, erhöhte Schwermetall- oder N-Gehalte, maßgeblich zu einem reduzierten Streuabbau beitragen, kann mit den zur Verfügung stehenden Informationen nicht geklärt werden. Vermutlich spielen alle Faktoren eine Rolle. Da sich die Gehalte der Makroelemente im Beobachtungszeitraum nicht signifikant verändert haben, ist anzunehmen, dass schon zu Beginn der Beobachtungen der Streuabbau gehemmt war. Die Humus- 
akkumulation hat damit auch zu einer Akkumulation von wichtigen Nährstoffen wie N, P und Ca in der organischen Auflage geführt.

Im Mineralboden der Monitoringfläche im Solling wurde dagegen eine Abnahme der Vorräte basischer Kationen beobachtet (MEESENBURG et al. 1999). Insbesondere für $\mathrm{Ca}$ wurde ein deutlicher Rückgang festgestellt. Zurückgeführt werden kann dies auf den Export im Zusammenhang mit dem Transport mobiler Anionen $\left(\mathrm{SO}_{4}, \mathrm{NO}_{3}, \mathrm{Cl}\right)$.

Im Ergebnis bedeutet die Akkumulation von Nährstoffkationen im Auflagehumus und die Verarmung am Austauschkomplex im Mineralboden eine Umverteilung innerhalb des Bodens. Dabei ist die Speicherung von Nährstoffen im Auflagehumus als eine weniger stabile Form anzusehen, da Störungen des Waldökosystems (Windwurf, Schädlingskalamitäten, Endnutzung) zu einem raschen Abbau des Auflagehumus führen und die darin gespeicherten Nährstoffe dem Ökosystem verloren gehen können.

\section{Stoffhaushalt}

Der Haushalt vieler quantitativ bedeutsamer Nähr- und Schadstoffe in Waldökosystemen wird einerseits durch Einträge aus der Atmosphäre und die Freisetzung durch die Mineralverwitterung, andererseits durch Austräge mit dem Sickerwasser und über die Biomassenutzung geprägt (UlRICH 1994). Für einige Stoffe sind jedoch auch andere Pfade von Bedeutung. Beispielsweise werden N-Komponenten in gasförmiger Form an die Atmosphäre abgegeben (z. B. $\mathrm{N}_{2} \mathrm{O}, \mathrm{N}_{2}, \mathrm{NO}$ ).

Durch eine Bilanzierung der Stoffeinträge und -austräge können Aussagen zur Stabilität von Waldökosystemen getroffen werden. Reichern sich Schadstoffe an oder gehen Nährstoffvorräte zurück, ist zumindest langfristig mit einer Destabilisierung zu rechnen. Ziel der Forstwirtschaft muss es sein, nach dem Prinzip der Nachhaltigkeit die Bewirtschaftung so zu gestalten, dass sich langfristig ausgeglichene Stoffbilanzen einstellen.

\subsection{Stoffeinträge}

Die seit dem letzten Jahrhundert anthropogen verursachte Emission von Säuren und N-Verbindungen in die Atmosphäre hat die globalen Stoffkreisläufe stark verändert. Insbesondere für Wälder, die aufgrund ihrer großen äußeren Oberfläche eine bedeutende Senke für atmosphärische Inhaltsstoffe darstellen und die meist nicht gedüngt werden, stellen atmogene Stoffeinträge eine dominante Komponente ihrer Stoffkreisläufe dar. In weiten Bereichen der nördlichen Hemisphäre sind infolge von Säure- und N-Einträgen Böden versauert und Waldökosysteme mit $\mathrm{N}$ gesättigt worden. 
Nachdem seit Beginn der Industrialisierung und insbesondere seit den 1950er Jahren die Deposition von Luftverunreinigungen in Wälder stark angestiegen waren, sind seit Mitte der 1970er Jahre aufgrund von Luftreinhaltemaßnahmen die Schwefel- und Säuredepositionen in Mitteleuropa und so auch auf den Monitoringflächen deutlich zurückgegangen (s. Abb. 2, MEESENBURG et al. 1995, BALÁZS 1998, GAUGER et al. 2002). Die N-Einträge nahmen in diesem Zeitraum nur leicht ab oder blieben konstant (s. Abb. 3).

Tabelle 4: $\quad$ Mittlere Stoffeinträge mit dem Freilandniederschlag (OF) und Gesamtdeposition (TD, berechnet nach ULRICH 1994) auf den Monitoringflächen im Zeitraum 1990 - 2002 (SL = Solling, $G W=$ Göttinger $W$ ald, $Z B=$ Zierenberg; Datenquelle: Nordwestdeutsche Forstliche Versuchsanstalt)

\begin{tabular}{c|c|c|cccccccccc|cc}
\hline & Fluss & $\begin{array}{c}\mathrm{H}_{2} \mathrm{O} \\
{[\mathrm{mm}]}\end{array}$ & $\mathrm{Na}^{+}$ & $\mathrm{K}^{+}$ & $\mathrm{Mg}^{2+}$ & $\mathrm{Ca}^{2+}$ & $\begin{array}{c}\mathrm{H}^{+} \\
{\left[\mathrm{mmol}_{\mathrm{c}} \mathrm{m}^{-2} \mathrm{a}^{-1}\right]}\end{array}$ & $\mathrm{SO}_{4}^{2-}$ & $\mathrm{Cl}^{-2}$ & $\mathrm{NH}_{4}^{+}$ & $\mathrm{NO}_{3}$ & & $\begin{array}{c}\mathrm{N}_{\text {tot }} \\
{\left[\mathrm{mmol} \mathrm{m}^{-2} \mathrm{a}^{-1}\right]}\end{array}$ \\
\hline $\mathrm{SL}$ & OF & 1184 & 40 & 5 & 7 & 16 & 17 & 0.5 & 59 & 33 & 63 & 51 & 126 & 12 \\
$\mathrm{SL}$ & TD & 931 & 55 & 8 & 10 & 22 & 55 & 0.8 & 102 & 59 & 94 & 73 & 179 & 21 \\
\hline $\mathrm{GW}$ & OF & 684 & 24 & 3 & 7 & 19 & 11 & 0.2 & 48 & 20 & 47 & 45 & 100 & 8 \\
$\mathrm{GW}$ & TD & 615 & 35 & 5 & 9 & 27 & 37 & 0.4 & 79 & 41 & 75 & 68 & 151 & 13 \\
\hline ZB & OF & 752 & 26 & 7 & 19 & 38 & 18 & 0.2 & 45 & 22 & 39 & 36 & 85 & 9 \\
ZB & TD & 604 & 53 & 14 & 39 & 77 & 61 & 0.4 & 91 & 63 & 81 & 76 & 171 & 19 \\
\hline
\end{tabular}

Die drei Monitoringflächen unterscheiden sich hinsichtlich der Höhe und des zeitlichen Musters der Stoffeinträge nur wenig (s. Tab. 4). Aufgrund der höheren Niederschlagsraten liegen die Stoffeintragsraten im Solling allgemein etwas höher als auf den beiden anderen Untersuchungsflächen. Die Einträge basischer Kationen sind in Zierenberg, vermutlich aufgrund der höheren Anteile landwirtschaftlich genutzter Flächen in der Umgebung, am höchsten. Gemessen an anderen europäischen Buchenwäldern sind die N-Einträge auf einem überdurchschnittlichen Niveau (EICHHORN et al. 2001). Im Vergleich zu Fichtenbeständen liegen die Einträge in Buchenwälder allgemein niedriger (BALÁZS 1998, ROTHE et al. 2002). Dies kann auch im Solling beobachtet werden, wo in einem benachbarten Fichtenbestand insbesondere die Säure- und N-Einträge deutlich höher als im Buchenbestand sind (MEESENBurg et al. 1995).

Die Gesamtdepositionsraten (berechnet mit dem Kronenraumbilanzmodell nach UlRICH 1994) sind gegenüber den Einträgen mit dem Freilandniederschlag deutlich erhöht. Der Kronenraum der Waldbestände wirkt als effektive Senke für die partikulären und gasförmigen Luftinhaltsstoffe. Ungefähr $50 \%$ der N-Deposition findet in reduzierter (d. h. als $\mathrm{NH}_{4}$ ) und zwischen 7 und $15 \%$ in organischer Form statt. Die $\mathrm{NH}_{4}$-Deposition trägt damit erheblich zum Eintrag potenzieller 
Säure bei (Göttinger Wald 75-95 \%, Zierenberg 40 75\%, Solling 50-85 \%). Für die $\mathrm{N}$-Komponenten muss davon ausgegangen werden, dass die Berechnung der Gesamtdeposition nach ULRICH (1994) eine konservative Schätzung darstellt und somit die realen Eintragsraten wesentlich höher liegen (GAUGER et al. 2002, MeEsenburg et al. 2005). In einem dem Buchenbestand im Solling benachbarten Fichtenbestand fanden IBROM et al. (1995) mittels mikrometeorologischer Methoden eine gegenüber der Kronenraumbilanzierung um $60 \%$ höhere N-Deposition. Auch bei konservativer Schätzung liegen die N-Einträge weit über dem Bedarf der Waldbestände für den Zuwachs der Holzbiomasse von 10 bis $16 \mathrm{~kg} \mathrm{ha}^{-1} \mathrm{a}^{-1}$ (RADEMACHER et al. 2008).

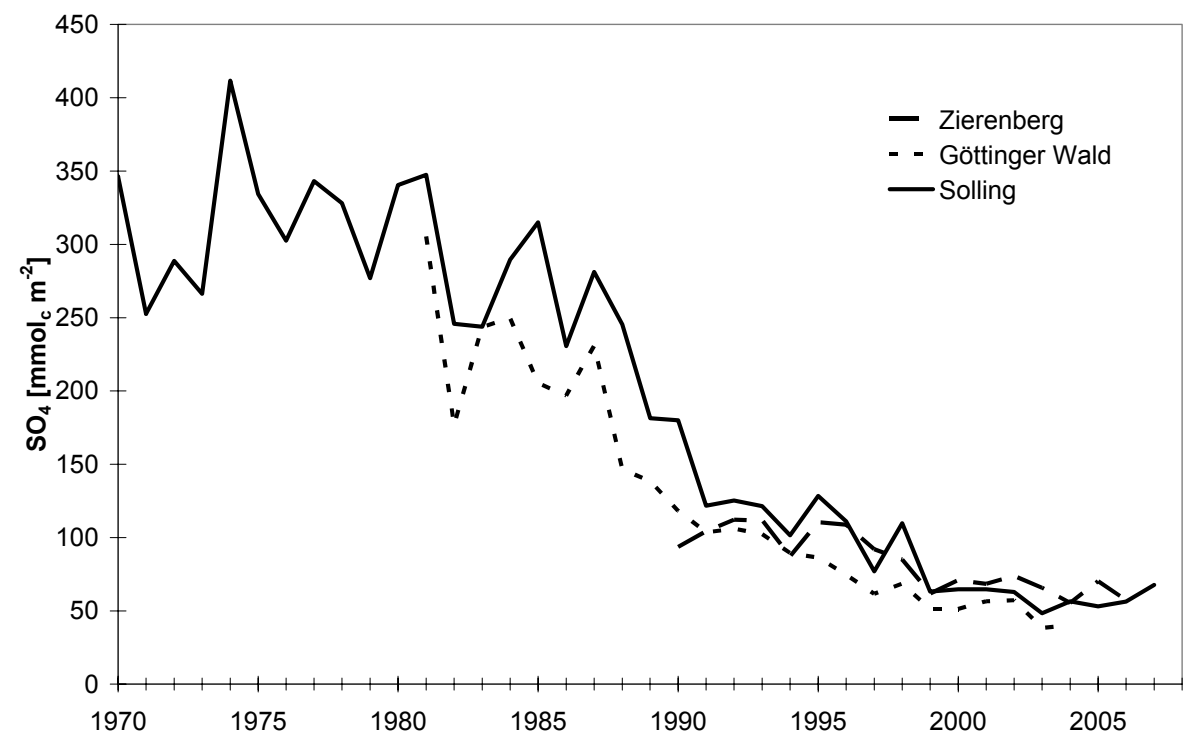

Abbildung 2: Zeitreihen der $\mathrm{SO}_{4}$-Einträge (Gesamtdeposition) auf den Monitoringflächen

Im Zeitraum 1990 bis 2002 gingen auf allen Flächen die Einträge von SO , freier Säure $\left(\mathrm{H}^{+}\right)$und Ca zurück. Dies kann auf verschiedene Maßnahmen zur Luftreinhaltung zurückgeführt werden. Die N-Komponenten zeigten in diesem Zeitraum keinen Trend. In Zierenberg konnte ein Anstieg der Niederschlagsmengen verzeichnet werden.

\subsection{Stoffbilanzen}

Die Bilanzierung von Stoffflüssen stellt ein wichtiges Hilfsmittel zur Beurteilung der Stabilität von Ökosystemen und ein Frühwarnsystem zur Erkennung von Umweltveränderungen dar (ULRICH 1994, MEESENBURG et al. 2002). Für die drei Monitoringflächen wurden für den Zeitraum 1990-2002 Stoffbilanzen nach 
ULRICH (1994) berechnet. Für die Bilanzierung wurden als Input-Flüsse atmogene Stoffeinträge und die Freisetzung aus der Mineralverwitterung, als Output-Flüsse der Austrag mit dem Sickerwasser und die Speicherung in der Biomasse berücksichtigt.

$\mathrm{N}$ wird zu annähernd gleichen Anteilen in reduzierter und oxidierter Form eingetragen, während der Austrag mit dem Sickerwasser fast ausschließlich in oxidierter Form als $\mathrm{NO}_{3}$ stattfindet (s. Abb. 3). Dies bedeutet, dass $\mathrm{NH}_{4}$ entweder aufgenommen oder nitrifiziert wird.

Im Mittel fand auf der Untersuchungsfläche Solling im Zeitraum 1990 - 2002 eine Speicherung von $\mathrm{N}$ statt, da nahezu kein $\mathrm{N}$ mit dem Sickerwasser ausgetragen wurde. Der mittlere N-Bilanzüberschuss von $25 \mathrm{~kg} \mathrm{ha}^{-1} \mathrm{a}^{-1}$ korrespondiert mit der beobachteten N-Vorratszunahme in der Humusauflage (s. Kap. 3.2). Trotz der hohen N-Retention ist von einem weit fortgeschrittenen Zustand der N-Sättigung im Solling auszugehen, wie in einem N-Düngungsexperiment gezeigt wurde (MEESENBURG et al. 2004).

Im Göttinger Wald war die Bilanz nahezu ausgeglichen, d. h. die N-Speicherung im Zuwachs des Waldbestandes und der Sickerwasseraustrag kompensierten die N-Deposition. Bei leicht abnehmenden N-Einträgen traten Anfang der 1980er, sowie Ende der 1980er und 1990er Jahre Phasen erhöhter $\mathrm{NO}_{3}$-Austräge auf.

In Zierenberg war der N-Output im Mittel größer als der N-Input. Dabei kann ein signifikanter Trend eines zunehmenden Bilanzdefizits festgestellt werden, was auf zunehmende $\mathrm{NO}_{3}$-Austräge bei nahezu konstanten $\mathrm{N}$-Einträgen zurückgeführt werden kann. Dies kann nach EICHHORN (1995) als Anzeichen eines Humusvorratsabbaus im Mineralboden (Humusdisintegration nach ULRICH 1981) gedeutet werden. Betroffen hiervon sind insbesondere Standorte mit hoher Basensättigung, die aber durch Versauerungsprozesse den Silikat-Pufferbereich verlassen haben (EICHHORN 2000, BRUMME u. KHANNA 2008).

Für Zierenberg und Göttinger Wald können bei hohen Verwitterungs- und Austragsraten ausgeglichene $\mathrm{Mg}$ - und $\mathrm{Ca}$-Bilanzen unterstellt werden. Im Solling ist im Mittel eine Mg-Akkumulation zu beobachten, für Ca wurden bis ca. 1990 positive und danach negative Bilanzen ausgewiesen. Ein ähnliches Muster ist für $\mathrm{SO}_{4} \mathrm{zu}$ beobachten, was darauf hindeutet, dass der Ca-Austrag durch $\mathrm{SO}_{4}$ als mobilem Anion gesteuert wird. 

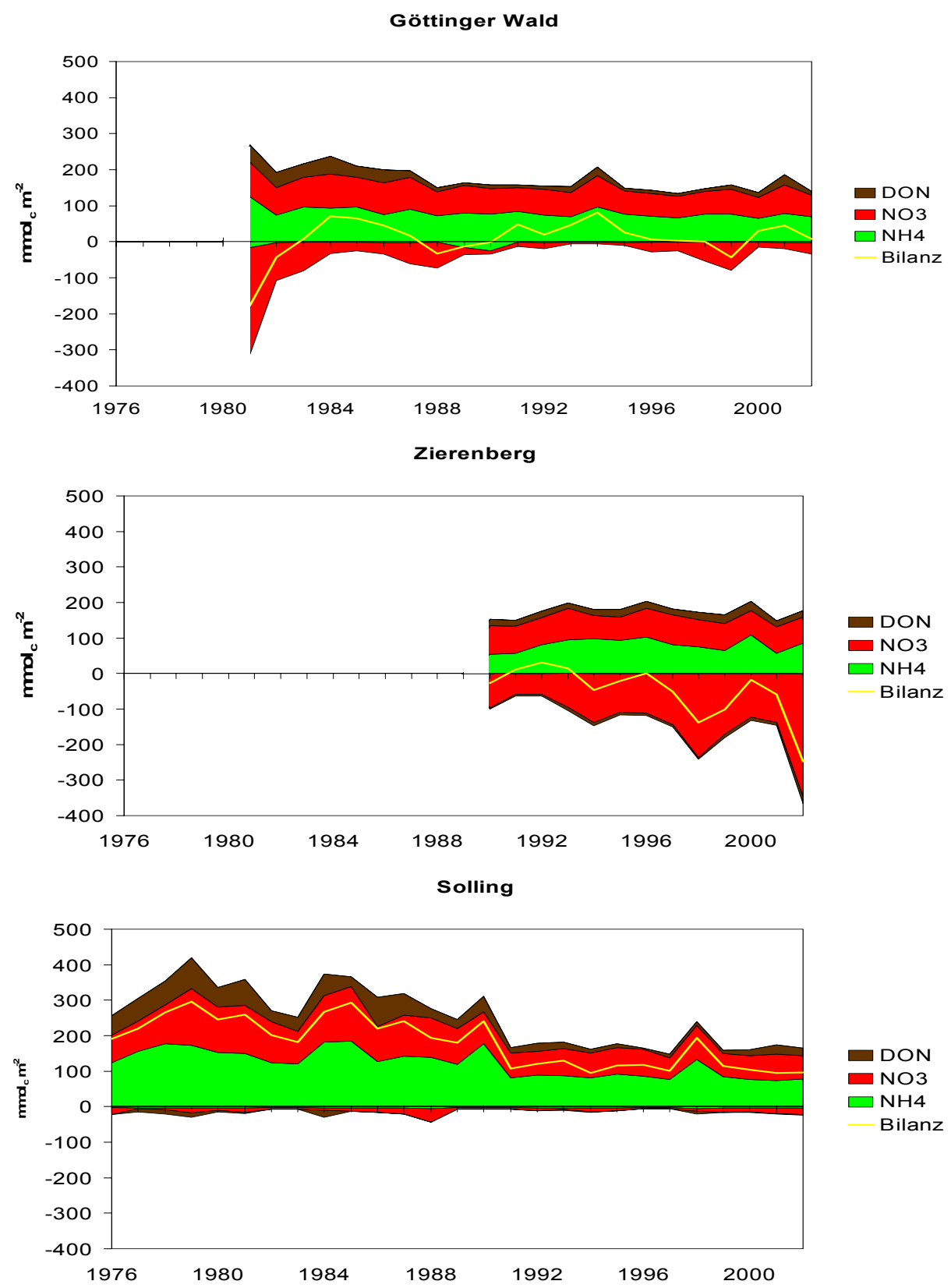

Abbildung 3: Zeitreiben der N-Deposition, N-Sickerwasserausträge und N-Bilanzen auf den Monitoringflächen Göttinger Wald, Zierenberg und Solling (DON = gelöster organischer N) 


\section{Schlussfolgerungen}

Die untersuchten Monitoringflächen Göttinger Wald, Zierenberg und Solling unterschieden sich hinsichtlich des Säure-/Base-Status ihrer Böden deutlich. Dies wird vorwiegend durch die unterschiedlichen Substrate, aus denen sich die Böden entwickelt haben, hervorgerufen. Im Solling wurde im Beobachtungszeitraum (1966 - 2001) anhand einer Abnahme der Basensättigung eine Bodenversauerung durch den atmogenen Säureeintrag festgestellt. In Zierenberg und Göttinger Wald sind nur im Oberboden Versauerungstendenzen zu erkennen, da das basenreiche Ausgangssubstrat bisher eine ausreichende Pufferung gewährleistet.

Die im Solling beobachtete Zunahme der Auflagehumusmächtigkeit steht vermutlich im Zusammenhang mit hohen Säure-, Schwermetall- und N-Einträgen in der Vergangenheit, da hohe Gehalte der genannten Stoffe zu einem verringerten Humusabbau führen können. Die beobachtete mittlere N-Akkumulationsrate von $21 \mathrm{~kg} \mathrm{ha-1} \mathrm{a}^{-1}$ kann ohne entsprechend hohe N-Einträge kaum realisiert werden. In der Folge der Humusakkumulation hat sich die Verteilung der Vorräte der Nährstoffkationen $\mathrm{K}, \mathrm{Mg}$ und $\mathrm{Ca}$ zwischen Humusauflage und Mineralboden verschoben, so dass aktuell in der Humusauflage ungefähr die Hälfte dieser Nährstoffe gespeichert sind. Dies birgt das Risiko entsprechender Nährstoffverluste im Falle eines beschleunigten Humusabbaus.

Wesentliche Quellen der atmogenen Stoffeinträge sind landwirtschaftliche Aktivitäten, Verkehr und industrielle Prozesse. Aufgrund von Luftreinhaltemaßnahmen haben sich die Einträge von Säurebildnern in den vergangenen Jahrzehnten drastisch reduziert. Die N-Einträge zeigen ebenfalls einen rückläufigen Trend, jedoch fallen die Reduktionen deutlich geringer aus. Ungefähr $50 \%$ der aktuellen N-Einträge entfallen auf reduzierten N. Die drei Buchenbestände unterscheiden sich hinsichtlich der Höhe der N-Einträge, des zeitlichen Musters und der Verteilung auf die verschiedenen N-Spezies nur wenig. Derzeit liegt die N-Deposition noch weit über dem Bedarf der Bestände für den Biomassezuwachs.

Bezüglich der N-Bilanzen weisen die drei Buchwaldökosysteme deutliche Unterschiede auf. Während der Buchenbestand im Solling die N-Einträge fast vollständig zurückhält, werden im Göttinger Wald und insbesondere in Zierenberg erhebliche N-Mengen mit dem Sickerwasser ausgetragen. Die über die Stoffflussbilanzierung ermittelten N-Retentionsraten im Solling stehen im Einklang mit der beobachteten N-Akkumulation in der Humusauflage. Damit kann gezeigt werden, dass sich Stoffflussanalysen als sensitiver Indikator für Veränderungen in Waldökosystemen eignen. Negative N-Bilanzen in Zierenberg können als Zeichen eines Humusvorratsabbaus (Humusdisintegration) interpretiert werden. Möglicherweise ist dies charakteristisch für Waldökosysteme, bei denen sich im Zuge der Bodenversauerung die Humusdynamik auf ein neues Gleichgewicht einstellt.

Im Solling wurde seit etwa 1990 eine negative Ca-Bilanz festgestellt. Der Austrag von Kationen wird durch den Transport mobiler Anionen gesteuert. Da 
für die nächsten Jahrzehnte weiterhin mit hohen $\mathrm{SO}_{4}$-Austrägen aufgrund der Remobilisierung von zwischengespeichertem Schwefel zu rechnen ist, werden die CaAusträge und damit das Risiko einer Nährstoffverarmung auch weiterhin hoch bleiben. Die Forstwirtschaft sollte darauf mit einer angepassten Biomasseentnahme beispielsweise bei der Energieholznutzung reagieren (MEIWES et al. 2008).

Die negativen Ca-Bilanzen des Buchenwaldökosystems Solling implizieren, dass innerhalb weniger Jahrzehnte die beobachtete Ca-Vorratsabnahme im Mineralboden nicht rückgängig gemacht werden kann; die Ca-Zufuhr über Verwitterungsprozesse und atmogenen Eintrag reicht dazu nicht aus. Die Bodenschutzkalkung bietet die Möglichkeit, den Ca-Verlust kurzfristig zu kompensieren und die Elastizität des Systems gegenüber Versauerung zu erhöhen (vgl. EvERS et al. in diesem Band).

Die Möglichkeiten der Einflussnahme auf den Stickstoffhaushalt von Buchenwäldern durch forstliche Maßnahmen sind insgesamt begrenzt. Das Risiko erhöhter N-Austräge kann am wirksamsten durch eine Reduktion der atmogenen Einträge verringert werden.

Die drei Buchen-Ökosysteme reagieren in Abhängigkeit vom Ausgangssubstrat der Böden sehr verschieden auf den atmogenen Eintrag versauernder und eutrophierender Stoffe. Die Säurepuffersysteme der Böden bestimmen mit ihren chemischen Reaktionen, Pufferraten und -kapazitäten die Stoffbilanzen. Das System auf Kalkstein reagiert sehr elastisch auf den atmogenen Säureeintrag. Das System auf Basalt reagiert aufgrund der hohen Säureneutralisationskapazität ebenfalls elastisch, es verliert jedoch große Mengen an Stickstoff. Hinsichtlich der Stickstoffvorräte strebt das System vermutlich einem neuen Gleichgewichtszustand auf einem niedrigeren N-Niveau zu. Bei der Pufferung des Säureeintrages in dem System auf Buntsandstein kommt es zur Verarmung basischer Kationen und zur Freisetzung von Aluminium. Um den Verlust an basischen Kationen durch Verwitterung wieder auszugleichen, werden bei verringerter Säuredeposition lange Zeiträume erforderlich sein. Darüber hinaus werden infolge der Auflagehumusakkumulation Nährstoffe festgelegt, die dem aufstockenden Bestand nicht unmittelbar zugänglich sind. Das System auf Buntsandstein besitzt von den drei Buchenwald-Ökosystemen die geringste Elastizität; mit der Bodenschutz- bzw. Kompensationskalkung lässt sich der Säure-Base-Haushalt solcher bodensaurer Buchenwälder auf Buntsandstein wieder stabilisieren. 


\section{Literatur}

Aber, J.D.; Nadelhoffer, K.J.; Steudler, P. u. Melillo, J.M. (1989): Nitrogen saturation in northern forest ecosystems: Hypotheses and implications. Bioscience 39, 378-386

Aber, J.D.; McDowell, W.; Nadelhoffer, K.J.; Magill, A.; Berntson, G.; Kamakea, M.; McNulty, S.; Currie, W.; Rustad, L. u. FernandeZ, I. (1998): Nitrogen saturation in temperate forest ecosystems: Hypotheses revisited. BioScience 48, 921-934

BALÁzs, A. (1998): 14 Jahre Niederschlagsdeposition in Hessischen Waldgebieten: Ergebnisse von den Meßstationen der Waldökosystemstudie Hessen. Hessische Landesanst. Forsteinrichtung, Waldforschung und Waldökologie Forschungsbericht 25, $129 \mathrm{~S}$.

Barth, N.; Brandtner, W.; Cordsen, E.; Dann, T.; Emmerich, K.-H.; Feldhaus, D.; KleEFisch, B.; SCHIlling, B. u. UtermanN, J. (2002): Boden-Dauerbeobachtung: Einrichtung und Betrieb von Boden-Dauerbeobachtungsflächen. In: Rosenkranz, D.; BaCHMann, G.; KÖnIG, W. u. EINSELE G. (Hrsg.): Bodenschutz, 3. Bd. 9152, 1-127

BERG, B. (2000): Litter decomposition and organic matter turnover in northern forest soils. Forest Ecol. Managem. 133, 13-22

Berg, B. u. MATZner, E. (1997): Effect of $\mathrm{N}$ deposition on decomposition of plant litter and soil organic matter in forest systems. Environ. Rev. 5, 1-25

BÖTTCHER, J. u. Springob, G. (2001): A carbon balance model for organic layers of acid forest soils. J. Plant Nutr. Soil Sci. 164, 399-405

Brumme, R. u. KHANNA, P.K. (2008): Ecological and site historical aspects of $\mathrm{N}$ dynamics and current $\mathrm{N}$ status in temperate forests. Global Change Biol. 14, 125-141

Covington, W.W. (1981): Changes in forest floor organic matter and nutrient content following clearcutting in northern hardwoods. Ecology 62, 41-48

De Vries, W.; Reinds, G.; Gundersen, P. u. STERBA, H. (2006): The impact of nitrogen deposition on carbon sequestration in European forests and forest soils. Global Change Biol. 12, 1151-1173

DisE, N.B. u. WRIGHT, R.F. (1995): Nitrogen leaching from European forests in relation to nitrogen deposition. Forest Ecol. Managem. 71, 153-161

EichHORN, J. (Hrsg.) (1992): 10 Jahre Waldökosystemstudie Hessen: Ergebnisse und Perspektiven. Forschungsber. Hessische Forstliche Versuchsanstalt 15

EICHHORN, J. (1995): Stickstoffsättigung und ihre Auswirkungen auf das Buchenwaldökosystem der Fallstudie Zierenberg. Ber. Forschungszentrum Waldökosysteme A 124

EICHHORN, J. (2000): Kennwerte zur Beurteilung eines möglichen Humusvorratsabbaus. In: BUNDESMINISTERIUM FÜR ERNÄHRUNG, LANDWIRTSCHAFT UND FORSTEN (Hrsg.): Kennwerte zur Charakterisierung des ökochemischen Bodenzustandes und des Gefährdungspotentials durch Bodenversauerung und Stickstoffsättigung an Level II-Waldökosystem-Dauerbeobachtungsflächen. 81-93

Eichhorn, J.; Haussmann, T.; PaAr, U.; Reinds, G.J. u. DE VRies, W. (2001): Assessments of impacts of nitrogen deposition on beech forests. TheScientificWorld 2001

Ellenberg, H.; MAyer, R. u. Schauermann, J. (Hrsg.)(1986): Ökosystemforschung: Ergebnisse des Solling-Projekts. Stuttgart, Ulmer

Gauger, T.; Anshelm, F.; Schuster, H.; Erisman, J.W.; Vermeulen, A.T.; Draajers, G.P.J.; BleEker, A. u. NAGEL, H.-D. (2002): Mapping of ecosystem specific long-term trends in deposition loads and concentration of air pollutants in Germany and their comparison with Critical Loads and Critical Levels, Part 1: Deposition Loads 1990 - 1999. Inst. f. Navigation Stuttgart, 1-207

Haussmann, T. u. Lux, W. (1997): Dauerbeobachtungsflächen zur Umweltkontrolle im Wald Level II: Erste Ergebnisse. Bundesministerium für Ernährung, Landwirtschaft und Forsten (Hrsg.)

HÖRmANN, G. (1997): SIMPEL - Ein einfaches, benutzerfreundliches Bodenwassermodell zum Einsatz in der Ausbildung. Dtsch. Gewässerkundl. Mitt. 41, 67-72 
Ibrom, A.; Oltchev, A.; Constantin, J.; Marques, M. u. Gravenhorst, G. (1995): Die Stickstoffimmission und -deposition in Wäldern. IMA-Querschnittseminar Wirkungskomplex Stickstoff und Wald, Umweltbundesamt Texte 28/95, 20-29

KAhle, H.-P.; SpieKer, H.; Pérez MARTíneZ, P.J. u. UnSeld, R. (2005): Causes of changes in growth of European forests: analysis of the roles of climatic factors and nitrogen nutrition. Forests in the Balance: Linking Tradition and Technology, XXII IUFRO World Congress, 8-3 August 2005, Brisbane, Australia, Abstracts. The International Forestry Review 7, 89

Khanna, P.K.; Fortmann, H.; Meesenburg, H.; Eichhorn, J. u. Meiwes, K.J. (2008): Biomass and Element Content of Foliage and Aboveground Litterfall on the three Long-Term Experimental Beech Sites - Dynamics and Significance. In: Brumme, R. u. KhannA, P.K. (Hrsg.): Functioning and Management of European Beech Ecosystems: Results from site specific long-term studies. Ecol. Studies, in Vorber.

Leuschner, C.; Meier, I.C. u. Hertel, D. (2006): On the niche breadth of Fagus sylvatica: soil nutrient status in 50 Central European beech stands on a broad range of bedrock types. Ann. For. Sci. 63, 355-368

Meesenburg, H.; Meiwes, K.J. u. Rademacher P. (1995): Long term trends in atmospheric deposition and seepage output in northwest German forest ecosystems. Water, Air and Soil Pollution $85,611-616$

Meesenburg, H.; MeiWes, K.J.; Schulze, A. u. Rademacher, P. (1997): Bodendauerbeobachtungsflächen auf forstlich genutzten Flächen (BDF-F). In: KLEEFISCH, B. u. KUES, J. (Hrsg.): Das Bodendauerbeobachtungsprogramm von Niedersachsen: Methodik und Ergebnisse, Arb.-H. Boden 2/1997, 77-95

Meesenburg, H.; Meiwes, K.J. u. Bartens, H. (1999): Veränderung der Elementvorräte im Boden von Buchen- und Fichtenökosystemen im Solling. Ber. Freiburger Forstl. Forschung 7, 109-114

Meesenburg, H.; Dammann, I.; Evers, J.; Schulze, A.; Rademacher, P.; Mindrup, M.; König, N.; Fortmann, H.; EberL, C. u. Meiwes, K.J. (2002): Forstliches Umweltmonitoring als Entscheidungshilfe für die Forstwirtschaft und Umweltpolitik. Forst und Holz 57, 707-712

Meesenburg, H.; Merino, A.; Meiwes, K.J. u. Beese, F.O. (2004): Effects of long-term application of ammonium sulphate on nitrogen fluxes in a beech ecosystem at Solling, Germany. Water, Air, and Soil Pollution: Focus 4, 415-426.

Meesendurg, H.; Mohr, K.; DÄmmgen, U.; SchaAf, S.; Meiwes, K.J. u. Horváth, B. (2005): Stickstoff-Einträge und -Bilanzen in den Wäldern des ANSWER-Projektes - eine Synthese. In: DÄmmgen, U. (Hrsg): Bestimmung von Ammoniak-Einträgen aus der Luft und deren Wirkung auf Waldökosysteme (ANSWER-Projekt), Landbauforschung Völkenrode Sonderheft 279, 95108

Meiwes, K.J. u. BeEse, F. (1988): Ergebnisse der Untersuchung des Stoffhaushaltes eines Buchenwaldökosystems auf Kalkgestein. Ber. Forschungsz. Waldökosysteme B 9

Meiwes, K.J.; Meesendburg, H.; Bartens, H.; Rademacher, P. u. Khanna, P.K. (2002): Akkumulation von Auflagehumus im Solling: Mögliche Ursachen und Bedeutung für den Nährstoffkreislauf. Forst und Holz 57, 428-433

Meiwes, K.J.; Asche, N.; Block, J.; KAllweit, R.; KÖlling, C.; RABen, G. u. V. Wilpert, K. (2008): Potentiale und Restrikitionen der Biomassenutzung im Wald. AFZ - Der Wald 62, 598 - 603

Rademacher, P.; Khanna, P.K.; Eichhorn, J. u. Guericke, M. (2008) Tree Growth, Biomass, Element Stocks. Brumme, R. u. Khanna, P.K. (Hrsg.): Functioning and Management of European Beech Ecosystems: Results from site specific long-term studies. Ecol. Studies, in Vorber.

Rothe, A.; Huber, C.; Kreutzer, K. u. Weis, W. (2002) Deposition and soil leaching in stands of Norway spruce and European beech. Results from the Höglwald research in comparison with other European case studies. Plant Soil 240, 1-14.

SEIDLING, W. u. FISCHER, R. (2008): Deviances from expected Ellenberg indicator values for nitrogen are related to $\mathrm{N}$ throughfall deposition in forests. Ecol. Indicators 8, 639-646 
SVERDRuP, H. u. WARFVINGE, P. (1993): Calculating field weathering rates using a mechanistic geochemical model PROFILE. Appl. Geochem. 8, 273-283

TYLER, G. (1992): Critical concentrations of heavy metals in the mor horizon of Swedish forests. Solna, Sweden, Swedish Environmental Protection Agency, Report 4078, 1-38

UlRICH, B. (1981): Zur Stabilität von Waldökosystemen. Forstarchiv 52, 165-170

UlRICH, B. (1994): Nutrient and acid/base budget of central European forest ecosystems. HÜtTERmann, A. u. GodBold, D.L. (Hrsg.): Effects of acid rain on forest processes. New York, Wiley, 1-50

UN/ECE (2004): Manual on methods and criteria for harmonized sampling, assessment, monitoring and analysis of the effects of air pollution on forests. International Co-operative Programme on assessment and monitoring of air pollution effects on forests ICP-FORESTS, http://www.icpforests.org

Korrespondierender Autor:

Dr. Henning Meesenburg

Nordwestdeutsche Forstliche Versuchsanstalt

Grätzelstr. 2

37079 Göttingen

E-Mail: Henning.Meesenburg@nw-fva.de

URL: www.nw-fva.de

Dr. Karl Josef Meiwes

Heike Fortmann

Birte Scheler

Prof. Dr. Johannes Eichhorn

Nordwestdeutsche Forstliche Versuchsanstalt 



\title{
Auswirkungen von Bodenschutzkalkungen auf Buchenwälder (Fagus sylvatica L.)
}

\author{
Effects of ameliorative liming on beech forests \\ (Fagus sylvatica L.) \\ Jan Evers, Inge Dammann, Axel Noltensmeier und Ralf-Volker Nagel
}

\section{Zusammenfassung}

Anhand über 25-jähriger Versuchsreihen wurde der Einfluss von Bodenschutzkalkungen auf Buchenbestände in Schleswig-Holstein, Niedersachsen, Hessen und Rheinland-Pfalz untersucht. Es konnte ein positiver Effekt der Kalkung auf das Höhenwachstum, den Kronenzustand der Buche sowie auf die Nährstoffgehalte von Buchenblättern nachgewiesen werden. Zusätzlich wurde das Nährstoffangebot im Mineralboden deutlich verbessert und die Stoffumsätze im Auflagehumus und oberen Mineralboden aktiviert. Unter gekalkten Parzellen kam es trotz geringeren Kohlenstoffvorräten im Auflagehumus im Vergleich zu den jeweiligen Kontrollparzellen insgesamt nicht zu Kohlenstoffverlusten, da bei gekalkten Parzellen die Kohlenstoffvorräte im Mineralboden höher waren. Damit konnte Kohlenstoff im Waldboden durch die Kalkung stabilisiert werden. Die mit der Kalkung ausgebrachten Calciumvorräte fanden sich im Auflagehumus und Mineralboden wieder und gingen somit dem System nicht verloren. Die durch die Kalkung erhöhte Säureneutralisationskapazität kann für die untersuchten Flächen jedoch noch nicht als ausreichend angesehen werden, mittlere Verhältnisse unbelasteter Waldöko- 
systeme bezüglich der Nährstoffversorgung wieder herzustellen. Die Calcium- und Magnesiumgehalte der Buchenblätter auf den gekalkten Parzellen lagen im hohen bis sehr hohen Bewertungsbereich, die entsprechenden Gehalte auf den Kontrollparzellen im mittleren bis geringen Bereich. Die Kaliumgehalte der Buchenblätter auf den ungekalkten Parzellen waren höher als auf den gekalkten Parzellen. Insgesamt ergaben die Ergebnisse, dass die derzeit durchgeführten Bodenschutzkalkungen geeignet sind, die Vitalität von Buchenbeständen und die Stabilität der Waldböden zu erhöhen. Auch nach über 10 Jahren hatte die Kalkung noch nachweisbare positive Effekte.

Stichworte: Rotbuche, Bodenschutzkalkung, Höhenzuwachs, Kronenzustand, Kohlenstoff, Nährstoffe

\begin{abstract}
Based on the experimental trial series spanning more than 25 years, the effect of soil ameliorative liming on beech stands in Schleswig-Holstein, Lower Saxony, Hesse and Rhineland-Palatinate was investigated. Liming was found to have a positive effect on height growth, beech crown condition, and on the nutrient content of beech leaves. In addition, nutrient availability in the mineral soil improved markedly, and the nutrient turnover in the raw humus and upper mineral soil was activated. Overall, on the limed plots, carbon losses did not eventuate, despite the lower carbon supply in the raw humus compared to the control plots because the carbon supply in the mineral soil was higher on the limed plots. Thus liming stabilised the carbon supply in the forest soil. The calcium supply, enhanced through liming, was located in the raw humus and mineral soil, and hence was not lost to the system. The increase in acid neutralisation capacity of the plots investigated as a result of liming was inadequate, however, in re-establishing the average levels of nutrient availability of unaffected forest ecosystems. The calcium and magnesium content of beech leaves on limed plots was high to very high, whereas the corresponding content on control plots was moderate to low. The potassium content of beech leaves on the unlimed plots was higher than that on the limed plots. Overall, the results indicate that the soil ameliorative liming carried out in the past was appropriate for increasing the vitality of beech stands and the stability of forest soils. Even after more than 10 years, the positive effects of liming are still evident.
\end{abstract}

Keywords: beech, ameliorative liming, height increment, crown condition, carbon, nutrients 


\section{Einleitung}

In den Wäldern Deutschlands ist die Rotbuche (Fagus sylvatica L.) die bestimmende Baumart der potenziellen natürlichen Vegetation mit einem Anteil von über $80 \%$ (LEUSCHNER 1998). Aktuell hat die Buche in Hessen $30 \%$, in Niedersachsen $14 \%$ und in Sachsen-Anhalt 6\% Anteil an der Holzbodenfläche im Hauptbestand (BMVEL 2004). Die Bedeutung der Buche kommt in verschiedenen Waldprogrammen zum Ausdruck, wie z. B. im Programm zur Langfristigen Ökologischen Waldentwicklung (LÖWE-Programm) in Niedersachsen: hier wird explizit eine Erhöhung der Buchenanteile vorgesehen, überwiegend in Mischbeständen (NLF 2007). Grundsätzlich beinhalten alle Waldprogramme das Leitbild standortsgemäßer, struktur- und artenreicher, leistungsstarker, gesunder und stabiler Wälder. Ebenso ist der Erhaltungszustand von Buchenwäldern aus Naturschutzsicht im Rahmen der FFH-Lebensraumtypen europaweit von besonderer Bedeutung. Die Schadstoffbelastung aus der Luft stellt eine erhebliche Belastung des Waldökosystems Wald dar. Konsequente Maßnahmen zur Minderung derselben werden z. B. in der „Leitlinie Wald“ Sachsen-Anhalts (MRLU 1997) angefordert. Der Erhalt der natürlichen Leistungskraft auch der Waldböden ist ein vorrangiges Ziel waldbaulicher Richtlinien der Länder. Bodenschutzkalkungen zur Minderung der negativen Auswirkungen der durch Stoffeinträge bedingten Versauerung von Waldböden werden unter anderem in den Richtlinien von Niedersachsen, Hessen und Sachsen-Anhalt, den Trägerländern der NW-FVA, eingefordert (LÖWEErlass 2007, RIBES 2002, MRLU 1997). Die anhaltende Überforderung der natürlichen Pufferkapazität der Waldböden wird durch die Ergebnisse der Bodenzustandserhebungen der Länder belegt: Bundesweit ist eine flächendeckende, weitgehend substratunabhängige Versauerung und Basenverarmung der Oberböden im Wald nachgewiesen worden (WOLFF et al. 1997). Daher sind Ergebnisse von Untersuchungen zur Wirkung von Bodenschutzkalkungen und deren Bewertungen derzeit und auch zukünftig von großer Bedeutung (z. B. SPELLMANN u. MEIWES 1995).

Zur Untersuchung der langfristigen Auswirkungen der Bodenschutzkalkung auf Bestandes- und Bodenparameter wurden in Niedersachsen, Hessen und Schleswig-Holstein seit Anfang der 1980er Jahre Vergleichspärchen gekalkt und ungekalkt für die Baumarten Rotbuche, Rotfichte (Picea abies (L.) Karst.), Waldkiefer (Pinus sylvestris L.) sowie Trauben- und Stieleiche (Quercus petraea (Mattuschka) Liebl.; Quercus robur L.) angelegt (BMVEL 2001). Diese Flächen sind periodisch alle 4-5 Jahre ertragskundlich aufgenommen worden. Auf einem Teil der Flächen wurde jährlich der Kronenzustand erfasst, in mehrjährigen Abständen Auflagehumus- und Bodenproben analysiert und zur Beurteilung der Ernährungssituation Nadel- bzw. Blattanalysen durchgeführt. In diesem Beitrag werden die Ergebnisse zur Buche vorgestellt. 


\section{Material und Methoden}

\subsection{Beschreibung der Versuchsflächen}

\subsubsection{Lage}

Die nördlichste Versuchsfläche liegt in Eutin (Schleswig-Holstein, Anlage 1987, s. Abb. 1), es folgen weiter südlich Sellhorn und Göhrde in der niedersächsischen Heide sowie Dassel im niedersächsischen Bergland. Die in Hessen 1983 angelegten Versuche zur Buche reichen von Hess. Lichtenau im Norden (Kaufunger Wald) über Frankenberg (Schiefergebirge), Königstein (Taunus), Jossgrund (Spessart) nach Lampertheim (Odenwald) im Süden. Der Kalksteigerungsversuch Dhronecken in Rheinland-Pfalz wurde wegen seiner inhaltlich ähnlichen Fragestellung in diese Auswertung einbezogen. Damit ist insgesamt ein relativ guter Nord-SüdTransekt für verschiedene Buchenwaldstandorte in Deutschland abgedeckt.

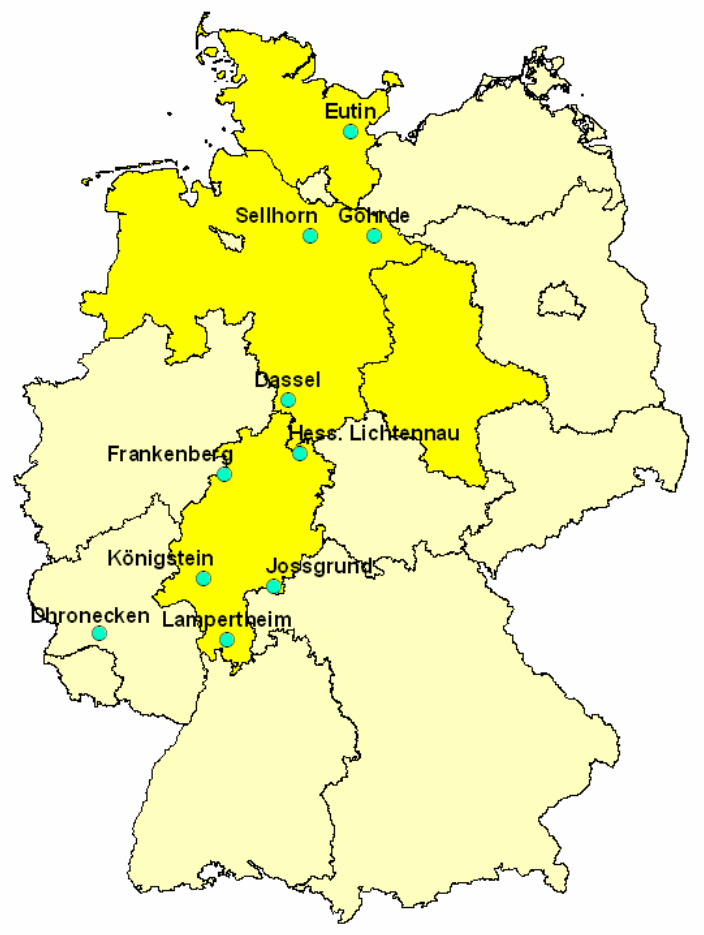

Abbildung 1: Lage der Versuchsflächen (Geobasisinformation (C) Bundesamt für Kartographie und Geodäsie (www.bkg.bund.de)), dunkelgelb: Zuständigkeitsbereich der NW-FVA 


\subsubsection{Standorte}

In Tabelle 1 sind die wichtigsten allgemeinen Angaben zu den jeweiligen Versuchsflächen aufgeführt:

Tabelle 1: Allgemeine Angaben zum Standort, dem Bestandesalter, Parzellenzabl, Exposition, Höhe $\ddot{u}$. NN sowie allgemeine Klimadaten

\begin{tabular}{|c|c|c|c|c|c|c|c|c|}
\hline Fläche & Region & $\begin{array}{l}\text { Buchen- } \\
\text { alter } \\
\text { (2008) }\end{array}$ & $\begin{array}{c}\text { Größe } \\
\text { (ha) }\end{array}$ & $\begin{array}{l}\text { Expo- } \\
\text { sition }\end{array}$ & $\begin{array}{l}\text { Nei- } \\
\text { gung } \\
(\%)\end{array}$ & $\begin{array}{l}\text { Höhe } \\
\text { ü. NN } \\
\text { (m) }\end{array}$ & $\begin{array}{c}\text { Jahres- } \\
\text { Nieder- } \\
\text { schlag* } \\
\text { (mm) }\end{array}$ & $\begin{array}{c}\text { Jahres- } \\
\text { Mittel- } \\
\text { temp.* } \\
\left({ }^{\circ} \mathrm{C}\right)\end{array}$ \\
\hline Eutin & Holst. Hügelland & 109 & $2 \times 0.25$ & Ebene & 0 & 55 & 742 & 8,3 \\
\hline Sellhorn & Hohe Heide & 99 & $2 \times 0.25$ & Ebene & 0 & 110 & 837 & 8,1 \\
\hline Göhrde & Ostheide & 111 & $2 \times 0.25$ & Ebene & 0 & 100 & 676 & 8,5 \\
\hline Dassel & Solling & 99 & $2 \times 0.25$ & $\mathrm{SO}$ & 3 & 430 & 1145 & 6,6 \\
\hline Hess. Lichtenau & Kaufunger Wald & 124 & $5 \times 0.30$ & $\mathrm{NO}$ & 10 & 460 & 902 & 7,1 \\
\hline Frankenberg & Schiefergebirge & 121 & $5 \times 0.25$ & $\mathrm{SO}$ & 10 & 420 & 667 & 7,5 \\
\hline Königstein & Taunus & 118 & $4 \times 0.25$ & S & 25 & 600 & 900 & 6,6 \\
\hline Jossgrund & Spessart & 130 & $4 \times 0.25$ & W & 14 & 420 & 969 & 7,0 \\
\hline Lampertheim & Odenwald & 128 & $4 \times 0.25$ & $\mathrm{~S}$ & 7 & 550 & 1047 & 7,9 \\
\hline Dhronecken & Hunsrück & 73 & $10 \times 0.25$ & $\mathrm{NW}$ & 5 & 670 & 875 & 6,5 \\
\hline
\end{tabular}

* Klimadaten Periode $1961-1990$ aus WolfF et al. 2003, für Dhronecken aus SpeLLmann u. MEIWES 1995

Die untersuchten Buchenbestände sind zwischen 73 Jahre (Versuch Dhronecken) und 130 Jahre (Jossgrund) alt. Damit fehlen in den Versuchen Buchen-Jungbestände in Wiederholungen und Altbestände über 130 Jahre. In Deutschland befinden sich in der Alterspanne 60-140 Jahre 60\% aller Buchenbestände (BMVEL 2004), so dass diese Versuche den mittleren Altersbereich der Buche in Deutschland gut abdecken.

Die Höhenstufen der untersuchten Buchenflächen reichen von der planaren Stufe (Eutin, Sellhorn und Göhrde), der submontanen Stufe (Dassel, Frankenberg, Jossgrund) über die montane Stufe in Hess. Lichtenau, Lampertheim und Königstein bis hin zur obermontanen Stufe in Dhronecken. Damit kommen alle Höhenstufen in diesen Versuchen vor, mit Ausnahme der kollinen Stufe des unteren Hügellandes.

Die durchschnittlichen Jahresniederschläge reichen von knapp $700 \mathrm{~mm}$ bis über 1100 mm (Periode 1961 - 1990). Dieser Bereich liegt vollständig in der ökologischen Standortsamplitude der Buche (LEUSCHNER 1998). 
Im Folgenden werden kurz Geologie und Böden der einzelnen Flächen charakterisiert:

Eutin: schwach wechselfeuchter, staufrischer Geschiebelehm über wurzelerreichbarem, nährstoffreichem Geschiebemergel, gut nährstoffversorgt;

Sellhorn: frisch bis vorratsfrischer, noch gut mesotropher, über $50 \mathrm{~cm}$ mächtiger, verlehmter Geschiebesand über Geschiebelehm;

Göhrde: mäßig sommertrockener, schwach mesotropher, verlehmter Geschiebesand über unverlehmtem Schmelzwassersand;

Dassel: $\quad$ lehmig-sandige, ca. $20-30 \mathrm{~cm}$ mächtige Lösslehmdecke über basenarmem Buntsandstein, insgesamt frisch bis vorratsfrisch und mäßig nährstoffversorgt;

Hess. Lichtenau: skelettreicher, teilweise toniger, mittlerer Buntsandstein mit schluffig-lehmigem Decksediment, mesotrophe, mäßig frische, podsolige Braunerde;

Frankenberg: $\quad$ mittel bis stark grusiger, schluffig-sandiger Lehm über Tonschiefer und Grauwacken-Skelettboden, insgesamt frisch und mesotroph;

Königstein: skelettreicher Schiefer und Taunusquarzit mit löss- und bimsreichem Decksediment, insgesamt frisch und mesotroph;

Jossgrund: $\quad$ mittlerer Buntsandstein unter Schlufflehm-Fließerden mit geringer Nährstoffversorgung, frische bis mäßig frische, podsolige Braunerde, insgesamt mesotroph;

Lampertheim: Fließerde aus karbonatfreien Lehmen über Hangschutt aus Plutoniten, betont frisch und eutroph;

Dhronecken: diluvialer Decklehm aus sandigem Lehm mit 10-20\% Grus und Steinen über lehmigem Sand und Grus aus Gehängeschutt; tiefgründige, podsolige, basenarme Braunerde, die ganzjährig mit saurem Hangwasser versorgt ist, insgesamt geringe Nährstoffversorgung.

\subsection{Kalkungsmaßnahmen}

In Tabelle 2 sind die jeweiligen Bodenschutzkalkungen auf den 10 Versuchsflächen nach Zeitpunkt, Menge, Kalktyp mit den jeweiligen elementbezogenen Ausbringungsmengen an Calcium, Magnesium, Phosphor und der Säureneutralisationskapazität (SNK) angegeben. Die in den letzten beiden Spalten aufgeführte theoretisch zu kompensierende Säuremenge ergibt sich auf Grundlage der modellierten potenziellen Netto-Säuredeposition (GAUGER et al. 2002) für die letzten 25 Jahre sowie für die atmogene Deposition für den Zeitraum 1790 bis 1982 in Anlehnung an Zeitreihen für den Standort Rothamsted in England 
(SVERDRUP et al. 1995), jeweils abzüglich der jeweiligen geschätzten Pufferraten für den effektiven Wurzelraum der Böden in den jeweiligen Versuchen (zwischen 0,2-0,4 kmolc je ha und Jahr). Die von GAUGER et al. (2002) zwischen 1990 - 1999 modellierten Werte wurden für den Untersuchungszeitraum 1983 bis 2007 extrapoliert. Insgesamt wurde von einer konstanten Freisetzung von Nährstoffkationen durch Silikatverwitterung über die angegebenen Zeiträume ausgegangen. Kam es zu Überschreitungen dieser Pufferraten durch die potenzielle Netto-Säuredeposition, wurde von einer Säureakkumulation im Mineralboden ausgegangen. Austräge mit dem Sickerwasser wurden nicht berücksichtigt. Diese in der Tabelle aufgeführten Angaben können nur Größenordnungen darstellen, die die ungefähre Säurebelastung dieser Standorte verdeutlichen sollen.

Die Bodenschutzkalkungen in den Versuchen sind überwiegend per Hand ausgebracht worden, um die erforderliche Sorgfalt sicherzustellen (Ausnahme Göhrde 1983: Ausbringung mit dem Schlepper und Schleuderstreuer).

Insgesamt sind zwischen 1,0 (Hess. Lichtenau und Königstein) und 8,5 (Sellhorn) Tonnen Kalk je Hektar ausgebracht worden. Im Kalksteigerungsversuch Dhronecken wurden bis zu 15 Tonnen Kalk je Hektar verschiedener Zusammensetzung ausgebracht. Wegen umfangreicherer Kalkungsmaßnahmen ist der Kalksteigerungsversuch Dhronecken von der Haupttabelle abgesetzt. 
Jan Evers, Inge Dammann, Axel Noltensmeier, Ralf-Volker Nagel

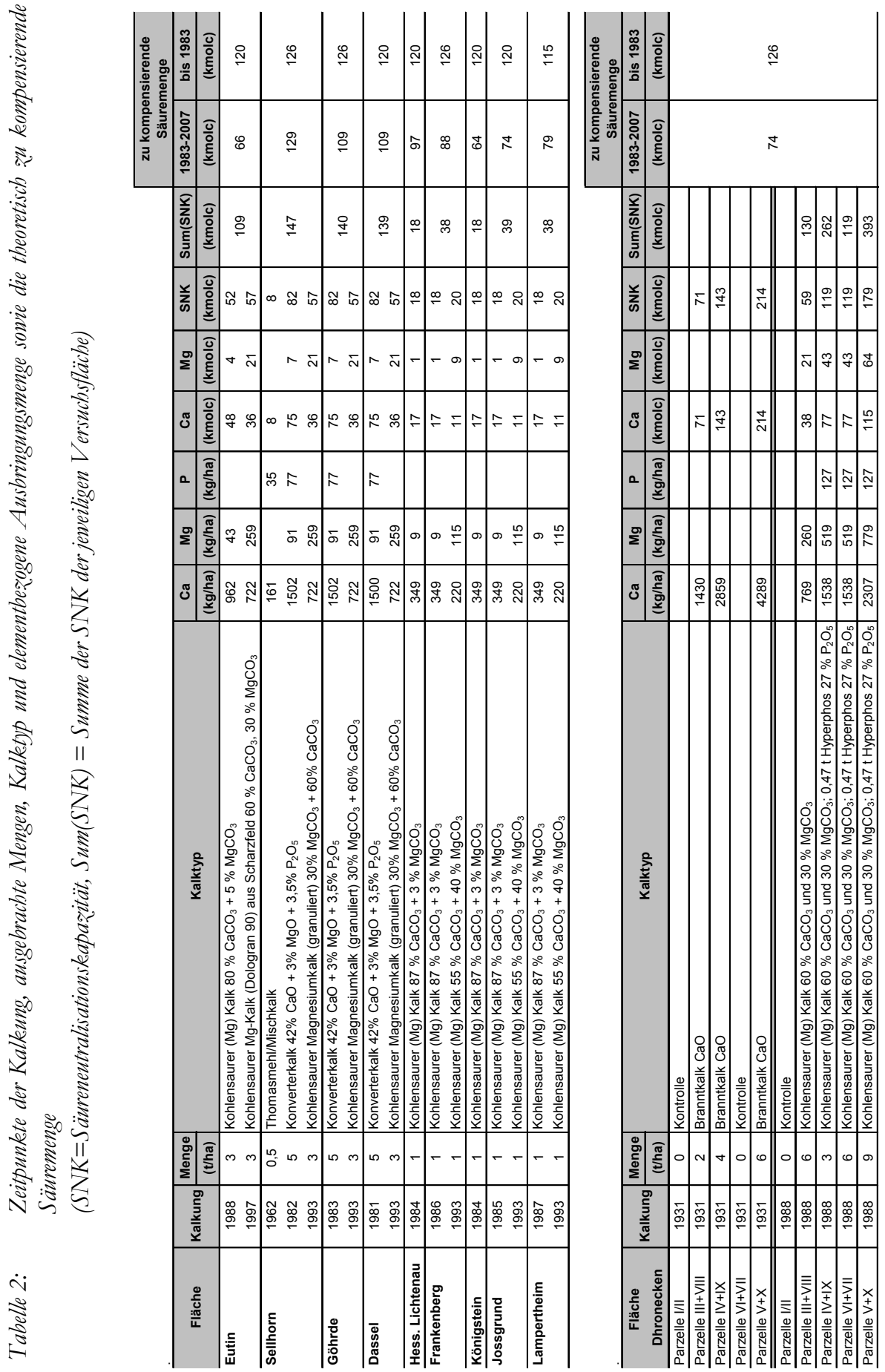




\subsection{Bestandesaufnahmen, Beprobung und Analysen}

Die ertragskundlichen Aufnahmen wurden im 4- bis 5-jährigen Zyklus als BHDVollaufnahme mit Umfangmessband und als repräsentative Höhenmessung an einem festen Baumkollektiv zur Herleitung der Bestandeshöhenkurve durchgeführt. Die Versuchsbestände wurden nach den Vorgaben einer schwachen Hochdurchforstung unter Förderung der jeweils besten Bäume behandelt. Die Eingriffsstärken waren allgemein gering. Der Kronenzustand der Buchen auf den Flächen Eutin, Sellhorn, Göhrde und Dassel ist nach dem Verfahren der Dauerbeobachtungsflächen Waldschäden (BMVEL 2001) erhoben worden. Die Kronenverlichtung und die Vergilbung sind je Parzelle an 50 (soweit vorhanden) vorherrschenden bis mitherrschenden Buchen in 5\%-Stufen eingeschätzt worden. Die erste Erhebung erfolgte 1984 (ohne Eutin), ab 1987 (inkl. Eutin) wurde der Kronenzustand jährlich erhoben.

Der Auflagehumus und der Mineralboden der Flächen Eutin, Sellhorn, Göhrde und Dassel wurden 1990 und 1998 beprobt. In Dassel und Göhrde folgte 2004 eine weitere Beprobung. Pro Parzelle wurden 1990 und 1998 jeweils drei Einzelproben zu drei Mischproben zusammengefasst, 2004 wurden je Parzelle vier Mischproben aus je drei Einzelproben gebildet. Der Mineralboden wurde in den Tiefenstufen 0-5, 5-10, 10-20 und 20-40 cm, in Dassel und Göhrde 2004 zusätzlich auch in 40-60 cm Bodentiefe beprobt. Profilansprachen lagen nicht vor, so dass Trockenrohdichten und Skelettanteile für die Vorratsberechnung geschätzt werden mussten.

Die Blattprobennahme erfolgte an herrschenden Buchen aus der Lichtkrone. 1990 und 1997 wurden je ungekalkter/gekalkter Parzelle drei Buchen beprobt. Auf den Flächen Eutin, Sellhorn, Göhrde und Dassel wurden in den Jahren 2004 und 2007 je Parzelle 8 Buchen geprobt. Je zwei Einzelproben wurden zu einer Mischprobe vereinigt und entsprechend vier Mischproben je Parzelle analysiert. Ergebnisse bodenchemischer Analysen und Analysen von Blattgehalten von den hessischen Flächen waren zum Zeitpunkt der Auswertung noch nicht verfügbar.

Bei der Probenvorbereitung und den chemischen Analysen wurden folgende Methoden angewandt: Effektive Austauschkapazität (Perkolation mit $1 \mathrm{~N} \mathrm{NH}_{4} \mathrm{Cl}$ ), Elementaranalyse für Kohlenstoff und Stickstoff sowie SCHEIBLER für Carbonat und $\mathrm{HNO}_{3}$-Druckaufschluss bei den Blattproben. Diese Methoden sind zusammen mit den jeweils zugehörigen Probenvorbereitungsmethoden in KÖNIG u. FORTMANN (1996, 1999a und 1999b) beschrieben.

Die statistische Datenauswertung erfolgte mit SPSS 15.0.1 Inc. 


\section{Ergebnisse}

\subsection{Höhenwachstum}

In den Abbildungen 2, 3 und 4 ist die Oberhöhe $\mathrm{H}_{100}$ über dem jeweiligen Alter der Buchen für die gekalkten und ungekalkten Parzellen (=Kontrolle) der Versuchsflächen dargestellt. Der Parameter $\mathrm{H}_{100}$ wurde gewählt, da die Oberhöhe stärker vom Standort und damit von einer Bodenschutzkalkung beeinflusst wird als von der Bestandesbehandlung wie Durchforstungsart und -stärke.
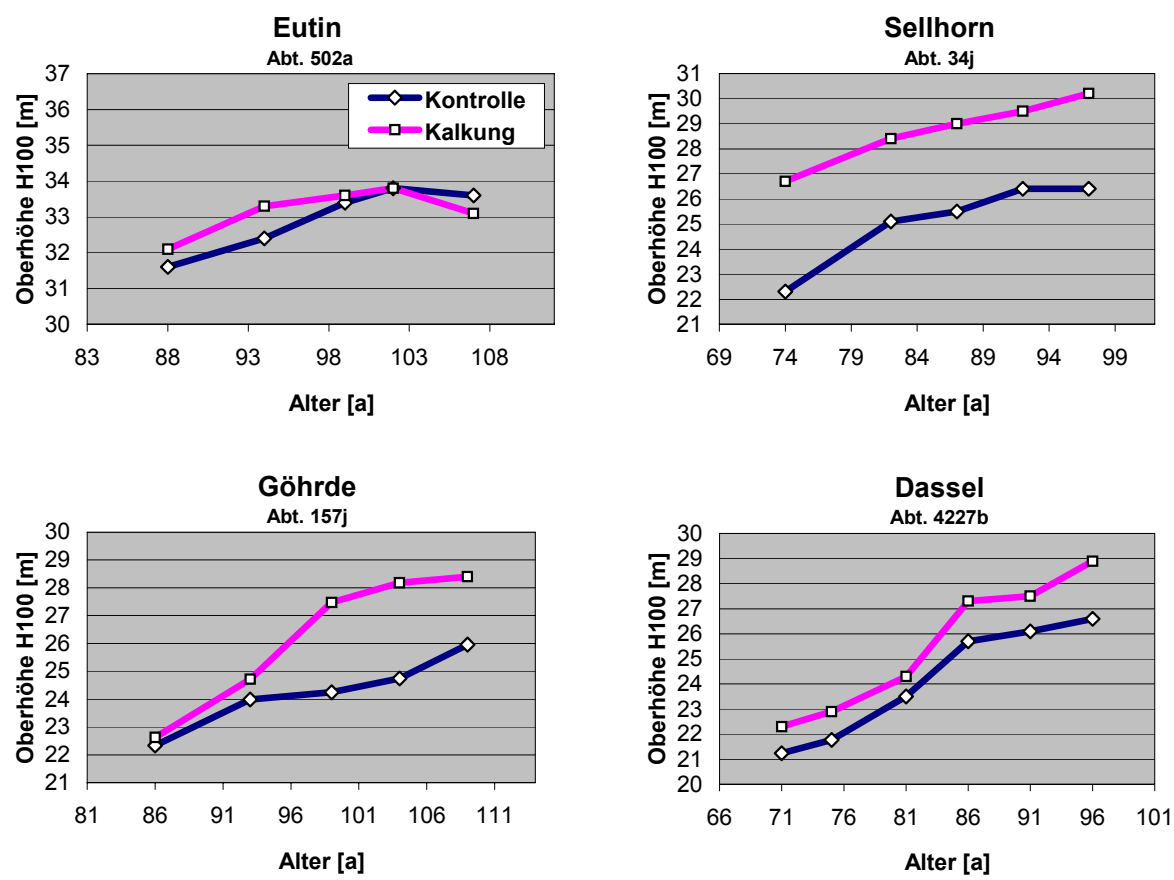

Abbildung 2: Oberböhe $H_{100}$ über dem Alter der gekalkten und ungekalkten Buchen in den Flächen Eutin, Sellhorn, Göhrde und Dassel

Am deutlichsten zeigt sich ein Anstieg der Oberhöhe auf der gekalkten im Vergleich zur ungekalkten Parzelle auf der Fläche Göhrde (s. Abb. 2), hier sind bei etwa gleicher Ausgangshöhe im Alter 86 die gekalkten Buchen seit der dritten Aufnahme im Alter 99 durchschnittlich 2-3 m höher als die ungekalkten. Diese Tendenz weisen auch die gekalkten Buchen in Dassel auf. In Sellhorn ist bereits das Ausgangsniveau zu Beginn der Versuchsreihe sehr unterschiedlich. Dies lässt auf entsprechende standörtliche Unterschiede der Parzellen bereits zu Beginn des Versuches schließen. In Eutin liegen die Oberhöhen der gekalkten und ungekalkten Parzelle im gesamten Beobachtungszeitraum eng zusammen, hier flachen beide 
Oberhöhenkurven ab dem Alter 103 ab, was auf die gekalkte Parzelle etwas stärker zutrifft.
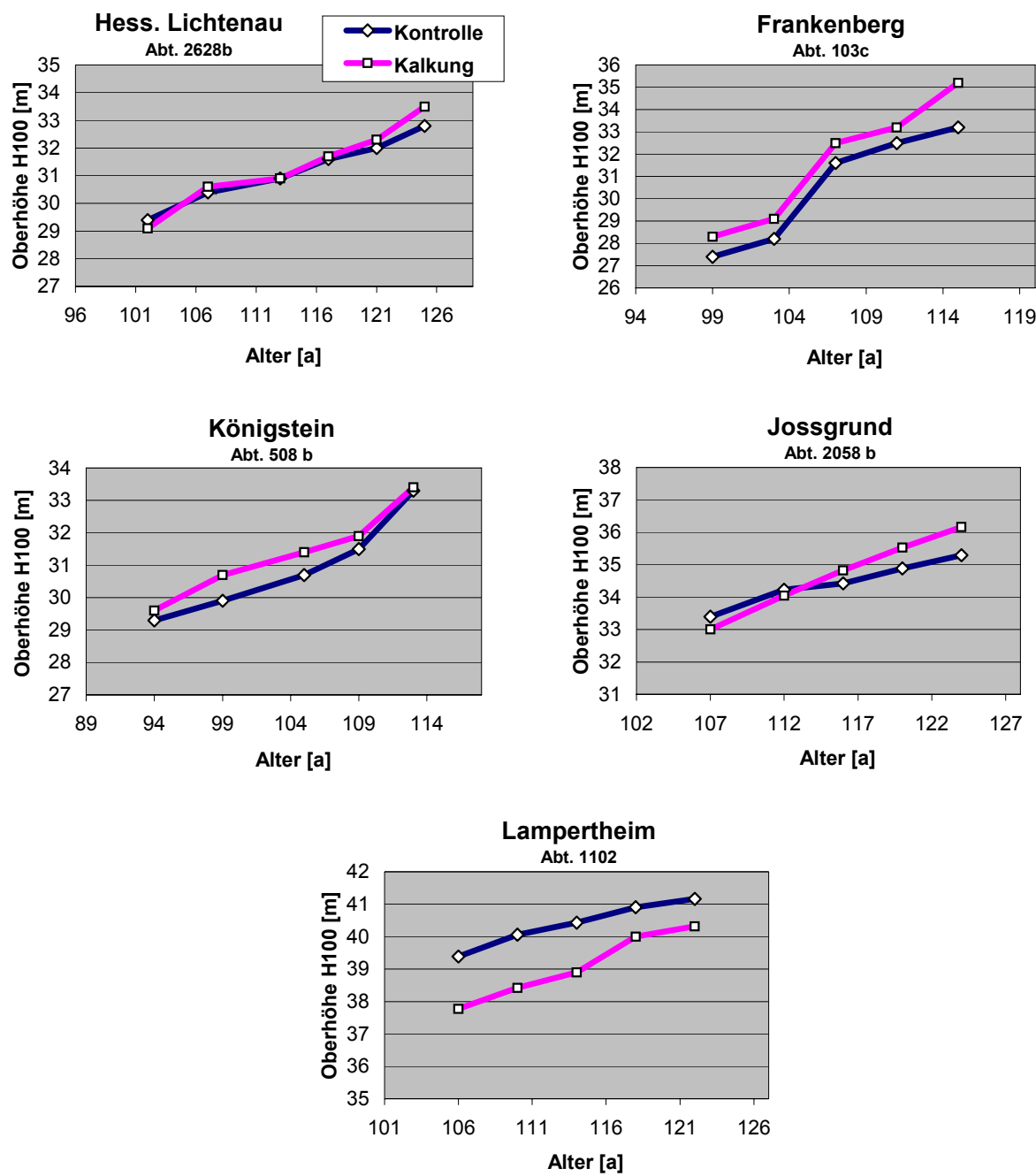

Abbildung 3: Oberböhe $H_{100}$ über dem Alter der gekalkten und ungekalkten Buchen in den Flächen Hess. Lichtenau, Frankenberg, Königstein, Jossgrund und Lampertheim

Ein gegenüber der Kontrolle gesteigertes Höhenwachstum zeigt sich bei den hessischen Versuchen bei den gekalkten Buchen in der Fläche Jossgrund, Frankenberg und relativ auch in Lampertheim (s. Abb. 3). In Hess. Lichtenau und Königstein sind die Unterschiede zwischen gekalkten und ungekalkten Buchen gering. 


\section{Buchen-Kalksteigerungsversuch Dhronecken}

Abt. 148 b

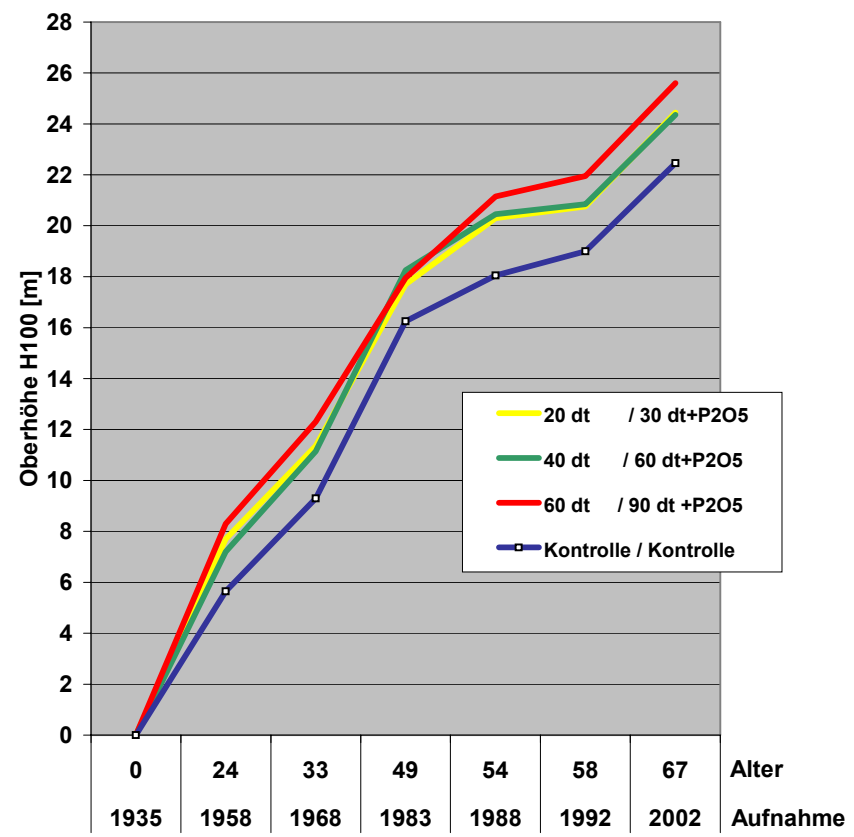

Abbildung 4: Oberböhe $H_{100}$ über dem Alter der gekalkten und ungekalkten Buchen im Kalksteigerungsversuch Dhronecken

Die Oberhöhen der gekalkten Buchen im Kalksteigerungsversuch Dhronecken liegen über denen in den Kontrollflächen (s. Abb. 4). Die Variante mit den höchsten Oberhöhen ist zugleich die mit der höchsten Kalkausbringungsmenge, allerdings sind die Unterschiede $\mathrm{zu}$ den Varianten mit geringeren Kalkausbringungsmengen nicht sehr deutlich. In Dhronecken konnten SPELLMANN und MEIWES (1995) bereits deutliche Zuwachseffekte bei der Gesamtwuchsleistung von über $40 \%$ Mehrleistung durch die Kalkung nachweisen.

$\mathrm{Da}$ die Kalkungen und Bestandesaufnahmen in unterschiedlichen Jahren durchgeführt wurden und die Bestände unterschiedliche Alter haben, wurde für einen summarischen Vergleich des Kalkungseffektes auf die Oberhöhe $\mathrm{H}_{100}$ die Differenz zwischen End- und Anfangsoberhöhe gebildet. Im t-Test für abhängige Stichproben ließ sich unter dem $5 \%$-Niveau über alle Flächen ein Unterschied von 0,7 m zugunsten der gekalkten Buchen nachweisen (s. Tab. 3). Unter Ausschluss von Eutin (mergelbeeinflusst) ergab sich ein höheres Signifikanzniveau von 0,01 für knapp $1 \mathrm{~m}$ höhere $\mathrm{H}_{100}$ bei den gekalkten Buchen. 
Tabelle 3: Höhenquwachs $\left(H_{100}\right)$ im Beobachtungszeitraum nach Flächen und Behandlung (Kalk $\%=$ Höhenzuwachs $H_{100}$ der gekalkten Flächen in \% der Kontrolle)

\begin{tabular}{lcccc}
\hline \multicolumn{5}{c}{ Höhenzuwachs (m) im Beobachtungszeitraum } \\
\hline Flächen & Kalkung & Kontrolle & Kalk\% & p \\
\hline Eutin & 1,0 & 2,0 & 50 & \\
Sellhorn & 3,5 & 4,1 & 85 & \\
Göhrde & 5,8 & 3,6 & 159 & \\
Dassel & 6,6 & 5,4 & 123 & \\
Hess. Lichtenau & 4,4 & 3,4 & 129 & \\
Frankenberg & 6,9 & 5,8 & 119 & \\
Königstein & 3,8 & 4,0 & 95 & \\
Jossgrund & 3,2 & 1,9 & 167 & \\
Lampertheim & 2,6 & 1,5 & 172 & \\
Dhronecken & 17,1 & 15,9 & 108 & 0,045 \\
\hline Mittel & 5,5 & 4,8 & 120,8 & \\
Mittel (ohne Eutin) & 6,0 & 5,1 & 128,7 & \\
\hline
\end{tabular}

Zusammenfassend zeigt sich ein positiver Einfluss der Bodenschutzkalkung auf die Entwicklung der Oberhöhen der Buchen, am deutlichsten in den Versuchen Göhrde und Dhronecken. Ein Kalkungseffekt auf das Höhenwachstum ist jedoch nicht auf allen Flächen festzustellen. Eutin ist die einzige Fläche, bei der ein Rückgang der Oberhöhe auf der gekalkten gegenüber der ungekalkten Parzelle aufgetreten ist. Bei diesem Standort war ein Kalkungseffekt auf das Höhenwachstum auch nicht zu erwarten, da von einer Durchwurzelung von nährstoffreichen Schichten (hier Mergel) auszugehen ist.

\subsection{Kronenverlichtung und Vergilbung}

Die mittleren Kronenverlichtungen und Vergilbungen der Buchenkronen liegen überwiegend zwischen 20 und $40 \%$ und sind damit nach der Nomenklatur der Waldzustandserhebung schwach bis mittelstark geschädigt (s. Abb. 5). Während auf den Flächen Sellhorn, Göhrde und Dassel die Kronenverlichtungen der Buchen zu Beginn der Messreihen 1984 auf jeweils beiden Parzellen bei rund 10\% lagen und sich im Zeitverlauf auf durchschnittlich $25 \%$ erhöhten, haben die Buchen auf der Fläche Eutin bereits bei der ersten Erhebung 1987 eine relativ hohe durchschnittliche Kronenverlichtung aufgewiesen und bis 2005 beibehalten.

Die mittleren Kronenverlichtungen der gekalkten Buchen sind geringer als die Werte der ungekalkten Buchen. Bei den Versuchsflächen Eutin und Sellhorn weichen die jährlichen Mittelwerte der durchschnittlichen Kronenverlichtung zwischen den gekalkten und ungekalkten Buchen kaum voneinander ab. In Sellhorn ist das Niveau der Verlichtungen der gekalkten Buchen zwischen 1993 und 
1998 deutlich geringer als die mittleren Verlichtungswerte der ungekalkten Buchen. In den Versuchsflächen Göhrde und Dassel sind die gekalkten Buchen bei gleichem Ausgangsniveau besser belaubt als die ungekalkten Buchen. In Göhrde ist dies am deutlichsten.
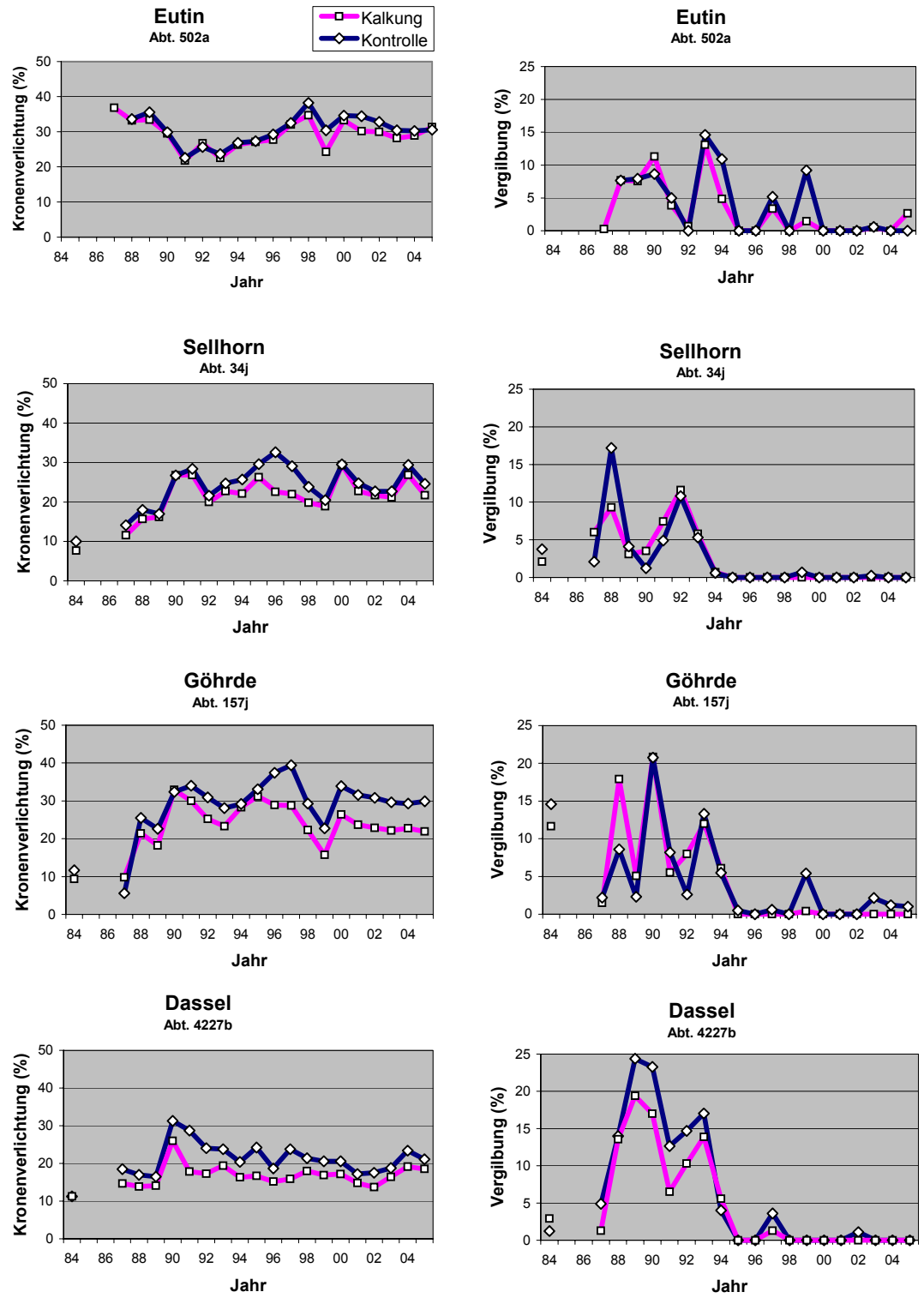

Abbildung 5: Mittlere Kronenverlichtung (links) und mittlere Vergilbung (rechts) der gekalkten und ungekalkten Buchen in den Flächen Eutin, Sellhorn, Göhrde und Dassel 
Bei der mittleren Vergilbung der Buchen auf allen Parzellen der Versuchsflächen zeigt sich ein klarer Rückgang unabhängig von der Bodenschutzkalkung. Ab 1994 sind Vergilbungen nur noch vereinzelt zu beobachten. Die Unterschiede zwischen dem Vergilbungsgrad der gekalkten und ungekalkten Buchen sind gering, lediglich in Dassel weisen die gekalkten Buchen zeitweise geringere Vergilbungen auf als die ungekalkten Buchen. In Einzelfällen waren die gekalkten Buchen stärker vergilbt, so z. B. in Göhrde 1988.

In Tabelle 4 sind die mittlere Kronenverlichtung und Vergilbung über den gesamten Beobachtungszeitraum für die jeweiligen Flächen und im Mittel nach Behandlung gekalkt/ungekalkt angegeben. Im t-Test für abhängige Stichproben ergaben sich hochsignifikante geringere mittlere Kronenverlichtungen bei den gekalkten Buchen im Vergleich zu den ungekalkten. Diese Unterschiede waren in Göhrde und Dassel am höchsten, in Sellhorn geringer und in Eutin am geringsten. Im Mittel ergab sich eine um 3,4\%-Punkte geringere Kronenverlichtung bei den gekalkten Buchen; diese Differenz ist zwar relativ gering, aber bei einem Standardfehler von 0,29 und dem hohen Signifikanzniveau gut abgesichert. Bezüglich der Vergilbung zeigten sich zwischen den gekalkten und jeweils ungekalkten Buchen nur sehr geringe Unterschiede, die sich nur in Dassel mit geringer vergilbten Buchen in der Kalkungsparzelle absichern ließen. Bei den geringen Werten der durchschnittlichen Vergilbung sind diese Unterschiede von geringer Relevanz.

Zusammenfassend zeigt sich ein relativ geringer, aber positiver Einfluss der Bodenschutzkalkung auf die Belaubungsdichte der Buche. Die Unterschiede bei der Vergilbung zwischen gekalkten und ungekalkten Buchen sind gering, ab 1994 werden für beide Behandlungen nur selten Vergilbungen festgestellt.

Tabelle 4: $\quad$ Mittlere Kronenverlichtung und mittlere Vergilbung im Beobachtungszeitraum nach Flächen und Behandlung (Diff = mittlere Differenz, $S D=$ Standardabweichung, $S D F=$ Standardfebler der Diff., $p=$ Signifikanzniveau)

\begin{tabular}{lccccccc} 
& & \multicolumn{7}{c}{ Mittlere Kronenverlichtung 1984 - 2005 (\%) } \\
\hline Flächen & $\mathbf{N}$ & Kalkung & Kontrolle & Diff. & SD & SDF & p \\
\hline Eutin & 18 & 28,9 & 30,5 & 1,52 & 1,79 & 0,42 & 0,002 \\
Sellhorn & 20 & 21,2 & 23,7 & 2,40 & 2,58 & 0,58 & 0,001 \\
Göhrde & 20 & 23,3 & 28,3 & 5,08 & 3,58 & 0,80 & 0,000 \\
Dassel & 20 & 16,7 & 20,9 & 4,26 & 2,41 & 0,54 & 0,000 \\
\hline Mittel & 78 & 22,4 & 25,7 & 3,40 & 3 & 0,29 & 0,000 \\
\hline
\end{tabular}

\begin{tabular}{lccccccc}
\cline { 3 - 8 } & \multicolumn{7}{c}{ Mittlere Vergilbung 1984 - 2005 (\%) } \\
\hline Flächen & $\mathbf{N}$ & Kalkung & Kontrolle & Diff. & SD & SDF & p \\
\hline Eutin & 18 & 3,2 & 3,9 & 0,70 & 2,54 & 0,60 & 0,256 \\
Sellhorn & 20 & 2,5 & 2,5 & 0,07 & 2,23 & 0,50 & 0,894 \\
Göhrde & 20 & 4,5 & 4,4 & $-0,01$ & 3,03 & 0,68 & 0,991 \\
Dassel & 20 & 4,6 & 6,1 & 1,46 & 2,45 & 0,55 & 0,015 \\
\hline Mittel & 78 & 3,7 & 4,2 & 0,50 & 2,6 & 0,29 & 0,065 \\
\hline
\end{tabular}




\subsection{Bodenchemische Untersuchungen}

\subsubsection{Auflagehumus}

Die aus den Beprobungen und chemischen Analysen berechneten Kohlenstoffund Calciumvorräte sind in der Abbildung 6, die Mittelwerte mit statistischen Kennwerten und Testergebnissen in Tabelle 5 dargestellt. Diese beiden Elemente wurden beispielhaft aus den Analyseergebnissen zur Darstellung von Behandlungseffekten beim Auflagehumus und Mineralboden ausgewählt: Kohlenstoff deswegen, weil es in allen organischen Geweben als wichtigstes Element vertreten und im Rahmen der Kohlenstoffbindung von besonderem Interesse ist und Calcium, weil hier die stärksten Kalkungseffekte zu erwarten waren.
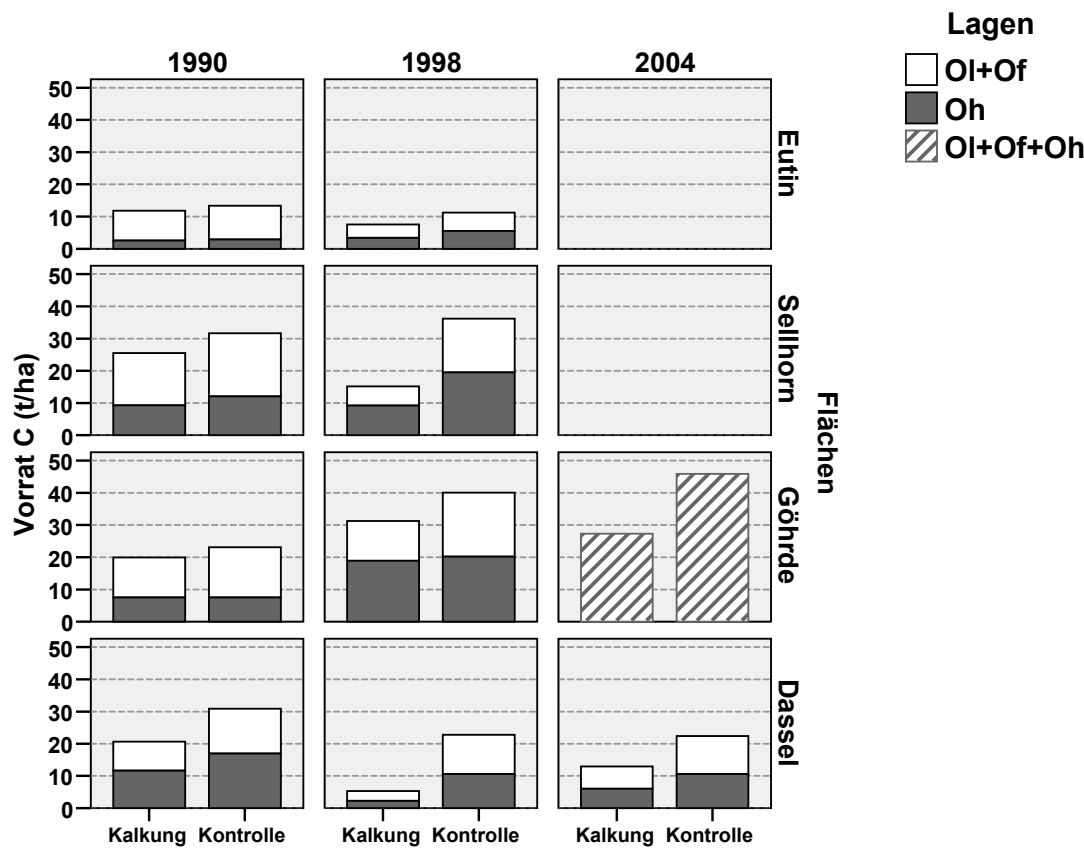

Abbildung 6: Koblenstoffvorrat im Auflagebumus (t/ha) getrennt nach Humuslagen für die Jabre 1990, 1998 und 2004 für die Flächen Eutin, Sellhorn, Göhrde und Dassel nach Kalkung und ungekalkter Kontrolle

In allen gekalkten Parzellen sind die Kohlenstoffvorräte zum jeweiligen Zeitpunkt der Probennahme geringer als in den ungekalkten. Die Unterschiede finden sich nicht nur bei den jeweiligen Ol- und Of-Lagen, sondern auch in der Oh-Lage. In der Versuchsfläche Göhrde konnten infolge intensiven Wühlens durch Schwarzwild die Lagen des Auflagehumus 2004 nicht mehr sinnvoll getrennt werden, daher 
sind die Auflagehumusvorräte dort nicht mehr getrennt nach ihren Lagen dargestellt.

Bezogen auf die jeweiligen Probennahmen und Einzelflächen ist die Entwicklung der Kohlenstoffvorräte unterschiedlich: In Eutin nehmen die Vorräte leicht ab, wobei 1998 die Kohlenstoffvorräte in der Ol- und Of-Lage geringer, in der Oh-Lage höher sind. In der Fläche Göhrde dagegen nehmen die Kohlenstoffvorräte sowohl in der gekalkten als auch in der ungekalkten Parzelle im Auflagehumus eher zu. Dies trifft auch für Sellhorn bei der ungekalkten Parzelle zu, in der gekalkten Parzelle nehmen die Vorräte hingegen ab. In Dassel dagegen kommt es zu einer Abnahme der Humusvorräte in der Kontrolle von 1990 zu 1998, von 1998 zu 2004 bleibt der Kohlenstoffvorrat in der Kontrolle konstant. In der gekalkten Parzelle ist zunächst eine Abnahme, dann eine Zunahme der Vorräte zu beobachten.

Die Unterschiede zwischen den Humusvorräten insgesamt auf den gekalkten zu den ungekalkten Parzellen nehmen von 1990 zu 1998 zu (s. Tab. 5). Zwischen den Behandlungen Kalkung gegen Kontrolle lassen sich diese Unterschiede nicht für 1990, doch für die Jahre 1998 und 2004 und für alle Jahre insgesamt signifikant absichern.

Tabelle 5: $\quad$ Mittlere Koblenstoff- und Calciumvorräte im Auflagehumus, statistische Kennwerte und Testergebnisse für die Jahre 1990, 1998 und 2004 sowie den gesamten Zeitraum nach Versuchsbehandlung $(\mathrm{N}=$ Anzabl der Mischproben, $M W=$ Mittelwert, $S D=$ Standardabweichung, $S T F=$ Standardfehler des $M W, p=$ Signifikanzniveau)

\begin{tabular}{|c|c|c|c|c|c|c|c|c|c|c|c|c|}
\hline \multirow[b]{2}{*}{ Jahr } & \multirow[b]{2}{*}{ Behandlung } & \multirow[b]{2}{*}{$\mathbf{N}$} & \multicolumn{5}{|c|}{ C t/ha Auflagehumus } & \multicolumn{5}{|c|}{ Ca kg/ha Auflagehumus } \\
\hline & & & MW & SD & STF & $\mathrm{p}$ & & MW & SD & STF & $\mathrm{p}$ & \\
\hline \multirow{2}{*}{1990} & Kalkung & 12 & 19,1 & 6,8 & 2,0 & \multirow{2}{*}{0,103} & \multirow{2}{*}{ t-Test } & 907 & 407 & 117 & \multirow{2}{*}{0,000} & \multirow{2}{*}{ Mann-Whitney-U } \\
\hline & Kontrolle & 12 & 24,5 & 8,8 & 2,5 & & & 273 & 244 & 70 & & \\
\hline \multirow{2}{*}{1998} & Kalkung & 12 & 14,5 & 11,3 & 3,3 & \multirow{2}{*}{0,014} & \multirow{2}{*}{ t-Test } & 932 & 746 & 215 & \multirow{2}{*}{0,000} & \multirow{2}{*}{ Mann-Whitney-U } \\
\hline & Kontrolle & 12 & 27,6 & 12,6 & 3,6 & & & 163 & 36 & 10 & & \\
\hline \multirow{2}{*}{2004} & Kalkung & 8 & 20,1 & 7,8 & 2,8 & \multirow{2}{*}{0,026} & \multirow{2}{*}{ t-Test } & 1040 & 555 & 196 & \multirow{2}{*}{0,000} & \multirow{2}{*}{ Mann-Whitney-U } \\
\hline & Kontrolle & 8 & 34,1 & 13,9 & 4,9 & & & 175 & 54 & 19 & & \\
\hline \multirow{2}{*}{ Mittel } & Kalkung & 32 & 17,6 & 9,0 & 1,6 & \multirow{2}{*}{0,000} & \multirow{2}{*}{ t-Test } & 950 & 573 & 101 & \multirow{2}{*}{0,000} & \multirow{2}{*}{ Mann-Whitney-U } \\
\hline & Kontrolle & 32 & 28,1 & 11,9 & 2,1 & & & 207 & 158 & 28 & & \\
\hline
\end{tabular}

Insgesamt steigen die mittleren Kohlenstoffvorräte der ungekalkten Parzellen von 24,5 t/ha im Jahre 1990 über 27,6 t/ha 1998 auf 34,1 t/ha 2004 an, wogegen der Kohlenstoffvorrat bei den gekalkten Parzellen von 19,1 (1990), 14,5 (1998) und 20,1 (2004) t/ha eher auf ähnlichem Niveau geblieben ist. Über alle Jahre hinweg ergibt sich bei den ungekalkten Parzellen ein mittlerer Kohlenstoffvorrat von 28,1 t/ha, bei den gekalkten Parzellen ein Mittel von 17,6 t/ha. Hier sind jedoch die hohen Standardabweichungen im Zusammenhang mit den grundsätzlichen Schwierigkeiten einer repräsentativen Humusbeprobung im Wald zu beachten, die die Interpretation dieser Ergebnisse einschränken. 
Die Calciumvorräte im Auflagehumus sind mit Ausnahme der Fläche Dassel 1990 in allen gekalkten Parzellen erwartungsgemäß deutlich höher als in den Kontrollparzellen (s. Abb. 7). Dies lässt sich für alle Jahre hochsignifikant absichern (s. Tab. 5). Im zeitlichen Verlauf verlagert sich der Hauptanteil des Calciumvorrats von der Ol- und Of-Lage (1990) hin zur Oh-Lage (1998, vor allem Sellhorn und Göhrde). Die Calciumvorräte bleiben in den einzelnen Jahren je Fläche und Variante relativ konstant mit Ausnahme der gekalkten Fläche Göhrde.
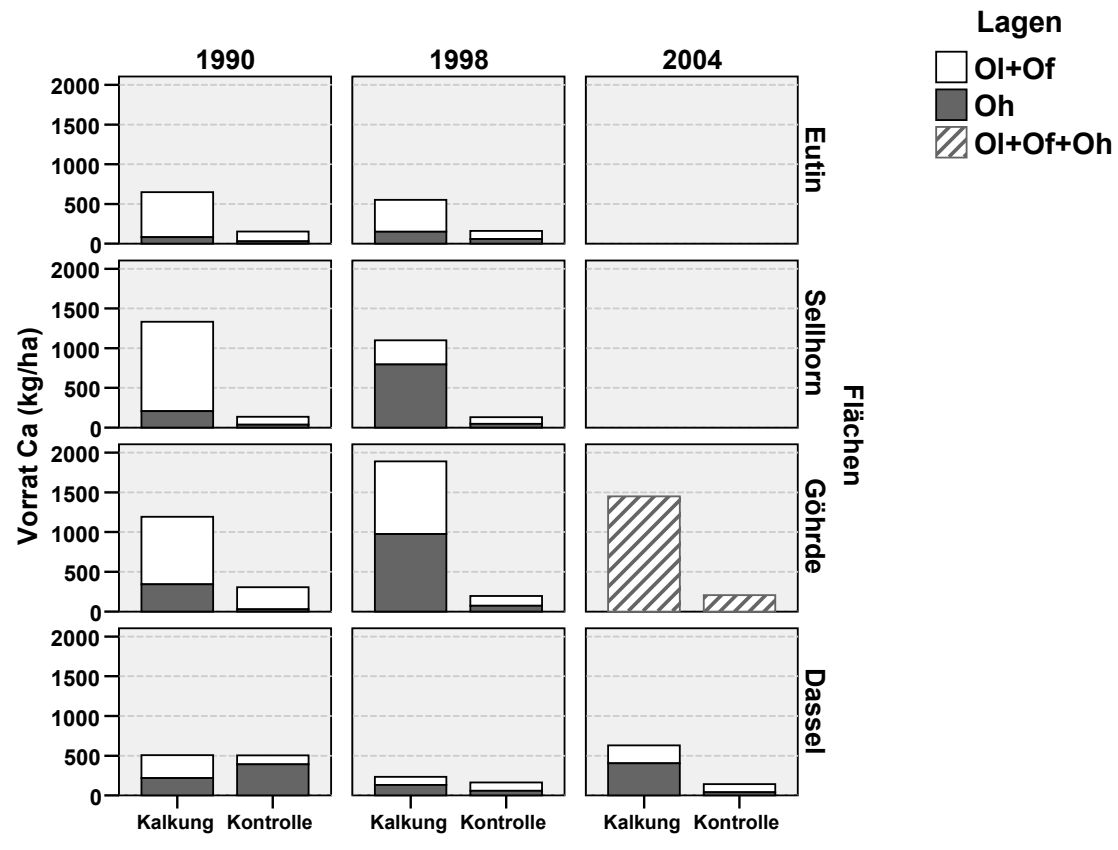

Abbildung 7: Calciumvorrat im Auflagehumus (kg/ ha) getrennt nach Humuslagen für die Jahre 1990, 1998 und 2004 für die Flächen Eutin, Sellhorn, Göhrde und Dassel nach Kalkung und ungekalk.ter Kontrolle

\subsubsection{Mineralboden}

Für die Beschreibung der Unterschiede der chemischen Eigenschaften des Mineralbodens hinsichtlich Kalkung und Kontrolle wurden die Basensättigung sowie analog zum Auflagehumus die Kohlenstoff- und Calciumvorräte ausgewählt.

Die Basensättigung der Kontrollflächen liegt in allen Erhebungsjahren in den Tiefenstufen unter $20 \%$, die Standorte sind damit nach dem ARBEITSKREIS STANDORTSKARTIERUNG (2003) als basenarm zu klassifizieren. Die Ausnahme bildet die Basensättigung von 30\% der Tiefenstufe 0-5 cm 1990 auf der Fläche 
Sellhorn. Insgesamt variieren die Basensättigungswerte der Kontrollflächen kaum zwischen den Erhebungszeitpunkten (s. Abb. 8).

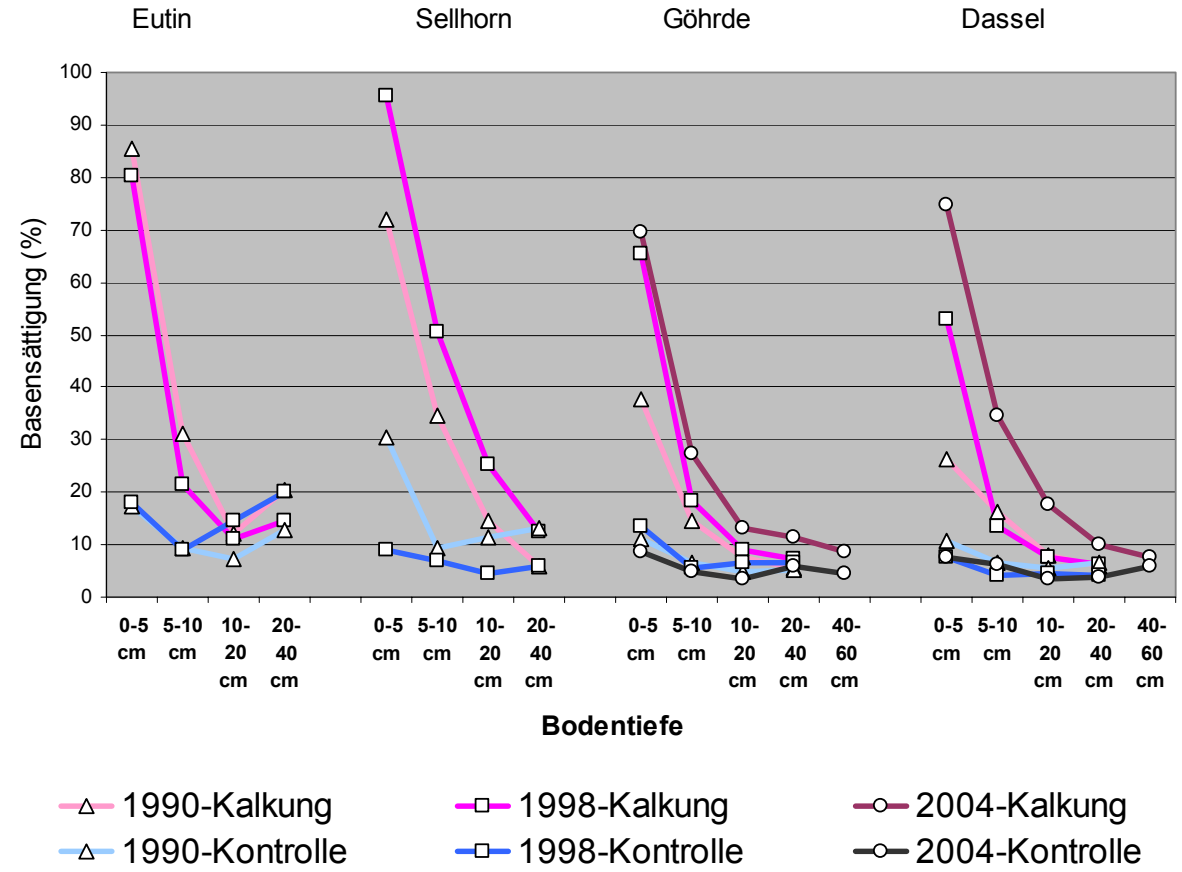

Abbildung 8: Basensättigung nach Tiefenstufen in den Jabren 1990, 1998 und 2004 für die Flächen Eutin, Sellhorn, Göhrde und Dassel nach Varianten Kalkung und ungekalkter Kontrolle

Nach der Kalkung ist für alle Kalkungsparzellen eine Verbesserung der Basensättigung festzustellen. Die Kalkung hat vor allem in den oberen Bodenschichten (bis $10 \mathrm{~cm}$ ) eine deutliche Steigerung der Basensättigung bewirkt, so dass diese Tiefenstufen in den Beprobungsjahren 1998 und 2004 als basenreich bezeichnet werden können. Auf der Fläche Sellhorn ist eine deutliche Erhöhung der Basensättigung auch in einer Bodentiefe von 10-20 cm im Jahre 1998 erkennbar. Die Flächen Göhrde und Dassel zeigen 2004 sogar einen Anstieg der Basensättigung bis in die Tiefenstufe $20-40 \mathrm{~cm}$ und teilweise auch 40-60 cm. Mit Ausnahme von Eutin erhöht sich in allen Flächen die Basensättigung in den jeweiligen Tiefenstufen mit jeder zusätzlichen Kalkung. Eine Basensättigung zwischen 30 und $50 \%$ wird als Gleichgewichtszustand mittlerer Waldstandorte angesehen (ARBEITSKREIS STANDORTSKARTIERUNG 2003).

In Abbildung 9 sind die Kohlenstoffvorräte im Mineralboden nach Tiefenstufen bis $10 \mathrm{~cm}$ Bodentiefe dargestellt. Die Grenze bis $10 \mathrm{~cm}$ Bodentiefe wurde 
gewählt, da vor allem in oberen Mineralbodenbereichen von Kalkungseffekten auszugehen ist.
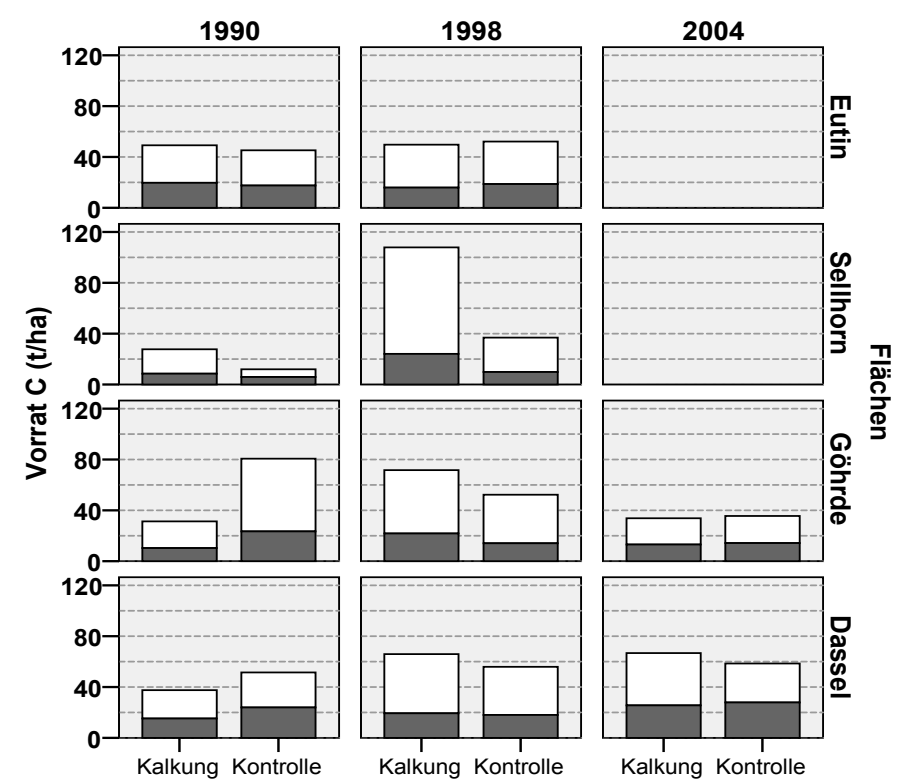

Tiefe von (cm)

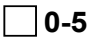

5-10

Abbildung 9: Koblenstoffvorrat im Mineralboden (t/ ha) getrennt nach Tiefenstufen für die Jabre 1990, 1998 und 2004 für die Flächen Eutin, Sellhorn, Göhrde und Dassel nach Kalkung und ungekalk.ter Kontrolle

1990 sind die Kohlenstoffvorräte im Mineralboden bis $10 \mathrm{~cm}$ Bodentiefe in den gekalkten Parzellen in Eutin und Sellhorn höher, in Göhrde und Dassel geringer als in der Kontrolle. 1998 liegen die Kohlenstoffvorräte dieser Bodenschicht in den gekalkten Parzellen über den Kontrollen (Ausnahme Eutin). 2004 sind die Kohlenstoffvorräte zwischen Kalkung und Kontrolle in Göhrde nahezu gleich, in Dassel in der Kalkungsvariante höher im Vergleich zur Kontrolle. Der überhöhte Kohlenstoffvorrat der gekalkten Parzelle in Sellhorn 1998 in 0-5 cm Bodentiefe ist nicht plausibel. Da alle 3 Wiederholungen ähnlich hohe Kohlenstoffgehalte aufweisen, die Laborwerte überprüft und keine besonderen Ereignisse bei der Beprobung gemeldet wurden, ist der Wert mit dargestellt. Von 1990 zu 1998 gab es insgesamt über alle Flächen bei den Kalkungsparzellen eine Zunahme der Kohlenstoffvorräte (rechnerisch von 36,5 auf 73,8 t/ha bzw. 62,4t/ha ohne Sellhorn), von $1998 \mathrm{zu}$ 2004 wieder eine Abnahme auf rechnerisch 50,3 t/ha. Die Kohlenstoffvorräte der Kontrollparzellen sind über diesen Zeitraum mit Werten zwischen 45 und $48 \mathrm{t} / \mathrm{ha}$ relativ konstant geblieben. Die Unterschiede zwischen den gekalkten und ungekalkten Parzellen lassen sich für 1998 auch statistisch absichern (s. Tab. 6). 
Die austauschbaren Calciumvorräte bis in $40 \mathrm{~cm}$ Bodentiefe sind für die Beprobungsjahre und Flächen nach Kalkung und Kontrolle in Abbildung 10 dargestellt. Im Jahr 1990 sind die Calciumvorräte in allen Parzellen mit $60 \mathrm{~kg} / \mathrm{ha}$ Calcium für alle gekalkten Parzellen und $18 \mathrm{~kg} / \mathrm{ha}$ für die jeweiligen Kontrollen nach der Bewertung des ARBEITSKREISES STANDORTSKARTIERUNG (2003) sehr gering. 1998 und 2004 sind die Calciumvorräte der gekalkten Parzellen mit durchschnittlich knapp 1000-1400 kg/ha im mittleren Bereich, und zwar überwiegend in der Tiefenstufe 0-5 cm. Die hohen Calciumvorräte in 20-40 cm Bodentiefe 1998 sowohl in der Kontrolle als auch in der gekalkten Parzelle in Eutin sind vermutlich auf den dort vorkommenden Mergel zurückzuführen, der 1990 bei der Beprobung nicht getroffen wurde.
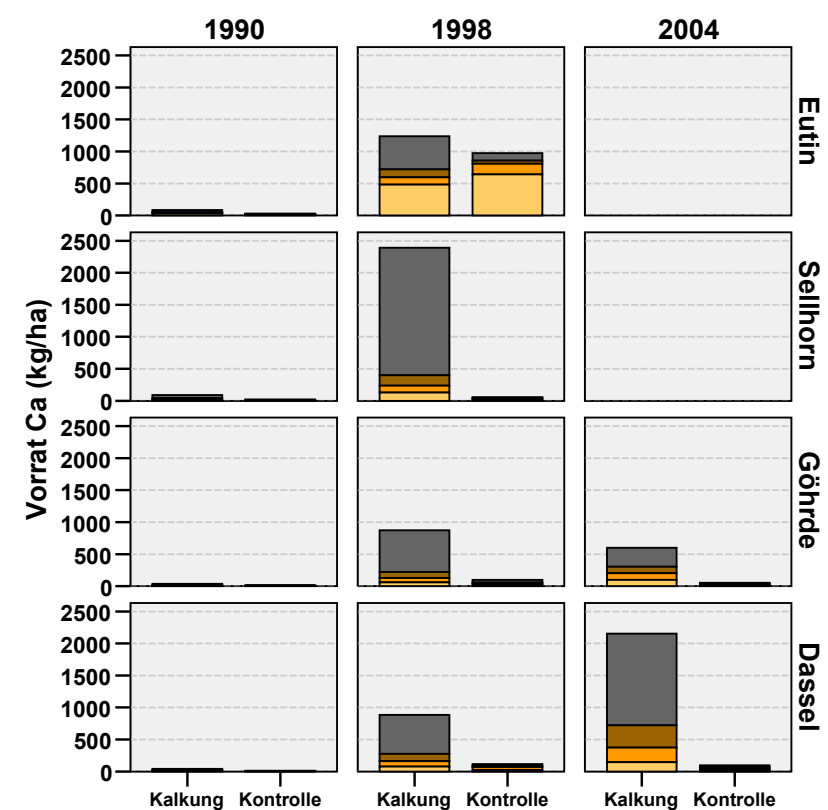

Tiefe von $(\mathrm{cm})$
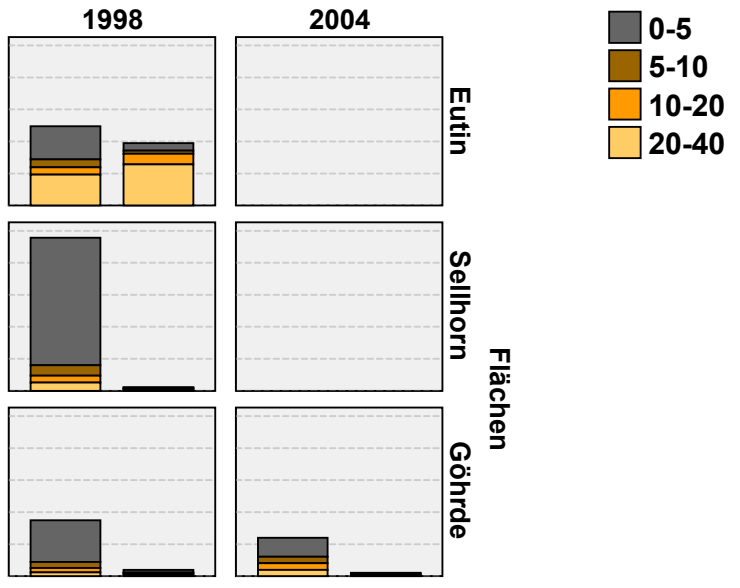

Abbildung 10: Calciumvorrat im Mineralboden (kg/ ha) getrennt nach Tiefenstufen für die Jahre 1990, 1998 und 2004 für die Flächen Eutin, Sellhorn, Göhrde und Dassel nach Kalkung und ungekalkter Kontrolle

Die Calciumvorräte zwischen den gekalkten und ungekalkten Parzellen sind für alle Jahre und das Gesamtergebnis hochsignifikant abgesichert (s. Tab. 6). 
Tabelle 6: $\quad$ Mittlere Koblenstoff- und Calciumvorräte im Mineralboden, statistische Kennwerte und Testergebnisse für die Jahre 1990, 1998 und 2004 sowie den gesamten Zeitraum nach Versucbsbehandlung ( $N=$ Anzabl der Mischproben, $M W=$ Mittelwert, $S D=$ Standardabweichung, $S T F=$ Standardfehler des $M W, p=$ Signifikanzniveau)

\begin{tabular}{|c|c|c|c|c|c|c|c|c|c|c|c|c|}
\hline \multirow[b]{2}{*}{ Jahr } & \multirow[b]{2}{*}{ Behandlung } & \multirow[b]{2}{*}{$\mathbf{N}$} & \multicolumn{5}{|c|}{$\begin{array}{c}\text { C t/ha Mineralboden } \\
0-10 \mathrm{~cm}\end{array}$} & \multicolumn{5}{|c|}{$\begin{array}{c}\text { Ca kg/ha Mineralboden } \\
0-40 \mathrm{~cm}\end{array}$} \\
\hline & & & MW & SD & STF & $\mathrm{p}$ & & MW & SD & STF & $\mathrm{p}$ & \\
\hline \multirow{2}{*}{1990} & Kalkung & 9 & 36,5 & 10,1 & 3,4 & \multirow{2}{*}{0,340} & \multirow{2}{*}{$\mathrm{t}$-Test } & 60 & 29 & 10 & \multirow{2}{*}{0,000} & \multirow{2}{*}{ Mann-Whitney-U } \\
\hline & Kontrolle & 9 & 47,8 & 32,7 & 10,9 & & & 18 & 10 & 3 & & \\
\hline \multirow{2}{*}{1998} & Kalkung & 12 & 73,8 & 26,0 & 7,5 & \multirow{2}{*}{0,003} & \multirow{2}{*}{ t-Test } & 1346 & 705 & 204 & \multirow{2}{*}{0,000} & \multirow{2}{*}{$\mathrm{t}$-Test } \\
\hline & Kontrolle & 12 & 44,6 & 16,5 & 4,8 & & & 307 & 505 & 146 & & \\
\hline \multirow{2}{*}{$1998^{*}$} & Kalkung & 9 & 62,4 & 14,6 & 4,9 & \multirow{2}{*}{\multicolumn{2}{|c|}{$0,069 \mathrm{t}$-Test }} & & & & & \\
\hline & Kontrolle & 9 & 47,2 & 18,3 & 6,1 & & & & & & & \\
\hline \multirow{2}{*}{2004} & Kalkung & 8 & 50,3 & 25,8 & 9,1 & \multirow{2}{*}{0,758} & \multirow{2}{*}{ t-Test } & 1377 & 1181 & 418 & \multirow{2}{*}{0,000} & \multirow{2}{*}{ Mann-Whitney-U } \\
\hline & Kontrolle & 8 & 47,1 & 13,2 & 4,7 & & & 74 & 37 & 13 & & \\
\hline \multirow{2}{*}{ Mittel } & Kalkung & 29 & 55,7 & 27,0 & 5,0 & \multirow{2}{*}{0,145} & \multirow{2}{*}{$\mathrm{t}$-Test } & 956 & 958 & 178 & \multirow{2}{*}{0,000} & \multirow{2}{*}{$\mathrm{t}$-Test } \\
\hline & Kontrolle & 29 & 46,3 & 21,4 & 4,0 & & & 153 & 344 & 64 & & \\
\hline
\end{tabular}

* ohne Sellhorn

\subsection{Calcium-, Magnesium- und Kaliumgehalte der Buchenblätter}

Die Calciumgehalte der Buchenblätter der gekalkten Buchen liegen überwiegend im Bereich einer sehr hohen Calciumversorgung, die entsprechenden Gehalte in der Kontrolle mit Ausnahme der analysierten Buchenblätter in Eutin im mittleren bis geringen Bereich (s. Abb. 11) nach der Bewertung des ARBEITSKREISES STANDORTSKARTIERUNG (2003). Diese Unterschiede lassen sich für jedes Probennahmejahr signifikant absichern, ab 1997 hochsignifikant (s. Tab. 7).

Die Probennahme in der Fläche Eutin in den Jahren 1990 und 1997 erfolgte versehentlich für beide Parzellen aus ungekalkten Bereichen der Versuchsfläche, daher sind diese Werte aus der Auswertung ausgeschlossen worden. 

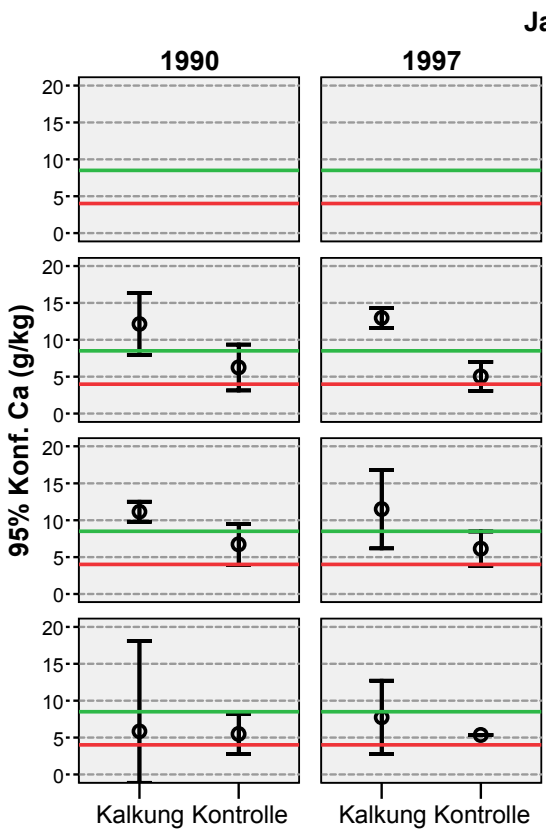

Jahr
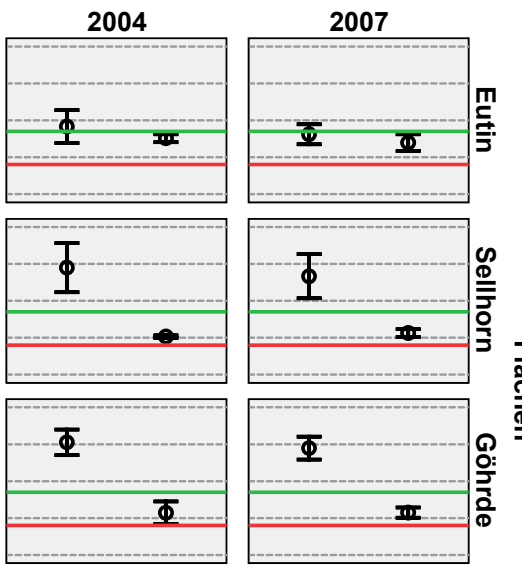

\section{$\frac{\pi}{\frac{\pi}{0}}$}
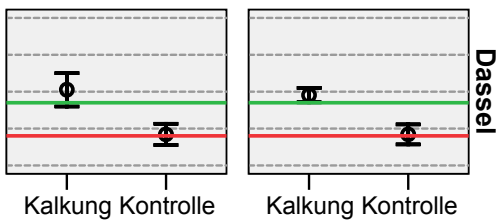

Abbildung 11: Konfidenzintervalle der Calciumgehalte in Buchenblättern für die Jahre 1990, 1997, 2004 und 2007 für die Flächen Eutin, Sellhorn, Göhrde und Dassel nach Kalkung und ungekalkter Kontrolle (unterbalb der roten Linie sehr geringe, oberhalb der grünen Linie sehr hohe Calcium-Versorgung der Buchenblätter)

Die Magnesiumgehalte der Buchenblätter auf den gekalkten Parzellen sind 1990 noch nahezu gleich im Vergleich zur Kontrolle, doch ab 1997 höher als diese (s. Tab. 7). Dies lässt sich für 1997 signifikant und 2004, 2007 sowie über alle Jahre hinweg hochsignifikant absichern. Ab 2004 liegen die Magnesiumgehalte der Buchenblätter in den gekalkten Parzellen im sehr hohen, die der Kontrollen im mittleren Bewertungsbereich (Bewertung nach ARBEITSKREIS STANDORTSKARTIERUNG 2003).

Bei den Kaliumgehalten zeigt sich ein anderes Bild: Die Kaliumgehalte der Buchenblätter sind in den gekalkten Parzellen 1990 und 1997 noch im hohen, 2004 und 2007 nur noch im (oberen) geringen Bewertungsbereich. Die entsprechenden Werte der Kontrollparzellen behalten im Auswertungszeitraum ein einheitliches Niveau bei und liegen damit stets über den jeweiligen Werten der Kalkungsparzellen. Diese Unterschiede lassen sich ab 1997 über alle Jahre hochsignifikant absichern. 
Tabelle 7: $\quad$ Mittlere Calcium-, Magnesium- und Kaliumgehalte in Buchenblättern, statistische Kennwerte und Testergebnisse für die Jahre 1990, 1997, 2004 und 2007 sowie den gesamten Zeitraum nach Versuchsbehandlung $N=$ Anzabl, $M W=$ Mittelwert, SD $=$ Standardabweichung, $S T F=$ Standardfehler des $M W, p=$ Signifikanzniveau)

\begin{tabular}{|c|c|c|c|c|c|c|c|}
\hline \multirow{2}{*}{ Jahr } & \multirow{2}{*}{ Behandlung } & \multirow{2}{*}{$\mathbf{N}$} & MW & SD & STF & \multirow{2}{*}{$\mathrm{p}$} & \\
\hline & & & \multicolumn{3}{|c|}{$\mathrm{g} / \mathrm{kg}$} & & \\
\hline \multicolumn{8}{|l|}{1990} \\
\hline \multirow[t]{2}{*}{$\mathrm{Ca}$} & Kalkung & 9 & 9,71 & 3,93 & 1,31 & \multirow{2}{*}{0,028} & \multirow{2}{*}{ T-Test } \\
\hline & Kontrolle & 9 & 6,16 & 1,14 & 0,38 & & \\
\hline \multirow[t]{2}{*}{ Mg } & Kalkung & 9 & 0,93 & 0,47 & 0,16 & \multirow{2}{*}{0,703} & \multirow{2}{*}{ T-Test } \\
\hline & Kontrolle & 9 & 0,86 & 0,27 & 0,09 & & \\
\hline \multirow[t]{2}{*}{$\mathbf{K}$} & Kalkung & 9 & 6,90 & 1,68 & 0,56 & \multirow{2}{*}{0,436} & \multirow{2}{*}{ T-Test } \\
\hline & Kontrolle & 9 & 7,65 & 2,26 & 0,75 & & \\
\hline
\end{tabular}

1997

\begin{tabular}{r|l|l|lll|l|l}
\hline $\mathbf{C a}$ & Kalkung & 12 & 10,73 & 2,77 & 0,92 & \multirow{2}{*}{0,000} & \multirow{2}{*}{ T-Test } \\
& Kontrolle & 12 & 5,51 & 0,78 & 0,26 & & \\
\hline $\mathbf{M g}$ & Kalkung & 12 & 0,89 & 0,26 & 0,09 & \multirow{2}{*}{0,042} & \multirow{2}{*}{ T-Test } \\
& Kontrolle & 12 & 0,66 & 0,18 & 0,06 & & \\
\hline \multirow{2}{*}{$\mathbf{K}$} & Kalkung & 12 & 7,02 & 2,05 & 0,68 & \multirow{2}{*}{0,006} & \multirow{2}{*}{ T-Test } \\
& Kontrolle & 12 & 9,68 & 1,46 & 0,49 & & \\
\hline
\end{tabular}

2004

\begin{tabular}{r|l|l|lll|l|l}
\hline $\mathbf{C a}$ & Kalkung & 16 & 12,29 & 3,05 & 0,76 & \multirow{2}{*}{0,000} & \multirow{2}{*}{ T-Test } \\
& Kontrolle & 16 & 5,66 & 1,41 & 0,35 & & \\
\hline $\mathbf{M g}$ & Kalkung & 16 & 2,82 & 0,37 & 0,09 & \multirow{2}{*}{0,000} & \multirow{2}{*}{ T-Test } \\
& Kontrolle & 16 & 1,05 & 0,34 & 0,09 & & \\
\hline \multirow{2}{*}{$\mathbf{K}$} & Kalkung & 16 & 5,47 & 0,53 & 0,13 & \multirow{2}{*}{0,000} & \multirow{2}{*}{ T-Test } \\
& Kontrolle & 16 & 7,52 & 1,01 & 0,25 & & \\
\hline
\end{tabular}

2007

\begin{tabular}{|c|c|c|c|c|c|c|c|}
\hline \multirow[t]{2}{*}{$\mathrm{Ca}$} & Kalkung & 16 & 11,36 & 2,91 & 0,73 & \multirow{2}{*}{0,000} & \multirow{2}{*}{ T-Test } \\
\hline & Kontrolle & 16 & 5,64 & 1,15 & 0,29 & & \\
\hline \multirow[t]{2}{*}{ Mg } & Kalkung & 16 & 2,20 & 0,40 & 0,10 & \multirow{2}{*}{0,000} & \multirow{2}{*}{ T-Test } \\
\hline & Kontrolle & 16 & 0,95 & 0,27 & 0,07 & & \\
\hline \multirow[t]{2}{*}{$\mathbf{K}$} & Kalkung & 16 & 5,43 & 1,07 & 0,27 & \multirow{2}{*}{0,000} & \multirow{2}{*}{ T-Test } \\
\hline & Kontrolle & 16 & 7,72 & 1,31 & 0,33 & & \\
\hline
\end{tabular}

Mittel

\begin{tabular}{r|l|c|ccc|c|c}
\hline $\mathbf{C a}$ & Kalkung & 50 & 11,25 & 3,17 & 0,44 & \multirow{2}{*}{0,000} & \multirow{2}{*}{ U-Test } \\
& Kontrolle & 50 & 5,71 & 1,17 & 0,13 & & \\
\hline $\mathbf{M g}$ & Kalkung & 50 & 1,93 & 0,90 & 0,13 & \multirow{2}{*}{0,000} & \multirow{2}{*}{ U-Test } \\
& Kontrolle & 50 & 0,91 & 0,31 & 0,04 & & \\
\hline \multirow{2}{*}{$\mathbf{K}$} & Kalkung & 50 & 6,00 & 1,46 & 0,21 & \multirow{2}{*}{0,000} & \multirow{2}{*}{ T-Test } \\
& Kontrolle & 50 & 8,00 & 1,63 & 0,23 & & \\
\hline
\end{tabular}




\section{Diskussion}

Die Ergebnisse dieser Untersuchung zeigen insgesamt eine positive Wirkung der Kalkung auf die Vitalität der Buche: Über alle Versuchsflächen hinweg konnte für eine Beobachtungsperiode von 25 Jahren (Dhronecken 70 Jahre) eine knapp $1 \mathrm{~m}$ höhere Oberhöhe $\left(\mathrm{H}_{100}\right)$ für die gekalkten Buchen nachgewiesen werden. Auch die mittleren Kronenverlichtungen der gekalkten Buchen sind signifikant geringer als die der ungekalkten Kontrollen, allerdings sind die Unterschiede nicht sehr hoch. Geringere Vergilbungen der gekalkten Buchen konnten bei einer Versuchsfläche abgesichert werden. Die Blattgehalte der gekalkten Buchen sind für Calcium und Magnesium signifikant erhöht und befinden sich im Bewertungsbereich mittlerer bis sehr hoher Gehalte, wogegen die jeweiligen Blattgehalte der Buchen auf den ungekalkten Parzellen im geringen bis teilweise im mittleren Versorgungsbereich liegen.

In Niedersachsen konnten DAMMANN et al. (2003) bereits bei Rotfichten bei der Untersuchung von acht Vergleichsflächen eine ähnliche Wirkung der Bodenschutzkalkung nachweisen. Auf den gekalkten Flächen stellten sich günstigere Benadelungsdichten, geringere Vergilbungen und erhöhte Grundflächenzuwächse im Vergleich zu den nicht gekalkten Kontrollen ein. Rotfichten mit dichterer Benadelung wiesen zudem bessere Zuwachsraten auf als Rotfichten mit höherem Verlichtungsgrad. Es wird aber auch von Versuchen zur Rotfichte und Tanne berichtet, wo die Bodenschutzkalkung keine oder nur vereinzelt positive Effekte hatte (AlDiNGER u. KREMER 1985, AldingER 1983).

Nach KRAMER (1988) gibt es zahlreiche ertragskundliche Untersuchungen über den Einfluss des Standortes auf das Wachstum der Buche (u. a. RÖHE 1985). Danach beeinflussen die Nährstoffverhältnisse des Bodens, insbesondere der Stickstoffvorrat, den Zuwachs der Buchen stärker als beispielsweise den der Fichte. SPELLMANN u. MEIWES (1995) zeigten, dass im Voranbau- und Kalksteigerungsversuch Dhronecken durch Bodenbearbeitung ein begrenzter, durch Kalkung mit zunehmender Dosierung ein deutlicher Volumenzuwachseffekt erzielt werden kann. Gleichzeitig kam es zu einem Verlust an Feinhumusvorrat und Stickstoff im Auflagehumus, zumal bei der ersten Kalkung sich schnell auflösender Branntkalk ausgebracht wurde. Damit induzierte die Kalkung einen beschleunigten Abbau der Rohhumusdecken und eine Stickstoffmobilisierung. Die größte Wirkung zeigte sich beim Höhenzuwachs: Bis zum Ende des Dickungsstadiums lagen die Buchen der Variante mit der größten Kalkausbringungsmenge um 1,5 Ertragsklassen über den Buchen der Kontrolle.

Ein derart starker Effekt der Kalkung konnte auf den hier betrachteten Vergleichspaaren der Monitoringflächen aus dem Bodenschutzkalkungsprogramm mit praxisüblicher Ausbringung von kohlensaurem Magnesiumkalk und ungekalkter Kontrolle nicht nachgewiesen werden. Die entsprechenden Versuchsflächen wurden in einem Bestandesalter zwischen 71 und 107 Jahren angelegt und liegen 
demzufolge in einem Altersbereich weit jenseits der Kulmination des Höhenzuwachses der Buche, der je nach Standort bei ungefähr 40 Jahren liegt. Nach KRAMER (1988) ist die überlegene Höhenwuchsleistung von Bäumen auf guten Standorten vor allem auf das sehr hohe Jugendwachstum zurückzuführen. In höherem Bestandesalter sind deshalb durch meliorative Maßnahmen wie eine Kalkung nicht mehr so hohe Zuwachssteigerungen zu erwarten. Zudem sind die Kalkgaben in den betrachteten Vergleichsflächenpaaren geringer und milder in ihrer Wirkung als im Kalksteigerungsversuch Dhronecken. Dennoch entspricht der durchschnittlich nachgewiesene Höhenunterschied von 1,0 m im Alter 100 bei der Buche ungefähr einer viertel Ertragsklasse, dies wären rechnerisch bei Vollbestockung ungefähr $4 \%$ mehr Derbholzmasse bei gekalkten gegenüber ungekalkten Beständen. In den Versuchen Sellhorn, Göhrde und Dassel ist zusätzlich Phosphor mit der Kalkung ausgebracht worden, dies kann ebenfalls zur Vitalitätssteigerung der Buchen beigetragen haben.

Da mit der Bodenversauerung ein Verlust an Nährstoffen durch Auswaschung verbunden ist, hat die Kalkung eine Verbesserung der Nährstoffversorgung der Waldbäume zum Ziel (MEIWES 1994, UlRICH 1995, BEESE 1996). Weiterhin soll durch die Kalkung eine Neutralisierung eingetragener Säurebildner, eine Stärkung der Pufferkapazität des Bodens zur Vermeidung einer weiteren Versauerung sowie eine moderate Aktivierung der Stoffumsätze im Auflagehumus erreicht werden (MeIWES 1994, Leube 2000). Die Erreichung dieser Ziele konnte in den hier untersuchten Versuchen zur Buche nachgewiesen werden.

Deutlich höhere Basensättigungen im Mineralboden und Calciumvorräte im Auflagehumus und Mineralboden der gekalkten gegenüber den ungekalkten Parzellen konnten in dieser Untersuchung belegen, dass die ausgebrachten Nährstoffe im Waldökosystem verbleiben. Die mit der Kalkung zugeführten Calciumvorräte finden sich in ähnlichen Größenordnungen im Auflagehumus und Mineralboden, wobei es im Zeitverlauf zu einer Verlagerung des Calciums von der Ol- und Of-Lage über die Oh-Lage in den oberen Mineralboden kommt. Von dort wird es über die Wurzeln aufgenommen, was sich an den hohen Nährstoffgehalten der gekalkten Buchenblätter zeigt. Die höhere Basensättigung und höheren Calciumvorräte im effektiven Wurzelraum (Auflage und Mineralboden bis $40 \mathrm{~cm}$ ) in den Kalkungsflächen zeigen, dass die Bodenschutzkalkung zu einer Annäherung an die Wuchsverhältnisse mittlerer, unbelasteter Waldbestände geführt hat. Die Bodenschutzkalkung kann deshalb sowohl als konsequente Maßnahme zur Melioration versauerter Waldböden als auch zur Vorbeugung entkoppelter Stoffkreisläufe angesehen werden. Die nach einer Kalkung langfristige Speicherung von Calcium im Auflagehumus und oberen Mineralboden ist mehrfach belegt (ALDINGER 1987, SPELLMANN u. MeIWES 1995, HÖLSCHER et al. 1999, MEESENBURG et al. 2001, WAGNer 2007, MOORE et al. 2008). Wird jedoch die mit der Kalkung ausgebrachte Säureneutralisationskapazität mit der theoretisch zu kompensierenden Säuremenge (Eintrag über Deposition abzüglich Pufferrate) verglichen, so zeigt 
sich mit Ausnahme des Kalksteigerungsversuches in Dhronecken, dass die ausgebrachten Kalkmengen noch nicht als ausreichend angesehen werden können, um ein mittleres Niveau der Nährstoffversorgung unbelasteter Waldböden wiederherzustellen.

In den ungekalkten Kontrollen kommt es über alle Versuche hinweg von 1990 bis $2004 \mathrm{zu}$ einem Humusaufbau von durchschnittlich 10 t/ha Kohlenstoff, dies entspricht einer jährlichen Zunahme von rechnerisch $0,7 \mathrm{t} / \mathrm{ha}$. Bei den gekalkten Parzellen dagegen wird zunächst Auflagehumus abgebaut (1998), wobei 2004 wieder das durchschnittliche Niveau von 1990 erreicht wird. Gleichzeitig findet auf den gekalkten Parzellen verstärkt eine Verlagerung von Kohlenstoff in den Mineralboden statt. Zum einen zeigt dies die kalkungsbedingte Förderung der biologischen Aktivität im Auflagehumus und oberen Mineralboden beziehungsweise deren Hemmung durch langfristige Säureeinträge bei den Kontrollen, zum anderen eine langfristigere Festlegung von Kohlenstoff durch die Verlagerung in den Mineralboden im Zuge der Bodenschutzkalkung. MEESENBURG u. MEIWES (1999) berichten ebenfalls aus einer ungekalkten Level II-Fläche mit 160-jähriger Buche (B1 Solling) von einer Zunahme der Kohlenstoffvorräte im Auflagehumus. 1/3 der Streu wird dort rechnerisch nicht mineralisiert, was zu jährlichen Kohlenstoffzunahmen von 0,5 t/ha führte. Diese Größenordnung wird in den hier untersuchten Flächen bestätigt. Untersuchungen an Rotfichten- und Tannenbeständen im Schwarzwald belegen ebenfalls einen durch Kalkung induzierten Humusabbau, welcher ebenfalls nicht mit einem Kohlenstoffverlust verbunden war, da der Kohlenstoff im Mineralboden angereichert wurde (ALDINGER 1987).

Die zweimalige Zufuhr von magnesiumhaltigen Kalken auf den Versuchsflächen Sellhorn, Göhrde und Dassel hat dazu geführt, dass Defizite in der Calcium- und Magnesiumversorgung der Buchenblätter ausgeglichen werden. Die nachgewiesene Kalkungswirkung hält auch zehn Jahre nach der letzten Kalkung noch an: ab 1997 werden für die Calciumgehalte, ab 2004 auch für die Magnesiumgehalte auf den Kalkungsparzellen etwa doppelt so hohe Werte in den Blattgehalten verzeichnet wie auf den Kontrollparzellen. Über eine erfolgreiche Erhöhung der Calcium- und Magnesiumgehalte bei Buchenblättern nach Kalkung auf basenarmen Standorten berichten auch BÜTTNER et al. (1993) und ASCHE (1994).

Interkostale Vergilbungen stehen im engen Zusammenhang mit Magnesiummangel. BÜTTNER et al. (1993) nennen als Grenzwert für das Auftreten von Vergilbungserscheinungen an Buchenblättern einen Gehalt von 0,6 g/kg Magnesium in der Trockenmasse. Mitentscheidend für das Auftreten und die Intensität der Vergilbung sind Trockenheit und Strahlung. Obwohl Magnesiumgehalte unterhalb des genannten Grenzwertes auf der Kontrollfläche in Dassel im gesamten Auswertungszeitraum vorkommen, werden ab 1998 kaum noch Vergilbungen registriert.

Die Kalium-Gehalte der Buchenblätter der gekalkten Parzellen sinken im Beobachtungszeitraum ab. Eine Erklärung bietet der Kalium/Calcium-Antagonismus 
der Wurzeln bei der Aufnahme dieser Ionen (HÜTTL 1991). Während auf den Flächen Sellhorn und Dassel die Buchen der gekalkten Parzellen ab 2004 nur gering mit Kalium versorgt sind bzw. Kalium-Mangel aufweisen, wird auf allen Kontrollparzellen eine gute bis sehr gute Kalium-Versorgung beibehalten. Aus der Literatur ergibt sich für Buche und Fichte kein einheitliches Bild über die KaliumGehalte in den Blattorganen nach Kalkung. Während BÜTTNER et al. (1993), AsCHE (1994) und MEESENBURG et al. (2001) explizit darauf verweisen, dass die Kalium-Gehalte der Buchenblätter durch die Kalkung nur gering beeinflusst wurden, wurde ein Rückgang der Kalium-Gehalte nach Kalkungsmaßnahmen in anderen Untersuchungen häufiger festgestellt (ALDINGER 1987, v. WILPERT 2003, WAGNER 2007, DAMMANN et al. 2008). Dieser Begleiterscheinung der Kalkung sollte weiter nachgegangen werden, um die Kalium-Versorgung bei der Kalkungsplanung angemessen berücksichtigen zu können. Die nach der Bodenschutzkalkung deutlich bessere Versorgung der Buchenblätter mit Calcium und Magnesium gegenüber teilweise kritischen Gehalten der Blätter des Kontrollkollektives unterstreicht jedoch den positiven Effekt und die Notwendigkeit der Bodenschutzkalkung.

Die Ergebnisse der in dieser Arbeit untersuchten Versuchsreihen konnten zeigen, dass die durchgeführten Kalkungen positive Wirkungen auf die Vitalität von Buchenbeständen und die Stabilität des Waldbodens hatten. Bei Kalkungsmaßnahmen sollten die standörtlichen Gegebenheiten, die Ertragssituation und die bereits ausgebrachten Kalkmengen unbedingt berücksichtigt werden, um die natürliche Leistungskraft der Waldstandorte möglichst wiederherzustellen und nachhaltig zu erhalten. Ergebnisse langfristig angelegter Versuche können hier zur Entscheidungsunterstützung beitragen.

\section{Literatur}

ALDINGER, E. (1983): Gesundheitszustand von Nadelholzbeständen auf gedüngten und ungedüngten Standorten im Buntsandstein-Schwarzwald. AFZ 38, 794-796

ALDINGER, E. (1987): Elementgehalte im Boden und in Nadeln verschieden stark geschädigter Fichten-Tannen-Bestände auf Praxiskalkungsflächen im Buntsandstein-Schwarzwald. Freiburger Bodenkundliche Abhandlungen Heft 19, Freiburg im Breisgau, 266 S.

Aldinger, E. u. KREMER, W.L. (1985): Zuwachsuntersuchungen an gesunden und geschädigten Fichten und Tannen auf alten Praxiskalkungsflächen. Forstw. Cbl. 104, 360-373

ARBEITSKREIS STANDORTSKARTIERUNG (2003): Forstliche Standortsaufnahme. Arbeitskreis Standortskartierung in der Arbeitsgemeinschaft Forsteinrichtung, IHW-Verlag Eching bei München, $352 \mathrm{~S}$.

ASCHE, N. (1994): Elementgehalte von Buchenblättern auf ausgewählten Kalkversuchsflächen. AFZ $14,792-794$

BEESE, F.O. (1996): Indikatoren für eine multifunktionale Waldnutzung. Forstw. Cbl. 115, 65-79

BMVEL (2001): Dauerbeobachtungsflächen Waldschäden im Level II-Programm - Methoden und Ergebnisse der Kronenansprache seit 1983. Arbeitskreis „Krone“ der Bund-Länder Arbeitsgruppe Level II, Bundesministerium für Verbraucherschutz, Ernährung und Landwirtschaft, Referat 533, Bonn, 84 S. und Anhang 
BMVEL (2004): Die zweite Bundeswaldinventur - BWI². Das Wichtigste in Kürze. Bundesministerium für Verbraucherschutz, Ernährung und Landwirtschaft, Referat Öffentlichkeitsarbeit, Berlin

BütTner, G.; Hartmann, G. u. Thomas, M. (1993): Vorzeitige Vergilbung und Nährstoffgehalte des Buchenlaubes in Südniedersachsen. Forst und Holz 48, 627-630

Dammann, I.; Helbig, M.; PaAr, U.; Gawehn, P.; Eichhorn, J.; Schröck, H.W.; Mayer, F.-J. u. ZIEGLER, CH. (2003): Ausgewählte Auswertungen zu regionalen Entwicklungen des Kronezustandes, Ergebnisse und Hypothesen zu Ursachen und Wirkungen. Workshop Integrierende Auswertung der Daten des Forstlichen Umweltmonitoring (Level I/II) vom 24.-26.Februar 2003 in Bonn-Röttgen, BMVEL/IFOM Projekt des BMBF, 155-181

DAmmann, I.; Mindrup, M. u. SCHÖNFELDER, E. (2008, angenommen): Entwicklung der Kalium-, Calcium- und Magnesium-Nährstoffversorgung von ausgewählten Waldbeständen in Niedersachsen und ihre Bedeutung für die Biomassenutzung. Forst und Holz

Gauger, Th.; Anshelm, F.; Schuster, H.; Erisman, J.W.; Vermeulen, A.T.; Draaijers, G.P.J.; BleECKer, A. u. NAGEL, H.-D. (2002): Mapping of ecosytem specific long-term trends in deposition loads and concentrations of air pollutants in Germany and their comparison with critical Loads and Critical Levels. Teil 1 Depositon Loads 1990 - 1999. Institut für Navigation der Universität Stuttgart und Umweltbundesamt (Final Repot 29942 210), 207 S.

Hölscher, D.; Asche, N. u. BeESE, F. (1999): Langfristige Effekte einer Waldkalkung auf bodenchemische Parameter, mikrobielle Biomasse und Regenwurmbesatz. Forstarchiv 70, 127-132

HÜTTL, R.F. (1991): Die Nährelementversorgung geschädigter Wälder in Europa und Nordamerika. Freiburger Bodenkundl. Abhandlungen, Habilitationsschrift, 28, $440 \mathrm{~S}$.

KÖnIG, N. u. ForTmann, H. (1996): Probenvorbereitungs-, Untersuchungs- und Elementbestimmungs-Methoden des Umweltanalytik-Labors der Nieders. Forstl. Versuchsanstalt, Teil 1-3: Elementbestimmungsmethoden A-Z, Gerätekurzanleitung und Datenverarbeitung, Berichte des Forschungszentrums Waldökosysteme Göttingen, Reihe B, Bd. 46, 47, 48

KÖNIG, N. u. ForTMAnN, H. (1999a): Probenvorbereitungs-, Untersuchungs- und Elementbestimmungs-Methoden des Umweltanalytik-Labors der Nieders. Forstl. Versuchsanstalt, 1. Ergänzung: 1996 - 1998 Teil 1-3: Elementbestimmungsmethoden A-Z, Untersuchungs- und Vorbereitungsmethoden, Gerätekurzanleitungen, Qualitätskontrolle, Datenverarbeitung. Berichte des Forschungszentrums Waldökosysteme Göttingen, Reihe B, Bd. 58, 59 und 60

KÖnIG, N. u. ForTMAnn, H. (1999b): Probenvorbereitungs-, Untersuchungs- und Elementbestimmungsmethoden des Umweltanalytik-Labors der Niedersächsischen Forstlichen Versuchsanstalt und des Zentrallabors II des Forschungszentrums Waldökosysteme. Teil 4: Probenvorbereitungs- u. Untersuchungsmethoden, Qualitätskontrolle und Datenverarbeitung. Berichte des Forschungszentrums Waldökosysteme Göttingen, Reihe B, Bd. 49, 1996, 435 S.

Kramer, H. (1988): Waldwachstumslehre, Parey Verlag, Hamburg Berlin, 374 S.

LEuBE, F. (2000): Leitfaden Forstliche Bodenschutzkalkung in Sachsen. Schriftenreihe der Sächsischen Landesanstalt für Forsten, Heft 21, $58 \mathrm{~S}$.

LeusChNeR (1998): Mechanismen der Konkurrenzüberlegenheit der Buche. Ber. d. Reinh.-TüxenGes. Hannover 10, 5-18

LÖWE-ERLASS (2007): Langfristige, ökologische Waldentwicklung in den Niedersächsischen Landesforsten. RdErl. d. ML v. 20.3.2007 -405- 64210-56.1-

Meesenburg, H. u. MeiWes, K.J. (1999): Veränderung der Elementvorräte im Boden von Buchenund Fichtenökosystemen im Solling. Berichte Freiburger Forstliche Forschung 7, 109-114

Meesenburg, H.; Meiwes, K.J.; Wagner, M. u. Prenzel, J. (2001): Ecosystem effects after ameliorative liming of a catchment at the Harz mountains, Germany. Plant nutrition, 914-915

MeIWEs, K.J. (1994): Meliorationsmöglichkeiten. In: MatschulLat et al. (1994): Gefahr für Ökosysteme und Wasserqualität. Springer Berlin, 415-431

Beiträge aus der NW-FVA, Band 3, 2008 
Moore, J.D.; Duchesne, L. u. Ouimet, R. (2008): Soil properties and maple-beech regeneration a decade after liming in a northern hardwood stand. Forest Ecology and Management 255, 34603468

MRLU (1997): Verbindlichkeit der Leitlinie zur Erhaltung und nachhaltigen Entwicklung des Waldes in Sachsen-Anhalt (Leitlinie Wald). RdErl. des Ministeriums für Raumordnung, Landwirtschaft und Umwelt Sachsen-Anhalts. MBl LSA Nr. 51/1997 vom 17.11.1997, 1871-1894

NLF (2007): Das LÖWE-Programm. 15 Jahre langfristig ökologische Waldentwicklung. Niedersächsische Landesforsten, Braunschweig

RiBeS 2002: Richtlinie für die Bewirtschaftung des Hessischen Staatswaldes. www.Hessenforst.de/service/download/RiliRibes.pdf

RÖHE, P. (1985): Untersuchungen über das Wachstum der Buche in Baden-Württemberg, Schriftenreihe der Landesforstverwaltung Baden- Württemberg, Stuttgart, Bd. 61

Spellmann, H. u. MeIWES, K.J. (1995): Positive Auswirkung der Kalkung. Kalksteigerungs- und Bodenbearbeitungsversuch Dhronecken 148. AFZ 50, 71-73

Sverdrup, H.; Warfinge, P.; BLAKe, L. u. Goulding, K. (1995): Modelling recent and historic soil data from the Rothamsted Experimental Station, UK using SAFE. Agriculture, Ecosystems and Environm. 53, 161-177

UlRICH, B. (1995): Der ökologische Bodenzustand - seine Veränderung in der Nacheiszeit, Ansprüche der Baumarten. Forstarchiv 66, 117-127

WAGNER, M. (2007): Analyse und Modellierung langfristiger Auswirkungen einer hoch dosierten Kalkungsmaßnahme auf den Stoffaustrag im Einzugsgebiet der Steilen Bramke (Oberharz). Cuvillier Verlag Göttingen, 160 S.

WiLPERT, K. V. (2003): Drift des Stoffhaushalts im Fichten- Düngeversuch Pfalzgrafenweiler. AFJZ $174,21-30$

WolfF, B.; Erhard, M.; Holzhausen, M. u. Kuhlow, T. (2003): Das Klima in den Forstlichen Wuchsgebieten Deutschlands. Mitteilungen der Bundesforschungsanstalt für Forst- und Holzwirtschaft Hamburg, Nr. 211

WolfF, B.; RIEK, W.; BARITZ, R. u. HeNNIG, P. (1997): Deutscher Waldbodenbericht 1996. BMELF Bonn, $141 \mathrm{~S}$.

Korrespondierender Autor:

Dr. Jan Evers

Nordwestdeutsche Forstliche Versuchsanstalt

37079 Göttingen

Grätzelstr. 2

E-Mail: Jan.Evers@nw-fva.de

URL: www.nw-fva.de

Inge Dammann

Axel Noltensmeier

Ralf-Volker Nagel

Nordwestdeutsche Forstliche Versuchsanstalt 


\section{Genetische Vielfalt nordwestdeutscher Buchenwälder}

\section{Genetic diversity of beech forests in northwest Germany}

Alwin Janßen, Karl Gebhardt und Wiffried Steiner

\section{Zusammenfassung}

Seit 1991 wird die genetische Variation der Buche in Hessen und Niedersachsen mit dem Ziel untersucht und erfasst, Entscheidungshilfen für ein genetisches Management auf Bestandesebene zur Verfügung zu stellen. Dazu wurde die genetische Variation von 19 hessischen Beständen in 12 Wuchsgebieten und in verschiedenen Höhenlagen beschrieben. Daneben wurden der Einfluss verschiedener Saatguternte- und Anzuchtverfahren sowie der Effekt unterschiedlicher Bewirtschaftungskonzepte und Intensitätsgrade von Durchforstungen auf die genetischen Strukturen untersucht.

Insbesondere vor dem Hintergrund stärkerer Witterungsextreme und der prognostizierten Klimaänderung, die hohe Anpassungsleistungen der Baumart Buche erfordern werden, sollte ein genetisches Monitoring erfolgen, das Auskunft über die Intaktheit ihres genetischen Systems und über dessen mögliche zeitliche und räumliche Veränderungen geben kann.

Stichworte: Buche, genetische Variation, Anpassungsfähigkeit, waldbaulicher Einfluss, genetisches Monitoring 


\begin{abstract}
In Hesse and Lower Saxony, Germany, the genetic variation in beech has been investigated and recorded since 1991 to provide decision support for the management of this forest genetic resource. Thus, the genetic variation of 19 stands in Hesse, located in 12 ecological regions at different elevations, has been described. In addition, the effect of different seed harvest and propagation methods, and of different management practices and thinning intensities on genetic structures were investigated.

In the face of greater weather extremes and predicted climate change, which will call for high adaptability from beech, genetic monitoring of beech should be undertaken to gain a better understanding of its genetic integrity, and identify any temporal and spatial variation.
\end{abstract}

Keywords: beech, genetic variation, adaptability, influence of silviculture, genetic monitoring

\title{
1 Einleitung
}

Die 1992 bei der UNCED-Konferenz in Rio de Janeiro beschlossene Konvention über die Biologische Vielfalt beinhaltet den Schutz und die nachhaltige Nutzung der Biodiversität (SECRETARIAT OF THE CONVENTION ON BIOLOGICAL DiveRSITY 2005). Die genetische Vielfalt ist ein essentieller Bestandteil der Biodiversität, auf der die Anpassungsfähigkeit von Baumarten an sich ändernde Umweltbedingungen und damit ihre Überlebensfähigkeit basieren (ZIEHE et al. 1999). Die Vielfalt genetischer Strukturen bestimmt neben der Anpassungsfähigkeit auch die Leistungsfähigkeit von Wäldern. Sie ist Grundlage einer nachhaltigen und stabilen Entwicklung von Waldbeständen.

Im Zuständigkeitsbereich der Nordwestdeutschen Forstlichen Versuchsanstalt (Bundesländer Hessen, Niedersachsen, Sachsen-Anhalt und Schleswig-Holstein) bilden die Buchenwälder unter den jetzigen Standortbedingungen in weiten Teilen die Klimaxgesellschaften. Etwa $75 \%$ der betreuten Fläche wären unter natürlichen Bedingungen mit Buchenwald bedeckt. Nach FIRBAS (1949, 1952) ist für Hessen seit etwa 5.000 Jahren von einem geschlossenen Buchenwald auszugehen. Die Buche konnte sich also über rund 20 Buchengenerationen an die in Nordwestdeutschland herrschenden Umwelt- und Standortbedingungen anpassen. Dabei muss in diesem Bereich für die Buche momentan noch von optimalen Wuchsbedingungen ausgegangen werden.

Viele unserer heimischen Wälder waren Anfang der 1980er Jahre akut durch Luftschadstoffimmissionen gefährdet. In den Hochlagen des Erzgebirges waren zu dieser Zeit bereits ganze Bestände abgestorben. Der Deutsche Bundesrat hatte deshalb 1985 „Maßnahmen zur Erhaltung der genetischen Vielfalt der Waldbaum- 
arten“ beschlossen (BUNDESRAT 1985). Daraufhin hat eine Bund-Länder-Arbeitsgruppe ein entsprechendes Konzept zur Erhaltung forstlicher Genressourcen erstellt (MELCHIOR et al. 1989), das inzwischen aufgrund der veränderten politischen Konstellation und aufgrund neuer Erkenntnisse angepasst worden ist (PAUL et al. 2001). Neben der Erhaltung seltener Baumarten lag ein Schwerpunkt in Hessen und Niedersachsen auf der Beerntung von Buchenbeständen. Das Buchensaatgut wurde zum Teil in Forstgenbanken eingelagert, zum Teil wurden Erhaltungsflächen als „genetische Kopien“ der Erntebestände angelegt. Vor dem Hintergrund der zu dieser Zeit herrschenden Unkenntnis über die genetische Variation der Buche in Deutschland und über die genetischen Strukturen der Buchenbestände wurde sowohl in Hessen als auch in Niedersachsen ein umfassendes Untersuchungsprogramm zur Klärung genetischer Fragestellungen begonnen, deren wichtigste Ergebnisse im Folgenden dargestellt werden.

Gerade auch im Hinblick auf die prognostizierten Klimaänderungen ermöglichen die genetischen Untersuchungen zusammen mit den Ergebnissen aus Herkunftsversuchen und Nachkommenschaftsprüfungen eine Aussage aus genetischer Sicht über die zukünftige Eignung von Beständen und daraus geerntetem Vermehrungsgut.

\section{Untersuchungen zur genetischen Vielfalt von Buchen- beständen}

\subsection{Untersuchungen hessischer Bestände}

Die genetischen Untersuchungen wurden mit Hilfe von Isoenzymanalysen durchgeführt. Variation an Isoenzymgenorten lässt sich über elektrophoretische Untersuchungen und anschließende enzymspezifische Anfärbungen von Isoenzymen sichtbar machen (MÜLLER-STARCK u. STARKE 1993, ROTHE 1994). 19 Buchenbestände aus allen Wuchsgebieten in Hessen wurden in den Jahren 1991 bis 1996 in die Untersuchungen einbezogen (s. Abb. 1). Ziel war die Klärung folgender Fragestellungen:

- Wie groß ist die genetische Vielfalt der Buche in Hessen?

- Ist diese genetische Vielfalt ausreichend groß oder müssen spezielle Erhaltungsmaßnahmen durchgeführt werden?

- Gibt es regionale oder höhenspezifische Unterschiede zwischen den untersuchten Beständen? 


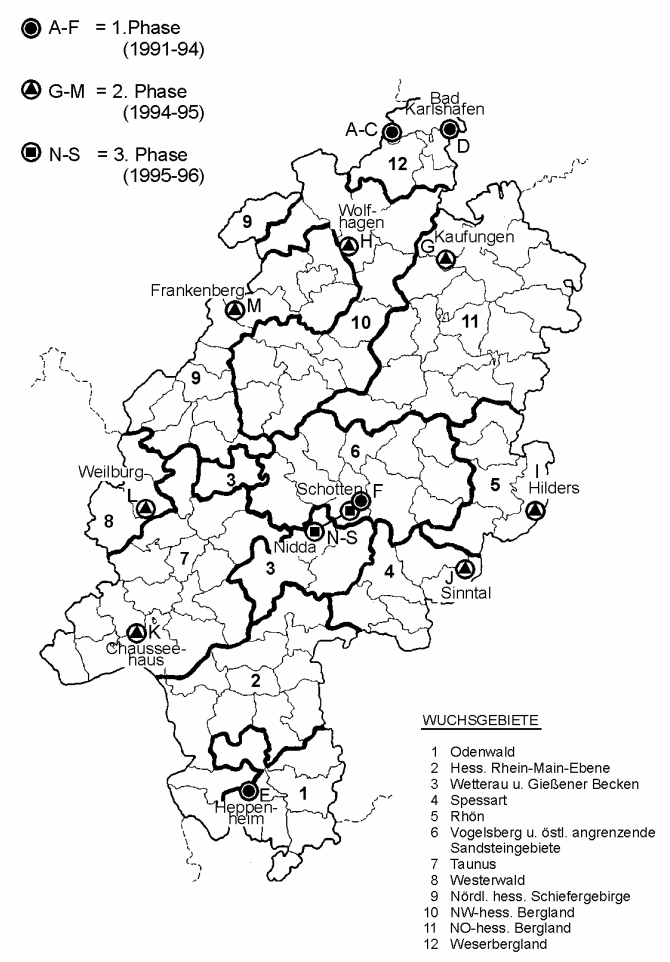

Abbildung 1: Hessische Wuchsgebiete (1-12) und Lage der 19 untersuchten Buchenbestände (A-S) (JANBEN 1998)

Die genetische Variation ist innerhalb der untersuchten Bestände (99\%) weitaus größer als zwischen den Beständen (1\%). Dieses deutet auf eine ausreichend große genetische Vielfalt der Buche in Hessen hin. Spezielle Erhaltungsmaßnahmen wie Saatgutbeerntungen, Saatguteinlagerungen in der Forstgenbank oder die Anlage von Ex-situ-Beständen sind daher nicht erforderlich (SANDER et al. 2001).

Die relativ hohen Subpopulationsdifferenzierungen (s. Abb. 2) resultieren aus den markanten Familienstrukturen in den Beständen. Der Verwandtschaftsgrad nimmt dabei mit der räumlichen Entfernung stark ab. Die Ergebnisse decken sich mit denen anderer bundesdeutscher Untersuchungen (KONNERT et al. 2000, ZIEHE et al. 2002).

Bei der Analyse von sechs autochthonen Beständen entlang eines Höhengradienten am Vogelsberg (150-660 m ü. NN) korrelierte die Häufigkeit bestimmter Genotypen mit der Höhenlage (s. Abb. 3). Beim Enzymsystem AAT ist eine Abnahme des Genotyps C1C1 von 17\% im Tieflagenbestand R (150-180 m ü. NN) bis hin zu 9\% im am höchsten gelegenen Bestand O (600-660 m ü. NN) zu beobachten. Beim Genotyp A2A3 des Enzymsystems SKDH findet sich ein 
Anstieg in der Häufigkeit von $1 \%$ in den Beständen R, S und Q bis zu $13 \%$ im Bestand O. Umgekehrt verhält es sich beim Genotyp A2A2, der von $98 \% \mathrm{im}$ Bestand R bis auf 87 \% im Bestand O abnimmt (SANDER et al. 2000). Eine höhenspezifische Anpassung bestimmter Genotypen und Allele wird auch bei vergleichbaren Untersuchungen am Schauinsland im Schwarzwald attestiert (LÖCHELT u. FRANKE 1995).

Dieser nachgewiesene Zusammenhang hat in das Forstsaatgutrecht bereits Eingang gefunden. Seit Mitte der 1990er Jahre werden die Herkunftsgebiete regional größer und zusätzlich nach Höhenstufen getrennt ausgewiesen.

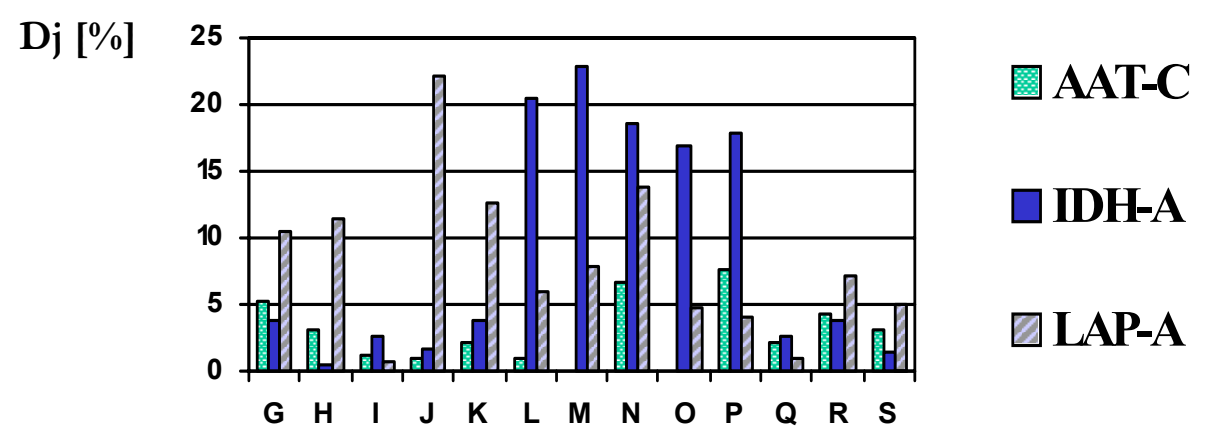

Abbildung 2: Subpopulationsdifferenzierung (Dj) von 13 hessischen Buchenbeständen an drei IsoenzymGenorten (AAT-C, IDH-A, LAP-A) (SANDER et al. 2001)

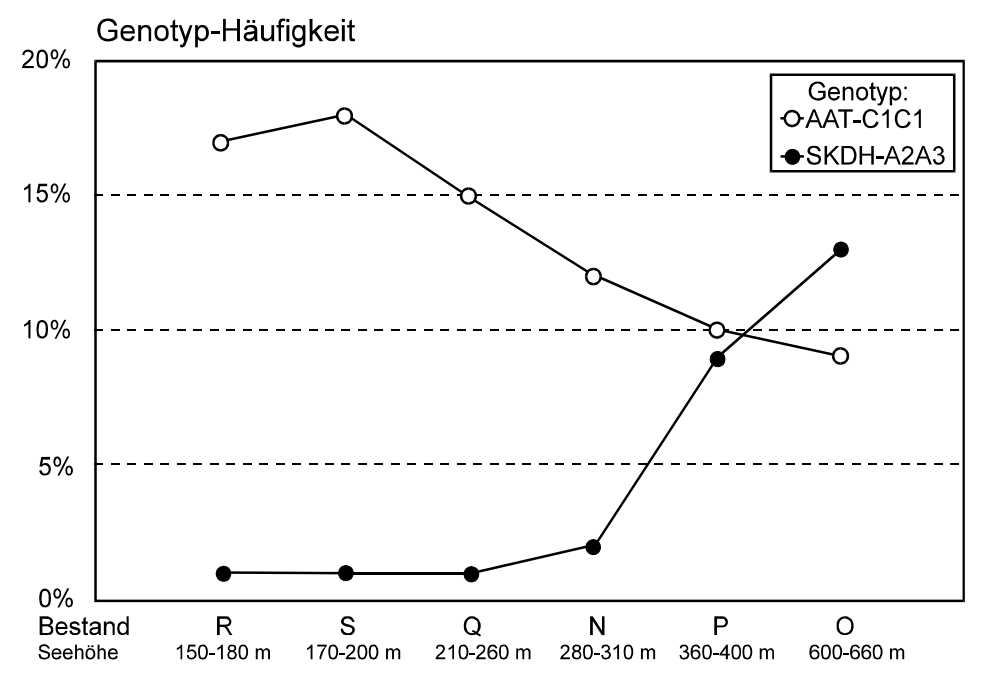

Abbildung 3: Genetische Differenzierung von Rotbuche in sechs Höhenzonen in Hessen, dargestellt an den Genorten AAT-C und SKDH-A (JANBEN 1998) 


\subsection{Untersuchungen in einem Naturwaldreservat}

Diese Untersuchungen wurden im Jahr 2001 begonnen und sind längerfristig im Sinne eines genetischen Monitorings angelegt. Zunächst wurden im 1988 ausgewiesenen Naturwaldreservat „Niestehänge“ im damaligen Hessischen Forstamt Witzenhausen, die Buchen an den $100 \times 100$ m - Gitternetzpunkten sowohl in der Kernzone als auch in einem angrenzenden bewirtschafteten Vergleichsbestand genetisch analysiert. Danach wurden sowohl im Naturwaldreservat als auch im Wirtschaftswald jeweils 4 Hektar große Vergleichsflächen ausgewählt und alle dort vorhandenen 648 Altbuchen genetisch untersucht.

Die Maßzahlen für die Genpool-Diversität der identifizierten EinzellocusGenotypen erwiesen sich als relativ ähnlich zwischen den untersuchten Beständen (s. Tab. 1). Einzig die höhere hypothetische gametische Multilocus-Diversität des Naturwaldreservats Niestehänge $\left(\nu_{\text {gam }}=65,1\right)$ verweist auf das bessere Bildungsvermögen unterschiedlicher Genotypen in diesem Bestand. Der Anteil der Heterozygoten war wiederum in der bewirtschafteten Vergleichsfläche des Staatswaldes höher $(4,4 \%)$ als im Naturwaldreservat.

Neben den Isoenzymanalysen kamen bei Buchen auch DNA-Analysen zum Einsatz (GEBHARDT 2003). Mit PCR-basierten Techniken der DNA-Analytik ist es gelungen, sogenannte genetische Fingerabdrücke zu erzeugen. Damit können einzelne Individuen eines Buchenbestandes an zahlreichen ISSR-Genorten (InterSimple Sequenz Repeats) unterschieden werden. Es wird so möglich, einen Einzelbaum zweifelsfrei zu identifizieren. 2003 wurde mit dem Aufbau einer Bank mit genomischer DNA von Buchen aus unterschiedlichen hessischen Beständen begonnen. Die Bank umfasst derzeit 1.250 Akzessionen, die für diverse Untersuchungen verfügbar sind.

Tabelle 1: Genetische Maße (GILLET 1994) der Vergleichsflächen im Naturwaldreservat „Niestebänge" (NWR-Nieste) und angrenzendem Wirtschaftswald (VGl-Nieste), Quelle: GEBHARDT (2003)

\begin{tabular}{|c|c|c|c|}
\hline \multirow[b]{2}{*}{ Genetische Parameter } & \multirow[b]{2}{*}{ Kurzform } & \multicolumn{2}{|c|}{ Bestände } \\
\hline & & $\begin{array}{l}\text { NWR- } \\
\text { Nieste }\end{array}$ & VG1-Nieste \\
\hline Genpool-Diversität & $v$ & 1,491 & 1,496 \\
\hline $\begin{array}{l}\text { Hypothetisch gametische } \\
\text { Multilocus-Diversität }\end{array}$ & $v_{\text {gam }}$ & 65,1 & 59,1 \\
\hline Totale Populationsdifferenzierung & $\delta_{\mathrm{T}}$ & 0,33 & 0,332 \\
\hline
\end{tabular}




\subsection{Untersuchungen in waldwachstumskundlichen Versuchen}

Im Rahmen eines Forschungsprojektes zu genetischen Auswirkungen von Durchforstungen (siehe Abschnitt 3.6) wurden insgesamt sieben waldwachstumskundliche Versuche mittels Isoenzymen genetisch charakterisiert. Drei Versuchsflächen liegen in Niedersachsen (Lauterberg, Uslar, Unterlüß), jeweils zwei in RheinlandPfalz (Mayen, Winnweiler) und Sachsen (Hirschberg, Olbernhau). Die genetischen Strukturen der unbehandelten Nullflächen unterscheiden sich dabei zum Teil deutlich. Die mittlere Differenzierung liegt mit $\delta=6,1 \%$ etwas höher, als die bei anderen Untersuchungen festgestellten Werte für Buche in Mitteleuropa mit rund $4 \%$ (KONNERT et al. 2000). Tabelle 2 zeigt die genetischen Variationsmaße der sieben unbehandelten Versuchsbestände sowie die Maßzahlen für genetische Vielfalt, allelische Diversität, aktuelle Heterozygotie und Differenzierung.

Die Werte lassen auf ausgeprägte genetische Unterschiede schließen. Die mittleren genotypischen Abstände liegen zwischen 6,1 \% und 19,3\%. Die mittleren allelischen Abstände variieren zwischen 4,1 \% und 13,9\% mit dem überwiegenden Teil der Werte über $6 \%$. An allen Genorten sind die genetischen Unterschiede signifikant auf dem $99,9 \%$ Niveau.

Tabelle 2: $\quad$ Genetische Variation von sieben unbehandelten Versuchsflächen in drei Bundesländern (Maße siehe GILLET 1994)

\begin{tabular}{|c|c|c|c|c|c|c|c|c|c|}
\hline \multirow[t]{2}{*}{ Bestand } & \multirow[t]{2}{*}{$\mathbf{N}$} & \multicolumn{3}{|c|}{ genetische Vielfalt } & \multicolumn{2}{|c|}{$\begin{array}{l}\text { allelische } \\
\text { Diversität }\end{array}$} & \multirow{2}{*}{$\begin{array}{c}\text { aktuelle } \\
\text { Hetero- } \\
\text { zygotie } \\
\mathrm{H}_{\mathrm{a}}[\%]\end{array}$} & \multicolumn{2}{|c|}{$\begin{array}{c}\text { Differen- } \\
\text { zierung }\end{array}$} \\
\hline & & $\mathrm{A} / \mathrm{L}$ & $\mathrm{P}[\%]$ & Gp & $v_{\text {pool }}$ & $\nu_{\text {gam }}$ & & $\delta_{\mathrm{T}}[\%]$ & Dj $[\%]$ \\
\hline Hirschberg & 500 & 2,3 & 100 & $8,75^{*} 10^{3}$ & 1,498 & 13,7 & 29,5 & 29,1 & 6,2 \\
\hline Olbernhau & 504 & 2,4 & 100 & $1,75^{*} 10^{4}$ & 1,443 & 11,3 & 26,7 & 28,0 & 8,1 \\
\hline Lauterberg & 832 & 2,4 & 100 & $1,09 * 10^{4}$ & 1,400 & 9,2 & 24,1 & 25,8 & 4,8 \\
\hline Mayen & 179 & 2,7 & 100 & $5,83 * 10^{4}$ & 1,414 & 9,4 & 15,6 & 25,6 & 7,7 \\
\hline Unterlüß & 345 & 2,3 & 89 & $7,29 * 10^{3}$ & 1,421 & 9,8 & 23,7 & 26,1 & 2,5 \\
\hline Uslar & 307 & 2,9 & 100 & $9,72 * 10^{4}$ & 1,423 & 9,9 & 19,9 & 26,4 & 8,8 \\
\hline Winnweiler & 149 & 2,6 & 100 & $2,9 * 10^{4}$ & 1,442 & 10,8 & 21,8 & 27,0 & 8,0 \\
\hline
\end{tabular}

\section{Beeinflussungen der genetischen Variation}

\subsection{Untersuchungen zur Art der Beerntung}

Bei Bestandesbeerntungen interessiert vor allem die Frage, inwieweit das geerntete Saatgut den Ausgangsbestand genetisch repräsentiert. Um dies zu überprüfen wurde der Genfluss in einem der unter Abschnitt 2.1 genannten Bestände im 
damaligen Hessischen Forstamt Bad Karlshafen (Bestand A, s. Abb. 1) untersucht. Nach genetischer Analyse der Altbäume und nach Aufnahme des Blühverhaltens wurde systematisch anhand eines Rasterverfahrens Saatgut geerntet und genetisch analysiert. Aus den Daten wurde ein Computersimulationsmodell erstellt, an dem unterschiedliche Beerntungsverfahren getestet wurden (JANßEN 2000).

Daraus konnten Empfehlungen zur Saatguternte abgeleitet werden, die im Beitrag von KLEINSCHMIT et al. in diesem Band dargestellt sind.

\subsection{Abstammungsrekonstruktion}

Für Zwecke der Zertifizierung und Kontrolle von Saatgutpartien, die in den Handel gelangen oder aus denen Pflanzen angezogen werden, kann eine Abstammungsrekonstruktion mithilfe genetischer Marker vorgenommen werden. Wie WYPUKOL et al. (2008) berichten, war es für die rückwirkende Unterscheidung von fünf hessischen Saatguterntebeständen erforderlich, vier Kern-MikrosatellitenGenorte an 14 bis 15 potenziellen Erntebäumen zu charakterisieren. Die Zuordnung des mit Netzen geernteten Saatgutes zum Erntebestand wurde durch DNAAnalyse der Bucheckern-Schalen möglich, da der Genotyp der Schalen dem des Mutterbaumes 1:1 entspricht (s. Abb. 4). Außerdem wiesen alle Bestände an unterschiedlichen Loci private Allele auf, allerdings in sehr geringen Häufigkeiten.

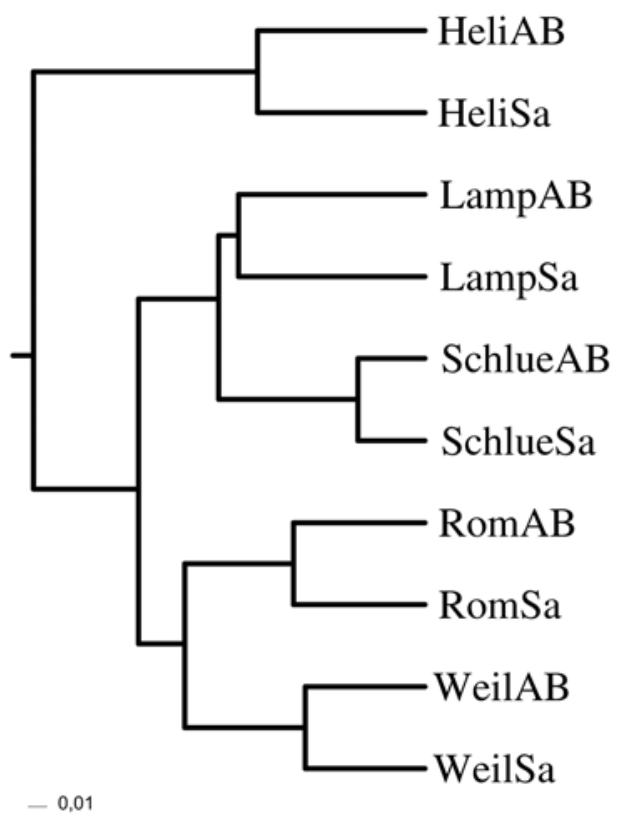

Abbildung 4: Gruppierung (UPGMA-Methode) der Multilocus-Genotypen der Altbäume (AB) und Samenschalen (Sa) von 5 hessischen Buchenbeständen (Heli: Hess. Lichtenau, Lamp: Lampertheim, Schlue: Schlüchtern, Rom: Romrod, Weil: Weilburg) 
Unter der Vorraussetzung, dass bei der Saatguternte eine repräsentative Stichprobe eingelagert wird, ergibt sich nach der Anzucht von Pflanzen die Möglichkeit der Rückverfolgung, da die Allelhäufigkeiten der im Saatgut vorhandenen Embryonen denen der daraus angezogenen Pflanzen entsprechen müssen.

\subsection{Einflüsse in der Anzuchtphase}

In der Anzuchtphase können Einflüsse wie beispielsweise die Größensortierung oder unterschiedliche Beschattung die genetischen Strukturen des Vermehrungsgutes verändern. Dazu wurden Bucheckern aus 16 verschiedenen Beständen (Saatgut aus 12 hessischen Beständen sowie aus je einem Bestand aus Schweden, Belgien, Bayern und Niedersachsen), während dreier Jahre im Freiland und unter einer Beschattung von $60 \%$ bzw. $80 \%$ angezogen. Dabei überlebten unter den jeweiligen Beschattungen nur ein Drittel bzw. ein Viertel der Buchenpflanzen im Vergleich zu den ohne Beschattung aufgewachsenen Buchen. Dabei gab es sehr große Unterschiede zwischen den Mortalitätsraten der Herkünfte. Dies weist auf ein hohes Selektionspotenzial durch die Beschattung bzw. die damit einhergehenden Umweltbedingungen (z. B. Infektionsdruck durch Pilze) hin.

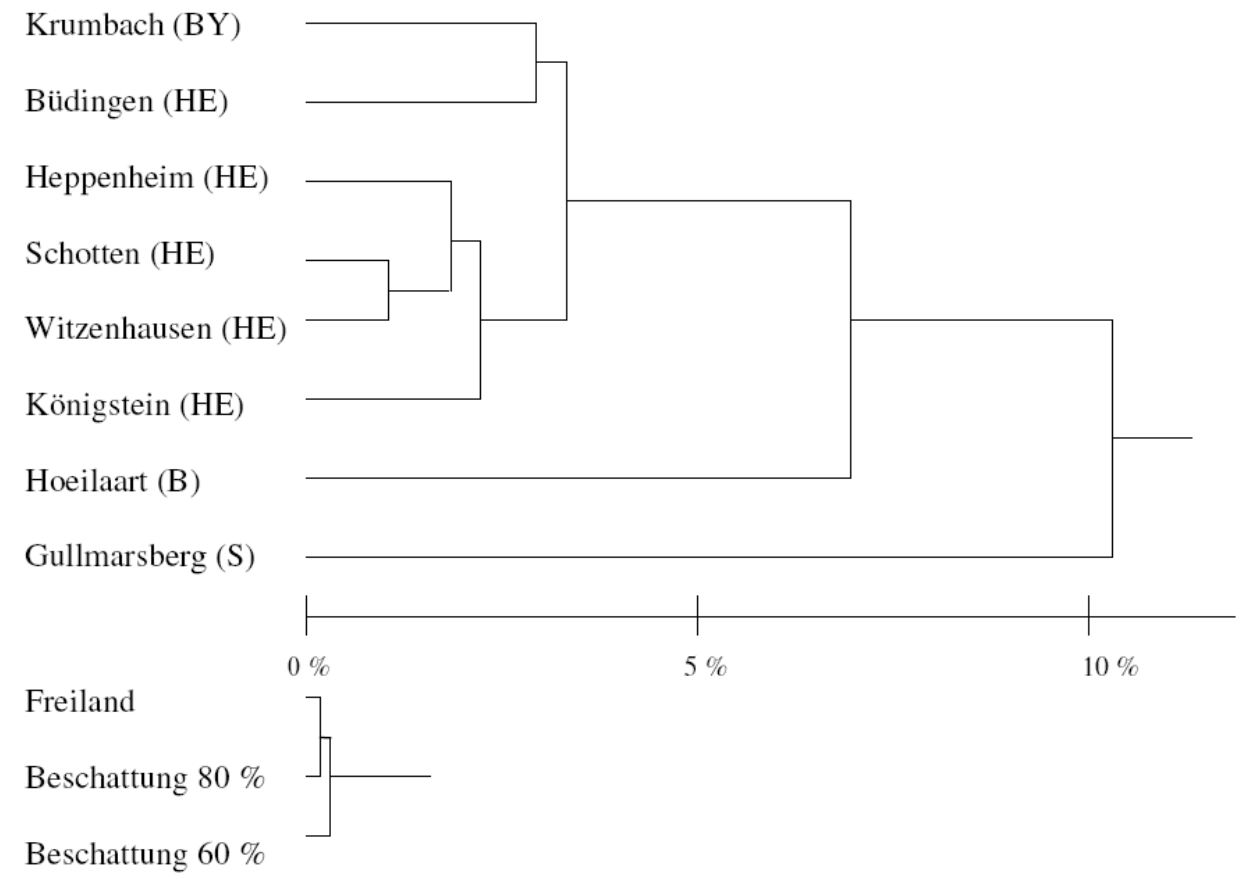

Abbildung 5: Genetische Abstände zwischen den Bestandesabsaaten (oben) und zwischen den Anzuchtvarianten mit unterschiedlichem Beschattungsgrad (unten) (JANßEN et al. 2002) (Abk.: $B=$ Belgien, $B Y=$ Bayern, $H E=H e s s e n, S=S$ chweden $)$ 
Auch der Höhenwuchs und das Austriebverhalten wurden signifikant von der Beschattung beeinflusst. Darüber hinaus wiesen die verschiedenen Herkünfte zueinander in ihren phänotypischen Merkmalen deutliche Unterschiede auf. Die genetischen Untersuchungen ergaben, berechnet über alle Beschattungsvarianten, einen signifikanten Unterschied zwischen den einzelnen Beständen, was auf die Herkunftsunterschiede zurückzuführen war (JANßEN et al. 2002). So waren beispielsweise die Buchenpflanzen der schwedischen Herkunft mit einem genetischen Abstand von 10,2 \% deutlich von den anderen Herkünften zu unterscheiden. Die Beschattung führte, berechnet über alle Herkünfte, an den untersuchten IsoenzymGenorten trotz der extrem unterschiedlichen Mortalitätsraten zu keinen Unterschieden an den untersuchten Genorten (s. Abb. 5).

\subsection{Vergleich phänotypisch unterschiedlicher Absaaten}

Im Allgemeinen gibt es im Forstbereich keine genetischen Marker, die für die forstlich relevanten Parameter, insbesondere Wuchs- und Qualitätsleistung, verantwortlich sind. Die zur Verfügung stehenden Marker erlauben jedoch eine allgemeine Beschreibung der genetischen Strukturen hinsichtlich Vielfalt und Diversität auf allelischer und genotypischer Ebene. Es lässt sich die Variation innerhalb von Populationen beschreiben und auch die Differenzierung zwischen Populationen. $\mathrm{Ob}$ dabei die forstlich relevanten und erwünschten Merkmale (Qualität und Wuchsleistung) mit besonderen genetischen Strukturen einhergehen, ist nur in wenigen Fällen untersucht.

Im Buchen-Herkunftsversuch von 1959 im Niedersächsischen Forstamt Münden (KLEINSCHMIT u. SVOLBA 1996) wurden vier Versuchsglieder genetisch untersucht, darunter die Provenienzen Zwiesel-Ost und Metzingen, die sich bezüglich der Stammqualität extrem unterscheiden. An den Ergebnissen zeigte sich, dass die auffallenden Qualitätsunterschiede hier mit genetischen Unterschieden an den Isoenzym-Genorten korrelieren (STEINER et al. 2008).

Die hervorragende Herkunft „Zwiesel-Ost“ wies bei der allelischen Diversität (sowohl bei der Genpool-Diversität als auch bei der hypothetisch gametischen Multilocus-Diversität) und der Heterozygotie die höchsten Werte auf. Die qualitativ sehr schlechte Herkunft Metzingen hatte diesbezüglich die niedrigsten Werte (s. Abb. 6). Die genetischen Untersuchungen unterstützen damit die phänotypisch-morphologischen Beobachtungen: Forstlich relevante Qualität ist nicht nur auf waldbauliches Handeln, sondern zu beträchtlichen Teilen auch auf genetisch vorhandene Unterschiede zurückzuführen.

Bei zwei weiteren Provenienzen aus Niedersachsen (Knobben und Kupferhütte) konnten auch die in den ursprünglichen Erntebeständen entstandenen Naturverjüngungen beprobt und genetisch untersucht werden. Auffällig ist, dass die unterschiedlichen Wuchsbedingungen während der letzten 45 Jahre (Freiflächenpflanzung nach Anzucht im Kamp gegenüber Naturverjüngung unter 
Schirm) nur zu sehr geringen Unterschieden in den populationsgenetischen Merkmalen führten.

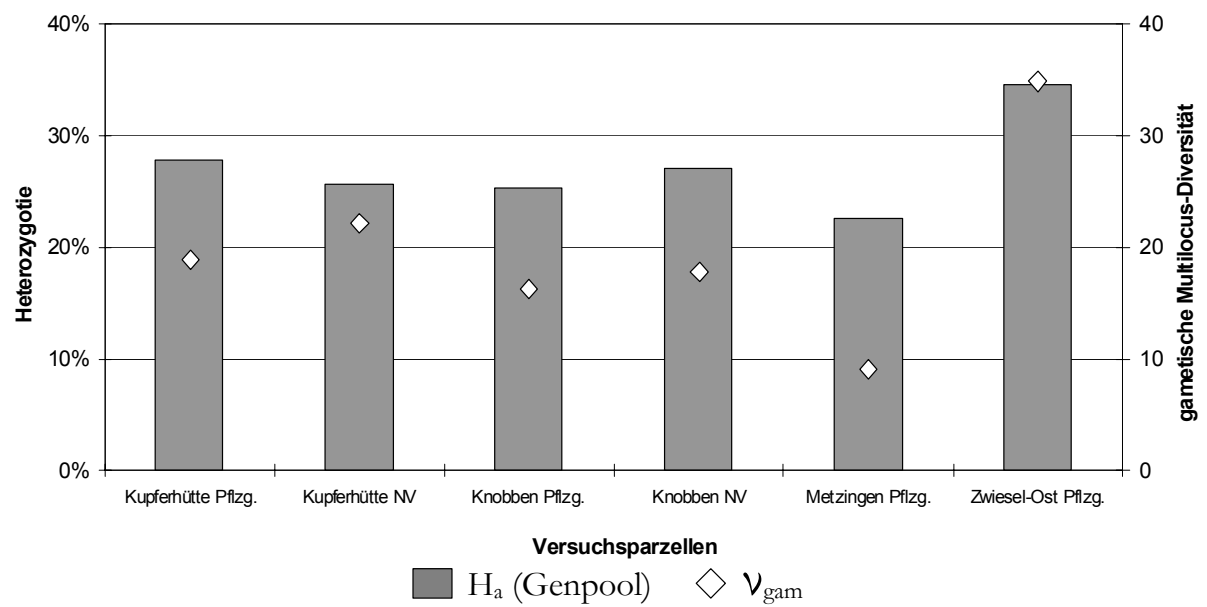

Abbildung 6: Heterozygotie und hypothetisch gametische Multilocus-Diversität ( $\left.v_{\text {gan }}\right)$ gepflanzter und naturverjüngter Buchenherkë̈fte (STEINER et al. 2008)

\subsection{Einfluss der Bewirtschaftungsart}

Zwei direkt benachbarte etwa 120-jährige Buchenbestände im Hessischen Forstamt Wehretal zeigen deutliche Unterschiede in phänotypischen Merkmalen wie Höhenwuchs, Kronenansatzhöhe, Astreinigung, Durchmesser oder Zwieselbildung (s. Tab. 3). Beide Bestände sind im gleichen Zeitraum durch Naturverjüngung entstanden und konnten sich während der Entstehungsphase über Polleneinträge genetisch austauschen. Während der eine Bestand im Kleinprivatwaldbesitz mittelwaldartig mit der hauptsächlichen Zielsetzung der Brennholzentnahme bewirtschaftet wurde, ist der benachbarte Bestand, der sich im Landesbesitz befindet, als Hochwald zur Stammholzproduktion bewirtschaftet worden.

Tabelle 3: $\quad$ Phänotypische Merkmale der Altbuchen im Privat- und Staatswald

\begin{tabular}{|c|c|c|c|c|c|c|c|}
\hline & \multirow{2}{*}{$\begin{array}{c}\text { Baum- } \\
\text { höhe } \\
\text { [m] }\end{array}$} & \multirow{2}{*}{$\begin{array}{l}\text { BHD } \\
{[\mathrm{cm}]}\end{array}$} & \multirow{2}{*}{$\begin{array}{c}\text { Kronen- } \\
\text { ansatz } \\
{[\mathrm{m}]}\end{array}$} & \multirow{2}{*}{$\begin{array}{c}\text { Kronen- } \\
\text { prozent } \\
{[\%]}\end{array}$} & \multirow{2}{*}{$\begin{array}{c}\text { Zwiesel } \\
{[\%]}\end{array}$} & \multicolumn{2}{|c|}{$\begin{array}{c}\text { Bäume mit } \\
\text { Astreinigung [\%] }\end{array}$} \\
\hline & & & & & & mäßig & schlecht \\
\hline alle Parzellen & 30,2 & 36,4 & 11,9 & 60,6 & 28,0 & 31,6 & 10,6 \\
\hline Staatswald & 33,6 & 40,4 & 15,5 & 53,7 & 25,2 & 13,7 & 4,6 \\
\hline Privatwald & 26,7 & 32,2 & 8,2 & 69,4 & 30,8 & 49,7 & 16,6 \\
\hline
\end{tabular}


Beide Bestände wurden mit Hilfe von Isoenzymanalysen genetisch untersucht. Allerdings wurden kaum genetische Unterschiede zwischen den Beständen gefunden (s. Tab. 4). Die unterschiedliche Bewirtschaftung über 120 Jahre, die durch die phänotypischen Merkmale deutlich belegt ist, ließ sich an den untersuchten Isoenzymgenorten nicht nachweisen (JANßEN u. SCHULZ 2002).

Tabelle 4: $\quad$ Genetische Maße bei unterschiedlicher Bewirtschaftung

\begin{tabular}{lccc}
\hline & Genpool-Diversität & $\begin{array}{c}\text { Hypothetisch gametische } \\
\text { Multilocus-Diversität }\end{array}$ & $\begin{array}{c}\text { Genpool- } \\
\text { Distanz }\end{array}$ \\
\hline alle Parzellen & 1,469 & 48,4 & \\
Staatswald & 1,469 & 48,8 & $2,3 \%$ \\
Privatwald & 1,467 & 47,7 & \\
\hline
\end{tabular}

\subsection{Genetische Untersuchungen in Durchforstungsversuchen in Niedersachsen und Rheinland-Pfalz}

Um zukünftig Aspekte der genetischen Anpassungsfähigkeit bei waldbaulichen Eingriffen berücksichtigen zu können, wurden auf fünf waldwachstumskundlichen Versuchsflächen in Niedersachsen und Rheinland-Pfalz undurchforstete Teilbestände (Nullflächen) mit durchforsteten Teilbeständen verglichen. Zusätzlich wurden zwei undurchforstete Bestände aus Sachsen in die Untersuchungen einbezogen.

Außerdem wurden auf den unbehandelten Nullflächen Simulationen verschiedener Durchforstungsvarianten durchgeführt und ihre Auswirkungen auf die genetischen Strukturen des ausscheidenden bzw. verbleibenden Bestandes untersucht. Die Ergebnisse sind bei DOÚNAVI et al. (2002), SCHÜTE u. RUMPF (2003) sowie CREMER et al. (2005) dokumentiert.

Insgesamt betrachtet scheinen einmalige Pflegeeingriffe anhand der vorliegenden Untersuchungsergebnisse einen eher geringen Einfluss auf die genetischen Strukturen von Buchenbeständen bzw. geringe Selektionswirkungen zu haben. Außerdem ist kein Trend dahingehend zu erkennen, dass mit Zunahme der Durchforstungsstärke die genetische Variation wesentlich geringer wird.

Ein sehr ähnliches Bild ergibt sich, wenn Durchforstungseingriffe innerhalb der Nullflächen simuliert werden. Erst bei extrem starken Eingriffen ist ein deutlicher Verlust an allelischer Diversität nachweisbar. Ansonsten sind die durch Durchforstungen verursachten Veränderungen viel geringer als die bereits ursprünglich vorhandenen Unterschiede zwischen den Versuchsflächen.

Die Entnahme der qualitativ guten und starken Bäume (Wertträger) im Zuge der Zielstärkennutzung hat keine wesentlichen Auswirkungen auf die genetischen 
Strukturen des verbleibenden Bestandes und folglich auch nicht auf die des Folgebestandes an den untersuchten Genorten. Geht man allerdings von regelmäßigen, immer wiederkehrenden Durchforstungsmaßnahmen aus, die sich über einen Zeitraum von mehreren Jahrzehnten erstrecken, kann aufgrund dieser Ergebnisse nicht völlig ausgeschlossen werden, dass es zu einer Einengung der genetischen Variation kommen kann.

Ein gleichmäßiger waldbaulicher Eingriff bzw. eine gleichmäßige Entnahme von Bäumen über die Fläche reduziert für seltene Allele, die vorwiegend geklumpt vorkommen, die Gefahr, dass sie als Gruppe entnommen werden.

\subsection{Untersuchungen in hessischen Durchforstungsversuchen}

Um den Einfluss von Durchforstungen auf die genetischen Strukturen von Buchenbeständen zu untersuchen, wurden in zwei waldwachstumskundlichen Versuchen im Stadtwald Laubach und im Staatswald Schotten die nicht oder kaum bewirtschafteten Bestände (Nullparzellen) mit durchforsteten Beständen bezüglich ihrer genetischen Strukturen verglichen. Die genetische Diversität ist dabei in den durchforsteten Beständen etwas höher als in den Nullparzellen. Der Anteil heterozygoter Buchen ist hier ebenfalls in beiden Versuchen höher (s. Tab. 5).

In Bezug auf die genetische Diversität der untersuchten Isoenzym-Genorte ist daher die Bewirtschaftung eher positiv zu sehen. Während unter natürlichen Bedingungen ohne menschlichen Einfluss in den Nullparzellen nur nach dem Gesichtspunkt der Vitalität selektiert wird, kommt in den durchforsteten Parzellen der menschliche Einfluss in Form von qualitativen Selektionskriterien hinzu. Eine qualitativ gute, aber weniger vitale Buche hat hier unter Mithilfe des selektierenden Försters eine größere Überlebenswahrscheinlichkeit (JANBEN u. NOWACK 2001).

Tabelle 5: $\quad$ Genetische Maße in den waldwachstumskundlichen Versuchen Laubach und Schotten

\begin{tabular}{l|cc|cc}
\hline & \multicolumn{2}{|c|}{ Laubach } & \multicolumn{2}{c}{ Schotten } \\
& Nullparzelle & Durchforstung & Nullparzelle $\quad$ Durchforstung \\
\hline $\begin{array}{l}\text { Multilocus- } \\
\text { Diversität }\end{array}$ & 17,3 & 23,1 & 20,6 & 22,2 \\
Differenzierung & 29,8 & 32,3 & 31,5 & 32,0 \\
Heterozygotenanteil & $29,3 \%$ & $30,6 \%$ & $30,2 \%$ & $32,0 \%$ \\
\hline
\end{tabular}




\section{Diskussion und Ausblick}

Für eine heimische Hauptbaumart wie der Buche ist die Kenntnis über die genetischen Strukturen innerhalb der Bestände und über von außen einwirkende Effekte auf die genetische Struktur unbedingt notwendig, um diese zielgerichtet bewirtschaften und verjüngen zu können. In Zeiten prognostizierter Klimaänderungen in relativ kurzer Zeit und in erheblichem Umfang mit bis zu $4{ }^{\circ} \mathrm{C}$ Durchschnittstemperaturanstieg in den nächsten 50 bis 100 Jahren sind diese Kenntnisse außerordentlich wichtig. Aber auch andere Baumarten, die, wie beispielsweise die Traubeneiche, von solchen Klimaänderungen profitieren könnten, dürfen bei den genetischen Untersuchungen nicht außer Acht gelassen werden. Dies gilt natürlich auch für Baumarten wie der Fichte, für die die vorhergesagten Klimaänderungen mit einem Risikoanstieg behaftet sind.

In großen, der Natur überlassenen Gebieten wie dem Buchennationalpark Kellerwald-Edersee oder dem Nationalpark Harz sollte ein genetisches Monitoring installiert werden, um möglichst frühzeitig Auswirkungen von Klimaänderungen, die nicht von unmittelbaren menschlichen Eingriffen überdeckt werden, feststellen zu können. Änderungen der Anpassungsfähigkeit sind von essentieller Bedeutung für die Überlebensfähigkeit. Genetisches Monitoring, wie es von einer Expertengruppe der Bund-Länder-Arbeitsgruppe „Forstliche Genressourcen und Forstsaatgutrecht" beschrieben wurde, dient somit der Bewahrung der Intaktheit der genetischen Systeme bzw. einer ausreichend hohen Anpassungsfähigkeit (bttp:// www.genres.de/fgrdeu/genetisches-monitoring/). Durch die wiederholte Erfassung des Zustandes der genetischen Systeme wird es möglich, Veränderungen und Entwicklungen aufzuzeigen, die Einflussgrößen zu charakterisieren und Maßnahmen zur Erhaltung der genetischen Vielfalt abzuleiten.

Neben dem Einsatz bewährter Isoenzymanalytik besteht die Möglichkeit eine nahezu unbegrenzte Anzahl von DNA-Markern anzuwenden. Da es sich bei den bisher eingesetzten DNA-Markern, siehe auch DOÚNAVI (2000), SCHELTER u. KRABEL (2002) sowie GEBHARDT et al. (2002), vielfach um anonyme Marker (simple sequence repeats $=$ SSR und Inter-SSR) handelt, deren Bedeutung für das pflanzliche Wachstum und insbesondere für die Entwicklung waldbaulich relevanter Eigenschaften (z. B. Geradschaftigkeit) noch ungeklärt ist, muss ihre Eignung für die Beantwortung spezifischer Fragestellungen jeweils überprüft oder entsprechende Entwicklungsarbeit geleistet werden. Für Biodiversitätsstudien haben sich neben Isoenzymen vielfach kernkodierte Mikrosatelliten und extrachromosomale, uniparentale DNA-Marker bewährt (GILLET 1999).

Zur Klärung der Autochthonie müssen die räumlichen Muster genetischer Diversität, beispielsweise der chloroplastischen Erbinformation (cp-DNA), analysiert werden. Die möglichen Methodiken sind von mehreren Autoren für die Baumarten Eiche, Tanne sowie für die südliche Buche (Nothofagus nervosa) in der Literatur hinreichend beschrieben worden (DUMOLIN-LAPEQUE et al. 1997, 
Konnert u. BERgmann 1995, Vendramin u. ZiEgEnHAgEN 1997, MARCHELLI et al. 1998). Bei diesen Baumarten hat es sich gezeigt, dass der Genpool einer Baumart ganz wesentlich durch die bei der nacheiszeitlichen Rückwanderung wirksamen populationsgenetischen Prozesse (Flaschenhalseffekte) und durch den Rückwanderungsweg beeinflusst ist.

Autochthonieuntersuchungen sollten durchgeführt werden, um zum einen die Herkunftssicherheit in Zukunft zu erhöhen und zum anderen Saatgutverbringungen über größere Entfernungen zu ermitteln. Sollten Makrofossilien (Holz, Blätter, Samen) gefunden werden, könnten paläogenetische Untersuchungen Hinweise zur Rückwanderung der Buche und zur Anpassungsgeschichte geben.

Nach heutigem Kenntnisstand wird die Bedeutung der Anpassungsfähigkeit unserer Waldbaumpopulationen mit zunehmender Umweltvariabilität noch steigen. Da wir nicht wissen, welche Gene im Einzelnen unter zukünftigen Umweltbedingungen die Überlebens- und Leistungsfähigkeit sichern werden, kommt der genetischen Diversität als Basis der Anpassungsfähigkeit eine zentrale Bedeutung zu. Die Forstwirtschaft braucht Informationen und Empfehlungen, wie verschiedene Bewirtschaftungsmethoden unter dem Aspekt der genetischen Vielfalt zu bewerten sind. Unter Umständen wird es sogar eines aktiven GenpoolManagements bedürfen, um nicht allein auf die natürlich, aber sehr langsam ablaufenden Anpassungsvorgänge der Mutation, Selektion und Migration angewiesen zu sein.

\section{Literatur}

BundeSRAT (1985): Entschließung des Bundesrates über Maßnahmen zur Erhaltung der genetischen Vielfalt der Waldbaumarten vom 8.2.1985. Drucksache 573/85

Cremer, E.; RumpF, H.; Wolf, H.; MAurer, W. u. Steiner, W. (2005): Führen Durchforstungen zu Veränderungen der genetischen Strukturen von Buchenbeständen? Forst und Holz 60, 184-188

Doúnavi, A. (2000): Familienstrukturen in Buchenbeständen (Fagus sylvatica). Dissertation Universität Göttingen, $142 \mathrm{~S}$.

DOÚNAVI, K.; STEINER, W. u. MAURER, W.D. (2002): Effects of different silvicultural treatments on the genetic structure of European beech populations (Fagus sylvatica L.). In: GADOW, K. V.; NAgel, J. u. SABOrowsKi, J. (eds.): Managing Forest Ecosystems (vol. 4, special issue): Continuous Cover Forestry - Assessment, Analysis, Scenarios. Kluwer Academic Publishers: Dordrecht, Boston, London, 81-90

Dumolin-Lapegue, S.; Demesure, B.; Fineschi, S.; Le Corre, V. u. Petit, R.J. (1997): Phylogeographic structure of white oaks throughout the European continent. Genetics 146, 1475-1487

FirBAS, F. (1949): Waldgeschichte Mitteleuropas, Bd. 1. Gustav Fischer, Jena

FIRBAS, F. (1952): Waldgeschichte Mitteleuropas, Bd. 2. Gustav Fischer, Jena

GEBHARDT, K. (2003): Genetische Diversität der Buche (Fagus sylvatica L.) im hessischen Staatswald. Mitt. aus der Forschungsanstalt für Waldökologie und Forstwirtschaft Rheinland-Pfalz 52/04, 189-196

Gebhardt, K.; Pohl, A. u. Vornam, B. (2002): Genetic inventory of Black Poplar populations in the Upper Rhine floodplains: conclusions for conservation of an endangered plant species. In: VAN DAM, B.C. u. BORDÁCS, S. (eds.): Proceedings of an Int. Symposium „Genetic diversity in 
river populations of European Black Poplar“ held 16. - 20. May 2001 in Szekszárd, Hungary, printed by C. Nyomda Ltd. Budapest, Hungary, 145-156

GILLET, E.M. (1994): GSED - Genetic structures from electrophoresis data. User's Manual version 1.0, Universität Göttingen, 49 S.

GiLlet, E.M. (ed.) (1999): "Which Marker for Which Purpose?" Final Compendium of the Research Project "Development, optimisation and validation of molecular tools for assessment of biodiversity in forest trees"; http://webdoc.sub.gwdg.de/ebook/y/1999/whichmarker/index. htm , gedruckt als: Mitteilungen der Bundesforschungsanstalt für Forst- und Holzwirtschaft, Hamburg, Nr. 198, 164 + iv pp., Kommissionsverlag Max Wiedebusch, Hamburg, 2000

JANßEN, A. (1998): Untersuchungen zur genetischen Variation der Buche in Hessen. In: HLFWW (Hrsg.): Jahresberichte 1996/1997. Hessische Landesanstalt für Forsteinrichtung, Waldforschung und Waldökologie, Hann. Münden, 15-17

JANBEN, A. (2000): Der Einfluß von Ernteverfahren auf die genetische Struktur von Saatgut eines Buchenbestandes. Forschungsberichte der Hessischen Landesanstalt für Forsteinrichtung, Waldforschung und Waldökologie, Band 27, $142 \mathrm{~S}$.

JANBEN, A. u. NOWACK, S. (2001): Beeinflussen Durchforstungen die genetische Struktur von Buchenbeständen? AFZ/Der Wald 56, 614-615

JANBEN, A. u. SCHULZ, R.-D. (2002): Einfluss der Bewirtschaftungsweise aufgrund unterschiedlicher Besitzart? AFZ/Der Wald 57, 1272-1275

JANßEN, A.; SChulz, R.-D. u. HolzBerg, H. (2002): Einfluß verschiedener Beschattungsvarianten in der Anzuchtphase auf die genetischen und phänotypischen Strukturen von Bestandesabsaaten. Forst und Holz 57, 338-341

KLEINSCHMit, J. u. SvOlBA, J. (1996): Ergebnisse der Buchenherkunftsversuche von KrAHL-Urban. AFZ/Der Wald 14, 780-782

Konnert, M. u. Bergmann, F. (1995): The geographical distribution of genetic variation of silver fir (Abies alba, Pinaceae) in relation to its migration history. Plant Systematics and Evolution 195, $19-30$

KonnerT, M.; Ziehe, M.; TrÖBer, U.; MAurer, W.; JANßEn, A.; SANDER, T.; HusSendÖRfer, E. u. Hertel, H. (2000): Genetische Variation der Buche (Fagus sylvatica L.) in Deutschland: Gemeinsame Auswertung genetischer Inventuren über verschiedene Bundesländer. Forst und Holz 55, 403-408

LÖCHELT, S. u. FranKE, A. (1995): Bestimmung der genetischen Konstitution von Buchen-Beständen (Fagus sylvatica L.) entlang eines Höhentransektes von Freiburg auf den Schauinsland. Silvae Genetica 44, 947-949

Marchelli, P.; Gallo, L.; Scholz, B. u. Ziegenhagen, B. (1998): Chloroplast DNA markers revealed a geographical divide across Argentinean southern beech Nothofagus nervosa (Phil.) Dim. et Mil. distribution area. Theoretical and Applied Genetics 97, 642-646

Melchior, G.H.; Becker, A.; Behm, A.; Doerflinger, H.; Kleinschmit, J.; Muhs, H.J.; SCHMitT, H.-P.; STEPHAN, B.R.; TABEL, U.; WeIsGerber, H. u. Widmaier, T. (1989): Erhaltung forstlicher Genressourcen in der Bundesrepublik Deutschland. Forst und Holz 44, 379-404

MÜlLER-STARCK, G. u. STARKE, R. (1993): Inheritance of isozymes in European beech (Fagus sylvatica L.). J. Heredity $84,291-296$

Paul, M.; Hinrichs, T.; Janßen, A.; Schmitt, H.-P.; Soppa, B.; Stephan, B.R. u. Dörflinger, H. (2001): Konzept zur Erhaltung und nachhaltigen Nutzung forstlicher Genressourcen in der Bundesrepublik Deutschland. Schriftenreihe der Sächsischen Landesanstalt, 66 S.

Rothe, G.M. (1994): Electrophoresis of Enzymes: Laboratory Methods. Springer Verlag, BerlinHeidelberg. $307 \mathrm{~S}$.

SAnder, T.; König, S.; Rothe, G.M.; Janßen, A. u. Weisgerber, H. (2000): Genetic variation of European beech (Fagus sylvatica L.) along an altitudinal transect at mount Vogelsberg in Hesse, Germany. Molecular Ecology 9, 1349-1361 
Sander, T.; Rothe, G.M.; Weisgerber, H. u. Janben, A. (2001): Allelic and genotypic variation of 13 European beech (Fagus sylvatica L.) populations in Hesse, Germany. Forest Genetics 8, 1324

SCHelter, D. u. KRABel, D. (2002) Genetische Charakterisierung von Laubholz-Naturverjüngung unter Fichten-Reinbeständen mittels molekulargenetischer Erhebungen. GWPG-Tagung 6.-9. Okt. 2002, Hamburg, Poster PS-1-13

SCHÜte, G. u. RumpF, H. (2003): Untersuchungen waldbaulicher Einflüsse auf die genetische Struktur naturverjüngter Buchenbestände (Fagus sylvatica L.). Forstarchiv 74, 90-96

Secretariat of the Convention on Biological Diversity (2005): Handbook of the Convention on Biological Diversity Including its Cartagena Protocol on Biosafety, 3rd edition, (Montreal, Canada)

Steiner, W.; Rumpf, H. u. GAerTner, U. (2008): Genetische und phänotypische Unterschiede an einigen Provenienzen im Buchenherkunftsversuch von 1959. (in Vorbereitung)

Vendramin, G.G. u. Ziegenhagen, B. (1997): Characterization and inheritance of polymorphic plastid microsatellites in Abies. Genome 40, 857-864

Wypukol, H., Liepelt, S., Ziegenhagen, B. u. Gebhardt, K. (2008): Genetische Methoden zur Abstammungsanalyse und Prüfung von Sortenechtheit und -reinheit. In: Tagungsbericht des Symposiums „Herkunftskontroll““, 7.-8.2.08 in Kassel (im Druck)

Ziehe, M.; Hattemer, H.H.; MÜLler-Starck, R. u. MÜLLer-Starck, G. (1999): Genetic structures as indicators for adaptation and adaptational potentials. In: MÀTYAS, Cs. (ed.): Forest Genetics and Sustainability. Kluwer Academic Publishers, Dordrecht, The Netherlands, pp. 75-89

Ziehe, M.; Vornam, B.; Müller-Starck, R.; Turok, J.; Hattemer, H.H.; Maurer, W.D. u. Tabel, U. (2002): Genetische Strukturen der Buche in Rheinland-Pfalz. In: MAURER, W.D.: Vom genetischen Fingerabdruck zum gesicherten Vermehrungsgut: Untersuchungen zur Erhaltung und nachhaltigen Nutzung forstlicher Genressourcen in Rheinland-Pfalz. Mitteilungen aus der Forschungsanstalt für Waldökologie und Forstwirtschaft Rheinland-Pfalz (ISSN 1610-7705), Nr. 49/02, 99-118

Korrespondierender Autor:

Dr. Alwin Janßen

Nordwestdeutsche Forstliche Versuchsanstalt

Abteilung Waldgenressourcen

Prof.-Oelkers-Str. 6

34346 Hann. Münden

E-Mail: Alwin.Janssen@nw-fva.de

URL: www.nw-fva.de

Dr. Karl Gebhardt

Dr. Wilfried Steiner

Nordwestdeutsche Forstliche Versuchsanstalt 



\title{
Schutz durch Nutzung forstlicher Genressourcen der Buche (Fagus sylvatica L.) in Nordwest- deutschland
}

\author{
Protection through utilisation of the forest genetic \\ resources of beech (Fagus sylvatica L.) in northwest \\ Germany
}

Jörg Kleinschmit, Hans-Martin Rau und Karl Gebhardt

\section{Zusammenfassung}

Genetische Variation ist die Voraussetzung für die Anpassungsfähigkeit an sich verändernde Umweltbedingungen und für die nachhaltige Leistungsfähigkeit unserer Wälder. Eine nachhaltige Nutzung ohne Erhaltung ist daher nicht möglich. Die Nordwestdeutsche Forstliche Versuchsanstalt (NW-FVA) stellt dem Waldbesitzer Herkunftsempfehlungen auf der Basis gesicherten Wissens zur Verfügung. Prüfungen von Buchen-Nachkommenschaften zeigen deutliche genetische Unterschiede bei phänotypischen Merkmalen wie Vitalität und Wüchsigkeit. Dies bietet dem Forstbetrieb die Chance zur Steigerung der Produktivität durch die Wahl des besten Vermehrungsgutes. Naturverjüngung ist nicht immer die beste Wahl. Die Auswahl der besten Bestände ist entscheidend.

Die Erfassung von Objekten zur Erhaltung forstlicher Genressourcen liefert wertvolle Daten für die nachhaltige Nutzung und den Schutz der Buche. Dazu 
wurde über die Länder Nordwestdeutschlands verteilt ein Netz phänotypisch herausragender Bestände ausgewählt, in welchen die Weitergabe der bestehenden genetischen Information an die folgende Bestandesgeneration besondere Bedeutung hat. Neben dieser In-situ-Erhaltung werden weitere Maßnahmen zur Erhaltung und Bereitstellung besonderer genetischer Information getroffen.

Die Methoden der Herkunftssicherung: amtliche Kontrolle und Dokumentation, genetischer Vergleich mit Referenzproben und Stabilisotopen-Untersuchungen geben dem Verbraucher und den Behörden Instrumente an die Hand, um Zweifel an der Identität des genutzten Vermehrungsgutes zu überprüfen.

Stichworte: Erhaltung, Produktivitätssteigerung, forstliche Genressourcen, nachhaltige Nutzung, Herkunftssicherung, Buche

\begin{abstract}
Genetic variation is essential for the ability to adapt to changing environmental conditions, and for the sustainable productivity of our forests. Therefore, sustainable utilisation in the absence of conservation is not possible. Based on their scientific knowledge, the Northwest German Forest Research Station (NW-FVA) provides forest owners with provenance recommendations. Tests of beech progenies reveal clearly the genetic differences in relation to phenotypic characteristics such as vitality and vigour. This gives forest enterprises the opportunity to increase productivity by selecting the best reproductive material. Natural regeneration is not always the best choice. The selection of the best stands is crucial.

The survey of units for the conservation of forest genetic resources provides valuable data for the sustainable use and conservation of beech. To this end, a network of phenotypically excellent stands has been selected across the states of northwest Germany so that the existing genetic information can be passed on to subsequent forest generations. In addition to this in situ conservation, other measures for the conservation and provision of special genetic information have been undertaken.

The methods to be able to verify the origin of a provenance, including state control and documentation, genetic comparisons with reference samples, and stable isotope investigations provide users and government departments with the tools needed to check any uncertainty about the genetic identity of reproductive material used.
\end{abstract}

Keywords: conservation, productivity increase, forest genetic resources, sustainable utilisation, provenance verification, beech 


\section{Einleitung}

Die biologische Vielfalt ist die Grundlage für die Stabilität und Anpassungsfähigkeit und damit die nachhaltige Produktivität unserer Wälder. Biologische Vielfalt ist daher sowohl ökologisch wie auch ökonomisch wichtig. Die biologische Vielfalt umfasst die Vielfalt an Lebensräumen, die Artenvielfalt und die genetische Vielfalt. Der vorliegende Artikel befasst sich mit der genetischen Vielfalt der Buche (Fagus sylvatica L.) in den Bundesländern Hessen, Niedersachsen, Sachsen-Anhalt und Schleswig-Holstein.

Die besondere Bedeutung der genetischen Vielfalt liegt darin begründet, dass die Erbinformation Basis der Informationsweitergabe über die Lebensspanne einzelner Individuen hinaus ist (GREGORIUS 1997). Durch die in den Genen gespeicherte Information ist Anpassung von Populationen an sich ändernde Umweltbedingungen erst möglich. Aus Sicht des Menschen als Nutzer der Natur ist die Erhaltung forstlicher Genressourcen daher zum einen notwendige Voraussetzung als Basis für eine Auswahl möglichst wüchsiger und stabiler Genotypen zur Produktion nachwachsender Rohstoffe, zum anderen ist die Erhaltung aber auch Daseinsvorsorge im Hinblick auf sich verändernde, nicht genau vorhersagbare Umweltbedingungen. Erhaltung und nachhaltige Nutzung ergänzen sich. Eine nachhaltige Nutzung ohne gleichzeitige Erhaltung ist nicht möglich. In diesem Sinne sollen im Folgenden Ergebnisse aus der angewandten forstgenetischen Buchenforschung vorgestellt werden.

Um dem Ziel Schutz durch Nutzung der forstgenetischen Ressourcen gerecht zu werden, müssen die Erkenntnisse der forstgenetischen Forschung über genetische Variation zwischen Herkünften, Beständen und innerhalb von Beständen im Rahmen der forstlichen Bewirtschaftung berücksichtigt werden. Zu diesen Erkenntnissen gehört die Tatsache, dass die heute zu sehenden Muster genetischer Variation bei der Buche zum einen durch die Rückzugs- und Ausbreitungsereignisse während und nach den Eiszeiten geprägt sind (MAGRI et al. 2006). Zum anderen spiegeln sie den Einfluss des Menschen in der neueren Forstgeschichte wider. Hier sind neben waldbaulichen Eingriffen besonders anthropogen bedingte Arealveränderungen der Buche durch Rodungen oder Ersatz der Buche durch andere Baumarten und die künstliche Wiederverbreitung der Buche durch Pflanzungen zu nennen.

\section{Prüfung von Buchen-Nachkommenschaften}

Bei der Prüfung von Buchen-Nachkommenschaften wird das Ausmaß genetisch bedingter Unterschiede phänotypischer Merkmale wie z. B. Vitalität, Qualität, Stabilität und Anpassungsfähigkeit geschätzt. Dazu werden Nachkommenschaften (Familien oder Bestandesabsaaten) verschiedener Ausgangsbestände unter gleichen Umweltbedingungen in speziellen Versuchsanordnungen nebeneinander angebaut. 
Die besten Ausgangsbestände sollen später als „geprüft“ nach dem Forstvermehrungsgutgesetz zur Saatguternte zugelassen werden.

Die Buche hat sich in solchen Versuchen als relativ schwieriges Untersuchungsobjekt erwiesen. Teilweise treten starke Einflüsse der Versuchsumwelten auf, sodass dadurch die veranlagungsbedingten Unterschiede in Leistung und Qualität überlagert werden. Des Weiteren hat sich herausgestellt, dass es bei Buche sehr lange dauern kann, bis man die genetische Veranlagung, forstwirtschaftlich wichtige Eigenschaften wie Gerad- oder Wipfelschäftigkeit auszubilden, mit ausreichender Sicherheit bewerten kann. Deswegen müssen Buchenversuche über Jahrzehnte hinweg beobachtet werden. Das hat zur Folge, dass man relativ große Parzellen benötigt, in denen auch nach ersten Durchforstungen noch genügend Individuen verbleiben. Daraus ergibt sich wiederum, dass man auf einer gegebenen Fläche weniger verschiedene Prüfglieder testen kann. Die für den Versuch beernteten Bestände sollten nicht zu alt sein, sonst besteht die Gefahr, dass sie nach Abschluss der Prüfung nicht mehr existieren.

Im Vergleich zu ihrer heutigen forstwirtschaftlichen Bedeutung ist die Buche, abgesehen von den 19 durch Krahl-Urban in den 50- und 60er Jahren des vorigen Jahrhunderts begründeten Herkunftsversuchen mit 133 Herkünften (KLEINSCHMIT u. SVOLBA 1996), erst relativ spät in größere Vergleichsprüfungen aufgenommen worden. So sind zwischen 1986 und 1998 auf Initiative des Institutes für Forstgenetik und Forstpflanzenzüchtung der damaligen Bundesforschungsanstalt für Forst- und Holzwirtschaft 62 Feldversuche mit insgesamt 350 Herkünften in 21 europäischen Ländern begründet worden, davon 21 in Deutschland (LIESE$\mathrm{BACH}$ et al. 1998). Die Vorgängerinstitutionen der NW-FVA haben schwerpunktmäßig zwischen 1986 und 1999 ca. 40 Versuchsflächen angelegt, die vorrangig der Prüfung von über 250 Bestandesnachkommenschaften vor allem in Hessen, Niedersachsen und Schleswig-Holstein dienen.

Aus den Herkunfts- und Bestandesprüfungen bei Buche lassen sich trotz der beschriebenen Schwierigkeiten wichtige Erkenntnisse für den Waldbau und die Erhaltung besonders wertvoller Genressourcen ableiten: Die Vorkommen der Buche zeigen eine ihrem ausgedehnten natürlichen Verbreitungsgebiet entsprechende große Variabilität in ihren Leistungs- und Qualitätsmerkmalen wie auch in ihrer Toleranz gegenüber Umwelteinflüssen wie Spätfrost oder Trockenheit (CZAJOWSKI u. BOLTE 2006a und 2006b, LIESEBACH 2000, MUHS u. VON WÜHLISCH 1992, KLEINSCHMIT u. SVOlBA 1996, ThOMASIUS u. GÄrTNER 1988). Das kann auch für relativ nahe beieinander liegende Bestände gelten. Bei der Pflanzung von Buchen kann daher nicht nur, wie bei den meisten anderen Baumarten auch, die Herkunft, sondern vielfach sogar der einzelne Erntebestand entscheidenden Einfluss auf den Anbauerfolg haben. Die räumliche Nähe von Ursprung des Vermehrungsgutes und Anbauort (hier Versuchsflächen) ist nicht unbedingt Garant für optimales Wachstum. Das heißt, dass lokales Material nicht notwendigerweise das Beste ist. So wird die ortsnahe Bestandesnachkommenschaft aus dem Bram- 
wald in einem von Krahl-Urban dort angelegten Versuch sowohl in ihrer Vitalität als auch Qualität im Alter von 50 Jahren von mehreren weit entfernten Herkünften sehr deutlich übertroffen.

Wichtiger als die horizontale Nähe scheint zu sein, dass Buchen aus vergleichbaren Höhenlagen verwendet werden (LIESEBACH 2000). Dies passt zu Ergebnissen von genetischen Untersuchungen in Hessen. Sie haben gezeigt, dass an den untersuchten Isoenzymgenorten dann eher Unterschiede zwischen Beständen auftreten, wenn diese aus sehr unterschiedlichen Höhenstufen stammen (SANDER et al. 2000). Konsequenterweise sind bei Buche in der FORSTVERMEHRUNGSGUTHERKUNFTSGEBIETSVERORDNUNG (1994) nicht nur horizontale Herkunftsgebiete, sondern für Berglandregionen auch Höhenzonen ausgewiesen.
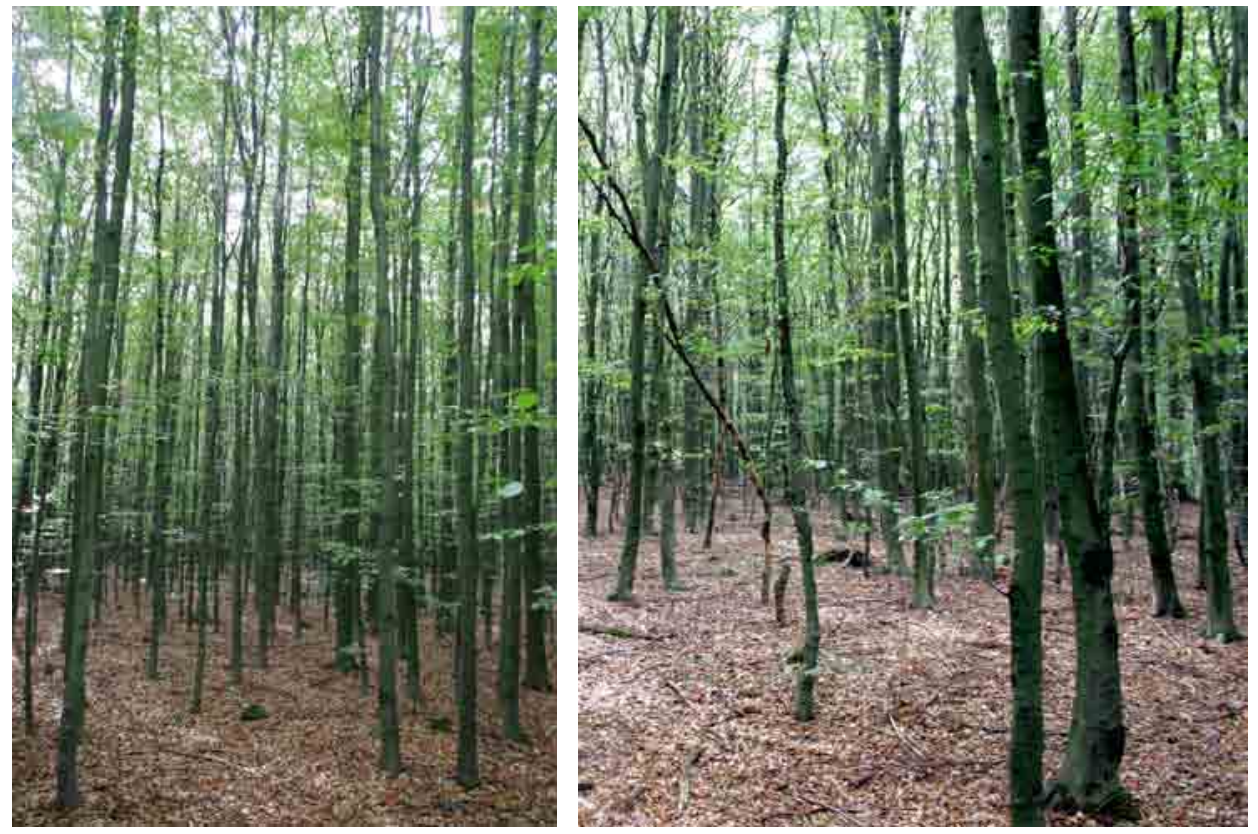

Abbildung 1: Vergleich der Schaftform der Herkunft Zwiesel-Ost (links) zur Herkunft Metzingen (rechts) im Versuch von Krabl-Urban im Niedersächsischen Forstamt Münden, Abteilung 136 im Alter 39

Welchen entscheidenden Einfluss der Ausgangsbestand beispielsweise auf die Geradschaftigkeit haben kann, lässt sich auf Flächen des Buchenversuches von Krahl-Urban zeigen. Es gibt dort ohne schützende Beschirmung Parzellen mit etwa 90\% weitgehend geraden Buchen (Herkunft „Zwiesel-Ost“, Bayerischer Wald) und andere, bei denen nicht eine einzige gerade Buche zu finden ist (s. Abb. 1). Aus einer Reihe von Versuchen zeichnet sich ab, dass Buchenherkünfte aus höheren Lagen tendenziell eher gerade Formen entwickeln als Tieflagenherkünfte. Versuche mit Nachkommen einzelner Buchen zeigen darüber 
Jörg Kleinschmit, Hans-Martin Rau, Karl Gebhardt

hinaus, dass es auch auf individueller Ebene beträchtliche Unterschiede geben kann.

\section{Herkunftsempfehlungen, verfügbares Vermehrungsgut}

Aufbauend auf den Erkenntnissen aus der Herkunftsforschung gibt es für die meisten Bundesländer baumartenweise gegliederte Herkunftsempfehlungen für die dort jeweils vorkommenden Anbaugebiete. Schaut man sich die von der Versuchsanstalt erarbeiteten Empfehlungen für Hessen, Niedersachsen und SchleswigHolstein an, so fällt auf, dass es für Buche nur sehr wenige Empfehlungen von Vermehrungsgut der besten Kategorie ,geprüft“ gibt, deren Zulassung auf Ergebnissen von langjährigen Vergleichsanbauten beruht. Das hängt mit den oben geschilderten Problemen bei der Buchenherkunftsforschung zusammen.

Während für Hessen an zweiter Stelle bewährtes, autochthones Vermehrungsgut aus vergleichbaren Lagen im gleichen Forstbetrieb empfohlen wird, folgen für die beiden anderen Länder sogenannte „Sonderherkünfte“ und „Spitzenbestände“. „Sonderherkünfte“ sind von der Gütegemeinschaft für forstliches Vermehrungsgut nach phänotypischen Merkmalen ausgewählte Bestände, welche die amtlich vorgeschriebenen Zulassungsvoraussetzungen für die Saatguternte in besonderem Maße erfüllen. Bei den „Spitzenbeständen“ handelt es sich um eine kleine Gruppe amtlich zugelassener Saatguterntebestände, die aus Sicht der für die niedersächsischen Landesforsten zuständigen Saatgutberatungsstelle beim Forstamt Oerrel für die Bereitstellung von forstlichem Vermehrungsgut besonders geeignet erscheinen. In beiden Fällen ist die erbliche Überlegenheit von Vermehrungsgut aus solchen Beständen nicht durch vorangehende Vergleichsprüfungen belegt. Im Gegensatz zu den bestandesweise abgegrenzten ,geprüften“ Beständen und den „Spitzenbeständen“ handelt es sich bei „Sonderherkünften“ meist um Gruppen von verschiedenen Beständen zwischen 3 und 1.156 ha. Bei sehr großen „Sonderherkünften“ ist damit zu rechnen, dass die Qualität des Vermehrungsgutes je nach Ort des einzelnen beernteten Bestandes schwankt, weil bei so großen Komplexen auch eine entsprechende genetische Variabilität innerhalb des Komplexes zu erwarten ist.

Das weitaus meiste Vermehrungsgut von Buche, das in Deutschland verwendet wird, stammt aus den 5.709 amtlich in der Kategorie „,ausgewählt“ zugelassenen Beständen, die mit ihren rd. 75.000 ha ungefähr $5 \%$ der Buchenfläche in Deutschland ausmachen (BUND-LÄNDER-ARBEITSGRUPPE „FORSTLICHE GENRESSOURCEN UND FORSTSAATGUTRECHT“ 2006). Die Kriterien für die amtliche Zulassung von Beständen sind in einer Bundesverordnung geregelt (FORSTVERMEHRUNGSGUT-ZULASSUNGSVERORDNUNG 2002). Sie beinhalten für die Kategorie ,ausgewählt“ neben den Mindestforderungen zur Sicherung einer ausreichenden genetischen Vielfalt - Alter (70 Jahre), Fläche (2,5 bzw. in höheren Lagen auch nur 1 oder $1 / 4$ ha) und Baumzahl (40 bzw. 20) - Kriterien wie Gesundheit, Schaft- 
und Kronenform, Zuwachs und Homogenität. Bei der Zulassung ist es wichtig, nicht nur die absolut phänotypisch besten Bestände einer Baumart in einem Land zuzulassen, sondern in dem gesamten potenziellen Anbaugebiet die relativ besten auszuwählen. Damit soll erreicht werden, dass auch für ganz unterschiedliche Standortbedingungen das voraussichtlich am besten angepasste Vermehrungsgut geerntet werden kann. Andernfalls könnte beispielsweise Vermehrungsgut von hochproduktiven Tieflagenstandorten auch in ökologisch stark abweichende Hochlagen verbracht werden, an die es womöglich nicht ausreichend angepasst ist.

Nach Angaben der BUNDESANSTALT FÜR LANDWIRTSCHAFT UND ERNÄHRUNG 2007 sind seit 1997 in Deutschland pro Jahr ca. $91.000 \mathrm{~kg}$ Bucheckern in als „ausgewählt“ zugelassenen Beständen gesammelt worden. Während die Buche früher nur etwa alle zehn Jahre eine sogenannte Vollmast ausbildete, haben sich die Abstände - vermutlich in Zusammenhang mit der Klimaerwärmung - deutlich verringert. Allerdings schwanken die Mengen von Jahr zu Jahr sehr stark (2005/6 nur $11 \mathrm{~kg}$ ). Zu den genannten Mengen kommen jährlich jeweils rund $800 \mathrm{~kg}$ aus „geprüften“ Beständen und aus Beständen im Ausland. Zieht man die Mengen ab, die in andere Länder verbracht werden, so ergibt sich ein Saldo von jährlich $95.000 \mathrm{~kg}$, was einer theoretisch möglichen Ausbeute an 1-jährigen Sämlingen von etwa 95 Mio. entspräche.

\subsection{Empfehlungen zur Saatguternte}

Wie JANßEN (2000) mithilfe von Isoenzymanalysen zeigen konnte, beeinflussen Saatgut-Ernteverfahren die genetischen Strukturen der daraus hervorgehenden Nachkommen. Zu berücksichtigen ist ferner, dass aus Naturverjüngung entstandene Bestände der schwersamigen Buche teilweise Familienstrukturen aufweisen, benachbarte Bäume also genetisch ähnlicher sind (VORNAM et al. 2004). Da sich die Ziele von Beerntungen unterscheiden, ergeben sich je nach Ziel unterschiedliche Empfehlungen:

Beerntungen zur Erhaltung forstlicher Genressourcen sollen die genetischen Strukturen der Ausgangspopulation möglichst genau repräsentieren. Deshalb soll in einem Vollmastjahr auf möglichst großer Fläche mit Netzen geerntet werden. Ist eine Beerntung der Gesamtfläche nicht möglich, kann ein Stichprobenraster verwendet werden, dessen Maximalabstand der Erntepunkte $25 \mathrm{~m}$ betragen sollte, da dies dem effektiven Genfluss durch Samenverbreitung entspricht. Für Beerntungen zur Anlage von Provenienz- und Nachkommenschaftsprüfungen gelten dieselben Vorgaben.

Saatgutbeerntungen für die Versorgung der Forstbetriebe müssen den Anforderungen des Forstvermehrungsgutgesetzes entsprechen. Es sind mindestens 20 Bäume je Bestand zu beernten. Diese Bäume sollten über den ganzen Bestand verteilt sein. Durch Mischung des Saatgutes verschiedener Erntebestände desselben Herkunftsgebietes kann zwar die Anpassungsfähigkeit der Mischung erhöht 
Jörg Kleinschmit, Hans-Martin Rau, Karl Gebhardt

werden, jedoch ist anzunehmen, dass sich der Vorteil der Angepasstheit verringert. Bei Einsatz von Sauggeräten und bei der Wildlingswerbung muss darauf geachtet werden, dass ein repräsentativer Teil des Bestandes einbezogen ist, damit die vorhandenen Familienstrukturen in der Folgegeneration nicht zu Inzuchteffekten führen.

Beerntungen zur Durchführung genetischer Untersuchungen müssen bei geringem Stichprobenumfang die genetische Struktur des Ausgangsbestandes repräsentieren. Nach ZIEHE et al. (1998) liefert das Verfahren der Saatgutsammlung unter jedem Altbaum gegenüber dem Verfahren der Sammlung mit Netzen unter stark fruktifizierenden Bestandespartien die besseren Schätzwerte. GILLET (1999) berechnete an einem Genort mit drei verschiedenen Genotypen für eine qualifizierte Schätzung genotypischer Werte einen Mindeststichprobenumfang von 499 Eckern.

\section{Programm zur Erhaltung forstlicher Genressourcen}

Damit Bäume sich an veränderte Umweltbedingungen, wie z. B. an die erwartete Klimaänderung, anpassen können, müssen sie über ausreichende genetische Variabilität verfügen. Für das Überleben der Arten ist es also nicht nur wichtig, dass sie an die aktuellen Bedingungen angepasst sind, was u. a. in Anbauversuchen untersucht wird, sondern auch, dass sie sich ihre genetische Anpassungsfähigkeit bewahren können. Im Sinne der multifunktionalen Waldbewirtschaftung ist es daher ein Ziel der im Jahr 2000 verabschiedeten Neufassung des Konzeptes zur Erhaltung und nachbaltigen Nutzung forstlicher Genressourcen in der Bundesrepublik Deutschland der Bund-Länder-Arbeitsgruppe „Forstliche Genressourcen und Forstsaatgutrecht" (PAUL et al. 2000), die Erhaltung und nachhaltige Nutzung der genetischen Vielfalt der Wälder großflächig miteinander zu verbinden. Dazu muss die genetische Vielfalt in ihrer aktuellen Verteilung erfasst werden. Anschließend soll sie in den verschiedenen Wuchsgebieten und Wuchsbezirken repräsentativ erhalten und damit langfristig für eine nachhaltige Nutzung zur Verfügung gestellt werden.

Die langfristige - über die Lebensspanne eines Individuums hinausgehende Erhaltung von genetischer Information ist nur über die Weitergabe dieser Information von Generation zu Generation möglich. Dies geschieht im Normalfall durch Samenproduktion (generative Vermehrung) und natürliche Verjüngung. Dabei haben die jeweils an die Umwelt angepassten genetischen Varianten eine bessere Überlebenschance. Gleichzeitig wird durch die Neukombination der Gene im Rahmen der generativen Vermehrung die Anpassungsfähigkeit erhöht.

Geeignete Objekte zur Erhaltung forstlicher Genressourcen sind daher qualitativ herausragende Bestände, die sowohl aktuell angepasst sind als auch eine ausreichende genetische Vielfalt besitzen und sich natürlich verjüngen können, damit die besten Bestandesglieder ihre genetische Information vor ihrem Aushieb aus dem Bestand an die Folgegeneration weitergeben können. Dieser dynamischen 
Erhaltung forstlicher Genressourcen in-situ (vor Ort) wird oberste Priorität eingeräumt.

Nur dort, wo keine langfristig überlebensfähigen Populationen mehr vorhanden sind, soll die genetische Vielfalt über Erhaltung ex-situ (an anderer Stelle) im Rahmen von Erhaltungspflanzungen, Klonarchiven oder der Lagerung von Saatgut in einer Forstgenbank gesichert und hinterher der forstlichen Praxis wieder zur Verfügung gestellt werden. Dies erforderte teilweise methodische Neu- oder Weiterentwicklungen, wie z. B. die vegetative Vermehrung für die Buche.

Aktuell sind für die Baumart Buche in den Ländern Hessen, Niedersachsen, Sachsen-Anhalt und Schleswig-Holstein die in Tabelle 1 genannten Objekte ausgewählt, gesichert und neu angelegt worden. Die Information zu den ausgewiesenen Objekten und geplanten Erhaltungsmaßnahmen wird den Waldbesitzern von der NW-FVA als Datenbankauszug zur Verfügung gestellt. Landes- und waldbesitzerspezifisch erfolgt darüber hinaus eine Anbindung an das jeweilige forstliche Informationssystem und über eine Digitalisierung der Objekte die Darstellung in den geographischen Informationssystemen der Waldbesitzer.

Tabelle 1: Erhaltungsobjekte Buche in den Ländern Hessen, Niedersachsen, Sachsen-Anhalt und Schleswig-Holstein in der Datenbank der NW-FVA (Sachstand 31.12.2007)

\begin{tabular}{lcc}
\hline Art des Erhaltungsobjektes & Anzahl & Größe/Menge \\
\hline Bestandesobjekte $i n$-situ & 149 & 2773 ha \\
Einzelobjekte $i n$-situ & 86 & 259 Individuen \\
Bestandesobjekte $e x$-situ & 51 & 120 ha \\
Samenplantagen & 5 & 4,6 ha; 87 Klone \\
Saatgut in der Forstgenbank & 324 Partien & $566 \mathrm{~kg}$ insgesamt \\
\hline
\end{tabular}

\subsection{Erhaltung in-situ}

Im Rahmen des o. g. Konzeptes sollen von einer Hauptbaumart wie der Buche 1-2\% der Fläche als In-situ-Bestandesobjekte erfasst und langfristig gesichert werden. Die Auswahl von In-situ-Bestandesobjekten erfolgt in der Regel wegen ihrer Angepasstheit und herausragenden Qualität. Die Auswahl geschieht nach einer Verschneidung mit Daten aus der Forsteinrichtung, der Waldbiotopkartierung und des Waldnaturschutzes.

Die notwendigen Erhaltungsmaßnahmen werden durch die NW-FVA geplant und in den Landesforsten durch die Forsteinrichtung im Rahmen der periodischen Betriebsregelung berücksichtigt. Dabei ist eine Erfolgskontrolle der geplanten Insitu-Maßnahmen und eine angepasste Maßnahmenfortschreibung vorzunehmen. Die Ausweisung als In-situ-Bestandesobjekte schließt eine Nutzung der Bestände im Anschluss an eine erfolgte Sicherung durch Naturverjüngung oder aber 
Verjüngung mit Material aus dem Objekt nach dem Prinzip Schutz durch Nutzung ausdrücklich ein.

Neben den In-situ-Bestandesobjekten sind besondere Einzelindividuen oder Gruppen erfasst worden, die entweder durch ihr hohes Alter oder aber durch besondere Wuchsform (z. B. Süntelbuchen) auffallen.

\subsection{Erhaltung ex-situ}

Bei ex-situ zu sichernden Erhaltungsobjekten soll der NW-FVA die Gelegenheit zur Sicherung der genetischen Information vor einer Nutzung oder anderweitigen Veränderung des Erhaltungsobjektes gegeben werden.

Unter dem Eindruck des Waldsterbens und der hohen Belastung durch Luftschadstoffe wurden die Buchenwälder in Hessen Ende der 1980er Jahre in vier Prioritätsstufen eingeteilt. Eine repräsentative Anzahl von Buchenbeständen der Prioritätsstufen 1 und 2 sollte unverzüglich gesichert werden. Zur Priorität 1 gehörten alle zugelassenen Buchenbestände über $400 \mathrm{~m}$ Meereshöhe in den Hauptschadgebieten, zur Priorität 2 alle zugelassenen Bestände über $400 \mathrm{~m}$ ü. NN in den übrigen Wuchsgebieten. Von diesen Objekten wurde über Netzernte Saatgut gewonnen und ausgesät. Mit den angezogenen Pflanzen legte die Versuchsanstalt 51 Ex-situ-Buchen-Erhaltungsobjekte bis zum Jahr 2008 mit einer Fläche von rund 120 ha an.

Für die Baumart Buche wurden in den Jahren 1956/57 und 1961 in Niedersachsen fünf Plusbaum-Samenplantagen mit einer Fläche von 4,6 ha angelegt. Die Plusbäume stammen aus Hessen, Niedersachsen, Schleswig-Holstein und Rheinland-Pfalz. Ziel der Anlage dieser Pfropflingssamenplantagen ist neben der Erhaltung phänotypisch ausgewählter Genotypen die Produktion von geprüftem Vermehrungsgut. Dazu wurden 1994 von den Plantagen zwei Nachkommenschaftsprüfungen angelegt. Anders als bei anderen Baumarten spielt bei der Buche Vermehrungsgut aus Samenplantagen oder von Klonen bisher keine nennenswerte Rolle.

\subsubsection{Langzeitlagerung von Bucheckern in der Forstgenbank}

Das sehr gute Mastjahr 1989/90 und die Teilmasten in den Jahren 1990 - 1995 boten die Chance, in den Kühlräumen der Forstgenbank in Hann. Münden gröBere Mengen von Buchensaatgut zur Erhaltung der genetischen Vielfalt langfristig einzulagern. Dazu wurden in Hessen insgesamt 67 Bestände der Prioritätsstufen 1 und 2 (s. o.) in 50 Forstämtern ausgewählt. Pro Bestand erntete man insgesamt etwa $20 \mathrm{~kg}$ von mindestens 20 Bäumen mit jeweils gleichen Anteilen an der Gesamtmenge. Nach der Netzernte in den ausgewählten Beständen und der Reinigung des Rohsaatgutes wurde das Saatgut auf 8-9\% Wassergehalt heruntergetrocknet, um die Stoffwechselaktivitäten des Saatgutes soweit wie möglich zu ver- 
ringern, ohne eine spätere Keimfähigkeit nachteilig zu beeinflussen. Anschließend erfolgte die Einlagerung bei $-10^{\circ} \mathrm{C}$.

Der Versuch der Langzeitlagerung bei Buche zielte darauf ab, die Einlagerung in der Forstgenbank als Doppelsicherung neben der Ausweisung bzw. Anlage von Erhaltungsbeständen zu erproben und die Überbrückung von Saatgutengpässen in Jahren ohne Vollmast zu ermöglichen. Die Keimfähigkeit der in der Genbank verbliebenen Saatgutpartien wird jährlich überprüft. Abbildung 2 verdeutlicht, dass das Saatgut aus der Vollmast 1989 über ein sehr hohes Keimprozent verfügte, das in den ersten fünf Jahren der Einlagerung weitgehend unverändert blieb und nach 10-jähriger Einlagerung im Mittel noch bei $71 \%$ lag. Nach 18 Jahren Lagerung unter optimierten Bedingungen zeigen die Partien immerhin noch Keimprozente von im Mittel 54 \%. Bei den geringeren Masten in den Folgejahren zeigte sich dagegen von Anfang an eine verminderte durchschnittliche Keimfähigkeit. Dies mag daran liegen, dass in Jahren mit Teilmasten nur Teile der Baumpopulationen an der Blüte und Fruchtbildung beteiligt waren. Die geringere Lebenskraft spiegelt sich auch in der Lagerfähigkeit wider. Bei einigen dieser Partien hielt sich die Keimkraft etwa fünf bis sieben Jahre lang auf dem Ausgangsniveau, um danach teilweise deutlich abzusinken.

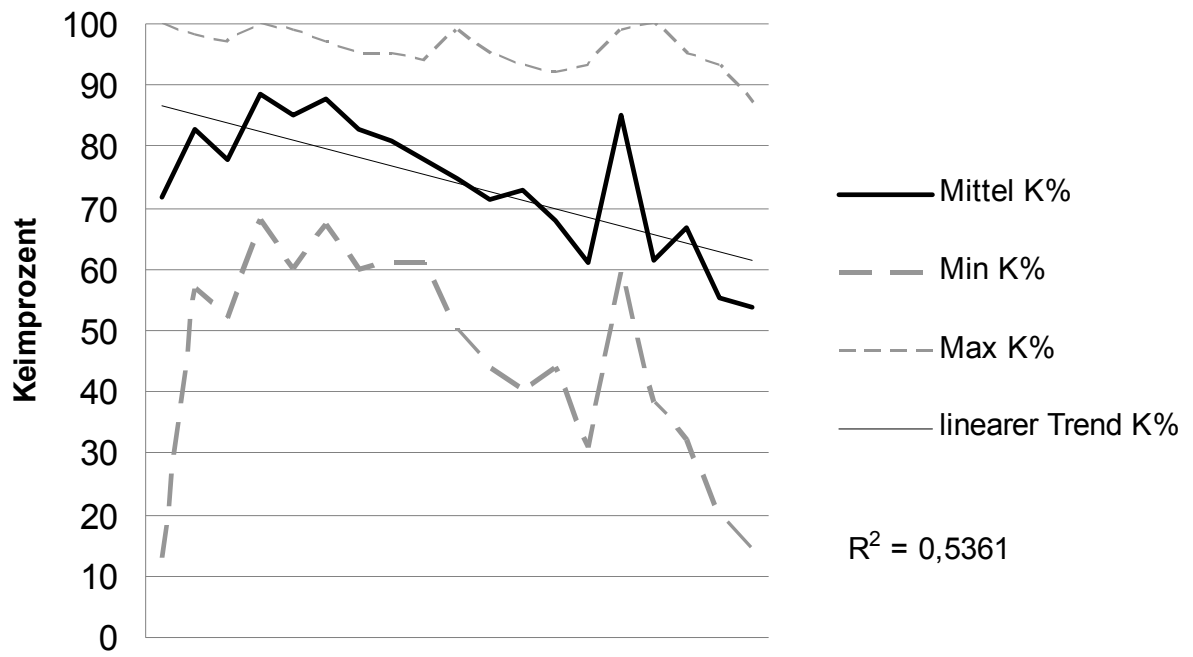

'89 '91' 93 '95' 97 '99 '01 '03 '05 '07 Jahr der Keimprüfung

Abbildung 2: Entwicklung des Keimprozentes (K\%) von Buchensaatgut nach mehrjähriger Einlagerung bei-10 ${ }^{\circ} \mathrm{C}$, Reifejabr 1989, $N=30$ Saatgutpartien

Mit der beschriebenen Einlagerungsmethode erscheint es nunmehr möglich, unter Berücksichtigung der individuellen Reaktion jeder Saatgutpartie auf die Behand- 
lungsmaßnahmen auch lange Zeitspannen von mehr als zehn Jahren zu überbrücken und der Praxis jederzeit hochwertiges Buchensaatgut zur Verfügung zu stellen.

\subsection{Vegetative Vermehrungsmethoden}

Die vegetative Vermehrung hat die Erzeugung von genetisch identischen Kopien des Ausgangsmaterials zum Ziel. Dies kann sinnvoll sein, wenn spezielle Genotypen über die Lebensspanne eines Individuums hinaus erhalten werden sollen, künstliche Populationen mit ausgewählten Genotypen zusammengestellt werden sollen (s. Abschnitt: Erhaltung ex-situ), oder aber im Rahmen einer sogenannten bulk, propagation, wenn nicht genügend geeignetes generatives Vermehrungsgut zur Verfügung steht (KLEINSCHMIT 1989).

Die Möglichkeiten von vegetativen Vermehrungstechniken unterliegen allerdings einigen gravierenden Beschränkungen. Erstens geht die Alterung von Gehölzen mit einer abnehmenden Vermehrbarkeit einher (BONGA 1987). Die Dedifferenzierung von Geweben und die anschließende Regeneration fehlender Organe wird durch zunehmendes physiologisches Alter des Ausgangsmaterials erschwert (Zyklophysis). Zweitens beschränkt das Forstvermehrungsgutgesetz die Verwendung von vegetativ vermehrtem Material für forstliche Zwecke auf geprüftes Vermehrungsgut. Drittens sind die Kosten von vegetativ erzeugtem Vermehrungsgut i. d. R. höher als die von Sämlingen.

\subsubsection{Stecklingsvermehrung}

Im Rahmen einer Diplomarbeit wurden die Voraussetzungen für eine erfolgreiche Stecklingsvermehrung der Buche untersucht (BAUMGARTEN 1991). Es zeigte sich, dass eine befriedigende Bewurzelung von Steckreisern von über $90 \%$ erzielt werden kann! Als Steckhölzer werden noch unverholzte Triebe genommen. Diese werden mit 0,5\% iger Indolylbuttersäure im Steckbereich behandelt und im Kiessubstrat in einem Gewächshaus mit Sprühanlage gesteckt. Das verwendete Material muss physiologisch jung sein! In dem Versuch wurden Steckreiser von dreijährigen Sämlingen gewonnen. Verschiedene Schnitttechniken im basalen Bereich steigerten den Bewurzelungserfolg dagegen nicht.

\subsubsection{Mikrovegetative Vermehrung und Langzeitlagerung}

Die In-vitro-Vermehrung zielt auf die jahreszeitlich unabhängige Regeneration neuer Sprosse und Pflanzen unter sterilen Bedingungen. Dies gelingt entweder über die Förderung des Austriebs von Achselknospen, über die Produktion von Adventivknospen oder auf dem Wege der somatischen Embryogenese. Bis dato wird jedoch im Gegensatz zu vielen anderen Laubbaumarten keines der genannten Verfahren für die Massenvermehrung von Buche genutzt. 
Im Rahmen eines BMBF-Forschungsvorhabens (WEISGERBER u. GEBHARDT 1995) gelang es zwar mit hinreichendem Erfolg, somatische Embryonen an steril präparierten zygotischen Embryonen von Buchen zu induzieren, und auch die Induktion haploider Pflanzen aus Antherenkultur wurde beschrieben (JOERGENSEN 1988, 1991). Die Induktion von Achsel- oder Adventivsprossen an Knospen oder Kallusgewebe adulter Buchen war jedoch nur mit mäßigem Erfolg möglich (GEBHARDT 1990, MEIER u. REUTHER 1994).

Der Einfluss der Topophysis war nachzuweisen und resultierte in einer besseren Vermehrbarkeit von Explantaten aus Stockausschlägen gegenüber solchen aus dem äußeren Kronenbereich (GEBHARDT 1990). Nach LANG u. KOHLENBACH (1988) wurden aus Blättern juveniler und adulter Buchen lebensfähige Protoplasten isoliert, deren Entwicklung jedoch im Kallusstadium verblieb.

\section{Herkunftssicherung}

Nach dem in Deutschland geltenden Recht darf forstliches Saatgut nur in amtlich zugelassenen Beständen, Samenplantagen etc. gewonnen werden. Die Erntebestände sind in einem amtlichen Erntezulassungsregister dokumentiert, und die Beerntung ist meldepflichtig. Amtliche Kontrollen sind dann eine Vorraussetzung für die Ausstellung sogenannter Stammzertifikate.

Nachlassende oder ungenügende Kontrolle begünstigt die Verwendung von falsch deklariertem Vermehrungsgut. Dies wiederum kann zu Schäden der Waldentwicklung, Gewinneinbußen der Forstbetriebe und hohen Folgekosten für Ersatzaufforstungen führen. Die Kontrolle von Saatgutaufkommen und -verbringung durch amtliche Kontrollen und Dokumentation wird erheblich erschwert durch:

- einen zunehmenden Saatgut- und Pflanzenhandel über Landesgrenzen hinweg

- $\quad$ hohen Zeit- und Kostenaufwand der amtlichen Kontrolle

- $\quad$ immer weniger Personal in den Behörden und bei den Forstbetrieben

Da Saatgutpartien nach der Ernte häufig in kleinen Teilmengen gehandelt werden und die Pflanzenanzucht in unterschiedlichen Betrieben erfolgen kann, ist eine Rückverfolgung letztendlich nur dann möglich, wenn ein genetischer Vergleich der gehandelten Saatgutpartien mit einer gelagerten Rückstellprobe erfolgen kann. Die Überprüfbarkeit der Herkunftsidentität mithilfe von Rückstellproben findet auf freiwilliger Basis nach den Verfahren des Fördervereins für forstliches Vermehrungsgut e. V. (HAASE et al. 2007) oder des Zertifizierungsringes für überprüfbare forstliche Herkunft Süddeutschland e. V. (www.zuef-forstpflanzen.de) statt.

Referenzproben dienen sowohl der Ermittlung des Anteils an reinem Saatgut und nach Keimprüfung auch einer Abschätzung der möglichen Menge des zu erzeugenden Vermehrungsgutes als auch für Vergleichsuntersuchungen zum Nach- 
weis der Authentizität. Mit genetischen Analysen kann die Übereinstimmung von Art und Verteilung genetischer Marker im Saatgut und in den daraus erzeugten Pflanzen geprüft werden. Eine Zuordnung des geernteten Saatgutes zum Erntebestand ist in Grenzen (WYPUKOL et al. 2008) auch durch DNA-Analyse der Bucheckern-Schalen möglich, da der Genotyp der Schalen dem des Mutterbaumes 1:1 entspricht, und sich deren Abstammung nicht ändert.

Im Rahmen des BMBF-Verbundforschungsprojektes „Herkunftskontrolle“ (FKZ 330587A) erwies sich darüber hinaus die Analytik von Stabilisotopen als tauglich zur Unterscheidung von Saatgutpartien zahlreicher Baumarten. Mithilfe einer Diskriminanzanalyse war es möglich, nicht hessische Saatgutpartien von Buche anhand der Stabilisotopen-Signaturen (13C/15N) und Elementgehalte von Kohlenstoff und Stickstoff von Saatgutpartien aus fünf hessischen Beständen zu unterscheiden (GEBHARDT 2008).

Letztendlich wird eine Kombination aus amtlicher Kontrolle und Dokumentation, der Möglichkeit von genetischen Vergleichen mithilfe von Referenzproben und die Untersuchung von Stabilisotopen die Herkunftssicherung wirkungsvoll unterstützen.

\section{Diskussion}

Mit den Herkunftsversuchen zur großräumigen genetischen Variation der Buche und der Erfassung eines Netzes von Buchenerhaltungsobjekten sind wichtige Schritte zu einer genetisch nachhaltigen Nutzung der Buchenwälder unternommen worden.

Wie bei allen anderen Baumarten auch unterliegen die heute vorhandenen zugelassenen Saatguterntebestände der Buche ständiger Veränderung. Mit dem Älterwerden ändert sich ihre Zusammensetzung durch natürliche Absterbe- und Verjüngungs-Prozesse aber auch durch den Einfluss der Bewirtschaftung. Wenn beispielsweise die wüchsigsten und qualitativ besten Buchen zuerst und bevor sie sich flächendeckend verjüngen konnten, genutzt werden, dann kann dies erhebliche Auswirkungen auf die genetische Zusammensetzung des Folgebestandes haben. Noch gravierender wären die Folgen, wenn Teile des für die Saatguternte zugelassenen Bestandes mit Buchen aus einem anderen Bestand unterpflanzt würden. Aufgrund solcher Veränderungen in den Ausgangsbeständen verlieren zum einen Ergebnisse von Vergleichsprüfungen irgendwann ihre Aussagekraft. Zum anderen lassen sich in natürlicher Verjüngung befindliche oder unterpflanzte Buchenbestände nicht mehr mit den üblichen Netzen zur Saatguternte unterlegen. Deshalb müssen immer wieder neue Bestände in Vergleichsprüfungen einbezogen und bei Bewährung für die Saatguternte zugelassen werden. Auch das Register der nicht geprüften Saatguterntebestände muss laufend überprüft und aktualisiert werden, damit nur wirklich phänotypisch geeignet erscheinende und technisch beerntbare Bestände darin enthalten sind. 
Die Kriterien der Forstvermehrungsgut-Zulassungsverordnung geben zwar Mindestanforderungen an zuzulassende Bestände vor, eine Zulassung aller geeigneten Bestände ist damit aber nicht gewährleistet. Forschungsbedarf besteht darin, inwieweit bereits für alle Herkunftsgebiete eine ausreichende Anzahl von Beständen zugelassen ist und ob diese Bestände tatsächlich qualitativ über die übrigen in jedem Herkunftsgebiet herausragen. Im Sinne einer Sicherung der genetischen Vielfalt muss ferner darauf geachtet werden, dass die zugelassenen Bestände auch tatsächlich alle und nicht nur besonders einfach und billig beerntbare Bestände beerntet werden. Die Informationen über die tatsächlich beernteten Bestände und die Erntemengen liegen bei den zuständigen Landesstellen nach Forstvermehrungsgutgesetz vor und müssen nur diesbezüglich ausgewertet werden.

Der Datenbestand „Erhaltung forstlicher Genressourcen“ stellt eine einmalige Quelle detaillierter Informationen dar, welche es dem Forstbetrieb erlaubt, Prioritäten im Naturschutzbereich zu setzen oder aber weitere qualitativ herausragende Bestände für die Zulassung nach dem Forstvermehrungsgutgesetz vorzuschlagen. Die Herausforderung für die Zukunft wird in der Fortschreibung und Aktualisierung dieses Datensatzes im Zusammenspiel zwischen Waldeigentümer/Forsteinrichtung und NW-FVA liegen. Außerdem soll mithilfe der Ex-situErhaltungsplantagen herkunftsgesichertes Vermehrungsgut für die Forstwirtschaft und den Waldnaturschutz bereitgestellt werden.

Aktuell werden Auswirkungen des Klimawandels auf die Wälder modelliert. Dabei sollte zwingend das durch genetische Variation innerhalb der Art Fagus sylvatica L. gegebene evolutionäre Anpassungspotenzial berücksichtigt werden. Dies ist bisher nicht der Fall. Ohne diesen Aspekt sind die Modelle aber unvollständig, da sie den ggf. großen genetischen Einfluss nicht abbilden können. Im gleichen Zuge sollten die bestehenden Herkunftsempfehlungen um Aspekte des Klimawandels erweitert werden.

Ergebnisse von punktuellen und kurzfristigen Fallstudien zeigen, dass natürliche und anthropogene Einflüsse populationsgenetische Prozesse verändern können. Besondere Gefährdungen für die genetische Vielfalt und damit für die langfristige Anpassungsfähigkeit der Buche stellen Umweltbelastungen wie Immissionen und anthropogen verursachte Klimaänderungen dar, welche in ihrem Ausmaß und ihrer Geschwindigkeit das genetische Anpassungspotenzial der Buche vor besondere Herausforderungen stellen. Der Einfluss solcher Umweltveränderungen auf die genetische Variation sollte durch genetisches Monitoring u. a. für die Baumart Buche als Teil des forstlichen Monitorings untersucht werden. Dazu ist ein Netz von Intensivmessflächen notwendig, auf welchen die genetischen Prozesse auf bewirtschafteten und unbewirtschafteten Flächen langfristig untersucht werden. Eine Anbindung an das Netz des europaweiten forstlichen Umweltmonitorings ist anzustreben. Das genetische Monitoring soll Aussagen über die Veränderungen und mögliche Gefährdung des genetischen Systems durch Bewirtschaftungsmaßnahmen, Schadstoffeinträge und Klimawandel ermöglichen. Außer- 
dem soll exemplarisch der Zusammenhang zwischen der genetischen Variation und den physiologischen Grundlagen der Anpassungsfähigkeit untersucht werden. Dazu muss der aktuelle genetische Zustand von ausgewählten Altbeständen charakterisiert und die Weitergabe der genetischen Information an die Folgegeneration beobachtet werden. Dort, wo die lokale genetische Ausstattung nicht ausreicht, sind mögliche positive Auswirkungen von ,genetischer Anreicherung“ mit Material anderer Herkünfte zu prüfen und ggf. entsprechende Maßnahmen zu ergreifen.

Nachhaltige Waldwirtschaft wird nur dann gelingen, wenn Ökosysteme auf verschiedenartige Änderungen der Rahmenbedingungen reagieren können und gleichzeitig die Waldfunktionen gewahrt werden. Ein idealer Wald ist optimal an die jeweiligen Bedingungen angepasst, hoch produktiv und besitzt eine hohe Anpassungsfähigkeit an sich ändernde Standort- und Klimabedingungen. Zugelassene Saatguterntebestände und In-situ-Bestandesobjekte zur Erhaltung forstlicher Genressourcen werden u. a. unter dem Aspekt der Angepasstheit und Anpassungsfähigkeit ausgewählt und spezielle Maßnahmen zu ihrer Behandlung vorgeschlagen. Die Nutzung dieser zugelassenen Saatguterntebestände und die spezielle Bewirtschaftung der In-situ-Bestandesobjekte als Genressource trägt damit in besonderer Weise zum Schutz der genetischen Vielfalt bei. Die Integration von Schutz und nachhaltiger Nutzung ist für die Forstwirtschaft daher besonders sinnvoll.

\section{Literatur}

Baumgarten, S. (1991): Möglichkeiten und Einflüsse der Anwendung von Wuchsstoffen bei der Stecklingsvermehrung der Rotbuche (Fagus sylvatica L.). Diplomarbeit, Fachhochschule Weihenstephan, Fachbereich Forstwirtschaft, 105 S., Band 2 mit Anlagen

BONGA, J.M. (1987): Clonal propagation of mature trees: problems and possible solutions. In: BONGA J.M. u. DurZan D.J. (Hrsg.): Cell and Tissue Culture in Forestry. Martinus Nijhoff Publ. Dordrecht, Vol. I, 249-271

BUNDESANSTALT FÜR LANDWIRTSCHAFT UND ERNÄHRUNG (2007): Erhebung zur Versorgungssituation von forstlichem Vermehrungsgut im Bundesgebiet (Erfassungszeitraum 01.07.2006 30.06.2007), 324-2.2, $118 \mathrm{~S}$,

http://www.ble.de/cln_116/nn_427084/SharedDocs/Downloads/02_Kontrolle_ZZulassung /06_SaatUndPflanzgut/Erhebung06_07,templateId=raw,property=publicationFile.pdf/Erhe bung06_07.pdf

BUND-LÄNDER-ARBEITSGRUPPE „ForSTLICHE GENRESSOURCEN UND FORSTSAATGUTRECHT“ (2006): Tätigkeitsbericht für den Zeitraum 01.01.2001 - 01.01.2004, 152 S.

CZAJKowski, T. u. Bolte, A. (2006a): Unterschiedliche Reaktion deutscher und polnischer Herkünfte der Buche (Fagus sylvatica L.) auf Trockenheit. Allg. Forst- u. J.-Ztg. 177, 30-40

CZAJKowski, T. u. BOlTE, A. (2006b): Frosttoleranz deutscher und polnischer Herkünfte der Buche (Fagus sylvatica L.) und ihre Beeinflussung durch Trockenheit. Archiv für Forstwesen u. Landschaftsökologie 40,119-126 
ForSTVERMEHRUNGSGUT-HERKUNFTSGEBIETSVERORDNUNG (1994): Forstvermehrungsgut-Herkunftsgebietsverordnung vom 07.10.1994, Bundesgesetzblatt I S. 3578, geändert durch Verordnung vom 15.01.2003, Bundesgesetzblatt I, S. 238

FORSTVERMEHRUNGSGUT-ZULASSUNGSVERORDNUNG (2002): Forstvermehrungsgut-Zulassungsverordnung vom 20.12.2002, Bundesgesetzblatt I, S. 4721, berichtigt am 07.01.2003 Bundesgesetzblatt I, S. 50

GEBHARDT, K. (1990): In-vitro-Kultur bei Waldbäumen: Untersuchungen über In-vitro-Techniken zur Züchtung und Anzucht von Waldbäumen. Schriften des Forschungsinstitutes für schnellwachsende Baumarten, Hann. Münden, Band 6

GEBHARDT, K. (2008): Unterscheidung von Saatgutpartien anhand der Stabilisotopen-Signaturen und Elementgehalte. Tagungsband des Symposiums „Herkunftskontrolle“ 7.-8.2.08, Kassel, im Druck

GILLET, E.M. (1999): Minimum sampling size for sampling genetic marker distributions. In: Gillet, E.M. (Hrsg.): Final compendium of the Research Project „Development, optimisation and validation of molecular tools for assessment of biodiversity in forest trees" in the European Union DGXII, http://webdoc.sub.gwdg.de/ebook/y/1999/whichmarker/index.htm

GrEgORIUS, H.-R. (1997): Genetische Grundlagen der Ökosystemstabilität. Forstwissenschaftliches Centralblatt 116, 29-38

HaAse, B.; Hosius, B. u. Leinemann, L. (2007): Das FfV-Verfahren stellt sich vor. AFZ/Der Wald $62,852-853$

Janßen, A. (2000): Der Einfluss von Ernteverfahren auf die genetische Struktur von Saatgut eines Buchenbestandes. Diss. Univ. Göttingen, Forschungsbericht der HLFWW, Bd. 27

Joergensen, J. (1988): Embryogenesis in Quercus petraea and Fagus sylvatica., J. Plant Physiol. 132, 638640

Joergensen, J. (1991): Androgenesis in Quercus petraea, Fagus sylvatica and Aesculus hippocastanum. In: Ahuja, M.R. (Hrsg.): Woody Plant Biotechnology, Plenum Press, New York, 353-354

KLeinschmit, J. (1989): Perspektiven und Grenzen der vegetativen Vermehrung forstlichen Pflanzenmaterials. Forstarchiv 60, 139-145

KleinsChmit, J. u. Svolba, J. (1996): Ergebnisse der Buchenherkunftsversuche von Krahl-Urban. AFZ/Der Wald 51, 780-782

LANG, H. u. KOHLENBACH, W. (1988): Callus formation from mesophyll protoplasts of Fagus sylvatica L.. Plant Cell Reports 7, 485-488

LIESEBACH, M. (2000): Genetic value and management of beech in mountainous regions in Germany. Zeszyty Naukowe Akademii Rolniczej im. H. Kollataja w Krakowie nr 358, Sesja Naukowa Zeszyt 69, 79-91

LiesebaCH, M.; WÜHlisCH, G. v. u. MuHS, H.-J. (1998): Anlage des Internationalen Buchen-Herkunftsversuches 1996/1998. In: Jahresbericht 1998 der Bundesforschungsanstalt für Forst- und Holzwirtschaft, Hamburg, S. 14

Magri, D.; Vendramin, G.G.; Comps, B.; Dupanloup, I.; Geburek, T.; GÖmÖry, D.; Latalowa, M.; Litt, T.; Paule, L.; Roure, J.M.; Tantau, I.; Van Der Knaap, W.O.; Petit, R.J. u. De BEAUlieu, J.-L. (2006): A new scenario for the Quaternary history of European beech populations: palaeobotanical evidence and genetic consequences. New phytologist 171, 199-221

Meier, K. u. Reuther, G. (1994): Factors controlling micropropagation of mature Fagus sylvatica. Plant Cell, Tissue and Organ Culture 39, 231-238

MuHs, H.-J. u. WÜHLISCH, G. v. (1992): Research on the improvement of beech in the last decade. In: Rossello, R. E. (Hrsg.): Investigación Agraria, Sistemas y Recursos Forestales, Proceedings of the International Congress on Beech, Pamplona, 63-89

Paul, M.; Hinrichs, T.; Janben, A.; SchmitT, H.P.; Soppa, B.; Stephan, B.R. u. Dörflinger, H. (2000): Konzept zur Erhaltung und nachhaltigen Nutzung forstlicher Genressourcen in der Bundesrepublik Deutschland. Neufassung 2000, bestätigt durch die Forstchefkonferenz am 
26./27.10.2000 in Augsburg, Sächsische Landesanstalt für Forsten, Pirna, http://www.genres.de /fgrdeu/konzeption/

Sander, T.; König, S.; Rothe, G. M.; Janßen, A. u. Weisgerber, H. (2000): Genetic variation of European beech (Fagus sylvatica L.) along an altitudinal transect at mount Vogelsberg in Hesse, Germany. Molecular Ecology 9, 1349-1361

Thomasius, H. u. GÄRTNER, H. (1988): Auswertung eines Buchenprovenienzversuches von Münch hinsichtlich Wachstum, Qualität und Phänologie. In: KorPel, S. u. PAule, L. (Hrsg.): 3. IUFRO-Buchensymposium. Hochschule für Forstwirtschaft und Holztechnologie, Zvolen, $31-45$

Vornam, B.; DeCARLi, N. u. GaILING, O. (2004): Spatial distribution of genetic variation in a natural beech stand (Fagus sylvatica L.) based on microsatellite markers. Conservation Genetics (5): 561570

Weisgerber, H. u. Gebhardt, K. (1995): Ergebnisse und Perspektiven der Mikrovermehrung von Waldbäumen. Allg. Forst- und Jagdzeitung 166, 99-105

WÜHLISCH, G. V. (2007): Series of international provenance trials of European beech. In: Improvement and Silviculture of Beech, Symposium IUFRO Research Group 1.10.00, Research Institute of Forests and Rangelands (RIFR), Teheran, Iran 2007, 135-144

Wypukol, H.; Liepelt, S.; Ziegenhagen, B. u. Gebhardt, K. (2008): Genetische Methoden zur Abstammungsanalyse und Prüfung von Sortenechtheit und -reinheit. In: Tagungsbericht des Symposiums "Herkunftskontrolle", 7.-8.2.08 in Kassel (im Druck)

Ziehe, M.; STARKe, R.; HATtemer, H.H. u. Turok, J. (1998): Genotypische Strukturen in BuchenAltbeständen und ihren Samen. Allgemeine Forst- und Jagd-Zeitung 169, 91-99

Korrespondierender Autor:

Dr. Jörg R. G. Kleinschmit

Nordwestdeutsche Forstliche Versuchsanstalt

Abteilung Waldgenressourcen

Professor-Oelkers-Str. 6

34346 Hann. Münden

E-Mail: Joerg.Kleinschmit@nw-fva.de

URL: www.nw-fva.de

Hans-Martin Rau

Dr. Karl Gebhardt

Nordwestdeutsche Forstliche Versuchsanstalt 


\title{
Komplexe Erkrankungen an Buche
}

\author{
Complex diseases in beech
}

Ulrich Bressem

\section{Zusammenfassung}

Die Rotbuche besiedelt unterschiedlichste Lebensräume, besitzt laut Lehrmeinung eine hohe Anpassungsfähigkeit an umweltbedingte Veränderungen, gilt als konkurrenzstark und dominant, aber auch als Beispiel für eine plastische Baumart im Klimawandel. Sie soll auch in Zukunft eine wichtige, zentrale Rolle in der Forstwirtschaft spielen.

Es stellt sich die Frage, ob diese Einschätzung angesichts der Klimaänderung und der teilweise bedenklichen Vitalitätsentwicklung der Buche in den letzten Jahren nicht doch zu optimistisch ist.

In letzter Zeit werden an Buche vermehrt unterschiedliche Erkrankungen beobachtet. Im ersten Teil dieses Beitrages werden (a) die Buchenkomplexerkrankung/Buchenrindennekrose, (b) Phytophthora cambivora an Buche und (c) die sogenannte Vitalitätsschwäche der Buche mit Prachtkäferbefall beschrieben. Näher eingegangen wird jeweils auf typische Symptome, die Umstände des Krankheitsauftretens, den Krankheitsverlauf und mögliche forstliche Gegenmaßnahmen.

Im zweiten Teil wird kurz über die Ergebnisse einer Umfrage zu den genannten Schäden im Zuständigkeitsgebiet der Nordwestdeutschen Forstlichen Versuchsanstalt (Niedersachsen, Hessen, Sachsen-Anhalt, Schleswig-Holstein) 
berichtet und es werden weitere in Nordwestdeutschland auftretende Schadbilder an Buche und die besonderen Rahmenbedingungen für deren Entstehung beschrieben. Nach einer abschließenden Wertung der Ergebnisse werden Empfehlungen für die Behandlung von Buchenbeständen aus Sicht des Waldschutzes gegeben.

Stichworte: Buche, Buchenkomplexerkrankung, Phytophthora cambivora, Buchenvitalitätsschwäche, Klimaänderung

\begin{abstract}
Beech currently occupies very different habitats, and, according to expert opinion, is highly adaptable to environmentally induced changes, regarded as highly competitive and dominant, but also to have a very high adaptation potential in the changing climate. It is expected to play an important, central role in forest management in the future as well.

In view of the climate change, and the somewhat critical changes in beech vitality in recent years, the question, whether this estimation of beech is perhaps too optimistic, should be posed.

Recently, different diseases have been observed more frequently in beech. In the first part of this paper, (a) the beech bark disease, (b) Phytophthora cambivora in beech, and (c) the so-called reduced beech vitality accompanied by Agrilus viridis are described. The typical symptoms, conditions under which the diseases occur, disease development and possible forestry counter-measures are discussed in more detail.

In the second part, the results of a questionnaire about the above-mentioned damage conducted in the Northwest German Forest Research Station's areas of responsibility (Lower Saxony, Hesse, Saxony-Anhalt, Schleswig-Holstein) are reported in brief. In addition, the diseases found to affect beech in northwest Germany, and the particular conditions for their emergence are described. Finally, after an evaluation of the results, forest protection recommendations for the management of beech stands are made.
\end{abstract}

Keywords: beech, beech bark disease, Phytophthora cambivora, reduced beech vitality, climate change 


\section{Einleitung}

Die Rotbuche besiedelt unterschiedlichste Lebensräume und besetzt diese dauerhaft (DFWR 2008). Sie hat eine breite genetische Basis und besitzt eine hohe Anpassungsfähigkeit an umweltbedingte Veränderungen. Sie gilt als ein Beispiel für pflanzliche Konkurrenzstärke (NIESAR 2007) und Dominanz im Bestand, aber auch als Beispiel für eine plastische Baumart im Klimawandel.

Viele Fachleute sind der Meinung, dass natürliche und naturnahe Buchenwälder für den Klimawandel gut gerüstet sind (KöLLING 2006a). Die Buche soll auch in der Zukunft in vielen Bereichen eine wichtige, zentrale Rolle spielen.

Ist diese Einschätzung uneingeschränkt gerechtfertigt oder müsste sie angesichts der sich abzeichnenden klimabedingten Verschiebungen bei den Konkurrenzverhältnissen der Baumarten und der teilweise bedenklichen Vitalitätsentwicklung der Buche in der letzten Zeit nicht doch stärker relativiert werden?

In den letzten Jahren werden vermehrt unterschiedliche Erkrankungen und Schadbilder an Buche beobachtet. Im Rahmen dieses Beitrages sollen zunächst die wesentlichen Schadenstypen beschrieben und vorgestellt werden, nämlich die klassische Buchenkomplexerkrankung (Buchenrindennekrose), Phytophthora cambivora an Buche und die sogenannte Buchenvitalitätsschwäche.

Darüber hinaus soll erörtert werden, inwiefern bei solchen Schadbildern aus Sicht des Waldschutzes und Waldbaus steuernd eingegriffen werden kann bzw. muss, um ggf. größere Schäden zu verhindern.

Im Spätherbst 2007 wurde im Zuständigkeitsgebiet der Nordwestdeutschen Forstlichen Versuchsanstalt (NW-FVA; Niedersachsen, Hessen, Sachsen-Anhalt und Schleswig-Holstein) eine Umfrage zu entsprechenden Schäden an Buche durchgeführt. Die wichtigsten Ergebnisse sollen hier ebenfalls vorgestellt werden.

Am Schluss des Beitrages wird der Versuch unternommen, die Ergebnisse mit Blick auf den Klimawandel zu werten.

\section{Beschreibung der drei wichtigsten Schadenstypen}

\subsection{Klassische Buchenkomplexerkrankung (Buchenrindennekrose)}

Die Buchenkomplexerkrankung / Buchenrindennekrose ist seit vielen Jahrzehnten bekannt. Sie tritt meist in Erkrankungswellen auf, die mehrere Jahre anhalten. Die letzte große Buchensterbenswelle liegt über 40 Jahre zurück (NIESAR 2007).

Ab 1999/2000 ist die Erkrankung überregional und zeitgleich in höheren Lagen z. B. in Nordrhein-Westfalen (Sauerland) (EMSCHERMANN et al. 2001), Rheinland-Pfalz (EISENBARTH 2001) und angrenzenden EU-Ländern stark und teilweise mit flächigen Schäden aufgetreten. Seit 2002 wurde auch im Zuständig- 
keitsbereich der NW-FVA lokal bis regional eine bedeutsame Zunahme der Erkrankung beobachtet.

\subsubsection{Typische Symptome der Buchenkomplexerkrankung}

Vorausgehender Befall durch die Buchenwollschildlaus (Cryptococcus fagisuga; Massenvermehrung vor (!) dem eigentlichen Krankheitsbeginn; bewirkt Rindenverletzungen und Prädisposition).

- Langgestreckte, spindelförmige, oft mehrere Meter lange Rindennekrosen, meist auf der Schattenseite der Stämme (nördliche bis nordöstliche Richtungen), oft unterhalb des Kronenansatzes beginnend und nach unten fortschreitend, mit Schleimfluss.

- Die Rindennekrosen werden hervorgerufen durch rindenzerstörende Pilze, wie z. B. Neonectria coccinea (früher: Nectria coccinea). Neonectria ist gekennzeichnet durch winzige, nur stecknadelkopfgroße, rötliche, kugelförmige Fruchtkörper auf der Rinde (s. Abb. 1). Bei starker Fruchtkörperbildung können Befallsstellen rötlich schimmern.

- Im fortgeschrittenen Stadium an geschädigten Stellen am Stamm aufreißende / abblätternde Rinde und Bohrlöcher von Holzbrütern, z. B.: Nutzholzborkenkäfer (Xyloterus domesticus), Sägehörniger Werftkäfer (Hylecoetus dermestoides); dort Eindringen von Weißfäuleerregern mit Verlust der Holzfestigkeit und rascher Entwertung des Holzes, z. B. durch: Zunderschwamm (Fomes fomentarius), Angebrannter Rauchporling (Bjerkandera adusta). Vereinzelt tritt auch der Rotrandige Baumschwamm (Fomitopsis pinicola) auf, der eine Braunfäule verursacht.

- Im Endstadium am Stamm: Pilzkonsolen (u. a. Zunderschwamm, Angebrannter Rauchporling). Pilzbefall in größerer Stammhöhe zeigt den Beginn der Zerfallsphase an.

- Stammbrüche oft in Form typischer flacher, waagerechter, relativ glatter Bruchstellen in 5-8 m Höhe (s. Abb. 2) Gelegentlich brechen Stämme auch bei noch grüner Krone.

- Auf der Schattenseite der Stämme: oft am Stammfuß beginnende und mehrere Meter hoch reichende, auffällige Rau-/Grobborkigkeit. Stammfüße häufig recht stark mit Moos bewachsen.

- Zuweilen Befall durch holzentwertende Käfer (Xyloterus domesticus) auch an sonst äußerlich (noch) symptomlosen Buchen. ( $\mathrm{Zu}$ den Symptomen insgesamt siehe z. B. auch: EISENBARTH 2001, EMSCHERMANN u. NIESAR 2001; PETERCORD 2006, AREND et al. 2006. Die schädigende Wirkung der Buchenwollschildlaus haben u. a. KUNKEL 1968, BRAUN 1976 u. 1977 sowie PETERCORD 1999 untersucht.) 

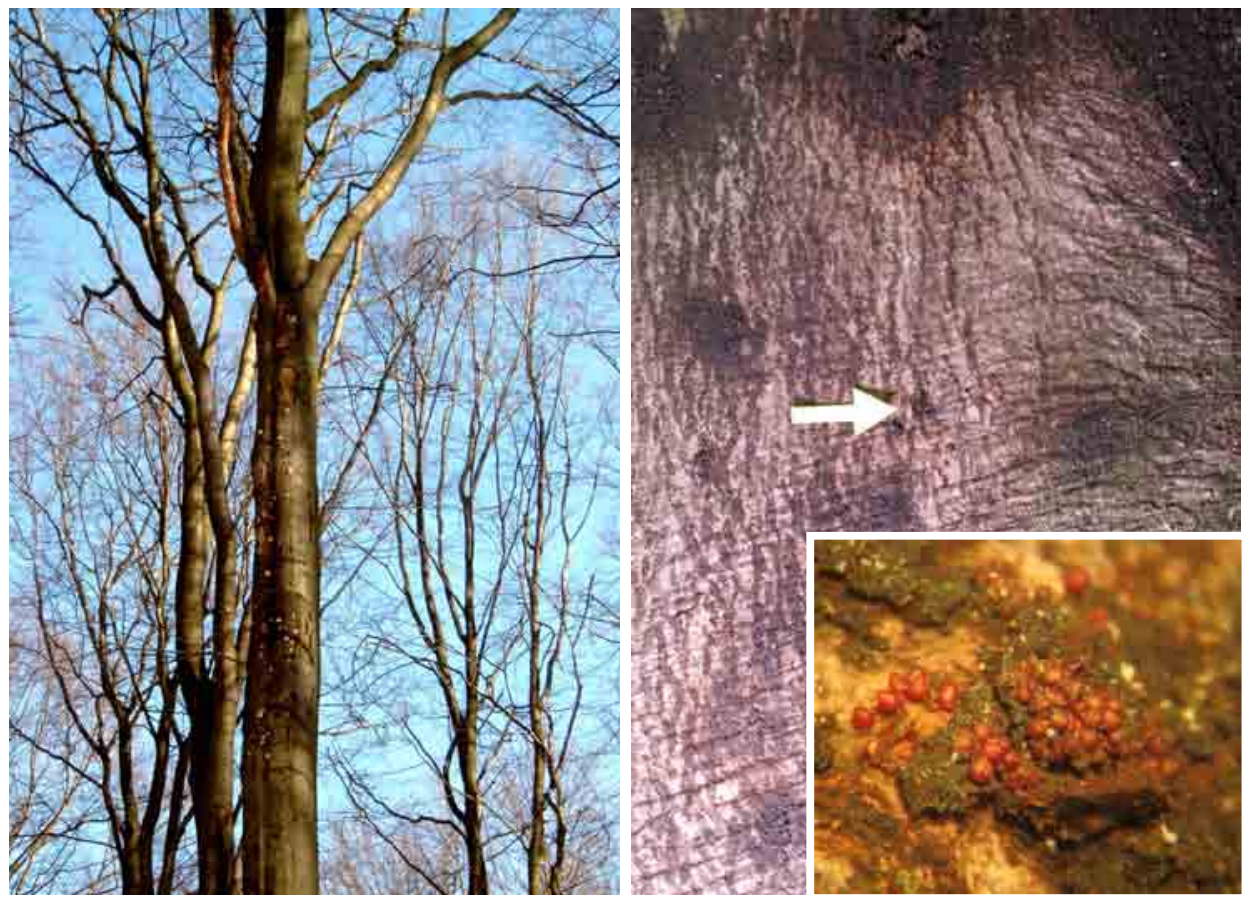

Abbildung 1: Buchenkomplexerkrankung, links: Rindenschädigung hoch oben am Stamm; rechts: Schleimflussflecken und Fruchtkörper von Neonectria coccinea
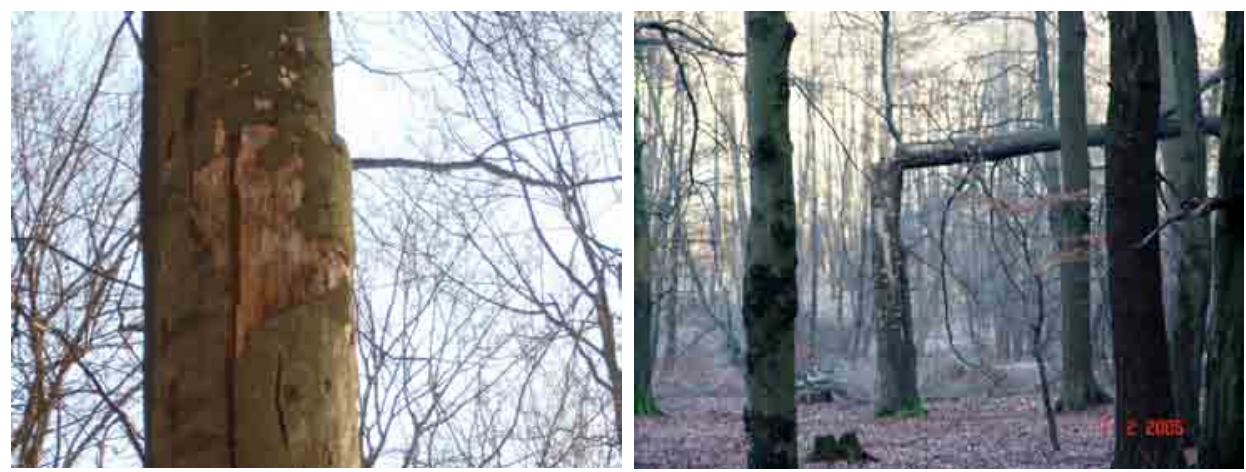

Abbildung 2: Buchenkomplexerkerankung, links: abplatzende Rinde im mittleren Stammbereich, Weißfäule und bereits angedeuteter Stammbruch; rechts: typischer waagerechter Stammbruch

Beiträge aus der NW-FVA, Band 3, 2008 


\subsubsection{Umstände des Auftretens / Krankheitsverlauf}

Untersuchungen in höheren Mittelgebirgslagen Nordrhein-Westfalens (NIESAR et al. 2007, NiESAR 2007) lassen den Schluss zu, dass hohe Niederschlagssummen und anhaltend hohe Feuchtigkeit in milden Wintern sowie vorheriger oder zeitgleicher Befall durch die Buchenwollschildlaus wesentliche Vorraussetzungen für die Entstehung der Erkrankung sind. Die genannten Faktoren ermöglichen die Infektion des Baumes und das Wachstum des eigentlichen Krankheitsverursachers Neonectria coccinea.

Die Buchenwollschildlaus leitet den Krankheitskomplex ein. Sie verursacht kleinflächige Rindennekrosen, die im mittleren Stammbereich unter geschlossener Rinde zunächst unbemerkt bleiben. Durch diese Schädigungen des Rindengewebes wird Pilzbefall durch Neonectria-Arten möglich. Sind die winzigen, rötlichen Fruchtkörper des Pilzes zu beobachten, ist die Rinde in diesen Bereichen bereits abgestorben. Die Entwicklung der Rindennekrosen findet vor allem im Winterhalbjahr statt. Zu dieser Zeit sind die Abwehrmechanismen lebender Buchenrinde z. T. inaktiv (NIESAR et al. 2007, NIESAR 2007). Holzbrütende Käfer und Holzfäulepilze dringen an den Schadstellen ein, was zu Weißfäule und letztendlich Stammbrüchen führt.

Schadensschwerpunkte fanden sich in Rheinland-Pfalz, Luxemburg und Nordrhein-Westfalen bisher in höheren Lagen (meist ab etwa $400 \mathrm{~m}$ ) und in über 120-jährigen Beständen (AREND et al. 2006, NIESAR 2007). AREND et al. (2006) belegen mit Hilfe von Jahrringanalysen, dass die Kambiumnekrosen der letzten Erkrankungswelle bereits Mitte der neunziger Jahre entstanden sind. Die Krankheit kam also bereits seit mehreren Jahren unerkannt in den Beständen vor.

Es wird ein Zusammenhang mit Unregelmäßigkeiten im Wasserhaushalt und den Folgen der Klimaänderung angenommen: Gute Entwicklungsbedingungen liegen für die Buchenwollschildlaus in wärmeren, trockenen Sommern und für den Pilz Neonectria in milden, feuchten Wintern vor (NIESAR 2007, PETERCORD 1999).

\subsubsection{Maßnabmen zur Schadensbegrenzung vor Ort}

In Risikobereichen der Buchenkomplexerkrankung (Höhenlagen der Mittelgebirge) sollten ältere Buchenbestände laufend auf Erkrankungssymptome überwacht werden.

Buchen mit flächig abgestorbener Rinde, mit Bohrmehlhäufchen und vor allem mit Pilzfruchtkörpern (z. B. Zunderschwamm) weisen bereits eine aggressive und fortgeschrittene Holzfäule auf. Sie brechen leicht.

Dem Ausbruch der Erkrankung kann kaum gezielt entgegengewirkt werden. Das Ausmaß der Folgeschäden lässt sich jedoch eindämmen, wenn die Erkrankung rechtzeitig erkannt wird. Nutzungen im Frühstadium können Wertverluste vermeiden. Der rechtzeitige Einschlag ist allerdings oft schwierig, da die Anfangs- 
symptome kaum wahrgenommen werden und die Erkrankung scheinbar plötzlich in den Beständen auftritt (AREND et al. 2006).

Regelmäßige Durchforstungen können das Infektionsrisiko mindern, da sie die Luftbewegung in Beständen erhöhen. Dadurch trocknen schattseitige, feuchte Rindenpartien, die bevorzugt von Pilzen besiedelt werden, schneller ab und sind dann für (Rinden-)Pilze weniger attraktiv.

\subsection{Phytophthora cambivora an Buche}

Wurzel- und Wurzelhals-Erkrankungen der Buche durch den pilzähnlichen Krankheitserreger Phytophthora cambivora können örtlich/regional von Bedeutung sein. Die Krankheit tritt insbesondere auf basenreichen, lehmigen, sehr frischen bis stauoder wechselfeuchten Standorten auf (HARTMANN 2007) und kann über einzelstamm- und gruppenweise Ausfälle bis zur Auflösung von Bestandesteilen führen.

Regenreiche Witterungsperioden mit zeitweise länger anhaltendem Wasserüberschuss verstärken die Erkrankung auf den genannten Standorten. Folgen auf Perioden mit Wasserüberschuss ausgeprägte Trockenphasen so begünstigt dies den Befall, ebenso wie milde Winter.

Auf sauren, sandigen und eher trockenen Böden fehlt die Erkrankung bisher weitgehend.

\subsubsection{Typische Symptome des Befalls durch Phytophthora cambivora}

- Schleimflussflecken am Stammfuß (s. Abb. 3, links), teilweise mit Rindenrissen; nach vorsichtigem Abschälen der Rinde sind typische, aus dem Wurzelbereich aufsteigende Nekrosen an Wurzelanläufen und am Stammfuß sichtbar (s. Abb. 3, Mitte und rechts).

- Vergilbtes, schütteres Laub in der Oberkrone als Frühsymptom (aber: zunächst unspezifisch, Verwechselung mit anderen Schadursachen möglich!).

- Langsames Zurücksterben der Krone, Totäste, Ablösen der Rinde an Kronenästen und am oberen Stamm.

- Begleitender Befall durch rinden- und holzbrütende Käfer.

- Zuweilen sekundäres Hineinwachsen/Überwachsen der Phytophthora-Stammfußnekrosen z. B. vom Hallimasch, auch der Brandkrustenpilz ist oft beteiligt.

\subsubsection{Umstände des Auftretens / Krankheitsverlauf}

Phytophthora cambivora kann als aggressiver Parasit im Oberboden der genannten Standorte vorkommen und verbreitet sich vor allem durch im Bodenwasser frei bewegliche Zoosporen. Risikostandorte sind flache, schattseitige Unterhänge. 
Der Pilz befällt Fein- und Schwachwurzeln und bringt diese zum Absterben. Aus dem Wurzelbereich kommend steigt Phytophthora bis zu 2 Meter am Stamm hinauf (in Einzelfällen auch höher) und verursacht große zungenförmige, nach oben hin auslaufende Rindennekrosen mit dunklen Schleimflussflecken.

Sichtbare Schäden am Baum treten erst auf, wenn größere Teile der oberflächennahen Wurzeln infiziert sind. Letzte Sicherheit hinsichtlich des Befalls gibt nur der Nachweis des Erregers im Labor.

Das Absterben des Baumes erfolgt i. d. R. erst nach mehrjährigem Kränkeln, dann sind auch zunehmend Sekundärschädiger zu beobachten. Die fortgeschrittene Wurzelfäule hat neben Absterbeerscheinungen und einer Holzentwertung auch oftmals Windwurf zur Folge. Phytophthora-Wurzelhalsnekrosen können ebenfalls an Jungbuchen auftreten und zum Absterben der Pflanzen führen. Neben der Buche ist auch der Bergahorn anfällig. Andere Edellaubhölzer (z. B. Esche) scheinen weitgehend resistent zu sein.
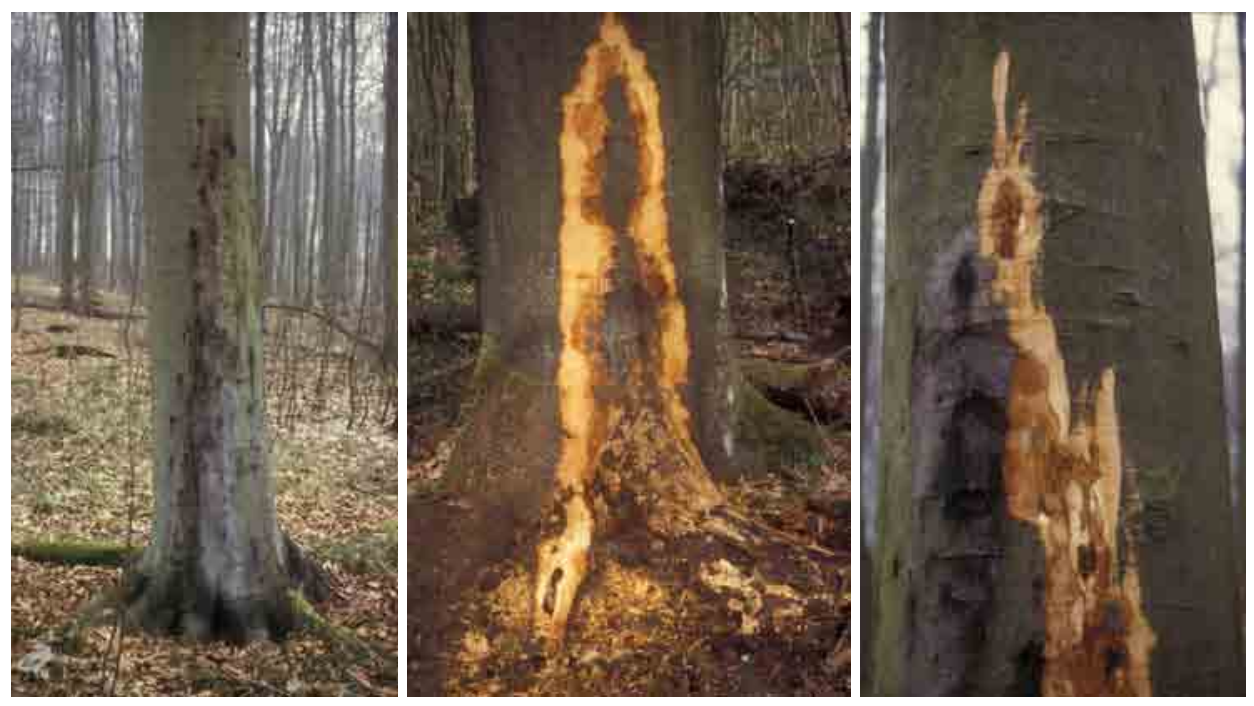

Abbildung 3: Links: Schleimflussflecken am Stammfuß durch Phytophthora cambivora; Mitte und rechts: freigelegte Nekrosen am Stammfuß

\subsubsection{Maßnabmen zur Schadensbegrenzung vor Ort}

Stärker befallene Buchen sollten rechtzeitig, d. h. vor der Entwertung durch Sekundärschädiger (z. B. Sägehörniger Werftkäfer (Hylecoetus dermestoides) und/oder Laubnutzholzborkenkäfer (Xyloterus domesticus)), entnommen werden. Die Holzentwertung schreitet nicht so schnell voran wie bei der Buchenkomplexerkrankung. Sie betrifft zunächst vor allem den Stammfußbereich. Rechtzeitiger Einschlag rettet somit große Teile des wertvollen Stammholzes. 
Hat der Erkrankungsprozess auf einer Fläche erst einmal begonnen, kann er durch waldbauliche oder auch Forstschutz-Maßnahmen kurzfristig nicht mehr gestoppt werden. Da der Pilz im Boden nicht bekämpft werden kann, bleibt bei der Verjüngung der betroffenen Bestände letztlich nur der Baumartenwechsel. Edellaubhölzer (außer Bergahorn), sofern sie standörtlich in Frage kommen, sind deutlich weniger anfällig. Keinesfalls sollte auf infizierten Standorten wieder auf Buche gesetzt werden, weder aus Naturverjüngung noch aus Pflanzung.

\subsection{Buchenvitalitätsschwäche (mit Buchenprachtkäfer)}

Von der Vitalitätsschwäche sind insbesondere stärker aufgelichtete mittelalte und alte Buchen-Bestandesteile, mehr oder minder frei stehende Altbuchen und Buchen an sonnenexponierten Rändern betroffen. Buchen in flachgründigen und in wechselfeuchten Standortsbereichen mit ggf. Extremsituationen hinsichtlich der Wasserversorgung (insbes. starke Austrocknung) scheinen besonders gefährdet zu sein.

\subsubsection{Typische Symptome der Buchenvitalitätsschwäche}

- Schüttere Belaubung, Kleinblättrigkeit, vorzeitiger Laubfall aus der Lichtkrone.

- Verlust des Feinreisigs in der Oberkrone, Absterbeerscheinungen (Totäste), Zopftrocknis (s. Abb. 4).

- $\quad$ Rinde im geschädigten Kronenbereich und am Stamm flächig aufgerissen und abgeplatzt.

- Befall der Starkäste durch Buchenprachtkäfer (Agrilus viridis; Merkmale: Schleimflussflecke auf der Rinde, darunter sich schlängelnde (s. Abb. 5), immer breiter werdende und mit festgepresstem Bohrmehl gefüllte Fraßgänge der Prachtkäfer-Larven, querovale Ausfluglöcher), teilweise mit Verfärbungen im Holzkörper, die in Faserrichtung stammabwärts verlaufen.

\subsubsection{Umstände des Auftretens / Krankheitsverlauf}

Die Häufung von Witterungsextremen (Hitze/ Trockenheit) in Verbindung mit Bestandesauflichtungen (Windwurf) bzw. starken Freistellungen führt bei älteren Buchen häufig zu einer deutlichen Reduktion der Vitalität. Sekundärbefall durch rindenbrütende Käfer, insbesondere Buchenprachtkäfer, ist häufig die Folge (s. auch FVA BADEN-WÜRTTEMBERG 2008). Dem Buchenprachtkäfer kommt auf vielen Standorten eine Schlüsselstellung bei den beobachteten Absterbeerscheinungen im Zuge der Buchenvitalitätsschwäche zu (PETERCORD et al. 2007). Örtlich wird auch stärkerer Befall durch den Kleinen Buchenborkenkäfer (Taphrorychus bicolor) beobachtet. 
Untersuchte Buchen zeigten allerdings häufiger noch vitale Blattknospen in der Lichtkrone, so dass die an „Dürreschäden“ erinnernden Schadbilder ohne Prachtkäferbefall vermutlich hätten ausheilen können. Auffällig waren entsprechende Schäden nach dem Trockenjahr 2003. Der heiße Sommer 2006 verschärfte die Situation. Stressfördernd dürfte sich auch der extrem warme April 2007 ausgewirkt haben. Es muss davon ausgegangen werden, dass die Abnahme der Vitalität der Buchen mit anschließendem Prachtkäferbefall weiter anhalten wird.
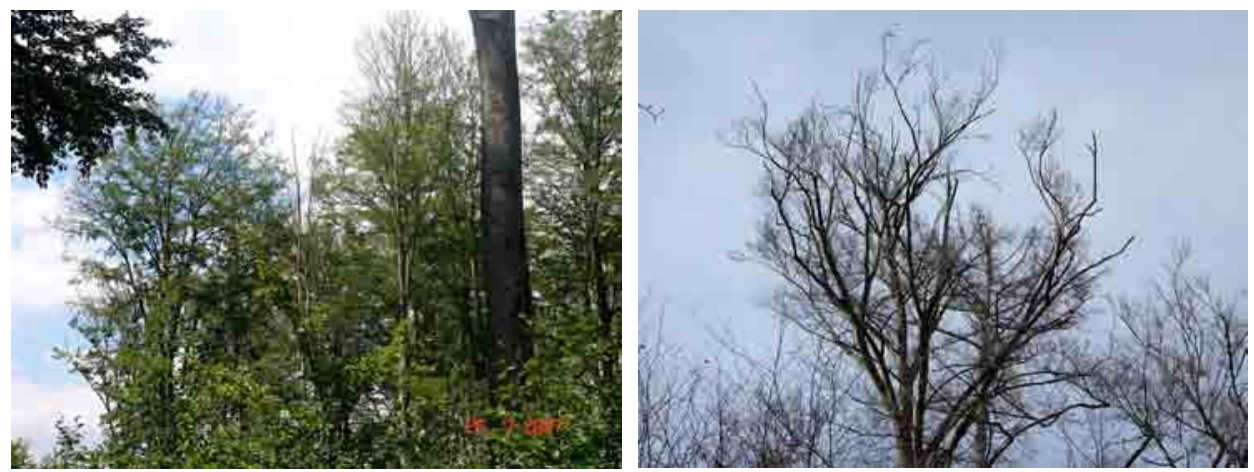

Abbildung 4: Buchenvitalitätsschwäche: verlichtete Oberkeronen mit Totästen (links: Sommer, rechts: Winter)

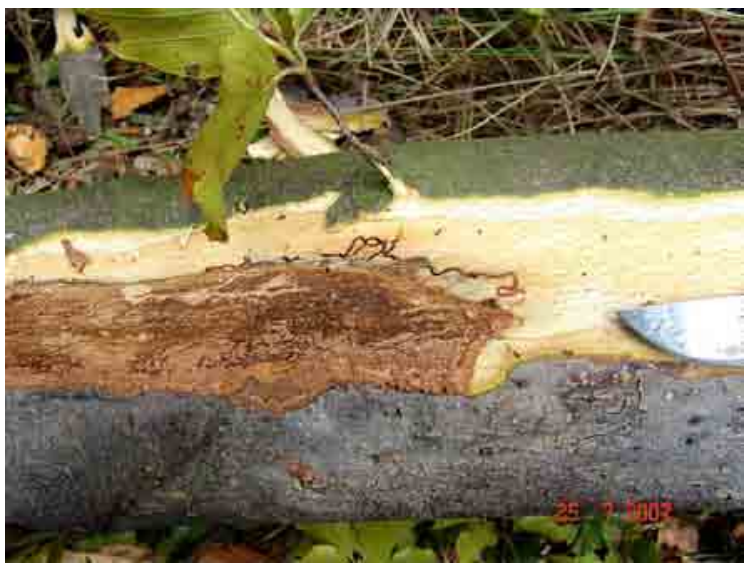

Abbildung 5: Buchenvitalitätsschwäche: Befall durch Buchenprachtkëfer an Starkästen Probefällung erforderlich!) 


\subsubsection{Maßnahmen zur Schadensbegrenzung vor Ort}

Die negativen Auswirkungen von Witterungsextremen (Hitze, Trockenheit) werden zusätzlich durch starke Eingriffe in den Altbestand, z. B. in der Verjüngungsphase, verstärkt. Auf betroffenen Standorten wird dazu geraten, die Bestände nicht zu stark aufzulichten und ggf. den Seitenschutz zu erhalten. Da zunächst nur die Kronen von den Schadsymptomen betroffen sind, besteht im Bezug auf die Holzqualität im Stammbereich in der ersten Phase keine akute Entwertungsgefahr.

Gezielte Sanitärhiebe sind angeraten, wenn mehr als 50 \% der Krone abgestorben sind bzw. akut absterben. Sobald Prachtkäfer auch im Stammbereich auftreten, kommt es zur technischen Holzentwertung u. a. durch Weißfäulepilze. Solche Bäume sind möglichst zeitnah im Zuge von Sanitärhieben (,saubere Waldwirtschaft" zu entnehmen.

\section{Umfrage zu Schäden an Buche im Zuständigkeitsbereich der NW-FVA}

\subsection{Vorbemerkungen, Beschreibung des Vorgehens}

Um für den Zuständigkeitsbereich der NW-FVA (Niedersachsen, Hessen, Sachsen-Anhalt, Schleswig-Holstein) einen Überblick über Schäden und ggf. entsprechende Schadensschwerpunkte zu erlangen, wurde Ende 2007 eine Umfrage zu den Schäden an Buche durchgeführt.

Die drei zuvor erläuterten Buchen-Erkrankungen wurden zu diesem Zweck steckbriefartig beschrieben, mit Bildern versehen und als Kurzinformation den Forstämtern bzw. Revierleitern zur Verfügung gestellt.

Sehr bald zeigte sich, dass die Ansprache und Abgrenzung der Schadbilder in der Praxis nicht immer ganz einfach war, weil z. B. bestimmte Schadsymptome wie Schleimfluss bei verschiedenen Schadbildern vorkommen können oder im Einzelfall auch verschiedene Schadtypen in einem Bestand zu beobachten sind. Teilweise wurden auch weitere, nicht abgefragte Schadbilder (z. B. Krebserkrankungen oder Sonnenbrand) gemeldet. Bei unklaren Erkrankungsfällen wurden deshalb Schadensschwerpunkte bereist, Schadbilder vor Ort diskutiert und Meldungen ggf. auch korrigiert.

\subsection{Beteiligung an der Umfrage}

Befragt wurden 155 Forstämter und Forstverwaltungen. Die Meldungen sollten auf Revierebene abgegeben werden. Rückmeldungen erfolgten aus $48 \%$ der Forstämter und aus $23 \%$ der Revierförstereien. Insgesamt kamen Meldungen aus 273 Revieren. 
Die Qualität der Melderückläufe war sehr unterschiedlich. Einige wenige Rückläufe konnten nicht ausgewertet werden. In Einzelfällen gab es Hinweise auf hohe Arbeitsbelastungen und auf Personalengpässe, so dass die Forstämter auf detaillierte Angaben verzichteten. Insofern können - auch in Anbetracht der geringen Rücklaufquote - im Ergebnisteil nur einige Zusammenhänge dargestellt werden.

\subsection{Ergebnisse der Schadflächenmeldungen zu den drei abgefragten Erkrankungstypen}

83 Reviere meldeten Beobachtungen bzw. Schäden zur Buchenkomplexerkrankung, 43 Reviere zu Phytophthora cambivora an Buche und 73 Reviere meldeten die Vitalitätsschwäche der Buche.

Die gemeldeten Schadflächen (Hektar, gerundet) verteilen sich auf die 4 erfassten Bundesländer wie folgt (s. Tab. 1):

Tabelle 1: Gemeldete Schadflächen [ha] nach Erkrankungstyp und Bundesländern sowie deren Gesamtbuchenwaldfläche $>80$ Jahre

\begin{tabular}{lccccc}
\hline Land & $\begin{array}{c}\text { Buchen- } \\
\text { komplex- } \\
\text { erkr. } \\
\text { (ha) }\end{array}$ & $\begin{array}{c}\text { Phytoph- } \\
\text { thora an } \\
\text { Buche } \\
\text { (ha) }\end{array}$ & $\begin{array}{c}\text { Buchen- } \\
\text { vitalitäts- } \\
\text { schwäche } \\
\text { (ha) }\end{array}$ & $\begin{array}{c}\text { Gesamt- } \\
\text { schad- } \\
\text { fläche } \\
\text { (ha) }\end{array}$ & $\begin{array}{c}\text { Gesamt- } \\
\text { buchen- } \\
\text { waldfläche } \\
\text { > 80 Jahre } \\
\text { (ha)* }\end{array}$ \\
\hline Niedersachsen & 435 & 50 & 415 & 900 & 92.286 \\
Hessen & 525 & 90 & 1635 & 2250 & 141.483 \\
Sachsen-Anhalt & 415 & 25 & 670 & 1110 & 12.697 \\
Schleswig-Holst. & 65 & 35 & 120 & 220 & 18.553 \\
\hline Gesamt & $\mathbf{1 4 4 0}$ & $\mathbf{2 0 0}$ & $\mathbf{2 8 4 0}$ & $\mathbf{4 4 8 0}$ & $\mathbf{2 6 5 . 0 1 9}$
\end{tabular}

* Daten: Bundeswaldinventur 2, Stichjahr 2002 (BMELV 2008)

Mit $63 \%$ entfallen die meisten Schadflächenmeldungen auf die Buchenvitalitätsschwäche, $32 \%$ auf die Buchenkomplexerkrankung und $5 \%$ auf Phytophthora an Buche.

Schadensschwerpunkte für die Buchenkomplexerkrankung liegen in Niedersachsen im Solling, im Harz und im Raum Wolfenbüttel, in Hessen im Waldeckischen Upland (Willingen), im Raum Herborn (Ausläufer des Westerwaldes) sowie im Vogelsberg, in Sachsen-Anhalt im Bereich des Ostharzes. Bei einigen wenigen bislang noch nicht vor Ort besichtigten Schadensfällen muss (noch) davon ausgegangen werden, dass es sich zwar um komplexe Schäden an Buche handelt, die aber nicht in jedem Falle der sogenannten „Buchenkomplexerkrankung“ zugeord- 
net werden können. Dennoch wurden diese geringen Flächenmeldungen vorerst bei der Buchenkomplexerkrankung verbucht.

Phytophthora an Buche ist in Niedersachsen örtlich ein Problem in der Berglandschwelle. In Hessen gibt es Verdachtsfälle im Raum Darmstadt, in Mittelhessen und im Vogelsberg, die aber noch überprüft werden müssen. Sachsen-Anhalt meldet Phytophthora-Fälle im Harz, Schleswig-Holstein in nordöstlichen Landesteilen.

Die Buchenvitalitätsschwäche ist sehr verbreitet, vor allem in Hessen. Teilweise gab es entsprechende Meldungen „für das ganze Revier“ ohne Flächenangabe und letztlich ohne Flächenberücksichtigung bei der Auswertung. Schadensschwerpunkte liegen nach den vorliegenden Meldungen in Niedersachsen im Bergland. In hessischen Wäldern werden entsprechende Probleme im Rhein-Main-Gebiet sowie in Teilen des Vogelsberges, des Taunus und in Mittelhessen festgestellt. In Sachsen-Anhalt wird dieses Schadbild vor allem im (Ost-)Harz und im Raum Flechtingen beobachtet.

\subsection{Sondersituationen bzw. weitere Schadbilder}

Örtlich bzw. regional wurden erhebliche Schäden an Buche gemeldet, die sich aufgrund von Sondersituationen ergeben haben oder auf weitere Krankheitserreger zurückzuführen sind. Diese Schadbilder waren mit der Abfrage nicht abgedeckt. Sie sollen aber dennoch erwähnt werden:

\subsubsection{Absterbeerscheinungen, insbesondere aufgrund von Grundwasserabsenkung und Maikäfer-Engerlingsfraß}

Schwerpunktmäßig werden in den hessischen Forstämtern Darmstadt und Lampertheim, in geringem Umfang auch in Gross-Gerau, massive Absterbeerscheinungen in Buchenbeständen ab Alter 60-80 Jahre beobachtet, die maßgeblich neben einer Vielzahl anderer, zusätzlich beteiligter Faktoren im Ballungsraum Rhein-Main - auf Grundwasserabsenkungen und Maikäfer-Engerlingsfraß an den Wurzeln zurückzuführen sind (s. Abb. 6).

Es handelt sich meist um sandige Standorte (mäßig frisch, teilweise wechselfeucht/wechseltrocken; mesotroph, teilweise eutroph; Höhenlage: 50-150 m ü. NN). Die Jahresniederschläge liegen oft unter $600 \mathrm{~mm}$, die Niederschläge in der Vegetationszeit unter $300 \mathrm{~mm}$. Der ehemals gegebene und nutzbare Anschluss der Baumwurzelsysteme ans Grundwasser ist hier in vielen Bereichen abgerissen. Örtlich wurden die Bestände durch die 1990er Windwürfe („Wiebke“ usw.) aufgerissen mit der Folge von Sonnenbrandschäden an der Buchenrinde, Vergrasung etc. Die jüngste Maikäfer-Gradation begann Ende der 1980er Jahre. Die Schadflächen (Wurzelfraß) haben sich seitdem kontinuierlich vergrößert. 


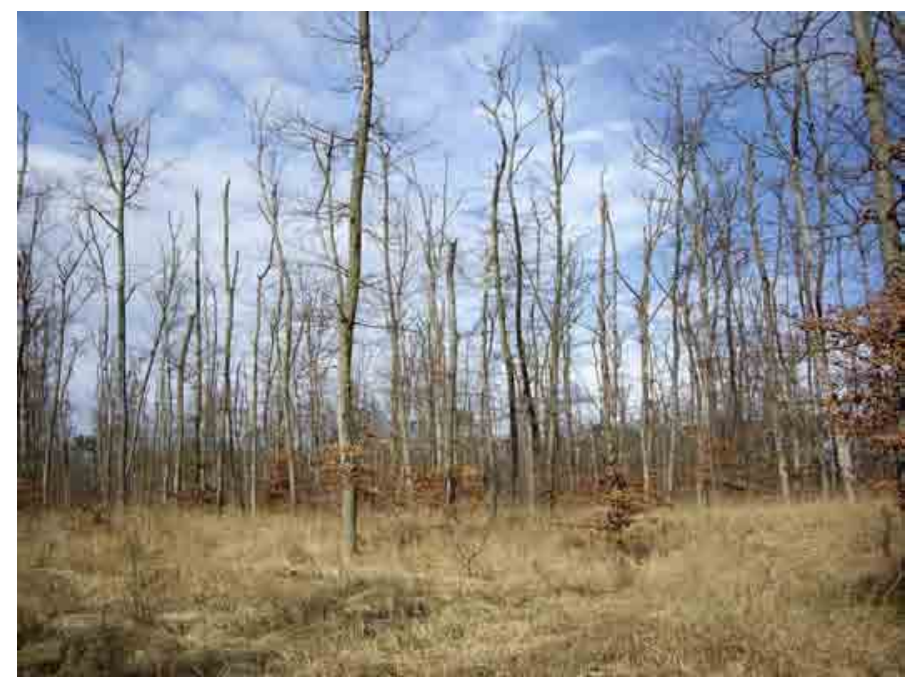

Abbildung 6: Absterbeerscheinungen aufgrund von Grundwasserabsenkung, Maikä̈er-Engerlingsfraß etc.

Bereits vor dem Trockenjahr 2003 gab es Ausfälle in den Buchen-Beständen. Nach 2003 war eine starke Zunahme der Abgänge mit Auflösungserscheinungen zu verzeichnen. Wenn dieser Prozess erst einmal begonnen hat, schreitet er insbesondere nach Trockenjahren unaufhaltsam fort. Auch dies ist eine Art komplexer Schaden an Buche, wobei menschliche Einflüsse (aktiv: Grundwasserabsenkung, passiv: Unterlassung der Maikäferbekämpfung) allerdings eine maßgebliche Rolle spielen.

Die derzeitige Buchen-Schadfläche aufgrund von Grundwasserabsenkung und Maikäferfraß wird im hessischen Ried insgesamt auf etwa 1700 ha geschätzt.

\subsubsection{Absterbeerscheinungen aufgrund extremer Trockenstresssituationen}

Im Norden des Frankfurter Flughafens werden auf tiefgründigen Sandstandorten (meist schluffiger Sand über tonigem Sand, mäßig frisch, mesotroph, Zentrale Eichen-Mischwaldzone) gravierende Absterbeerscheinungen an Buche aufgrund von Trocknis beobachtet. Flächig abblätternde Rinde am Stamm und in der Krone ohne weitere maßgebliche Einflussfaktoren (z. B. Borkenkäfer oder Pilze) ist hier typisch.

Die Standorte liegen für Buche, insbesondere hinsichtlich ihrer Wasserversorgung, im Grenzbereich. In den relativ trockenen und warmen 1990er Jahren (siehe auch DWD-Station Flugwetterwarte Frankfurt/M.; PAAR et al 2007) waren bereits vereinzelt Absterbeerscheinungen zu beobachten. Das Trockenjahr 2003 hat einen schwerwiegenden Erkrankungsschub ausgelöst und die Absterbeerscheinungen verschärft. 
Auch Alteichen sind hier erheblich geschädigt und zeigen in den Kronen die bekannten Symptome des „Eichensterbens“, zudem starken Prachtkäferbefall und derzeit auffällige Spechtabschläge. Lediglich eingemischte ältere Kiefern vermitteln einen noch weitgehend vitalen Eindruck.

\subsubsection{Stammschäden durch Neonectria ditissima}

Bekannt sind Neonectria-Krebsschäden an Buchenästen, die durch ungleich gestaltete, spindelförmige Astverdickungen zu erkennen sind (BUTIN 1996).

Örtlich treten diese Krebsschäden aber auch am Stamm auf. Sie gehen im Frühstadium oft mit Schleimfluss einher und sind durch beulige, unregelmäßig rissige Auftreibungen gekennzeichnet (s. Abb. 7, links). Später führt die Erkrankung zu kraterartigen Krebswunden (s. Abb. 7, rechts; HARTMANN et al. 2007).
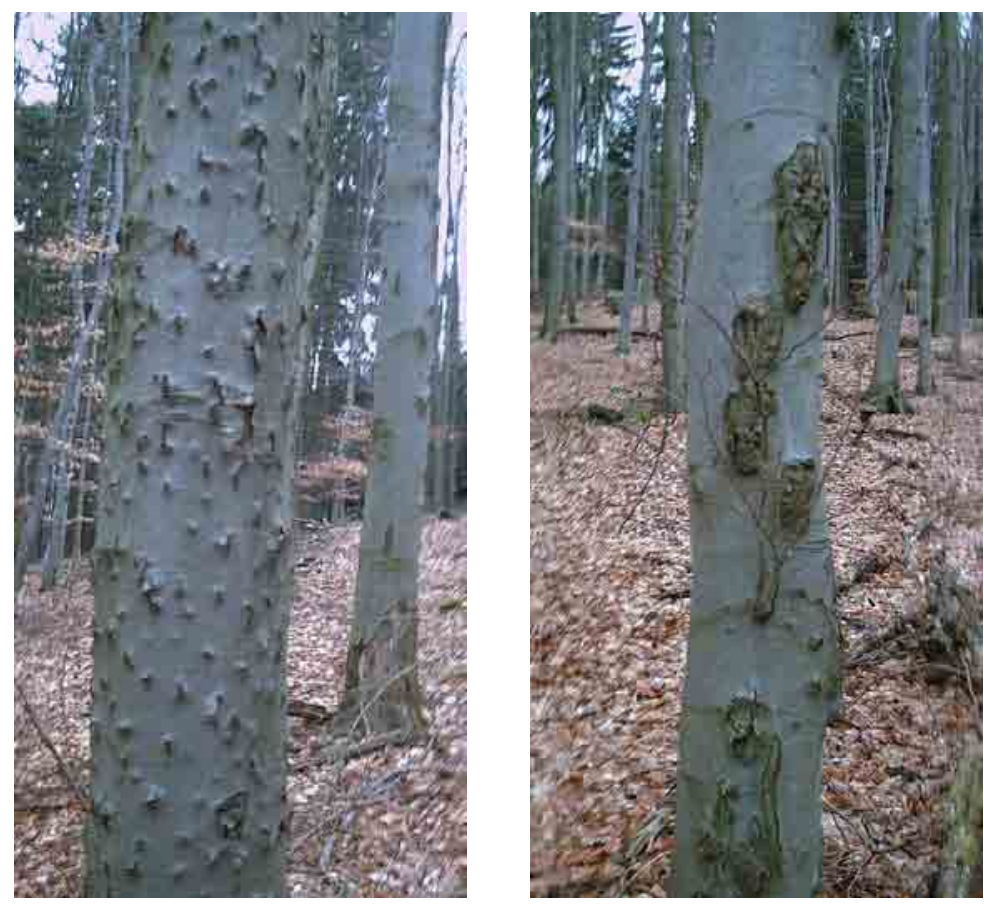

Abbildung 7: Links u. rechts: Krebsschäden am Stamm, hervorgerufen durch den Pilz Neonectria ditissima 


\section{Diskussion und Wertung der Ergebnisse}

\subsection{Allgemeine Betrachtungen zur Klimaerwärmung und zur Buche}

Die Erhaltung stabiler, multifunktionaler und nachhaltig nutzbarer Wälder wird auch für die Zukunft von großer Bedeutung sein. Der Schutz vor biotischen und abiotischen Schadfaktoren und die Minderung von Produktionsrisiken sind dabei zentrale Aufgaben.

Der Klimawandel ist keine ferne Zukunft mehr, er findet bereits statt (UMWELTBUNDESAMT 2007). Es zeichnen sich in Nordwestdeutschland niederschlagsarme, heiße Sommer und milde, niederschlagsreichere Winter ab (BRECKLE 2005, MAYER et al. 2005). Bereits jetzt wird eine Zunahme extremer Wetterereignisse wie Hitzeperioden, Stürme, Starkregen und Überschwemmungen beobachtet.

Problematisch für die Pflanzen sind weniger die meteorologisch gemessenen Temperatur-Mittelwerte, sondern vielmehr die auftretenden Extreme (BRECKLE 2005). Ähnlich ist es beim Niederschlag. Der meteorologisch gemessene JahresMittelwert ist zunächst von nachrangiger Bedeutung. Wichtiger sind die jahreszeitliche Verteilung der Niederschläge und wiederum auftretende Extreme (Maxima, Minima).

Für die Waldbäume allgemein besteht ein Dilemma darin, dass der prognostizierte Klimawandel mit häufiger auftretenden atmosphärischen Extrembedingungen sehr rasch - eigentlich zu rasch - ablaufen wird (MAYER et al. 2005, BRECKLE 2005, KÖLLING et al. 2007). Unangepasste Baumarten oder bereits vorgeschädigte Bestandesteile werden generell immer weniger Zeit haben, sich zu regenerieren. Alte Buchenbestände sind regional schon jetzt stärker geschädigt und vitalitätsschwach. Kurzfristige Anpassungen an veränderte Klimabedingungen sind in Altbeständen zumindest fraglich.

Die Buche dürfte in Zukunft vor allem von der veränderten Niederschlagsverteilung mit zunehmender Sommertrockenheit bei gleichzeitig feuchteren und milderen Wintern negativ betroffen sein. Auf entsprechenden Standorten wird im Einzelfall auch zunehmende Staunässe aufgrund von Starkregenereignissen im Herbst und Frühjahr eine Rolle spielen (s. auch RENNENBERG et al. 2004). Als trockenstress- und überflutungsempfindliche Baumart leidet die Buche schon heute auf solch extremen Standorten unter Stress, zunehmender Krankheitsanfälligkeit und reduzierter Konkurrenzkraft.

\subsection{Konkrete Krankheitsbeobachtungen und Erklärungsansätze}

Die offensichtliche Zunahme der hier vorgestellten Krankheitsbilder lässt sich vor diesem Hintergrund bereits hinreichend erklären: 
Die weit verbreitete Buchenvitalitätsschwäche wird maßgeblich durch Sommertrockenheit und Hitze-Stress gefördert. Die letzten Jahre waren geprägt von entsprechenden ungewöhnlichen Witterungsverläufen und Extremsituationen. Auffällig war bereits die im Vergleich zum langjährigen Mittel starke Erwärmung in den 1990er Jahre. Besonders einschneidend war das Trockenjahr 2003 mit dem oft zitierten ,Jahrhundertsommer". Weitere Extreme folgten in kurzen zeitlichen Abständen, nämlich die außergewöhnliche Hitze/Trockenheit im Juni/Juli 2006, der heiße April 2007 und der sehr warme, trockene und sonnenreiche Mai 2008.

Für die Buchenkomplexerkrankung und auch Phytophthora an Buche spielen neben allgemeinen Stresssituationen erhöhte Temperaturen und gute Feuchtigkeitsverhältnisse im Herbst und Winter eine herausragende Rolle, weil sie günstige Bedingungen für Pilzinfektionen und Pilzwachstum schaffen. Solche Bedingungen lagen in den letzten Jahren vor. Zunehmend mildere und regenreichere Winter in Kombination mit extrem warmen Sommern lassen eine weitere Zunahme von Erkrankungswellen der Buchenkomplexerkrankung/ Buchenrindennekrose erwarten (NIESAR 2007).

Die vorliegenden Erhebungen zu drei Krankheitsbildern der Buche zeigen, dass insbesondere ältere Buchen ab etwa 120 Jahren zunehmend Erkrankungssymptome und schlechte Vitalitätszustände zeigen. Die ältere Buche vermittelt vielerorts nicht mehr den Eindruck ausgesprochener Plastizität, die sie noch vor wenigen Jahrzehnten ausgezeichnet hat. Die Schadbilder der letzten Jahre deuten darauf hin, dass die Buche anfälliger ist, als lange Zeit angenommen wurde, und dass örtlich sehr wohl erhebliche Probleme vor dem Hintergrund der verschiedenen Facetten der Klimaänderung zu erwarten sind.

Über diese Beobachtungen hinaus belegen schon seit Jahren die Ergebnisse der Waldzustandserhebungen (WZE) die schlechter werdenden Vitalitätszustände älterer Buchen. Die WZE Hessen dokumentiert bei älteren Buchen über 60 Jahre im Zeitraum 1984 - 2007 einen Anstieg der mittleren Kronenverlichtungen von $15 \%$ auf $33 \%$ (PAAR et al. 2007).

Zudem zeigen Untersuchungen zur Fruchtbildung seit 1988 die Tendenz, dass die Buche in kürzeren Abständen und vielfach stärker fruktifiziert als es nach früheren Angaben zu erwarten gewesen wäre. Dies steht nach Überzeugung der Autoren des o. g. Waldzustandsberichtes 2007 im Zusammenhang mit einer Häufung warmer Jahre wie auch einer erhöhten Stickstoffversorgung der Bäume. Die Fruchtbildung der Buche wird als ein Schlüsselindikator für den Nachweis von Umweltveränderungen angesehen. Die Häufung von Samenjahren ist für die Bäume mit erheblichem Reservestoffverbrauch und Stress verbunden, gleichzeitig auch mit verringerter Widerstandskraft gegen biotische Schaderreger.

Ganz offenbar haben veränderte Umweltbedingungen die derzeitige Schadenssituation bei der Buche mitgeprägt. Einerseits sind es Schadstoffeinträge mit ihren Folgewirkungen, andererseits haben aber Witterungsbedingungen und klimatische 
Veränderungen mit Extremsituationen älteren Buchen unter bestimmten Bedingungen stark zugesetzt.

Insbesondere in standörtlichen Grenzbereichen hinsichtlich der Wasserversorgung sind in Zukunft vermehrt Probleme zu erwarten. Eindrucksvoll zeigt der Waldkomplex nördlich des Frankfurter Flughafens, in welche Richtung sich die Buche bei zusätzlicher witterungsbedingter Trockenheit/Hitze auf ohnehin mit Wasser schwach versorgten Standorten entwickelt: sie reagiert mit Trocknis und massiven Absterbeerscheinungen.

Eine etwas anders gelagerte, in der Konsequenz aber ähnliche Situation ist im hessischen Ried durch Grundwasserabsenkung, Maikäfer-Engerlingsfraß und weitere Ballungsraum-Stressfaktoren (z. B. Randschäden) gegeben. Die Buche zeigt aufgrund ungenügender Wasserversorgung auch hier Zopftrocknis und starke Absterbeerscheinungen.

Besorgniserregend sind auch die weit verbreiteten Schadbilder der BuchenVitalitätsschwäche. Sie werden gefördert durch sonnenexponierte Randstellung und Freistellung der Buchenkronen. Schäden entstehen durch starke Sonneneinstrahlung und Überhitzung bei unzureichendem Schutz durch Nachbarbäume. Oftmals wird schließlich eine Kettenreaktion mit nachfolgendem Käfer- und Pilzbefall ausgelöst, die zum Absterben der Bäume führen kann.

\subsection{Hat die Buche unter diesen Vorzeichen eine Chance?}

BOLTE (2005) äußert, dass eine vorschnelle Abkehr von der Buche vor dem Hintergrund der Klimaerwärmung und der sich ändernden Rahmenbedingungen sicherlich der falsche Weg wäre. Er rät zu einem risikobewussten Waldbau mit der Buche, der die Naturverjüngung bestehender Buchen- und Mischbestände ebenso wie die Anpflanzung getesteter lokaler oder eingeführter Herkünfte bei An- und Umbaumaßnahmen nutzt. Dies sei eine wichtige Ergänzung zur allgemeinen Risikostreuung durch Baumartenmischung. Er betont auch, dass einiges dafür spricht, dass sich die Buche in gewissem Rahmen an die laufende Klimaerwärmung in Mitteleuropa anpassen kann. Dies sollte aber nicht davon abhalten, immer wieder neu über die Anpassungsgrenzen und die Schädigungsgefahr der Buche kritisch nachzudenken.

BRECKLE (2005) fordert insgesamt für Waldbestände behutsame Eingriffe unter Beachtung ökologischer Regeln mit dem Ziel der Erhaltung bzw. Schaffung artenreicher, naturnaher Mischwälder. Er stellt heraus, dass dies voraussichtlich über längere Zeit die Ökosystemfunktionen erhält, möglichst viele Optionen offen lässt und die nachhaltigen Nutzungsmöglichkeiten verbessert.

Auch KÖLLING (2006b) unterstreicht, dass sich die Wälder von morgen vor allem auf Veränderungen des Wasserhaushaltes einstellen müssen. Er hebt hervor, dass wir dabei auch die Veränderungen im Blick haben müssen, die das oft labile Gleichgewicht von Schädlingen und Waldbäumen betreffen. 
Trotz der auf manchen Standorten zu erwartenden, teilweise recht ungünstigen Rahmenbedingungen für die Buche und offenkundiger, doch stärkerer Krankheitsanfälligkeit kann und wird die Buche auch in zukünftigen Waldbildern eine wichtige Rolle spielen. Verschiebungen hinsichtlich der Konkurrenzverhältnisse der Baumarten und Veränderungen der Buchenareale sind aber zu berücksichtigen.

Die Beobachtungen zu den Schadentwicklungen der letzten Zeit in Nordwestdeutschland zwingen dazu, zukünftig noch stärker darauf zu achten, dass die Buche nicht in standörtlichen Grenzbereichen (Wasserhaushalt) angebaut oder nachgezogen wird.

Insbesondere an älteren Buchen ist eine Zunahme von Schäden zu beobachten. Hierauf muss ggf. kurzfristig durch Sanitärhiebe und mittel- bis langfristig durch rechtzeitige und konsequente (Kronen-)Pflegemaßnahmen zur frühzeitigen Vitalitätserhaltung und -steigerung reagiert werden. Andererseits sind lange Beibehaltung des Kronenschlusses und Vermeidung zu schneller Freistellung (z. B. im Zuge der Verjüngung) angeraten. Zu starke Kronenfreistellung bedeutet zusätzlichen Stress und muss ebenso vermieden werden wie Stress aufgrund unterlassener Pflegemaßnahmen. Aber auch Stress durch die Maßnahme selbst ist durch pflegliche Waldbewirtschaftung und Vermeidung von Stamm- und Wurzelverletzungen zu minimieren. Hier muss aktiv steuernd und unterstützend für die Buche eingegriffen werden.

Insgesamt ist für die Zukunft mehr denn je das „Fingerspitzengefühl“ des Waldbewirtschafters gefordert, um der Buche durch gezielte Maßnahmen Perspektiven zu erhalten und unnötigen Stress von dieser Baumart abzuwenden. Die Leitlinie ist: Schutz und Entwicklung durch forstliche Eingriffe und Pflege, aber auch Schutz durch Nutzung.

\section{Literatur}

Arend, J.-P.; Eisenbarth, E. u. Petercord, R.. (2006): Buchenkomplexkrankheit in Luxemburg und Rheinland-Pfalz - Schadsymptome, Ausmaß und Entwicklung der Schäden. Mitteilungen aus der Forschungsanstalt für Waldökologie und Forstwirtschaft Rheinland-Pfalz, Nr. 59, 11-22

BMELV (BUNDESMINISTERIUM FÜR ERNÄHRUNG, LANDWIRTSCHAFT UND VERBRAUCHERSCHUTZ) (2008): Bundeswaldinventur 2 - Ergebnisdatenbank. www.bundeswaldinventur.de

Bolte, A. (2005): Die klimatische Anpassungsfähigkeit der „Mutter des Waldes“. Zur Zukunft der Buche in Mitteleuropa. AFZ/Der Wald 60, 1077-1078

Braun, H. J. (1976): Das Rindensterben der Buche, Fagus sylvatica L., verursacht durch die Buchenwollschildlaus Cryptococcus fagi Bär. I. Die Anatomie der Buchenrinde als Basis-Ursache. Eur. J. For. Path. 6, 136-146

BRAun, H. J. (1977): Das Rindensterben der Buche, Fagus sylvatica L., verursacht durch die Buchenwollschildlaus Cryptococcus fagi Bär. II. Ablauf der Krank-heit. Eur. J. For. Path. 7, 76-93

BRECKLE, S.-W. (2005): Möglicher Einfluss des Klimawandels auf die Waldvegetation Nordwestdeutschlands ? LÖBF-Mitteilungen 30, 19-24

Butin, H. (1996): Krankheiten der Wald- und Parkbäume. Diagnose - Biologie - Bekämpfung. Georg Thieme Verlag, Stuttgart, NEW York. 261 S. 
DEUTSCHER FORSTWIRTSCHAFTSRAT (DFWR) (2008): Buchenwälder - vielfältig, einmalig, nachhaltig. Broschüre, 55 Seiten

EISENBARTH, E. (2001): Buchen-Komplexkrankheit in Rheinland-Pfalz. AFZ/Der Wald 56, 12201221

Emschermann, F. u. NiESAR, M. (2001): Einfluss von Schadorganismen auf die Vitalität der Waldbäume. In: Bericht über den ökologischen Zustand des Waldes, Waldschutz. Landesanstalt für Ökologie, Bodenordnung und Forsten (NRW), Nov. 2001

Emschermann, F.; Geisthoff, N.; Niesar, C. M. u. Strothbäumer, H. W. (2001): Waldschutzsituation 2000/2001 in Nordrhein-Westfalen. AFZ/Der Wald 56, 342-344

FVA (Forstliche Versuchs- Und Forschungsanstalt) BADEN-WÜrtTEMBERG (2008): Waldschutzbericht 2007/2008

HARTMANN, G. (2007): Untersuchungsberichte zur Buchen-Phytophthora im Elm (Niedersachsen), Teile 1 und 2 (unveröffentlicht)

Hartmann, G.; Nienhaus, F. u. Butin, H. (2007): Farbatlas Waldschäden. Diagnose von Baumkrankheiten. 3. Auflage, Verlag Eugen Ulmer, 269 S.

KÖLLING, C. (2006a): Wälder im Klimawandel: Einwirkung, Anfälligkeit, Anpassung. UBA-Workshop am 17.10.2006

KÖLLING, C. (2006b): Waldbau im Klimawandel - Eine Herausforderung für die Forstliche Standortserkundung. Forstinfo Nr. 3, 1-4

KÖlding, C.; Zimmermann, L. u. WALENTOWSKI, H. (2007): Entscheidungshilfen für den klimagerechten Waldumbau in Bayern. Klimawandel: Was geschieht mit Buche und Fichte? AFZ/Der Wald 62, 584-588

KunKel, H. (1968): Untersuchungen über die Buchenwollschildlaus Cryptococcus fagi Bär. (Insecta, Coccina), einen Vertreter der Rindenparenchymsauger. Z. ang. Ent. 61, 373-380

Mayer, H.; Holst, TH.; Brugger, U. u. KirChgässner, A. (2005): Trends der forstlich relevanten Klimavariablen Lufttemperatur und Niederschlag im Südwesten Deutschlands von 1950 bis 2000. Allg. Forst- u. Jagd-Ztg. 176, 45-56

NiESAR, M. (2007): Symptom- und Ursachenanalyse der aktuellen Buchenerkrankung in höheren Lagen von Nordrhein-Westfalen. Dissertation zur Erlangung des Doktorgrades der Mathematisch-Naturwissenschaftlichen Fakultäten der Georg-August-Universität zu Göttingen. Cuvillier Verlag Göttingen, 115 S.

Niesar, M.; Hartmann, G.; Kehr, R.; Pehl, L. u. Wulf, A. (2007): Symptome und Ursachen der aktuellen Buchenrindenerkrankung in höheren Lagen von Nordrhein-Westfalen. Forstarchiv 78, $105-116$

Paar, U.; Dammann, I.; Gawehn, P.; Wendland, J. u. Eichhorn, J. (2007): Waldzustandsbericht 2007. Nordwestdeutsche Forstliche Versuchsanstalt (Hrsg.), 31 S.

PETERCORD, R. (1999): Entwicklung bewirtschafteter Buchen-Edellaubholz-Mischbestände unter dem Einfluß der Buchenwollschildlaus (Cryptococcus fagisuga Lind.) unter besonderer Berücksichtigung physiologischer und genetischer Aspekte. Dissertation, Hainholz Verlag Göttingen

Petercord, R. (2006): Die Buchenwollschildlaus (Cryptococcus fagisuga LIND.) als Auslöser der Buchenrindennekrose. Mitteilungen aus der Forschungsanstalt für Waldökologie und Forstwirtschaft Rheinland-Pfalz, Nr. 59, 53-62

Petercord, R.; Delb, H. u. SChröter, H. (2007): Vermeintliche Dürreschäden an Buche entpuppen sich als Käferbefall: Schwere Schäden durch den Buchen-Prachtkäfer in Baden-Württemberg. AFZ/Der Wald 62, 686-690

Rennenberg, H.; Seiler, W.; Matyssek, R.; Gessler, A. u. Kreuzwieser, J. (2004): Die Buche (Fagus sylvatica L.) - ein Waldbaum ohne Zukunft im südlichen Mitteleuropa? Allg. Forst- u. Jagd-Ztg. 175, 210-224

Umweltbundesamt (2007): Neue Ergebnisse zu regionalen Klimaänderungen. Das statistische Regionalisierungsmodell WETTREG. 27 S. 
Autor:

Dr. Ulrich Bressem

Nordwestdeutsche Forstliche Versuchsanstalt

Grätzelstr. 2

37079 Göttingen

E-Mail: Ulrich.Bressem@nw-fva.de

URL: www.nw-fva.de 



\title{
Untersuchungen zur Trockenheitstoleranz der Buche am Beispiel des witterungsextremen Jahres 2003
}

\author{
Assessment of the drought resistance of beech \\ exemplified by the 2003 extreme weather conditions \\ Johannes Eichborn, Inge Dammann, Egbert Schönfelder, Mascha Albrecht, \\ Wolfgang Beck und Uwe Paar
}

\section{Zusammenfassung}

Jahre mit extremer Witterung können Risiken für die Buche bedeuten. Am Beispiel des in der Reihe der letzten Jahre ganz besonders warmen und niederschlagsarmen Jahres 2003 wird das Reaktionsmuster der Buche auf Flächen des forstlichen Umweltmonitorings in Nordwestdeutschland untersucht.

Der Biomassezuwachs für Derbholz und Äste wird im Jahr 2004 um ca. 50 \% reduziert, die Entwicklung der Höhentriebe bleibt mehrere Jahre eingeschränkt. Mit der Reduktion der Höhen- und der Durchmesserleistung geht eine forstbetrieblich relevante Verringerung des Holzertrages einher. Bei aufeinanderfolgenden extrem warm-trockenen Jahren kann die Produktion von Buchenholz als nachwachsender Rohstoff mit allen ökologischen und ökonomischen Konsequenzen weitere Einschränkungen erfahren. 
In Bezug auf die Nettoprimärproduktion wird die Minderleistung im Stammholz durch eine 2004 sehr umfangreiche Mast ausgeglichen. Die Biomasse in Blättern reagiert nur unwesentlich, so dass insgesamt die oberirdische Nettoprimärproduktion 2004 nur um etwa $3 \%$ verringert ist; Wurzelsysteme sind nicht berücksichtigt. Ausgelöst durch die Witterungsbedingungen des Jahres 2003 ändert sich damit im Nachfolgejahr die Baumstrategie von Holzproduktion, Raumeroberung und baumindividueller Stabilität hin zur Fruchtbildung, natürlicher Regeneration und Arterhaltung. Die aktive Anpassung der Kohlenstoff-Allokation an veränderte Umweltbedingungen ist ein wesentliches Indiz für eine hohe Stresstoleranz der Buche. Die Buche weist im Vergleich der Hauptbaumarten gegenwärtig die niedrigste Absterberate auf. Es ergeben sich gegenwärtig keine Hinweise auf eine schwerwiegende Einschränkung der Vitalität der Buche. Inwieweit eine unmittelbare Abfolge mehrerer Extremjahre wie 2003 zu Grenzen der Anpassungsfähigkeit der Buche führen wird, bleibt offen.

Stichworte: Resilienz, Vitalität, Wachstum, Mortalität, Fruktifikation, KohlenstoffAllokation

\begin{abstract}
The occurrence of extreme weather conditions may create risks for beech. Following 2003, which stands out from other years as being a particularly hot year with low precipitation, the response patterns of beech were investigated at forest monitoring plots in northwest Germany.

In 2004, the growth of beech merchantable wood and branch biomass declined by $50 \%$. After 2003, the development of apical shoots was restricted for a number of years. The decrease in height and diameter growth may lead to a reduction in timber yield. In the event of a series of successive extreme hot dry years, the production of the renewable beech wood resource may decrease even further with severe ecological and economic consequences.
\end{abstract}

Overall the net primary production was balanced as an extensive mast in 2004 compensated for the reduced height and diameter growth. The difference in leaf biomass was minimal, resulting in an overall decrease in the aboveground net primary production in 2004 of only about $3 \%$; root systems were not considered. As a consequence of the 2003 extreme weather conditions, the beech growth strategy changed in the following year from one of wood production, spatial acquisition and individual tree stability to one of pronounced fruit development, natural regeneration and species maintenance. The high ability of beech to adapt its allocation of carbon in response to changes in environmental conditions is an important indicator of its high stress tolerance. Currently, beech has the lowest mortality rate of the main tree species. To date, the criteria investigated show no evidence of a serious reduction in beech vitality. The extent to which the occurrence of extreme 
weather conditions such as in 2003 in successive subsequent years would affect the adaptability of beech is still uncertain.

Keywords: resilience, vitality, growth, mortality, fructification, C-allocation

\section{$1 \quad$ Einleitung}

\subsection{Klimawandel durch $\mathrm{CO}_{2}$-Freisetzung aus fossilen Energieträgern}

Die vom IPCC (2007) vorgelegten Indizien einer globalen Erwärmung begründen eine gesellschaftliche Diskussion des Klimawandels auf wissenschaftlicher Grundlage.

Die Kohlendioxid-( $\left.\mathrm{CO}_{2}\right)$-Freisetzung aus fossilen Energieträgern ist nach IPCC die Ursache des Klimawandels. Nach HORN et al. (2007) und ZIESING (2007, 2008) steigen die $\mathrm{CO}_{2}$-bezogenen Emissionen global seit 1990 bis heute exponentiell an (1990: 21,6; 2000: 24,0; 2006: 29,0 Mio. t $\mathrm{CO}_{2}$ je Jahr). Die Jahre 2005 und 2007 hatten global sogar die höchsten $\mathrm{CO}_{2}$ Steigerungsraten seit Beginn der Aufzeichnungen (NOAA 2008). Neben $\mathrm{CO}_{2}$ sind auch andere klimarelevante Gase zu beachten. Trotz aller Anstrengungen nach dem Kyoto-Protokoll, treibhauswirksame Methan- $\left(\mathrm{CH}_{4}\right)$-Emissionen $\mathrm{zu}$ reduzieren, ist in den Jahren 2007 und 2008 nach einer Phase der Stabilisierung sogar eine Trendwende hin zu steigenden $\mathrm{CH}_{4}$-Konzentrationen festzustellen.

Das von der EU im Jahr 2008 vorgelegte Klimapaket 2020 stellt die Grundlage für eine neue Energiepolitik in der EU dar. Bis 2020 soll der $\mathrm{CO}_{2}$-Ausstoß um $20 \%$ verringert werden, der Anteil an erneuerbarer Energie auf $20 \%$ ansteigen und $20 \%$ Energie eingespart werden. Allerdings lässt eine um jährlich etwa 80 Millionen Menschen steigende Weltbevölkerung sowie die wirtschaftliche Entwicklung - nicht nur in den Schwellenländern China und Indien - global einen weiterhin steigenden Energiebedarf erwarten.

Die von der EU eingeleitete Politik soll dazu beitragen, dass Temperaturänderungen von global $2{ }^{\circ} \mathrm{C}$ im Jahresdurchschnitt nicht überschritten werden. Diese Differenz bedeutet allerdings bereits - auch in Verbindung mit damit einhergehenden Extremereignissen - eine für die Ökologie der Wälder grundlegende Veränderung ihrer Lebensbedingungen (EICHHORN u. PAAR 2008, SPELLMANN et al. 2007).

\subsection{Ausgangssituation für Buchenwälder}

Mit der Nutzung fossiler Energie entstand schon vor der Wahrnehmung des Klimawandels eine Vielzahl wesentlicher Veränderungen für die Ökologie der Buchenwälder. Schwefelverbindungen begründeten eine gerichtete Veränderung 
des Säurehaushaltes (ULRICH et al. 1979). Der Säureeintrag (potenzielle Säuredeposition nach GAUGER et al. 2002) in Buchenwälder lag etwa im Solling im Mittel der 1970er Jahre bei etwa $6,0 \mathrm{kmol}_{\mathrm{c}} \mathrm{ha}^{-1} \mathrm{a}^{-1}$ und nahm auf ca. 2,5 $\mathrm{kmol}_{\mathrm{c}} \mathrm{ha}^{-1} \mathrm{a}^{-1}$ im Mittel der 1990er Jahre ab. Ein abnehmender Trend der Säureeinträge konnte von MEESENBURG et. al (1995) auch auf anderen intensiven Versuchsflächen in Niedersachsen gefunden werden. Der Eintrag von Stickstoffverbindungen (MEESENBURG et al. 2002) konnte insbesondere auf manchen ehemals streugenutzten Standorten die grundlegende Stickstoffunterversorgung ausgleichen, führte andererseits bei Stickstoffüberschüssen zu belastenden Bedingungen für Buchenwälder (EICHHORN 1995), teilweise mit Nitratanreicherungen in die Hydrosphäre und N-Abgabe in Form von treibhausrelevanten Spurengasen in die Atmosphäre (BRUMME 1999). Mit Blick auf die Waldgeschichte ab Ende des Mittelalters mit Bedarfsdeckung im Wald wie Köhlerei, Waldweide und vor allem Streunutzung wird deutlich, dass die durch die Umwelt beeinflussten Wachstumsfaktoren für Buchenwälder stets variabel und in vielfältiger Weise durch menschliche Einflüsse geprägt waren.

Was bedeutet aber eine derartige Variabilität der standörtlichen Bedingungen für die Buche? Die Buche findet zunächst auf einer großen Zahl mitteleuropäischer Wuchsorte gut geeignete Bedingungen. Die Standortsamplitude von Buchenwäldern reicht von sehr sauren Böden bis zu reinen Kalkstandorten. Die Buche gedeiht sowohl in Regionen mit einer mittleren Jahresniederschlagssumme von weniger als $550 \mathrm{~mm}$ als auch von über $2000 \mathrm{~mm}$ (LEUSCHNER et al. 2006), allerdings nimmt unter $650 \mathrm{~mm}$ das Trockenstressrisiko zu (MANTHEY et al. 2007). Im nördlichen Teil Deutschlands kommt die Buche bis an die Küste vor, im Südharz bis in Höhen von $800 \mathrm{~m}$ ü. NN, in den Nordalpen bis zu $1700 \mathrm{~m}$ ü. NN (ОTTO 1994). In Europa wird das Verbreitungsgebiet der Buche im Osten vor allem durch kontinental kalte Winter begrenzt, im Süden durch Sommerdürre und im Norden durch zu lange Winter und Spätfrost (SCHULZE et al. 2002). Dabei erhalten die Buchenwälder in Mittel- und Ostpolen etwas mehr Niederschlag als die im Nordostdeutschen Tiefland. Nach ElLENBERG (1996) ist die Buche die einzige Baumart, die alle übrigen aus ihrem physiologischem Optimalbereich ganz oder fast ganz verdrängen kann. Die hohe interspezifische Konkurrenzkraft beruht zum einen auf der Fähigkeit der Buche, Schatten zu tolerieren und Schatten zu erzeugen und zum anderen auf ihrem flexiblen Höhenwachstum. Zusätzlich ist die Buche in der Lage, mit ihrem Wurzelwerk den Bodenraum erfolgreicher zu erschließen als beispielsweise die Konkurrenzbaumart Traubeneiche (LEUSCHNER 1998). LEUSCHNER (2006) bewertet die Buche aufgrund ihrer ökologischen Potenz in Zentraleuropa als besonders erfolgreiche Pflanzenart. In der Mehrzahl der Fälle stellen unsere heutigen Buchenwälder autochthone Restpopulationen dar, die in der jeweiligen Region über viele Generationen standortsangepasst sind. Unter den gegenwärtigen ökologischen Rahmenbedingungen lässt sich danach auf eine große Anpassungsfähigkeit der Buche schließen. 
Tatsächlich ist das Vorkommen der Buche in der jüngeren Vergangenheit wesentlich durch waldbauliche Ziele geprägt. Die Ergebnisse der Bundeswaldinventur (BMVEL 2004) belegen eine Zunahme der Buchenfläche in den alten Ländern um $2 \%$ im Zeitraum von 15 Jahren. Heute nehmen Buchenwälder einen Anteil von rd. $15 \%$ der Waldfläche in Deutschland ein. Besonders hoch ist der Anteil in Hessen (30\%), in Niedersachsen sind 14\% und in Sachsen-Anhalt begründet in menschlichen Aktivitäten sowie regional ausgeprägter Sommertrockenheit - nur 6\% der Waldfläche mit Buche bestockt.

Wie kann nun beurteilt werden, ob witterungsextreme Jahre ein Risiko für die Stabilität der Buche und ihre Bewirtschaftung darstellen? Welche Indikatoren sind zu erfassen? Hat sich nach dem witterungsextremen Jahr 2003 die Allokation des assimilierten Kohlenstoffs der Buche charakteristisch verändert? Sind nach 2003 Einschränkungen der Buchenvitalität nachweisbar? Wie schnell klingen die Reaktionen der Buche auf 2003 wieder ab und erreichen normale Werte? Wie anpassungsfähig ist die Buche bei Witterungsextremen?

\subsection{Der außerordentlich trocken-heiße Sommer 2003 - Beispiel für Wirkungen eines witterungsextremen Jahres auf Buchenwälder}

In der Reihe der letzten Jahre war 2003 ganz besonders warm und niederschlagsarm. Die Durchschnittstemperatur lag mehr als $3{ }^{\circ} \mathrm{C}$ über dem langjährigen Mittel und war damit die höchste seit 1949.

Zwischen Oktober 2002 bis Januar 2003 sowie im Mai 2003 fielen überdurchschnittliche Niederschläge; allein im Oktober $200270 \%$ mehr als im langjährigen Mittel. Nachdem die Bodenspeicher in der Nichtvegetationszeit 2002/2003 gut mit Wasser gefüllt waren, fielen in den Monaten Februar bis April sehr geringe Niederschläge. Ende Juni waren die Bodenwasservorräte bereits weitgehend aufgebraucht. Daher kommt dem Ausbleiben der Niederschläge von Juni bis August 2003 eine besondere Bedeutung zu. Sichtbare Auswirkungen an den Bäumen zeigten sich ab Mitte August auf flachgründigen, wechselfeuchten, und wechseltrockenen sowie auf sonnenexponierten Standorten. Hier war an einigen Orten ein frühzeitiger Laubfall mit in der Regel noch grünen Blättern zu beobachten. Die Vegetationsperiode 2003 erwies sich im großräumig bewaldeten forsthydrologischen Forschungsgebiet Elsterbach (Nordhessen) als Phase mit der geringsten Abflusshöhe seit Messbeginn Ende der 1960er Jahre. Erstmals wurde in der Vegetationszeit des Jahres 2003 weniger als die Hälfte des langjährigen AbflussMittelwerts erreicht. 


\section{Resilienzeigenschaften als Bewertungskriterium für die Vitalität der Buche}

Störungen sind den Waldökosystemen zugehörige Erscheinungen, ihr Eintreten ist für die Walddynamik von ausschlaggebender Bedeutung (OTTO 1994). Ohne Anpassung ist für Bäume aufgrund ihrer Ortsgebundenheit kein Überleben möglich. Optimierung und zugleich Anpassung sind daher bei Bäumen besonders wichtig (ROLOFF 2005). Waldökosysteme besitzen als komplexes biologisches System die Fähigkeit, auf Veränderungen und Störungen widerstandsfähig oder resilient zu antworten. Als Resilienz wird die Fähigkeit definiert, auf unterschiedliche Bedingungen bzw. Störungen zu reagieren, diese aber elastisch zu tolerieren, ohne dass sich langfristig ein anderer Ökosystemzustand einstellt (WALKER 1995, WALKER et al. 1999, CROPP et al. 2002). Bei Bäumen sind dabei art- und standortsspezifische Unterschiede zu erwarten.

\subsection{Vitalitätsdreieck „Langlebigkeit - Funktion - Stresstoleranz“}

Bezogen auf die Gesundheit von Bäumen und Wäldern definierte SCHWERDTFEGER (1970), dass ,unter Krankheit gemeinhin eine Abweichung vom normalen Verlauf der Lebensvorgänge im Organismus verstanden wird, die ihn oder Teile von ihm in Gedeihen oder Dasein bedrohen. Nicht jede Abweichung vom Normalen braucht Gedeihen oder Dasein des Lebewesens zu gefährden. Im Einzelfall kann es schwer sein, eine Grenze zwischen krank und gesund zu ziehen." Im paneuropäischen ICP Forests Manual zur visuellen Ansprache des Zustandes von Baumkronen (EICHHORN ET AL. 2006) definiert ROSKAMS (2006) Schaden als Abweichung eines Kompartiments eines Baumes mit einem nachteiligen Effekt für die vom Baum zu erfüllenden biologischen Funktionen.

GEHRIG (2004) setzt an den Eigenschaften des Systems Baum an und definiert in seinem Vitalitätsdreieck "Vitalität als Zustand im Spannungsdreieck: Langlebigkeit - Funktion - Stresstoleranz". Ein Organismus ist nach GEHRIG vital, wenn er unter den herrschenden Umweltbedingungen die zur Verfügung stehende Energie in diesem Dreieck optimal einsetzt. Das Vitalitätsdreieck bietet Ansätze zu einer praktisch durchführbaren, quantifizierenden Erfassung von Resilienzeigenschaften an Bäumen und Wäldern.

Die Absterberate in bewirtschafteten Wäldern wird als Hinweis verwendet, inwieweit Einschränkungen des natürlichen Alters langlebiger Baumarten zu erwarten sind. Anhand der Erfassung der annuellen Mortalität von Probebäumen einer systematischen Stichprobe aller Altersstufen wird die Langlebigkeit zu einer zahlenmäßigen Größe.

Grundlegende biologische Funktionen des Baumlebens sind Wachstum und Fruchtbildung bzw. Vermehrung: Beide Schlüsselprozesse sind Ausdruck der Kohlenstoffallokation der Bäume bzw. stehen auch für wichtige Formen der Kohlenstoffspeicherung im globalen C-Haushalt. 
Gegenüber den äußeren Faktoren ist als Stresstoleranz die Fähigkeit zu verstehen, abiotische und biotische Einflüsse zu ertragen und sich an diese anzupassen. Wie ist etwa die Stärke der Reaktion der Pflanzen auf Phasen hoher Temperatur oder Trockenheit (auch Extremereignisse), wie schnell erfolgt die Erholung nach einer solchen Reaktion? Ergeben sich in Abhängigkeit von der Stärke der Einflussgröße reversible bzw. nicht reversible Reaktionen? Ein klassisches Beispiel für pflanzengruppenbezogene Stresstoleranz mit Wirkung auf die Evolution der Bäume im Übergang von Gymnospermen zu Angiospermen ist die Empfindlichkeit gegenüber der Phase der Frosttrocknis ausgangs des Winters: Die sich erwärmende bodennahe Luft übt einen Transpirationsanreiz aus, während der Boden noch gefroren ist - eine Situation, an die laubabwerfende Bäume besser angepasst sind als die immergrünen.

\section{Konzept zur Erfassung der Resilienz von Bäumen und Wäldern}

Ziel eines Konzepts zur Erfassung der Resilienz ist es, Reaktionen von Bäumen und Beständen in ihrem Zeitbezug zu erfassen und diese im Hinblick auf ökologische und ökonomische Risiken zu bewerten. Den hier vorgeschlagenen Indikatoren ist gemein, dass die Methoden definiert sind (z. B. Manual ICP Forests, EICHHORN et al. 2006), ihre Anwendung qualitätsgesichert erfolgt, nur wissenschaftlich geprüfte Merkmale vorgeschlagen werden und die Zeitachse der jahresbezogenen Ergebnisse auch in die Vergangenheit reicht. Einige der für eine Bewertung der Resilienzeigenschaften von Wäldern wesentlichen Merkmale sind bislang für die Dauerbeobachtung der Wälder nicht als verpflichtend eingestuft. Eine Weiterentwicklung der europaweiten forstlichen Dauerbeobachtung ist deshalb erforderlich (DOBBERTIN u. DE VRIES 2008, EICHHORN u. SZEPESI 2008).

Nachfolgend werden die Reaktionsmuster der Buche anhand konkreter Indikatoren zahlenmäßig ermittelt. Dabei findet für „Langlebigkeit“ die annuelle Mortalität von Probebäumen Verwendung. Für die biologische Funktion der Buche werden insbesondere die Indikatoren Durchmesser- und Höhenzuwachs, Fruktifikation, Belaubung der Oberkrone sowie Blattbiomasse herangezogen. Aus der zeitlichen Entwicklung der Messwerte erfolgen Rückschlüsse auf die Stresstoleranz der Buche.

\subsection{Langlebigkeit}

Im Rahmen der systematischen Stichproben der Waldzustandserhebung wird jährlich der Ausfall von Probebäumen registriert und dessen Ursache ermittelt. Auf diese Weise können Baumentnahmen im Rahmen von Durchforstungen oder Endnutzungen vom echten Absterben unterschieden werden. Die Stichprobenpunkte unterliegen der normalen Waldbewirtschaftung. Ausgefallene Bäume 
werden jeweils nach objektiven Prinzipien ersetzt, die Zahl der Bäume in der Stichprobe bleibt grundsätzlich konstant (Stichprobe Buche 2007 in Hessen: 1397 Bäume, in Niedersachsen: 2315 und in Sachsen-Anhalt: 492). Das Stichprobenkonzept ermöglicht etwa für Bundesländer flächenbezogene Aussagen, erfasst jedoch unzureichend lokale Absterbeprozesse durch regional begrenzte biotische bzw. standörtliche Faktoren (vgl. BRESSEM in diesem Band). Messgröße ist die annuelle Mortalität in Prozent des Stichprobenkollektives.

Ein Vergleich aller Baumarten und Altersstufen im systematischen Stichprobennetz des ICP Forests-Systems Level I $(16 \mathrm{~km}$ x $16 \mathrm{~km})$ der Jahre 1987 2004 ergibt für Spanien eine annuelle Mortalität von 0,24\%, für Frankreich $0,20 \%$, für Deutschland 0,25\% und für die Tschechische Republik 0,61\%. Im Vergleich dazu bleibt die annuelle Mortalität in Finnland mit 0,17 \% relativ gering. Insgesamt ergibt sich zwischen 1987 und 2004 eine geringe Mortalität. Im stärker von Säurebelastungen betroffenen kontinentalen Europa sind die Werte etwas erhöht. Auf europäischer Ebene liegt die Mortalitätsrate der Buche niedriger als die von Fichte, Kiefer und Eiche (UN/ECE u. EC 2000).

Die Zeitreihen zeigen für Hessen, Niedersachsen und Sachsen-Anhalt durchschnittliche Absterberaten zwischen $0,1 \%$ in Niedersachsen und 0,3\% in Hessen sowie Sachsen-Anhalt, die Ergebnisse entsprechen also etwa dem europäischen Niveau.

Zwischen den Hauptbaumarten bestehen Unterschiede. Deutlich ist in allen drei Ländern die besondere Überlebensfähigkeit der Buche erkennbar, der Hauptbaumart mit der niedrigsten jährlichen Absterberate. Zwischen 1984 und 2007 sind stets nur einzelne Bäume betroffen, so dass die durchschnittliche Absterberate für die Buche (Alter über 60 Jahre) im Mittel unter 0,05\% liegt. Nach den Sturmwürfen Anfang der 1990er Jahre wie auch nach dem außerordentlichen Sommer 2003 sind in Hessen wie auch in Sachsen-Anhalt und Niedersachsen vereinzelt Mortalitätsraten der Buche von immer noch sehr geringen 0,3\% festzustellen. Auch auf intensiv betreuten Versuchsflächen bestätigt sich die geringe Mortalität der Buche (DAMMANN et al. 2000).

Eine Analyse der geringen Buchenmortalität auf dem $4 \mathrm{~km}$ x $4 \mathrm{~km} \mathrm{Netz} \mathrm{der}$ Waldzustandserhebung Hessen zeigt, dass die geringe Buchenmortalität in allen Landesteilen ein vergleichsweise homogener Befund ist.

Im Vergleich dazu reichen bei der Fichte die Mortalitätsraten bis zu 3,3\% (s. Abb. 1), ein Unterschied um den Faktor 10 gegenüber der Buche. Phasen mit erhöhten Absterberaten sind für diese Baumart insbesondere in den Jahren nach Sturmwürfen, Borkenkäferbefall sowie im Anschluss an besonders trocken/heiße Jahre wie $2003 \mathrm{zu}$ verzeichnen. Maximale Werte wurden dabei in der Periode 2004/2005 erreicht, also eineinhalb Jahre nach dem außerordentlichen Sommer. Ähnliche Befunde liegen für Frankreich vor. Nach 2003 trat in Frankreich das bisherige Maximum der Mortalität der Zeitreihe 1989 bis 2004 auf. Laubbaumarten 
erreichten 0,5\%, Nadelbaumarten 1,3\% Mortalität. Der Kurvenverlauf kann auch durch Maßnahmen zur Steuerung der Borkenkäferpopulation bestimmt sein.

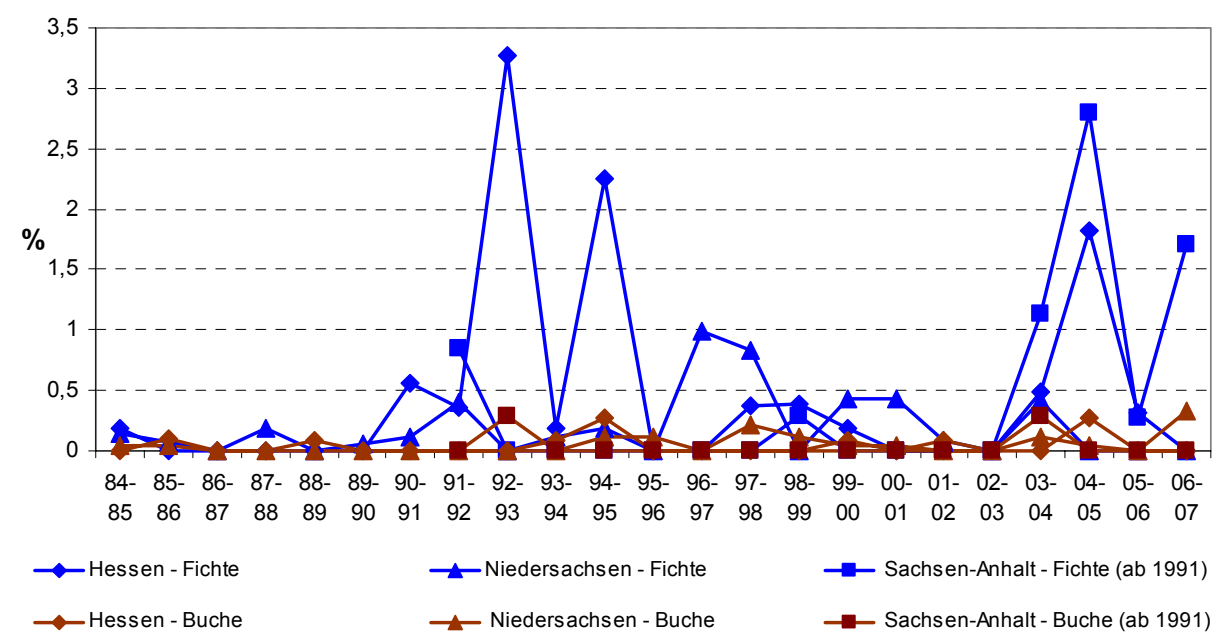

Abbildung 1: Jährliche Absterberate (\%) von Fichte und Buche (Alter über 60 Jabre) in Niedersachsen, Hessen und Sachsen-Anhalt (Waldzustandserhebung 1984 - 2007, in SachsenAnhalt ab 1991)

\subsection{Wachstum und Fruktifikation}

Im Sinne von GIVNISH (1988) sind Produkte der Kohlenstoffassimilation und deren Zuordnung zu verschiedenen Baumkompartimenten (C-Allokation) Ausdruck der biologischen Funktionalität des Baumlebens. Werden die Indikatoren der C-Allokation wie beispielsweise das Baumwachstum oder die Fruktifikation in jährlichen Zeitintervallen erfasst, ermöglicht dies eine zeitabhängige, quantitative Bewertung der Reaktion von Bäumen auf äußere Faktoren, also der Toleranz von Bäumen gegenüber Stressoren. Nachfolgend werden daher die Reaktionen des Baumwachstums und der Fruktifikation auch im Zusammenhang mit der Fragestellung Stresstoleranz diskutiert.

Zum Zuwachs an Biomasse von Bäumen zählen insbesondere die Kompartimente Blätter, Früchte mit den zugehörigen Knospen und Fruchtschalen, Äste und Derbholz sowie Wurzeln. Im Rahmen von Untersuchungen auf Dauerbeobachtungsflächen liegen für die Buche längere Zeitreihen vor, insbesondere zum Höhen- und Durchmesserwachstum, zur Belaubung und Fruchtbildung. Die hessischen intensiven Beobachtungsflächen weisen optimale Wuchsbedingungen für die Buche auf [Hünfeld: $625 \mathrm{~mm}$ Jahresniederschlag, Braunerde-Pseudogley, Buntsandstein; Kirchhain: 650 mm Jahresniederschlag, Parabraunerde-Pseudogley, Basalt mit Lößlehm; Kellerwald: 650 mm, Braunerde, Tonschiefer u. Grauwacke in Wechsellagerung; Homberg/Efze: 700 mm Jahresniederschlag, Parabraunerde- 
Pseudogley, Basalt; Zierenberg: 700 mm Jahresniederschlag, Braunerde, Basalt; Kalbach: 825 mm Jahresniederschlag, Braunerde, Buntsandsteinsubstrat; Spessart: $963 \mathrm{~mm}$ Jahresniederschlag, podsolierte Braunerde, Buntsandstein; Weilburg: 950 mm Jahresniederschlag, Lockerbraunerde, Schieferton].

\subsubsection{Durchmesserquwachs}

Der Durchmesserzuwachs (in 1,30 m Höhe) wurde auf den im Mittel 140 Jahre alten Buchenflächen der intensiven Dauerbeobachtung exemplarisch in Hessen (Hünfeld, Kirchhain, Kellerwald, Homberg/Efze, Zierenberg, Spessart und Weilburg) anhand von je 2 Kernbohrungen von 20 herrschenden Buchen je Fläche erfasst (s. Abb. 2). Kernbohrungen ermöglichen einen Vergleich von aktuellen mit retrospektiven Befunden.

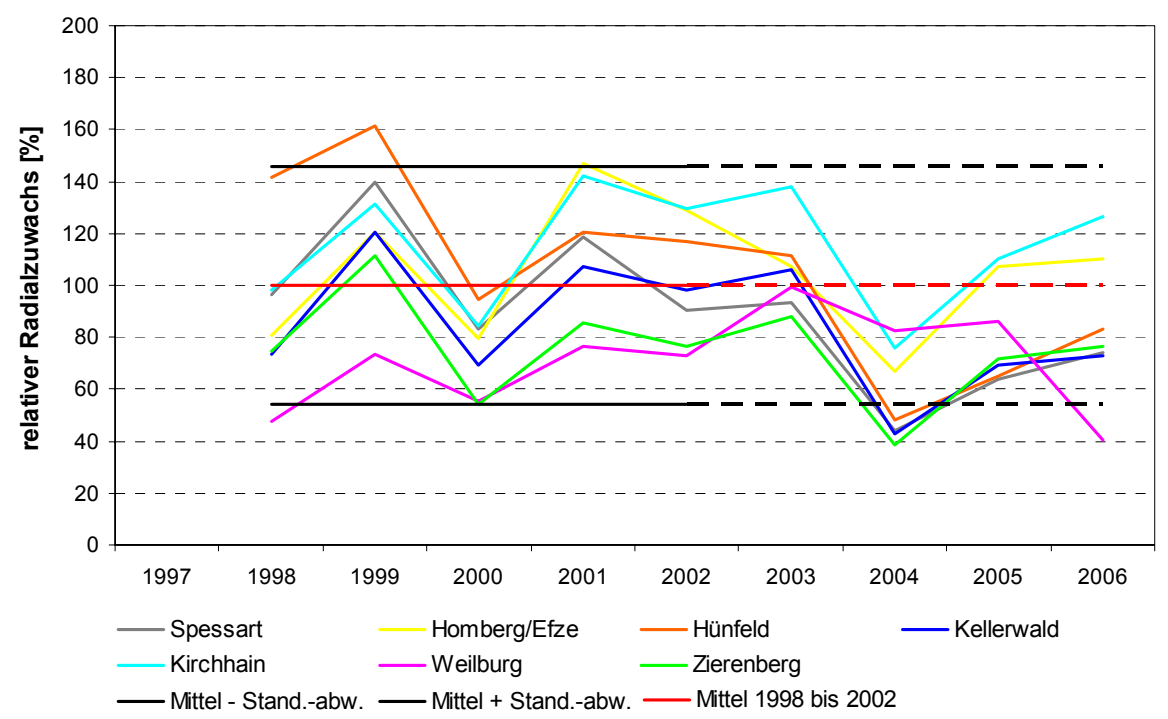

Abbildung 2: Entwicklung des mittleren relativen Radialzuwachses von Buche auf sieben Flächen der intensiven Dauerbeobachtung (Level II) in Hessen. Vergleich der Jahreswerte ab 2003 mit dem Zeitraum 1998 - 2002.

Auf den Buchenflächen beträgt 2003 der Radialzuwachs 107 \% des Mittels 1998 2002. Bereits vor dem Eintritt der anhaltenden Trockenheit und Hitze war in diesem Jahr der größte Teil der Jahrringbreite ausgebildet. In der Vegetationszeit 2004, also ein Jahr nach dem „Jahrhundertsommer“ 2003, zeigt sich ein signifikanter Einbruch des Radialzuwachses auf $57 \%$ des Mittels von 1998 - 2002. Der Radialzuwachs der Buchen erholt sich im darauf folgenden Jahr 2005 wie auch 2006 auf $84 \%$ des Mittels. Trotz Erholung wird das Ausgangsniveau nicht ganz erreicht. 


\subsubsection{Höhen₹uwachs}

Ende Juli/Anfang August 2007 wurden auf den Buchenflächen des intensiven Monitorings in Hessen auf jeder Fläche von sechs vorherrschenden und herrschenden Bäumen je zwei Triebe aus der Lichtkrone gewonnen und anschließend die einzelnen Trieblängen der Jahre 1997 - 2007 am Haupttrieb vermessen. Anhand der Triebbasisnarben war eine retrospektive Bewertung der Trieblängen möglich.

Analog zum Radialzuwachs in 1,30 m Höhe tritt erst in der Vegetationszeit nach dem besonders warmen-trockenen Jahr auf allen Flächen eine massive Wachstumsreduktion auf (s. Abb. 3). Die Höhentriebe erreichen 2004 nur $70 \%$ der Länge der Vorjahre. Nach einem gemischten Modell mit den Probebäumen und Flächen als zufällige Faktoren ist der Rückgang der Trieblängen 2003/2004 von $34 \mathrm{~mm}$ höchst signifikant $(\mathrm{p}=0$,0004). Im Gegensatz zur Durchmesserentwicklung setzt sich diese Depression länger fort (2005: $55 \%$ ). Nach günstigen Höhentrieben im Jahr 2006 tritt 2007 erneut ein sehr kurzer Trieb auf. Die Nachwirkungen nach dem Jahr 2003 sind bis 2007 zu erkennen. Im Mittel erreichen die Höhentriebe in diesen vier Jahren nur 66 \% des Mittels der Jahre 1998 bis 2002, ein Hinweis auf eine längerfristige Wirkung des Jahres 2003 für die Entwicklung der Höhentriebe. Nach ROLOFF (2001) führen Trockenphasen zu einer Bildung von Kurztrieben, die sich bei Buchen jüngeren und mittleren Alters im nachfolgenden Jahr wieder normalisieren sollte. Eine Abfolge einer Reihe von Kurztrieben, sog. Kurztriebketten, wird als Vitalitätseinschränkung definiert. 2003 hat großräumig einen Impuls zur Bildung von Kurztriebketten ausgelöst

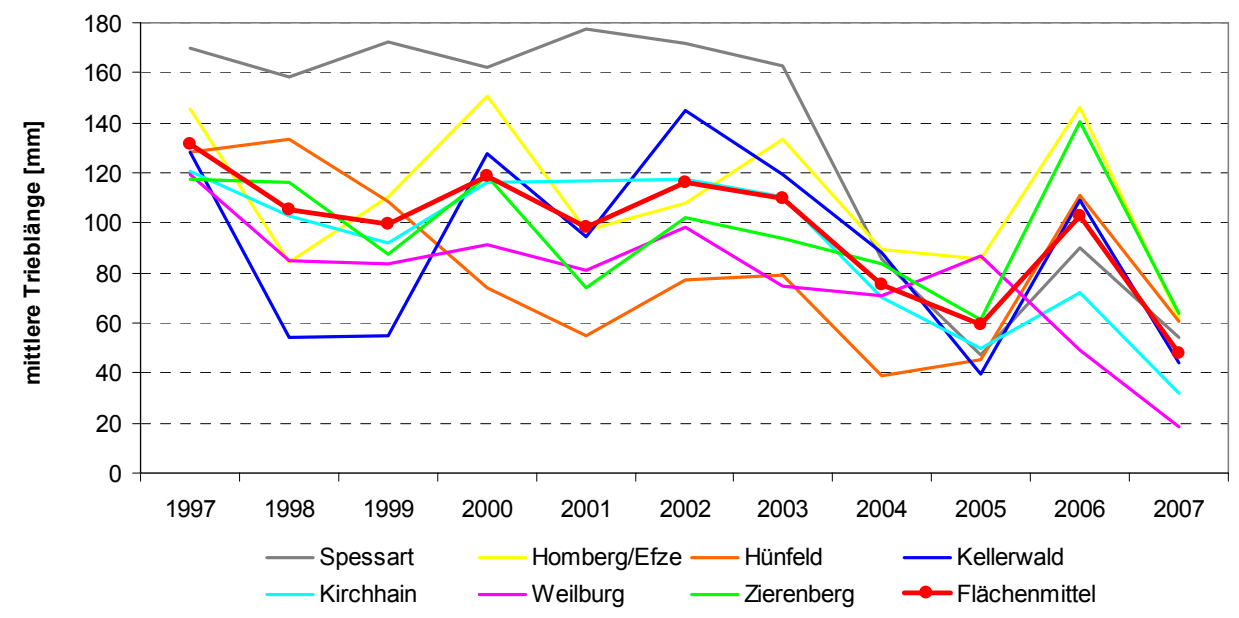

Abbildung 3: Entwicklung apikaler Trieblängen von Buche auf sieben Flächen der intensiven Dauerbeobacbtung (Level II) in Hessen 1997 - 2007 
Nach dem gleichen Verfahren wie auf den Flächen des intensiven Monitorings wurden die Trieblängen von Bäumen der Stichproben-Flächen der extensiven Dauerbeobachtung in Hessen ermittelt (s. Abb. 4). Von drei vorherrschenden oder herrschenden Bäumen pro Fläche wurden je zwei Triebe aus der Lichtkrone geworben. Die Stichprobe umfasst 240 Buchen von 80 Flächen. Als Teilstichprobe des systematischen $8 \mathrm{~km}$ x $8 \mathrm{~km}$ Netzes ermöglicht diese Auswertung eine flächenrepräsentative Aussage für die Buche in Hessen.

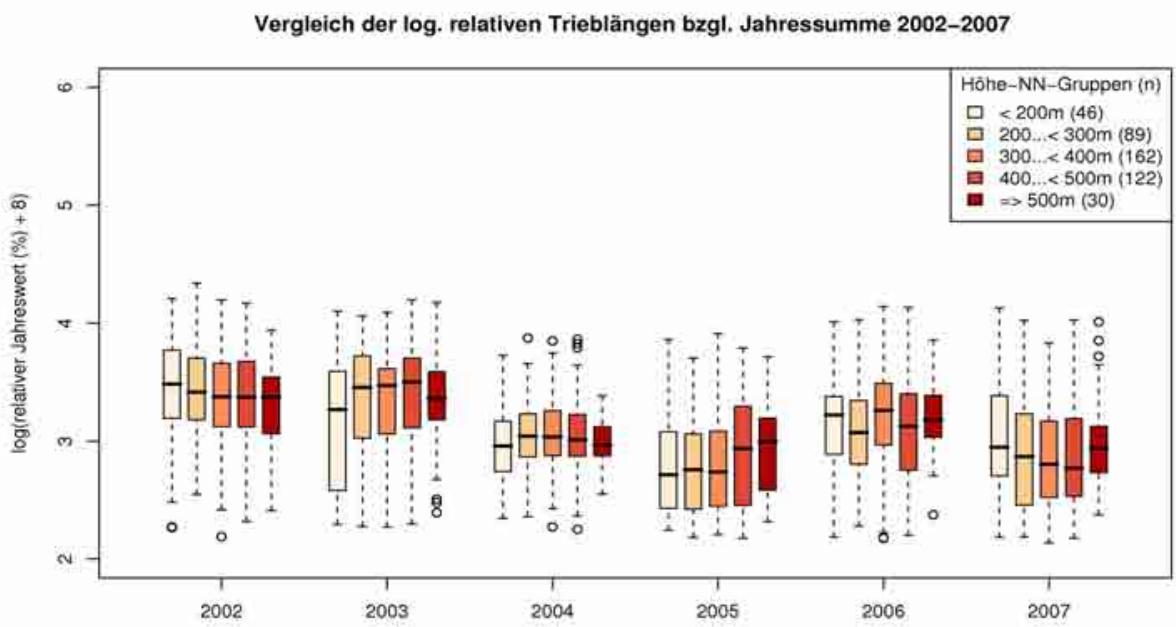

Abbildung 4: Entwicklung der Trieblängen der obersten Kronenspitze von Buche auf 80 Flächen der extensiven Dauerbeobachtung (8 km $\times 8 \mathrm{~km}$; Level I) in Hessen 2002 - 2007 nach Höhenstufen. Absolute Trieblängen in Relation zum Mittel der Jabre 2002 - 2007 im Hinblick auf Normalverteilung logarithmiert; Box and Whisker-Plots mit Mediandarstellung.

Zunächst bestätigt die Auswertung der systematischen Stichprobe den Befund der Intensivmessflächen. Die Buchen in Hessen zeigen im Jahr 2003 keine Reduktion der Höhentriebentwicklung. Der Höhentrieb war schon relativ zeitig abgeschlossen, also noch vor dem Eintritt der Trockenheitsphase (vgl. auch MITSCHERLICH 1978).

Dagegen erscheinen die Werte in den beiden nachfolgenden Jahren und insbesondere 2005 deutlich verringert. In diesem Jahr ist im Gegensatz zu anderen bemerkenswert, dass die Reduktion der Höhentriebe in größeren Höhenlagen (über $400 \mathrm{~m}$ bzw. über $500 \mathrm{~m}$ über NN) geringer ausfällt als in den tieferen Lagen (s. Abb. 4). Kurze Triebe sind 2004 und verstärkt 2005 vor allem auf Standorten mit geringer Summe aus klimatischer Wasserbilanz und nutzbarer Feldkapazität $(<50 \mathrm{~mm})$ anzutreffen. Buchenbestände sind auf nährstoffreichen Standorten (Basalt, tertiäre Tone, aber auch kalkbeeinflusste Böden) mit vielfach höherer nutzbarer Feldkapazität tendenziell weniger von dem Witterungseinfluss 2003 betroffen 
als solche auf nährstoffarmem Buntsandstein und vor allem auf Sandstandorten mit geringer Wasserspeicherung wie im Rhein-Main Gebiet. Nach günstigen Höhentrieben im Jahr 2006 tritt 2007 erneut ein sehr kurzer Trieb auf.

\subsubsection{Fruktifikation}

Auch bei der Einstufung der Fruktifikation ergänzen sich die Ergebnisse der flächenrepräsentativen Übersichtserhebung und der intensiven Dauerbeobachtung. Die bei der jährlichen Übersichtserhebung (Level I) einzelbaumweise durchgeführte Einstufung der Fruktifikation gibt vor allem Aufschluss über Vorkommen und Intensität der Fruchtbildung. Quantitative Daten aus Streufalluntersuchungen der intensiven Dauerbeobachtung auf Buchenflächen zu Knospen, Blüten, Schalen und Früchten ermöglichen dagegen Beiträge zum Verständnis der Kohlenstoffallokation der Buche in Jahren mit und ohne Fruchtbehang.

Die Blüten- und Fruchtbildung ist ebenso wie das Sprosswachstum oder die Laubbildung eine Stoffwechselleistung der Buche, für die erhebliche Baustoffe beansprucht werden. Im Allgemeinen unterbrechen intensive Mast- oder Fruchtjahre bei der Buche mehrjährige Phasen ohne Mastanhang. In einem Buchenbestand fruktifizieren die besonders wuchskräftigen Buchen im vollen Lichtgenuss sowie großkronige Randbäume meist häufiger und intensiver als die übrigen Bestandesmitglieder.

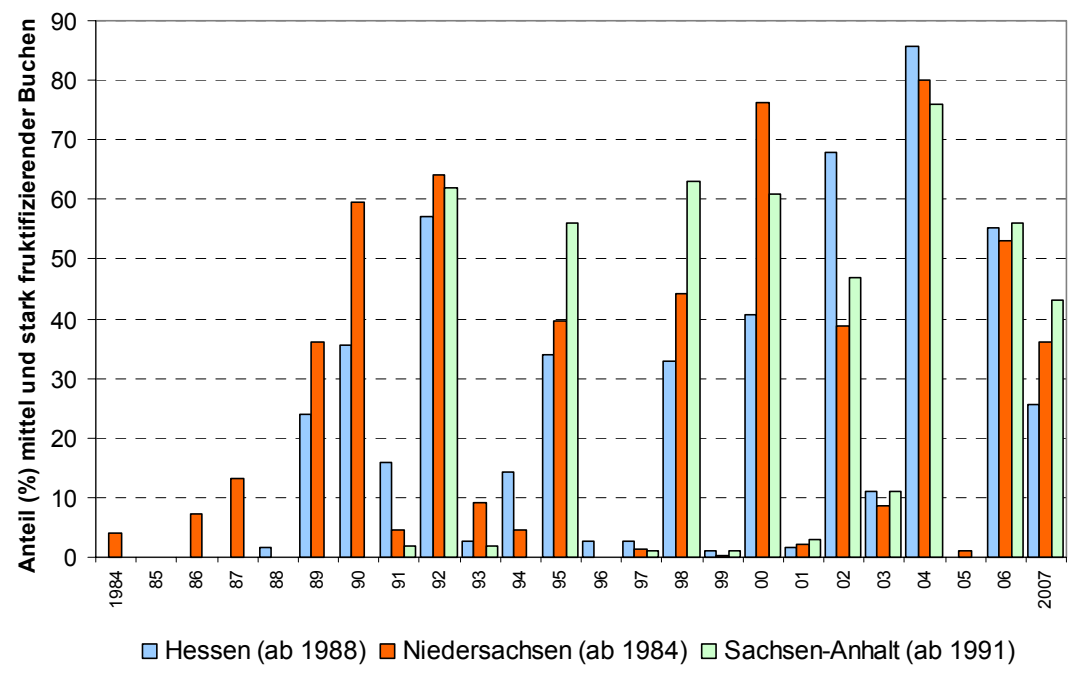

Abbildung 5: Anteil der Buchen (Alter über 60 Jahre) mit mittlerem bis starkem Fruchtanhang (Waldzustandserbebung 1984 - 2007, Hessen ab 1988, Sachsen-Anhalt ab 1991)

Aus Abbildung 5 ergibt sich, dass die Fruchtbildung in den letzten Jahren häufiger auftritt (PAAR et al. 2000) als noch in den 1980ern bzw. als dies aufgrund früherer 
Angaben zu erwarten gewesen wäre. So geben diesbezüglich SCHWAPPACH (1895), BORCHERS (1954), ROHMEDER (1967) und BURSCHEL (1966) Zeitintervalle von 7-8 Jahren an. Für Niedersachsen ist nachzuweisen, dass die intensive Fruktifikation im Jahr 1989 im Anschluss an eine fünfjährige Phase ohne nennenswerte Fruchtbildung aufgetreten ist. Nach 1989 sind 1990, 1992, 1995, 1998, 2000, 2002, 2004, 2006 und 2007 jeweils Jahre mit einer intensiven Fruchtbildung. Fast ohne Eckern waren die Buchen dagegen beispielsweise in den Jahren 1997, 1999, 2001 und 2005. Damit hat sich seit 1998 ein etwa 2-jähriger Turnus intensiver Buchenmasten entwickelt. Die Fruchtbildung der Buche verläuft in Hessen, Niedersachsen und in Sachsen-Anhalt weitgehend synchron, daher ist von großräumig gleichlaufenden Steuergrößen wie Großwetterlagen auszugehen (WACHTER 1964). Im Jahr 2004 wurde in allen drei Ländern der im Vergleich der Zeitreihe umfangreichste Bucheckernanhang festgestellt.

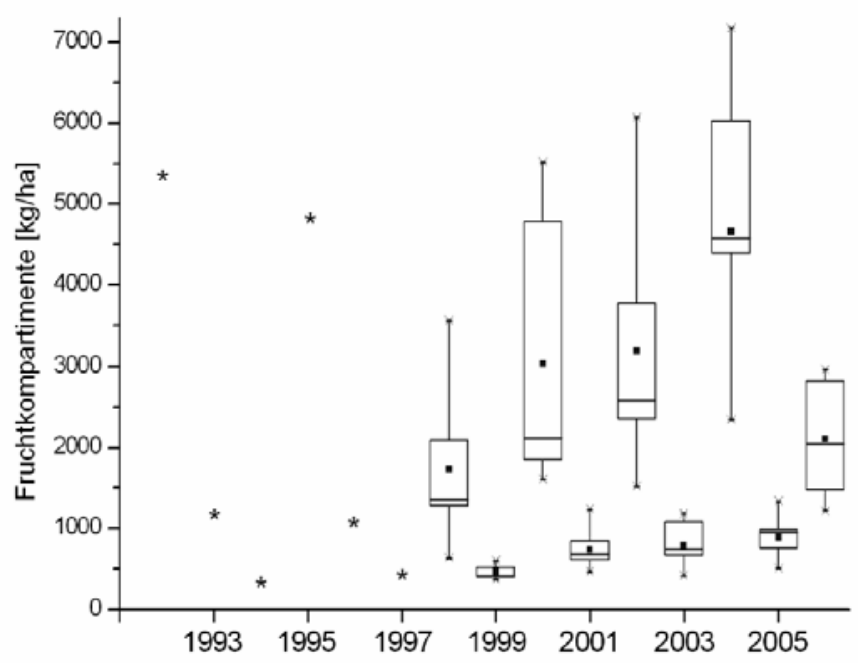

Abbildung 6: Fruchtkompartimente (Summe aus Knospen, Blüten, Früchten und Schalen) im Streufall der Buchen der Level II-Flächen (1998 - 2006): Kalbach (bis 2004), Hünfeld, Kirchbain, Zierenberg, Homberg/Efze, Spessart und Weilburg; Sterne: Zierenberg (1992 - 1997); Box-und Whisker-Plots (Querstriche in der Box: Median, Quadrate: arithmetisches Mittel)

Die im Streufall gemessenen Fruchtkompartimente (Summe aus Früchten, Blüten, Knospen und Schalen) der sieben intensiv beobachteten Buchenflächen in Hessen (s. Abb. 6, Tab. 1) ergeben eine mittlere Fruchtbiomasse von $2090 \mathrm{~kg} \mathrm{ha}^{-1} \mathrm{a}^{-1}$. Bemerkenswert ist aber die im Vergleich zur Blattmasse (s. Abb. 9) außerordentlich große Variabilität von Jahr zu Jahr (1999: $463 \mathrm{~kg} \mathrm{ha}^{-1} \mathrm{a}^{-1}$, dagegen 2004 ein 10 facher Wert von $\left.4668 \mathrm{~kg} \mathrm{ha}^{-1} \mathrm{a}^{-1}\right)$. Die Abfolge von starker und geringer Fruktifikation auf den Level II-Fallstudien ist mit der Rhythmik der flächenbezogenen Übersichtserhebung (s. Abb. 5) vergleichbar. Dies trifft insbesondere für die Mast- 
jahre 1998, 2000, 2002, 2004 und 2006 wie auch im Falle von Zierenberg für 1992 und $1995 \mathrm{zu}$.

Tabelle 1: $\quad$ Oberirdische Nettoprimärproduktion (in t Biomasse $h a^{-1} a^{-1}$ ) 1998 bis 2004 im Mittel von sieben Level II-Flächen in Hessen

\begin{tabular}{l|ccccccc|c}
\hline & $\mathbf{1 9 9 8}$ & $\mathbf{1 9 9 9}$ & $\mathbf{2 0 0 0}$ & $\mathbf{2 0 0 1}$ & $\mathbf{2 0 0 2}$ & $\mathbf{2 0 0 3}$ & $\mathbf{2 0 0 4}$ & Mittel \\
\hline Blätter & 3,003 & 3,614 & 3,291 & 3,625 & 2,971 & 3,186 & 3,563 & 3,322 \\
Früchte & 0,378 & 0,007 & 0,917 & 0,029 & 0,879 & 0,046 & 1,480 & 0,534 \\
Knospen & 0,301 & 0,276 & 0,442 & 0,292 & 0,440 & 0,302 & 0,484 & 0,363 \\
Blüten & 0,119 & 0,032 & 0,305 & 0,039 & 0,251 & 0,049 & 0,440 & 0,176 \\
Schalen & 0,935 & 0,148 & 1,375 & 0,386 & 1,625 & 0,393 & 2,264 & 1,018 \\
\hline $\begin{array}{l}\text { Summe } \\
\begin{array}{l}\text { Frucht- } \\
\text { kompartimente }\end{array}\end{array}$ & 1,733 & 0,463 & 3,039 & 0,745 & 3,194 & 0,790 & 4,668 & 2,090 \\
\hline
\end{tabular}

In den Mastjahren (1998, 2000, 2002, 2004) betrug die im Streufall gemessene Biomasse an Blüten im Mittel $279 \mathrm{~kg} \mathrm{ha}^{-1} \mathrm{a}^{-1}$; 2004 erreichten die Blüten mit $440 \mathrm{~kg} \mathrm{ha} \mathrm{a}^{-1} \mathrm{a}^{-1}$ den höchsten Wert der Zeitreihe. Früchte fanden sich in Mastjahren im Mittel $913 \mathrm{~kg} \mathrm{ha}^{-1} \mathrm{a}^{-1}$ (2004: 1481), dagegen in Nichtmastjahren im Mittel nur $27 \mathrm{~kg} \mathrm{ha}^{-1} \mathrm{a}^{-1}$. Bemerkenswert ist, dass in Nichtmastjahren nicht nur weniger Blüten entstehen, sondern sich zudem aus gebildeten Blüten auch deutlich weniger Früchte entwickeln. In der Regel entsteht in diesen Jahren je Kilogramm Blüten sogar weniger als ein Kilogramm Früchte (Quotient Früchte/Blüten s. Abb. 7, links).
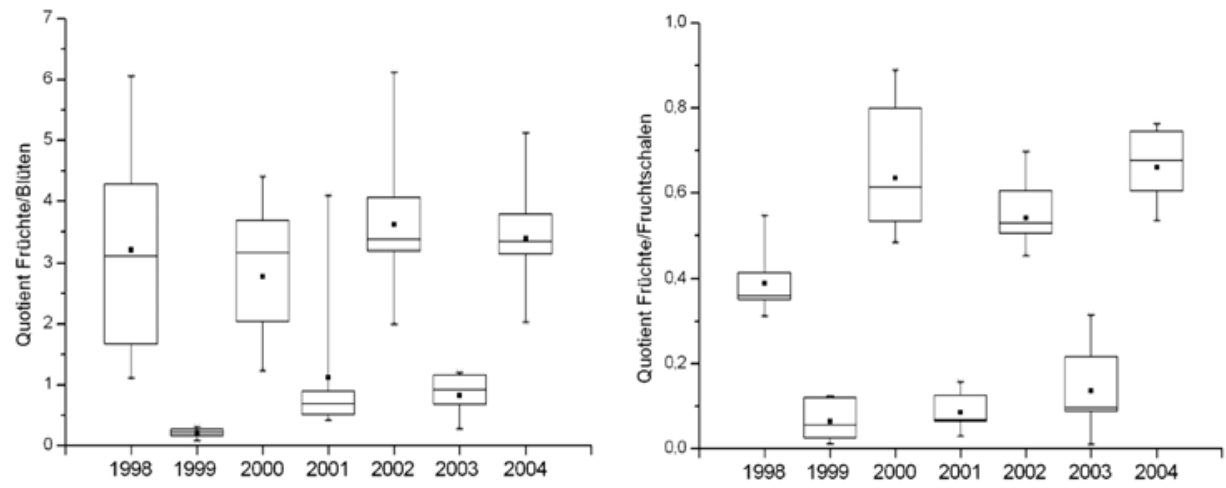

Abbildung 7: Quotient Früchte/Blüten (links) und Früchte/Fruchtschalen (rechts), Biomasse im Streufall der Buchen der Level II-Flächen (1998 - 2004): Kalbach, Hünfeld, Kirchbain, Zierenberg, Homberg/Efze, Spessart und Weilburg; Box- und Whisker-Plots (Querstriche in der Box: Median, Quadrate: arithmetisches Mittel) 
Auch der Quotient aus Früchten und Fruchtschalen unterscheidet sich in den beiden Jahresgruppen (s. Abb. 7, rechts). In Mastjahren sind deutlich mehr Früchte je Einheit Schalen vorhanden als in Nichtmastjahren.

Eine Erklärung ermöglicht das Verhältnis von Bucheckern-Schalen und Blüten. Dieser Quotient variiert in den beiden Gruppen vergleichsweise wenig (Quotient Mastjahre: 6,1; Nicht-Mastjahre 7,8). Während sich die Größe von Bucheckern von Jahr zu Jahr kaum unterscheidet, entwickeln sich in NichtmastJahren aus einem nennenswerten Teil der Blüten Früchte mit Cupula, aber keine vollständig ausgebildeten Bucheckern. Nichtmastjahre zeichnen sich demnach einerseits durch weniger Blüten, andererseits auch durch eine sehr gehemmte Entwicklung von Früchten aus.

Die veränderte Abfolge von Mastjahren bei Buche ist Ausdruck einer veränderten Zyklisierung und Verfügbarkeit von Bau- und Reservestoffen. Zwar wird der Impuls zu einer Fruktifikation durch Temperaturkriterien der Vorjahre ausgelöst (GRUBER 2004), die weitere Entwicklung bis hin zu vitalen Früchten erfordert jedoch in großem Umfang verfügbare Baustoffe und chemische Energie. Dies wird anschaulich in der chemischen Zusammensetzung von Bucheckern (gesättigte und ungesättigte Fettsäuren wie Linolsäure und Ölsäure, Stärke sowie Aminosäuren einschließlich pflanzlichen Eiweißes), aber auch im Gehalt von Elementen wie Stickstoff $(\mathrm{N})$ in den Fruchtkompartimenten der Streu. So wurde beispielsweise für die Level II-Fläche Zierenberg ein N-Gehalt von $150 \mathrm{~kg} \mathrm{ha}^{-1}$ im Jahr 2004 in der Streu gemessen, davon $120 \mathrm{~kg}$ in Fruchtkompartimenten (Früchte, Fruchtschalen und Blüten) und nur $30 \mathrm{~kg} \mathrm{~N}$ in der Blattbiomasse.

\subsection{Belaubung}

\subsubsection{Verlichtung der Oberkrone}

Die Zeitreihe der mittleren Oberkronenverlichtung ${ }^{1}$ fängt für die über 60-jährigen Buchen 1984 in Hessen und auch in Niedersachsen mit einem Ausgangswert von etwa $15 \%$ an. Bis Mitte der 1990er Jahre verdoppeln sich die Werte. 1995 fehlen im Vergleich zu einer wuchsplatzbezogenen Referenz einer vollbelaubten Oberkrone der Buche im Mittel etwa 1/3 der Blätter. Seit Mitte der 1990er Jahre sind Jahre erkennbar, die unterschiedlich vom mehrjährigen Niveau abweichen. Beispielsweise ist die mittlere Oberkronenverlichtung insbesondere in Hessen und

\footnotetext{
${ }^{1}$ Nach dem ICP-Forests-Manual zur Ansprache von Baumkronen (EICHHORN et al. 2006) werden in der Waldzustandserhebung die von Baumnachbarn unbeeinflussten oberen Teile von vorherrschenden, herrschenden und mitherrschenden Bäumen beurteilt.
} 
in Sachsen-Anhalt in den Jahren 2000 und 2004 sprunghaft erhöht; beides Jahre mit einem besonders hohen Fruchtanhang (s. Abb. 5).

Im Anschluss an das warm-trockene Jahr 2003 ist die markanteste Steigerung der Verlichtungswerte seit Beginn der Beobachtungen 1984 festzustellen.

Bei der Buche besteht seit Beginn der Erhebung ein differenzierter Trend zwischen jungen und alten Bäumen (s. Abb. 8). Die Oberkronenverlichtung der jüngeren (bis 60-jährigen) Buchen liegt weit unter den Werten der Älteren (über 60-jährigen). Insbesondere fehlen die sprunghaften Anstiege der Jahre 1992 und 2000.

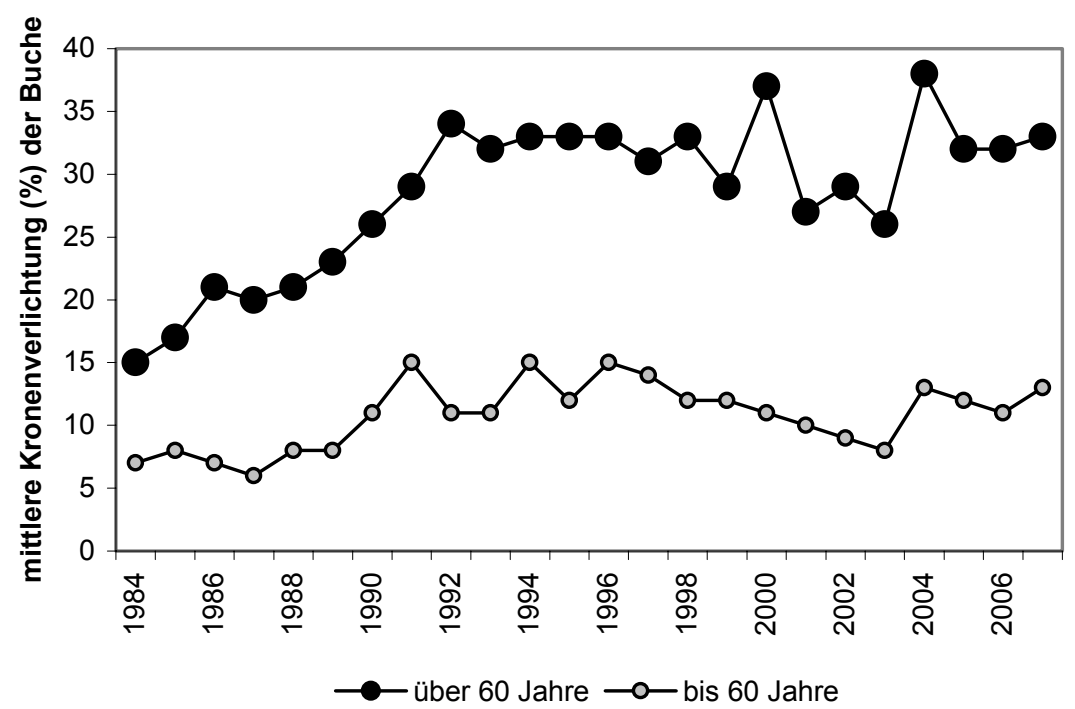

Abbildung 8: Entwicklung der mittleren Kronenverlicbtung (\%) in der von Nachbarbäumen unbeeinflussten Oberkrone der Buche in Hessen getrennt nach Altersstufen (8 km $\times 8 \mathrm{~km}$ Netv)

Da die Blühreife der Buche überwiegend erst ab dem Alter 40 einsetzt, wirken sich intensive Fruchtjahre auf den Verlauf der mittleren Oberkronenverlichtung der jüngeren Buche kaum aus. Eine gewisse Besonderheit stellt das Jahr 2004 dar, da in diesem Jahr auch bei der jüngeren Buche ein sprunghafter Anstieg der Kronenverlichtung festzustellen ist, allerdings schwächer ausgeprägt im Vergleich zu den älteren Bäumen.

Für Eichenbestände besteht in der Rhein-Main-Ebene ein gesicherter statistischer Zusammenhang zwischen hohen Blattverlusten in der Oberkrone und nachfolgender Mortalität. Der Anteil von über 60-jährigen Bäumen mit mehr als $60 \%$ Blattverlust erreicht hier - wie in keinem anderen hessischen Wuchsgebiet oder bei keiner anderen Baumart - seit 1994 häufig Werte zwischen $10 \%$ und $22 \%$. Während die annuelle Mortalität bei Eichen mit einem Blattverlust unter $60 \%$ bei unter $1 \%$ liegt, steigt die Wahrscheinlichkeit des Absterbens ab $60 \%$ Blattverlust 
rasch und überproportional an. Das Beispiel zeigt, dass bei der Eiche starke Blattverluste der Oberkrone ein sicherer Weiser für Absterberisiken sind.

Wie veränderte sich im Vergleich zur Eiche bei der Buche die Häufigkeit der Blattverluststufe über 60 \% nach 2003? Im Mittel der Jahre 1998 bis 2002 erreicht der Anteil starker Oberkronenverlichtung in Hessen, Niedersachsen und SachsenAnhalt lediglich $3 \%$. Der Wert bleibt auch 2003 unauffällig. 2004 verdreifacht bis vervierfacht sich jedoch der Anteil der Buchen mit über $60 \%$ Blattverlust in der Oberkrone in Hessen und in Sachsen-Anhalt. In Sachsen-Anhalt tritt 2006 abermals ein hoher Wert auf. Trotz erhöhter Anteile starker Oberkronenverlichtung zeigt die Buche gegenwärtig nur unwesentlich erhöhte Mortalitätsraten.

\subsubsection{Blattbiomasse}

Im Gegensatz zur Belaubungsschätzung, bei der per Definition eine Beurteilung des nicht von Nachbarbäumen beeinflussten Teils der Lichtkrone der vorherrschenden, herrschenden und mitherrschenden Buchen erfolgt, gibt der Streufall eine bestandesbezogene Information wieder. Es werden Blätter aller Bäume aus allen Kronenbereichen erfasst, insbesondere auch die größeren und schwereren Schattblätter aus den unteren Kronenteilen.

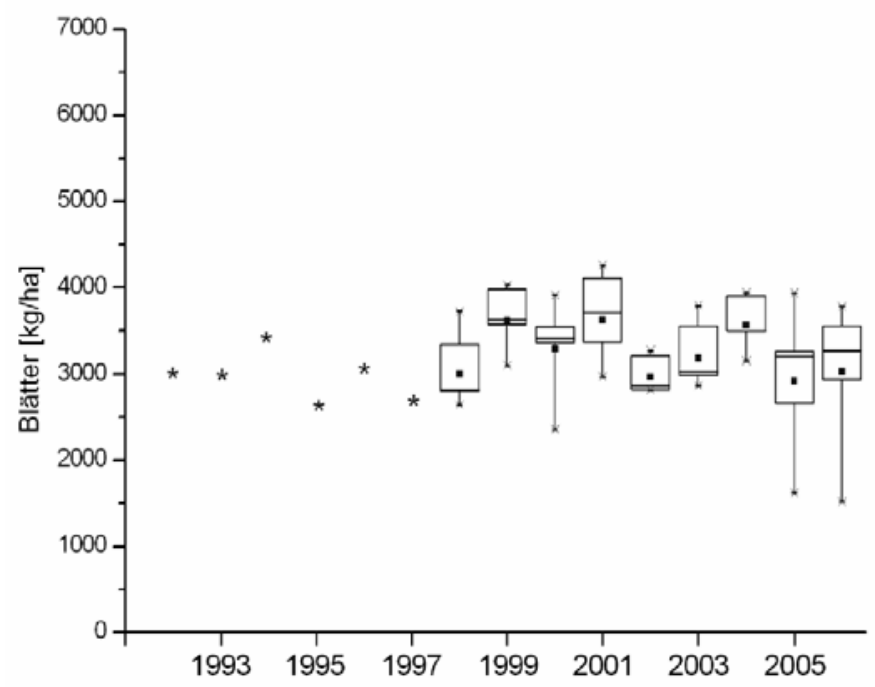

Abbildung 9: Entwicklung der Blattbiomasse auf sieben Level II-Flächen in Hessen 1998 - 2006, dargestellt als Box- und Whisker-Plots (Querstriche in der Box: Median, Quadrate: arithmetisches Mittel). Für die Fläche Zierenberg (Sterne) liegt die Zeitreihe seit 1992 vor.

Im Mittel werfen die sieben Buchenbestände $3.301 \mathrm{~kg} \mathrm{ha}^{-1} \mathrm{a}^{-1}$ Laubstreu ab (s. Abb. 9). Die Variabilität von Jahr zu Jahr ist mit einer Spannweite zwischen $2971 \mathrm{~kg} \mathrm{ha}^{-1} \mathrm{a}^{-1}$ (2002) und $3625 \mathrm{~kg} \mathrm{ha}^{-1} \mathrm{a}^{-1}$ (2001) eher gering. Der Laubabfall des 
Jahres 2003 entspricht einem mittleren Wert, 2004 lag die Gesamtmasse der Blätter sogar über dem Durchschnitt. Dagegen zeigt der Box- und Whisker-Plot des Jahres 2005 mit im Mittel $2920 \mathrm{~kg} \mathrm{ha}^{-1} \mathrm{a}^{-1}$ eine niedrige Blattbiomasse an (vgl. Abschnitt 3.2).

Am Beispiel der Level II-Fläche Zierenberg (s. Abb. 10) wird deutlich, dass in Fruktifikationsjahren die Biomasse der Fruchtkompartimente nicht aus einer jeweils reduzierten Blattmasse resultiert, wie dies aus einer bei Buche biologisch konkurrierenden Bildung von Blatt- und Blütenknospen denkbar wäre.

\section{Zierenberg}

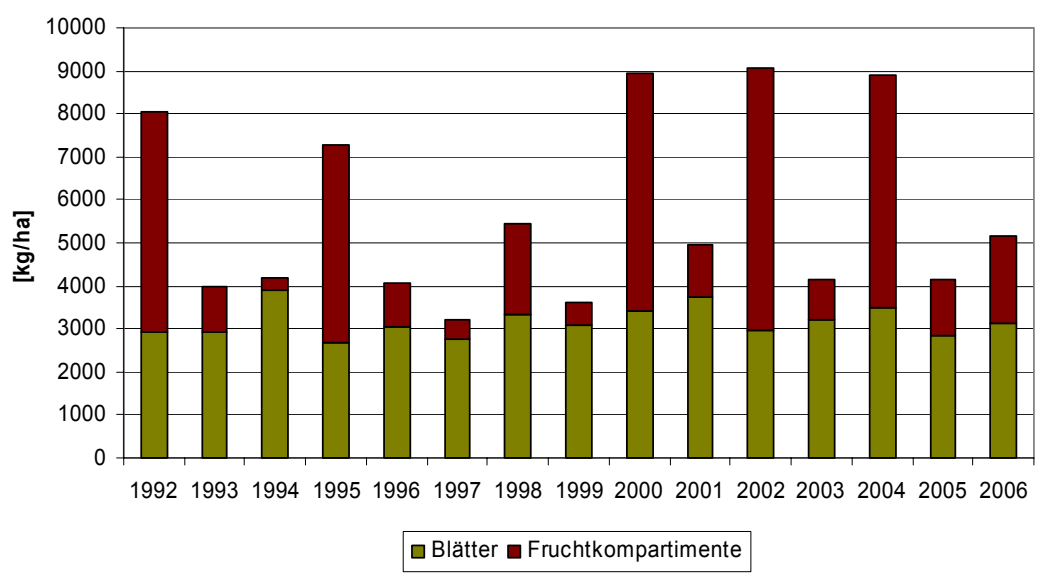

Abbildung 10: Blatt- und Fruchtbiomasse auf der Level II-Fläche Zierenberg 1992 - 2006. Gleicher Befund auf den Vergleichsflächen.

\subsection{Blattvergilbung, Ernährungszustand und biotische Faktoren}

Der grüne Blattfarbstoff, das Chlorophyll, ist Sitz der Kohlenstoff-Assimilation. Seit Beginn der Waldzustands-Übersichtserhebung wird deshalb die Häufigkeit und Intensität der Vergilbung von Nadeln und Blättern erfasst. Vergilbungen an nahezu einem Drittel der beobachteten Bäume traten vorzugsweise in der zweiten Hälfte der 1980er und der ersten Hälfte der 1990er Jahre auf. Seit 1995 ist Vergilbung nur an rd. $5 \%$ der Buchen feststellbar, in den letzten Jahren liegen die Werte oft nur bei 1-2\%. 2004 und 2005 sind die Werte auf sehr niedrigem Niveau leicht erhöht.

Zur Beurteilung der Ernährungssituation der Buche in Hessen und Niedersachsen werden an elf Buchenflächen jährlich Blattanalysen durchgeführt. Die untersuchten Buchenbestände repräsentieren eine breite Amplitude von Standorten. Eine Bewertung der Elementgehalte anhand der Referenzwerte des ARBEITSKREISES STANDORTSKARTIERUNG (2003) ergibt im Mittel der Jahre 1995 - 2005, dass die Stickstoff-Gehalte der Buchenblätter im Bereich hoher Werte 
liegen. Nach 2003 ergibt sich entgegen der Annahme von RENNENBERG (2004), der für 1-jährige Buchenkeimlinge unter kontrollierten Bedingungen eine reduzierte N-Aufnahme bei reduzierter Wasserverfügbarkeit beschreibt, kein Abfall der N-Gehalte in den Buchenblättern. Für die Kalium-, Calcium- und Magnesium-Versorgung werden überwiegend Werte im mittleren bis sehr hohen Bereich festgestellt, lediglich auf versauerten, basenarmen Standorten werden in Blättern Defizite in den Calcium- und Magnesium-Gehalten beobachtet. Die Phosphor-Versorgung der Blätter ist auf der Mehrzahl der Flächen unzureichend. Die Stickstoffquotienten - bewertet nach BURG $(1985,1990)$ zitiert nach BÜTTNER et al. (1993) - belegen, dass aufgrund einer standortsübergreifenden StickstoffEutrophierung vielfach disharmonische Ernährungssituationen vorliegen.

Für eine Bewertung der Resilienzeigenschaften ist darüber hinaus eine quantitative Darstellung biotischer Faktoren wie Insekten und Pilze notwendig. Seit Beginn der Waldzustandserhebung werden Insekten und Pilze an der Buche erfasst, bislang ist deren Einfluss auf die Buchenvitalität in Hessen, Niedersachsen und Sachsen-Anhalt jedoch eher gering. ROSKAMS (2006) führte im europaweiten Level I- und Level II-System ein, für Probebäume die beobachteten biotischen und abiotischen Schäden gegliedert nach dem betroffenen Baumteil, Ausmaß der Symptome und der Schadursache zu dokumentieren.

\section{4 Übersicht Hauptergebnisse}

Tabelle 2: $\quad$ Übersicht der Indikatoren von Resilienzeigenschaften der Buche nach dem warmen, trockenen Jabr 2003. Werte der Jabre 2003 bis 2006 (2007) in Relation zum Mittel der Jabre 1998 - 2002.

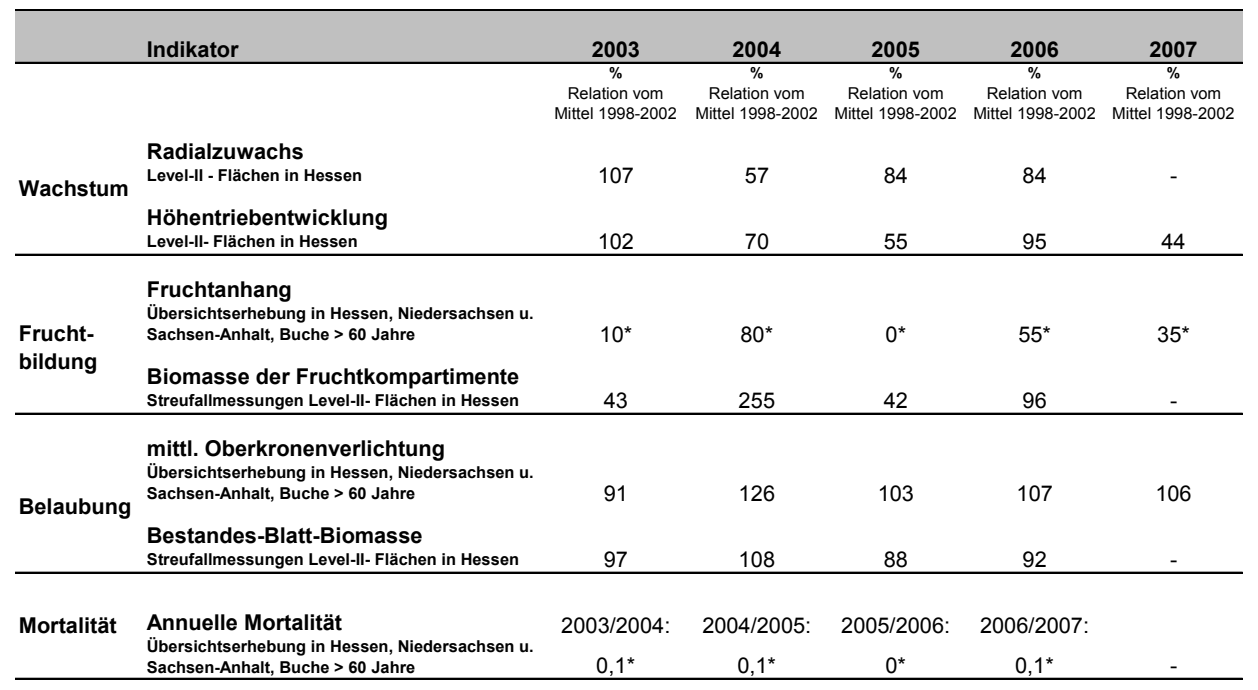

* Prozent der Stichprobenbäume je Jahr (Jahreswerte) 
Tabelle 3: $\quad$ Oberirdische Nettoprimärproduktion (in t Biomasse ba $a^{-1} a^{-1}$ ) im Jabr 2004 im Vergleich zum Mittel der Jahre 1998 bis 2002, sieben Level II-Flächen in Hessen

\begin{tabular}{l|c|cc}
\hline $\begin{array}{l}\text { Bildung oberirdischer } \\
\text { Biomasse }\end{array}$ & Mittel 1998 - 2002 & \multicolumn{2}{|c}{2004} \\
& Biomasse $\mathrm{t} \mathrm{ha}^{-1} \mathrm{a}^{-1}$ & Biomasse $\mathrm{t} \mathrm{ha}^{-1} \mathrm{a}^{-1}$ & $\begin{array}{c}\text { \% vom Mittel } \\
1998-2002\end{array}$ \\
\hline $\begin{array}{l}\text { Derbholz, Äste } \\
\text { Annahme: } 12 \mathrm{~m}^{3} \mathrm{ha}^{-1} \mathrm{a}^{-1}\end{array}$ & 6,7 & 3,3 & 49 \\
Blätter & 3,3 & 3,6 & 108 \\
Fruchtkompartimente & 1,8 & 4,6 & 255 \\
\hline Summe & 11,8 & 11,5 & 97 \\
\hline
\end{tabular}

\section{Bewertung Resilienzeigenschaften, Vitalität und Risiko der Buche}

Die Buche wird von BACKES u. LEUSCHNER (2000) zitiert in RENNENBERG et al. (2004) als vergleichsweise trockenheitssensitive Baumart bewertet, für die zu erwarten ist, dass sie in ihrer physiologischen Konkurrenzkraft durch längere Trockenphasen besonders negativ beeinflusst wird. Wie kann aber das tatsächliche Risiko der Buche gegenüber Jahren mit extremer Witterung zahlenmäßig erfasst und bewertet werden? Dazu wird der außerordentlich trocken-heiße Sommer 2003 als Beispiel herangezogen und Reaktionsmuster der Buche auf Flächen des forstlichen Umweltmonitorings untersucht. Um die Reaktionsmuster zu veranschaulichen, wird auf das Vitalitätsdreieck von GEHRIG (2004) mit seinen Kriterien Langlebigkeit, biologischer Funktion und Stresstoleranz Bezug genommen. Für jedes der Kriterien werden Indikatoren genutzt, die in den Zeitreihen des forstlichen Umweltmonitorings vorliegen. So wird für die „Langlebigkeit“ die annuelle Mortalität von Probebäumen verwendet, für die „biologische Funktion“ insbesondere die Indikatoren Durchmesser- und Höhenzuwachs, Fruktifikation, Belaubung der Oberkrone sowie Blattbiomasse ermittelt. Eine Integration der sektoralen Betrachtung erfolgt mit der Bewertung der Zuordnung von assimiliertem Kohlenstoff in verschiedene Baumkompartimente. Aus dem zeitlichen Ablauf der Reaktionen wird auf Eigenschaften der Stresstoleranz der Buche geschlossen.

Langlebigkeit: Die annuelle Mortalität der Buche liegt im Mittel für Hessen, Niedersachsen und Sachsen-Anhalt (Buche über 60 Jahre) in den Jahren nach 2003 unverändert bei lediglich 0,1 \% (s. Tab. 2). Auf Länderebene betragen die Mortalitätsraten in Einzeljahren bis 0,3\% (Sachsen-Anhalt 2003/2004, Hessen 2004/2005, Niedersachsen 2006/2007). Gegenwärtig ist die Buche in dem für größere Regionen flächenrepräsentativen Level I-Beobachtungsnetz die Hauptbaumart mit der niedrigsten jährlichen Absterberate. Wie die Mortalitätsrate und 
Schäden an Buchen durch mehrfach wiederholte Trockenheitsjahre beeinflusst werden, ist jedoch nicht abzusehen (vgl. RENNENBERG et al. 2004, MANTHEY et al. 2007).

Bereits heute wird auf einzelnen, für Trockenheit besonders disponierten Standorten in extremen Perioden vorzeitiger Blattabfall und das Absterben alter Buchen beobachtet (MEIER u. LEUSCHNER 2007, BREDA et al. 2006). LEUSCHNER u. HERTEL (2003) haben mit zunehmendem Trockenstress eine reduzierte Feinwurzelmasse festgestellt. Dies kann auf trockenheitsdisponierten Standorten negative Auswirkungen auf die Vitalität der Buche haben.

Funktion: Die Höhentriebe erreichen im Jahr 2004 nur $70 \%$ der Trieblängen des Zeitraums 1998 - 2002. Die Wuchsdepression setzt sich auch 2005 deutlich fort. Die nach dem Jahr 2006 erneut sehr niedrige Höhenentwicklung 2007 deutet darauf hin, dass bei einer Häufung warm-trockener Jahre mit einer länger anhaltenden Begrenzung der Höhenwuchsleistung zu rechnen ist. Daraus können sich Folgerungen für Wachstumsverläufe, aber auch für die Konkurrenzkraft der Buche gegenüber Baumarten wie Eiche, Hainbuche oder Linde ergeben. Bei anhaltendem Wasserstress wird ein Zurücksetzen von Buchenkronen und spätere Sekundärkronenbildung beobachtet.

Der Radialzuwachs zeigt 2004, also ein Jahr nach 2003 eine Reduktion auf $57 \%$ des Mittels 1998 - 2002, erholt sich jedoch im Gegensatz zur Entwicklung der Höhentriebe bereits im darauf folgenden Jahr weitgehend. Dieses Ergebnis steht im Einklang mit der Erwartung von FELBERMEIER (1994), dass die Buche auf Trockenstress 1-2 Jahre mit verringertem Jahrringzuwachs reagiert, um danach wieder zum ursprünglichen Wachstumsniveau zurückzufinden.

Der für die Level II-Flächen hergeleitete mittlere Zuwachs an Biomasse für Derbholz und Äste von etwa $12 \mathrm{~m}^{3} \mathrm{ha}^{-1} \mathrm{a}^{-1}$ wird im Jahr 2004 ca. um $50 \%$ reduziert im Vergleich zum Mittel von 1998 bis 2002, erholt sich danach wieder, erreicht jedoch nicht ganz das Ausgangsniveau. Mit diesem Sachverhalt geht eine forstbetrieblich relevante deutliche Verringerung des Holzertrages einher. Dagegen ist 2004 mit 255 \% des Mittels der Jahre 1998 bis 2002 die im Beobachtungszeitraum umfangreichste Mehrproduktion an Früchten, Knospen und Fruchtschalen festzustellen. Bemerkenswert ist, dass die Verlagerung in die Früchte mit einer in etwa gleich bleibenden Biomasse in Blättern einhergeht. Im Vergleich zur Referenzperiode 1998 bis 2002 ist die oberirdische Nettoprimärproduktion 2004 insgesamt nur um etwa $3 \%$ verringert (ohne Berücksichtigung der Wurzelsysteme) (s. Tab. 3).

Stresstoleran₹: Nach dem außerordentlich warm-trockenen, aber in seiner Charakteristik singulären Jahr 2003 ergeben sich für die Buche kein großräumig feststellbares Ansteigen der Mortalität und keine wesentliche Reduktion der gesamten oberirdischen Biomasseproduktion. Im Hinblick auf die Zuordnung des assimilierten Kohlenstoffs ist jedoch eine drastische Verlagerung von Stamm- in Fruchtbiomasse festzustellen. Dies ist gleichzusetzen mit einem völligen Um- 
steuern der Baumstrategie von Raumeroberung und baumindividueller Stabilität hin zur Mast für die Arterhaltung. Deutliche Reduktionen der Höhen- und der Durchmesserentwicklung sind nachweisbar. Damit wird die Produktion von Holz als nachwachsendem Rohstoff mit aller ökologischen und ökonomischen Konsequenz eingeschränkt. Dem steht jedoch die Sicherung der Art durch Fruchtbildung und natürliche Verjüngung gegenüber.

Wichtig für die Bewertung der Stresstoleranz ist es, die Zeitdauer der Reaktionen auf eine Störung zu beschreiben. Einige Indikatoren lassen ein rasches Zurückschwingen in den Normbereich erkennen. So hat die Zunahme der Verlichtung von Oberkronen im Anschluss an das warm-trockene Jahr 2003 in Hessen und Sachsen-Anhalt 2004 ein sprunghaftes Erscheinungsbild. Ab 2005 entsprechen die Verlichtungswerte wieder dem Mittel der Jahre 1998-2002. Die durch die extremen Witterungsereignisse ausgelöste starke Änderung der Oberkronenbelaubung erreicht nach vergleichsweise wenigen Jahren wieder das Niveau der - allerdings gegenüber 1984 erhöhten - Vergleichsjahre. Ähnliches trifft für die Durchmesserentwicklung zu, dagegen zeichnet sich bei den Höhentrieben eine länger wirksame Beeinträchtigung ab. Auch die in Folge einer Reihe von warmen Jahren und guter Stickstoffverfügbarkeit deutlich zunehmende Häufigkeit von Mastjahren bei der Buche ist als Indiz für längerfristig wirksame Veränderungen bauminterner Kreisläufe und Regelkreise zu interpretieren.

Ein Organismus ist nach GEHRIG (2004) vital, wenn er unter den herrschenden Umweltbedingungen die zur Verfügung stehende Energie in dem Dreieck aus Langlebigkeit, biologische Funktion und Stresstoleranz optimal einsetzt. Die Ergebnisse zu diesen Kriterien können nach 2003 nicht als Hinweis auf eine schwerwiegende Einschränkung der Vitalität der Buche gewertet werden. Vielmehr zeigt das deutliche Umsteuern der Kohlenstoff-Allokation der Buche eine aktive Anpassung an geänderte Umweltbedingungen und damit ein hohes Maß an Stresstoleranz. Auf Grund dieser besonderen Anpassungsfähigkeit ist im Einklang mit der Bewertung von LEUSCHNER (1998) und HERTEL et al. (2004) das generelle Bild einer ausgesprochen trockenheitsempfindlichen Baumart Buche zu revidieren. Inwieweit eine unmittelbare Abfolge mehrerer Extremjahre wie $2003 \mathrm{zu}$ Grenzen der Anpassungsfähigkeit der Buche führen wird, bleibt offen.

\section{Literatur}

ARBEITSKREIS STANDORTSKARTIERUNG IN DER ARBEITSGEMEINSCHAFT FORSTEINRICHTUNG (2003): Forstliche Standortaufnahme, IHW-Verlag, $352 \mathrm{~S}$.

BACKes, K. u. LEUSCHNER, C. (2000): Leaf water relations of competitive Fagus sylvatica and Quercus petraea trees during 4 years differing in soil drought. Can. J. Forest Res. 30, 335-346

BMVEL (2004): Die zweite Bundeswaldinventur - BWI2. Zu den Bundeswaldinventur-Erhebungen 2001 bis 2002 und 1986 bis 1988. Bundesministerium für Verbraucherschutz, Ernährung und Landwirtschaft (Hrsg.), 87 S. 
Borchers, K. (1954): Zur Technik der Buchennaturverjüngung in Niedersachsen, Forst- und Holzwirt, 416-421

Breda, N.; Huc, R.; Granier, A. u. Dreyer, E. (2006): Temperate forest trees and stands under severe drought: a review of ecophysiological responses, adaptation processes and long-term consequences. Ann. For. Sci. 63. 625-644

BRumme, R. (1999): Hierarchical control on nitrous emission in forest ecosystems. Global Biochemical Cycles, Vol 13, 1137-1148

Büttner, G.; Hartmann, G. u. Thomas, F. (1993): Vorzeitige Vergilbung und Nährstoffgehalte des Buchenlaubes in Südniedersachsen. Forst u. Holz 48, 627-630

BURG, J. van den (1985): Foliar analysis for determination of tree nutrient status - a compilation of literature data. Rijksinstitut voor Onderzoek in de Bosen Landschapsbouw „De Dorschkamp“, Wageningen, Rapport Nr. 414

BURG, J. van den (1990): Foliar analysis for determination of tree nutrient status - a compilation of literature data. 2. Literature 1985 - 1989 „De Dorschkamp“, Institute for Forestry and Urban Ecology, Wageningen, Rapport Nr. 591

BURSCHEL, P. (1966): Untersuchungen in Buchenmastjahren. Fortsw. Cbl. 85, 204-219

Cropp, R. u. GABriC, A. (2002): Ecosystem adaptation: Do ecosystems maximize resilience? Ecology 83, 2019-2026

Dammann, I.; SChröcK, H.W. u. Herrmann, T. (2000): Ansätze zur integrierten Auswertung von Kronenzustandsdaten im Rahmen des Level-II-Programms. Forstarchiv 71, 59-64

DobBERTIN, M. u. DE VRIES, W. (2008): Interactions between climate change and forest ecosystems. In: FISCHER, R. (Report ed.): Forest ecosystems in a changing environment: Identifying future monitoring and research needs. Cost Workshop Istanbul, Turkey. COST www.cost.esf.org. 8-11

EICHHORN, J. (1995): Stickstoffsättigung und ihre Auswirkungen auf das Buchenwaldökosystem der Fallstudie Zierenberg (Habilitationsschrift), Ber. d. Forsch. Zentrums Waldökosysteme, Reihe A. Göttingen, 186 S.

EichHorn, J.; Szepesi, A.; Ferretti, M.; Durrant, D. u. Roskams, P (1999/updated: 2006): ICP Forests Manual Part II Visual Assessment of Crown Condition, UNECE ICP Forests. $69 \mathrm{~S}$.

EichHORN, J. u. PAar, U. (2008): Klimaänderung und Waldentwicklung in Hessen. Hess. Forstverein Jahrestagung 2006. Mitt. d Hess. Forstvereins. (im Druck)

EICHHORN, J. u. SZEPESI, A. (2008): Tree reactions to environmental changes. In: FISCHER, R. (Report ed.): Forest ecosystems in a changing environment: Identifying future monitoring and research needs. Cost Workshop Istanbul, Turkey. COST www.cost.esf.org. 12-13

Ellenberg, H. (1996): Vegetation Mitteleuropas mit den Alpen. Ulmer Verlag Stuttgart, 1095 S.

Felbermeier, B. (1994): Die klimatische Belastbarkeit der Buche, Forstwissenschaftliches Centralblatt 113, 152-174

Gauger, T.; Anshelm, F.; Schuster, H.; Erisman, J.W.; Vermeulen, A.T.; Draaijers, G.P.J.; BleEker, A. u. NAGel, H.D. (2002): Mapping of ecosystem specific long-term trends in deposition loads and concentrations of air pollutants in Germany and their comparison with Critical Loads and Critical Levels. Part 1: Deposition Loads 1990 - 1999, Final Report 29942 210, Institut für Navigation der Universität Stuttgart und Umweltbundesamt, 207 S.

GEHRIG, M. (2004): Methoden zur Vitalitätsbeurteilung von Bäumen. Vergleichende Untersuchungen mit visuellen, nadelanalytischen und bioelektrischen Verfahren. Diss. ETH Zürich Nr. 15341, $255 \mathrm{~S}$.

GivnisH, T.J. (1988): Adaptation to sun and shade: A whole-plant perspective. Aust. J. Plant Physiol., 15, 63-92

Gruber, F. (2004): Über die Vitalität der Rotbuche (Fagus sylvatica L.) In: Schriftenreihe Dendrologie, Baumpflege und Waldbotanik aus dem Inst. F. Forstbotanik u. Baumphysiologie der Georg-August-Universität Göttingen. 215 S. 
Hertel, D.; Coners, H.; Muhs, A.; SchipkA, F. u. Strobel, J. (2004): Zur Trockenheitsgrenze der Buche in Mittel- und Osteuropa: Eine ökosystemare Transektstudie an Buchen-Altbeständen. Berichte des Landesamtes für Umweltschutz Sachsen-Anhalt, Sonderheft 2, 28-37

Horn, M.; Wernicke, I. u. Ziesing H.-J. (2007): CO 2 Emissionen in Deutschland 2007. Energiewirtschaftliche Tagesfragen. WASO Verlags- und Versicherungsservice GmbH

IPCC (2007): 4. Sachstandsbericht (AR 4) des IPCC (2007) über Klimaänderungen. Synthesebericht. www.ipcc-wg1.ucar.edu, $52 \mathrm{~S}$.

LeuschneR, C. (1998): Mechanismen der Konkurrenzüberlegenheit der Rotbuche. Ber. d. Reinh.Tüxen-Ges. 10, 5-18

Leuschner, C. u. Hertel, D. (2003): Fine root biomass of temperate forests in relation to soil acidity and fertility, climate, age and species. Progress in Botany 64, 405-438

Leuschner, C.; MeIer, I. u. HerTel, D. (2006): On the niche breadth of Fagus sylvatica: soil nutrient status in 50 Central European beech stands on a broad range of bedrock types. Ann. For. Sci 63, $355-368$

MANThey, M.; Leuschner, C. u. HÄRdLE, W. (2007): Buchenwälder und Klimawandel. Natur und Landschaft 82, 441-445

Meesenburg, H.; Meiwes, K.J. u. Rademacher, P. (1995): Long term trends in Deposition and Seepage Output in Northwest German Forest Ecosystems. Water, Air and Soil Pollution 85: 611-616

Meesenburg, H.; Dammann, I.; Evers, J.; Schulze, A.; Rademacher, P.; Mindrup, M.; König, N.; Fortmann, H.; EberL, C. u. Meiwes, K.J. (2002): Forstliches Umweltmonitoring als Entscheidungshilfe für die Forstwirtschaft und Umweltpolitik. Forst und Holz 57, 707-712

MEIER, I.C. u. LEUSCHNER, C. (2007): The belowground drought response of European beech: fine root biomass and production in 14 mature stands across a precipitation gradient. Submitted

Mitscherlich, G. (1978) Wald, Wachstum und Umwelt, 1. Band. J.D. Sauerländer's Verlag. Frankfurt pp. 144

NOAA (2008): www.noaa.gov. National Oceanic and Atmospheric Administration, USA

ОтTо, H.J. (1994): Waldökologie. Ulmer Verlag Stuttgart, $391 \mathrm{~S}$.

PaAr, U.; KirchHoff, A.; Westphal, J. u. Eichorn, J. (2000): Fruktifikation der Buche in Hessen. AFZ-DerWald 25, 1362-1363

RennenberG; H.; Seiler, W.; Matyssek, R.; Gessler, A. u. Kreuzwieser, J. (2004): Die Buche (Fagus sylvatica L.) - ein Waldbaum ohne Zukunft im südlichen Mitteleuropa. Allgem. Forst- u. Jagdzeitung 175, 210-224

ROHMEDER, E. (1967): Beziehungen zwischen Frucht- bzw. Samenerzeugung und Holzerzeugung der Waldbäume. AFZ, 33-39

Roloff, A. (2001): Baumkronen: Verständnis und praktische Bedeutung eines komplexen Naturphänomens. Ulmer Verlag, $164 \mathrm{~S}$.

Roloff, A. (2005): Phänomene der Anpassung und Optimierung bei Bäumen. AFZ-DerWald, 641645

Roskams, P. (2006): Assessments of Damage Causes. In: EICHhorn, J.; Szepesi, A.; Ferretti, M.; Durrant, D. u. Roskams, P. (2006): Manual on Methods and Criteria for harmonized sampling, assessment, monitoring and analysis of the effects of air pollution on forests. ICP Forests. www.icp-forests.org.

Schulze, E.D.; Beck, E. u. MÜller-Hohenstein, K. (2002): Pflanzenökologie. Spektrum Akad. Verlag, $846 \mathrm{~S}$.

SCHWAPpach, A. (1895): Die Samenproduktion der wichtigsten Waldbäume in Preußen. Zeitschr. Forst- und Jagdwesen, 147-174

SChwertfeger, F. (1970): Die Waldkrankheiten. Verlag Paul Parey, Hamburg und Berlin, 509 S. 
Spellmann, H., Sutmöller, J. u. Meesenburg, H. (2007): Risikovorsorge im Zeichen des Klimawandels. Vorläufige Empfehlungen der NW-FVA am Beispiel des Fichtenanbaus. AFZ/Der Wald 62, 1246-1249

Ulrich, B.; Mayer, R. u. Khanna, P.K. (1979): Deposition von Luftverunreinigungen und ihre Auswirkungen in Waldökosystemen im Solling. Schriften Forstl. Fakult. Göttingen. 256 S.

UN/ECE u. EC (2000): Forest Condition in Europe. Results. 2000 Technical Report, Federal Research Centre for Forestry and Forest Products, Geneva and Brussels, 86 S.

WaCHTER, H. (1964): Über die Beziehung zwischen Witterung und Buchenmastjahren. Forstarchiv $35,69-78$

WALKer, B (1995): Conserving Biological Diversity through Ecosystem Resilience. Conservation Biology 9, 747-752

WalKer, B.; Kinzig, A. u. LANGridge, J. (1999): Plant attribute diversity, resilience, and ecosystem function: The nature and significance of dominant and minor species. Ecosystems 2, 95-113

ZIESING, H.-J. (2007): Nach wie vor weltweit steigende $\mathrm{CO}_{2}$-Emissionen. Energiewirtsch. Tagesfragen 57. Jg. Heft 9, 58-68

Ziesing, H.-J. (2008): $\mathrm{CO}_{2}$-Emissionen in Deutschland 2007: Milde Temperaturen und hohe Energiepreise lassen Emission sinken. Energiewirtsch. Tagesfragen 58. Jg. Heft 4, 2-8

Korrespondierender Autor:

Prof. Dr. Johannes Eichhorn

Nordwestdeutsche Forstliche Versuchsanstalt

Grätzelstraße 2

37079 Göttingen

E-Mail: Johannes.Eichhorn@nw-fva.de

URL: www.nw-fva.de

Inge Dammann

Dr. Egbert Schönfelder

Mascha Albrecht

Dr. Uwe Paar

Nordwestdeutsche Forstliche Versuchsanstalt

Dr. Wolfgang Beck

Johann Heinrich von Thünen-Institut (vTI) 


\title{
Der Klimawandel und seine Auswirkungen auf die Buchenwälder in Deutschland
}

\author{
The effects of climate change on beech forests in \\ Germany
}

Johannes Sutmöller, Hermann Spellmann, Caroline Fiebiger und Matthias Albert

\section{Zusammenfassung}

Nach den Klimaprojektionen des Intergovernmental Panel on Climate Change (IPCC) werden für die meisten Regionen Deutschlands bis zum Ende des Jahrhunderts höhere Temperaturen und geringere Niederschläge in der Vegetationszeit erwartet. Außerdem wird mit einem häufigeren Auftreten von Witterungsextremen gerechnet.

Der Schwerpunkt der natürlichen Verbreitung der Buche liegt in Mittel- und Westeuropa mit seinem atlantischen bis subkontinentalen Klima. In Deutschland fehlt die Buche lediglich in den Hochlagen der Mittelgebirge und der Alpen, auf azonalen Standorten sowie auf extrem nassen und trockenen Standorten.

Die klimatischen Verhältnisse im natürlichen Verbreitungsgebiet der Buche reichen für eine Beurteilung ihrer Anpassungsfähigkeit an die sich ändernden Klimabedingungen nicht aus. Hierzu bedarf es weiterer Informationen, 
insbesondere zum Bodenwasserhaushalt, zur Lage und zu ihrem jeweiligen Leistungsvermögen.

In Zukunft werden die Wälder in der Vegetationszeit voraussichtlich vermehrt Trockenstressbedingungen ausgesetzt sein, die ihre Vitalität und Produktivität massiv beeinträchtigen können. Es werden daher wichtige Untersuchungen zum Trockenstressrisiko der Buche vorgestellt. Zudem wird der heute relativ geringe Einfluss klimatischer Faktoren auf die Höhenwuchsleistung der Buche an ihren derzeitigen Anbaustandorten mit Hilfe von Daten der zweiten Bundeswaldinventur verdeutlicht.

Die konkreten Auswirkungen des Klimawandels auf den Wasserhaushalt eines Buchenbestandes werden am Beispiel einer Versuchsfläche im Hessischen Ried vorgestellt. In Zukunft würde sich unter den klimatischen Bedingungen der feuchten Variante des A1B-Klimaszenarios (nach IPCC) eine leichte und unter denen der trockenen Variante eine deutliche Verschlechterung in der Wasserversorgung einstellen.

In der Gesamtschau wird deutlich, dass der derzeitige Kenntnisstand noch keine abschließende Bewertung der Folgen klimatischer Veränderungen auf die Sensitivität, Stabilität und Elastizität von Buchen-Ökosystemen erlaubt. Die Betrachtungen münden daher in einer vorläufigen Abgrenzung der künftigen Anbaustandorte der Buche und in ersten Empfehlungen zur waldbaulichen Steuerung und Entwicklung von Buchenbeständen.

Stichworte: Buche, Klimawandel, Regionalisierung, Trockenstress, Wasserhaushalt

\begin{abstract}
The climate projections from the Intergovernmental Panel on Climate Change (IPCC) predict higher temperatures and lower precipitation during the growing season for most regions in Germany by the end of the century. Furthermore, a more frequent occurrence of weather extremes is expected.

The natural distribution of beech is concentrated in central and western Europe where an Atlantic to sub-continental climate prevails. In Germany, beech is absent at high elevations in the central mountain ranges and the alps, on azonal sites, as well as on extremely wet and dry sites.

The climatic conditions in the natural range of beech are insufficient for an assessment of the adaptability of beech to the changing climatic conditions. Here, additional information is needed, in particular about the soil water budget, the site conditions, and the productivity of beech in each case.

In future, forests will be subject to increasing water stress during the growing season, which may have considerable impact on beech vitality and productivity. Consequently, important investigations into the risk to beech of drought stress are
\end{abstract}


presented. Moreover, the relative low influence of climatic factors on beech height growth at the existing beech sites today is shown clearly with data from the second national forest inventory.

The actual effects of climate change on the water budget of a beech stand are presented for a study site in "Hessisches Ried" (Hesse). In future, under the climatic conditions of the moist, and the dry variant in the A1B-climate scenario (from IPCC) a slight worsening, and a conspicuous worsening in the water availability would result respectively.

Overall, it is evident that current knowledge still is inconclusive about the consequences of climate change in relation to the sensitivity, stability and flexibility of beech ecosystems. These considerations provide a preliminary delimitation of sites of future beech occurrence, and the first recommendations for silvicultural management and development of beech stands.

Keywords: beech, climate change, regionalisation, drought stress, water budget

\section{Einleitung}

Der Klimawandel lässt für Deutschland einen deutlichen Temperaturanstieg bei gleichzeitig veränderter Niederschlagsverteilung erwarten. Die Klimaänderungen werden regional unterschiedlich stark ausgeprägt sein (SPEKAT et al. 2007). Bereits seit Beginn der 1990er Jahre hat sich die Jahresmitteltemperatur landesweit im Vergleich zur Klimanormalperiode 1961 - 1990 um annähernd $1{ }^{\circ} \mathrm{C}$ erhöht. Bis zur Mitte des 21. Jahrhunderts wird nach dem A1B-Szenario die Temperatur im Jahresmittel um $1,6^{\circ} \mathrm{C}$ im Norden Deutschlands und bis zu 2,4 ${ }^{\circ} \mathrm{C}$ in Süddeutschland zunehmen (GERSTENGARBE 2008). Das Umweltbundesamt geht davon aus, dass bis zum Jahr 2100 die mittlere Erwärmung in Deutschland zwischen $2,5^{\circ} \mathrm{C}$ und $3,5^{\circ} \mathrm{C}$ betragen wird (UBA 2006). Die Projektionen der Niederschläge zeigen für Deutschland keinen einheitlichen Trend. Allerdings werden für die Jahreszeiten markante Veränderungen in der Niederschlagsverteilung vorhergesagt. Während die Sommermonate deutlich trockener ausfallen sollen, wird für die Wintermonate eine Zunahme der Niederschläge erwartet. Neben den jahreszeitlichen Veränderungen werden in Zukunft die Häufigkeit und Stärke von Extremereignissen zunehmen. Dies betrifft sowohl Starkregenereignisse und Dürreperioden als auch Sturmereignisse.

Für die in langen Zeiträumen produzierende Forstwirtschaft stellen Ausmaß und Geschwindigkeit des erwarteten Klimawandels eine besondere Herausforderung dar. In Zukunft ist vor allem damit $\mathrm{zu}$ rechnen, dass es vermehrt $\mathrm{zu}$ Trockenstressbedingungen kommt, die die Vitalität, Stabilität und Produktivität der Wälder massiv beeinträchtigen können. Des Weiteren werden die warmen und niederschlagsreicheren Winter das Auftreten von Pilzerkrankungen begünstigen, die trockeneren Sommer die Massenvermehrung von Schadinsekten fördern und 
der frühere Beginn der Vegetationsperiode das Spätfrostrisiko erhöhen. Außerdem wird sich das Artenspektrum verändern, weil sich die Konkurrenzverhältnisse zwischen den Arten ebenso verschieben werden wie ihre Verbreitungsgrenzen. Zudem werden die waldbaulichen Bewirtschaftungskonzepte an die veränderten ökologischen Rahmenbedingungen anpasst werden müssen. Dies wird nicht ohne Folgen für den Holzmarkt und die Ertragssituation der Forstbetriebe bleiben (SPELLMANN et al. 2007).

Angesichts dieser tief greifenden Veränderungen bedarf es langfristig ausgerichteter Anpassungsstrategien, um die Risiken zu verteilen bzw. zu begrenzen. Die wichtigste forstbetriebliche Weichenstellung ist diesbezüglich die Baumartenwahl. Es stellt sich die Frage nach den Zukunftsaussichten unserer Hauptwirtschaftsbaumarten Buche, Eiche, Fichte und Kiefer. Ihre heutigen Flächenanteile und Anbauschwerpunkte sind ein Spiegelbild der Standorte und Regionalklimate, aber auch der sich im Laufe der Zeit ändernden eigentümerspezifischen und gesellschaftlichen Ansprüche an den Wald. In den letzten 20 Jahren wurde mit Einführung des naturnahen Waldbaus im Staatswald und dessen Förderung im Privatwald der Laubwaldvermehrung Vorrang eingeräumt und insbesondere die Buche verstärkt angebaut. Ob diese Entwicklung angesichts der erwarteten Klimaänderungen anhalten wird, hängt vom Anpassungspotenzial der Buche ab, das seit einiger Zeit kontrovers diskutiert wird (u. a. LEUSCHNER et al. 2001, SCHRAMEL u. RENNENBERG 2002, RENNENBERG et al. 2004, V. LÜPKE 2004, AMMER et al. 2005, BRÈDA et al. 2006, CZAJKOWSKI 2006, KÖLLING et al. 2005, KÖLLING u. ZIMMERMANN 2007, SPELLMANN et al. 2007, BOLTE et al. 2007, MANTHEY et al. 2007).

Mit diesem Beitrag wird der Versuch unternommen, wesentliche Gesichtspunkte der laufenden Diskussion aufzugreifen und zusammenzuführen.

\section{Klimatische Ansprüche der Buche}

\subsection{Verbreitungsgebiet der Buche}

Der Schwerpunkt der natürlichen Verbreitung der Buche (Fagus sylvatica L.) liegt in Mittel- und Westeuropa mit seinem atlantischen bis subkontinentalen Klima (s. Abb. 1). In Süd- und Südosteuropa ist sie auf die montane Stufe beschränkt, wobei sie in der Mitte und im Süden der iberischen Halbinsel ebenso fehlt wie im südlichen Griechenland. Im Osten reicht ihr Areal vom westlichen und südlichen Polen, über die Westukraine und Rumänien bis nach Bulgarien. Ausgeklammert bleiben die Tieflagen in Zentral- und Ostpolen sowie die ungarische und rumänische Tiefebene. Die Nordgrenze der Buche verläuft von Südengland entlang der Südküste Norwegens und reicht bis in den Süden Schwedens (RÖHRIG u. BARTSCH 1992). 


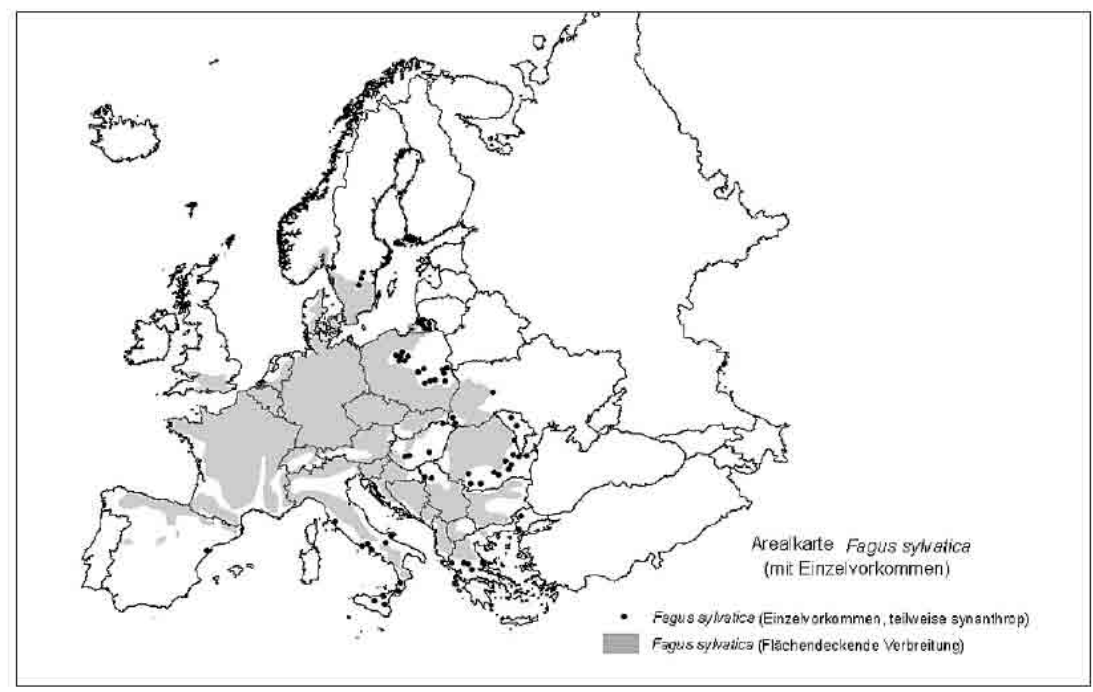

Abbildung 1: Arealkarte von Fagus sylvatica in Europa mit Einzelvorkommen (Punkte) (Institut für Geobotanik der Martin Luther Universität Halle-Wittenberg, Arbeitsgruppe Chronologie und Biogeographie der Gefäßpflanzen 2006; aus CZAJKOWSKI 2006)

In Deutschland ist die Buche fast flächendeckend vertreten. Ausgenommen sind lediglich die Hochlagen der Mittelgebirge und der Alpen, azonale Standorte (Auen, Brücher, Moore), Standorte mit hoch anstehendem Grundwasser, ausgeprägte Stauwasserstandorte und zu trockene Standorte wie Sandpodsole sowie extrem flachgründige Böden (vgl. OTTO 1994, ElLENBERG 1996). Der Hauptanteil der heutigen Buchenstandorte (ca. 75 \%) befindet sich in einer Höhenlage von 200 bis $800 \mathrm{~m}$ (kolline bis mittelmontane Höhenstufe) (BMVEL 2004). Im Nordharz wächst die Buche bis in eine Höhe von etwa $600 \mathrm{~m}$ ü. NN, im südlichen Harz bis $800 \mathrm{~m}$ ü. NN. Im Schwarzwald reicht sie von 150 bis $1400 \mathrm{~m}$ ü. NN, im Bayerischen Wald bis $1300 \mathrm{~m}$ ü. NN und in den Nordalpen von 300 bis $1700 \mathrm{~m}$ ü. NN (OTTO 1994, FELBERMEIER 1993).

Angesichts des erwarteten Klimawandels sind die klimatischen Verhältnisse im natürlichen Verbreitungsgebiet der Buche von besonderem Interesse, um ihr heutiges klimatisches Anpassungsvermögen einschätzen zu können. Buchenwälder existieren in Mitteleuropa bei Jahresniederschlägen zwischen 460 bis $>2000 \mathrm{~mm}$ (LEUSCHNER 1998). Die Verbreitung wird vor allem durch die Bodenwasserverfügbarkeit begrenzt (RENNENBERG et al. 2004). Ist diese gering, so wird die Buche durch verschiedene Eichenarten ersetzt, im feuchten Bereich treten an ihre Stelle die Arten der Hartholzaue (ELLENBERG 1996). Im nordöstlichen Mitteleuropa spricht vieles dafür, dass als weitere begrenzende Faktoren die thermische Kontinentalität mit heißen Sommern (Juli-Mitteltemperatur $>19^{\circ} \mathrm{C}$ ) und kalten, langen Wintern $\left(>141\right.$ Tage mit $<0{ }^{\circ} \mathrm{C}$ Mitteltemperatur und einer Januar-Mitteltemperatur $\left.<-3^{\circ} \mathrm{C}\right)$ sowie die kurzen Wachstumsperioden $\left(<217\right.$ Tage $\left.\geq 7{ }^{\circ} \mathrm{C}\right)$ hinzu- 
kommen (CZAJKOWSKI 2006). Darüber hinaus sind auch noch zeitlich diskret auftretende Extremeinflüsse (Hitze, Dürre, Frost, Überflutungen) zu beachten (CZAJKOWSKI et al. 2006, RENNENBERG et al. 2004).

Derartige klimatische Informationen allein reichen allerdings nicht aus, um die Anpassungsfähigkeit der Buche oder anderer Baumarten an klimatische Veränderungen zu bewerten und Entscheidungshilfen für die Baumartenwahl zu geben. Dies gilt auch für die derzeit viel diskutierten Klimahüllen, bei denen das natürliche Vorkommen von Baumarten in Abhängigkeit von aktuellen und künftig erwarteten Jahresmitteltemperaturen und Jahresniederschlagssummen dargestellt wird (KÖLLING u. ZIMMERMANN 2007). Dieser statische Ansatz greift zu kurz, weil die natürlichen Verbreitungsgrenzen oft ungewiss sind und die künstlichen Verbreitungsgrenzen ebenso vernachlässigt werden wie die Extremstandorte. Außerdem bleiben die Anpassungspotenziale verschiedener Herkünfte wie auch die Konkurrenzeinflüsse anderer Baumarten unberücksichtigt. Des Weiteren wird nicht von den aussagefähigeren klimatischen Verhältnissen in der Vegetationszeit ausgegangen und es findet der lokale Bodenwasserhaushalt keine Beachtung (KÄTZEL 2008, BOLTE et al. 2008).

\subsection{Trockenstressrisiko der Buche}

In Zukunft ist damit zu rechnen, dass es vermehrt zu Trockenstressbedingungen kommt, die die Vitalität und Produktivität der Wälder massiv beeinträchtigen können. Hierzu wird auch die bereits zu beobachtende Verlängerung der Vegetationszeiten beitragen (MENZEL 1997).

Wie bei allen anderen Baumarten hängt bei der Buche die Effizienz der Wasserversorgung eines jeden Baumes von seinen Möglichkeiten ab, Wasser aufzunehmen und in Zeiten hoher Transpiration Wasserdefizite zu vermeiden. Relevant für die Beurteilung von Trockenstressgefährdung ist die tatsächliche Bodenfeuchte. Da es je nach Standort und Baumart spezifische Grenzen für Trockenstress gibt, ist eine pauschale Bewertung des Gefährdungspotentials nicht möglich.

Die Wasseraufnahme der Bäume wird von der räumlichen Ausdehnung des Wurzelwerks und der Feinwurzeldichte bestimmt. Bei abnehmendem Bodenwassergehalt wird die tatsächliche Verdunstung zunehmend eingeschränkt. Der Wasserpotenzialgradient zwischen Boden und Atmosphäre verändert sich, die zunehmende Saugspannung mindert die pflanzeninterne Wasserverfügbarkeit, das Sprosswasserpotenzial nimmt ab (RENNENBERG et al. 2004, BRÈDA et al. 2006). Als Abwehrreaktion schließt die Buche ihre Stomata. Hierdurch wird zum einen die Photosynthese eingeschränkt, zum anderen aber auch die Transpiration. Letztere beeinflusst entscheidend den Wasserpotenzialgradienten zwischen Boden und Wurzel und damit die Wasseraufnahme. Sind die Potenzialunterschiede zu groß, reißt die Wasserversorgung ab und es kommt zum Absterben der Feinwurzeln (Leuschner u. Hertel 2003). Bei hohen Temperaturen und geschlossenen Sto- 
mata fehlt zudem die Verdunstungskälte, so dass die Blätter direkt geschädigt werden. Es kommt zu einem vorzeitigen Blattabfall und in extremen Trockenperioden zu einem teilweisen oder vollständigen Absterben alter Bäume (BRÈDA et al 2006). Eine besondere Gefährdung der Buche ist zu erwarten, wenn sich Dürreereignisse wie im Jahre 2003 in aufeinander folgenden Jahren wiederholen sollten, weil dann die Bäume weder ihr Feinwurzelwerk noch ihre Belaubung ausreichend regenerieren können (vgl. RENNENBERG et al. 2004, BRÈDA et al. 2006). Nach solchen trockenen, heißen Sommern kommen im Folgejahr als weitere Stressfaktoren die vermehrte Blüte und Fruktifikation der Buche hinzu, die in einem erheblichen Umfang Reservestoffe binden.

MANTHEY et al. (2007) gehen davon aus, dass Buchenbestände auf sommertrockenen Standorten in Gebieten mit heute weniger als $650 \mathrm{~mm}$ Jahresniederschlag oder auf flachgründigen Böden in Zukunft bei trockenerem Klima nachhaltig geschädigt werden können. Dies kann, muss aber nicht so sein, weil es im Zusammenhang mit den Klimaänderungen zahlreiche Unwägbarkeiten gibt, der örtliche Bodenwasserhaushalt eine entscheidende Rolle spielt und bei der Buche eine große genetische Variation besteht. Ergebnisse aus Freiland- und Laboruntersuchungen haben gezeigt, dass es deutliche Unterschiede in der Trockenstresstoleranz sowohl entlang eines West-Ost-Klimagradienten zwischen deutschen und polnischen Herkünften (CZAJKOWSKI u. Bolte 2006) als auch zwischen Lokalpopulationen in Baden-Württemberg (SCHRAML u. RENNENBERG 2002) gibt.

\subsection{Höhenbonität der Buche in Abhängigkeit von Klimafaktoren}

Die standortsbezogene Baumartenwahl erfolgt in der Forstpraxis auf ökologischer und ökonomischer Grundlage. Zu den wichtigsten ökonomischen Kriterien zählt die Ertragskraft der Baumarten, die im Allgemeinen durch absolute Höhenbonitäten (Höhe im Alter 100) angegeben wird. Im Gegensatz zur Fichte, bei der das Bestandeswachstum vor allem von den Niederschlags- und Wärmeverhältnissen beeinflusst wird (MITSCHERLICH 1950a), hat das Großklima im Allgemeinen keinen signifikanten Einfluss auf die Höhenwuchsleistung der Buche (MITSCHERLICH 1950b). Die Variablen Nährstoffangebot, Durchwurzelbarkeit des Bodens und Luftfeuchtigkeit erklären zu mehr als $90 \%$ die Zusammenhänge zwischen der Leistung der Buche und den Standortsfaktoren in Baden-Württemberg (RÖHE 1985)

Dieser Sachverhalt lässt sich anhand der Ergebnisse der zweiten Bundeswaldinventur (BWI 2) in den Bundesländern Niedersachsen, Hessen, Sachsen-Anhalt und Schleswig-Holstein weitgehend bestätigen (BMVEL 2004). Für diese Bundesländer liegen die Temperaturwerte und die berechnete Klimatische Wasserbilanz (KWB $=$ Differenz zwischen Niederschlag und potenzieller Evapotranspiration) auf einem 200 x 200 m Raster vor. Die potenzielle Evapotranspiration beschreibt die Wassermenge, die eine Pflanze bei uneingeschränkter Wasserverfügung ver- 
dunsten kann. Der Bilanzierungszeitraum umfasst die Vegetationszeiten (VZ) der Klimanormalperiode 1961 - 1990.

Die Bonitierung der Buchenvorkommen erfolgte an jedem BWI-Punkt. Die zweidimensionale Dichteverteilung (Temperatur und KWB) wurde mit der Buchengrundfläche pro Hektar gewichtet, so dass vollbestockte Reinbestände mit größerem Gewicht in die Dichteverteilung eingehen als einzelne Buchen in Mischbeständen. In Abbildung 2 werden die $95 \%$-Quantile der Dichteverteilung für drei Bonitätsstufen und das 95\%-Quantil aller von der BWI in den vier Bundesländern erfassten Waldstandorte verglichen.

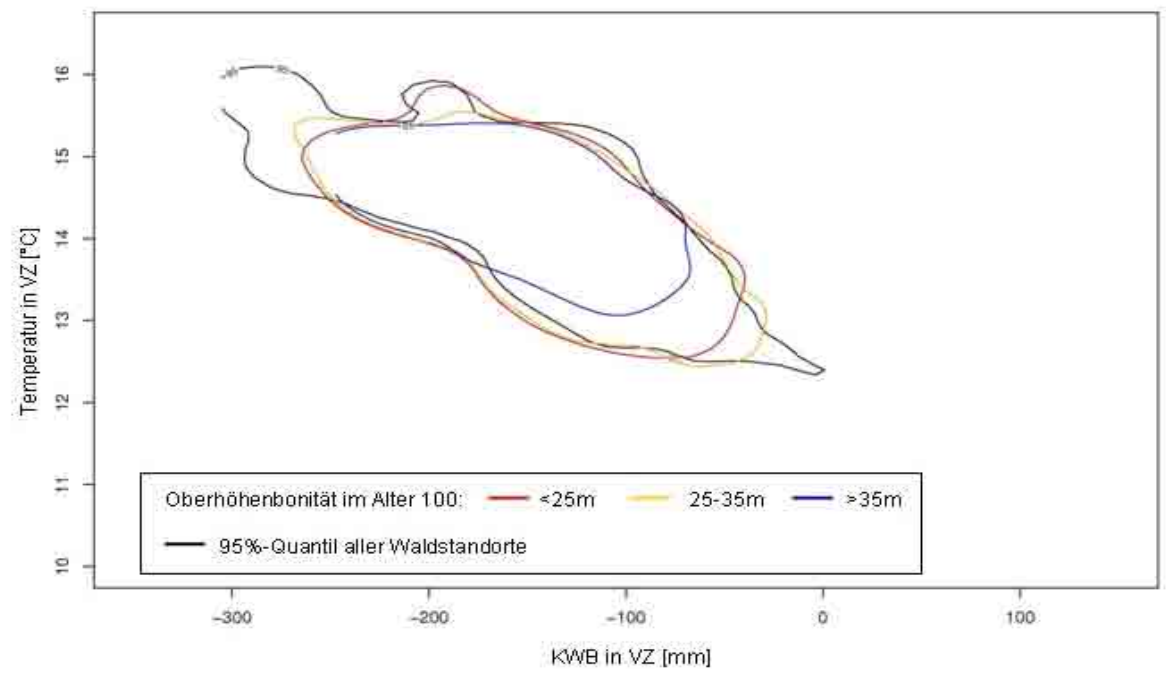

Abbildung 2: Die $95 \%$-Quantile für 3 Bonitätsstufen der BWI-Buchen-Stichprobenpunkte in Niedersachsen, Hessen, Sachsen-Anhalt und Schleswig-Holstein (KWB = Klimatische Wasserbilanz; $V Z=V$ egetationszeit)

Das Buchenvorkommen in den vier untersuchten Bundesländern deckt unter den Klimaverhältnissen der Normalperiode weite Teile des dortigen Standortsspektrums ab. Nur auf den extrem warm-trockenen (hohes Wasserbilanzdefizit) und den äußerst kühl-nassen Standorten (geringes Defizit in der KWB) ist die Buche in Nordwestdeutschland nicht vertreten. Auffallend ist, dass einige Buchenvorkommen unter den bisherigen klimatischen Verhältnissen in der Vegetationszeit trotz größerer Wasserbilanzdefizite beachtliche Leistungen zeigen. Geht man von einem negativen Effekt auf das Höhenwachstum bei Mangel an pflanzenverfügbarem Wasser aus, heißt dies im Umkehrschluss, dass die Buchenbestände auf relativ gut wasserversorgten Standorten stocken müssen. Die Trockenheitsgrenze von ca. $275 \mathrm{~mm}$ KWB-Defizit in der Vegetationszeit hat keinen Einfluss auf die Höhenbonität der Buche, weil andere Standortsfaktoren kompensatorisch wirken. Auch die Wärmegrenze von rd. $15,5^{\circ} \mathrm{C}$ in der Vegetationszeit hat keinen 
Einfluss auf die Höhenleistung, jedoch wird der positive Effekt des Temperaturanstiegs im Bereich von ca. $12,5^{\circ} \mathrm{C}$ bis $13,5^{\circ} \mathrm{C}$ sichtbar.

\section{Klimaszenarien}

\subsection{Einleitung}

Der vierte Sachstandsbericht des Intergovernmental Panel on Climate Change (IPCC) fasst den aktuellen Kenntnisstand zur globalen Erwärmung zusammen (IPCC 2007). Er dokumentiert die Ergebnisse verschiedener Arbeitsgruppen, die sich mit den wissenschaftlichen Grundlagen, möglichen Anpassungsstrategien und der Verminderung des Klimawandels beschäftigten. Der Arbeitsbereich „Wissenschaftliche Grundlagen“ kommt zu dem Ergebnis, dass der globale Klimawandel fast ausschließlich auf menschliches Handeln zurückzuführen ist. Daraus ergeben sich für die Zukunft verschiedene Projektionen der Klimaentwicklung. Die Szenarien basieren auf unterschiedlichen Annahmen zur Bevölkerungsentwicklung, zum Wirtschaftswachstum und zur Umweltentwicklung. Aus der Kombination dieser Faktoren resultieren Emissionsszenarien, die einen mehr oder minder starken Anstieg der Globaltemperatur im 21. Jahrhundert erwarten lassen (s. Abb. 3).

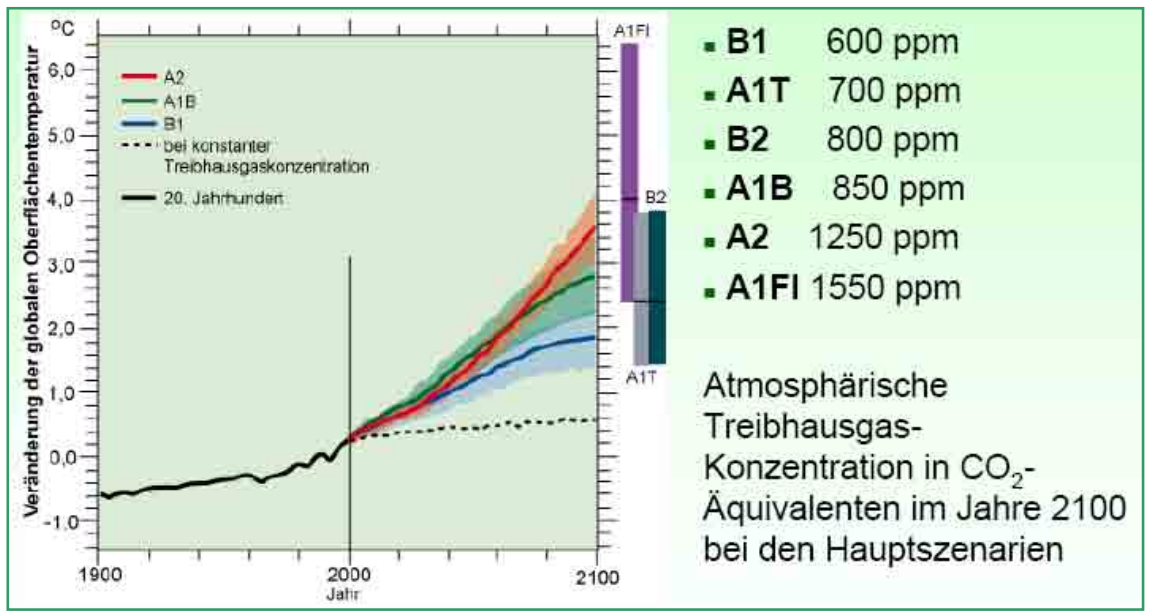

Abbildung 3: Erwarteter Temperaturanstieg bei verschiedenen Klimaszenarien (IPCC 2007) 
Die Bandbreite der erwarteten globalen Erwärmung reicht von knapp $2{ }^{\circ} \mathrm{C}$ beim moderaten B1-Szenario bis über $6{ }^{\circ} \mathrm{C}$ beim A1Fl-Szenario. Auffällig ist, dass etwa bis zur Mitte des 21. Jahrhunderts die Schwankungsbreite der prognostizierten Temperaturerhöhung zwischen allen Szenarien nur bei rund $1{ }^{\circ} \mathrm{C}$ liegt und eine Erwärmung der Globaltemperatur um ca. $2{ }^{\circ} \mathrm{C}$ erwartet wird. Erst in der zweiten Hälfte des 21. Jahrhunderts nehmen die Unterschiede zwischen den Szenarien deutlich zu (s. Abb. 3). Erklären lässt sich diese Entwicklung durch die lange Verweilzeit der Treibhausgase in der Atmosphäre, die z. B. beim Kohlendioxid $\left(\mathrm{CO}_{2}\right)$ über einhundert Jahre beträgt. Dies hat zur Folge, dass bereits emittierte Treibhausgase auch zur Mitte des 21. Jahrhunderts größtenteils noch nicht abgebaut sein werden und damit zur weiteren Erwärmung beitragen. Die Verhältnisse am Ende des 21. Jahrhunderts werden entscheidend davon abhängen, ob die Treibhausgasemissionen in den nächsten Jahrzehnten signifikant reduziert werden können, um den Temperaturanstieg langfristig auf maximal $2{ }^{\circ} \mathrm{C}$ zu stabilisieren.

Zur Berechnung möglicher Klimaentwicklungen werden globale Klimamodelle eingesetzt. Ihre maximale räumliche Auflösung beträgt 200 x 200 Kilometer. Für viele Fragestellungen ist diese räumliche Auflösung unzureichend, so dass für bestimmte Gebiete Regionalmodelle entwickelt wurden. Bei ihnen wird zwischen dynamischen und statistischen Modellen unterschieden. Dynamische Verfahren nutzen die Ergebnisse der Globalmodelle als Eingangsdaten und simulieren die Klimaentwicklung auf einem höher aufgelösten regionalen Gitternetz. Als Beispiel sei hier das Regionalmodell REMO des Hamburger Max-Planck-Instituts für Meteorologie genannt (JACOB et al. 2007, JACOB 2001). Statistische Verfahren gehen davon aus, dass die globalen Klimamodelle die großräumigen Zirkulationsmuster (Wetterlagen) treffend beschreiben. Über die Kausalkette von sich verändernden Häufigkeitsverteilungen der Großwetterlagen werden lokale Auswirkungen auf meteorologischen Größen wie Niederschlag und Temperatur abgeleitet.

Das statistische Modell WETTREG (SPEKAT et al. 2007) liefert regionale Klimaprojektionen für Deutschland. Es nutzt die Ergebnisse, die mit dem Globalmodell ECHAM5/MPI-OM des Hamburger Max-Planck-Institut für Meteorologie erzeugt wurden (ROECKNER et al. 2003, ROECKNER et al. 2004). Über statistische Beziehungen werden für Stationen des Deutschen Wetterdienstes (DWD) Zeitreihen berechnet. Die mit WETTREG generierten Zeitreihen liegen für den Zeitraum von 1960 bis 2100 in verschiedenen Varianten der Szenarien B1, A1B und A2 vor. Damit das Modell für Szenariozwecke eingesetzt werden kann, wurde es für den Zeitraum von 1961 bis 1990 anhand von gemessenen Beobachtungsdaten validiert. Anschließend wurden die Stationsdaten der WETTREG-Simulationen mithilfe von Regionalisierungsverfahren auf ein Flächenraster interpoliert, um flächenhafte bzw. regionalspezifische Aussagen zum zukünftigen Klima treffen zu können. 


\subsection{Klimawandel in Deutschland}

Das IDP-Modul der Potsdamer Firma CEC (Climate \& Environment Consulting Potsdam GmbH) kombiniert verschiedene Verfahren zur Regionalisierung der WETTREG-Stationsdaten auf ein Modellgitter (KREIENKAMP u. SPEKAT 2006). Während höhenunabhängige Wetterelemente, wie z. B. die Sonnenscheindauer mit dem abstandsgewichteten IDW-Verfahren (Inverse-Distance-Weighting) in die Fläche interpoliert werden, wird zur Regionalisierung der Lufttemperatur und der Niederschlagswerte die IDW-Methode mit einem höhenabhängigen Regressionsverfahren kombiniert.

Nachfolgend werden die wesentlichen klimatischen Veränderungen, die bei dem höheren Emissionsszenario A1B und dem moderaten Szenario B1 für Deutschland bis zum Ende des 21. Jahrhunderts erwartet werden, kurz vorgestellt. Der Vergleich der jeweiligen Szenarien wird für die Periode 2071 - 2100 und der Klimanormalperiode 1961 - 1990 durchgeführt. Die Ergebnisse wurden der Veröffentlichung von SPEKAT et al. 2007 entnommen.

Die Jahresmitteltemperatur für Deutschland betrug in der Klimanormalperiode $8,2{ }^{\circ} \mathrm{C}$. Thermisch bevorzugt waren die großen Flusstäler in West- und Südwestdeutschland (Rhein, Main etc.) sowie die tieferen Lagen in Westdeutschland, Teile Brandenburgs und Sachsen-Anhalts. Hier erreichte die Jahresmitteltemperatur Werte von über $9{ }^{\circ} \mathrm{C}$, während unterdurchschnittliche Temperaturwerte in den höheren Lagen der Gebirgsregionen auftraten. Bis zum Ende des 21. Jahrhunderts wird für ganz Deutschland eine Temperaturzunahme von $1,8^{\circ} \mathrm{C}$ (B1) bis $2,3{ }^{\circ} \mathrm{C}$ (A1B) erwartet. Regional sind die Unterschiede jedoch deutlich ausgeprägter. Eine vergleichsweise geringe Erwärmung wird für die Küstengebiete von Nord- und Ostsee, die Mittelgebirge und Ostbayern prognostiziert, überdurchschnittlich hoch wird die Temperaturzunahme im gesamten Norden Deutschlands und dem Alpenvorland mit annähernd $3{ }^{\circ} \mathrm{C}$ ausfallen. Im Jahresgang fällt die Temperaturzunahme sehr unterschiedlich aus. Die Wintermonate werden bis zu $3,5^{\circ} \mathrm{C}$ (z. T. bis $4{ }^{\circ} \mathrm{C}$ ) wärmer, das Frühjahr erfährt dagegen nur eine Erhöhung der Temperatur von unter $1{ }^{\circ} \mathrm{C}$ und die Sommer- und Herbstmonate folgen dem Jahrestrend mit einem Anstieg von $2-2,5^{\circ} \mathrm{C}$.

Beim Niederschlag zeigen die WETTREG-Simulationen einen klaren Trend. Während bei den Sommerniederschlägen mit teilweise erheblichen Rückgängen gerechnet werden muss, wird für die Wintermonate infolge der Zunahme von Westwindwetterlagen ein deutlicher Niederschlagsanstieg erwartet. Gebiete mit derzeit geringen Niederschlägen von teilweise unter $200 \mathrm{~mm}$ in den Sommermonaten sind im Osten und Nordosten Deutschlands sowie am Niederrhein zu finden. Der Süden, insbesondere die Alpen mit dem Alpenvorland, zeichnet sich durch überdurchschnittlich hohe Sommerniederschläge in der Periode 1961 - 1990 aus. Im Winter sind die regionalen Unterschiede in den Niederschlagsverhältnissen, abgesehen von den Mittelgebirgsregionen und den Alpen, relativ gering. Für die Periode 2071-2100 wird je nach Szenario im Mittel mit einer Zunahme der 
Winterniederschläge von $20-30 \%$ gerechnet. Allerdings wird insbesondere die Westhälfte Deutschlands von der Niederschlagszunahme profitieren (teilweise bis zu 80 \% höhere Niederschläge). Weite Bereiche Ostdeutschlands und der Alpenraum erfahren hingegen nach den Klimasimulationen keine wesentliche Erhöhung der Winterniederschläge. In den Sommermonaten ist dagegen landesweit mit einer Abnahme der Niederschläge von rund $20 \%$ zu rechnen, wobei für Teile Ostdeutschlands (Vorpommern) nach dem höheren A1B-Szenario ein Niederschlagsrückgang von bis zu $40 \%$ prognostiziert wird. Dies bedeutet, dass in denjenigen Regionen Deutschlands, die bereits jetzt unter einem erhöhten Niederschlagsdefizit in den Sommermonaten zu leiden haben, in Zukunft die Gefahr von Trockenstress- und Wassermangelsituationen erheblich zunehmen wird.

\subsection{Pflanzenverfügbares Wasser}

Klimatische Wasserbilanz (KWB) und nutzbare Feldkapazität (nFK) sind wesentliche klimatologische und hydrologische Kenngrößen, um die Menge des pflanzenverfügbaren Wassers charakterisieren zu können. Die nutzbare Feldkapazität entspricht der Wassermenge, die ein Boden in unveränderter Lagerung maximal gegen die Schwerkraft zurückhalten kann, abzüglich des Totwassers.

Auf Grundlage der WETTREG-Simulationsergebnisse wurden für den Zuständigkeitsbereich der Nordwestdeutschen Forstlichen Versuchsanstalt mit den Ländern Hessen, Niedersachsen, Sachsen-Anhalt und Schleswig-Holstein die Stationsdaten auf ein Modellgitter mit einer horizontalen räumlichen Auflösung von $200 \times 200$ Metern interpoliert. Mit Hilfe des Wasserhaushaltsmodells WaSiM/ETH (SCHULLA 1997) wurde die potenzielle Evapotranspiration nach PENMAN-MONTEITH (MONTEITH 1965) ebenfalls für das $200 \mathrm{~m}$-Raster berechnet und die klimatische Wasserbilanz für alle vier Bundesländer ermittelt. Die Auswertung wurde für das Szenario A1B (erhöhtes Emissionsszenario) durchgeführt, da dieses Szenario derzeit als die wahrscheinlichste Projektion der Klimaentwicklung angesehen wird (GERSTENGARBE 2008). Für jedes Klimaszenario wurden 10 Realisierungen (Varianten) pro Dekade erzeugt (SPEKAT et al. 2007). Um die Unsicherheit der Szenarienbandbreite bei der Berechnung der KWB zu berücksichtigen und der forstlichen Praxis einen Handlungskorridor aufzuzeigen, wurde jeweils die trockene und feuchte Variante des A1B-Szenarios ausgewertet.

In Tabelle 1 ist für den Zeitraum 1961 - 1990 und für die Dekaden 2041 - 2050 sowie 2091 - 2100 die jeweils modellierte KWB getrennt nach den vier Bundesländern aufgeführt. Die KWB wurde für die Vegetationsperiode bestimmt, die für die Entwicklung der Pflanzen maßgeblich ist. Die Vegetationsperiode wurde nach der Methode von MENZEL (1997) getrennt für die Bilanzierungsperioden berechnet, so dass die in Zukunft erwartete Verlängerung der Vegetationszeit bei der KWB berücksichtigt werden konnte. 
Bereits in der Klimanormalperiode wird im Flächenmittel aller untersuchten Bundesländer ein mehr oder weniger hohes Wasserbilanzdefizit für die Vegetationszeit ausgewiesen. Die niedrigsten Defizite in der Klimanormalperiode weist das Land Hessen mit rund $120 \mathrm{~mm}$ auf (feuchte Variante A1B). Demgegenüber ist das mittlere Wasserbilanzdefizit von Sachsen-Anhalt bereits heute mit über $200 \mathrm{~mm}$ deutlich größer als in den anderen Bundesländern. In Zukunft werden sich die Wasserbilanzdefizite in allen vier Bundesländern weiter erhöhen und bis zum Ende des Jahrhunderts zwischen $250 \mathrm{~mm}$ und fast $400 \mathrm{~mm}$ (Sachsen-Anhalt, trockene Variante A1B) erreichen. Die Ursache sind trockene Sommer bei gleichzeitig zunehmender Verdunstungsleistung der Pflanzen infolge höherer Temperaturen.

Tabelle 1: $\quad$ Simulierte Klimatische Wasserbilan₹ $[\mathrm{mm}]$ für die feuchte und trockene Variante des A1B-Szenarios für die V egetationsperiode (März/ April bis Oktober)

\begin{tabular}{l|cc|cc|cr}
\hline $\begin{array}{l}\text { Klimatische } \\
\text { Wasserbilanz }\end{array}$ & \multicolumn{2}{|c|}{$\mathbf{1 9 6 1 - \mathbf { 1 9 9 0 }}$} & \multicolumn{2}{c|}{$\mathbf{2 0 4 1 - 2 0 5 0}$} & \multicolumn{2}{c}{$\mathbf{2 0 9 1 - 2 1 0 0}$} \\
\hline Hessen & feucht & trocken & feucht & trocken & feucht & trocken \\
Niedersachsen & -118 & -137 & -182 & -273 & -245 & -303 \\
Sachsen-Anhalt & -121 & -165 & -165 & -255 & -294 & -303 \\
Schleswig-Holstein & -139 & -246 & -286 & -353 & -369 & -395 \\
\hline
\end{tabular}

Ob der Bodenwasserspeicher das Wasserdefizit künftig ggf. ausgleichen kann, soll am Beispiel von Niedersachsen gezeigt werden (s. Abb. 4). Dazu wurde für die drei Untersuchungsperioden die nutzbare Feldkapazität zur KWB addiert. Die nutzbare Feldkapazität wurde aus der Bodenübersichtskarte im Maßstab 1:50.000 (BÜK50) abgeleitet. Am Beispiel der feuchten und trockenen Variante des A1B-Szenarios wird sichtbar, dass bereits heute in einigen Regionen Niedersachsens das Bodenwasser nicht mehr ausreicht, um eine uneingeschränkte Verdunstung der Pflanzen zu ermöglichen. Im Landesmittel beträgt das Defizit bei der trockenen Variante für die Vegetationszeit rund $30 \mathrm{~mm}$. Bei der feuchten Variante wird im Landesmittel noch ein leichter Überschuss berechnet. Das Defizit der KWB schwankt ohne Berücksichtung des nutzbaren Bodenwassers zwischen 120 und $165 \mathrm{~mm}$ (s. Tab. 1). Das Defizit ist allerdings nicht flächendeckend über das Land gleich verteilt. Besonders die Sandböden im Osten Niedersachsens weisen für die Periode 1961 1990 gebietsweise ein Wasserdefizit von über $100 \mathrm{~mm}$ auf. Westniedersachsen und das südniedersächsische Bergland verfügen hingegen zurzeit noch über einen Wasserüberschuss. Zur Mitte des Jahrhunderts wird das Wasserdefizit unter Berücksichtigung der nutzbaren Feldkapazität je nach Variante des A1B-Szenarios 
deutlich zunehmen und im Flächenmittel auf $30 \mathrm{~mm}$ (feuchte Variante) bis $120 \mathrm{~mm}$ (trockene Variante) steigen.

feuchte Variante A1B

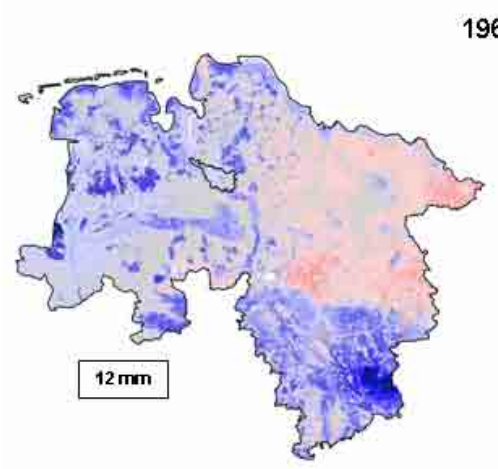

$2041-2050$

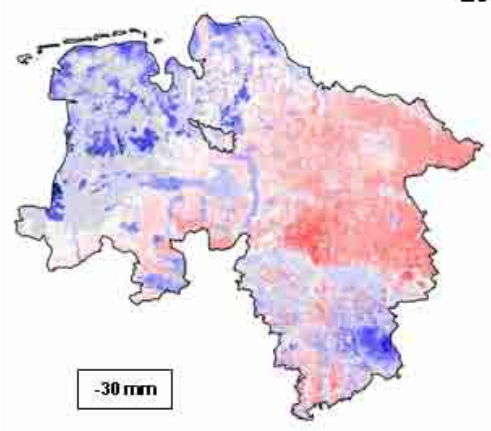

$2091-2100$
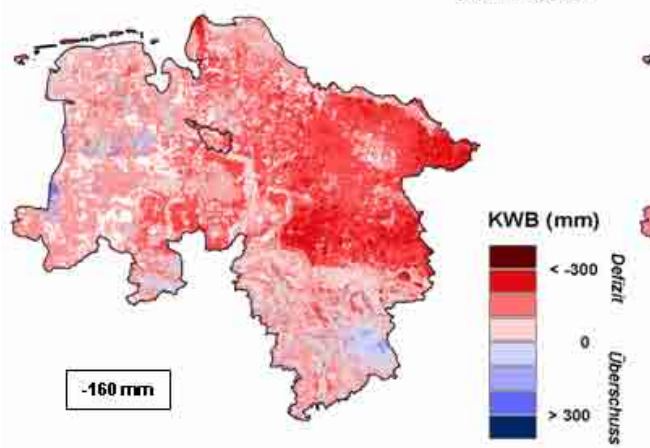

trockene Variante A1B
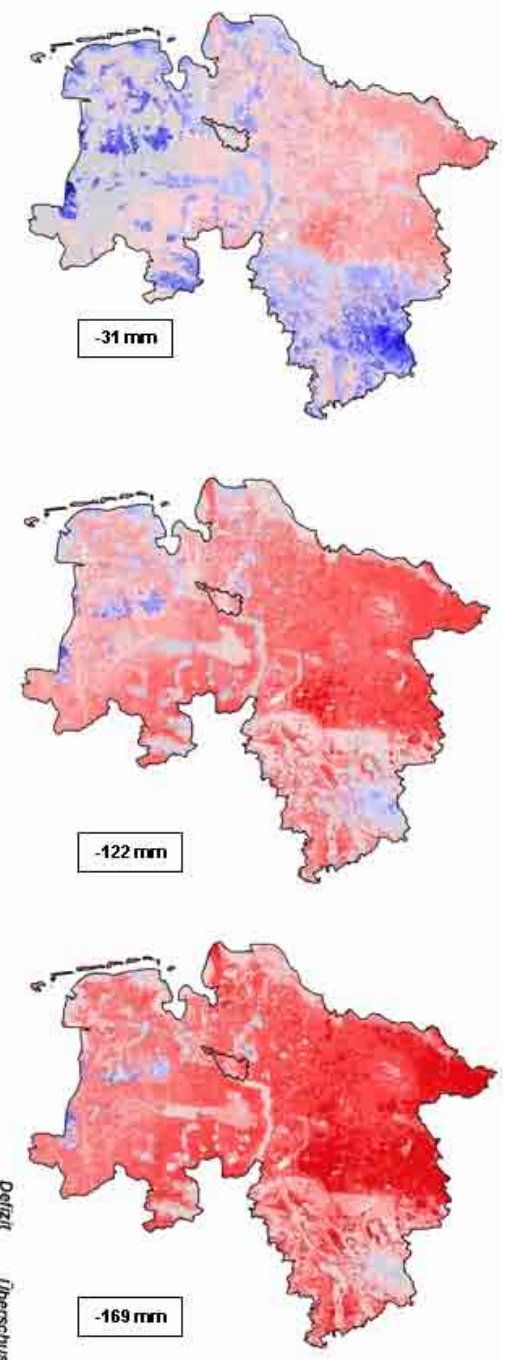

Abbildung 4: Periodische Veränderungen in der Wasserversorgung in Niedersachsen - Summe aus Klimatischer Wasserbilanz. (KWB) und nutz̧arer Feldkapazität (BÜK50) für die Vegetationszeit 
Weite Bereiche des niedersächsischen Tieflandes werden dann ein mehr oder minder hohes Defizit in der klimatischen Wasserbilanz aufweisen, und nur Standorte mit hohen Bodenwasservorräten im Westen des Landes und die höheren Mittelgebirgslagen sind noch durch einen leichten Wasserüberschuss gekennzeichnet. In der Dekade 2091 bis 2100 ist dann fast flächendeckend, mit Ausnahme der Hochlagen des Harzes, mit einem teilweise über $200 \mathrm{~mm}$ reichenden hohen Wasserdefizit in der Vegetationszeit zu rechnen. Im Flächenmittel beträgt das Wasserbilanzdefizit dann bei beiden Varianten rund $170 \mathrm{~mm}$.

In den anderen untersuchten Bundesländern ist mit einer vergleichbaren Erhöhung des Wasserbilanzdefizits während der Vegetationszeit zu rechnen (s. auch Tab. 1). Insbesondere in Sachsen-Anhalt und den angrenzenden ostdeutschen Bundesländern wird sich die Wasserversorgung der Pflanzen auf sandigen Böden mit geringer Wasserhaltefähigkeit deutlich verschlechtern.

\section{Folgen des Klimawandels für die Buche}

\subsection{Auswirkungen auf die Wasserversorgung - Beispiel Hessisches Ried}

Angaben zur klimatischen Wasserbilanz erlauben eine erste Einschätzung des Wasserangebotes für bestimmte Räume. Abgesicherte Erkenntnisse über das pflanzenverfügbare Wasser an einem bestimmten Standort lassen sich nur über Messungen zum Bodenwasserhaushalt und die Erfassung der örtlich wirksamen klimatischen Faktoren gewinnen. Um die Auswirkungen der erwarteten Klimaänderungen auf die Wasserversorgung eines Standortes beurteilen zu können, ist der Einsatz eines Wasserhaushaltsmodells notwendig, das die wesentlichen Komponenten des hydrologischen Prozessgeschehens abbildet. Am Beispiel einer Standortsimulation mit dem Wasserhaushaltsmodell WaSiM/ETH (SCHULLA 1997) werden die Folgen des Klimawandels auf einen Buchenbestand im Hessischen Ried untersucht.

Das Hessische Ried ist durch sehr geringe Reliefunterschiede und einen natürlicherweise geringen Grundwasserflurabstand charakterisiert. Bedingt durch großflächige Grundwasserabsenkungen entwickelten sich die ursprünglich stark grundwasserbeeinflussten Standorte aus tiefgründigem, sandigem Substrat zu trockenen Standorten. Auf vielen Flächen treten in unterschiedlichen Tiefen tonige Stauschichten auf. Mit rund $640 \mathrm{~mm}$ Jahresniederschlag und einer Jahresmitteltemperatur von $9,5^{\circ} \mathrm{C}$ gehört das Hessische Ried bereits heute zu den trockensten und wärmsten Regionen in Hessen.

Im Rahmen der Waldökosystemstudie Hessen wurden im Jahr 1998 im Hessischen Ried drei Intensivmessflächen in einem Kiefern-, Eichen- und Buchenbestand eingerichtet (EICHHORN 1992). Für die Standorte wurden bereits ab 1974 in einem Abstand von rund fünf Jahren waldwachstumskundliche Daten erhoben. 
Der Buchenbestand wies im Jahr 1998 ein mittleres Alter von 98 Jahren auf, die mittlere Bestandeshöhe betrug $27 \mathrm{~m}$ und der mittlere Brusthöhendurchmesser (BHD) $32 \mathrm{~cm}$. Der Buchenbestand war voll geschlossen und es gab am Boden keine Tendenz zur Vergrasung.

Die klimatische Wasserbilanz weist für die Klimanormalperiode 1961 - 1990 für den Buchenstandort in der Vegetationszeit ein mittleres Wasserdefizit von $275 \mathrm{~mm}$ auf (s. Tab. 2). Bei einer reinen Grasvegetation würde sich das Defizit auf knapp $165 \mathrm{~mm}$ reduzieren. Dies liegt deutlich über dem Flächenmittel von Hessen mit rund 120 mm (vgl. Tab. 1). Bis zum Jahr 2050 wird unter Berücksichtigung der Bestandesentwicklung das Defizit in der klimatischen Wasserbilanz des Buchenstandortes je nach Variante des A1B-Szenarios auf 315 bis $460 \mathrm{~mm}$ zunehmen (Gras: 200-320 mm Defizit). Die Bestandesentwicklung bis zum Jahr 2050 wurde mit dem Waldwachstumssimulator WaldPlaner (HANSEN 2006, NAGEL et al. 2006) fortgeschrieben. Dabei wurde die Annahme getroffen, dass sich der Buchenbestand ohne forstliche Eingriffe weiter entwickelt und ein Abgang nur durch natürliche Mortalität erfolgt.

Auf der Buchenfläche betrug die Verdunstung während der Vegetationszeit im Mittel der Referenzperiode 1961 - 1990 rund 75\% (ETR = $500 \mathrm{~mm}$ ) der aufgrund der klimatischen Verhältnisse maximal möglichen Verdunstungsleistung von $650 \mathrm{~mm}$ (ETP). Das Ergebnis der Wasserhaushaltssimulation zur realen Verdunstung liegt über den Werten, die von MüLLER (2003) für einen Buchenstandort im nordostdeutschen Tiefland gemessen wurden. Die überdurchschnittlich hohe Verdunstungsleistung der Buchenfläche im Hessischen Ried ist auf die hohe Bestandesdichte, die dort höheren Temperaturen und die im Vergleich zu Nordostdeutschland längere Vegetationszeit zurückzuführen.

Bei der feuchten Variante des A1B-Szenarios verdunsten in der Dekade 2041 2050 bei vergleichbarem Niederschlagsangebot von $380 \mathrm{~mm}$ ebenfalls rund $75 \%$ der maximal möglichen Verdunstungsmenge. Allerdings erhöht sich infolge der Temperaturzunahme und der verlängerten Vegetationsperiode die reale Verdunstung (ETR) auf $540 \mathrm{~mm}$. Dabei vergrößert sich das Wasserbilanzdefizit (Niederschlag minus realer Verdunstung) auf $160 \mathrm{~mm}$. Das Defizit wird durch das pflanzenverfügbare Bodenwasser kompensiert.

Bei der trockenen Variante des A1B-Szenarios fallen im Mittel der Jahre 2041 2050 rund $320 \mathrm{~mm}$ und damit knapp $20 \%$ weniger Niederschlag im Vergleich zur feuchten Variante und zur Referenzperiode. Ebenso wie bei der feuchten Variante findet keine Grundwasserneubildung statt (s. Tab. 2). Auffällig ist die stark eingeschränkte reale Verdunstungsleistung der Buchenfläche im Vergleich zur feuchten Variante. Im Mittel beträgt die reale Verdunstung $450 \mathrm{~mm}$ und liegt damit um $90 \mathrm{~mm}$ unter der Verdunstungsrate der feuchten A1B-Variante (s. Tab. 2). Rund zwei Drittel der Differenz werden durch die verminderte Niederschlagsmenge während der Vegetationszeit verursacht. Aber auch die eingeschränkte Auffüllung des Bodenspeichers während des Winterhalbjahres hat zur Folge, dass zu Beginn 
der Vegetationsperiode nicht genügend pflanzenverfügbares Wasser vorhanden ist, so dass die Verdunstungsleistung der Buchenfläche frühzeitig eingeschränkt wird. Die relative Verdunstung (ETR/ETP-Verhältnis) während der Vegetationszeit ist mit im Mittel rund 0,58 sehr niedrig und deutet darauf hin, dass unter trockenen A1B-Klimabedingungen die Buchenfläche im Hessischen Ried zukünftig einem hohen Trockenstressrisiko ausgesetzt sein würde.

Tabelle 2: $\quad$ Wasserbilanz für die Vegetationsperiode (März/ April bis Oktober) für den Buchenstandort im Hessischen Ried (alle Angaben in mm; ETR/ETP [-])

\begin{tabular}{l|cc|cc|cc}
\hline & \multicolumn{2}{|c|}{$\begin{array}{c}\text { Klimanormalperiode } \\
\text { 1961-1990 }\end{array}$} & \multicolumn{2}{c|}{ feuchte Variante } & \multicolumn{2}{c}{$\begin{array}{c}\text { trockene Variante } \\
\text { A1B, 2041 - 2050 }\end{array}$} \\
& Gras & Buche & Gras & Buche & Gras & Buche \\
\hline Niederschlag & 375 & 375 & 380 & 380 & 320 & 320 \\
ETR & 390 & 500 & 425 & 540 & 400 & 450 \\
Interzeption & 25 & 85 & 45 & 115 & 40 & 110 \\
Sickerwasser & 80 & 20 & 80 & 0 & 45 & 0 \\
\hline Wasserbilanz & -95 & -145 & -125 & -160 & -125 & -130 \\
\hline ETP & 540 & 650 & 580 & 695 & 640 & 780 \\
\hline ETR/ETP & 0.72 & 0.77 & 0.73 & 0.78 & 0.63 & 0.58 \\
\hline KWB & -165 & -275 & -200 & -315 & -320 & -460 \\
\hline
\end{tabular}

ETR = reale Verdunstung; ETP = potenzielle Verdunstung; KWB = klimatische Wasserbilanz; Wasserbilanz $=$ Niederschlag $-($ ETR + Sickerwasser $)$

Der Vergleich der KWB mit der Bilanz der Wasserhaushaltsmodellierung verdeutlicht, dass bereits heute die Buchenfläche im Hessischen Ried während der Vegetationszeit ein Wasserdefizit aufweist, das durch das pflanzenverfügbare Bodenwasser kompensiert werden muss. Die Buche reagiert mit einer eingeschränkten Verdunstungsleistung auf das Wasserdefizit. In Zukunft würde sich ohne den negativen Effekt der Grundwasserabsenkungen unter den klimatischen Bedingungen der feuchten Variante des A1B-Szenarios eine leichte und unter denen der trockenen Variante eine deutliche Verschlechterung in der Wasserversorgung der Buchenfläche im Hessischen Ried einstellen.

Im Hessischen Ried stellt die Trockenheit ein wesentliches Risiko für die Bestandesstabilität dar. Hohe Wasserbilanzdefizite während der Vegetationszeit können nur bedingt durch das pflanzenverfügbare Bodenwasser ausgeglichen 
werden. Auch in Zukunft muss von einem erhöhten Trockenstressrisiko für den Buchenbestand im Hessischen Ried ausgegangen werden. Hierbei liefert die Wasserhaushaltssimulation wertvolle Erkenntnisse, die eine im Vergleich zur KWB bessere Risikoabschätzung in der Wasserversorgung des Buchenbestandes unter heutigen und zukünftigen Klimabedingungen erlaubt.

\subsection{Auswirkungen auf die Anbauschwerpunkte}

Der Klimawandel wird sich vielfältig auf die Wälder in Deutschland auswirken. Zahlreiche Arbeiten der letzten Jahre belegen, dass sich die forstliche Forschung der Thematik angenommen hat (u. a. THOMASIUS 1991, LINDNER et al. 2002, RENNENBERG et al. 2004, v. LÜPKE 2004, WAGNER 2004, AMMER et al. 2005, BOlte 2005, BrÈDA et al. 2006, WOHLGEMUtH et al. 2006, SPELLMANN et al. 2007, BOlte u. IBISCH 2007, KÖLLING u. ZimMERMANN 2007, MANTHEY et al. 2007, KÄTZEL 2008).

Die Folgen des Klimawandels für die Buche werden unterschiedlich beurteilt. VON LÜPKE (2004) stuft unter Abwägung zahlreicher Kriterien die Buche neben Weißtanne und Fichte als relativ schlecht angepasste Baumart ein. Er empfiehlt, wo möglich, mit Mischungen zu arbeiten, um die Risiken zu verteilen und die Elastizität der Bestände nach Störungen zu erhöhen. Für RENNENBERG et al. (2004) ist die Buche eine trockenstress- und überflutungssensitive Baumart, deren einseitige Bevorzugung im Zuge des Waldumbaus der letzten 20 Jahre mit einem hohen Risikopotenzial für die Zukunft verbunden ist. Auch er empfiehlt, die Buche zur Risikoverminderung mit anderen Baumarten zu mischen, die entweder gegenüber Trockenheit oder gegenüber Staunässe toleranter sind. Im Gegensatz zu diesen Auffassungen stehen die Ansichten von AMMER et al. (2005) oder KÖLLING et al. (2005, 2007). Sie gehen davon aus, dass der erwartete Klimawandel dazu führen wird, dass die Buche zur Hauptbaumart der mitteleuropäischen Wälder wird.

Bei Abwägung der in diesem Beitrag zusammengetragenen Fakten lässt sich festhalten, dass die Buche ein großes Anpassungspotenzial hat, das ihr auch in Zukunft einen bedeutenden Flächenanteil an der Waldfläche in Deutschland sichern wird. Es werden jedoch nicht immer die gleichen Standorte sein, auf denen sie heute als führende Baumart stockt. Die Buche wird sich im Zuge des Klimawandels sowohl neue Bereiche erschließen als auch Gebiete mit extremen Standortbedingungen oder hohem Gefährdungspotenzial verlieren.

Die höheren Lagen (montane Höhenstufe) der Mittelgebirge und der Alpen werden mit zunehmender Erwärmung und Verlängerung der Vegetationszeit als potenzielle Buchenstandorte in Frage kommen. Hier wird die Buche aber auch in Zukunft mit der Fichte konkurrieren, da deren Wasserbedarf dort trotz abnehmender Sommerniederschläge in der Regel gedeckt sein wird. Demgegenüber werden Flächen mit einer schlechten Wasserversorgung zunehmend als potenzielle Anbaustandorte ausfallen, weil die Buche relativ trockenstressempfindlich ist. Dies 
betrifft Bereiche des ost- und nordostdeutschen Tieflandes, die bereits heute selten mit Buche bestockt sind, aber auch Gebiete im norddeutschen Tiefland (z. B. Lüneburger Heide) und möglicherweise in den tieferen Lagen Südwestdeutschlands (z. B. Rhein-Main-Gebiet, Oberrheingraben). Als gefährdete Standorte kommen die wechselfeuchten und wechseltrockenen Standorte hinzu, auf denen schon heute in bzw. nach Trockenperioden vermehrt Buchen in Altbeständen ausfallen bzw. die eine geringe Vitalität mit späterem Befall durch den Buchenprachtkäfer zeigen (s. Abb. 5) sowie die Mittelgebirgslagen mit hohen Niederschlagsmengen und anhaltend hoher Luftfeuchtigkeit in milden Wintern, wo die BuchenKomplexkrankheit unter Beteiligung der Buchenwollschildlaus und des Pilzes Neonectria coccinea ein großes Gefährdungspotenzial darstellt (vgl. AREND et al. 2006, NiESAR 2007, PETERCORD et al. 2007).

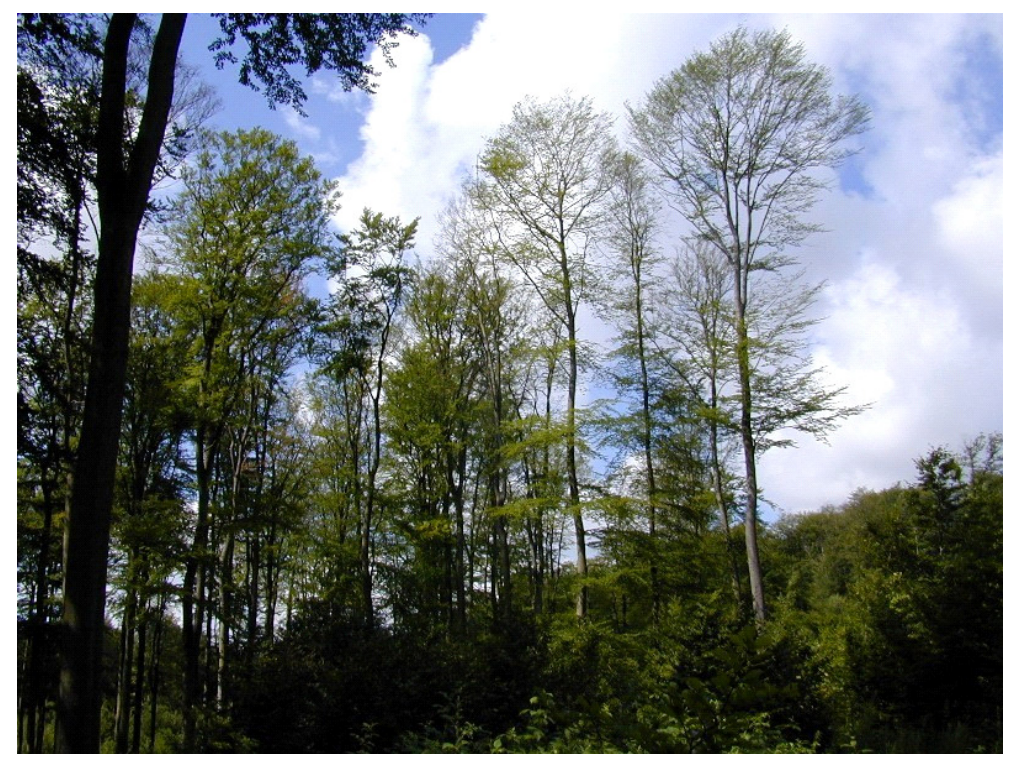

Abbildung 5: Buchenvitalitätsschwäche im NFA Reinhausen

Allgemein ist zu berücksichtigen, dass das Spätfrostrisiko für nicht überschirmte Buchenverjüngungen zunehmen wird, weil viele phänologische Beobachtungen (MENZEL 1997) belegen, dass seit Ende des letzten Jahrhunderts der Vegetationsbeginn bei vielen Pflanzenarten 1 bis 2 Wochen früher gegenüber der Klimanormalperiode eingesetzt hat. Die Klimaprojektionen lassen für die Zukunft einen noch früheren Beginn der Vegetationszeit erwarten, wobei nicht mit einem Rückgang des Spätfrostrisikos gerechnet wird. 


\section{$5 \quad$ Fazit}

Der erwartete Klimawandel wird maßgeblich durch das menschliche Handeln verursacht (IPCC 2007). Die weitere Entwicklung bis zum Ende des 21. Jahrhunderts wird davon abhängen, inwieweit die Treibhausgasemissionen in den nächsten Jahrzehnten signifikant reduziert werden können. Ein Anstieg der Globaltemperatur um $2{ }^{\circ} \mathrm{C}$ bis zum Jahr 2050 erscheint allerdings kaum noch abwendbar. Für die in langen Zeiträumen produzierende Forstwirtschaft stellt der Klimawandel daher eine besondere Herausforderung dar.

Die Klimaprojektionen lassen auch für Deutschland einen deutlichen Temperaturanstieg erwarten. Gleichzeitig werden die Sommermonate trockener als bisher, und in den Wintern ist mit einer Zunahme der Niederschläge zu rechnen. Das verminderte Niederschlagsangebot in der Vegetationszeit wird sich negativ auf die Wasserversorgung der Pflanzen auswirken. Trockenstresstolerantere Baumarten werden für die Forstwirtschaft an Bedeutung gewinnen.

Die Buche zählt nicht zu dieser Gruppe von Baumarten. Dennoch kann gegenwärtig nicht abgesichert beurteilt werden, welche Rolle diese Baumart mit ihrem heute ausgesprochen breiten Anbauspektrum in Zukunft spielen wird. Den zusätzlichen Chancen in den höheren Lagen des Berglandes stehen Risiken in den niederschlagsärmeren Regionen mit durchlässigen bzw. flachgründigen Böden gegenüber, die heute noch ihrem Anbaubereich zugeschrieben werden. Hinzu kommen die Standorte mit hoch anstehendem Grundwasser und stauenden Horizonten, die angesichts der erhöhten Winterniederschläge zu einem wachsenden Problem werden können. Auf die besonderen Gefahren einmaliger bzw. sich wiederholender Dürreereignisse selbst auf optimalen Buchenstandorten wurde verwiesen (s. Kap. 2.2).

Die Unsicherheiten in der Einschätzung der Zukunftsaussichten der Buche zeigen, dass die Klimafolgenforschung noch in ihren Anfängen steckt. Der derzeitige Kenntnisstand über die Auswirkungen des Klimawandels und über die Sensitivität, Stabilität bzw. Elastizität von Buchenwald-Ökosystemen muss noch erweitert werden. Hierzu sind Systembewertungen erforderlich, wie KÄTZEL (2008) sie fordert, die zahlreiche Faktoren einbeziehen. Zu ihnen zählen nicht nur ökologische, sondern auch ökonomische Einflussgrößen.

Für den forstlichen Praktiker, der nicht so lange warten will bzw. warten kann, gilt zunächst die allgemeine Empfehlung von BOLTE (2005), der zu einem risikobewussten Waldbau mit der Buche rät. Konkret heißt dies, strenge Beachtung der standörtlichen Verhältnisse (Klima, Boden, Lage), Berücksichtigung der biotischen und abiotischen Risiken, Begründung und Entwicklung von Mischbeständen, gestaffelte Hochdurchforstungen, die den bodenpfleglichen Unter- und Zwischenstand erhalten, Z-Baum-Zahlen, die Verluste berücksichtigen, und differenziert geführte Zielstärkennutzungen, die neben der Dimension und der Qualität auch die Vitalität und Bestandesstellung beachten. 


\section{Literatur}

Ammer, C.; Albrecht, L.; Borchert, H.; Brosinger, F.; Dittmar, C.; Elling, W.; Ewald, J.; Felbermeier, B.; von Gilsa, H.; Huss, J.; Kenk, G.; Kölling, C.; Kohnle, U.; Meyer, P.; Mosandel, R.; Moosmeyer, H.-U.; Palmer, S.; Reif, A.; Rehfuess, K.-E. u. Stimm, B. (2005): Zur Zukunft der Buche (Fagus sylvatica L.) in Mitteleuropa - Kritische Anmerkungen zu einem Beitrag von RENNENBERG et al. (2004). AFJZ, 176, 60-67

Arend, J.-P.; Eisenbarth, E. U. Petercord, R. (2006): Buchenkomplexkrankheit in Luxemburg und Rheinland-Pfalz - Schadsymptome, Ausmaß und Entwicklung der Schäden. Mitteilungen aus der Forschungsanstalt für Waldökologie und Forstwirtschaft Rheinland-Pfalz, Nr. 59, 11-22

BMVEL (BUNDESMINISTERIUM FÜR VERBRAUCHERSCHUTZ, ERNÄHRUNG UND LANDWIRTSCHAFT) (2004): Die zweite Bundeswaldinventur - BWI. Das Wichtigste in Kürze, Bonn, 87 S.

Bolte, A. (2005): Die klimatische Anpassungsfähigkeit der „Mutter des Waldes“. Zur Zukunft der Buche in Mitteleuropa. AFZ/Der Wald, 60, 1077-1078

Bolte, A. U. IBISCH, P.L. (2007): Neun Thesen zu Klimawandel, Waldbau und Waldnaturschutz. AFZ/Der Wald, 62, 572-576

Bolte, A.; CzAjKOwski, T. u. KOMPA, T. (2007): The north-eastern distribution range of European beech - a review. Forestry, 80, 413-428

Bolte, A.; Ibisch, P.; Menzel, A. u. Rothe, A. (2008): Was Klimahüllen uns verschweigen. AFZ/Der Wald, 63, 800-803

Brèda, N.; Huc, R.; Granier, A. u. Dreyer, E. (2006): Temperate forest trees and stands under severe draught: a review of ecophysiological responses, adaptation processes and long-term consequences. Ann. For. Sci., 63, 625-644

Czajkowski, T. (2006): Zur zukünftigen Rolle der Buche (Fagus sylvatica L.) in der natürlichen Vegetation. Diss., Universität Göttingen, 63 S.

CzAjKowski, T. u. Bolte, A. (2006): Unterschiedliche Reaktion deutscher und polnischer Herkünfte der Buche (Fagus sylvatica L.) auf Trockenheit. AFJZ, 117, 30-40

Czajkowski, T.; Kompa, T. U. Bolte, A. (2006): Zur Verbreitungsgrenze der Buche (Fagus sylvatica L.) im nordöstlichen Mitteleuropa. Forstarchiv, 77, 203-216

EichHORN, J. (HrsG.) (1992): 10 Jahre Waldökosystemstudie Hessen: Ergebnisse und Perspektiven. Forschungsber. Hessische Forstliche Versuchsanstalt 15

EllenberG, H. (1996): Vegetation Mitteleuropas mit den Alpen. 5. Auflage, Ulmer Verlag, Stuttgart

FelbermeIer, B. (1993): Der Einfluß von Klimaänderungen auf die Areale von Baumarten. Methodenstudie und regionale Abschätzung für die Rotbuche (Fagus sylvatica L.) in Bayern. Forstliche Forschungsberichte München, 134

Gerstengarbe, F.-W. (2008): Der Klimawandel - Ursachen und Veränderungen. Gartower Gespräche. Eigenverlag

HANSEN, J. (2006): Der WaldPlaner - Ein System zur Entscheidungsunterstützung in einer nachhaltigen Forstwirtschaft. In: DEGENHARDT, A. u. WunN, U. (Hrsg.): Sammlung der Beiträge von der 18. Jahrestagung der Sektion Biometrie und Informatik des DVFFA vom 25. bis 27.09.2006 in Trippstadt. Die Grüne Reihe, 112-119

IPCC (NTergovernmental Panel on Climate Change) (2007): Climate Change 2007 - The Physical Science Basis. Contribution of Working Group 1 to the Fourth Assessment Report of the IPCC

JACOB, D. (2001): A note to the simulation of the Annual and Interannual Variability of the Water Budget over the Baltic Sea Drainage Basin. Meteorology and Atmospheric Physics, Vol. 77, No. 1-4, 61-74.

Jacob, D.; Bärring, L.; Christensen, O.B.; Christensen, J.H.; Hagemann, S.; Hirschi, M.; Kjellström, E.; Lenderink, G.; Rockel, B.; Schär, C.; Seneviratne, S.I.; Somot, S.; van Ulden, A. u. VAN DEN HuRK, B. (2007): An inter-comparison of regional climate models for 
Europe: Design of the experiments and model performance. Climatic Change 81, 31-52. DOI 10.1007/s10584-006-9213-4

KÄTZEL, R. (2008): Klimawandel - Zur genetischen und physiologischen Anpassungsfähigkeit der Waldbaumarten. Archiv f. Forstwesen u. Landschaftsökologie, 42, 9 - 15

Kölling, C.; Walentowski, H. u. Borchert, H. (2005): Die Buche in Mitteleuropa. AFZ/Der Wald, 60, 696-699

Kölling, C. u. Zimmermann, L. (2007): Die Anfälligkeit der Wälder Deutschlands gegenüber dem Klimawandel. Gefahrstoffe - Reinhaltung der Luft, 67, 259-268

KÖlding, C.; ZimmermanN, L. u. Walentowski, H. (2007): Klimawandel: Was geschieht mit Buche und Fichte. AFZ/Der Wald, 62, 584-588

KreienkAmp, F. u. SPEKAT, A. (2006): IDP2006 - Ein Werkzeug zur explorativen Datenanalyse. Teilbericht zum Vorhaben: Ableitung von Transwetterlagen und Entwicklung eines interaktiven Diagnose- und Präsentationstools

Leuschner, C. (1998): Mechanismen der Konkurrenzüberlegenheit der Rotbuche. Ber. Reinh. Tüxen-Ges., 10, 5-18

Leuschner, C.; Backes, K.; Hertel, D.; Schipka, F.; Schmitt, U.; Terborg, O. u. Runge, M. (2001): Drought responses at leaf, stem and fine root levels of competitive Fagus sylvatica L. and Quercus petraea (Matt.) Liebl. Trees in dry and wet years. Forest ecology and Management, 149, $33-46$

Leuschner, C. u. Hertel, D. (2003): Fine root biomass of temperate forests in relation to soil acidity and fertility, climate, age and species. Progress in Botany, 64, 405-438

Lindner, M.; Badeck, F.-W.; Bartelheimer, P.; Bonk, S.; Cramer, W.; Dieter, M.; Döbbeler, H.; DurskÝ, J.; Duschl, C.; Fabrika, M.; Frömdling, D.; Gundermann, E.; Hennig, P.; Hölzer, W.; Lasch, P.; Leischner, B.; Liesebach, M.; Pommerening, A.; РоtT, M.; Pretzsch, H.; Schlott, W.; Scholz, F.; SPellmanN, H.; Suckow, F.; SudA, M. u. WolfF, B. (2002): Integrating Forest Growth Dynamics, Forest Economics and Decision Making to Assess the Sensitivity of the German Forest Sector to Climate Change. Forstwiss. Centralblatt, Volume 121, Supplement 1, 191-208

LÜPKE, B. V. (2004): Risikominderung durch Mischwälder und naturnaher Waldbau: ein Spannungsfeld. Forstarchiv, 75, 43-50

Manthey, M.; Leuschner, C. u. HÄrdTLE, W. (2007): Buchenwälder und Klimawandel. Natur und Landschaft, 82, 441-445

MenZel, A. (1997): Phänologie von Waldbäumen unter sich ändernden Klimabedingungen. Forstliche Forschungsberichte München, 164

Mitscherlich, G. (1950a): Die Bedeutung der Wuchsgebiete für das Bestandeswachstum von Buche, Eiche, Erle und Birke. Forstw. Cbl., 69, 27-51

MitsCherLiCH, G. (1950b): Die Bedeutung der Wuchsgebiete für das Bestandeswachstum von Fichte und Douglasie. Forstw. Cbl., 69, 184-211

MonTEITH, J.L. (1965): Evaporation and environment. Symp. Soc. Exp. Biol. 19: 205-224

MÜLLER, J. (2003): Wasserhaushalt von Kiefern- und Buchenreinbeständen und von Kiefern- und Buchenmischbeständen im nordostdeutschen Tiefland. 10. Lysimetertagung der Bundesanstalt für Alpenländische Landwirtschaft Gumpenstein, Irding/Österreich, BAL Bericht, 105-109

NAGEl, J.; DudA, H. u. Hansen, J. (2006): Forest Simulator BWINPro7. Forst und Holz, 61, 427-429

Niesar, M. (2007): Symptom- und Ursachenanalyse der aktuellen Buchenerkrankungen in höheren Lagen von Nordrhein-Westfalen. Diss. Universität Göttingen, 115 S.

Отто, H.-J. (1994): Waldökologie. Ulmer Verlag, Stuttgart, 391 S.

Petercord, R.; Delb, H. u. SCHRÖTER, H. (2007): Vermeintliche Dürreschäden an Buche entpuppen sich als Käferbefall: Schwere Schäden durch den Buchen-Prachtkäfer in Baden-Württemberg. AFZ/Der Wald, 62, 686-690 
Rennenberg, H.; Seiler, W.; MatysseK, R.; Gessler, A. u. Kreuzwieser, J. (2004): Die Buche (Fagus silvatica L.) - ein Waldbaum ohne Zukunft im südlichen Mitteleuropa? Allg. Forst- u. J.Ztg., 175, 210-224

Roeckner, E.; Baeuml, G.; Bonaventura, L.; Brokopf, R.; Esch, M.; Giorgetta, M.; Hagemann, S.; Kirchner, I.; Kornblueh, L.; Manzini, E.; Rhodin, A.; Schlese, U.; SchulZWEIDA, U. u. TOMPKINS A. (2003): The atmospheric general circulation model ECHAM5 - Part 1: Model Description. MPI-Berichte, Max-Planck-Institut für Meteorologie, Bd. 349

Roeckner, E.; Brokopf, R.; Esch, M.; Giorgetta, M.; Hagemann, S.; Kornblueh, L.; Manzini, E.; SCHlese, U. u. SCHUlzWEIDA, U. (2004): The atmospheric general circulation model ECHAM5 - Part 2: Sensitivity of simulated climate to horizontal and vertical resolution. MPIBerichte, Max-Planck-Institut für Meteorologie, Bd. 354

RÖHE, P. (1985): Untersuchungen über das Wachstum der Buche in Baden-Württemberg. Schriftenr. Landesforstverw. Baden-Württemberg, Nr. 61

RÖHRIG, E. u. BARTSCH, N. (1992): Der Wald als Vegetationsform und seine Bedeutung für den Menschen. Verlag Paul Parey, Hamburg u. Berlin, 350 S.

Schraml, C. u. Rennenberg, H. (2002): Ökotypen der Rotbuche (Fagus sylvatica L.) zeigen unterschiedliche Reaktionen auf Trockenstreß. Forstw. Cbl., 121, 59-72

SCHULLA, J. (1997): Wasserhaushalts-Simulationsmodell WaSiM-ETH, Anwender-Handbuch, Geogr. Inst. ETH Zürich

SPEKAT, A.; ENKE, W. u. KREIENKAMP, F. (2007): Neuentwicklung von regional hoch aufgelösten Wetterlagen für Deutschland und Bereitstellung regionaler Klimaszenarien auf der Basis mit dem Regionalisierungsmodell WETTREG auf der Basis von globalen Klimasimulationen mit ECHAM/MPI-OM T63L31 2010 bis 2100 für die SRES-Szenarios B1, A1B und A2; FuEVorhaben Förderkennzeichen 20441 138, Publikationen des Umweltbundesamtes

Spellmann, H.; Sutmöller, J. u. Meesenburg; H. (2007): Risikovorsorge im Zeichen des Klimawandels. AFZ/Der Wald, 52, 1246-1249

Thomasius, H. (1991): Mögliche Auswirkungen einer Klimaänderung auf die Wälder in Mitteleuropa. Forstw. Cbl., 110, 305-330

UBA (Umweltbundesamt) (2006): Künftige Klimaänderungen in Deutschland - Regionale Projektionen für das 21. Jahrhundert. Hintergrundpapier

WAGNER, S. (2004): Klimawandel - Konsequenzen für künftige Baumartenwahl und Bestandesstrukturierung. Tagungsbericht des Brandenburgischen Forstvereins e. V. zur Jahrestagung vom 26. Mai 2004 in Eberswalde, 56-66

Wohlgemut, T.; Bugmann, H.; Lischke, H. u. Tinner, W. (2006): Wie rasch ändert sich die Waldvegetation als Folge von raschen Klimaänderungen? Forum für Wissen, 7-16 
Korrespondierender Autor:

Johannes Sutmöller

Nordwestdeutsche Forstliche Versuchsanstalt

Grätzelstr. 2

37079 Göttingen

E-Mail: Johannes.Sutmoeller@nw-fva.de

URL: www.nw-fva.de

Prof. Dr. Hermann Spellmann

Dr. Caroline Fiebiger

Dr. Matthias Albert

Nordwestdeutsche Forstliche Versuchsanstalt 


\title{
Aspekte der Biodiversität von Buchenwäldern - Konsequenzen für eine naturnahe Bewirtschaftung
}

\author{
Aspects of the biodiversity of beech forests - \\ consequences for near to nature management
}

Peter Meyer und Marcus Schmidt

\section{Zusammenfassung}

Vor dem Hintergrund der aktuellen Debatte um den Schutz und die Nutzung von Buchenwäldern werden Aspekte ihrer Biodiversität beleuchtet und Kennzeichen einer naturnahen Bewirtschaftung abgeleitet. Dabei wird davon ausgegangen, dass sich naturnahe Lebensgemeinschaften durch eine typische und möglichst vollständige Arten- und Strukturausstattung auszeichnen.

Die Verbreitung von Buchenwäldern ist durch große naturräumliche Unterschiede mit einem Schwerpunkt im westdeutschen Hügel- und Bergland sowie einer erheblichen Fragmentierung im Tiefland gekennzeichnet. Anstrengungen zum Waldumbau in Richtung naturnaher Buchenwälder sollten auf die Defiziträume konzentriert werden. Im Tiefland sollten in die verbliebenen Restbestände gesellschaftsfremde Mischbaumarten nicht aktiv eingebracht werden.

Buchenwälder gelten zu Unrecht als artenarme Lebensgemeinschaften. Zwar sind für die weit verbreiteten Buchenwaldtypen relativ geringe Artenzahlen in der 
Bodenvegetation kennzeichnend, doch ist die Vielfalt der an Altbäume und Totholz gebundenen Arten ausgesprochen hoch.

Rund $80 \%$ der bewirtschafteten Buchenwälder mittlerer Standorte zeichnen sich durch das Auftreten von Störungszeigern und Offenlandarten in der Bodenvegetation aus. Diese werden insbesondere durch anthropogene Stoffeinträge sowie durch die Effekte forstwirtschaftlicher Maßnahmen (Bodenverwundung und -verdichtung, Auflichtungen) gefördert.

Hinsichtlich der an Totholz gebundenen Käferarten werden in bewirtschafteten Buchenwäldern Defizite festgestellt. Am Beispiel dieser Artengruppe lässt sich erneut zeigen, dass die Erhaltung und Entwicklung von Alt- und Totholz ein essenzieller Bestandteil einer naturnahe Buchenwaldbehandlung ist.

Anhand einer Analyse der Lücken- und Verjüngungsdynamik sowie der Waldentwicklungsphasen wird verdeutlicht, dass Buchenurwälder keineswegs strukturarm sind. Typisch ist hingegen ein kleinräumiges Mosaik verschiedener Entwicklungsphasen, die sich zudem zeitlich und räumlich überlappen. Einzelstamm- bis femelartige Behandlungskonzepte sowie lange Verjüngungszeiträume ähneln diesen natürlichen Mustern und können als Kennzeichen einer naturnahen Buchenwirtschaft bestätigt werden. Auch für Gefäßpflanzen, Moose und Flechten stellt dies die günstigste Bewirtschaftungsform dar.

Insgesamt wird empfohlen, die naturschutzfachliche und waldbauliche Planung stärker als bisher landschaftsökologisch auszurichten, um das Ziel einer wirksamen Erhaltung der biologischen Vielfalt mit einer ökonomisch tragfähigen Waldbewirtschaftung zu verbinden.

Stichworte: Hainsimsen-Buchenwald, Waldmeister-Buchenwald, Artenvielfalt, Strukturvielfalt, Naturnähe, Totholz

\begin{abstract}
In this paper, aspects of beech forest diversity relevant to the current debate about beech forest conservation are elaborated and characteristics of a near to nature management are derived. Conceptually it is assumed that the main characteristic of near to nature biotic communities is a typical, near complete assemblage of species and structural elements.

The distribution of beech forests, marked by considerable natural spatial variation, primarily is concentrated in the hilly and mountainous regions of western Germany. In the lowlands beech forests are highly fragmented. Thus, forest conversion efforts towards near to nature beech forests should be concentrated in the lowlands. In these remnant forests in the lowlands, the introduction of tree species atypical for these forest types should not be supported actively.
\end{abstract}


Beech forests unjustifiably are considered species poor communities. Although relatively few species are present in the ground vegetation in the widely distributed beech forest types, an extraordinarily high species diversity occurs in old trees and dead wood.

The presence of disturbance indicators and open land plant species is evident in the ground vegetation of about $80 \%$ of the commercially managed beech forests on moderate sites. These derive from anthropogenic nutrient inputs, in particular, as well as through the effects of forestry practices (soil damage and compaction, and opening up).

In managed beech forests, a deficit of saproxylic beetles was established. Consequently, the maintenance and development of old growth and woody debris is an important facet of a near to nature beech forest management regime.

An analysis of gap and regeneration dynamics, and the forest development phases shows that virgin beech forests are in no way structurally poor. In contrast, a mosaic of small forest patches in different phases of development with some temporal and spatial overlap is typical. Single-tree to group selection management systems, and long regeneration periods emulate these natural patterns. These management approaches also present favourable systems for vascular plants, mosses and lichens.

We recommend a strengthening of the landscape ecology emphasis in conservation and silvicultural planning in order to harmonise conservation of biological diversity and economically feasible forest management.

Key words: woodrush beech forest, woodruff beech forest, biodiversity, forest structure, near to nature forestry, dead wood

\section{Einleitung}

Vor dem Hintergrund der 2008 von Deutschland ausgerichteten 9. Vertragsstaatenkonferenz zur Konvention über die biologische Vielfalt hat sich die Debatte über den Schutz und die Nutzung von Buchenwäldern verschärft (z. B. PANEK 2007, 2008, BFN 2008, NABU 2008). Im Kern spitzt sich die Diskussion auf zwei Fragen zu:

- In welchem Umfang sollen Buchenwälder aus der Nutzung genommen werden?

- Was kennzeichnet eine multifunktionale und nachhaltige Buchenwaldbewirtschaftung, die naturschutzfachlichen und ökonomischen Zielen gleichermaßen gerecht wird?

Diese Diskussion ist nicht neu (vgl. LANA 1992, SCHERZINGER 1997, SACHVERSTÄNDIGENRAT FÜR UMWELTFRAGEN 2000, 2004). Seit mehreren Jahrzehnten wird in Deutschland intensiv über eine naturschutzgerechte Waldbewirtschaftung 
debattiert (KRUG et al. 2006). Ab Mitte der 1990er Jahre rückten dabei Buchenwälder verstärkt in den Fokus (z. B. ABs 1996).

In der Forstwirtschaft zeichnen sich dabei zwei Entwicklungslinien ab: Auf der einen Seite haben nach großflächigen Windwürfen und der Bedrohung durch das „Waldsterben“ fast alle Landesforstverwaltungen in den 1990er Jahren naturnahe Waldbauprogramme mit z. T. ambitionierten naturschutzfachlichen Zielsetzungen aufgelegt (DOHRENBUSCH u. ROSIN 2002). Naturnähe ist zu einem Leitbegriff der Waldbewirtschaftung in Deutschland und darüber hinaus geworden (BIELING u. Schraml 2003, Pommerening u. Murphy 2004). Auf der anderen Seite ist Forstwirtschaft in Deutschland durch erhebliche Rationalisierungsanstrengungen im Verbund mit gestiegenen Holzpreisen und erhöhten Holzeinschlägen wieder rentabel geworden. Auch zukünftig dürfte weltweit und in Deutschland die Nachfrage nach Holz erheblich steigen (FAO 2007), so dass die Verbindung von Schutz und Nutzung des Waldes weiterhin eine anspruchsvolle Aufgabe bleiben wird.

Auch aus Sicht des Naturschutzes sind kontrastierende Entwicklungen festzustellen: Die Anzahl und Flächengröße von (Buchenwald-)Schutzgebieten wurde in den letzten Jahrzehnten deutlich erhöht (BLAB 2003, BFN 2004). Naturschutzfachliche Regelungsinhalte spielen in der Rechtsetzung eine zunehmende Rolle - von der Umsetzung der FFH-Richtlinie in deutsches Recht bis hin zur Ausweitung der Umwelthaftung durch das im Jahr 2007 verabschiedete Umweltschadensgesetz. Der Kenntnisstand über die Biodiversität und ihre Gefährdungsursachen hat erheblich zugenommen (BFN 1997, BINOT-HAFKE et al. 2000, BLAB 2005, NiPKOW 2005, GÜNTHER et al. 2006). Dennoch konnte die Situation der biologischen Vielfalt in Deutschland bisher offenbar nicht entscheidend verbessert werden (ACHTZIGER et al. 2004, BINOT-HAFKE et al. 2003). Erfolge bei einzelnen Arten wie Kolkrabe, Schwarzstorch, Biber oder Luchs sind im Wesentlichen auf die Beendigung der direkten Verfolgung und aktive Stützungsmaßnahmen zurückzuführen. Die Wirksamkeit von Unterschutzstellungen ist nach den bisher vorliegenden Bilanzen unzureichend (HAARMANN u. PRETSCHER 1993, SSYMANK 1997).

In Wäldern wird die forstliche Bewirtschaftung nach wie vor als eine der wesentlichen Gefährdungsursachen für die biologische Vielfalt angesehen (REIF 1992, GÜNTHER et al. 2006). Dieser Feststellung wird von Seiten der Forstwirtschaft widersprochen (VOLK 1997, WILKE 2007) und stattdessen die Auffassung vertreten, dass die forstliche Bewirtschaftung ein hohes Niveau an biologischer Vielfalt gewährleistet (DFWR 2008).

Trotz der unterschiedlichen Auffassungen besitzen Naturschutz und Forstwirtschaft mit dem Bekenntnis zu einer naturnahen Waldwirtschaft ein gemeinsames Leitbild. Insbesondere für Buchenwälder, die natürlicherweise in Mitteleuropa vorherrschende Vegetationsform, gilt eine naturnahe Waldbehandlung als das vorrangige Ziel. Um als Wertmaßstab dienen zu können, bedarf der Begriff der Naturnähe allerdings einer Operationalisierung. 
Eine Lebensgemeinschaft kann als naturnah gelten, wenn sie sowohl typisch als auch möglichst vollständig ausgeprägt ist, d. h., wenn sie diejenigen Arten und Strukturen aufweist, die für das Naturraumpotenzial unter Berücksichtigung von natürlichen Störungen charakteristisch sind (vgl. REIF 2000). Diese Definition entspricht dem qualitativen Ansatz des Übereinkommens über die biologische Vielfalt und setzt sich von dem Ziel einer bloßen Maximierung der Artenzahl deutlich ab.

Im Rahmen des vorliegenden Beitrages sollen Kennzeichen naturnaher Buchenwälder auf der Basis von Untersuchungen in bewirtschafteten und unbewirtschafteten Beständen abgeleitet und Schlussfolgerungen für eine naturnahe Buchenwaldbewirtschaftung gezogen werden. Für eine Analyse des IstZustandes werden die Vegetationsdaten der Bodenzustandserhebung (BZE) II in den Bundesländern Hessen und Niedersachsen genutzt. Folgende Teilaspekte und Fragestellungen werden im Einzelnen behandelt:

1. Verbreitung von Buchenwäldern

- Welche Faktoren beeinflussen ihre heutige Verbreitung?

2. Artenvielfalt

- Gelten Buchenwälder zu Recht als artenarm?

- Wie naturnah ist derzeit die Artenzusammensetzung der Bodenvegetation?

- Wie wirkt sich die heutige forstliche Bewirtschaftung auf die Phytodiversität aus?

- Wie vollständig ist die Zoozönose? - Das Beispiel der Käfer

3. Strukturvielfalt

- Welches Störungsregime ist für Buchenwälder typisch?

- Was kennzeichnet die natürliche Verjüngungsdynamik?

- Wie verändert sich die Zusammensetzung nach Waldentwicklungsphasen mit steigender Naturnähe?

- Welchen Beitrag leisten ungenutzte Buchenwaldflächen für den Totholzaufbau?

\section{Verbreitung von Buchenwäldern}

\subsection{Historische Aspekte}

Der Schwerpunkt der Buchenwaldverbreitung in Deutschland liegt im mittleren Westdeutschland und hier besonders im hessischen und niedersächsischen Hügelund Bergland. Dieser Befund der Forsterhebung von 1927 (s. Abb. 1) gilt annähernd bis heute. Da Klima und Bodenbedingungen der Buche in Deutschland nur wenige Grenzen setzen (LEUSCHNER 1998), müssen vor allem historische Gründe zur Erklärung dieses Verbreitungsmusters herangezogen werden. Die Rot- 
buche selbst ist die durch anthropogene Nutzung in Deutschland am stärksten zurückgedrängte Baumart (HESMER 1937, LEUSCHNER 1998). Entscheidenden Einfluss auf die heutige Verbreitung von Buchenwäldern hatte vor allem die Zeit der extensiven Landwirtschaft und Waldverwüstung, die vom frühen Mittelalter bis zur Mitte des 18. Jahrhunderts andauerte (HESMER u. SCHROEDER 1963, SCHROEDER 1998). Nach umfangreichen Waldrodungen bis zum 13. Jahrhundert (JAHN 1996), haben vor allem die Nieder- und Mittelwaldwirtschaft, die Waldweide und -mast sowie der immense Holzbedarf der frühneuzeitlichen Industrie (Köhlerei, Glashütten, Eisenhütten etc.) zu einer Verringerung des Buchenanteils in Wäldern geführt. Hauptgründe sind die vergleichsweise schlechte Fähigkeit zum Stockausschlag und geringe Verbisstoleranz der Rotbuche sowie die aktive Förderung der Eiche als Mastbaum und Bauholz.

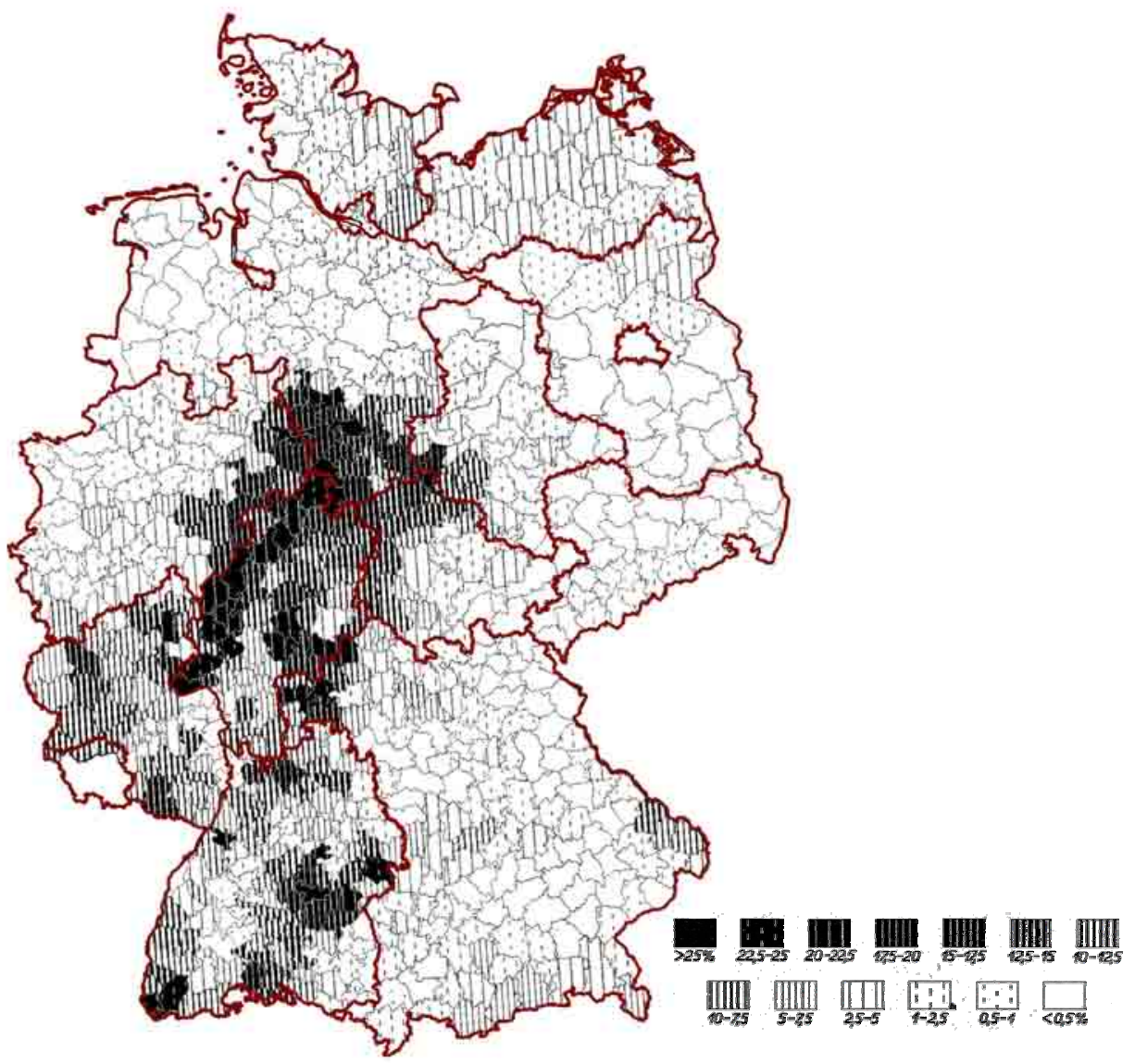

Abbildung 1: Vorkommen von Buchenwäldern in Deutschland 1927 nach Landkreisen in Prozent der Gesamtfläche (aus HESMER 1937, verändert) 
Die in der zweiten Hälfte des 18. Jahrhunderts begonnene Ablösung von Trift und Weiderechten (ABEL 1978) begünstigte eine Wiederausbreitung der Buche. Seit Beginn des 19. Jahrhunderts wurden Nieder- und Mittelwaldbestände in den meisten Regionen verstärkt in Hochwald überführt. Durch Pflanzung und natürliche Wiederausbreitung erhöhte sich seitdem der Buchenanteil beträchtlich (u. a. HESMER 1937, WINTERHOFF 1963, ZACHARIAS 1996). In einigen seit mehr als 100 Jahren ungenutzten ehemaligen Eichen-Hutewäldern wie dem „Neuenburger Urwald“ oder dem „Urwald Sababurg“ hat die Buche inzwischen wieder die Vorherrschaft übernommen (MEYER et al. 2006, RAPP u. SCHMIDT 2006). Größere Verluste gab es hingegen erneut im 19. und 20. Jahrhundert durch Umwandlung in Nadelwald. So nahm allein im Zeitraum zwischen 1883 und 1913 die Buchenwaldfläche in Deutschland zu Gunsten von Kiefer und Fichte um 175.000 ha ab (HESMER 1937).

Während im Hügel- und Bergland das Relief (in der montanen Höhenstufe auch das Klima) dem Ackerbau Grenzen setzten, hat im Tiefland auf Sandböden vor allem die Heidewirtschaft (BEHRE 2000, LEUSCHNER u. IMMENROTH 1994), auf Lehm- und Lössböden besonders der Ackerbau die Buche zurückgedrängt (HESMER 1937). Im niedersächsischen Tiefland sind nur relativ wenige Buchenwälder erhalten geblieben, von denen sich heute fast zwei Drittel im Staatswald befinden. Sie gehen überwiegend auf landesherrlichen Besitz zurück und befinden sich zu etwa $90 \%$ auf historisch alten Waldstandorten (HEINKEN 1995).

\subsection{Aktuelle Situation}

Zur Charakterisierung der aktuellen Situation der Buchenwälder und der Baumart Buche dienen hier die im Rahmen der BZE II in den Jahren 2006 und 2007 in den Bundesländern Hessen, Niedersachsen und Bremen auf einem systematischen Stichprobennetz (8 $8 \mathrm{~km}$-Raster) nach einheitlichem Verfahren gewonnenen Vegetationsaufnahmen. Auf $400 \mathrm{~m}^{2}$ großen Probeflächen (Plots) wurden alle Arten der Baum-, Strauch-, Kraut- und Kryptogamenschicht mit ihren Deckungsgraden erfasst. Der Datensatz erlaubt für die Flächen-Bundesländer repräsentative Aussagen. Als Buchenwälder werden nachfolgend alle Bestände aufgefasst, bei denen die Buche in der ersten oder zweiten Baumschicht einen relativen Deckungsgrad von mindestens $50 \%$ aufweist.

Von den insgesamt 312 Vegetationsaufnahmen der BZE II entfallen 202 auf das Hügel- und Bergland und 110 auf das Tiefland (Hessen: 139, Niedersachsen: 169, Bremen: 4). Davon werden 95 Waldbestände (30\%) nach den obigen Kriterien als Buchenwald eingestuft. Weitere 63 Vegetationsaufnahmen enthalten die Buche in der 1. und/oder 2. Baumschicht. In insgesamt 158 Beständen (51 \%) kommt demnach die Buche in der Baumschicht vor. In weiteren 75 Beständen ist Fagus sylvatica in Strauch- und/oder Krautschicht vertreten. Damit enthalten 
insgesamt $75 \%$ aller Vegetationsaufnahmen des BZE-Datensatzes die Pflanzenart Buche.

Von den 95 Buchenbeständen liegen 94 (99\%) im Hügel- und Bergland, davon $70(75 \%)$ in Hessen und $24(26 \%)$ in Niedersachsen. Nur einer befindet sich im niedersächsischen Tiefland. Das Hügel- und Bergland weist somit laut BZE II insgesamt einen Buchenwaldanteil von $47 \%$ (Hessen: $50 \%$, Niedersachsen: $38 \%$ ), das niedersächsische Tiefland von $1 \%$ auf.

Diese Ergebnisse zeigen die weite Verbreitung der Baumart Buche in Hessen und im niedersächsischen Hügel- und Bergland. Wertet man ihr Auftreten als Hinweis auf die potenzielle Verbreitung von Buchenwäldern, so ist für das untersuchte Gebiet von einem potenziellen Buchenwaldanteil von mindestens $75 \%$ auszugehen, ein Ergebnis, dass weitgehend mit anderen Erhebungen übereinstimmt (Niedersachsen: 67\%, s. NML 2004; Hessen: $90 \%$, s. HMULV 2006). Für die alten Bundesländer gibt LEUSCHNER (1998) einen potenziellen Anteil von $80 \%$ an. Den hohen Anteilen im Hügel- und Bergland steht im Tiefland ein in erster Linie historisch bedingter Mangel an Buchenwäldern gegenüber (JAHN 1979, HEINKEN 1995).

Die im Rahmen der BZE II gewonnenen Ergebnisse über die aktuelle Verbreitung von Buchenwäldern stimmen weitgehend mit den Erhebungen der Bundeswaldinventur $2\left(\mathrm{BWI}^{2}\right)$ in Niedersachsen überein. Deutliche Abweichungen ergeben sich allerdings in Hessen. Nach der BWI ${ }^{2}$ sind hier $30 \%$ (HMULV 2006), nach der BZE II $50 \%$ der Waldfläche von der Baumart Buche dominiert. Als Hauptgrund für die Abweichung müssen Unterschiede bei der Definition von Buchenwäldern angesehen werden. Die im Rahmen der BZE II-Auswertung verwendete Definition ist relativ weit gefasst und bezieht auch mit Buche unterbaute (zweite Baumschicht), jedoch von anderen Baumarten in der ersten Baumschicht geprägte Bestände ein. Da diese aus vegetationskundlicher Sicht bereits Buchenwald-Charakter besitzen und sich nach gegenwärtigem Kenntnisstand in Richtung Buchenwald entwickeln werden, erscheint diese Einstufung gerechtfertigt.

Die aufgenommenen Buchenwald-Bestände lassen sich mithilfe der bei SsYMANK et al. (1998) aufgeführten Arten den beiden FFH-Lebensraumtypen 9110 (Hainsimsen-Buchenwald) und 9130 (Waldmeister-Buchenwald) zuordnen. Dabei entfallen 56 (59\%) auf den Hainsimsen-Buchenwald (Hessen: 43, Niedersachsen 12 im Bergland, 1 im Tiefland) und 39 (41\%) auf den WaldmeisterBuchenwald (Hessen: 27, Niedersachsen Bergland: 12). Der ebenfalls im Untersuchungsgebiet vorkommende, jedoch wesentlich seltenere Lebensraumtyp 9150 (Mitteleuropäischer Orchideen-Kalk-Buchenwald) ist an den Probepunkten der BZE nicht anzutreffen. 


\section{Artenvielfalt}

Aufgrund der von der Rotbuche dominierten Baumschicht und einer vor allem auf bodensauren Standorten nur mit geringem Deckungsgrad und ohne auffällige Blühaspekte ausgebildeten Krautschicht gelten Buchenwälder oft als artenarm. Zwar sind Gefäßpflanzen eine Schlüsselartengruppe in Waldökosystemen, doch müssen bei der Betrachtung der Artenvielfalt auch andere Organismengruppen wie Moose, Flechten, Pilze oder Insekten einbezogen werden. Über die Gesamtartenzahl der in mitteleuropäischen Buchenwäldern lebenden Pflanzen- und Tierarten existieren bisher nur grobe Schätzungen. So geht BÜCKING (2003) von ca. 4.320 Pflanzen- und Pilzarten aus, von denen ca. 1.169 nur im Buchenwald leben. Die Zahl der Tierarten wird von ihm auf 6.715 geschätzt, davon 1.792 nur im Buchenwald. DOROw u. KOPELKE (2007) fanden in drei zwischen 51 und 74 ha großen hessischen Buchenwaldgebieten zwischen 1.582 und 2.328 Tierarten. Für vergleichbar große Gebiete erwarten sie 5.000-6.000 Tierarten. Alt- und totholzreiche Buchenwälder haben besondere Bedeutung für xylobionte Pilz- und Käferarten (KÖHLER u. KlaUSNITZER 1998, SCHMID u. HELFER 1999, UTSCHIK 2001).

\subsection{Bodenvegetation}

Die beiden weit verbreiteten Buchenwald-Lebensraumtypen mittlerer Standorte Hainsimsen-Buchenwald (auf stark sauren bis sauren Böden) und WaldmeisterBuchenwald (auf mäßig sauren bis alkalischen Böden) unterscheiden sich hinsichtlich der Struktur und Artenzusammensetzung ihrer Bodenvegetation deutlich. Während die Krautschicht des Hainsimsen-Buchenwaldes überwiegend weniger als ein Viertel und oft deutlich weniger als $10 \%$ der Probeflächen bedeckt, beträgt die Krautschicht-Deckung des Waldmeister-Buchenwaldes meist mehr als $20 \%$ und zeichnet sich insgesamt durch eine größere Variabilität aus (s. Abb. 2). Eine Moosschicht ist in $93 \%$ der Hainsimsen-Buchenwälder und in $72 \%$ der WaldmeisterBuchenwälder ausgebildet. Der Moosschicht-Deckungsgrad liegt in beiden Buchenwald-Lebensraumtypen meist bei unter $1 \%$ der Aufnahmefläche.

Auch bei den Artenzahlen der Gefäßpflanzen liegt der Waldmeister-Buchenwald (Median: 21, Mittelwert: 24) auf deutlich höherem Niveau als der Hainsimsen-Buchenwald (Median: 14, Mittelwert: 16, s. Abb. 3). Umgekehrt verhält es sich bei den Moosen; hier ist der Hainsimsen-Buchenwald etwas artenreicher (Median und Mittelwert: 4) als der Waldmeister-Buchenwald (Median und Mittelwert: 2). 


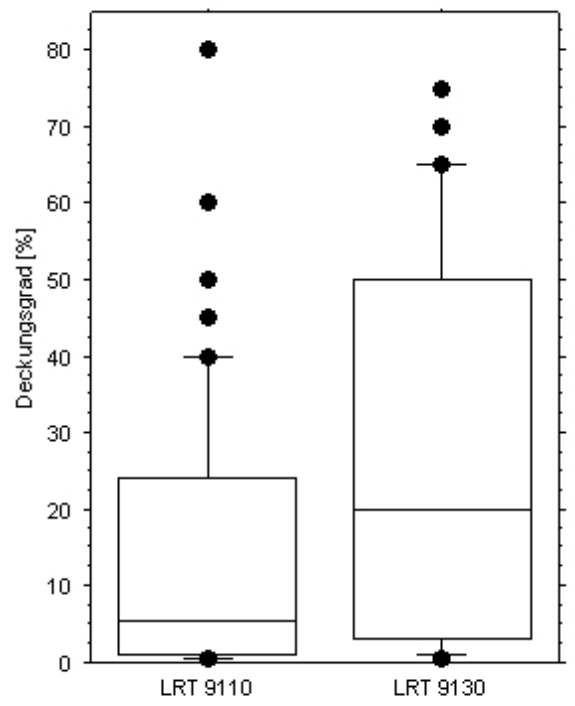

Abbildung 2:

Prozentuale Krautschicht-Deckungsgrade der FFH-Lebensraumtypen 9110 (HainsimsenBuchenwald) und 9130 (WaldmeisterBuchenwald); Datengrundlage: BZE II

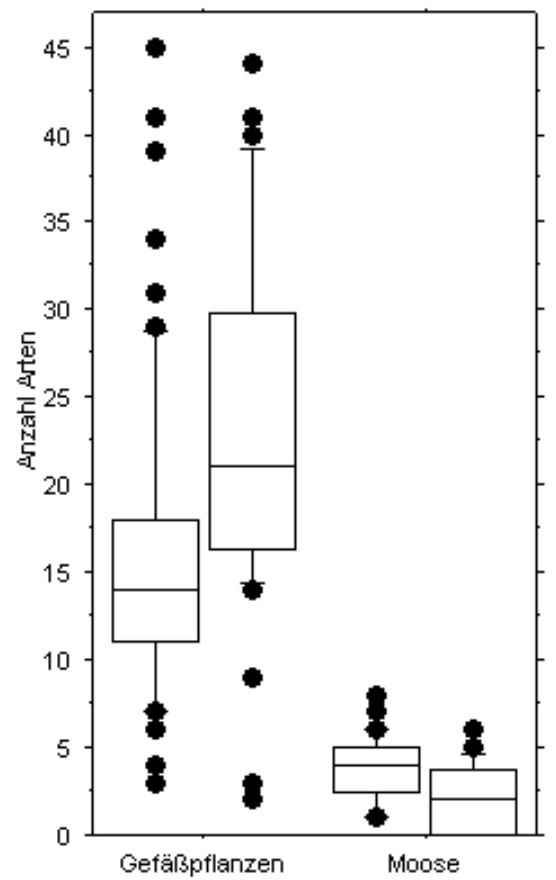

Abbildung 3:

Artenzablen der Gefäßpflanzen und Moose in den FFH-Lebensraumtypen 9110 (Hainsimsen-Buchenwald, jeweils links) und 9130 (Waldmeister-Buchenwald); Datengrundlage: BZE II 
Tabelle 1: $\quad$ Mittlere Gesamtartenzablen (GefäSpflanzen, Moose, Flechten) ausgewählter Waldgesellschaften auf der Grundlage überregionaler Vegetationsübersichten vorwiegend aus dem nördlichen und mittleren Deutschland. Die Anordnung erfolgt nach absteigender mittlerer Artenzabl. Die Vegetationstypen Waldmeister- und Waldgersten-Buchenwald entsprechen beide dem FFH-LRT Waldmeister-Buchenwald. Daher erfolgt hier keine getrennte Darstellung. Datengrundlage (als Hochzahl in Klammern angegeben): 1 DIERSCHKE (1989), 2 GOLISCH (2002), 3 HÄRDTLE (1995), 4 HEINKEN $u$. ZIPPEL (1999), 5 HEINKEN (1995), 6 HEINKEN (in prep.), 7 MAST (1999), 8 SCHMIDT (2000), 9 BZE II.

\begin{tabular}{lc}
\hline \multicolumn{1}{c}{ Vegetationstyp } & mittlere Gesamtartenzahl \\
\hline stark saure bis saure Standorte: & $30^{(7)}$ \\
Torfmoos-Erlenbruchwald & $26^{(6)}$ \\
Fichtenwald & $25^{(2)}$ \\
Habichtskraut-Eichenwald & $22^{(2)}, 24^{(5)}$ \\
Birken-Eichen-Wald & $20^{(5)}, 22^{(4)}$ \\
Drahtschmielen-Kiefernwald & $15^{(4)}, 22^{(5)}$ \\
Weißmoos-Kiefernwald & $19^{(4)}, 21^{(5)}$ \\
Flechten-Kiefernwald & $19^{(7)}$ \\
Scheidenwollgras-Moorwald & $16^{(2)}, 18^{(5)}, 20^{(9)}$ \\
Hainsimsen-Buchenwald & \\
mäßig saure bis alkalische Standorte: & $42^{(2)}$ \\
Felsenahorn-Eichenwald & $41^{(7)}$ \\
Winkelseggen-Erlen-Eschenwald & $39^{(8)}$ \\
Steinsamen-Eichenwald & $37^{(7)}$ \\
Hainsternmieren-Schwarzerlenwald & $36^{(8)}$ \\
Karbonat-Kiefernwald & $32^{(1)}, 35^{(8)}$ \\
Orchideen-Buchenwald & $29^{(7)}$ \\
Walzenseggen-Erlenbruchwald & $27^{(2)}$ \\
Waldlabkraut-Eichen-Hainbuchenwald & $21^{(1)}, 22^{(2)}, 24^{(3)}, 25^{(1)}, 26^{(9)}, 28^{(5)}$ \\
\hline Waldmeister-/Waldgersten-Buchenwald & \\
\hline &
\end{tabular}

Im Vergleich der Gesamtartenzahlen (Gefäßpflanzen, Moose, Flechten) mit anderen Waldgesellschaften (s. Tab. 1) zeigt sich, dass die beiden Buchenwaldgesellschaften mittlerer Standorte sowohl im Vergleich mit Waldtypen extremer Standorte (trocken, nass) als auch mit von anderen Baumarten geprägten Waldgesellschaften mittlerer Standorte (Ersatzgesellschaften von Buchenwäldern) relativ artenarm sind. So zeichnen sich Fichtenwälder oder Birken-Eichenwälder auf potenziellen Standorten des Hainsimsen-Buchenwaldes in der Regel durch eine 
höhere Gesamtartenzahl aus (GÄRTNER u. ENGELHARD 2005, HEINKEN 1995, SCHMIDT 1999, WECKESSER 2002). Hauptgrund ist die im Durchschnitt geringere Transmissivität der Baumschicht von Buchenwäldern (HEINKEN 1995, WECKESSER 2002), die zu lichtarmen und homogenen Bedingungen in der Bodenvegetation führt. Zwar herrscht auch im artenreicheren Orchideen-Buchenwald ein typisches Rotbuchen-Lichtklima (SCHMIDT 2000), doch wirkt sich der trockenheitsbedingt geringere Kronenschluss in dieser Waldgesellschaft positiv auf die Gesamtartenzahl aus. Unter den annähernd gleichen Lichtbedingungen im Hainsimsen- und im Waldmeister-Buchenwald (BRÜNN 1992, HEINKEN 1995) beeinflusst vor allem die Basenversorgung des Bodens die Artenzahl (SCHMIDT et al. 2002). Generell liegen die Gesamtartenzahlen auf basenreichen Standorten auf höherem Niveau als auf bodensauren (s. Tab 1, vgl. LEUSCHNER 1999).

Bei der Bewertung dieser Ergebnisse muss berücksichtigt werden, dass eine untypische Maximierung der Artenvielfalt aus naturschutzfachlicher Sicht nicht zielführend ist. Vielmehr gilt es, eine Vollständigkeit des Arteninventars im Hinblick auf waldtypische bzw. für das jeweilige Waldökosystem typische Arten zu bewahren oder zu entwickeln. Deutlich über den Durchschnittswerten liegende Gefäßpflanzen-Artenzahlen können auf ein vermehrtes Auftreten von Störungszeigern und Offenlandarten hindeuten und sind unter dem Aspekt der Naturnähe negativ zu bewerten. Als Störungszeiger gelten hier insbesondere Anzeiger für anthropogene Stoffeinträge (Stickstoff-Deposition, Kalkung), Bodenverwundung und -verdichtung (vgl. JENSCH 2004). Die Definition von Störungszeigern muss dabei für jeden Waldtyp gesondert erfolgen (SCHMIDT et al. 2003). Grundlage für eine Referenzliste von Störungszeigern sind hier die von GRABHERR et al. (1998) für verschiedene Buchenwaldtypen erarbeiteten Störungszeigerlisten in Kombination mit den bei EBRECHT (2005) genannten Arten mit Schwerpunkt im Bereich von Waldwegen. Außerdem gingen in die Auswertung alle Neophyten sowie alle bei SCHMIDT et al. (2003) aufgeführten Waldarten mit Schwerpunkt im Offenland und alle Offenlandarten ein.

Der Datensatz der BZE II gibt Auskunft über die aktuelle Situation von bewirtschafteten Buchenwäldern im Hinblick auf das Auftreten solcher Störungszeiger und Offenlandarten (im Folgenden unter dem Begriff „Störungszeiger“" zusammengefasst). Dabei ergeben sich hinsichtlich ihrer Artenzahl und ihres Deckungsgrades keine prinzipiellen Unterschiede zwischen Hainsimsen- und Waldmeister-Buchenwald. Nur $20 \%$ der Bestände weisen keine störungszeigende Gefäßpflanzenart auf. Die Mehrzahl der Buchenwälder (53\%) enthält 1-5 Störungszeiger auf $400 \mathrm{~m}^{2}$. Mit zunehmender Anzahl von Störungszeigern - maximal wurden 22 Arten in einem Plot gefunden - steigt auch ihr Anteil an der Gesamtdeckung der Krautschicht (s. Abb. 4). 


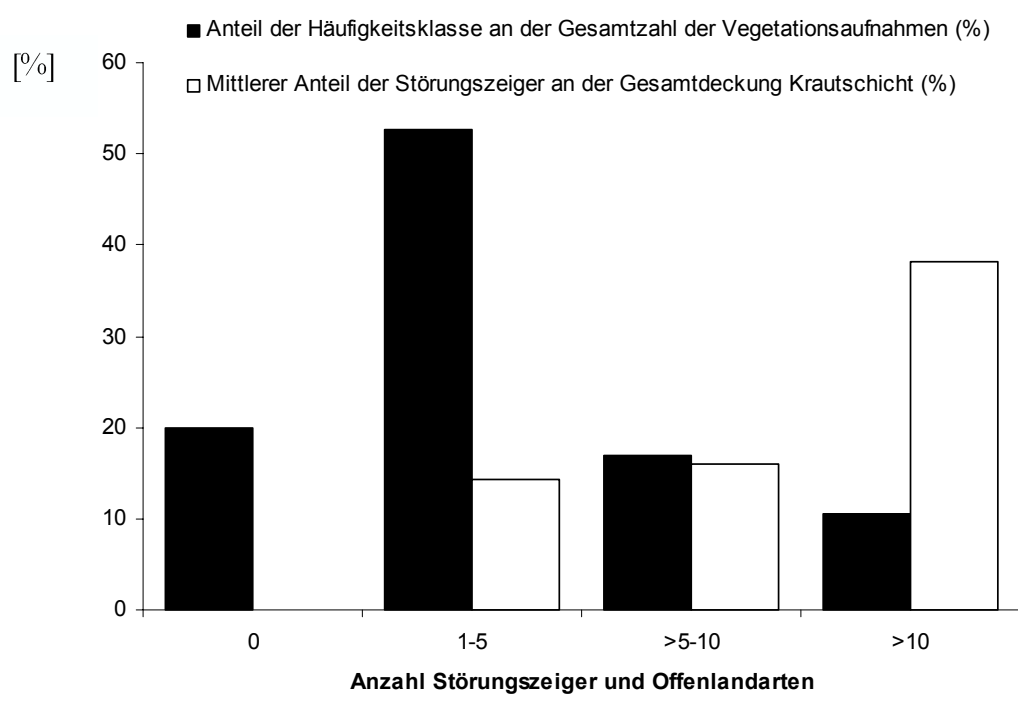

Abbildung 4: Anzabl von Störungszeigern und Offenlandarten in Buchenwäldern nach Häufigkeitsklassen und mittlerer Anteil der Störungszeiger an der Gesamtdeckung der Krautschicht; Datengrundlage: $400 \mathrm{~m}^{2}$-Plots der BZE II

Wegen ihrer geringen Zahl und Fläche ist über die spezifischen vegetationsökologischen Bedingungen mitteleuropäischer Buchen-Urwälder und die Unterschiede zu bewirtschafteten Wäldern nur wenig bekannt. Beim Vergleich von EichenHainbuchen-Urwäldern des Białowieża-Nationalparks mit Wirtschaftswäldern gleicher Baumartenzusammensetzung konnten ABS et al. (1999) in Letzteren ein gehäuftes Auftreten von Störungszeigern sowohl in der Bodenvegetation als auch in der Samenbank des Bodens nachweisen. Hauptgrund dafür ist, dass sich die in Urwäldern auftretenden natürlichen Störungen, etwa durch Windwurf oder Schwarzwild, in der Regel deutlich kleinflächiger auswirken als die in Wirtschaftswäldern.

Neben den wenigen aus der Urwaldforschung vorliegenden Daten kommt der Naturwaldreservateforschung eine besondere Bedeutung als Referenz zu. In hessischen Naturwaldreservaten (NWR) werden parallel zur waldkundlichen Inventur auch Vegetationsaufnahmen durchgeführt. Da zudem eine weiterhin bewirtschaftete sogenannte Vergleichsfläche für die meisten NWR besteht und ebenfalls untersucht wird, kann der Effekt der Bewirtschaftung auf die Artenvielfalt der Gefäßpflanzen der Krautschicht unmittelbar untersucht werden. In der nachfolgenden Auswertung für die Waldmeister-Buchenwälder des NWR Hohestein (SCHREIBER et al. 1999, SCHMIDT u. MEYER 2007) wird das Ausmaß forstlicher Eingriffe anhand der Veränderung der Grundflächenhaltung von 1996 bis 2007 quantifiziert. Datengrundlage sind die Inventurergebnisse in 44 Probekreisen. Zwischen Bewirtschaftungsintensität (Eingriffsstärke) und Gefäßpflanzen-Arten- 
zahl im Jahr 2007 ergibt sich ein signifikanter positiver Zusammenhang (s. Abb. 5). Bewirtschaftete und unbewirtschaftete Teilfläche unterscheiden sich deutlich in ihrer Artenvielfalt. Die Zunahme der Artenzahl an Gefäßpflanzen durch forstliche Bewirtschaftung bzw. deren Abnahme bei fehlender Bewirtschaftung ist ein vielfach bestätigter Befund (Lit. s. SCHMIDT u. SCHMIDT 2007). Veränderungen des Lichtklimas durch Auflichtung im Kronenraum, heterogene Bodenverhältnisse durch Rückearbeiten und eine höhere Wegedichte führen in Wirtschaftswäldern zu (mindestens vorübergehend) höheren Gefäßpflanzen-Artenzahlen pro Flächeneinheit.

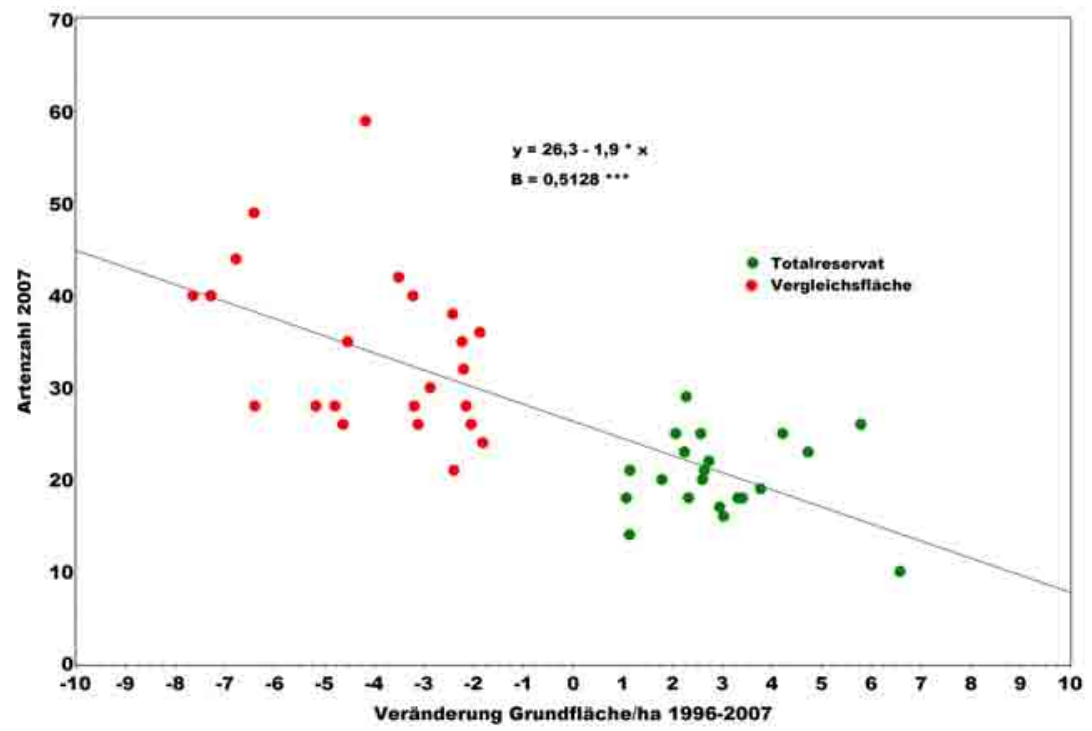

Abbildung 5: Artenzabl der Gefäßpflanzen (Krautschicht) in $250 \mathrm{~m}^{2}$ großen Vegetationsaufnahmeflächen im NWR Hohestein in Abhängigkeit von der Grundflächenveränderung von 1996 bis 2007(B = Bestimmtheitsmaß, *** $=p>F<0,001)$

Wird näher betrachtet, auf welche Arten die Zunahme der Vielfalt zurückgeht, so wird deutlich, dass es sich hierbei vor allem um Störungszeiger handelt (s. Abb. 6). Die Zunahme der Vielfalt ist demnach Ausdruck der Nivellierung von Unterschieden zwischen natürlicherweise verschiedenen Ökosystemen (s. o.). So ist die Zunahme von Bodenverdichtungszeigern nach Rückearbeiten (vgl. GAERTIG u. GREEN 2008), die ihren natürlichen Schwerpunkt in grundwassernahen Waldgesellschaften haben (z. B. Carex remota, Juncus effusus), in einem WaldmeisterBuchenwald ebenso untypisch wie die Zunahme von Ackerwildkräutern, Ruderaloder Grünlandarten, wie sie beispielsweise nach Kompensationskalkungen zu beobachten ist (SCHMIDT 2002). Nur wenige der hier für Buchenwälder als Störungszeiger zu bewerteten Arten werden auch durch natürliche Störungen (z. B. 
Windwurf, Auflichtung in der Zerfallsphase von Wäldern) gefördert (z. B. RubusArten).

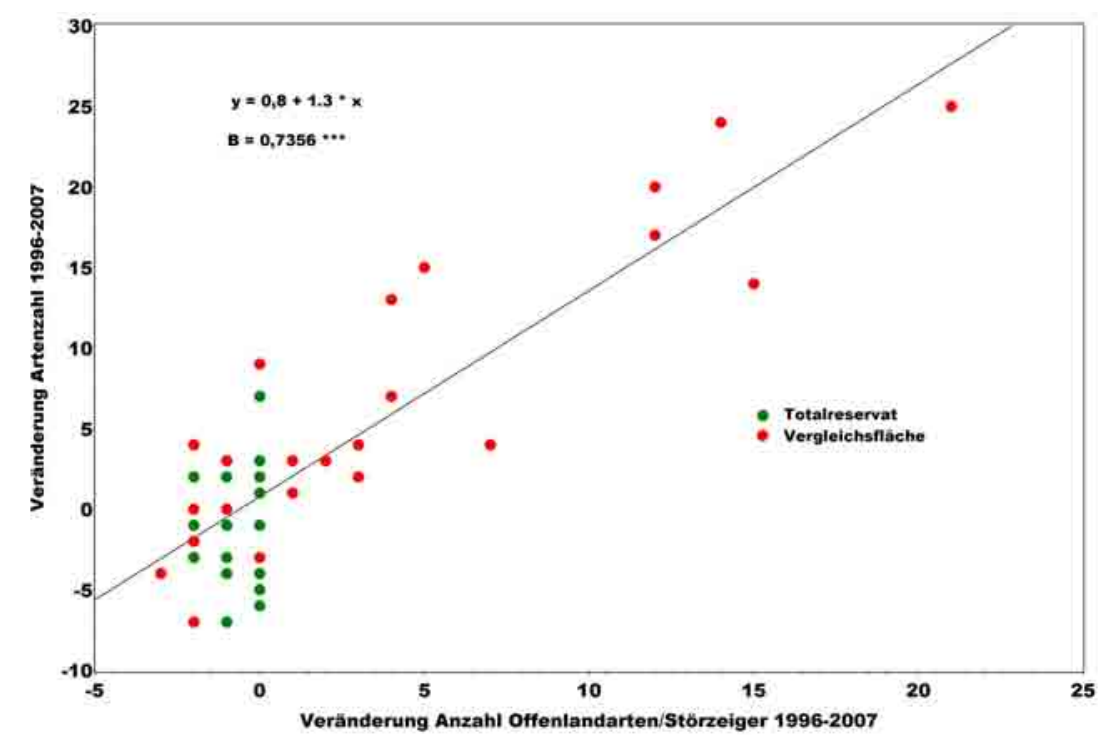

Abbildung 6: Veränderung der Artenzahl an Gefäßpflanzen in der Krautschicht in $250 \mathrm{~m}^{2}$ großen $V$ egetationsaufnahmeflächen im NWR Hohestein von 1996 bis 2007 in Abhängigkeit von der Veränderung an Arten des Offenlandes und von Störungszeigern $(B=$ Bestimmtbeitsmaß, *** $=p>F<0,001$ )

\subsection{Käfer}

Mittlerweile liegen für vier Buchen-NWR in Hessen umfangreiche Inventuren der Arthropodenfauna nach einer Standard-Methodik vor (DOROW et al. 1992). Die gefangenen Individuen wurden bis auf Artniveau bestimmt.

Die dabei ermittelten Artenzahlen können Aufschluss über die Vollständigkeit der untersuchten Lebensgemeinschaften geben. Dazu müssen sie anhand eines Vergleichsmaßstabs bewertet werden. Mindestvoraussetzungen für eine solche Bewertung ist die Kenntnis des Artenbestandes in den Wäldern des jeweiligen Bezugsraums (hier: Bundesland Hessen). Diese Voraussetzung ist für die Gruppe der Käfer gegeben. Hier ist der Kenntnisstand so weit fortgeschritten, dass KÖHLER u. KLAUSNITZER (1998) eine Liste aller in Deutschland vorkommenden Arten, ihrer Verteilung auf die Bundesländer und ihrer Habitat- und Biotoppräferenzen erarbeiten konnten. Als waldtypisch werden in der nachfolgenden Auswertung die dort mit der Biotoppräferenz „Wald-/Gehölzbiotope allgemein“ und „Wald-/Gehölzbiotope offene Strukturen“ eingestuften Arten angesehen. Durch diese Eingrenzung wird die Zahl der betrachteten Käferarten zwar erheblich einge- 
schränkt. Dies erscheint jedoch angesichts der hohen Zahl an Offenlandarten (s. Tab. 2) notwendig, um den Waldbezug der Auswertungen zu gewährleisten. Obwohl der Wissensstand bei den Käfern vergleichsweise weit fortgeschritten ist, ist aber zurzeit keine spezifische Analyse für Buchenwälder möglich.

Tabelle 2: $\quad$ Anzabl der Käferarten in vier Naturwaldreservaten und in Hessen nach Biotoppräferenz.

\begin{tabular}{lcccc}
\hline $\begin{array}{l}\text { Bezugs- } \\
\text { fläche }\end{array}$ & $\begin{array}{c}\text { Anzahl } \\
\text { Arten }\end{array}$ & \multicolumn{3}{c}{ Davon mit Biotoppräferenz: } \\
\cline { 3 - 5 } & & Offenland & $\begin{array}{c}\text { Wald/Gehölze } \\
\text { offene } \\
\text { Strukturen }\end{array}$ & $\begin{array}{c}\text { Geschlossene, } \\
\text { zonale Wälder }\end{array}$ \\
\hline GZ & 666 & 231 & 45 & 334 \\
SC & 736 & 270 & 56 & 334 \\
NI & 920 & 358 & 69 & 381 \\
HO & 698 & 238 & 53 & 326 \\
Hessen & 4.782 & 1.244 & 588 & 1.050 \\
\hline
\end{tabular}

Abkürzungen: GZ = NWR Goldbachs- und Ziebachsrück, SC = NWR Schönbuche, NI = NWR Niddahänge, $\mathrm{HO}=$ NWR Hohestein

Die Rote Liste der Käfer Deutschlands (GEISER 1998) wird als Maßstab zur Bewertung der Vollständigkeit der untersuchten Waldlebensgemeinschaften herangezogen. Wenn diese ein zutreffendes Bild von der Gefährdungslage der Käfer zeichnet, dann müssen die dort gelisteten Arten signifikant seltener gefunden werden als die nicht gefährdeten Arten. Ihr tatsächlich gefundener Anteil am Artenbestand wäre demnach unterproportional zu ihrem erwarteten Anteil, wie er sich aus dem Gesamtbestand an waldtypischen Käferarten in Hessen herleiten lässt. Als Maßzahl für die Abweichung vom Erwartungswert bietet sich die prozentuale Differenz zwischen dem tatsächlichen und dem erwarteten Artenanteil bei der gegebenen Artenzahl an (Gleichung 1). Mit dem Chiquadrat-Test wird geprüft, ob tatsächliche und erwartete Verteilung signifikant voneinander abweichen.

$\% \operatorname{Diff}_{\text {Erwartungswert }}=\left(\frac{\mathrm{pi}_{\text {beobachtet }}-\mathrm{pi}_{\text {erwartet }}}{\mathrm{pi}_{\text {erwartet }}}\right) \cdot 100 \quad$ (Gleichung 1)

wobei:

$\begin{array}{ll}\% \text { Diff }_{\text {Erwartungswert }}= & \text { prozentuale Abweichung vom Erwartungswert } \\ \text { pi }_{\text {beobachtet }} & \begin{array}{l}\text { beobachteter Anteil Arten des Gefährdungsgrades i am } \\ \text { gesamten beobachteten Artenbestand }\end{array} \\ \mathrm{pi}_{\text {erwartet }} & \begin{array}{l}\text { erwarteter Anteil Arten des Gefährdungsgrades i am gesamten } \\ \text { beobachteten Artenbestand }\end{array}\end{array}$


Die Ergebnisse bestätigen die Einstufung der Roten Liste (s. Abb. 7). Selbst bei außerordentlich hoher Untersuchungsintensität bleibt demnach die Zahl der gefundenen Arten mit Rote Liste-Status deutlich hinter der erwarteten Anzahl zurück. Überproportional vertreten sind die ungefährdeten Arten. Zwischen den NWR sind die Unterschiede äußerst gering.

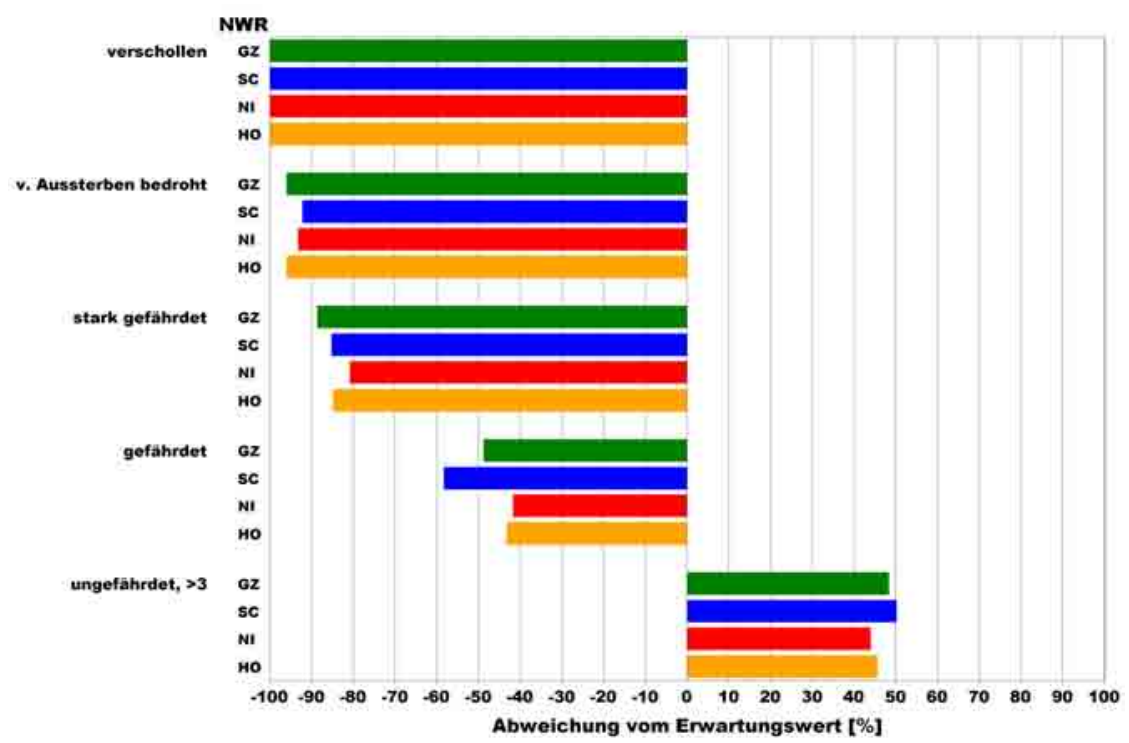

Abbildung 7: Prozentuale Abweichung zwischen gefundenen und erwarteten Artenzablen an Käfern je Gefährdungsgrad auf der Grundlage der Roten Liste der Käfer Deutschlands. Die Abweichungen zwischen der beobachteten und der erwarteten Artenzabl sind in jedem NWR signifikant ( $>$ C Chi-Quadrat <0,001). Abkürzungen der NWR s. Tab. 2, „ungefäbrdet, $>3 "=$ ungefährdete Arten oder Arten mit einem Rote Liste-Status > Kategorie 3.

Um zu klären, hinsichtlich welcher Habitate oder Strukturen die untersuchten Wald-Lebensgemeinschaften Defizite aufweisen, wird nach dem oben beschriebenen Verfahren die tatsächliche und die bei Vollständigkeit erwartete Verteilung auf die Habitatpräferenzen nach KÖHLER u. KLAUSNITZER (1998) betrachtet. Die Ergebnisse zeigen, dass die NWR insbesondere bei den Totholzbesiedlern deutlich unter dem Erwartungswert liegende Artenanteile aufweisen (s. Abb. 8).

Zum Zeitpunkt der Fauneninventuren entsprechen die erst wenige Jahre aus der Nutzung genommenen NWR typischen Buchen-Wirtschaftswäldern. Die Untersuchungsergebnisse belegen daher bewirtschaftungsbedingte Defizite in der Artenausstattung insbesondere im Hinblick auf die an Totholz gebundenen Arten. 


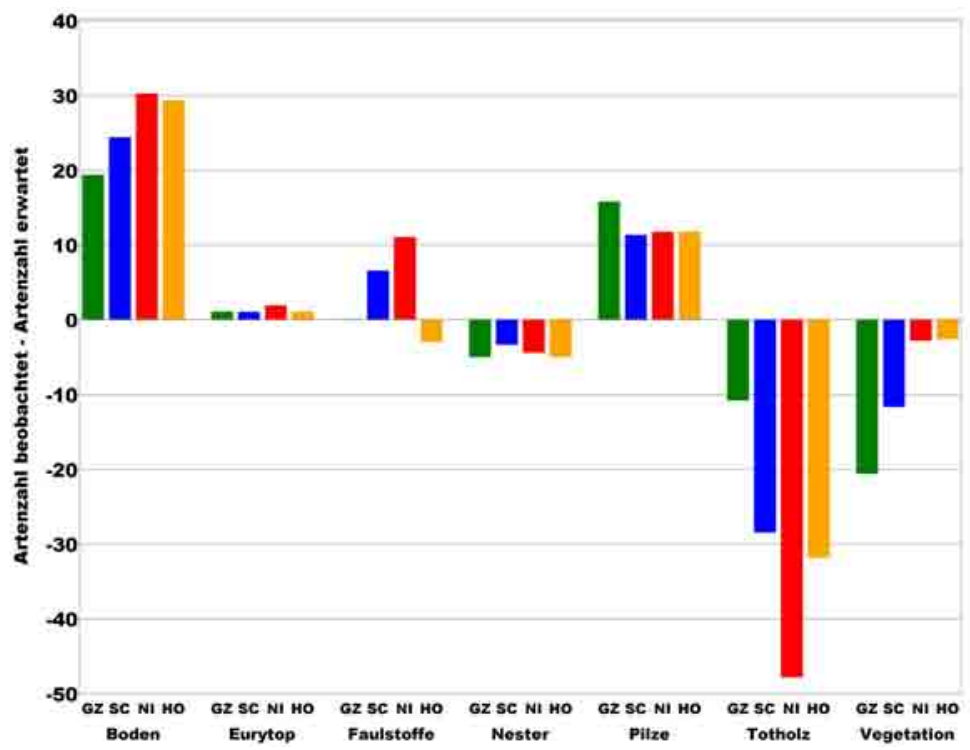

Abbildung 8: Prozentuale Abweichung zwischen gefundenen und erwarteten Artenzablen an Käfern je Habitatpräferen:. Die Abweichungen zwischen der beobachteten und der erwarteten Artenzahl sind in jedem NWR signifikant ( $p>$ Chi-Quadrat <0,001). Abkïrzungen der NWR s. Tab. 2, eurytop = obne spezifische Habitatpräferen:

\section{Strukturvielfalt}

Die Strukturvielfalt eines Waldes wird wesentlich durch die Häufigkeit und Größe von natürlichen und anthropogenen Störungen bestimmt (PICKETT u. WHITE 1985, AtTiWill 1994, Jax 1999, Dale et al. 1998, BENGTSSON et al. 2000, BÖNSEL u. MATTHES 2007). In mitteleuropäischen Wäldern führen natürlicherweise vor allem Stürme, Pilz- und Insektenbefall zur Ausbildung von Lücken und Löchern im Kronendach oder fallweise auch zum Absterben ganzer Waldbestände. Störungen lassen Strukturvielfalt entstehen - beispielsweise durch aufkommende Gehölzverjüngung oder Totholz. Nach Störungen werden aber auch die innerhalb der Lebensgemeinschaft zur Verfügung stehenden Ressourcen neu verteilt.

In mitteleuropäischen Buchenwäldern entwickelt sich die Gehölzverjüngung i. d. R. erst nach einer Störung. Dabei können zwei Grundmuster unterschieden werden: Großflächige Störungen führen häufig zu einer mehr oder weniger altersgleichen Verjüngung auf großer Fläche. Daraus gehen homogene, strukturarme Waldbestände hervor. Hingegen fördern kleinflächige Störungen die Gehölzverjüngung punktuell (und oft nur zeitlich befristet), so dass sich ein kleinräumiges Mosaik an Entwicklungsphasen und damit ein heterogen aufgebauter, struktur- 
reicher Wald herausbildet. Auch forstliche Eingriffe lassen sich als Störungen im ökologischen Sinne auffassen.

Diese Überlegungen machen deutlich, dass das Verständnis des Zusammenspiels von Störungen und Gehölzverjüngung wesentlich ist, um die Strukturvielfalt von Buchenwäldern zu charakterisieren. In den folgenden beiden Kapiteln werden daher die Struktur und Dynamik von Kronendachlücken und die Entwicklung der Gehölzverjüngung in mitteleuropäischen Buchenwäldern nach Störungen untersucht.

\subsection{Kronendachlücken}

In den zwei seit 1972 unbewirtschafteten niedersächsischen NWR Limker Strang und Lüßberg (s. Tab. 3) wurde die Lückendynamik des Kronendaches über mehrere Jahrzehnte auf der Basis von Luftbildern analysiert (MEYER u. ACKERMANN 2005). Dabei werden unter Lücken alle Unterbrechungen des geschlossenen Kronendaches mit einer Mindestgröße von $20 \mathrm{~m}^{2}$ verstanden, in denen der Nachwuchs weniger als die Hälfte der Höhe des umgebenden Bestandes erreicht hat (vgl. RUNKLE 1981). Die Luftbildzeitreihen umfassen 22 Jahre im NWR Limker Strang und 27 Jahre im NWR Lüßberg. Die zwei NWR waren von einem außergewöhnlich starken Orkan im November 1972 betroffen (KREMSER 1973). Die Kronendachlücken, die auf den Luftbildern Ende der 1970er und Anfang der 1980er Jahre zu erkennen sind, gehen auf dieses Störereignis und davor liegende Durchforstungen zurück.

Tabelle 3: $\quad$ Charakterisierung der untersuchten Naturwaldreservate

\begin{tabular}{lrr}
\hline & Limker Strang & Lüßberg \\
\hline Bezugsfläche Kronendach 2004 [ha] & 19,5 & 16,3 \\
\hline Baumarten & Buche, (Fichte) & Buche, (Eiche, Fichte) \\
\hline Alter im Jahr 2004 & 153 & 184 \\
\hline Waldtyp/FFH-LRT & $\begin{array}{r}\text { Hainsimsen- } \\
\text { Buchenwald }\end{array}$ & Hainsimsen-Buchenwald \\
\hline Wasserhaushalt & frisch - vorratsfrisch & mäßig frisch \\
\hline Nährstoffversorgung & (gut) mesotroph & schwach mesotroph \\
\hline Geologie & Löss über mittlerem & Schmelzwassersand über \\
Buntsandstein & Geschiebedecksand \\
\hline pH Oberboden Lücken & $3,2^{*}$ & $2,8^{*}$ \\
\hline C/N Oberboden in Lücken & $20^{*}$ & $27^{*}$ \\
\hline Organische Auflage in Lücken $[\mathrm{cm}]$ & $3,0^{*}$ & $5,5^{*}$ \\
\hline
\end{tabular}

* Mittelwerte nach Daten von KABER (2005) und LANGER (2006) 
Die wichtigsten Kenngrößen der Lückenstruktur zeigen, dass sich das Kronendach der untersuchten NWR nach den Störungen schließt (s. Tab. 4). So hat in beiden Gebieten die Lückenfläche und -anzahl stark abgenommen. Pro Jahrzehnt wurden durchschnittlich $38 \%$ (Limker Strang) bzw. 27\% aller Kronendachlücken komplett geschlossen. Hingegen sind, bezogen auf den jeweiligen Ausgangsbestand, nur $8 \%$ bzw. $10 \%$ neue Lücken entstanden. Auch visuell ist der zunehmende Kronenschluss deutlich zu erkennen (s. Abb. 9). Die mittlere Lückengröße ist vergleichsweise gering und liegt im Bereich der Fläche einer Altbuchenkrone (vgl. NAGEL 1999). Wie die Maximalwerte zeigen, gibt es aber in beiden Naturwäldern auch erheblich größere Öffnungen im Kronendach, die jedoch vergleichsweise selten auftreten.

Tabelle 4: $\quad$ Kennwerte der Lückenstruktur in den beiden Naturwaldreservaten und in den albanischen Buchen-Urwäldern (Mirdita, Puka und Rajca, Daten aus TABAKU 1999)

\begin{tabular}{|c|c|c|c|c|c|}
\hline Naturwald & Jahr & $\begin{array}{l}\text { Flächen- } \\
\text { anteil [\%] }\end{array}$ & $\begin{array}{l}\text { Anzahl } \\
\text { je ha }\end{array}$ & $\begin{array}{c}\text { Mittlere } \\
\text { Größe }^{* 1}\left[\mathrm{~m}^{2}\right]\end{array}$ & $\begin{array}{l}\text { Maximale } \\
\text { Größe }\left[\mathrm{m}^{2}\right]\end{array}$ \\
\hline \multirow{2}{*}{ Limker Strang } & 1982 & 10,8 & 8,5 & $127 \pm 230$ & 2.258 \\
\hline & 2004 & 2,5 & 3,2 & $77 \pm 96$ & 660 \\
\hline \multirow{2}{*}{ Lüßberg } & 1977 & 18,1 & 11,4 & $159 \pm 310$ & 3.174 \\
\hline & 2004 & 7,1 & 6,4 & $111 \pm 174$ & 1.289 \\
\hline Mirdita & 1997 & 6,6 & 9,0 & $74 \pm 54$ & 273 \\
\hline Puka & 1997 & 3,4 & 5,6 & $61 \pm 30$ & 133 \\
\hline Rajca & 1997 & 3,3 & 4,8 & $69 \pm 37$ & 209 \\
\hline
\end{tabular}

${ }^{*}$ Arithmetischer Mittelwert \pm Standardabweichung

Die Kennwerte der beiden NWR stimmen in ihrer Größenordnung recht gut mit denen aus albanischen Buchen-Urwäldern überein. Allerdings liegt dort die mittlere und maximale Lückengröße noch unter den entsprechenden Werten der NWR (zu methodischen Unterschieden s. TABAKU u. MEYER 1999).

Lückenerweiterungen treten nur selten auf. So beträgt die jährliche Rate, mit der Bäume am Lückenrand ausfallen, im NWR Limker Strang lediglich 0,12 \% und im NWR Lüßberg 0,45 \%. Im NWR Limker Strang fallen Bäume im geschlossenen Bestand mit einer fast identischen Wahrscheinlichkeit wie am Lückenrand aus $(0,10 \%)$. Im NWR Lüßberg ist die Ausfallrate im geschlossenen Bestand hingegen deutlicher geringer $(0,17 \%)$ als am Lückenrand.

Die Ergebnisse belegen die hohe Standfestigkeit und Reaktionsfähigkeit der Altbuchen in den beiden Untersuchungsgebieten, wobei allerdings die Werte des NWR Lüßberg - vermutlich alters- und/oder standortbedingt (s. Tab. 3) - deutlich unter denen im Limker Strang liegen. Dieser Unterschied setzt sich auch hinsichtlich des seitlichen Kronenwachstums der Randbäume fort (s. Tab. 5). 


\section{Limker Strang}

1982

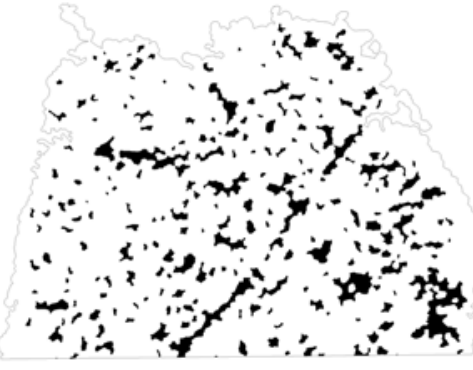

2004

\section{Lüßberg}

1977

2004
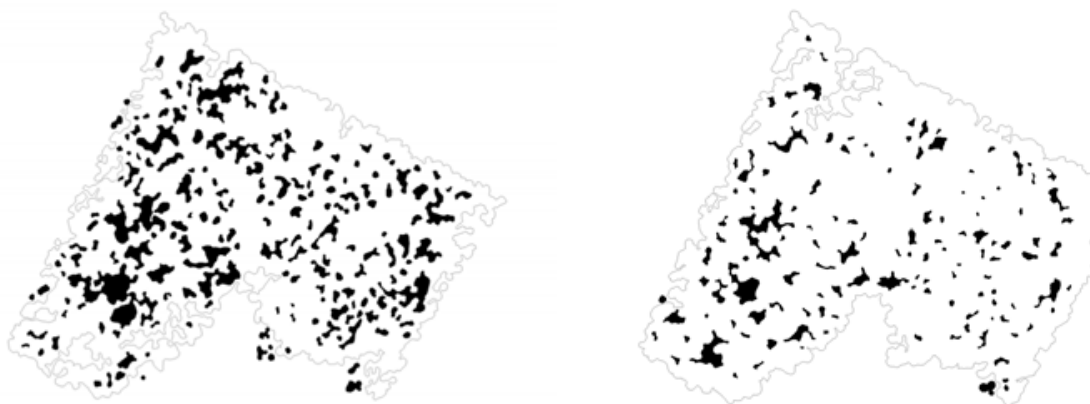

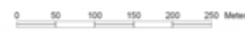

Abbildung 9: Entwicklung des Lückenmusters (Lücken in schwary) in den zwei untersuchten Naturwaldreservaten

Tabelle 5: $\quad$ Mittelwerte der seitlichen Lückenschlussrate je Jahr. Die Mittelwerte der beiden NWR sind signifikant unterschiedlich (proc npar1 way unter SAS 9.12, Mann Whitney U-Test $p>Z<0,001)$.

\begin{tabular}{lcc}
\hline Naturwald & $\begin{array}{c}\text { Anzahl Lücken } \\
\text { untersucht }\end{array}$ & $\begin{array}{c}\text { Mittlere Schlussrate } \pm \text { Standardabw. } \\
{[\mathbf{c m}]}\end{array}$ \\
\hline Limker Strang & 15 & $16,5 \pm 3,4$ \\
\hline Lüßberg & 20 & $11,9 \pm 3,2$ \\
\hline
\end{tabular}


Insgesamt wird deutlich, dass das natürliche Störungsregime in Buchenwäldern auf den meisten Standorten im Wesentlichen durch kleinflächige Störungsereignisse gekennzeichnet ist. Zusammenbrüche ganzer Bestände sind offenbar selten und treten vermutlich in Zeitabständen auf, die erheblich über der Lebensdauer einer Buchengeneration liegen. In dieser Hinsicht ähneln mitteleuropäische Buchenwälder anderen Laubmischwäldern der gemäßigten Zone. Kleinflächige, häufigere Störungen scheinen ein generelles Kennzeichen sommergrüner Laubwälder zu sein (RUNKLE 1991, TABAKU u. MEYER 1999).

\subsection{Gehölzverjüngung}

Im Jahr 2005 wurde in den beiden NWR in 30 (Limker Strang) bzw. 25 Lücken (Lüßberg) die Gehölzverjüngung untersucht. In beiden Untersuchungsgebieten besteht diese fast ausschließlich aus Rotbuchen. Die Wahrscheinlichkeit, dass sich Jungpflanzen über 1,5 m Höhe (= gesicherte Verjüngung) nach 33 Jahren (Lückenentstehung 1972, Aufnahme 2005) entwickelt haben, unterscheidet sich erheblich zwischen den beiden NWR. Obwohl der Lückenanteil im NWR Lüßberg deutlich höher ist, weisen hier nur rund ein Drittel aller beobachteten Lücken eine gesicherte Gehölzverjüngung auf. Hingegen hat sich im NWR Limker Strang in ca. $90 \%$ der Lücken gesicherte Gehölzverjüngung entwickelt. Als Erklärungsursache für den Unterschied bietet sich der Oberbodenzustand und insbesondere die unterschiedliche Höhe der organischen Auflage an. So ist der hemmende Einfluss der organischen Auflage auf die Verjüngung der Buche bekannt (BURSCHEL et al. 1964).

Trotz der erheblichen Unterschiede sind sich die beiden NWR in einem Punkt ähnlich: Gehölzverjüngung hat sich nur in Lücken, d. h. bei abgesenkter Dichte des Oberstandes entwickelt. Ihre Textur entspricht damit dem sog. „Schweizer Käse-Modell“": in die Grundmasse des dicht geschlossenen Bestandes sind scharf abgegrenzte Lücken eingesprengt, die fallweise mit Gehölzverjüngung gefüllt sind. Beobachtungen in weiteren NWR legen nahe, dass dieses Modell in gleichaltrigen, oligo- bis mesotrophen Buchenwäldern häufig zutrifft. Anders verläuft die Entwicklung allerdings auf eutrophen Standorten. Hier gewährleistet ein ständiges Vorkommen von Eschen- und Ahorn-Jungpflanzen, dass sich die Gehölzverjüngung unmittelbar im Anschluss an eine Störung entwickelt (KOMPA u. SCHMIDT 2005). Das „Schweizer Käse-Modell“ greift zu kurz, da sich unterschiedliche Entwicklungsphasen räumlich überlappen, statt horizontal scharf voneinander getrennt zu sein. Entscheidend für die Reaktionsfähigkeit eines Waldes auf Störungen ist daher vor allem die Vorverjüngung (SPURR 1956).

Die dargestellten Zusammenhänge gelten für gleichaltrige und erst wenige Jahrzehnte aus der Nutzung genommene Buchenwälder. Ob natürliche Buchenwälder eine ähnliche Verjüngungsdynamik zeigen, wird anhand der Ergebnisse von TABAKU (1999) sowie Verjüngungsinventuren in den NWR Vogelherd und Limker 
Strang (beide im Solling, Niedersachsen) aus den Jahren 1998 und 1999 untersucht. Als Vergleichsgröße für den Verjüngungserfolg wird die Trieblängensumme der Jungpflanzen je $\mathrm{m}^{2}$ Bebachtungsfläche (FEI et al. 2006) in Abhängigkeit von der Grundflächenhaltung des Oberstandes herangezogen (s. Abb. 10).

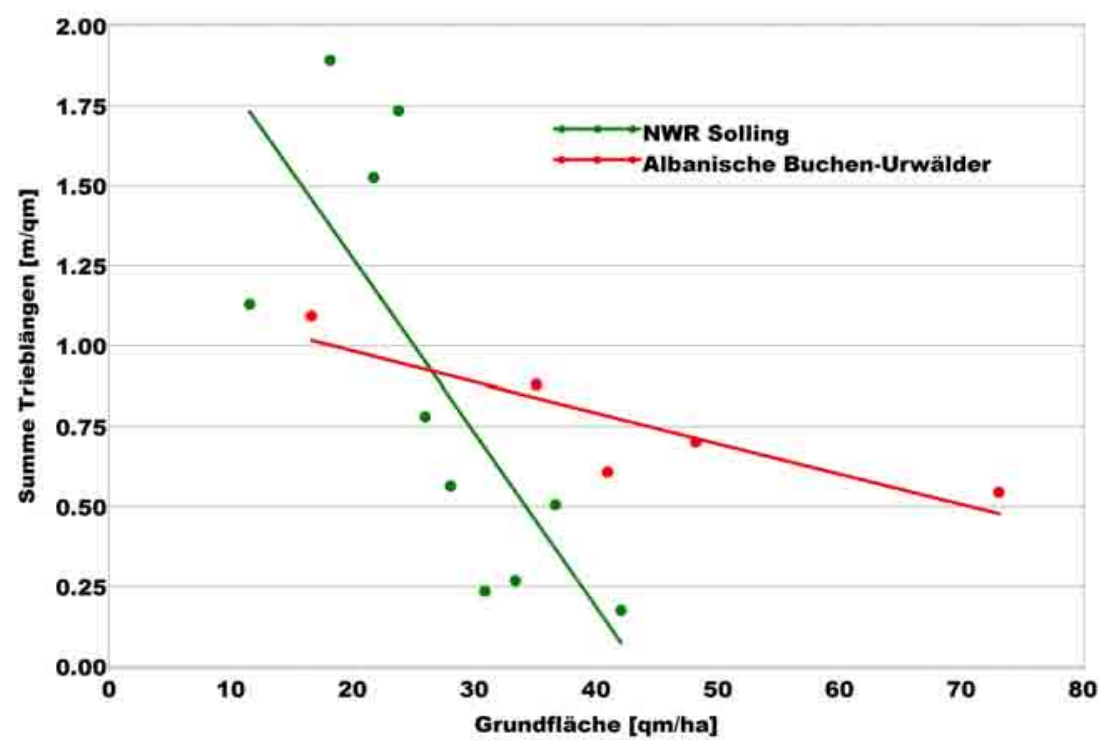

Abbildung 10: Beziebung zwischen kleinräumiger Grundflächenhaltung des Oberstandes (ermittelt in Probekereisen von $10 \mathrm{~m}$ Radius) und der Summe der Trieblängen der Gebölapflanzen < $7 \mathrm{~cm}$ BHD je $\mathrm{m}^{2}$ in Buchen-Naturwaldreservaten im Solling (NWR Solling) und Buchen-Urwäldern Albaniens. Die Wertepaare sind Mittelwerte von GrundflächenRangklassen. Die Regressionen sind in beiden Fällen signifikant (NWR: $p>F=0,0111$, Urwälder: $p>F=0,0465)$. Das Bestimmtheitsmaß beträgt für die NWR 0,5209 und für die Urwälder 0,7090.

Die Ergebnisse deuten auf erhebliche Unterschiede hinsichtlich der Dichteabhängigkeit des Verjüngungserfolgs hin. Während in den Buchen-NWR die Trieblängensumme deutlich negativ mit der Grundfläche des Derbholzbestandes korreliert, ist diese Beziehung in den Buchen-Urwäldern erheblich schwächer ausgeprägt. Selbst bei ausgesprochen hoher Grundflächenhaltung findet sich in den Urwäldern noch Gehölzverjüngung in einem erheblichen Ausmaß. Deren Präsenz selbst bei hoher Dichte des Altbestandes zeigt, dass sich in den südosteuropäischen Buchen-Urwäldern die verschiedenen Baumgenerationen sowohl zeitlich wie räumlich überlappen - offenbar ein typisches Kennzeichen (KORPEL 1995).

Als Erklärungsursache kommt vor allem der lange und vollständige Lebenszyklus von Urwäldern einschließlich älterer Entwicklungsphasen mit geringer Konkurrenzkraft des Oberstandes in Betracht. Hierdurch bestehen im Vergleich 
zu Wirtschaftswäldern bessere Bedingungen für die Etablierung und Entwicklung von Jungpflanzen. Entscheidend dürfte weiterhin sein, dass Buchen aufgrund ihrer Schattenfestigkeit über sehr lange Zeiträume unter einem geschlossenen Kronendach existieren können (DENGLER 1931). Diese Präsenz von Vorverjüngung garantiert schließlich ein unmittelbares Ineinandergreifen von Störereignis und Entwicklung der Verjüngung. Der Einfluss des Standortes auf das Zusammenspiel zwischen Störungen und Verjüngung wurde in Buchen-Urwäldern allerdings bisher nicht systematisch untersucht.

\subsection{Zusammensetzung nach Entwicklungsphasen}

Waldentwicklungsphasen klassifizieren den Entwicklungszustand eines in gewissen Grenzen homogenen und räumlich zusammenhängenden Baumkollektivs. Prinzipiell wird zwischen den Phasen Etablierung, Aufbau, Reife und Zerfall unterschieden. Dieses Grundschema wird allerdings je nach Untersuchungsansatz mehr oder weniger stark abgewandelt (vgl. LEIBUNDGUT 1959, ZUKRIGL et al. 1963, Korpel 1995, MeYer 1999, TABAKU 1999, Drössler u. MeYer 2006). Das Verteilungsmuster der Entwicklungsphasen spiegelt den Lebenszyklus, das Störungsregime und auch die Strukturvielfalt eines Waldes wider.

In der nachfolgenden Auswertung wird die von DRÖSSLER u. MEYER (2006) auf der Grundlage der Arbeit von TABAKU (1999) entwickelte Methodik zur numerischen Bestimmung von Waldentwicklungsphasen verwendet. Die Phasenausscheidung erfolgt auf 12,5 x 12,5 m großen Unterflächen.

Als Datengrundlage werden die Aufnahmen von TABAKU (1999) und DRÖSSLER (2006) in Buchen-Wirtschaftswäldern, -Naturwaldreservaten und -Urwäldern genutzt. Es handelt sich ausschließlich um von der Rotbuche dominierte Bestände. Betrachtet wird die relative Zusammensetzung nach Waldentwicklungsphasen (s. Abb. 11).

Die Phasenzusammensetzung der slowakischen und albanischen Urwälder ist sehr ähnlich (s. Abb. 11). Davon weichen die Wirtschaftswälder und das seit rund 35 Jahren ungenutzte Buchen-NWR Limker Strang erheblich ab. Plenter-, Altersund Zerfallsphase fehlen in der letztgenannten Gruppe weitgehend. Das seit rund 150 Jahren aus der Nutzung genommene NWR Heilige Hallen zeichnet sich durch Gleichaltrigkeit der fortschreitend zerfallenden Altbäume aus (TABAKU u. MEYER 1999). Diese Kohortenstruktur auf größerer Fläche dürfte der wichtigste Grund für den hohen Anteil an Lücken und jungen Entwicklungsphasen sein. Die Buchen-Wirtschaftswälder sind durch einen insgesamt deutlich verkürzten Lebenszyklus gekennzeichnet, in dem strukturreiche und fortgeschrittene Entwicklungsphasen kaum eine Rolle spielen. Infolge der Plenterbewirtschaftung erreicht allerdings der Buchenbestand Bleicherode einen mit Urwäldern vergleichbaren Anteil der Plenterphase. 


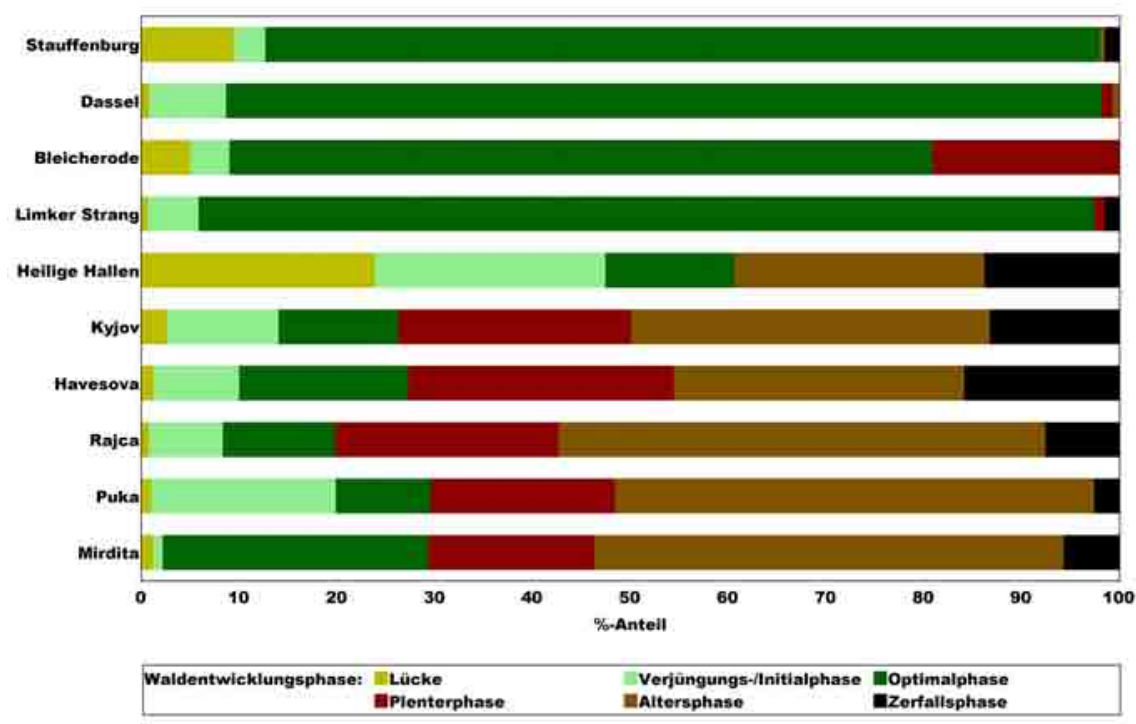

Abbildung 11: Anteile der verschiedenen Waldentwicklungsphasen in Buchen-Wirtschaftswäldern (Zielstärkennutzung = Stauffenburg und Dassel, Plenterwald = Bleicherode), Bu-Naturwaldreservaten (seit rd. 35 Jahren aus der Nutzung = Limker Strang, ca. 150 Jahre ungenutzt $=$ Heilige Hallen) und Bu-Urwäldern (Ostslowakei $=$ Kyjov und Havesova, Albanien = Rajca, Puka und Mirdita)

Die Ergebnisse verdeutlichen, dass der Entwicklungszyklus von Buchenwäldern durch die forstliche Bewirtschaftung verkürzt und um wesentliche Phasen reduziert wird. In den fünf Urwäldern ist bei bemerkenswert großer Übereinstimmung die Strukturvielfalt erheblich höher als in den Wirtschaftwäldern.

\subsection{Totholz}

Als wesentliches Strukturelement der Alters-, Zerfalls- und Verjüngungsphase spielt Totholz eine zentrale Rolle für die biologische Vielfalt in natürlichen Buchenwäldern (SCHUCK et al. 2004). Vergleichende Studien zeigen, dass ungenutzte Buchenwälder Totholzvorräte von durchschnittlich $130 \mathrm{~m}^{3} /$ ha enthalten (CHRISTENSEN et al. 2005). Welchen Effekt das Aussetzen der forstlichen Nutzung auf die Totholzdynamik hat, wird nachfolgend näher betrachtet.

In insgesamt 9 über rund 25 Jahre beobachteten Buchen-Untersuchungsflächen (1,0-1,5 ha Flächengröße) wurde die Totholzdynamik retrospektiv analysiert. Auf der Grundlage der Angaben von MÜLLER-USING (2005) über die Dauer von Zersetzungsgraden und den Volumenabbau mit fortschreitender Zersetzung wurden rückwirkend die Input- und Output-Raten modelliert. Aus der Bilanz der beiden Größen ergibt sich der im jeweiligen Jahr vorhandene Totholzvorrat (s. Abb. 12). 
Im Mittel der Jahre und Untersuchungsflächen beträgt die Differenz zwischen Input und Output 1,10 $\mathrm{m}^{3}$ je Hektar und Jahr (Spannweite <0,01-4,36). Nach 25 Jahren kann durchschnittlich der Aufbau einer Totholzmenge von rund $25 \mathrm{~m}^{3} \mathrm{je}$ Hektar erwartet werden. In diesem Zeitraum werden Mengen akkumuliert, die im Bereich der Schwellenwerte für das Vorkommen gefährdeter Arten liegen (BÜTLER u. SCHLÄPFER 2004, MÜLLER et al. 2007). Durch Nutzungsverzicht können demnach bereits mittelfristig signifikante Totholzmengen aufgebaut werden. Anlass für die aktive Schaffung von Totholz dürfte es daher nur in Ausnahmefällen geben.

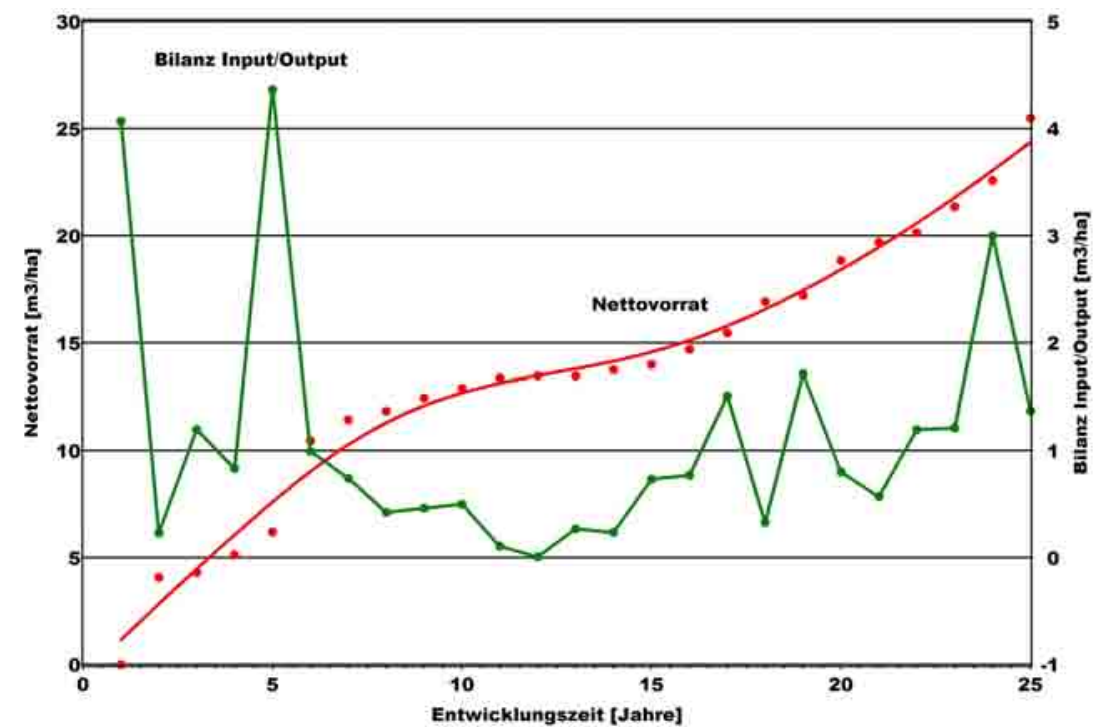

Abbildung 12: Nettovorrat und Differenz zwischen Input- und Output an Totholz $>7 \mathrm{~cm}$ Durchmesser in Abhängigkeit von der Dauer eigendynamischer Entwicklung (= Entwicklungszeit) in Buchen-Naturwaldreservaten. Mittelwerte aus 9 Buchen-Untersuchungsflächen (Altersspanne der Bestände im Beobachtungszeitraum 100-160 Jahre).

\section{Schlussfolgerungen}

Aus den vorgestellten Ergebnissen lassen sich folgende Eckpunkte für eine naturnahe Buchenwirtschaft im Sinne der Konvention zur Biologischen Vielfalt ableiten:

Eine einzelstamm- bis femelartige Bewirtschaftung kommt dem bisher bekannten natürlichen Störungsregime mitteleuropäischer Laubwälder am nächsten und kann daher als naturnahes Waldbauverfahren für Buchenwälder bestätigt werden. Einschränkend ist allerdings festzuhalten, dass die natürliche Häufigkeit großflächiger Störungen in mitteleuropäischen Buchenwäldern bisher nicht genau bekannt ist. Zudem können auch einzelstamm- bis femelartige Eingriffe bei einem aus ökonomischen Gründen nach wie vor verkürzten Lebenszyklus zu homogen aufgebauten 
Beständen führen (SCHÜTZ 2002). Kleinflächige Eingriffe verhindern eine übermäßige Förderung konkurrenzstarker Störungszeigerarten in der Bodenvegetation und schaffen Vielfalt der Wuchsbedingungen durch kleinräumige Wechsel. Die Ausbreitungspotenziale der einheimischen Waldarten werden nicht überfordert (BRUNET u. OHEIMB 1998, BRUNET 2007). Homogene Eingriffe auf großer Fläche wie Kahl- oder Schirmschläge haben hingegen einen negativen Einfluss auf die Artenvielfalt der Bodenvegetation wie auch der epiphytischen Moose und Flechten (ECKHARDT et al. 2003, OHEIMB 2003, TEUBER 2006).

Lange Verjüngungszeiträume greifen das für Urwälder typische Überlappen der Baumgenerationen auf und erhöhen gleichzeitig die Wahrscheinlichkeit für eine erfolgreiche Naturverjüngung.

Anhand der Vergleichsuntersuchungen von Naturwaldreservaten und Wirtschaftswäldern zeigt sich, dass die typische Ausprägung der Bodenvegetation wesentlich vom Ausmaß der Bodenstörungen abhängt. Hier liegt ein Konflikt zwischen Naturnähe und Bewirtschaftung vor, der nicht grundsätzlich gelöst, jedoch durch den Verzicht auf flächiges Befahren und einen möglichst weiten Abstand zwischen Rückegassen vermindert werden kann. Während sich der erstgenannte Grundsatz mittlerweile in allen üblichen Bewirtschaftungs- und Zertifizierungsstandards findet, ist bezüglich der derzeitig üblichen Abstände zwischen Rückegassen noch Diskussionsbedarf gegeben.

Naturnahe Buchenwälder sind häufig arm an Mischbaumarten. Diese Tatsache ist kein Mangel, sondern ein wesentliches Kennzeichen, das für die Erhaltung einer typischen Lebensgemeinschaft von großer Bedeutung ist (AMMER u. UTSCHICK 2004). Ausreichend große Komplexe von mebr oder weniger reinen Buchenwäldern obne gesellschaftsfremde Baumarten sind daher ein wichtiges Kennzeichen einer naturnahen Buchenwirtschaft. Die Auswertungen zur Buchenwaldverbreitung bestätigen eine starke Fragmentierung im nordwestdeutschen Tiefland. Die Einbringung von gesellschaftsfremden Mischbaumarten in die verbliebenen Restbestände sollte hier konsequent unterbleiben, um deren typische Vielfalt zu erhalten.

Durch ihre intensiven Anstrengungen, Nadelwälder in Richtung Laub- und Laubmischwälder umzubauen, hat die Forstwirtschaft bereits einen signifikanten Anstieg der Buchenwaldfläche erreicht. Während in einigen Gebieten naturräumlich und geschichtlich bedingt ein hoher Buchenanteil zu verzeichnen ist, bestehen in anderen Regionen noch erhebliche Defizite (z. B. nordwestdeutsches Tiefland). Die Anstrengungen zum Waldumbau sollten auf diese Defiziträume konzentriert werden.

Aus ökonomischen Gründen kann die Verkürzung des Lebenszyklus in Wirtschaftswäldern grundsätzlich nicht vermieden, sondern nur vermindert werden. Durch punktuellen oder flächenhaften Nutzungsverricht (Einzelbäume, Altholzinseln, Naturwaldreservate, Großschutzgebiete) lassen sich die damit einhergehenden Verluste der biologischen Vielfalt verhindern. Entsprechende Konzepte sind bereits entwickelt worden und befinden sich zurzeit in der Umsetzung (STEIN 
1981, NiedersäCHSISCHE LANDESFORSTEN 2000, MÜlLER et al. 2007). Ihre Wirksamkeit konnte bestätigt werden (JEDICKE 2006, BUSSLER et al. 2008). Um das Ziel eines ausreichenden Lebensraumangebotes für die an Altbäume und Totholz gebundenen Arten auf größerer Fläche zu erreichen, erscheint allerdings eine weitergehende Verbreitung und konsequentere Umsetzung erforderlich.

Die Schwellenwerte für ein Totholzangebot, das die Situation gefährdeter Arten wirksam verbessern kann, liegen auf einem so hohen Niveau $\left(>30 \mathrm{~m}^{3} / \mathrm{ha}\right.$, s. BÜTLER u. SCHLAEPFER 2004, MÜLLER et al. 2007), dass die entsprechenden Mengen kaum dauerhaft auf ganzer Fläche bereitgestellt werden können. Damit rückt die Frage der räumlichen Schwerpunktsetzung und der Vernetzung von Lebensräumen in den Vordergrund (Biotopverbund, vgl. JEDICKE 1990, AMLER et al. 1999, HARRISON u. BRUNA 1999, BURKHARDT et al. 2003, BAILLEY 2007). Eine stärkere Orientierung der waldbaulichen und naturschutafachlichen Planung an landschaftsökologischen Zusammenhängen erscheint insgesamt notwendig, um das Ziel einer Erhaltung der biologischen Vielfalt in unseren Wäldern bei gleichzeitiger Realisierung ökonomischer Ziele zu erreichen.

\section{Danksagung:}

Wir möchten uns bei allen Personen bedanken, die mit der Bereitstellung von Daten, der Mitwirkung bei der Auswertung und durch ihre Diskussionsbereitschaft einen besonderen Anteil am Zustandekommen des vorliegenden Beitrags haben. Besonders danken wir den Herren Frank Koehler (Bornheim), Theo Blick, Dr. Wolfgang H. O. Dorow und Dr. Jens-Peter Kopelke (Forschungsinstitut Senckenberg, Frankfurt), Dr. Jan Evers und Dr. Uwe Paar (Sachgebiet Waldzustand und Boden der NW-FVA) sowie Herrn Jörg Ackermann (Sachgebiet Luftbild und GIS der NW-FVA).

\section{Literatur}

ABEL, W. (1978): Geschichte der deutschen Landwirtschaft vom frühen Mittelalter bis zum 19. Jahrhundert. 3. Aufl., Stuttgart, 370 S.

ABS, C. (Red.) (1996): Buchenwälder. Ihr Schutz und ihre Nutzung. $100 \mathrm{~S}$.

ABS, C.; FISCHER, A. u. FALINSKI, J. B. (1999): Vegetationsökologischer Vergleich von Naturwald und Wirtschaftswald, dargestellt am Beispiel des Tilio-Carpinetum im Waldgebiet von Bialowieza/Nordost-Polen. Forstw. Cbl., 118, 181-196

AChtZiger, R.; Stickroth, H. u. Zieschank, R. (2004): Nachhaltigkeitsindikator für die Artenvielfalt - ein Indikator für den Zustand von Natur und Landschaft in Deutschland. Angewandte Landschaftsökologie, 63, 1-5 und 72-75

Amler, K.; Bahl, A.; Henle, K.; Kaule, G.; Poschlod, P. u. Settele, J. (Hrsg.) (1999): Populationsbiologie in der Naturschutzpraxis. Verlag Eugen Ulmer, 336 S.

AMMER, U. u. UTSCHICK, H. (2004): Folgerungen aus waldökologischen Untersuchungen auf hochproduktiven, nadelholzreichen Standorten für eine an Naturschutzzielen orientierte Waldwirtschaft. Forst und Holz, 59, 119-128 
ATTIWILL, P.M. (1994): The disturbance of forest ecosystems: the ecological basis for conservative management. Forest Ecology and Management, 63, 247-300

BAILEY, S. (2007): Increasing connectivity in fragmented landscapes: An investigation of evidence for biodiversity gain in woodlands. Forest Ecology and Management, 238, 7-23

BeHrE, K.-E. (2000): Der Mensch öffnet die Wälder - Zur Entstehung der Heiden und anderer Offenlandschaften. Rundgespr. Komm. Ökol., 18, 103-116

Bengtsson, J.; Nilsson, S.; Franc, A. u. Menozzi, P. (2000): Biodiversity, disturbances, ecosystem function and management of European forests. Forest Ecology and Management, 132, 39-50

BFN (Bundesamt FÜr NATURSChuTZ) (Hrsg.) (1997): Erhaltung der biologischen Vielfalt. Wissenschaftliche Analyse deutscher Beiträge. Landwirtschaftsverlag GmbH, Münster, 352 S.

BFN (Bundesamt FÜR NATURSCHUTZ) (2004): Daten zur Natur 2004. Landwirtschaftsverlag, Münster, 474 S.

BFN (BundesAmT FÜR NATURSCHUTZ) (2008): Bonner Thesen zum „Naturerbe Buchenwälder“.

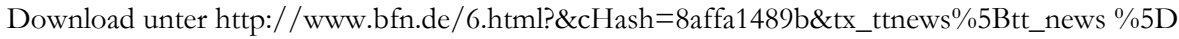
$=1115$

Bieling, C. u. SChramL, U. (2003): Was ist der Natur näher als der Wald? Zur Wahrnehmung des Waldzustandes durch private Eigentümer. Allgemeine Forst und Jagdzeitung, 175, 41-48

Binot-Hafke, M.; Gruttke, H.; Ludwig, G. u. Riecken, U. (2000): Bundesweite Rote Listen Bilanzen, Konsequenzen, Perspektiven. BfN-Schriftenvertrieb im Landwirtschaftsverlag, $255 \mathrm{~S}$.

Binot-Hafke, M.; Martens, H.; Boye, P.; Gruttke, H.; Haupt, H. u. Pretscher, P. (2003): Entwicklungen im zoologischen Artenschutz. Schriftenreihe des Deutschen Rates für Landespflege, $75,43-51$

BLAB, J. (2003): Leistungen im Gebietsschutz. Schriftenreihe des Deutschen Rates für Landespflege, $75,52-60$

BLAB, J. (2005): Rote Listen - Etappen und Meilensteine einer Erfolgsgeschichte. Naturschutz und Biologische Vielfalt, 18, 7-20

BÖnSEL, A. u. MAtTheS, J. (2007): Prozessschutz und Störungsbiologie - Naturschutzthesen seit dem ökologischen Paradigmenwechsel vom Gleichgewicht zum Ungleichgewicht der Natur. Natur und Landschaft, 82, 323-327

BRUNET, J. (2007): Plant colonization in heterogenous landscapes: an 80-year perspective on restoration of broadleaved forest vegetation. J. Appl. Ecol., 44, 563-572

BRUNET, J. u. OHEIMB, G. v. (1998): Migration of vascular plants to secondary woodlands in southern Sweden. J. Ecol., 86, 429-438

BRÜNN, S. (1992): Kleinräumige Vegetations- und Standortsdifferenzierung in einem Kalkbuchenwald. Diplomarb. Syst.-Geobot. Inst. Univ. Göttingen, 112 S.

BÜCKING, W. (2003): Naturwaldreservate. „Urwald in Deutschland“. Bonn, 66 S.

Burkhardt, R.; Baier, H.; Bendzko, U.; Bierhals, E.; Finck, P.; Jenemann, K.; Liegl, A.; Mast, R.; Mirbach, E.; Nagler, A.; Pardey, A.; Riecken, U.; Sachteleben, J.; Schneider, A.; SzeKely, S.; Ullrich, K.; Hengel, U. v. u. Zeltner, U. (2003): Naturschutzfachliche Kriterien zur Umsetzung des \3 BNatSchG "Biotopverbund". Natur und Landschaft, 78, 418-427

Burschel, P.; Huss, J. u. KALBhenN, R. (1964): Die natürliche Verjüngung der Buche. Schriftenr. Forstl. Fak. Univ. Göttingen, 34, 1-186

Bussler, H.; Blaschke, M. u. Walentowski, H. (2008): Das Rothenbucher Totholz- und Biotopbaum-Konzept. AFZ/Der Wald, 4, 200-203

BÜtLER, R. u. SCHLAEPFER, R. (2004): Wie viel Totholz braucht der Wald? Schweizerische Zeitschrift für Forstwesen, 155, 31-37

Christensen, M.; Hahn, K.; Mountford, E.; Ódor, P.; STAndovár, T.; ROZenbergar, D.; Diaci, J.; Wijdeven, S.; Meyer, P.; Winter, S. u. VRSKA, T. (2005): Dead wood in European beech (Fagus sylvatica) forest reserves. Forest Ecology and Management, 210, 267-282 
Dale, V. H.; Lugo, A. E.; MacAhon, J. u. Pickett, S. T. A. (1998): Ecosystem management on the context of large infrequent disturbances. Ecosystems, 1, 546-557

Dengler, A. (1931): Aus den südosteuropäischen Urwäldern. II: Die Ergebnisse einer Probeflächenaufnahme im Buchenwald Albaniens. Zeitschrift für Forst- und Jagdwesen, 62, 20 ff.

DFWR (2008): Biologische Vielfalt in Deutschlands Wäldern. Positionspapier des Deutschen Forstwirtschaftsrates e. V. (DFWR). Internetquelle: http://www.dfwr.de/download/DFWRPosition-Biologische $\% 20$ Vielfalt.pdf

DierschKe, H. (1989): Artenreiche Buchenwald-Gesellschaften Nordwest-Deutschlands. Ber. Reinh. Tüxen-Ges., 1, 107-148

Dohrenbusch, A. u. Rosin, A. (2002): Waldbaukonzepte im nationalen Vergleich. Tagung der Sektion Waldbau im Deutschen Verband Forstlicher Forschungsanstalten, 11.-13.09.2002, Berlin, 27-40

Dorow, W. H. O.; FlechtNer, G. u. KopelKe, J.-P. (1992): Naturwaldreservate in Hessen: Zoologische Untersuchungen Konzept. Naturwaldreservate in Hessen, 3, 1-159

Dorow, W. H. O. u. KopelKe, J.-P. (2007): Naturwaldreservate in Hessen. 7/2.2. Hohestein. Zoologische Untersuchungen 1994 - 1996, Teil 2. Mitt. Hess. Landesforstverw., 42, 1-341

DrösSLER, L. (2006): Struktur und Dynamik von zwei Buchenurwäldern in der Slowakei. Diss. Fak. Forstwiss. Waldökol. Univ. Göttingen, $101 \mathrm{~S}$.

DrÖsSLER, L. u. MEYER, P. (2006): Waldentwicklungsphasen in zwei Buchen-Urwaldreservaten in der Slowakei. Forstarchiv 77, 155-161

EBRECHT, L. (2005): Vegetation, Standortverhältnisse und Ausbreitungsbiologie von Pflanzen auf Rückegassen und Waldwegen im Göttinger Wald und im Solling. Göttingen, 317 S.

ECKHARDT, M.; GÜNZL, B.; SCHMIDT, M. u. KRIEBITZSCH, W.-U. (2003): Welche Faktoren beeinflussen die Artenvielfalt baumbewohnender Flechten in Laubwäldern? AFZ/DerWald, 58, 10831085

FAO (2007): State of the world's forests: 2007. FAO, Rom

Fei, S.; Gould, P. J.; STEINER, K. C. u. FInLEY, J. C. (2006): Aggregate height - a composite measure of stand density for tree seedling populations. Forest Ecology and Management, 223, 336-341

GAertig, T. u. GREeN, K. (2008): Die Waldbodenvegetation als Weiser für Bodenstörungen. AFZDerWald 6/2008, 300-301

GÄrTNER, S. u. ENGELHARD, J. (2005): Diversität versus Naturnähe - Einfluss von Baumarten auf die Bodenvegetation im Südschwarzwald und auf der Mittleren Schwäbischen Alb. Ber. Freib. Forstl. Forsch. 60, 37-47

Geiser, R. (1998): Rote Liste der Käfer. In: BinOT, M. et al.: Rote Liste gefährdeter Tierarten Deutschlands. Schriftenr. Landschaftspfl. Natursch. 55, 168-230

GolisCH, A. (2002): Waldgesellschaften grundwasserferner Standorte im südwestlichen Rheinischen Schiefergebirge unter besonderer Berücksichtigung wärmeliebender Wälder und ihrer Standortbedingungen. Diss. Bot. 357, 1-246

GrabHerR, G.; KOCH, G.; KIRChMEIR, H. u. ReITER, K. (1998): Hemerobie österreichischer Waldökosysteme. Veröff. Österr. MaB-Prog., 17, 1-493

Günther, A.; Nigmann, U.; Achtziger, R. u. Gruttke, H. (2006): Analyse der Gefährdungsursachen planungsrelevanter Tiergruppen in Deutschland. Natursch. Biol. Vielf., 21

HaArmann, K. u. Pretscher, P. (1993): Zustand und Zukunft der Naturschutzgebiete in Deutschland. Die Situation im Süden und Ausblicke auf andere Landesteile. Schriftenr. Landschaftspfl. Natursch., 39, 1-266

HÄRDTLE, W. (1995): Vegetation und Standort der Laubwaldgesellschaften (Querco-Fagetea) im nördlichen Schleswig-Holstein. Mitt. Arbeitsgem. Geobot. Schlesw.-Holst. Hamb., 48, 1-441

HARRISON, S. u. BRUNA, E. (1999): Habitat fragmentation and large-scale conservation: what do we know for sure? Ecography, 22, 225-232

Heinken, T. (in prep.): Synopsis der Pflanzengesellschaften Deutschlands. Dicrano-Pinion 
HEINKEN, T. (1995): Naturnahe Laub- und Nadelwälder grundwasserferner Standorte im niedersächsischen Tiefland: Gliederung, Standortsbedingungen, Dynamik.. Diss. Bot. 239, 1-311

Heinken, T. u. ZipPEL, E. (1999): Die Sand-Kiefernwälder (Dicrano-Pinion) im norddeutschen Tiefland: syntaxonomische, standörtliche und geographische Gliederung. Tuexenia, 19, 55-106

Hesmer, H. (1937): Die heutige Bewaldung Deutschlands. Berlin, 52 S.

Hesmer, H. u. Schroeder, F.-G. (1963): Waldzusammensetzung und Waldbehandlung im Niedersächsischen Tiefland westlich der Weser und in der Münsterschen Bucht bis zum Ende des 18. Jahrhunderts. Decheniana Beih., 11, 1-304

HMULV (HESSISCHES MINISTERIUM FÜR UMWELT, LÄNDLICHEN RAUM UND VERBRAUCHERSCHUTZ) (2006): Hessen - ein Buchenland. BWI² Bundeswaldinventur. Wiesbaden, 45 S.

JAHN, G. (1979): Zur Frage der Buche im nordwestdeutschen Flachland. Forstarchiv, 50, 85-95

JAHN, G. (1996): Von der ursprünglichen zur heutigen potentiellen Vegetation. Natur- \& Kulturlandschaft 1, 16-20

JAX, K. (1999): Natürliche Störungen: ein wichtiges Konzept für Ökologie und Naturschutz? Zeitschrift Ökologie u. Naturschutz, 7/4, 241 - 253

JEDICKE, E. (1990): Biotopverbund. Grundlagen und Maßnahmen einer neuen Naturschutzstrategie. Ulmer, Stuttgart, $254 \mathrm{~S}$.

JEDICKE, E. (2006): Altholzinseln in Hessen - Biodiversität in totem Holz. Grundlagen für einen Altund Totholz-Biotopverbund. HGON, $80 \mathrm{~S}$.

JENSCH, D. (2004): Der Einfluss von Störungen auf Waldbodenvegetation. Experimente in drei hessischen Buchenwäldern. Diss. Bot. 386: 1-388

KABER, T. (2005): Lückendynamik und Buchennaturverjüngung im Naturwald Limker Strang. Unveröff. Diplomarb. Fachhochsch. Hildesh.-Holzminden, Fachber. Forstwirtsch., Göttingen, $92 \mathrm{~S}$.

Krug, A.; Höltermann, A. u. Klein, M. (2006): Hundert Jahre Naturschutz und Landnutzung zwischen Konfrontation, Ideologie und neuen Allianzen. Natur und Landschaft, 81, 27-31

Köhler, F. u. Klausnitzer, B. (Hrsg.) (1998): Verzeichnis der Käfer Deutschlands. Entomol. Nachr. Ber., Beih. 4, 1-185

KOMPA, T. u. SCHMIDT, W. (2005): Buchenwald-Sukzession nach Windwurf auf Zechstein-Standorten des südwestlichen Harzvorlandes. Hercynia, 38, 233-261

KorPel, S. (1995): Die Urwälder der Westkarpaten. Stuttgart, Jena, New York, 310 S.

KREMSER, W. (1973): Lacerati turbine ventorum - vom Sturm zerfetzt! Ein Orkan verheert Niedersachsens Wälder. Neues Archiv für Niedersachsen, 22, 219 ff.

LANA (LÄNDERARBEITSGEMEINSCHAFT FÜR NATURSCHUTZ, LANDSCHAFTSPFLEGE UND ERHOLUNG) (1992): Lübecker Grundsätze des Naturschutzes. Schriftenreihe LANA, 3.

LANGER, M. (2006): Lückendynamik und Naturverjüngung im Naturwald Lüßberg. Unveröff. Diplomarb. Fachhochsch. Hildesh.-Holzminden, Fachber. Forstwirtsch., Göttingen, 85 S.

LeIBundguT, H. (1959): Über Zweck und Methodik der Struktur- und Zuwachsanalysen von Urwäldern.. Schweiz. Zeitschr. f. Forstwesen, 110, 111-124

LeuschneR, C. (1998): Mechanismen der Konkurrenzüberlegenheit der Rotbuche. Ber. Reinh.Tüxen-Ges., 10, 5-18

LEusCHNER, C. (1999): Zur Abhängigkeit der Baum- und Krautschicht mitteleuropäischer Waldgesellschaften von der Nährstoffversorgung des Bodens. Ber. Reinh.-Tüxen-Ges., 11, 109-131

LEusCHNER, C. u. IMMENROTH, J. (1994): Landschaftsveränderungen in der Lüneburger Heide 1770 1985. Dokumentation und Bilanzierung auf der Grundlage historischer Karten. Arch. Natursch. Landschaftsforsch., 33, 85-139

MAst, R. (1999): Vegetationsökologische Untersuchung der Feuchtwald-Gesellschaften im niedersächsischen Bergland. Arch. naturwiss. Diss., 8, 1-283

MeYer, P. (1999): Bestimmung der Waldentwicklungsphasen und der Texturdiversität in Naturwäldern. Allgemeine Forst- und Jagdzeitschrift, 170, 203-211 
Meyer, P. u. Ackermann, J. (2005): Muster und Dynamik von Kronendachlücken in drei bodensauren Buchen-Naturwäldern. Berichte Freiburger Forstliche Forschung, 60, 89-98

Meyer, P.; Wevell v. Krüger, A.; Steffens, R. u. Unkrig, W. (2006): Naturwälder in Niedersachsen - Schutz und Forschung. Band 1. Alfeld, 339 S.

MÜller, J.; Bußler, H. u. UtschicK, H. (2007): Wie viel Totholz braucht der Wald? - Ein wissenschaftsbasiertes Konzept gegen den Artenschwund der Totholzzönosen. Naturschutz und Landschaftsplanung, 39, 165-170

Müller-Using, S. (2005): Totholzdynamik eines Buchenbestandes im Solling. Ber. Forschungszentrums Waldökosysteme, Reihe A, 193, 1-175

NABU (Naturschutzbund Deutschland E.V., Bundesverband) (2008): Waldwirtschaft 2020. Perspektiven und Anforderungen aus Sicht des Naturschutzes. Berlin, 67 S.

NAGEL, J. (1999): Konzeptionelle Überlegungen zum schrittweisen Aufbau eines waldwachstumskundlichen Simulationssystems für Nordwestdeutschland. Schr. Forstl. Fak. Univ. Göttingen Nieders. Forstl. Versuchsanst., 128, 1-122

NipKOW, M. (2005): Zum Wert Roter Listen für den Artenschutz und die Naturschutzpolitik. Naturschutz und Biologische Vielfalt, 18, 187-197

NiEdERSÄCHSISCHE LANDESFORSTEN (2000): Habitatbäume und Totholz im Wald. Merkblatt Nr. 38 der Niedersächsischen Landesforsten, $16 \mathrm{~S}$.

NML (NIEDERSÄCHSISCHES Ministerium FÜR DEN LÄNDLICHEN RAUM, ERNÄHRUNG, LANDWIRTSCHAFT Und VerbraucherschutZ) (2004): Der Wald in Niedersachsen. Ergebnisse der Bundeswaldinventur II. Aus dem Walde. Schriftenr. Waldentwicklung Niedersachs., 55, 1-43

OHeImB, G. v. (2003): Einfluss forstlicher Nutzung auf die Artenvielfalt und Artenzusammensetzung der Gefäßpflanzen in norddeutschen Laubwäldern. - Schriftenr. Naturwiss. Forschungsergebnisse, 1-261

PANEK, N. (2007): Naturerbe im Würgegriff. Zur Situation der deutschen Buchenwälder im Natura 2000-Netz. Nationalpark, 2, 26-30

PANEK, N. (2008): Rotbuchenwälder in Deutschland. Beitrag zur Umsetzung einer Schutzstrategie. Naturschutz und Landschaftsplanung, 40, 140-146

PicketT, S. T. A. u. White, P. S. (1985): The ecology of natural disturbance and patch dynamics. Academic Press, Orlando, 472 S.

Pommerening, A. u. Murphy, S. T. (2004): A review of the history, definitions and methods of continuous cover forestry with special attention to afforestation and restocking. Forestry, 77, 2744

RApp, H.-J. u. SchMidt, M. (2006): Baumriesen und Adlerfarn. Der „Urwald Sababurg“ im Reinhardswald. Kassel, $192 \mathrm{~S}$.

ReIF, A. (1992): Zur Kritik von VOLK und SCHLENK in Forst und Holz, 46 (1991), Heft 24, S. 687-693: "Rote Listen und Forstwirtschaft. Der Wald - kein sicherer Schutz für gefährdete Pflanzen?" Forst und Holz, 47, 151-155

REIF, A. (2000): Das naturschutzfachliche Kriterium der Naturnähe und seine Bedeutung für die Waldwirtschaft. Z. Ökol. Natursch., 8, 230-250

RUNKLE, J. R. (1981): Gap regeneration in some old-growth forests of the eastern United States. Ecology, 62, 1041-1051

RunkLE, J. R. (1991): Gap Dynamics of Old-Growth Eastern Forests: Management Implications. Natural Areas Journal, 11 (1), 19-25

SACHVERSTÄNDIGENRAT FÜR UMWELTFRAGEN (2000): Umweltgutachten 2000: Schritte ins nächste Jahrtausend. Stuttgart, 114 S.

SACHVERSTÄNDIGENRAT FÜR UMWELTFRAGEN (Hrsg.) (2004): Umweltpolitische Handlungsfähigkeit sichern. $650 \mathrm{~S}$

SCHERZINGER, W. (1997): Kritische Formulierung einer Zieldiskussion zum Naturschutz im Wald. Schr. Aktivitäten des Ersten Voralberger Coleopterologischen Vereins, 10, 1-68 
SCHMid, H. u. HeLfer, W. (1999): Die Bedeutung der Naturwaldreservate für den Pilzartenschutz. NUA-Seminarber., 4, 140-146

Schmidt, M.; Ellenberg, H.; Heuveldop, J.; Kriebitzsch, W.-U. u. Oheimb, G. v. (2002): Wichtige Einflussfaktoren auf die Gefäßpflanzen-Artenvielfalt von Wäldern. Treffpunkt Biol. Vielfalt, 113-118

Schmidt, M.; Ewald, J.; Fischer, A.; Oheimb, G. V.; Kriebitzsch, W.-U.; Schmidt, W. u. ElLENBERG, H. (2003): Liste der Waldgefäßpflanzen Deutschlands. Mitt. Bundesforschungsanst. Forst- Holzwirtsch., 212, 1-34

SCHmidt, M. u. MeYer, P. (Red.) (2007): Hessische Naturwaldreservate im Portrait: Hohestein. Kassel, $34 \mathrm{~S}$.

SCHмidT, M. u. SCHмIDT, W. (2007): Vegetationsökologisches Monitoring in Naturwaldreservaten. Forstarchiv, 78, 205-214

SCHмidT, W. (1999): Bioindikation und Monitoring von Pflanzengesellschaften - Konzepte, Ergebnisse, Anwendungen, dargestellt an Beispielen aus Wäldern. Ber. Reinh.-Tüxen-Ges., 11, 133155

SCHмIDT, W. (2002): Einfluss der Bodenschutzkalkung auf die Waldvegetation. Forstarchiv, 73, 4354

SChreIBER, D.; KeItel, W. u. SCHMidT, W. (1999): Hohestein. Waldkundliche Untersuchungen (Schwerpunkt Flora und Vegetation). Naturwaldreservate in Hessen 7/1, 1-191

Schroeder, F.-G. (1998): Lehrbuch der Pflanzengeographie. Wiesbaden, 457 S.

SCHuCK, A.; MeYer, P.; Menke, N.; LIER, M. u. Lindner, M. (2004): Forest biodiversity indicator: Dead wood: a proposed approach towards operationalising the MCPFE indicator. In: Marchetti, M. (Hrsg.). Monitoring and indicators of forest biodiversity in Europe - from ideas to operationality. EFI Proceedings, 51, 49-77

SCHÜTZ, J. PH. (2002): Silvicultural tools to develop irregular and diverse forest structures. Forestry, $75,327-337$

SPURR, S. H. (1956): Natural restocking of forests following the 1938 hurricane in Central New England. Ecology, 37, 443-451

SsymanK, A. (1997): Schutzgebiete für die Natur: Aufgaben, Ziele, Funktionen und Realität. In: Erdmann, K.-H. u. Spandau, L. (Hrsg. 1997): Naturschutz in Deutschland. Strategien, Lösungen, Perspektiven. Stuttgart, 11-38

Ssymank, A.; Hauke, U.; RÜCKriem, C. u. SChröder, E. (1998): Das europäische Schutzgebietssystem NATURA 2000. Schriftenr. Landschaftspfl. Natursch., 53, 1-560

STEIN, J. (1981): Biotopschutzprogramm Altholzinseln im hessischen Wald. Beih. Veröff. Naturschutz Landschaftspflege Bad. Württ., 20, 91-110

TABAKU, V. (1999): Struktur von Buchen-Urwäldern in Albanien im Vergleich mit deutschen Buchen-Naturwaldreservaten und -Wirtschaftswäldern. Göttingen, 206 S.

TABAKU, V. u. MEYER, P. (1999): Lückenmuster in Buchenwäldern unterschiedlicher Nutzungsintensität. Forstarchiv, 70, 87-97

TEuber, D. (2006): Ergebnisse flechtenkundlicher Untersuchungen aus vier bodensauren Buchenwäldern. Mitt. Hess. Landesforstverw., 40, 1-86

UTSCHIK, H. (2001): Schutzstrategien für Waldpilze. LWF-Ber., 33, 14-17

VolK, H. (1997): Schutz der Buchenwälder in Deutschland - isoliert in Schutzgebieten oder integriert in die Forstwirtschaft? Forst und Holz, 52, 267-274

WeCKEsser, M. (2002): Die Bodenvegetation von Buchen-Fichten-Mischbeständen im Solling Struktur, Diversität und Stoffhaushalt. Göttingen, 157 S.

WiLKE, C. (2007): Waldland Hessen. AFZ/Der Wald, 62, 798-801

WiNTERHOFF, W. (1963): Vegetationskundliche Untersuchungen im Göttinger Wald. Nachr. Akad. Wiss. Göttingen Math.-Phys. Kl., 2, 21-79 
ZACHARIAS, D. (1996): Flora und Vegetation von Wäldern der Querco-Fagetea im nördlichen Harzvorland Niedersachsens - unter besonderer Berücksichtigung der Eichen-Hainbuchen-Mittelwälder. Natursch. Landschaftspfl. Nieders., 35, 1-150

Zukrigl, K.; ECKHARDT, G. u. NAther, J. (1963): Standortskundliche und waldbauliche Untersuchungen in Urwaldresten der niederösterreichischen Kalkalpen. Mitt. Forstl. Bundesversuchsanstalt, 62, Wien

Korrespondierender Autor:

Dr. Peter Meyer

Nordwestdeutsche Forstliche Versuchsanstalt

Grätzelstr. 2

37073 Göttingen

E-Mail: Peter.Meyer@nw-fva.de

URL: www.nw-fva.de

Dr. Marcus Schmidt

Nordwestdeutsche Forstliche Versuchsanstalt 


\section{Waldumbau mit Buche unter Berücksichtigung ihrer ökologischen Ansprüche}

\section{Forest conversion to beech in consideration of its ecological demands}

Hendrik Rumpf und Regina Petersen

\section{Zusammenfassung}

Die neuartigen Waldschäden in den 1980er Jahren haben in Deutschland bis heute zu einem verstärkten Umbau gleichaltriger Nadelholz-Reinbestände in Mischbestände im Wege des Buchen-Voranbaus geführt. Nach einem Abriss der wichtigsten verjüngungsökologischen Eigenschaften der Buche werden vor diesem Hintergrund zwei Versuche der NW-FVA zum Waldumbau exemplarisch vorgestellt und unter Würdigung einschlägiger Veröffentlichungen zu diesem Problemkreis diskutiert.

Auf der ersten Fläche wurden Wachstum und Qualität vorangebauter Buchen unter einem Kiefernbaumholz bei verschiedenen Dichten des Kronenschirmes und verschiedenen Pflanzverbänden untersucht. Mit Ausnahme des Höhenwachstums wurde die Entwicklung der Buchen bislang maßgeblich durch den Pflanzverband beeinflusst. Eine sehr starke Absenkung des Bestockungsgrades förderte das Durchmesserwachstum der Buchen nur geringfügig, führte aber zu erheblichen Zuwachsverlusten bei den Altkiefern. 
Auf der zweiten Fläche wurden die Auflaufergebnisse und die Überlebensrate einer Buchensaat unter Fichtenschirm analysiert. Des Weiteren wurde das Wachstum der gekeimten Buchen in Konkurrenz zur vorhandenen Fichten-Naturverjüngung bei unterschiedlichen Überschirmungsverhältnissen untersucht. Die Überlebensrate der Saat betrug nach 5 Jahren lediglich $5 \%$. Die etablierten Buchenpflanzen konnten sich im gesamten untersuchten Strahlungsbereich gegenüber der vorhandenen Fichtennaturverjüngung durchsetzen.

Stichworte: Waldumbau, Verjüngungsökologie, Buchenvoranbau, Pflanzverband, Buchensaat, Lichtsteuerung

\begin{abstract}
The forest dieback caused by acid rain in Germany in the 1980s led to the intensified conversion of pure evenaged coniferous stands to mixed stands by underplanting beech. After a summary of the most important characteristics of beech regeneration ecology, two forest conversion experiments conducted by the Northwest German Forest Research Station (NW-FVA) are introduced here by way of example, and the issues discussed in a review of the relevant literature.

At the first site, the growth and quality of the beech planted as underplantings in a Scots pine stand at different canopy cover densities and different plant spacings were investigated. So far, with the exception of height growth, primarily plant spacing has influenced the development of beech. A major reduction in the stocking level promotes beech diameter growth only minimally, yet leads to considerable losses in growth increment in mature Scots pine.

At the second site, the germination rate and survival rate of beech sown under a Norway spruce canopy have been analysed. In addition, the growth of newly germinated beech seedlings competing against the existing Norway spruce natural regeneration was investigated under different canopy cover conditions. After five years, the survival rate of the plants germinated from seed was only $5 \%$. The established beech seedlings competed successfully with existing Norway spruce natural regeneration across the range of radiation conditions investigated.
\end{abstract}

Keywords: forest conversion, regeneration ecology, beech underplanting, plant spacing, beech seed, light regulation 


\section{Einleitung}

\subsection{Waldumbau - Ziele und Tendenzen}

Unter Waldumbau wird das Ergreifen von Maßnahmen verstanden, welche Veränderungen des Waldgefüges, insbesondere der Baumartenzusammensetzung, zum Ziel haben. Vor dem Hintergrund sich bedrohlich entwickelnder immissionsbedingter Waldschäden (ULRICH 1986) wurde Mitte der 1980er Jahre in den Waldschadensschwerpunkten - beispielsweise im Harz oder im Erzgebirge - mit einem gezielten Waldumbau begonnen. Wichtigster Beweggrund für die Umwandlung von Fichtenreinbeständen in Mischbestände und den Übergang zu einzelstammweisen bis femelartigen Hiebsformen mit längeren Verjüngungszeiträumen war zunächst die Minderung bzw. Streuung des Betriebsrisikos (OTTO 1986).

In der Folge sind die Anforderungen an einen anspruchsvollen Waldbau, wie sie im Zuge der Ausrichtung forstlichen Handelns am Leitbild einer multifunktionalen, nachhaltigen Forstwirtschaft gestellt werden, immer vielgestaltiger geworden. Neben der Rio-Konvention bilden die Nachhaltigkeitskriterien von Helsinki, 1993, welche die Erhaltung der biologischen Vielfalt, der Produktivität, der Verjüngungsfähigkeit sowie der Vitalität der Waldbestände gewährleisten und damit auch für künftige Generationen die Option zur Multifunktionalität sicherstellen sollen, eine wichtige Entscheidungsgrundlage für Waldumbaumaßnahmen (vgl. WAGNER 2004 u. 2007).

In nahezu allen Waldbauprogrammen der Länder sowie in den Förderrichtlinien für den Privatwald wurde die Laub- und Mischwaldvermehrung als feste Zielgröße einer naturnahen Waldwirtschaft implementiert (u. a. OTTO 1995, WiCKEL u. ButTER 1998, V. TEUfFEL 1999, WOLLBORN 2000). Der Umbau von größtenteils nicht standortgerechten Nadelholzreinbeständen in naturnahe, standortgerechte und stabilere Laub- und Mischwälder wurde auf sehr großer Fläche vorangetrieben, wobei die Buche hierbei aufgrund ihrer ökologischen bzw. verjüngungsökologischen Eigenschaften bislang eine herausragende Stellung einnimmt. Die Zunahme der mit Buche bestockten Waldfläche von 12,9\% im Jahr 1987 (Bundeswaldinventur (BWI) I) auf 14,8 \% im Jahr 2002 (BWI II) unterstreicht deren Bedeutung für den Waldumbau (BMVEL 2004).

Der Umfang der Baumartenverschiebungen und die damit verbundenen Investitionen wurden anfangs recht selten kritisch hinterfragt (RIPKEN $1996 \mathrm{u}$. 1998, v. LÜPKE 1996, SPELLMANN 1997). Unter gestiegenem Kostendruck ist die Forstpraxis heute aber mehr denn je gezwungen Rationalisierungspotenziale auszuschöpfen. Zielsetzung und Umfang von Waldumbaumaßnahmen werden nicht zuletzt auch wegen der global zu beobachtenden Veränderungen am Holzmarkt zunehmend kritisch diskutiert (MÖHRING 2004, SPELLMANN 2005).

Bei der Bestandesbegründung wird deshalb heute bereits in vielen Forstbetrieben ein weitaus differenzierteres Vorgehen favorisiert, das die standörtliche 
und waldbauliche Ausgangssituation noch stärker berücksichtigt und sich an Leitbildern mit deutlich umrissenen Verjüngungs- und Bestandeszielen orientiert. Nicht zuletzt vor dem Hintergrund der verheerenden „Kyrill-Schäden“ sind die Forderungen nach einem zielorientierten Waldumbau mit effizientem Einsatz knapper Ressourcen lauter geworden.

Darüber hinaus zeichnet sich ab, dass die derzeit prognostizierten Klimaveränderungen und deren Auswirkungen auf die klimatische Wasserbilanz für künftige Waldumbaumaßnahmen in vielen Wuchsgebieten an Entscheidungsrelevanz gewinnen werden.

\subsection{Verjüngungsökologie der Buche}

Die Rotbuche (Fagus sylvatica L.) nimmt unter unseren heimischen Baumarten eine herausragende Stellung ein. Dank ihrer großen Konkurrenzkraft würde sie von Natur aus etwa $66 \%$ der Waldfläche in Deutschland bedecken, in den alten Bundesländern wären es sogar ca. 80 \% (LEUSCHNER 1998). Die Buche dominiert hierbei in einer weiten Standortamplitude sowohl hinsichtlich des Bodenfeuchtegehaltes als auch der Basensättigung (ELLENBERG 1996).

Für den in der Regel über Saat oder Pflanzung laufenden Waldumbau mit der Buche bildet die Verfügbarkeit genetisch hochwertigen Vermehrungsgutes eine wichtige Grundvoraussetzung (vgl. KLEINSCHMIT 1999). Daneben ist die Beachtung ihrer verjüngungsökologischen Eigenschaften von wesentlicher Bedeutung für den Verjüngungserfolg.

Die Buche blüht etwa von April bis Mai, wobei die Blütenbildung maßgeblich von der Witterung des Vorjahres bestimmt wird. Bei Fehlen einer Mast im Vorjahr führen überdurchschnittlich hohe Temperaturen im Juni und Juli verbunden mit deutlich geringeren Niederschlägen zu vermehrtem Blütenansatz (WACHTER 1964, SCHMIDT 2001). Nach GRUBER (2003) nehmen auf die Fruchtbildung der Buche die Witterungsfaktoren bestimmter Monate über einen Zeitraum von mindestens drei Jahren Einfluss. Im Zusammenwirken von steigenden Sommertemperaturen mit hohen N-Einträgen scheint sich die Fruktifikationsneigung der Buche in Häufigkeit und Ausmaß merklich zu erhöhen (PAAR et al. 2000, SCHMIDT 2001). Für das Ausreifen der Samen sind das Ausbleiben stärkerer Spätfröste und eine ausreichende Wasserversorgung während der Sommermonate Voraussetzung. Die Hauptfallzeit der Bucheckern liegt in den Monaten Oktober (Höhepunkt) und November. Der Samenertrag bei Vollmast kann bis zu $900 \mathrm{~kg} / \mathrm{ha}$ betragen.

Samen- und Keimlingsverluste durch Fressfeinde - Tauben, Finken, Mäuse, Wild - und Pilze, wie z. B. Rhizoctonia solani, Phytophthora spec., können sehr hoch sein (vgl. NIELSEN 1977), wobei die Gefahr des Verpilzens bei Mineralbodenkontakt allgemein geringer einzuschätzen ist (BRESSEM 1998, DUBBEL 1989 u. 1992). 
Im Gegensatz zur Eiche beeinflusst der Strahlungsgenuss bereits in der ersten Vegetationsperiode das Keimlingswachstum der Buche (u. a. WELANDER u. OTTOSSON 1998). Wie bei anderen Baumarten auch führt bei mehrjährigen Jungpflanzen bereits mäßige Beschattung zu einer Verlagerung der Biomasseproduktion von der Wurzel zum Spross, was zu einer Erweiterung des Spross/WurzelVerhältnisses führt (s. BURSCHEL u. SCHMALTZ 1965). Aus den Ergebnissen vieler Untersuchungen lässt sich eine nichtlineare Beziehung zwischen dem Wachstum junger Buchen und der Strahlungszufuhr ableiten. Innerhalb einer Spanne von etwa 10-20\% relativer Beleuchtungsstärke wird neben der Gesamtbiomasseproduktion auch das wesentlich weniger sensitiv auf die Beleuchtungsverhältnisse reagierende Höhenwachstum bereits erheblich eingeschränkt (u. a. GRALLA et al. 1997, LEDER u. WAGNER 1996, PAMPE 2000). Die Wachstumsreaktionen stehen häufig in enger Beziehung zur Nährstoff- und Wasserversorgung, wobei eine verbesserte Ressourcenverfügbarkeit die Photosyntheseleistung und damit die Schattentoleranz meist deutlich erhöht (u. a. BURSCHEL u. HuSS 1964, KLUMPP u. KAZDA 2000, KERKMANN 2007). Ein Überleben der Buche ist zweifelsohne auch bei relativen Beleuchtungsstärken unter $5 \%$ über längere Zeit möglich.

Insgesamt vermag die Buche in der Etablierungs- und frühen Jugendwachstumsphase auch bei sehr ungünstigen Verhältnissen zu überleben, was ihr insbesondere bei starker Beschattung Konkurrenzvorteile gegenüber lichtbedürftigeren Baumarten verschafft. Andererseits ist sie in der Lage, auf eine verbesserte Licht-, Nährstoff- und Wasserversorgung schnell mit gesteigertem Wachstum zu reagieren (MADSEN 1995).

\section{Versuche der NW-FVA zum Umbau von Nadelholzrein- beständen mit Buche}

Der Umbau von Nadelholzreinbeständen ist darauf ausgerichtet, standortgerechte, stabile und ertragreiche Wälder zu begründen. Eingriffe in den Stoffhaushalt sollen dabei minimiert und Finanzmittel sparsam eingesetzt werden. Bereits Anfang der 1990er Jahre wurde auf die potenziellen Möglichkeiten der „,biologischen Rationalisierung der Bestandesbegründung“ hingewiesen (z. B. RIPKEN 1992). Neben der Verwendung geeigneter Herkünfte und qualitativ hochwertigen Vermehrungsgutes wurden in der Reduzierung der Ausgangspflanzenzahlen, insbesondere über Voranbauten unter Schirm, durch Ausnutzung von Füll- und Treibholz oder Verwendung von Großpflanzen, größere Rationalisierungspotenziale gesehen. Auch die Saat, als ein mit der Naturverjüngung am ehesten vergleichbares Verjüngungsverfahren, kam als kostengünstige Alternative wieder mehr ins Gespräch.

Nachfolgend werden Versuchsergebnisse von zwei Versuchsanlagen zu den Themenkomplexen „Buchenvoranbau unter Kiefernschirm“ und „Buchenvoraussaat unter Fichtenschirm" vorgestellt. 
2.1 Versuch Unterlüß 1200 - Buchenvoranbau unter Kiefernschirm

\subsubsection{Versuchsanlass und Versuchsanlage}

Im nordwestdeutschen und nordostdeutschen Tiefland sollen beträchtliche Kiefernreinbestandsflächen in stabile Laub- oder Laub-Nadel-Mischbestände umgewandelt werden. Die Kiefer wird in ihrer führenden Position überwiegend durch Eiche, Buche oder Douglasie abgelöst. Neben ökologischen Vorteilen, wie der Verbesserung der Standorteigenschaften und der Reduzierung des Kalamitätsrisikos gegenüber biotischen und abiotischen Schadeinflüssen, versprechen sich die Forstbetriebe aus den zu entwickelnden Beständen eine Ertragssteigerung. Insbesondere für die Laubholzanteilflächen wird zunehmend gefordert, auf geeigneten Standorten qualitativ hochwertiges Holz zu erziehen. Entscheidende Weichenstellungen für das Wachstum und die qualitative Entwicklung erfolgen neben der Standortwahl über die Pflanzendichte, welche in erheblichem Maße die Höhe der Bestandesbegründungskosten bestimmt.

Zur Klärung der Frage, wie sich mit unterschiedlichen Pflanzverbänden und unter verschiedenen Schirmstellungen vorangebaute Buchen unter Kiefer entwickeln und inwieweit Wechselwirkungen zwischen Schirmdruck und Pflanzendichte zu erwarten sind, wurde 1994 im früheren Forstamt Sprakensehl ein Voranbauversuch angelegt. Neben der Buche umfasst der Versuch auch Traubeneiche und Douglasie. Die weiteren Ausführungen fokussieren sich jedoch ausschließlich auf die Buche.

Die Versuchfläche befindet sich im südöstlichen Teil des Wuchsbezirkes Hohe Heide, im heutigen Niedersächsischen Forstamt Unterlüß. Das Klima zeichnet sich durch einen Jahresniederschlag von $730 \mathrm{~mm}$ aus, von denen $330 \mathrm{~mm}$ in der forstlichen Vegetationszeit fallen. Die Jahresdurchschnittstemperatur beträgt $8,0^{\circ} \mathrm{C}$, in der forstlichen Vegetationszeit werden durchschnittlich $14,3{ }^{\circ} \mathrm{C}$ erreicht. Der Wuchsbezirk gilt als besonders spätfrostgefährdet. Das Ausgangssubstrat der Bodenbildung ist charakterisiert durch eine Deckschicht aus anlehmigem bis schwach anlehmigem Geschiebesand über in 40-50 cm Tiefe liegendem Schmelzwassersand, woraus sich eine podsolige Braunerde entwickelt hat. Es handelt sich um einen überwiegend mäßig frischen bis mäßig sommertrockenen Standort mit einer schwachen bis mäßigen Nährstoffversorgung, welcher in Niedersachsen für den vorrangigen Anbau von Douglasie mit Buche vorgesehen ist.

In dem bei Versuchsanlage 43-jährigen Kiefernbestand (0,7. Ekl. nach Ertragstafel Wiedemann, 1943) wurde die Buche im Sortiment $2+0$ in den Pflanzverbänden

$-\quad 1,5 \times 1 \mathrm{~m}$
$-\quad 2,0 \times 1 \mathrm{~m}$
$-\quad 3,0 \times 1 \mathrm{~m}$ 
in jeweils $20 \times 20$ m große Parzellen gepflanzt. Der Voranbau erfolgte versuchsbedingt in diesem frühen Entwicklungsstadium der Kiefer, um ausgehend von homogenen Bestandesverhältnissen ohne Störungslücken die Schirmstellung gezielt variieren zu können. Jeder Pflanzverband wurde unter den Zielbestockungsgraden (Ertragstafel Wiedemann, 1943)

$$
\begin{array}{ll}
\text { - } & B^{\circ} 0,4(72 \%) \\
\text { - } & B^{\circ} 0,6(58 \%) \\
\text { - } & B^{\circ} 0,8(46 \%)
\end{array}
$$

wiederholt. Zusätzlich wurden die Strahlungsverhältnisse mit Hilfe hemisphärischer Photos (WAGNER 1994a) quantifiziert. Die gemittelten Schätzwerte für die relative Beleuchtungsstärke sind den oben aufgeführten Zielbestockungsgraden in Klammern angehängt. Die Pflanzverbandsvarianten innerhalb gleicher Zielbestockungsgrade wiesen durchweg vergleichbare Strahlungsverhältnisse auf. Auf den Versuchsparzellen wurde jegliche ankommende Naturverjüngung in regelmäßigen Abständen entfernt, um den Einfluss von Pflanzverband und Schirm weitgehend „störungsfrei“ herausarbeiten zu können.

Auf den insgesamt 9 Versuchsparzellen wurden je Parzelle 5 Aufnahmeplots mit einem festen Radius von $3 \mathrm{~m}$ für die Erhebung der quantitativen und qualitativen Parameter ausgewählt. Die Messung von BHD und Höhe erfolgte an allen Buchen im Plot, die Erfassung der qualitätsbestimmenden Merkmale erfolgte an den 2 höchsten Bäumen je Aufnahmeplot. Folgende Qualitätsparameter wurden erfasst:

- Relative Ansaţ̧öhbe des ersten Grünastes

- Anzahl Grünäste auf den ersten 2 m Baumlänge

- Relative Ansatzhöhe des stärksten Grünastes auf den ersten 2 m Baumlänge

- Durchmesser des stärksten Grünastes

- Einschätzung der Stammform in 4 Stufen (gerade, bogig, knickig, verbuscht)

- Erfassung der Verzwieselung

Die statistische Analyse der Aufnahmedaten erfolgte mit dem Programm „SPSS“, Version 15.0. Die verschiedenen Parameter wurden einer mehrfaktoriellen Varianzanalyse unterzogen. Anwendung fand ein gemischtes lineares Modell unter Einbeziehung der Aufnahmeplots als sogenannter „,nested factor“. Die Zählwerte wurden vor der Analyse einer Wurzeltransformation unterzogen. Bei Vorliegen signifikanter Faktorwirkungen wurden Unterschiede zwischen den Faktorstufen unter Berücksichtigung der Sidak-Anpassung auf dem Niveau $\mathrm{p} \leq 0,05$ auf Signifikanz überprüft, wobei sich alle Vergleiche auf modellbasierte geschätzte Mittelwerte beziehen. Auf der Grundlage dieser Schätzwerte wurden auch die graphischen Darstellungen erstellt.

In einem kleineren Nebenversuch wurde bei einem Zielbestockungsgrad von 0,6 im Kiefernschirm und einem Pflanzverband der Buche von $2 \times 1 \mathrm{~m}$ auf 
$15 \times 15$ m großen Parzellen die ankommende Naturverjüngung - überwiegend Eberesche - nicht entfernt, um die Auswirkungen von Füll- und Treibholz auf die Entwicklung junger Buchen quantifizieren zu können. Aufnahme- und Auswertungsdesign entsprachen weitgehend dem bereits beschriebenen Vorgehen im Hauptversuch.

\subsubsection{Ergebnisse}

Die bis zum Alter 16 erreichten Pflanzenhöhen variieren in Abhängigkeit von Pflanzverband und Zielbestockungsgrad in einer Spanne von $587 \mathrm{~cm}$ bis $713 \mathrm{~cm}$ (s. Abb. 1), wobei nur für den Zielbestockungsgrad ein signifikanter Einfluss auf das Höhenwachstum nachgewiesen werden kann.

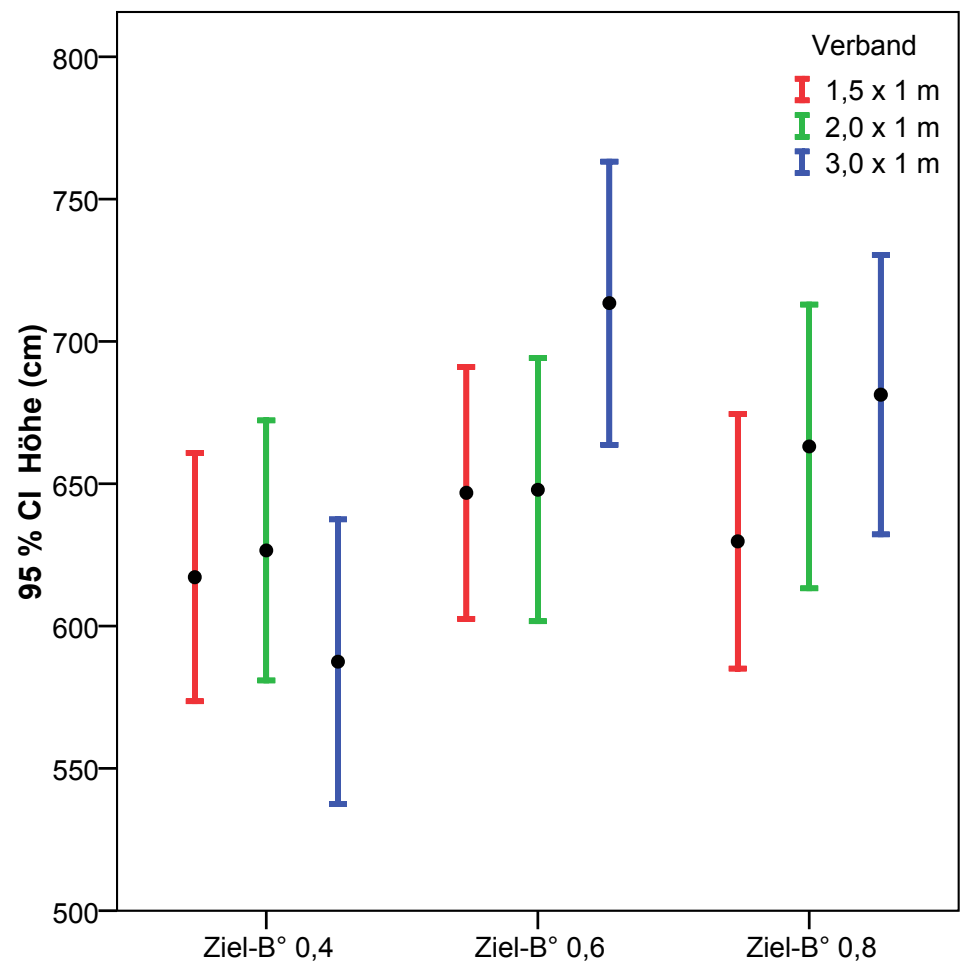

Abbildung 1: Höhendifferenzierung in Abhängigkeit von Pflanzverband und Zielbestockungsgrad

In der am lichtesten gestellten Überschirmungsvariante erreicht die Buche die geringste Höhe und unterscheidet sich signifikant $(\mathrm{p} \leq 0,05)$ von den Buchen bei höheren Zielbestockungsgraden. Die augenfälligste Differenzierung tritt beim Weitverband auf, wo die Höhenwuchsleistung bei sehr lichter Schirmstellung im Mittel um mehr als $100 \mathrm{~cm}$ nachlässt. 
Bei vergleichender Betrachtung des Höhenwachstums aller drei im Versuch Unterlüß 1200 integrierten Baumarten spiegeln sich die unterschiedlichen lichtökologischen Ansprüche von Buche, Traubeneiche und Douglasie deutlich wider (s. Abb. 2). Unter den gegebenen Standortsbedingungen und Anwendung eines praxisnahen Pflanzverbandes ist nur für Buche und Traubeneiche eine signifikante Wirkung des Zielbestockungsgrades nachzuweisen. Verglichen mit der „Schattbaumart“ Buche reagiert die „Lichtbaumart“ Traubeneiche auf eine Verbesserung des Lichtangebotes durchweg mit gesteigertem Wachstum, die „Halbschattbaumart“ Douglasie nimmt eine Mittelstellung ein.

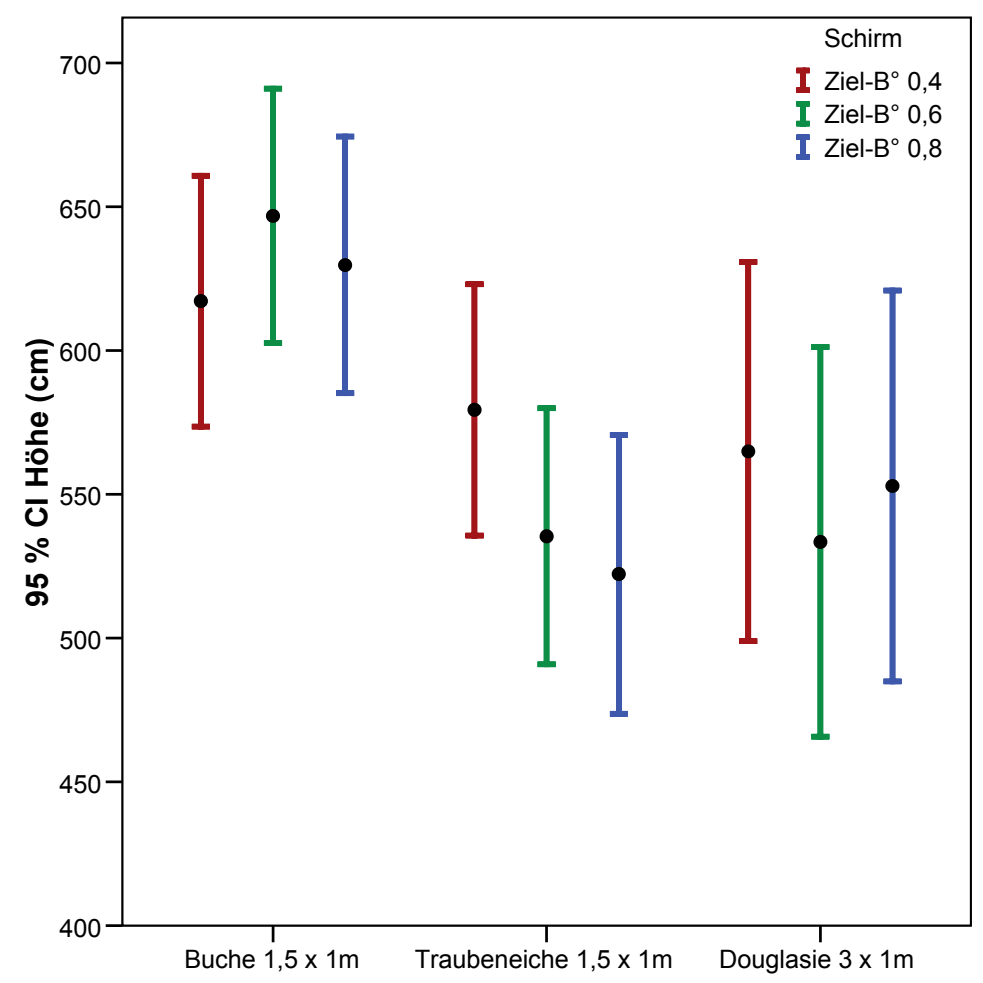

Abbildung 2: Höhendifferenzierung in Abhängigkeit von Baumart und Zielbestockungsgrad

Auch die ermittelten Brusthöhendurchmesser lassen eine starke Differenzierung mit einer Durchmesserspreitung von 36 bis $51 \mathrm{~mm}$ erkennen (s. Abb. 3), die aber überwiegend auf die signifikante Wirkung des Pflanzverbandes zurückzuführen ist. 


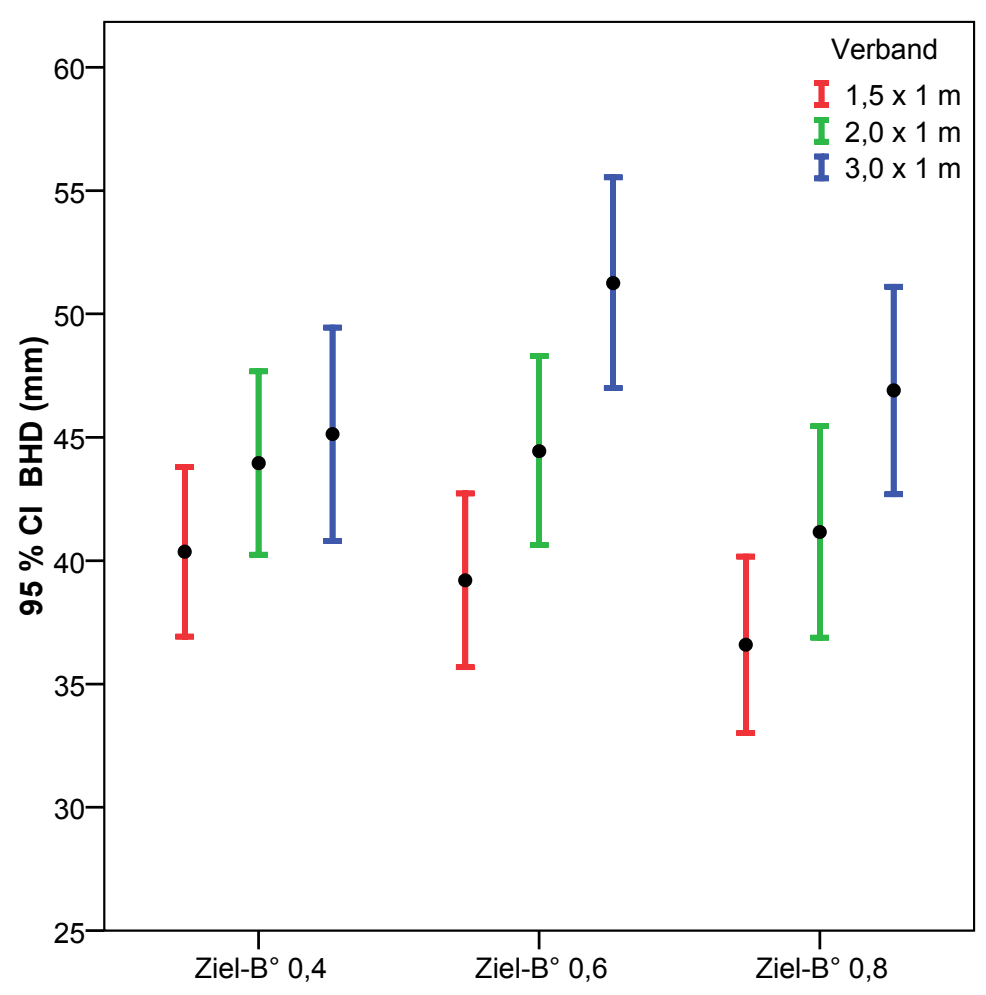

Abbildung 3: BHD-Differenzierung in Abhängigkeit von Pflanzverband und Zielbestockungsgrad

Der Vergleich der drei Pflanzverbände zeigt im Mittel aller Zielbestockungsgrade durchweg signifikante Unterschiede $(p \leq 0,05)$. Die Differenzen zwischen den Pflanzverbänden sind unter der lichtesten Schirmstellung am geringsten.

Die Erfassung der qualitätsbestimmenden Parameter führte zu recht indifferenten Ergebnissen. Unabhängig von Pflanzverband und Zielbestockungsgrad weisen $98 \%$ der untersuchten Buchen gerade Stammformen auf und nur $3 \%$ sind verzwieselt. Die mittlere relative Ansatzhöhe des ersten Grünastes variiert in Abhängigkeit von Pflanzverband und Überschirmung innerhalb einer vergleichsweise engen Spanne von 3,7 bis 10,1 \% der Baumlänge, wobei weder der Pflanzverband noch der Zielbestockungsgrad eine signifikante Wirkung zeigen. Über alle Zielbestockungsgrade hinweg kann zwischen dem Weit- und Engverband lediglich eine Verschiebung der relativen Astansatzhöhe von 4,9\% beim Weitverband auf 7,9\% beim Engverband festgestellt werden. Auch bei der relativen Ansatzhöhe des stärksten Grünastes ist kein signifikanter Einfluss des Pflanzverbandes und des Zielbestockungsgrades nachzuweisen. Die Spreitung zwischen den Pflanzverbänden beträgt lediglich 4,2\%, wobei der stärkste Grünast beim Weitverband mit 22,4\% der Baumlänge am tiefsten ansetzt. 
Statistisch absicherbar sind hingegen der Einfluss des Pflanzverbandes und des Zielbestockungsgrades auf die Anzahl lebender Äste auf den ersten $2 \mathrm{~m}$ Baumlänge (s. Abb. 4). Im Engverband entwickelten bzw. hielten sich mit durchschnittlich 11 Grünästen signifikant weniger Äste als in den weiteren Verbänden. Insbesondere bei sehr lichter Schirmstellung ist bei den weiteren Pflanzverbänden sprunghaft eine stärkere Astigkeit zu beobachten.

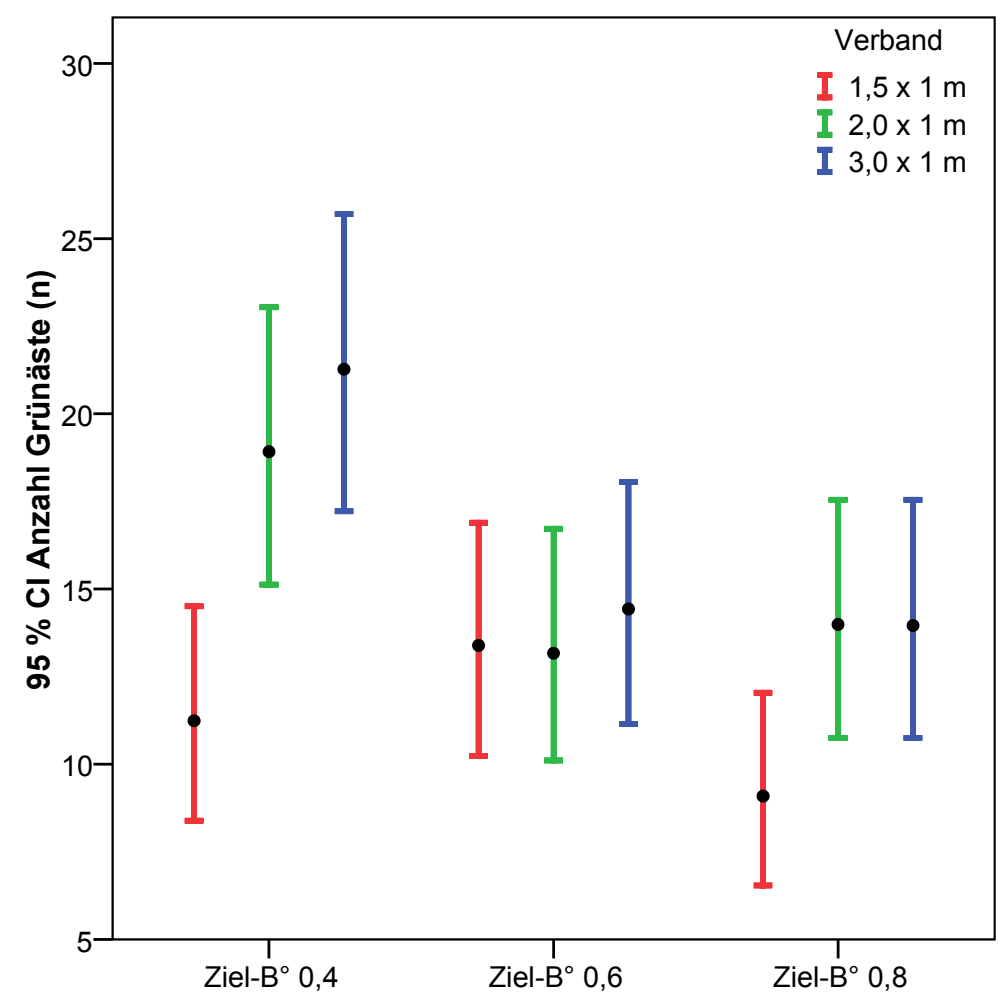

Abbildung 4: Anzabl Grünäste in Abhängigkeit von Pflanzverband und Zielbestockungsgrad

Auch der Astbasisdurchmesser ist im Weitverband mit 19,4 mm signifikant stärker als im Engverband mit 15,8 mm. Im Verband 2 x $1 \mathrm{~m}$ erreicht der Astbasisdurchmesser 16,6 mm. Eine signifikante Wirkung des Schirmes auf die Aststärke konnte nicht nachgewiesen werden.

Das Belassen von Weichlaubbäumen - nahezu ausschließlich Eberesche - führt gegenüber der konkurrenzfreien Variante bei gleichem Zielbestockungsgrad $(0,6)$ und Pflanzverband $(2 \times 1 \mathrm{~m})$ bei keinem der erhobenen Parameter zu signifikanten Unterschieden, wenngleich sich bei einigen Merkmalen zumindest Trends andeuteten (s. Abb. 5). 


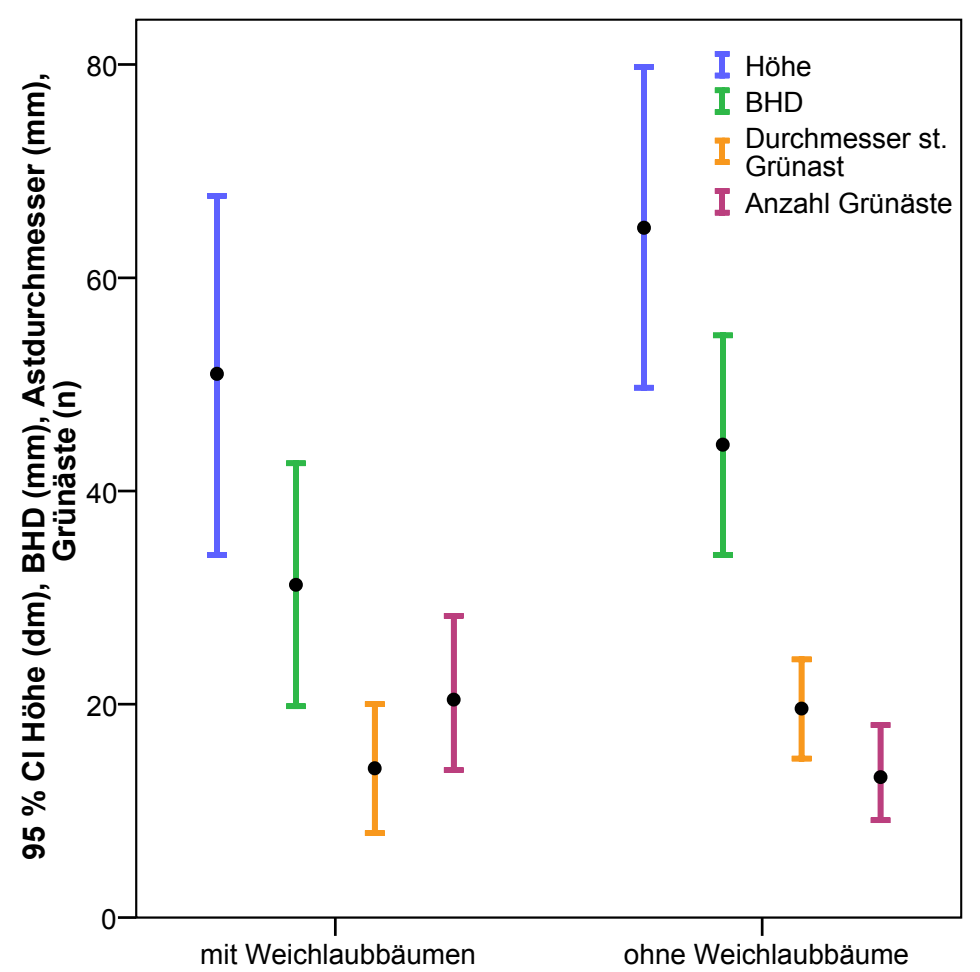

Abbildung 5: Höhe, BHD, Durchmesser stärkster Grünast und Anzabl Grünäste in Abhängigkeit von Weichlaubbaumkonkurrenz.

Die Höhen und Brusthöhendurchmesser der Buchen erreichen unter dem Einfluss der Weichlaubbaumkonkurrenz deutlich geringere Werte, wobei die weit gefassten Grenzen der Konfidenzintervalle auf insgesamt recht inhomogene Verhältnisse schließen lassen. Das Dickenwachstum der Äste wird etwas gebremst, die Grünastanzahl nimmt dagegen leicht zu. Eine fortgeschrittene Astreinigung kann nicht festgestellt werden. Die schärfsten Konkurrenten der Buchen, definiert durch einen maximalen Abstand zur Buche von $1 \mathrm{~m}$ und einem Sprosswachstum, dass mindestens $60 \%$ der Buchenhöhe erreicht, bleiben im Höhenwachstum durchschnittlich rund $70 \mathrm{~cm}$ hinter den Buchen zurück.

Die Entwicklung des Kiefernbestandes führte in Abhängigkeit vom Zielbestockungsgrad $\mathrm{zu}$ recht differenzierten waldwachstumskundlichen Ergebnissen (s. Tab. 1). Im Rahmen der grundflächenbezogenen Steuerung des Kiefernschirmes erfolgten seit Versuchsbeginn drei Durchforstungseingriffe. Insbesondere die sehr starke Absenkung der Bestandesgrundfläche auf $\mathrm{B}^{\circ} 0,4$ führt zu deutlichen Zuwachsverlusten im Gesamtbestand und zu einer im Gegensatz zum B ${ }^{\circ}$ 0,8 um über 100 Vorratsfestmeter geringeren Gesamtwuchsleistung. Der zusätzliche Standraum kann nicht für ein gesteigertes Durchmesserwachstum genutzt werden. 
Tabelle 1: $\quad$ Ertragskundliche Parameter des Kiefernbestandes in Abhängigkeit vom Zielbestockungsgrad nach 15-jähriger Beobachtungsdauer $\left(D_{100}=\right.$ Durchmesser der 100 stärksten Bäume je Hektar, $V=$ Volumen, GWL $=$ Gesamtwuchsleistung, $i_{V}=$ laufender Volumenzuwachs)

\begin{tabular}{ccccc}
\hline & $\begin{array}{c}\mathbf{D}_{\mathbf{1 0 0}} \\
{[\mathbf{c m}]}\end{array}$ & $\begin{array}{c}\mathbf{V} \\
{\left[\mathbf{m}^{3} / \mathbf{h a}\right]}\end{array}$ & $\begin{array}{c}\text { GWL* } \\
{\left[\mathbf{m}^{3} / \mathbf{h a}\right]}\end{array}$ & $\begin{array}{c}\text { iv } \\
{\left[\mathbf{m}^{\mathbf{3}} \mathbf{h}^{-1} \mathbf{a}^{-1}\right]}\end{array}$ \\
\hline Ziel-B $^{\circ} \mathbf{0 , 8}$ & 33,4 & 273 & 410 & 11,7 \\
Ziel-B & $\mathbf{0 , 6}$ & 199 & 382 & 10,1 \\
Ziel-B $^{\circ} \mathbf{0 , 4}$ & 34,4 & 127 & 303 & 5,9 \\
\hline
\end{tabular}

* Die bis zum Versuchsbeginn im Alter 43 angefallenen Durchforstungserträge sind in der Gesamtwuchsleistung nicht berücksichtigt.

\subsubsection{Diskussion}

Das beobachtete Wachstum und die qualitative Entwicklung der heute 16-jährigen Buchen lassen eine recht differenzierte Wirkung des Pflanzverbandes und des Zielbestockungsgrades erkennen. Bei keinem der erfassten Merkmale liegen signifikante Wechselwirkungen zwischen der Pflanzendichte der vorangebauten Buchen und dem Zielbestockungsgrad der Kiefer vor. Während das Höhenwachstum maßgeblich durch die Beleuchtungsverhältnisse gesteuert wird, entwickeln sich der Brusthöhendurchmesser und der Astbasisdurchmesser des stärksten Grünastes in straffer Abhängigkeit vom Pflanzverband, wobei die stärksten Dimensionen jeweils im weitesten Verband ( 3 x $1 \mathrm{~m}$ ) erzielt werden.

$\mathrm{Zu}$ teilweise vergleichbaren Ergebnissen kamen LEDER u. WEIHS (2000), die feststellten, dass das Durchmesserwachstum 12-jähriger Buchen unter dem Schirm 90-jähriger Kiefern ebenfalls im weitesten Pflanzverband (4 x $1 \mathrm{~m}$ ) am stärksten war. Mit abnehmender Überschirmungsintensität nahm insbesondere das Durchmesserwachstum, in geringerem Maße auch das Höhenwachstum aufgrund des zusätzlichen Lichtgenusses zu. Nach Analyse der Beziehung zwischen Konkurrenz und Wuchsleistung bei 9, 23, und 39 Jahre alten vorangebauten Buchen unter Kiefernschirm kommt RÖHLE (2001) zu dem vorsichtigen Schluss, dass mit zunehmendem Alter der Buche die Bedeutung des Schirmdrucks für den Höhenund Radialzuwachs abnimmt und die der intraspezifischen Konkurrenz innerhalb des Buchenvoranbaus zunimmt.

Im vorliegenden Versuch reagierten die Buchen auf die Abnahme der relativen Beleuchtungsstärke von $58 \%\left(\mathrm{~B}^{\circ} 0,6\right)$ auf $46 \%\left(\mathrm{~B}^{\circ} 0,8\right)$ mit nur unbedeutenden Veränderungen des Höhen- und Durchmesserwachstums. Verschiedene Untersuchungen haben verdeutlicht, dass mit stärker reduziertem Wachstum der Buche ohnehin erst bei wesentlich geringeren Beleuchtungsstärken ab etwa 10-20\% zu rechnen ist (s. Kap. 1.2). V. LÜPKE u. HAUSKELLER-BULLERJAHN (2004) zeigten anhand monomolekularer Wachstumsfunktionen (vgl. PRETZSCH 2001), dass sich 
das Höhenwachstum 8-jähriger gepflanzter Buchen unter einem alten BuchenEichenbestand bereits ab etwa 30-40 \% PAR (photosynthetisch aktive Strahlung) asymptotisch einem Maximalwert nähert.

Erstaunlicherweise führte im Versuch Unterlüß 1200 die Absenkung des Zielbestockungsgrades auf 0,4 - entsprechend $72 \%$ relativer Beleuchtungsstärke - zu signifikant geringerem Höhenwachstum und nahezu stagnierendem Durchmesserwachstum. Dieser Leistungsabfall wurde bereits bei einer früheren Auswertung des Versuches durch PETERSEN u. WAGNER (1999) aufgezeigt. SPELLMANN u. WAGNER (1993) berichteten hingegen von einem Voranbauversuch im niedersächsischen Harz, wo im Zuge eines Saumschlages in einem Fichtenaltbestand die Höhen und Wurzelhalsdurchmesser 9-jähriger Buchen entlang eines Gradienten von knapp $20 \%$ relativer Beleuchtungsstärke am westlichen Rand des Innensaumes bis hin zu über $60 \%$ relativer Beleuchtungsstärke am östlichen Rand des Außensaumes deutlich zunahmen. Auf einem eutrophen Basaltstandort in Nordhessen beobachteten WEIHS u. KLAENE (2000) an maximal 10-jährigen Buchen unter Fichtenschirm ein wesentlich gesteigertes Höhen- und Durchmesserwachstum ab einer relativen Beleuchtungsstärke von $60 \%$.

Möglicherweise kommen standörtliche Ursachen für das Wuchsverhalten der Buche in Unterlüß in Frage. Im Rahmen lichtökologischer Untersuchungen an vorangebauten jungen Traubeneichen unter Kiefernschirm in unterschiedlichen Regionen Brandenburgs analysierten KÄTZEL et al. (2006) die kleinklimatischen Verhältnisse in Abhängigkeit verschiedener Zielbestockungsgrade des Kiefernschirmes $(0,4 / 0,6 / 0,8)$. Der Bestandesniederschlag variierte dabei in einer recht engen Spanne zwischen $73 \%\left(\mathrm{~B}^{\circ} 0,4\right)$ und $60 \%\left(\mathrm{~B}^{\circ} 0,8\right)$ des Freilandniederschlages. Mit zunehmendem Bestockungsgrad kam es jedoch zu einer deutlichen Dämpfung der Temperaturmaxima und -minima. Bei einer Auflichtung des vollbestockten Kiefernbestandes auf $\mathrm{B}^{\circ}$ 0,4 erhöhte sich dagegen die potenzielle Evapotranspirationsrate bereits auf das vierfache. Wenngleich die Niederschlagsmengen im Bereich der Versuchsfläche Unterlüß 1200 mit rd. $330 \mathrm{~mm}$ in der forstlichen Vegetationszeit deutlich über denen im trockeneren Tieflandklima Brandenburgs liegen, kann periodischer Trockenstress trotz günstiger Beleuchtungsverhältnisse und geringerer Altholzwurzelkonkurrenz möglicherweise dennoch zu einem Rückgang der Assimilationsleistung der Buche geführt haben und Ursache für das geringe Höhen- und Durchmesserwachstum sein. Diese Vermutung liegt nahe, da der Standort in Unterlüß nicht zuletzt wegen des wenig bindigen sandigen Bodensubstrates als mäßig frisch, tendenziell sogar als mäßig sommertrocken eingestuft wurde. Im Rahmen eines Anbauversuches auf einem schwach nährstoffversorgten, sommertrockenen Sandstandort im Nordosten Niedersachsens beobachtete WAGNER (1994b) an jungen Buchen unter Kiefernschirm ebenso einen Wachstumsrückgang mit zunehmender relativer Beleuchtungsstärke. Diese Entwicklung hat sich auf der Versuchsfläche bis heute fortgesetzt und ist offensichtlich mit den für die Buche mit abnehmender Überschirmung ungünstiger werdenden klein- 
klimatischen Bedingungen zu erklären. Auch die mittlerweile häufiger zu beobachtenden Witterungsextreme lassen periodischen Trockenstress (z. B. Sommer 2003 u. 2006) als Ursache für die verminderte Wuchsleistung der Buche vermuten. Die Toleranz gegenüber stark eingeschränkter Wasserversorgung ist zumindest bei jungen Buchensämlingen geringer einzuschätzen als bei Eichen gleichen Alters. Bei Experimenten von V. HEES (1997) führte Trockenstress darüber hinaus zu einer vermehrten Biomasseallokation in den Feinwurzeln junger Buchen zu Lasten des Sprosswachstums.

Unabhängig von Pflanzverband und Zielbestockungsgrad zeigten die Buchen in Unterlüß fast durchweg orthotropes Wachstum. Die beobachtete geringe Abweichung der Sprossachse von der senkrechten Wuchsrichtung hat mit durchschnittlich $10-14^{\circ}$ vermutlich die Ausbildung überwiegend gerader Stammformen gefördert. Die gerade Wuchsform ist sicherlich in nicht unerheblichem Maße auch auf genetische Einflüsse zurückzuführen (KRAHL-URBAN 1953 u. 1958, HUSSENDÖRFER et al. 1996, KLEINSCHMIT u. SvOLBA 1996). Die über alle Pflanzverbände und Bestockungsgrade hinweg zu verzeichnende geringe Astreinigung und die indifferente Ansatzhöhe des stärksten Grünastes deuten darauf hin, dass der bislang erreichte Dichtschluss auch im Engverband noch nicht ausgereicht hat, den Astreinigungsprozess der 16-jährigen Buchen voranzutreiben. LEONHARDT u. WAGNER (2006) beobachteten an vorangebauten Buchen in Fichtenaltbeständen des Sauerlandes, dass in den ersten 10 Jahren nach der Pflanzung unabhängig von Schirmdruck und Pflanzendichte keine Qualitätsdifferenzierung stattgefunden hat, im Alter 11-15 konnte steigender Seitendruck nur in Verbindung mit hohem Schirmdruck die qualitative Entwicklung der Buchen beeinflussen. Erst im Zeitraum von 16 bis 20 Jahren wirkten sich Standraum und Schirmstellung deutlich auf die Qualitätsentwicklung aus, wobei ab Pflanzenzahlen von 5000 je ha mit abnehmender Pflanzendichte der Anteil schlechter Stammformen unabhängig vom Grad der Überschirmung deutlich zunahm.

Die Entwicklung der Astbasisdurchmesser und der Anzahl lebender Äste machen aber deutlich, dass auf der Fläche Unterlüß 1200 eine pflanzverbandsabhängige Qualitätsdifferenzierung bereits eingesetzt hat und sich diese für die Astigkeit mit der Senkung des Zielbestockungsgrades von 0,6 auf 0,4 verstärkt zu haben scheint. Soweit in diesem frühen Entwicklungsstadium einschätzbar, ist im Weitverband neben deutlich stärkeren Ästen mit einer verlangsamten Astreinigung zu rechnen, was künftig eine Qualitätsverschlechterung erwarten lässt. Der engere Pflanzverband von 1,5 x $1 \mathrm{~m}$ führte im Vergleich zum 2 × $1 \mathrm{~m}$ Verband bislang mit Ausnahme geringerer Grünastanzahlen zu keiner weiteren Qualitätsverbesserung.

Da die Wahl des Pflanzverbandes die Durchmesserentwicklung sowie die Ausbildung der Aststärken und damit die Geschwindigkeit der Astreinigung beeinflusst, wurde für Freiflächenkulturen die Frage des Ausgangsverbandes lange Zeit heftig diskutiert. Höhere Pflanzendichten von um die 10000 Pflanzen je ha lassen i. d. R. eine bessere Qualitätsentwicklung erwarten (KRAHL-URBAN 1963, 
RiCHTER 1990). MUHLE u. KAPPICH (1979) sowie FREIST (1980) berichten dagegen von 19 bzw. 29 Jahre alten Buchenbeständen, die bei Ausgangspflanzenzahlen von 6000 bzw. 5000 Pflanzen je ha sich qualitativ durchaus ansprechend entwickelt haben. OTT u. V. LÜPKE (2006) fordern jedoch für 10-jährige Buchenkulturen unter Freiflächenbedingungen eine Mindestpflanzenzahl von $5000 \mathrm{Bu}-$ chen je Hektar im Reinbestand.

Bei den bereits erwähnten Untersuchungen von LEDER u. WEIHS (2000) an Buchen unter Kiefernschirm erreichte der Astbasisdurchmesser des stärksten Grünastes sein Maximum wie im vorliegenden Versuch im weitesten Pflanzverband (4 x $1 \mathrm{~m}$ ). Die Ergebnisse zeigten darüber hinaus, dass ein Unterschreiten einer Pflanzendichte von 5000 Pflanzen je ha bei lockerer Überschirmung mit einer deutlichen Verzögerung der Astreinigung und abnehmender Wipfelschäftigkeit verbunden ist. Dagegen halten BERGERS et al. (2006) nach einer Überprüfung von Buchenvoranbauten unter Kiefer im niederrheinischen „Grenzwald“ bei einem Bestockungsgrad der Kiefer von 0,3 bis 0,5 einen Pflanzverband von $2 \times 1,5 \mathrm{~m}$ und bei einem Bestockungsgrad von 0,5 bis 0,7 einen Pflanzverband von $2 \times 2 \mathrm{~m}$ als zweckmäßig.

Das in Unterlüß beobachtete eingeschränkte Höhen- und Durchmesserwachstum der Buche unter Weichlaubbaumkonkurrenz bewegte sich bislang in einem tolerierbaren Rahmen. Ein um $4 \mathrm{~mm}$ geringerer Astbasisdurchmesser ließ zumindest ansatzweise eine qualitätsfördernde Wirkung der Ebereschen erkennen. Bei durchschnittlich einer konkurrenzstarken Eberesche je Buche bleibt abzuwarten, ob der gesteigerte Seitendruck ausreicht, die Astreinigung wesentlich voranzutreiben. Die Konkurrenzkraft der Eberesche ist im Vergleich zu Birke, Salweide und Aspe sicher deutlich geringer einzuschätzen, andererseits ist die Toleranz der Buche gegenüber Weichlaubbaumkonkurrenz, insbesondere gegenüber lichtdurchlässigen Arten, vergleichsweise stark ausgeprägt (vgl. PAMPE 2001). Mit zunehmender Salweidenkonkurrenz beobachtete LEDER (1995) erhöhte Anzahlen feinastiger und wipfelschäftiger Buchen, Durchmesser- und Höhenwachstum der Buchen ließen dagegen aber nach. Nach OTT u. V. LÜPKE (2006) sollten sich in Freiflächenkulturen im Alter von 10 Jahren bei einem Minimum von 2000 Buchen zusätzlich mindestens 4000 Misch-/Begleitbaumarten je Hektar etabliert haben, um im Stangenholz eine ausreichende Anzahl gut geformter Buchen erzielen zu können.

Ungeachtet dessen, dass nach dem heutigen Erfahrungs- und Wissensstand über die Konkurrenzkraft der Buche unter den gegebenen Standortsverhältnissen ein Buchenvoranbau wesentlich später eingeleitet würde, führte in Unterlüß die Absenkung des Zielbestockungsgrades auf $0,4 \mathrm{zu}$ unverhältnismäßig hohen Zuwachsverlusten in der Kiefer. Diese konnten weder durch ein gesteigertes Einzelbaumwachstum der Kiefern noch durch ein verbessertes Wachstum der vorangebauten Buchen kompensiert werden. DITTMAR u. KNAPP (1989) schlagen für die Bestandespflege der Kiefer mit Buchenunterbau/-voranbau im nordost- 
deutschen Tiefland bis zur Kulmination des dGZ Derbholz - je nach Bonität der Kiefer - im Alter von 70-85 Jahren, eine Absenkung des Bestockungsgrades auf 0,8 bis 0,7 (Ertragstafel Lembcke et al. 1975) vor. Untersuchungen in über 45- bis 120jährigen „Buchenunterbauten“ unter Kiefernschirm in den verschiedenen Klimaregionen Brandenburgs zeigten, dass die Buche in der Klimastufe „Tf“ (entsprechend 600 bis $>660 \mathrm{~mm}$ Jahresniederschlag) bei einem KiefernBestockungsgrad von 0,9 noch $90 \%$ der Durchmesserleistung des Buchenreinbestandes gleichen Alters, gleicher Bonität (Ertragstafel Dittmar et al. 1983) und gleichen Bestockungsgrades erreichen kann (KNAPP 1992). Die in Unterlüß beobachtete Entwicklung des Buchenvoranbaus lässt eine Absenkung des Bestockungsgrades der Kiefer unter 0,8 bislang nicht erforderlich erscheinen.

\subsubsection{Schlussfolgerungen}

Knapp 15 Jahre nach Einrichtung des Versuches befinden sich die vorangebauten Buchen in einer Entwicklungsphase, wo Selbstdifferenzierungsprozesse zunehmend stärker das Wachstum und die natürliche Schaftreinigung beeinflussen. Künftig sind noch deutlichere Differenzierungen zwischen den Versuchsvarianten $\mathrm{zu}$ erwarten. Die vorgestellten und diskutierten Versuchsergebnisse lassen zum heutigen Zeitpunkt aber dennoch einige vorsichtige Schlussfolgerungen zur Durchführung von Buchenvoranbauten unter Kiefernschirm zu:

- $\quad$ in Kiefernbeständen zweiter oder besserer Bonität sollten Voranbauten mit der konkurrenzstarken Buche erst ab einem Bestandesalter von 80 Jahren erfolgen

- unter den gegebenen Standortbedingungen sind übermäßig starke Absenkungen des Bestockungsgrades im Kiefernaltbestand unter $\mathrm{B}^{\circ}$ 0,6 nicht zielführend, da im Buchenvoranbau mit einem deutlichen Nachlassen des Höhenwachstums zu rechnen und eine weitere Durchmesserzunahme nicht zu erwarten ist. Darüber hinaus führt ein solcher Eingriff zu unverhältnismäßig hohen Zuwachsverlusten in der Kiefer, die im Laufe des Bestandeslebens nicht ausgeglichen werden können

- weitgehend unabhängig von den Beleuchtungsverhältnissen lässt der 2 x $1 \mathrm{~m}$ Pflanzverband bei noch gutem Durchmesserwachstum eine günstige qualitative Entwicklung der Buchen erwarten

- die Konkurrenz von Weichlaubbäumen - überwiegend Ebereschen - vermag es nur sehr beschränkt durch zusätzlichen Seitendruck die Qualität der Buchen zu fördern. Eine Konkurrenzsteuerung zugunsten der Buche ist unter den gegebenen Verhältnissen derzeit nicht erforderlich

Die weitere Entwicklung des Buchenvoranbaus wird zeigen, ob und inwieweit die Zielbestockungsgrade von 0,6 und 0,8 zu einem späteren Zeitpunkt abgesenkt 
werden müssen, um ein kräftiges Wachstum der Buche bei gleichzeitig hoher Vitalität und Qualität sicherzustellen.

\subsection{Umbau von Fichtenreinbeständen mithilfe der Buchensaat}

\subsubsection{Versuchsanlass und Versuchsanlage}

Angesichts der hohen Kosten, die herkömmliche Voranbauten aus Pflanzung verursachen, wird in den letzten Jahren verstärkt nach Alternativen gesucht. Ein in der Praxis vielerorts bewährtes Verfahren stellt die Saat dar. Ihren unbestrittenen Vorteilen - Erzielen hoher Pflanzendichten unter Vermeidung von Pflanzenschock und Wurzeldeformationen bei geringeren Kosten, Werbung von Wildlingen stehen jedoch auch gravierende Nachteile, insbesondere das schwer kontrollierbare Ausfallrisiko, entgegen. Um wissenschaftlich fundierte Praxisempfehlungen für Buchenvoraussaaten geben zu können, wurden auf Initiative des Deutschen Verbandes Forstlicher Forschungsanstalten in mehreren Bundesländern Voraussaatversuche angelegt, deren Ergebnisse u. a. in das Merkblatt „Erfolg von Buchensaaten steigern" (LWF 2004) eingegangen sind. Einer dieser Versuche befindet sich im Niedersächsischen Forstamt Clausthal (Harz). Hier sollen u. a. folgende Aspekte untersucht werden:

a) Dokumentation der Auflaufergebnisse und Überlebensraten der Saat

b) Entwicklung der Buchensaat in Konkurrenz zur vorhandenen Fichten-Naturverjüngung unter unterschiedlichen Überschirmungsverhältnissen

Standort: Höhenlage $540 \mathrm{~m}$ ü. NN (montan), Exposition Nord, Ausgangssubstrat Kulm-Tonschiefer, Bodentyp podsolige Braunerde. Frisch bis vorratsfrischer Schatthangstandort mit mäßiger Nährstoffversorgung, $30-70 \mathrm{~cm}$ mächtige Fließerde über basenarmen Silikatgestein.

Altbestand (Nov. 1997): Fichte 96-jährig, Ekl II,7 (Lkl 9), Grundfläche $39,9 \mathrm{~m}^{2} /$ ha $\left(\mathrm{B}^{\circ}=1,0\right)$ mit im Mittel 5-jähriger Fichten-Naturverjüngung.

Versuchsanlage: Im Frühjahr 1997 wurde in einem ein Hektar großen Fichtenbestand eine Buchen-Rillensaat mit der zweirilligen Sämaschine Ökosat/U schleppergestützt durchgeführt. Die Ausbringungsmenge betrug $65 \mathrm{~kg} / \mathrm{ha}$. Dabei wurden Rückegassen und ein bereits durch Fichten-Naturverjüngung bestocktes Bestandesloch nicht befahren, so dass insgesamt $82 \%$ der Fläche mit Buche eingesät wurden. Die Fläche wurde anschließend in einem 10 × 10 m-Raster verpflockt. An den Rasterpunkten wurde auf $3 \mathrm{~m}^{2}$ großen Probekreisen das Auflaufen und die Überlebensrate der gesäten Buchen fünf Jahre lang flächenrepräsentativ erfasst. Zudem wurde ausgehend von dem Bestandesloch auf drei Transekten in den geschlossenen Bestand hinein auf 68 Plots das Wachstum einzeln nummerierter Buchen und Fichten beobachtet. Dabei wurden Höhen und Durchmesser jährlich bis nunmehr zum Alter 10 gemessen. Des Weiteren wurde bei Versuchsbeginn zur 
Strahlungsschätzung über jedem Plot ein Fish-eye-Foto aufgenommen. Der daraus berechnete Diffuse Site Factor (DIFFSF) gibt den Anteil der diffusen Strahlung am Messpunkt in Prozent zur Freilandstrahlung an.

\subsubsection{Ergebnisse}

Auflaufergebnis der Buchensaat: Da die Fläche nicht vollständig befahren wurde, betrug die ausgebrachte Saatgutmenge bezogen auf die Nettofläche $79 \mathrm{~kg} / \mathrm{ha}$. Nach den ersten sechs Monaten waren 20.300 Buchen je ha besäter Fläche aufgelaufen, dies entspricht einem Auflaufergebnis von 12,0\%. Das Auflaufergebnis war nicht homogen, die Belegung der Aufnahmeplots reichte von 0 bis 31 Buchen.

Überlebensrate: In den nächsten 4 Vegetationsperioden nahm die Zahl der vorhandenen Buchen auf 11.450 Pflanzen/ha ab, dies entspricht einer Ausfallrate von $44 \%$, wenn man die Anzahl der vorhandenen Buchen im Herbst 1997 als Berechnungsgrundlage heranzieht. Gleichzeitig stieg der Anteil der Plots ohne Buchensämlinge von $38 \%$ auf $53 \%$. Die Überlebensrate der Saat betrug nach 5 Jahren lediglich $5 \%$ bezogen auf die Anzahl lebender Keime zum Zeitpunkt der Aussaat. Dabei konnte in den ersten drei Standjahren ein signifikanter Zusammenhang zwischen Überlebensrate und DIFFSF, welcher im Bereich zwischen 2,8\% und 22,8\% lag, nachgewiesen werden. Mit steigendem Lichtangebot nimmt die Ausfallrate degressiv ab (exponentielle Regression $\mathrm{r}^{2}=0,56$ ).

Von den bei Versuchsanlage vorhandenen Fichten aus Naturverjüngung waren bis zur Aufnahme $200793 \%$ vergangen. Auch hier stand die Ausfallrate in einem signifikanten Zusammenhang zum DIFFSF, je höher die relative Beleuchtungsstärke, desto niedriger die Ausfallrate (lineare Regression $r^{2}=0,65$ ).

Wachstum: Das Höhen- und das Durchmesserwachstum der Buchen wurde an 269 Pflanzen über einen Zeitraum von 10 Jahren gemessen. Die Buchen reagierten im Höhenwachstum positiv auf zunehmende Auflichtung. Der Effekt wirkte am stärksten ab einer relativen Beleuchtungsstärke von 10-15\%, darüber hinaus war keine weitere Höhenzunahme mit steigendem Lichtangebot $\mathrm{zu}$ verzeichnen (s. Abb. 5). Ähnlich verhielt es sich mit dem Durchmesserwachstum (s. Abb. 6). Auch hier waren deutliche Zuwachssteigerungen bei einer relativen Beleuchtungsstärke zwischen 10-15\% festzustellen, ein höheres Lichtangebot führte dagegen nur zu geringfügiger Durchmesserzunahme.

In beiden Abbildungen ist die im sechsten Standjahr vollzogene Durchforstung mit einer Eingriffsstärke von $82 \mathrm{Efm} / \mathrm{ha} \mathrm{zu}$ erkennen. Die Buchen reagierten mit einem Wachstumsschub, der möglicherweise durch die warme Sommerwitterung des Jahres 2003 noch unterstützt wurde. 


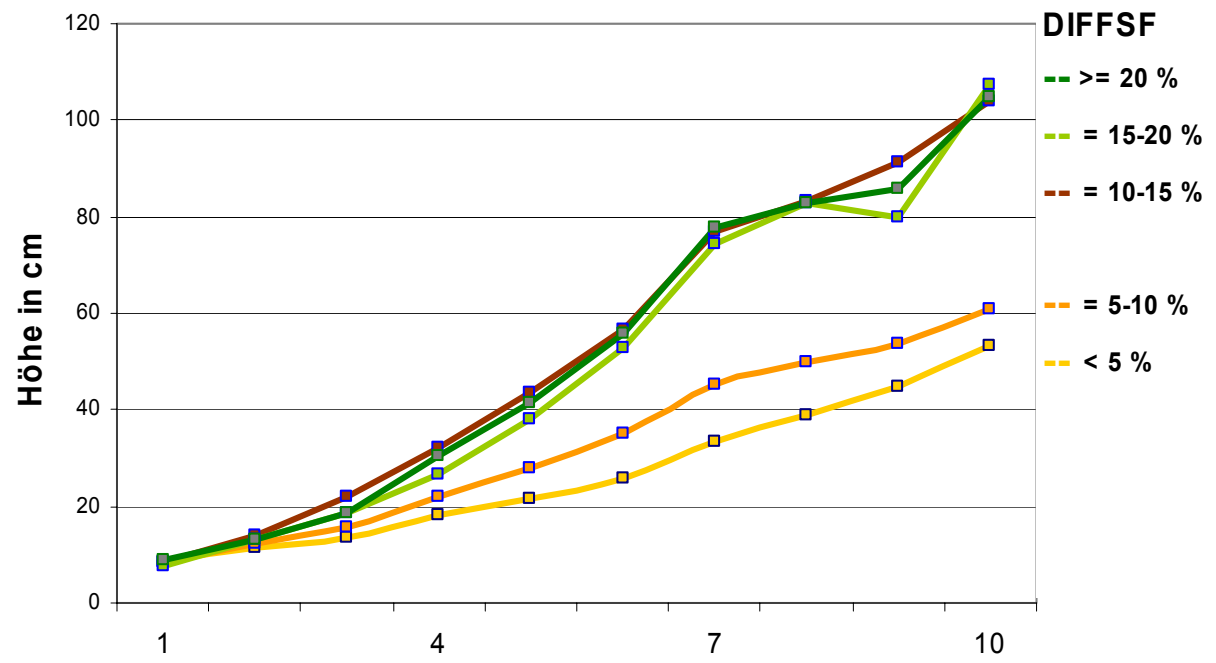

Alter in Jahren

Abbildung 5: Höhenwachstum der gesäten Buchen in Abbängigkeit von der Beleuchtungsstärke (DIFFSF)

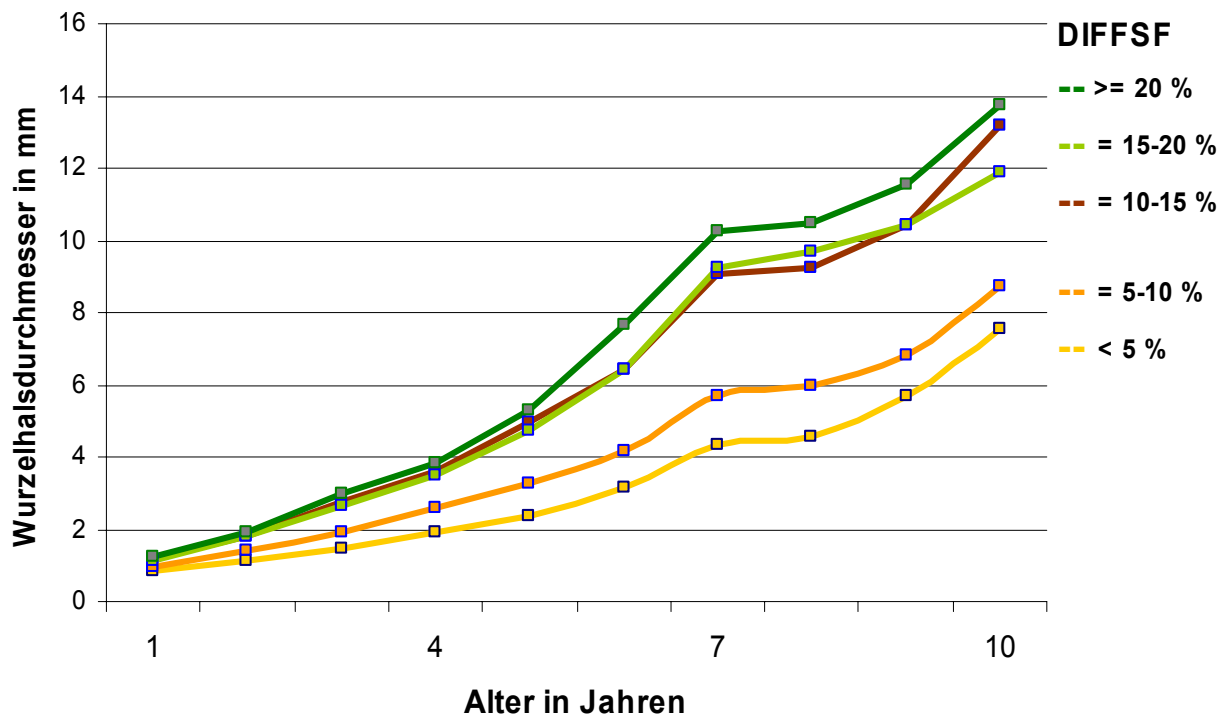

Abbildung 6: Durchmesserwachstum der gesäten Buchen in Abhängigkeit von der Beleuchtungsstärke (DIFFSF) 
Im Alter von 10 Jahren hatten die Buchen einen deutlichen Höhenvorsprung gegenüber der im Mittel 4 Jahre älteren und zum Zeitpunkt der Saat bereits etablierten Fichtennaturverjüngung erreicht (s. Abb. 7). Selbst am Rande des Bestandeslochs bei einer relativen Beleuchtungsstärke zwischen $20 \%$ und $25 \%$ geriet die Fichte gegenüber der Buche ins Hintertreffen.

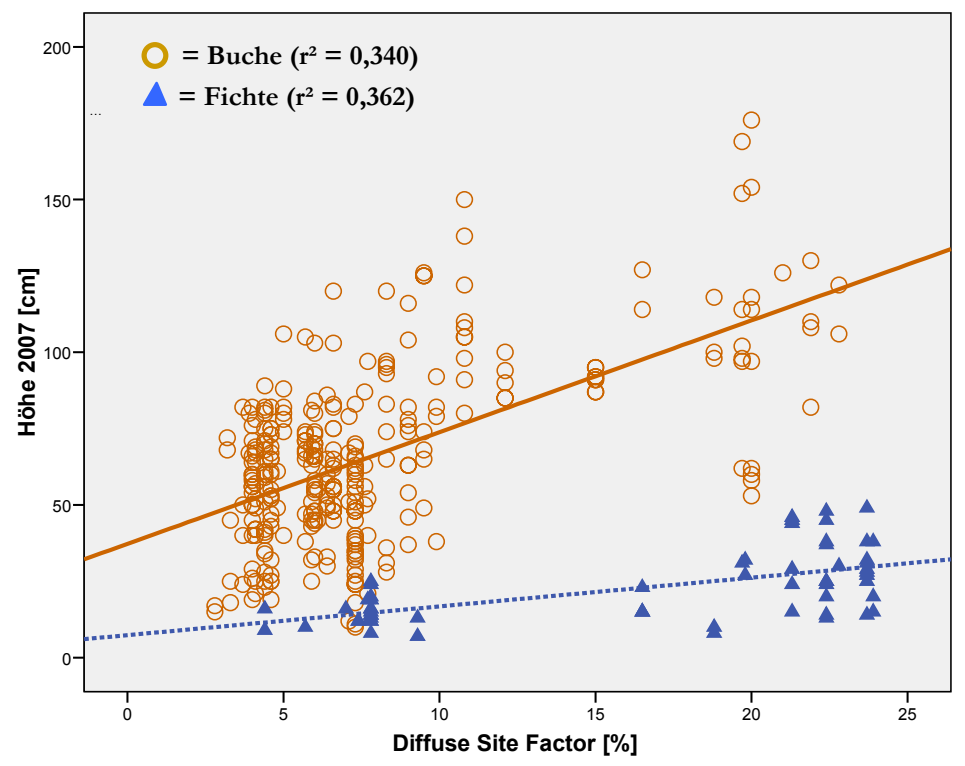

Abbildung 7: Einfluss des Diffuse Site Factors auf das Höhenwachstum von Buche und Fichte

\subsubsection{Diskussion}

Die Buchensaat stellt eine Alternative zur Pflanzung dar, verfügt allerdings nur über einen begrenzten Einsatzbereich und ist an mannigfaltige Voraussetzungen geknüpft. Geeignete Saatflächen sind stabile Bestände auf gut wasser- und nährstoffversorgten Böden mit schwacher Konkurrenzvegetation, guter Bodengare und geringer Rohhumusauflage. Eine der Saat vorausgehende Bodenbearbeitung mit Kalkung erhöht die Auflaufdichte signifikant (KÜBNER u. WICKEL 1998). AMMER und MOSANDL (2000) fanden deutlich bessere Auflaufergebnisse in einem Fichtenbestand mit einer Grundflächenhaltung von $41 \mathrm{~m}^{2} /$ ha als in einem sehr vorratsreichen Fichtenbestand mit $55 \mathrm{~m}^{2} /$ ha. Mit Modellen, die den Überschirmungsgrad bzw. die relative Altbaum-Feinwurzeldichte einbeziehen, konnten $72 \%$ bzw. $52 \%$ der Streuung der Auflaufergebnisse erklärt werden (AMMER et al. 2002). Nach GOMMEL (1994) sind nur vorgepflegte Bestände mit intaktem Bodenleben und aktiver Streuzersetzung für eine Buchen-Voraussaat geeignet. Er empfiehlt eine kräftige Durchforstung 4-5 Jahre vor der Saat sowie eine weitere Durchforstung 
sofort nach der Aussaat, wobei das Reisig als Schutz auf der Fläche belassen werden sollte. Ein Merkblatt der nordrhein-westfälischen Landesforstverwaltung (MURL 1998) sieht eine Durchforstung 2-3 Jahre vor der Saat vor. AMMER et al. (2001) sind hingegen der Meinung, dass erst möglichst kurz vor der Saat aufgelichtet werden sollte, damit sich die Bodenvegetation nicht zu stark entwickelt. Zeitpunkt und Stärke der Vorbereitungshiebe sollten im Einzelfall vom zu erwartenden Konkurrenzdruck durch Bodenvegetation und begleitende Naturverjüngung (z. B. Fichte) abhängig gemacht werden. Wie die Ergebnisse in Clausthal zeigen, führte eine Grundflächenhaltung von $39,9 \mathrm{~m}^{2} / \mathrm{ha}\left(\mathrm{B}^{\circ}=1,0\right)$ zu einem raschen Ausdunkeln der Fichten-Naturverjüngung, ohne dass das Auflaufen und Überleben der Buchensämlinge gefährdet war. Bis zu einem DIFFSF von $25 \%$ war die in diesem Versuch sogar ein paar Jahre ältere Fichte der Buche im Höhenwachstum deutlich unterlegen (s. Abb. 7). Untersuchungen von PAMPE (2000) im Harz machten deutlich, dass eine Angleichung der Höhenzuwächse erst ab einer Strahlungsklasse von DIFFSF 30-45\% festzustellen ist. Dies entspricht einem Bestockungsgrad des Fichtenbestandes von maximal 0,6. Des Weiteren konnte PAMPE (2002) für eine Buchensaat in Segeberg nachweisen, dass die auf den Plätzen gleichzeitig aufgelaufene Fichtennaturverjüngung bis zu einem DIFFSF von $45 \%$ nicht konkurrenzfähig war und bereits durch die 6-jährigen Saatbuchen ausgedunkelt wurde.

Die Durchforstung der Versuchsfläche Clausthal 6 Jahre nach Aussaat hatte mit der Absenkung der Grundfläche im Fichtenaltholz auf $36,5 \mathrm{~m}^{2} / \mathrm{ha}\left(\mathrm{B}^{\circ}=0,9\right)$ bereits einen deutlich positiven Effekt auf Durchmesser- und Höhenwachstum der Buchen, ohne dabei Gefahr zu laufen, die vorhandene Fichtennaturverjüngung übermäßig zu fördern. Eine weitere Auflichtung zugunsten der Buche wäre sicherlich möglich.

Saaten sollten gruppenweise in großen Beständen bei Vollmasten durchgeführt werden (ab 10 ha nach MURL 1998), um den Druck durch Fressfeinde, insbesondere Vögel und Mäuse (ENGLER et al. 1979), zu verteilen. Ein zusätzlicher Zaunbau ist dennoch in den meisten Fällen unumgänglich. Die Anforderungen an das zu verwendende Saatgut sind hoch, ebenso ist eine gute Logistik und eine hohe Flexibilität der Mitarbeiter erforderlich. Die in neuer Zeit empfohlenen Saatgutmengen variieren beträchtlich. Im Merkblatt der nordrhein-westfälischen Landesforstverwaltung (MURL 1998) werden für plätze- oder streifenweise BuchenVoraussaaten unter Fichtenschirm, basierend auf den Untersuchungen von LEDER u. WAGNER (1996) sowie LEDER (1998), Saatmengen von $60 \mathrm{~kg}$ je ha Buchenanteilfläche vorgeschlagen. TRAUTMANN (1996) empfiehlt unter Fichtenschirm $75 \mathrm{~kg} / \mathrm{ha}$, GOMMEL (1994) sogar 120-150 kg/ha. Nach einer Umfrage der Forstdirektion Oberbayern-Schwaben wurden in den 1990er Jahren zwischen 13 und $170 \mathrm{~kg} / \mathrm{ha}$ gesät, als gelungen wurden Saaten mit einer Saatgutmenge über $50 \mathrm{~kg} / \mathrm{ha}$ beurteilt (NÖRR 2004). Die Bayerische Landesanstalt (LWF 2004) empfiehlt als Kompromiss zwischen Kosten und Ausfallrisiko eine Menge 
zwischen 50 und $100 \mathrm{~kg} / \mathrm{ha}$ Buchensaatfläche. Im Ergebnis sollen möglichst 15.000 Buchen/ha die erste Vegetationsperiode überleben. Diese im Vergleich zur Pflanzung höheren Sämlingszahlen sind notwendig, da durch ungleichmäßiges Auflaufen höhere Ausfälle entstehen. Mit einer ausgebrachten Menge von $79 \mathrm{~kg} / \mathrm{ha}$ befindet sich der Versuch Clausthal im mittleren Bereich der empfohlenen Ausbringungsmengen. Nach der ersten Vegetationsperiode standen 20.300 Buchen/ha auf der Fläche, welches einer Auflaufrate von $12 \%$ entspricht. LEDER (1998) erreichte im ersten Standjahr bei einer Plätzesaat mit Mineralboden-Abdeckung eine durchschnittliche Pflanzenausbeute von rund $13 \%$, in einer Untersuchung von AMMER u. EL KATEB (2007) lag die Auflaufrate bezogen auf die ausgebrachte Menge keimfähiger Bucheckern zwischen 9,9 und 25,6\%. Ein noch besseres Ergebnis wies PAMPE (2002) ebenfalls auf Plätzesaat in einer Untersuchung in Segeberg nach, bei der ein Auflaufprozent von 31,4 erreicht wurde.

Die Überlebensrate der Buchen in Clausthal betrug nach 5 Jahren $5 \%$ und entspricht einer Pflanzenzahl von 11.450 Stück/ha. Dieses Ergebnis ist im Vergleich zu der Untersuchung von AMMER u. EL KATEB (2007) sowie PAMPE (2002) als eher schlecht einzustufen. Erstgenannte konnten in Abhängigkeit vom Standort nach acht Vegetationsperioden eine Überlebensrate zwischen 7,4\% und $22 \%$ nachweisen, während PAMPE (2002) für eine Saat unter Fichte in Segeberg nach 7 Jahren bezogen auf die Anzahl lebender Keime eine Auflaufrate von $9 \%$ ermittelte.

\subsubsection{Schlussfolgerungen}

Die Buchen-Voraussaat zur Umwandlung nicht standortsgerechter Fichtenreinbestände ist dann erfolgreich und entsprechend kostengünstig, wenn folgende Faktoren berücksichtigt werden:

- ausreichend aufgelichtete Bestände ohne Konkurrenzvegetation mit gut wasser- und nährstoffversorgten Böden und guter Bodengare

- Verwendung von hochwertigem Saatgut in ausreichender Menge $(\geq 60 \mathrm{~kg} / \mathrm{ha})$

- professionelle Vorbereitung und rasche Ausbringung des Saatgutes mit Verfahren, die den Mineralbodenanschluss der Bucheckern gewährleisten

Die gruppenweise in Altbeständen angelegten Saatflächen sollten dann in die helleren Bestandespartien eingebracht werden, wenn diese noch nicht mit FichtenNaturverjüngung besetzt sind. Befinden sich in den helleren Partien (größere Bestandeslücken, Störungslöcher) bereits höhere Fichtenkegel, kann auch in die dunkleren Bereiche gesät werden, nachdem ein Vorbereitungshieb zur Verbesserung der Wuchsbedingungen durchgeführt wurde. Die Konkurrenz gleichzeitig ankommender Fichten-Naturverjüngung ist für die Buche bis zu einer relativen Beleuchtungsstärke von $45 \%$ unproblematisch. 
Sind die Buchensämlinge in ausreichender Zahl aufgelaufen und haben sich etabliert, muss nachgelichtet werden, um den Wuchsvorsprung weiter auszubauen und die Qualitätsentwicklung zu fördern (Verhinderung des plagiotropen Wachstums). Bestände, deren Eignung für die Buchensaat zweifelhaft erscheint, sollten von vornherein bepflanzt werden, denn schlecht aufgelaufene Saaten führen nicht zu einem qualitativ befriedigenden Buchenanteil im Folgebestand.

\section{Literatur}

Ammer, CH. u. Mosandl, R. (2000): Zum Einfluss des Altbestandes auf das Keimergebnis gesäter Buchen. Deutscher Verband Forstlicher Forschungsanstalten, Sektion Waldbau, Jahrestagung in Dessau/Klieken, 79-94

Ammer, Ch.; Mosandl, R.; El KateB, H. u. Stölting, R. (2001): Die Entwicklung von Buchensaaten im Vergleich zu Pflanzungen. Allgemeine Forstzeitschrift/Der Wald, 1208-1210

Ammer, Ch.; Mosandl, R. u. El Kateb, H. (2002): Direct seeding of beech (Fagus sylvatica) in Norway spruce (Picea abies) stands - effects of canopy density and fine root biomass on seed germination. Forest Ecology and Management, 159, 59-72

Ammer, Ch. u. El KateB, H. (2007): Buchensaat oder -pflanzung: Vergleich zweier Optionen zum Umbau von Fichtenreinbeständen. LWF Wissen 58, 38-41

Bergers, Ch.; Frank, A. u. Kaiser, H. (2006): Voranbauten von Buche und Eiche unter Kiefer. Allgemeine Forstzeitschrift/Der Wald, 482-484

BMVEL (2004): Die zweite Bundeswaldinventur - BWI 2. Das Wichtigste in Kürze. Bundesministerium für Verbraucherschutz, Ernährung und Landwirtschaft (BMVEL), Bonn

BREsSEM, U. (1998): Förderung der Buchennaturverjüngung. Allgemeine Forstzeitschrift/Der Wald, 933-936

Burschel, P. u. Huss, J. (1964): Die Reaktion von Buchensämlingen auf Beschattung. Forstarchiv, 225-233

Burschel, P. u. SChMaltZ, J. (1965): Die Bedeutung des Lichtes für die Entwicklung junger Buchen. Allgemeine Forst- und Jagd-Zeitung, 193-210

DitTMar, O. u. KNapp, E. (1989): Waldbauliche Behandlung von Kiefernbeständen mit Buchenunterbau zwecks Übernahme der Buche als Hauptbestand. Sozialistische Forstwirtschaft, 146148

DubBel, V. (1989): Die Bedeutung des Bodenkontaktes für die Qualität des Buchensaatgutes. Forst und Holz, 512-516

DubBeL, V. (1992): Pilze an Bucheckern. Allgemeine Forstzeitschrift, 642-645

Ellenberg, H. (1996): Vegetation Mitteleuropas mit den Alpen in ökologischer, dynamischer und historischer Sicht. Stuttgart

Engler, J. M.; Le LOUARn, H. u. Le TACON, F. (1979): L'influence des oiseaux et des rongeurs sur la régénération naturelle du hêtre. Revue Forestière Francaise 31, 41-49

FrEIST, H. (1980): Beitrag zur Frage der Stammzahlhaltung am Beispiel eines Buchenjungbestandes im Bramwald. Forst und Holz, 21-22

Gommel, H. J. (1994): Umbau von Fichten-Beständen durch Buchen-Saat. Allgemeine Forstzeitschrift, 516-518

Gralla, T.; MÜller-Using, B.; Unden, T. u. Wagner, S. (1997): Über die Lichtbedürfnisse von Buchenvoranbauten in Fichtenbaumhölzern des Westharzes. Forstarchiv, 51-58

Gruber, F. (2003): Welche Witterung bestimmt die Fruchtbildung bei der Rotbuche? Allgemeine Forstzeitschrift/Der Wald, 246-249 
HEES, A. F. M. v. (1997): Growth and morphology of pedunculate oak (Quercus robur) and beech (Fagus sylvatica) seedlings to shading and drought. Annales de Science Forestière, 9-18

Hussendörfer, E.; SCHÜTZ, J.-P. u. SCHOlZ, F. (1996): Genetische Untersuchungen zu phänotypischen Merkmalen an Buche. Schweizerische Zeitschrift für Forstwesen, 785-802

KÄTZEL, R.; LÖFFLER; S.; WINTER, S. u. KALLWEIT, R. (2006): Zum Einfluss von Überschirmung und Begründungsverfahren auf den Entwicklungserfolg von Eichen- und Buchen-Voranbauten in der Initialphase. Eberswalder Forstliche Schriftenreihe, 23, 79-101

KERKMANN, M. (2007): Einflüsse von Niederschlagshöhe und Überschirmungsdichte auf das Wachstum und die Qualität junger Buchen aus Voranbauten in Fichtenaltbeständen. Berichte des Forschungszentrums Waldökosysteme, Reihe A, Band 200

KLEINSCHMIT, J. (1999): Ist Naturverjüngung immer die beste Lösung für den naturnahen Waldbau? In: Kohlstock, N.; STAuber, T.; ZASPEL, I. (Hrsg.): Erhaltung und Nutzung genetischer Ressourcen für den naturnahen Waldbau - Betriebswirtschaft und/oder Naturschutz. Mitteilungen der Bundesforschungsanstalt für Forst- und Holzwirtschaft, 199-214

KLeinschmit, J. u. SvolBA, J. (1996): Ergebnisse der Buchenherkunftsversuche von Krahl-Urban. Allgemeine Forstzeitschrift, 780-782

KLUMPP, K. u. KAZDA, M. (2000): Influence of nutrient amendment on photosynthetic parameters in Fagus sylvatica plants under a Norway Spruce Canopy. In: KLIMO, E.; HaGer, H. U. KulHavý; J. (Hrsg.): Spruce monocultures in Central Europe - Problems and Prospects, EFI-Proceedings 33, 161-165

KNAPP, E. (1992): Zur Wuchsleistung der Unterbaubuche im ungleichaltrigen Kiefern-BuchenMischbestand vor und nach ihrer Übernahme als Hauptbestand auf Standorten des nordostdeutschen Tieflandes. Schriftenreihe der Landesanstalt für Forstwirtschaft Nordrhein-Westfalen, Band 3, 29-46

KraHL-Urban, J. (1953): Rassefragen bei Eichen und Buchen. Allgemeine Forstzeitschrift, 478-480

KRAHL-Urban, J. (1958): Vorläufige Ergebnisse der Buchenprovenienzversuche. Allgemeine Forstu. Jagd-Zeitung, 242-251

KRAHL-UrBAN, J. (1963): Untersuchungen über Verbandsweiten bei Buchenpflanzungen. Forstarchiv, 157-164

KÜBNER, R. u. WiCKEL, A. (1998): Entwicklung einer Buchensaat unter Fichte im Osterzgebirge. Forstarchiv, 191-198

LEDER, B. (1995): Jugendwachstum und waldbauliche Behandlung von natürlich angesamten Weichlaubhölzern in Laubholzjungwüchsen. In: LÖBF (Hrsg.): Weichlaubhölzer und Sukzessionsdynamik in der naturnahen Waldwirtschaft. Schriftenreihe der Landesanstalt für Ökologie, Bodenordnung und Forsten/Landesamt für Agrarordnung Nordrhein-Westfalen, 29-44

LEDER, B. (1998): Pflanzenprozente nach Bucheckern-Voraussaaten unter Fichten-Schirm. Forst und Holz, 477-481

LEDER, B. u. WAGNER, S. (1996): Bucheckern/Streu-Voraussaat als Alternative beim Umbau von Nadelholzreinbeständen in Mischbestände. Forstarchiv, 7-13

LEDER, B. u. WeIHS, U. (2000): Wachstum und qualitative Entwicklung eines 8 Jahre alten BuchenVerbandsversuches unter Kiefernschirm im Niederrheinischen Tiefland. Forst und Holz, 172176

LeOnhardT, B. u. Wagner, S. (2006): Qualitative Entwicklung von Buchen-Voranbauten unter Fichtenschirm. Forst und Holz, 454-457

LeuschneR, C. (1998): Mechanismen der Konkurrenzüberlegenheit der Rotbuche. Berichte der Reinh.-Tüxen-Gesellschaft, 10, 5-18

LÜPKE, B. v. (1996): Waldbau unter ökonomischen Sachzwängen? Allgemeine Forst- u. Jagd-Zeitung, 178-184

LÜPKE, B. v. u. HAuSKELLER-BUlLERJAHN, K. (2004): Beitrag zur Modellierung der Jungwuchsentwicklung am Beispiel von Traubeneichen-Buchen-Mischverjüngungen. Allgemeine Forst- u. Jagd-Zeitung, 61-69 
LWF (2004): Erfolg von Buchensaaten steigern. Merkblatt 16 der Bayerischen Landesanstalt für Wald und Forstwirtschaft (LWF)

MAdSEN, P. (1995): Effects of soil water content, fertilization, light, weed competition and seedbed type on natural regeneration of beech (Fagus sylvatica). Forest Ecology and Management, 251264

MöHring, B. (2004): Betriebswirtschaftliche Analyse des Waldumbaus. Forst und Holz, 523-530

Muhle, O. u. Kappich, I. (1979): Erste Ergebnisse eines Buchen-Provenienz- und Verbandsversuchs im Forstamt Bramwald. Forstarchiv, 65-69

MuRL (1998): Merkblatt zur Bucheckern-Voraussaat unter Nadelholzschirm. Landesforstverwaltung Nordrhein-Westfalen

NiELSEN, B.O. (1977): Beech seeds as an ecosystem component. Oikos, 268-274

NÖRR, R. (2004): Buchensaat- ein Thema mit Zukunft. LWF aktuell, 1-3

OTT, B. u. v. LÜPKE, B. (2006): Erfolg von Buchenpflanzungen auf Sturmschadensflächen von 1990 im hessischen Vogelsberg. Forstarchiv, 119-126

Отто, H.-J. (1986): Standörtliche Voraussetzungen, Ziele und Waldbautechnik in Fichten-BuchenMischbeständen des Harzes, Allgemeine Forst- und Jagd-Zeitung, 157. Jg., 184-196, 214-222

Отто, H.-J. (1995): Die Verwirklichung des LÖWE-Regierungsprogramms. Allgemeine Forstzeitschrift/Der Wald, 1028-1031

PaAr, U.; KirchHoff, A. u. EichHorn, J. (2000): Fruktifikation der Buche in Hessen, Allgemeine Forstzeitschrift/Der Wald, 1362-1363

PAmpe, A. (2000): Zur Konkurrenz von Buche und Fichte in der montanen Stufe des Harzes. Deutscher Verband Forstlicher Forschungsanstalten, Sektion Waldbau, Jahrestagung in Dessau/Klieken, 95-109

PAmpe, A. (2001): Versuche zur Verwendung von Traubeneichen- und Buchen-Großpflanzen in Nordwestdeutschland. Forst und Holz, 331-337

PAmpe, A. (2002): Exkursionsführer Forschungsergebnisse für den Waldbau in Schleswig-Holstein. NW-FVA, 1-5

Petersen, R. u. WagneR, S. (1999): Erste Ergebnisse eine Voranbauversuchs unter Kiefer im östlichen Niedersachsen. Forst und Holz, 647-653

Pretzsch, H. (2001): Modellierung des Waldwachstums. Berlin

RichTER, J. (1990): Die Qualität von Buchenpflanzbeständen. Schriftenreihe der Landesanstalt für Forstwirtschaft Nordrhein-Westfalen, 1, 35-52

RIPKEN, H. (1992): Rationalisierungsmöglichkeiten in der biologischen Produktion des Forstbetriebes. Allgemeine Forstzeitschrift, 569-573

RIPKEN, H. (1996): Controlling der naturalen Leistungen und Kosten der Walderneuerung. BWLSeminar der Niedersächsischen Landesforstverwaltung, unveröffentlicht

RIPKEN, H. (1998): Ökonomische Nachhaltskriterien im Forstbetrieb. Forst und Holz, 155-160

RÖHLE, H. (2001): Wuchsverhalten und Konkurrenzdynamik in Waldbeständen in der Umbauphase. Beiträge für Forstwirtschaft und Landschaftsökologie, 182-187

SCHмIDT, W. (2001): Veränderungen der Samenproduktion und des Blattstreufalls in Buchenwäldern unter dem Einfluss von Umwelteinflüssen. Forschungsvorhaben im Auftrag der Forstlichen Versuchsanstalt Rheinland-Pfalz, $26 \mathrm{~S}$.

SpELlmann, H. (1997): Ertragsentwicklung im "LÖWE"-Wald der Niedersächsischen Landesforstverwaltung. Forst und Holz, 711-718

Spellmann, H. (2005): Produziert der Waldbau am Markt vorbei? Allgemeine Forstzeitschrift/Der Wald, 454-459

Spellmann, H. u. WagneR, S. (1993): Entscheidungshilfen für die Verjüngungsplanung in Fichtenbeständen zum Voranbau der Buche im Harz, Forst u. Holz, 483-490

TeufFEL, K. v. (1999): Waldentwicklungstypen in Baden-Württemberg. Allgemeine Forstzeitschrift / Der Wald, 672-676 
Thomasius, H. (1996): Geschichte, Theorie und Praxis des Dauerwaldes. ANW Bücherdienst Ebrach Trautmann, L. (1996): Saatkultur für Unterbau und Freifläche. Allgemeine Forstzeitschrift/Der Wald, 942-943

UlRiCH, B. (1986): Die Rolle der Bodenversauerung beim Waldsterben: Langfristige Konsequenzen und forstliche Möglichkeiten. Forstwissenschaftliches Centralblatt, 421-435

WACHTER, H. (1964): Über die Beziehung zwischen Witterung und Buchenmastjahren. Forstarchiv, 69-78

WAGNER, S. (1994a): Strahlungsschätzung in Wäldern durch hemisphärische Fotos. Dissertation Universität Göttingen

WAGNER, S. (1994b): Einbringung von Laubbaumarten in Kiefernbestände auf armen Sanden im Nordosten Niedersachsens. Forstarchiv 65, 3-9

WAGNER, S. (2004): Möglichkeiten und Beschränkungen eines funktionsorientierten Waldbaus. Forst und Holz, 105-111

WagneR, S. (2007): Rationaler Waldumbau - Fragen und Anregungen. Forst und Holz, 12-17

WeiHS, U. u. KLAENE, K. (2000): Wuchsdynamik und Qualität von Buchenvoranbauten unter Fichtenaltholz auf Basaltstandorten im Hessischen Forstamt Kassel. Forst und Holz, 177-181

Welander, N.T. u. OtTosson, B. (1998): The influence of shading on growth and morphology in seedlings of Quercus robur and Fagus sylvatica. Forest Ecology and Management, 117-126

WiCKEL, A. u. BUTTER, D. (1998): Ökologische Waldentwicklungsplanung in Sachsen. Allgemeine Forstzeitschrift/Der Wald, 220-222

WOLLBORN, P. (2000): Ist weniger mehr? Forst und Holz, 202-207

Korrespondierender Autor:

Dr. Hendrik Rumpf

Nordwestdeutsche Forstliche Versuchsanstalt

Grätzelstr. 2

37079 Göttingen

E-Mail: Hendrik.Rumpf@nw-fva.de

URL: www.nw-fva.de

Regina Petersen

Nordwestdeutsche Forstliche Versuchsanstalt 



\title{
Wachstum, Behandlung und Ertrag von Rein- beständen der Rotbuche (Fagus sylvatica L.) in Nordwestdeutschland
}

\author{
Growth, treatment and yield of pure beech \\ (Fagus sylvatica L.) stands in northwest Germany \\ Ralf-Volker Nagel und Hermann Spellmann
}

\section{Zusammenfassung}

$\mathrm{Zu}$ den wichtigsten Zielen der Buchenbestandespflege gehört die Gewährleistung eines hohen flächenbezogenen Wertertrages. Darauf abzielende Behandlungskonzepte müssen den Forstbetrieben angesichts sich ändernder ökonomischer und ökologischer Rahmenbedingungen gleichzeitig Anpassungsmöglichkeiten und Handlungsspielräume erhalten, die die Risiken der Buchenwirtschaft begrenzen.

Auf der Grundlage der ökologischen Eigenschaften der Buche und einschlägiger ertragskundlicher Forschungsergebnisse werden Maßnahmen zur Erreichung dieser Ziele analysiert und bewertet. Die Förderung einer ausreichenden Anzahl Z-Bäume durch früh einsetzende starke Hochdurchforstungen, die Verbesserung des Sorten- und Massenertrages des Füllbestandes sowie eine an die Wertentwicklung des Bestandes angepasste Zielstärkennutzung gehören zu den wichtigsten Empfehlungen. Gleichzeitig ist diese Art der Bestandesbehandlung geeignet, strukturreiche Buchenwälder zu entwickeln. 
Stichworte: Buche, ökologische Eigenschaften, Wachstum, Behandlung, Ertrag

\begin{abstract}
One of the most important aims of beech stand treatments is to attain a high value yield by area. Thus, given the changing economic and ecological conditions, the treatment strategies must afford forest enterprises both opportunities to adapt and the scope needed to limit the risks associated with beech management.

On the basis of the ecological characteristics of beech and the relevant yield science research results, measures for achieving these aims are analysed and evaluated. The promotion of an adequate number of future crop trees by the early implementation of heavy thinnings from above, the improvement of the assortment and volume yields of the other remaining trees in the stand, and the use of a target diameter harvesting regime appropriate for the optimum development of the stand value are amongst the most important recommendations. At the same time, this type of stand treatment is suitable for the development of highly structured beech forests.
\end{abstract}

Keywords: beech, ecological characteristics, growth, treatment, yield

\title{
1 Einleitung
}

Die Rotbuche (Fagus sylvatica L.) ist die wichtigste Laubbaumart in Deutschland. Sie stockt auf ungefähr 1,56 Mio. Hektar bzw. $15 \%$ der Waldfläche (BMVEL 2004). Die Verbreitungsschwerpunkte liegen in den stärker atlantisch geprägten Gebieten im Westen sowie im Süden Deutschlands. Ihr Flächenanteil wird sich mittelfristig deutlich erhöhen, wenn die in den vergangenen 20 Jahren durchgeführten umfangreichen Buchenvoranbauten unter dem Schirm von Nadelbaumbeständen in die führende Bestandesschicht übernommen werden. Auf vielen Standorten ist sie den anderen heimischen Baumarten überlegen und prägt ca. $74 \%$ der natürlichen Waldgesellschaften in Deutschland (BMVEL 2004).

Die Ansprüche an die Bewirtschaftung von Buchenwäldern haben im Laufe der Geschichte einen starken Wandel erfahren. Neben ihrer frühen Bedeutung als Futtergrundlage waren Buchenbestände bis weit in das 19. Jahrhundert hinein vor allem Energielieferant für die Bevölkerung und die frühen Industriebetriebe. Eine breite Verwendung des Buchenstammholzes setzte im Vergleich zu den anderen Hauptbaumarten erst relativ spät ein. Der steigenden Nachfrage passten sich auch die waldbaulichen Konzepte an, die fortan darauf abzielten, die Stamm- und insbesondere die Wertholzerträge der Buchenwirtschaft zu heben. Bereits SCHWAPPACH (1911) empfahl für die Buche die starke Durchforstung zur Steigerung des Massen- und vor allem des Wertertrages. Auch eine Reihe späterer Arbeiten stellte auf der Grundlage der Auswertung einer Vielzahl langfristig beob- 
achteter Buchen-Durchforstungsreihen die überlegene Wertleistung stärker im Herrschenden durchforsteter Buchenbestände klar heraus (u. a. SCHOBER 1972, ASSMANN 1976).

Weitergehende Konzepte zur Steigerung der Wertleistung der Buchenwirtschaft setzten bei der konsequenten Förderung ausgewählter Z-Bäume in der Durchforstungsphase an (ALTHERR 1971). Die qualitativ besten 100-120 herrschenden Buchen je ha sollten so in verkürzten Produktionszeiträumen und mit verringertem Entwertungsrisiko in die Stammholzklassen L4 bis L6 geführt werden. Nach einer bei $20 \mathrm{~m}$ Oberhöhe einsetzenden, ca. 20 Jahre währenden Lichtwuchsphase mit starker Grundflächenabsenkung war später eine abnehmende Durchforstungsstärke mit allmählichem Grundflächenanstieg vorgesehen. KLÄDTKE (1997) und HEIN et al. (2007) zeigten anhand von Auswertungen der Altherr'schen Versuchsreihen, dass die mit diesem Behandlungskonzept verfolgten Ziele erreichbar sind. HEIN et al. (2007) empfehlen darüber hinaus eine Verringerung der Z-Baumzahl auf 60 bis 80 Stück je ha und starke Eingriffe über die gesamte Durchforstungsphase.

Dieser Vorschlag geht in die Richtung eines von WILHELM et al. (1999a-d) vorgestellten Behandlungsmodells für Buchenbestände mit der ausschließlichen sehr starken Förderung von maximal 80, in der Regel jedoch nicht mehr als 50 ZBäumen je ha. Das als „Qualifizierung“ bezeichnete Erwachsen im Dichtschluss zur Förderung der natürlichen Astreinigung bis zum Erreichen einer astfreien Schaftlänge von ca. 6-9 m entspricht weitgehend früheren Praxisempfehlungen (V. LÜPKE 1986). Das Ziel der sich anschließenden Durchforstungen („Dimensionierung ${ }^{6}$ ) ist es, das Durchmesserwachstum der wenigen Z-Bäume stark zu beschleunigen und ihre Kronenentwicklung so zu fördern, dass ein Absterben von Ästen an der Kronenbasis dauerhaft vermieden wird. Dies setzt eine extreme Freistellung der Z-Bäume voraus, während in den Zwischenfeldern jegliche Bestandespflege unterbleibt. Bei entsprechender Behandlung sollen über $80 \mathrm{~cm}$ starke Wertbuchen in Produktionszeiträumen von 115 bis 120 Jahren erzeugt werden.

Anlass zur anhaltenden Kritik an diesem Behandlungskonzept sind vor allem die in dem Behandlungsmodell unterstellten Wuchsleistungen der Einzelbäume und der gewichtige Einwand, dass extrem geringe Z-Baum-Zahlen die flächenbezogene Wertleistung mindern (UTSCHIG 2000, GUERICKE 2002, KLÄDTKE 2001, 2002, SPELLMANN 2005). Darüber hinaus vernachlässigt das „Q/D-Konzept“ die Flächenproduktivität, die ökonomische Bedeutung der Vorerträge und die Produktionsrisiken angesichts des weltweit steigenden Holzbedarfs (DIETER et al. 2001, NABUURS et al. 2002, MANTAU et al. 2007) und der sich wandelnden Klimabedingungen (BRÈDA et al. 2006, SPEKAT et al. 2007, SPELLMANN et al. 2007, GERSTENGARBE 2008). Dessen ungeachtet haben mittlerweile neben Rheinland-Pfalz (LANDESFORSTEN RHEINLAND-PFALZ 2001, 2003) weitere Bundesländer Behandlungsrichtlinien für Buchenbestände erarbeitet, die das Behandlungskonzept von 
Wilhelm zu Grunde legen (u. a. V. TEUfFEL 2002, MLU BRANDENBURG 2004, SAARFORST 2004, AG „BUCHE“ LANDESBETRIEB WALD UND HOLZ NRW 2006).

Vor diesem Hintergrund greift der folgende Beitrag wichtige Aspekte der Pflege und Nutzung von Buchenbeständen erneut auf und leitet auf der Grundlage von Forschungsergebnissen der Nordwestdeutschen Forstlichen Versuchsanstalt (NW-FVA) Empfehlungen für die Forstpraxis ab.

\section{Ziele der Buchen-Bestandespflege}

Ein vorrangiges Ziel der Pflege von Buchenbeständen ist die Erhöhung des Anteils starken Stammholzes guter und bester Qualität. Hierzu zählt Wertholz im engeren Sinne (Güteklassen A mit F und TF), aber auch Stammholz der Güteklasse B. Bei der großen Gütedifferenzierung des Buchenholzes und den damit verbundenen erheblichen Preisunterschieden entscheidet die Erreichung dieses Ziels maßgeblich über den wirtschaftlichen Erfolg der Buchenwirtschaft (s. Abb. 1).
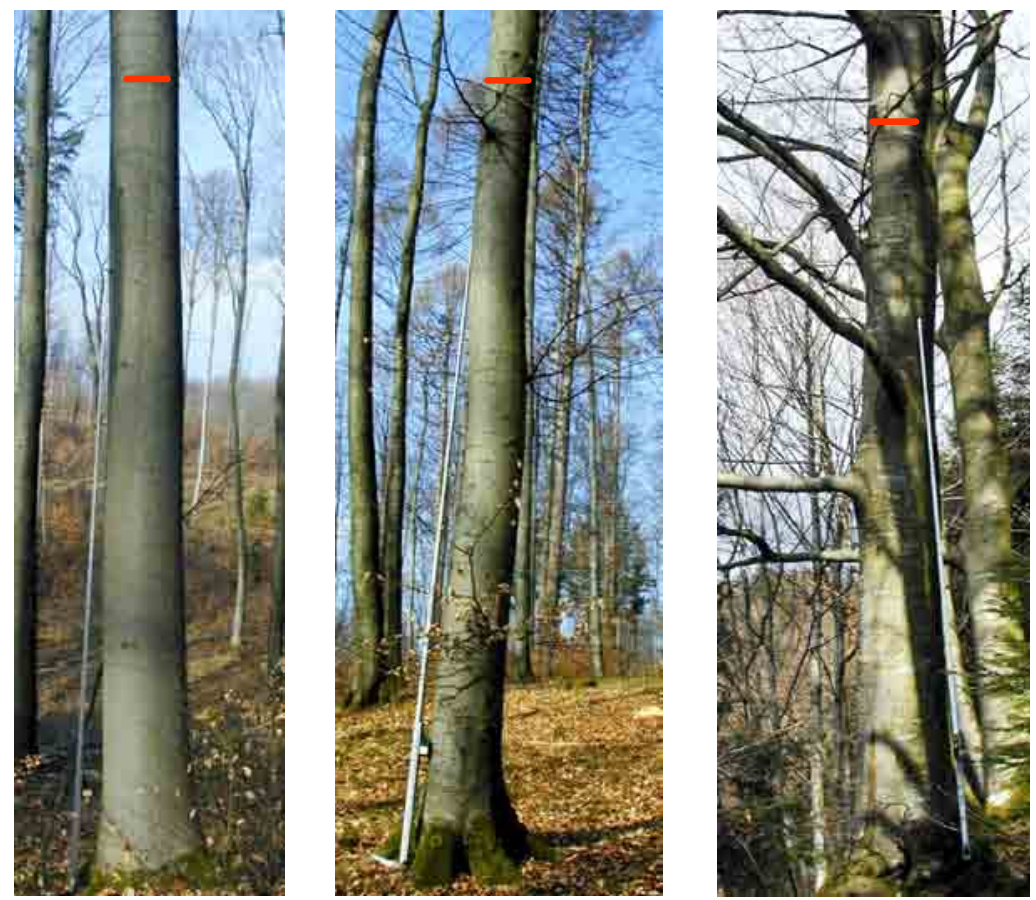

Abbildung 1: Starke Gütedifferenzierung von Buchenholz aufgrund der großen phänotypischen V ariabilität der Buche. Beispielhaft die Güteklassen 1(,sehr gut"), 3 (,,besserer Durchschnitt") und 6 (,schlecht") (aus Güteansprache am stehenden Stamm - Bundeswaldinventur II Baden-Württemberg, WILLMANN et al. 2001) 
Hierzu ist eine ausreichende Anzahl qualitativ guter und vitaler Wertträger ebenso notwendig, wie ein Pflegekonzept, dass die flächenbezogene Massenleistung nicht vernachlässigt. Darüber hinaus sind die Durchforstungen so zu führen, dass eine möglichst gute, risikoarme Entwicklung der Volumen- und Werterzeugung gewährleistet ist und nach Möglichkeit mehr Handlungsspielräume für die Verjüngungsphase geschaffen werden. Dies erfordert einen Bestandesaufbau, der durch vertikale und horizontale Strukturen mit einem dauerhaft vitalen Unterstand gekennzeichnet ist und zudem ein vielfältiges Lebensraumangebot für Tiere und Pflanzen bietet.

Des Weiteren müssen die Behandlungskonzepte aus übergeordneter Sicht so angelegt sein, dass sie bei den langen forstlichen Produktionszeiträumen den Forstbetrieben Handlungsspielräume erhalten, um auf Änderungen der ökologischen und ökonomischen Rahmenbedingungen angemessen reagieren zu können. Dazu tragen die Kenntnis und Berücksichtigung der ökologischen Eigenschaften und Ansprüche der Buche ebenso bei wie die Einbeziehung natürlicher Prozesse in die zielgerichtete Steuerung von Buchenbeständen. Beispiele sind die natürliche Verjüngung von Buchenbeständen mit Überschirmungszeiträumen von 30-50 Jahren, die weitgehende Selbstdifferenzierung junger Buchenbestände im Dichtschluss oder die auf eine optimale Wertleistung des Einzelbaums ausgerichtete Zielstärkennutzung.

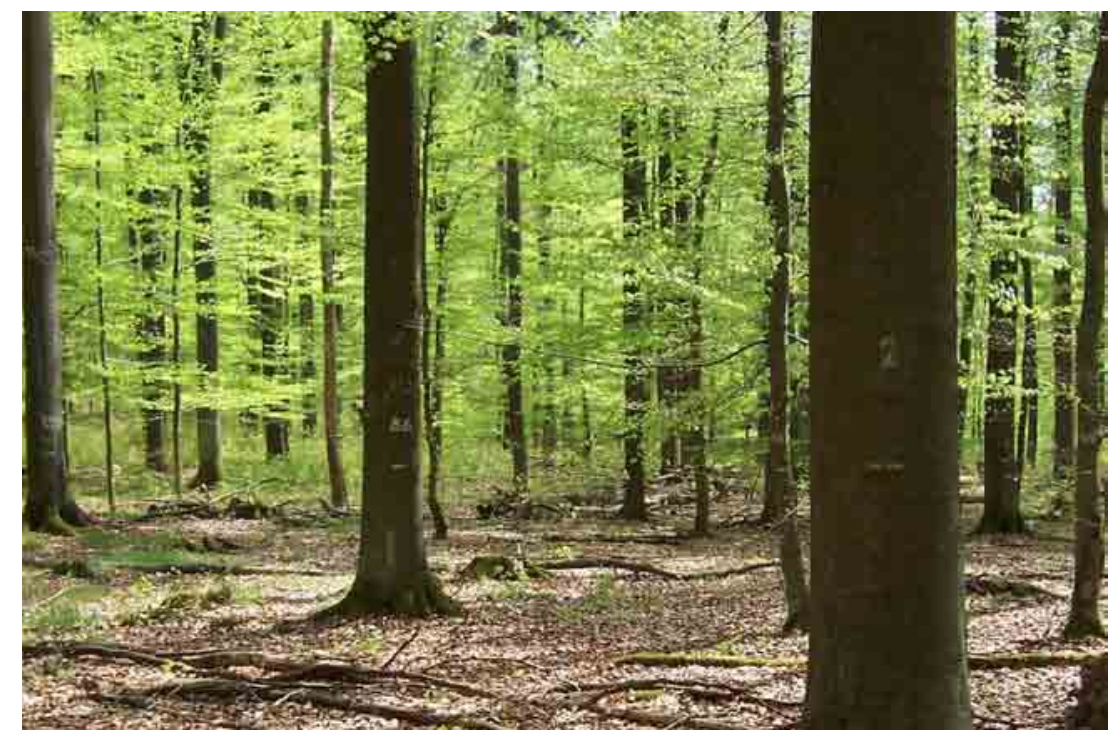

Abbildung 2: Strukturreicher Buchenbestand mit hohen Wertholzanteilen am Beginn der Zielstärkennutzing (Foto: NW-FVA) 


\section{Für die Bestandessteuerung wichtige ökologische Eigenschaften der Rotbuche}

Auf Dauer lassen sich waldbauliche Ziele nur erreichen, wenn die ökologischen Ansprüche und Eigenschaften der Baumarten beachtet werden. Charakteristisch für die Buche ist ihre breite Standortamplitude. Ihre Grenzbereiche werden nicht durch Nährstoffarmut, sondern durch extreme Wasserhaushaltsbedingungen bestimmt (OTTO 1994, ElLENBERG 1996, HEINKEN 1996, RENNENBERG et al. 2004). Es handelt sich um azonale Standorte (Auen, Brücher, Moore) sowie Standorte mit hoch anstehendem Grundwasser, ausgeprägte Stauwasserstandorte und zu trockene Standorte wie Sandpodsole sowie extrem flachgründige Böden. Als problematische Standorte kommen die wechselfeuchten und wechseltrockenen Standorte hinzu, auf denen in bzw. nach Trockenperioden eine erhöhte Disposition für Trocknisschäden, Buchen-Pilzerkrankungen und Buchenprachtkäferbefall besteht (vgl. HOLM 1995, KÖLLING et al. 2005, AREND et al. 2006, NiESAR 2007, PETERCORD et al. 2007).

Mit Blick auf die Ertragserwartung und den Klimawandel sollte sich die Buchenwirtschaft auf die tiefgründigen, frischen und mindestens mäßig mit Nährstoffen versorgten Standorte konzentrieren. Diese Böden erschließt die Buche mit ihrem intensiven Herzwurzelsystem hervorragend, was ihr eine gute Sturmfestigkeit verleiht. Der relativ großen Spätfrostgefahr in der Jugendphase kann durch Naturverjüngung unter dem Albestandsschirm oder Voranbau unter dem Schirm anderer Baumarten vorgebeugt werden.

Die Konkurrenzkraft der Buche beruht vor allem auf ihrer großen Schattentoleranz und der hohen Intensität ihres Bestandesschattens. Beide Eigenschaften verschaffen ihr Vorteile in der Verjüngungsphase. Das große Schattenerträgnis führt zu einer relativ langsamen Astreinigung, die einen längeren Schirmdruck und einen engen Dichtstand für eine gute Qualitätsentwicklung erforderlich macht (u. a. LEONHARDT u. WAGNER 2006). Weitere charakteristische Merkmale sind das lang anhaltende Höhenwachstum und ihre große Kronenplastizität bis ins hohe Alter (WAGNER 1999, UTSCHIG 2000). In Verbindung mit ihrem spät kulminierenden, dann jedoch lange auf hohem Niveau verbleibenden laufenden Volumenzuwachs (KRAMER 1988, S. 103) ist sie den meisten Mischbaumarten auf Dauer überlegen und neigt bei unbeeinflusster Bestandesentwicklung zur Bildung von Reinbeständen. Dieser Zuwachsgang und die gute Reaktionsfähigkeit auf Standraumerweiterungen bis ins hohe Alter ermöglichen es, bei der Buche die Grundsätze der Zielstärkennutzung betriebswirtschaftlich besonders wirksam umzusetzen (MiTSCHERLICH 1953/54, SPELLMANN 1999, RÖHE 2003).

Von allen heimischen Laubbaumarten zeigt die Buche die stärkste Selbstdifferenzierung und übertrifft darin auch die meisten Nadelbaumarten. Vor allem die starke Durchmesserdifferenzierung ist ein Merkmal auch unbehandelter Buchenbestände. Beispielhaft ist dafür in Tabelle 1 die Durchmesserdifferenzierung für je 
eine unbehandelte Buchen- und Fichten-Versuchsparzelle in vergleichbarem Alter und unter vergleichbaren Standortverhältnissen zu zwei Aufnahmezeitpunkten dargestellt. Die deutlich größeren Standardabweichungen bzw. Variationskoeffizienten der mittleren Brusthöhendurchmesser verdeutlichen die stärkere Differenzierung der Buchen-Parzelle, in der auch im Alter von 58 Jahren noch lebende Bäume mit $4 \mathrm{~cm}$ Brusthöhendurchmesser gefunden wurden.

Tabelle 1: Durchmesserdifferenzierung unbehandelter Buchen- und Fichtenparzellen auf vergleichbaren Buntsandstein-Standorten im Solling (Nullflächen Buchen-Df.-Versuch Münden 2028 u. Fichten-Df.-Versuch Neubaus 2271)

\begin{tabular}{|c|c|c|c|c|}
\hline \multirow{2}{*}{$\begin{array}{l}\text { Versuchsfläche } \\
\text { Alter [Jahre] }\end{array}$} & \multicolumn{2}{|c|}{$\begin{array}{c}\text { Buche } \\
\text { Münden 2028, Parz. } 6 \\
\text { 0,2 ha }\end{array}$} & \multicolumn{2}{|c|}{$\begin{array}{c}\text { Fichte } \\
\text { Neuhaus 2271, Parz. } 4 \\
0,2 \text { ha }\end{array}$} \\
\hline & 44 & 58 & 37 & 52 \\
\hline $\mathrm{N}$ (lebend) & 475 & 267 & 502 & 321 \\
\hline Mittelwert BHD [cm] & 11,6 & 17,7 & 14,3 & 20,8 \\
\hline $\min$. BHD $[\mathrm{cm}]$ & 4,0 & 4,0 & 4,4 & 9,9 \\
\hline $\max$. BHD $[\mathrm{cm}]$ & 25,0 & 34,2 & 25,5 & 36,4 \\
\hline Standardabweichung & 4,37 & 6,05 & 3,83 & 5,29 \\
\hline Variationskoeffizient [\%] & 37,6 & 34,2 & 26,8 & 25,4 \\
\hline
\end{tabular}

Eine weitere wichtige Eigenschaft der Buche ist ihre starke Qualitätsdifferenzierung. Auffallend an natürlich verjüngten Buchenbeständen ist das oftmals räumlich konzentrierte Auftreten qualitativ guter, aber auch schlechter Individuen (KATÓ u. MÜLDER 1979). Neben Dichteunterschieden in der Aufwuchsphase sind vielfach genetische Aspekte dafür ursächlich. In mehreren Untersuchungen konnte gezeigt werden, dass in Buchennaturverjüngungsbeständen die Bäume in einem Bereich mit einem Radius von 30-100 m genetisch ähnlicher sind, als man es bei einer zufälligen Verteilung der Genotypen im Raum erwarten würde (u. a. DoUNAVI 2000). Diese Strukturen erklärt man mit der begrenzten Pollen- und Samenverbreitung, die im Laufe der Zeit zu Familienstrukturen führt. 


\section{Wachstumstrends der Buche in Nordwestdeutschland}

\subsection{Die Buchen-Durchforstungsversuche der Nordwestdeutschen Forstlichen Versuchsanstalt (NW-FVA)}

Zur Einordnung des derzeitigen Wachstums von Buchenbeständen in Nordwestdeutschland im Vergleich zu den in der Praxis anerkannten und bewährten Bestandeswachstumsmodellen, wie der Buchenertragstafel von SCHOBER (1967) für mäßige und starke Durchforstung, und Einzelbaumsimulatoren wurden die Versuchsflächen der Nordwestdeutschen Forstlichen Versuchsanstalt hinsichtlich ihres Höhenwachstums, ihrer Grundflächenhaltung und ihres Durchmesserwachstums ausgewertet.

Gegenwärtig werden 14 Durchforstungsversuche in Buchenreinbeständen mit 77 Parzellen bei einer durchschnittlichen Parzellengröße von 0,208 ha beobachtet. Unter diesen Versuchen befinden sich die ,alten“ Versuche Chausseehaus 116, Herborn 1333, Laubach 130, Paderborn 616/618, Saarforst 1606 und Saupark 2080, deren Bestandesalter zur letzten ertragskundlichen Aufnahme zwischen 109 und 159 Jahren lag, mit Beobachtungszeiträumen zwischen knapp 80 und über 120 Jahren. Sie stocken auf mäßig frischen bis frischen Standorten mit überwiegend mäßiger Nährstoffversorgung. Nur Laubach 130 und Saupark 2080 sind gut nährstoffversorgt. Die Versuche wurden zwischen 1872 und 1934 von Bernhardt, Schwappach, Heß und Wiedemann angelegt mit dem Ziel, den Einfluss unterschiedlich starker Nieder- und Hochdurchforstungen auf die Dimensions- und Vorratsentwicklung vergleichen zu können. In den Versuchen Saarforst Süd, Paderborn und Chausseehaus wurden zudem mit Versuchsbeginn in den Hochdurchforstungsvarianten Z-Baum-Kollektive in der Größenordnung zwischen 170 bis 240 Z-Bäumen je ha markiert. Die Durchforstungen erfolgten auf Grundlage der Anweisung zur Auszeichnung von Durchforstungs- und Lichtungs-Versuchen des Vereins Deutscher Forstlicher Versuchsanstalten (ANONYMUS 1902).

Die ,jüngeren“ Buchen-Durchforstungsversuche wurden seit 1990 von Riebeling, Spellmann, Guericke, Nowack und Nagel angelegt (Schotten 393/395 im Jahre 1977) und befanden sich zur Erstdurchforstung in einem Altersbereich zwischen 26 und 44 Jahren. Für 24 Parzellen der zuletzt angelegten Versuche gibt es noch keine Wiederholungsaufnahme, weshalb sie nur in die Darstellung der Höhenentwicklung (Punktdarstellung) einbezogen wurden. Die jüngeren Versuche stocken schwerpunktmäßig auf frischen bis vorratsfrischen, ziemlich gut bis gut mit Nährstoffen versorgten Standorten. Zwei der Versuche liegen im niedersächsischen Tiefland, die anderen im Bergland von Niedersachsen, Hessen, RheinlandPfalz und im Saarland in Höhenlagen zwischen ca. 200 und 550 m. Die Gesamtbaumzahlen vor Durchforstungsbeginn lagen nach den Mittelwerten der Versuche zwischen 2.100 und 7.500 Stück je ha, im Durchschnitt bei 4.900 Stück je ha. Zum Oberstand wurden durchschnittlich 2.300 Bäume je ha gezählt. Die Oberhöhen 
$\left(\mathrm{h}_{100}\right)$ lagen bei durchschnittlich 16,6 m (Spannweite 10,8-20 m), die mittleren Durchmesser des Oberstandes $\left(\mathrm{d}_{\mathrm{g}}\right)$ zwischen 7,2 und 16,4 cm (Mittel 11,6 cm). Alle Bestände waren weitgehend aus Naturverjüngung entstanden. Nur in Ahlhorn und Lahnstein waren größere Fehlstellen in der Naturverjüngung mit Buchen ausgepflanzt worden.

Aufbauend auf den Untersuchungsergebnissen von SCHOBER (1972) wurden die neuen Versuche zur Beobachtung verschieden starker Hochdurchforstungsvarianten mit unterschiedlichen Z-Baum-Kollektiven von 80 bis max. 300 Stück je ha zu Versuchsbeginn angelegt. Die Durchforstungsstärke auf den Durchforstungsparzellen wird in allen Versuchen über Zielbestockungsgrade jeweils in Bezug zur Referenzgrundfläche einer undurchforsteten Nullfläche (natürlicher Bestockungsgrad) zahlenmäßig gesteuert. Je nach Durchforstungsvariante werden die Oberstandsgrundflächen zum Eingriffszeitpunkt auf 80\% (mäßige Durchforstung), $65 \%$ (starke Durchforstung) bzw. $50 \%$ (sehr starke Durchforstung) der Oberstandsgrundflächenhaltung der undurchforsteten Parzelle abgesenkt.

Die im Folgenden hinsichtlich des Einflusses der Bestandesbehandlung auf Struktur, Qualitätsentwicklung und Ertrag von Buchenbeständen weitergehend ausgewerteten Versuche sind in Tabelle 2 bezüglich ihrer Beobachtungsdauer und der standörtlichen Verhältnisse näher charakterisiert.

Tabelle 2: $\quad$ Standörtliche und klimatische Charakterisierung ausgewählter Buchen-Durchforstungsversuche der NW-FVA

\begin{tabular}{|c|c|c|c|c|c|c|c|c|c|}
\hline \multirow[t]{3}{*}{ Versuch } & \multirow{3}{*}{$\begin{array}{l}\text { Anlage } \\
\text { (Alter bei } \\
\text { Anlage) }\end{array}$} & \multirow{3}{*}{$\begin{array}{l}\text { Ekl. } \\
\text { (Ver- } \\
\text { suchs- } \\
\text { mittel } \\
2004 \text { ) }\end{array}$} & \multicolumn{3}{|c|}{ Standort } & \multicolumn{4}{|c|}{ Klima } \\
\hline & & & \multirow{2}{*}{$\begin{array}{l}\text { Höhe } \\
\text { ü. NN } \\
{[\mathrm{m}]}\end{array}$} & \multirow[t]{2}{*}{$\begin{array}{c}\text { Wasser- } \\
\text { versorgung }\end{array}$} & \multirow[t]{2}{*}{$\begin{array}{l}\text { Nährstoff- } \\
\text { versorgung }\end{array}$} & \multicolumn{2}{|c|}{$\begin{array}{c}\text { Temperatur } \\
{\left[{ }^{\circ} \mathrm{C}\right]}\end{array}$} & \multicolumn{2}{|c|}{$\begin{array}{l}\text { Niederschlag } \\
{[\mathrm{mm}]}\end{array}$} \\
\hline & & & & & & Jahr & VZ & Jahr & VZ \\
\hline $\begin{array}{l}\text { Saarforst } \\
1606\end{array}$ & $\begin{array}{c}1891 \\
(49)\end{array}$ & $\mathrm{I}, 3$ & 310 & $\begin{array}{l}\text { hangfrisch, } \\
\text { tiefgründig }\end{array}$ & $\begin{array}{c}\text { mäßig / } \\
\text { ziemlich gut }\end{array}$ & 8,8 & 15,1 & 819 & 354 \\
\hline $\begin{array}{c}\text { Paderborn } \\
616 / 618\end{array}$ & $\begin{array}{c}1898 \\
(52)\end{array}$ & $\mathrm{I}, 1$ & 300 & $\begin{array}{l}\text { mäßig frisch, } \\
\text { flachgründig }\end{array}$ & mäßig & 7,4 & 13,6 & 863 & 391 \\
\hline $\begin{array}{c}\text { Herborn } \\
1333\end{array}$ & $\begin{array}{l}1930 \\
(34)\end{array}$ & $\mathrm{II}, 2$ & 560 & $\begin{array}{l}\text { hangfrisch, } \\
\text { tiefgründig }\end{array}$ & mäßig & 5,9 & 12,3 & 922 & 382 \\
\hline $\begin{array}{c}\text { Ahlhorn } \\
106\end{array}$ & $\begin{array}{c}1991 \\
(26-31)\end{array}$ & $-\mathrm{I}, 5$ & 40 & $\begin{array}{c}\text { schwach } \\
\text { wechselfeucht }\end{array}$ & ziemlich gut & 8,6 & 14,7 & 617 & 315 \\
\hline $\begin{array}{c}\text { Unterlüß } \\
138\end{array}$ & $\begin{array}{c}1991 \\
(35)\end{array}$ & $-0,4$ & 110 & $\begin{array}{l}\text { mäßig frisch, } \\
\text { tiefgründig }\end{array}$ & $\begin{array}{l}\text { mäßig / } \\
\text { ziemlich gut }\end{array}$ & 7,6 & 14,6 & 775 & 359 \\
\hline $\begin{array}{c}\text { Münden } \\
2027\end{array}$ & $\begin{array}{c}1991 \\
(36)\end{array}$ & 0,6 & 380 & $\begin{array}{c}\text { frisch bis } \\
\text { vorratsfrisch, } \\
\text { mittelgründig }\end{array}$ & ziemlich gut & 7,5 & 13,9 & 840 & 380 \\
\hline $\begin{array}{c}\text { Münden } \\
2028\end{array}$ & $\begin{array}{l}1991 \\
(44)\end{array}$ & 0,7 & 380 & $\begin{array}{c}\text { frisch bis } \\
\text { vorratsfrisch, } \\
\text { mittelgründig }\end{array}$ & ziemlich gut & 7,5 & 13,9 & 840 & 380 \\
\hline
\end{tabular}




\subsection{Beobachtete Trends des Höhenwachstums}

Ein wichtiger Weiser für die Einschätzung der Leistungsfähigkeit einer Baumart ist ihre Höhenentwicklung über dem Alter auf gegebenem Standort. Zur Charakterisierung der Alters-Höhen-Beziehung werden heute i.d. R. Oberhöhen bzw. Spitzenhöhen verwendet, weil sie weniger von der Bestandesbehandlung beeinflusst werden als Mittelhöhen. Die Oberhöhenentwicklungen aller Parzellen der Buchen-Durchforstungsversuche der NW-FVA sind in Abbildung 3 dargestellt.

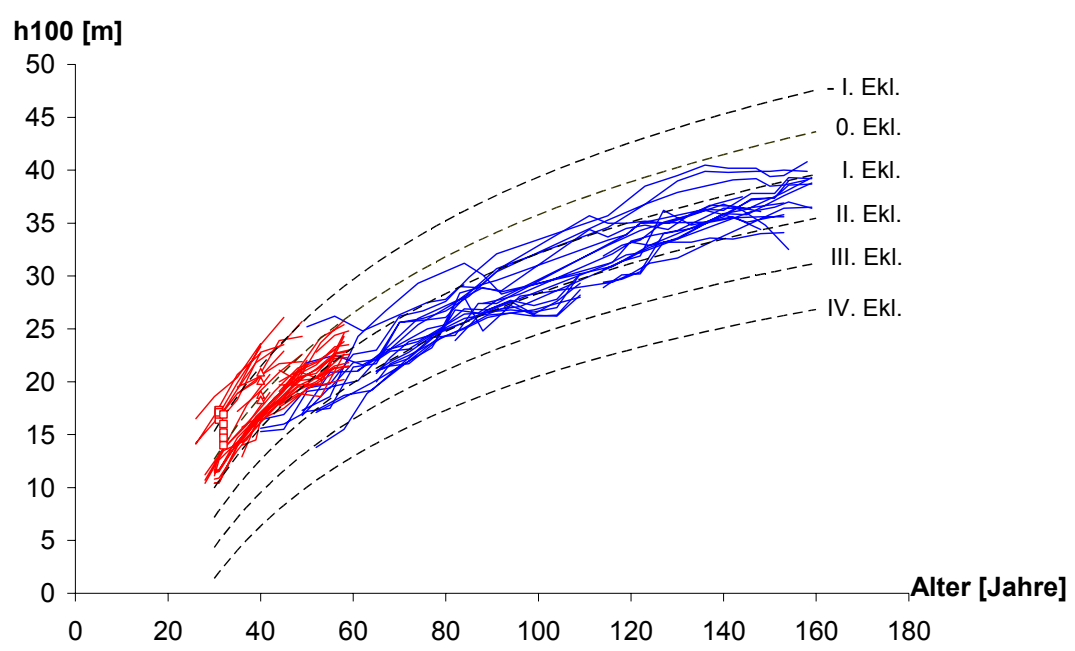

Abbildung 3: Höhenentwicklung über dem Alter für Buchen-Durchforstungsversuche der NW-FVA; rot: jüngere Versuche (Anlage ab 1990), blau: alte Versuche; Referenz: Bonitätsfächer der Buchen-Ertragstafel, mäß. Df., -I. und 0. Ertragsklasse extrapoliert (nach SCHOBER 1967, Regressionsfunktion n. DÖBBELER et al. 2003)

Als Vergleichsmaßstab dient der Bonitätsfächer der Buchen-Ertragstafel, mäß. Df. von SCHOBER (1967). Er wurde mit Hilfe einer Regressionsfunktion von DÖBBELER et al. (2003) um eine 0. und eine -I. Ertragsklasse erweitert. Die in blau dargestellten alten Versuche folgen weitgehend der Höhenentwicklung der Ertragstafel für die I. bis II. Ertragsklasse. Nur der Versuch Laubach 130 (besserer Standort) zeigt im Alter zwischen 120 und 150 Jahren eine deutlich steilere Höhenentwicklung. Das Absinken der Oberhöhen einiger Versuchsparzellen in höherem Alter ist durch die einsetzende Zielstärkennutzung zu erklären. Ein deutlich besseres Höhenwachstum zeigen die rot dargestellten jüngeren Versuche. Die Mehrzahl der Parzellen liegt im Niveau zwischen der I. und 0. Ertragsklasse. Die Versuche Lahnstein 9 und Saarforst 1668 (jeweils erst eine Aufnahme) sowie Unterlüß 138 liegen teilweise und Ahlhorn 106 vollständig oberhalb der Höhenentwicklung der -I. Ertragsklasse. Insgesamt zeigen die jungen Buchen-Durchforstungsversuche ein erstaunlich hohes Niveau im Höhenwachstum, wobei sie im Durchschnitt auf besseren Standorten stocken als die alten Versuche. Im Wachstumsgang folgen 
aber auch die jungen Versuche dem extrapolierten Bonitätsfächer der Schober`schen Ertragstafeln.

\subsection{Grundflächenentwicklung}

Die Bestandesgrundfläche charakterisiert bei gegebener Bonität die Wuchsleistung und die Dichte eines Bestandes. In Relation zu Modellvorgaben (Ertragstafeln, maximale Grundflächenhaltungen) ist sie eine wichtige Basisinformation für Nutzungsansätze bzw. -planungen. Die Grundflächenentwicklungen der mäßig bzw. stark und sehr stark durchforsteten Versuchsparzellen der alten Durchforstungsversuche (meist I. bis II. Ekl.) werden im Altersrahmen zwischen 80 und 120 Jahren weitaus besser durch die Grundflächenhaltungen der Ertragstafeln I. Bonität bei mäßiger oder starker Durchforstung abgebildet als davor und danach (s. Abb. 4). In der Jugend sind sie deutlich zu hoch und im höheren Alter, vor Einsetzen der Zielstärkennutzungen, zu gering. Die jüngeren Versuche im Altersrahmen zwischen 30 und 60 Jahren liegen fast ausnahmslos über der Grundflächenentwicklung der I. Ertragsklasse, mäßige Durchforstung. Auch unter Berücksichtigung ihrer besseren Bonitäten liegen die Tafelvorgaben deutlich zu niedrig, denn zu diesen Versuchen gehören auch mehrere Parzellen mit starker $(65 \%)$ bzw. sehr starker (50\%) Durchforstung im Verhältnis zu den jeweiligen Nullflächen (100\%), die in ihrem Bestandesbild ausnahmslos den Behandlungsvorgaben entsprechen.

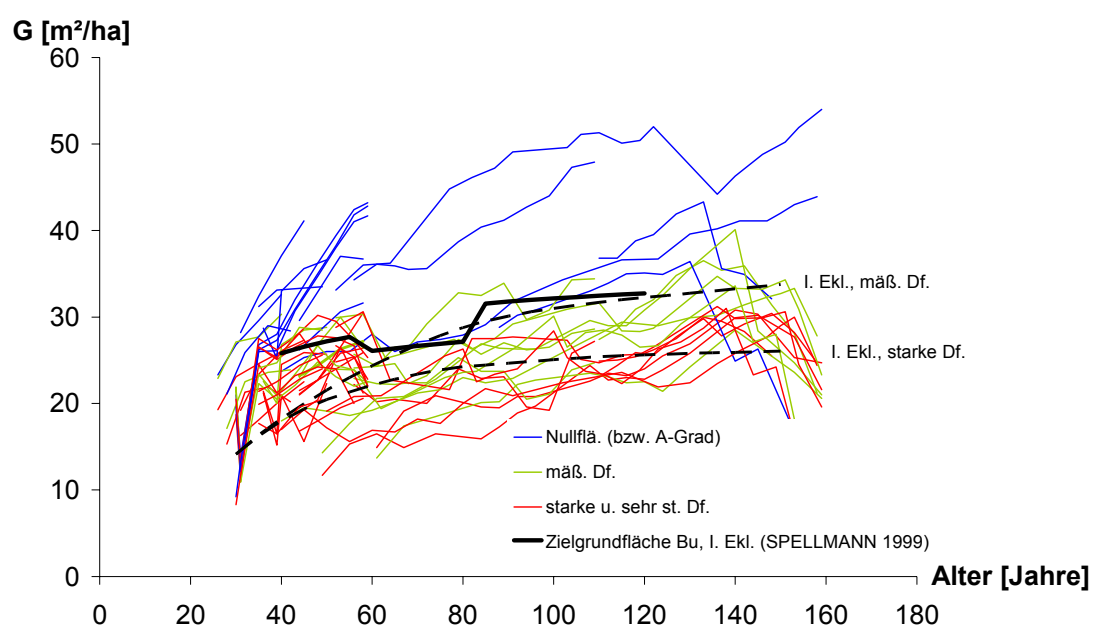

Abbildung 4: Grundflächenhaltung (verbl. Bestand) über dem Alter für Buchen-Durchforstungsversuche der NW-FVA; Referenz: Grundflächenbaltung der Buchenertragstafel, I. Ekl., mäß. und starke Df. (nach SCHOBER 1967) 
Auffallend sind die Grundflächenentwicklungen der blau dargestellten Nullflächen. Sie ragen deutlich aus der Kurvenschar heraus. Die Grundfläche des A-Grads des Versuchs Paderborn 616/618 erreicht im Alter 159 Jahre $54 \mathrm{~m}^{2}$ je ha, die des AGrads in Herborn $49 \mathrm{~m}^{2}$ je ha im Alter 109 und die drei Nullflächen des 59 Jahre alten Versuchs in Schotten nahezu $45 \mathrm{~m}^{2}$ je ha. Die Werte der Versuche Paderborn und Herborn liegen teilweise selbst deutlich über den maximalen Grundflächen für Buchenbestände in Nordwestdeutschland, die DÖBBELER (2004) mit Hilfe eines Verfahrens von STERBA $(1975,1981,1987)$ aus der Stammzahl-DurchmesserBeziehung unbehandelter bzw. schwach durchforsteter Versuchsflächen herleitete. Danach liegen die maximalen Grundflächen bei einer Oberhöhe ( $\left.h_{100}\right)$ von 32,4 m (für I. Ekl., mäß. Df. n. SCHOBER im Alter 100 erreicht) bei ca. $43 \mathrm{~m}^{2}$ je ha, die bis zu einer Oberhöhe $\left(\mathrm{h}_{100}\right)$ von $40 \mathrm{~m}$ noch auf knapp $45 \mathrm{~m}^{2}$ je ha ansteigen.

SPELLMANN et al. (1999) leiteten Durchforstungsvorgaben zur Festlegung summarischer Nutzungsansätze für die Forsteinrichtung mit Bezug zu den maximalen Grundflächen nach DÖBBELER (2004) her. Für die Buche wurden für drei an bestimmte Oberhöhenbereiche festgemachte Pflegephasen Zielbestockungsgrade von 0,70 - 0,65 - 0,75 in Bezug zur maximalen Grundfläche definiert. Der Verlauf der Grundflächenhaltung über dem Alter nach diesem Durchforstungskonzept mit gestaffelter Eingriffsstärke ist für die I. Ekl. in Abbildung 4 mit dargestellt. Er entspricht besser den Nutzungsmöglichkeiten, wie sie sich aus dem Wachstumsgang der Buche ergeben.

\subsection{Durchmesserzuwachspotenzial der Buche}

$\mathrm{Zu}$ den besonderen Merkmalen der Buche zählt ihre Zuwachsplastizität. Die durchschnittlichen jährlichen Durchmesserzuwächse in der 5-jährigen Aufnahmeperiode von 1994 bis 1999 bzw. 1995 bis 2000 machen deutlich, dass die Buche in der Lage ist, bis ins hohe Alter hohe jährliche Durchmesserzuwächse zu leisten (s. Abb. 5). So wurden in den älteren Durchforstungsversuchen bei einem BHD zwischen 60 und $70 \mathrm{~cm}$ noch jährliche Durchmesserzuwächse von bis zu $8 \mathrm{~mm}$ ermittelt.

In den über 100-jährigen Durchforstungsversuchen fällt die große Durchmesserspreite von $7 \mathrm{~cm}$ bis $80 \mathrm{~cm}$ BHD auf, die für die Buche charakteristisch ist (s. Abb. 5). Der durchschnittliche jährliche Durchmesserzuwachs der unterständigen Bäume (BHD $<20 \mathrm{~cm}$ ) ist erwartungsgemäß gering. Er liegt zwischen 0 und $3 \mathrm{~mm}$ im Jahr. In den jüngeren Versuchsflächen konnten hingegen im Durchmesserbereich zwischen 15 und $25 \mathrm{~cm}$ Maximalzuwächse von bis zu $12 \mathrm{~mm}$ im Jahr festgestellt werden. Als Referenz wurde in die Abbildung die aus bayerischem Versuchsflächenmaterial abgeleitete Potenzialfunktion des Einzelbaumwachstumssimulators SILVA (maximaler Durchmesserzuwachs) für die Baumart Buche mit aufgenommen (KAHN u. PRETZSCH 1997, UTSCHIG 2000). Die gemessenen Zuwächse in den über 100-jährigen Durchforstungsversuchen werden abgesehen 
von einzelnen Ausreißern über das ganze Durchmesserspektrum hinweg von der Durchmesserzuwachspotenzialkurve umschlossen. Es lässt sich aber auch erkennen, dass die jüngeren Durchforstungsversuche eine deutlich schnellere Durchmesserentwicklung aufweisen und hier das Durchmesserzuwachspotenzial im Bereich von Ausgangsdurchmessern zwischen 10 und $25 \mathrm{~cm}$ zum Teil durch die Potenzialfunktion unterschätzt wird.

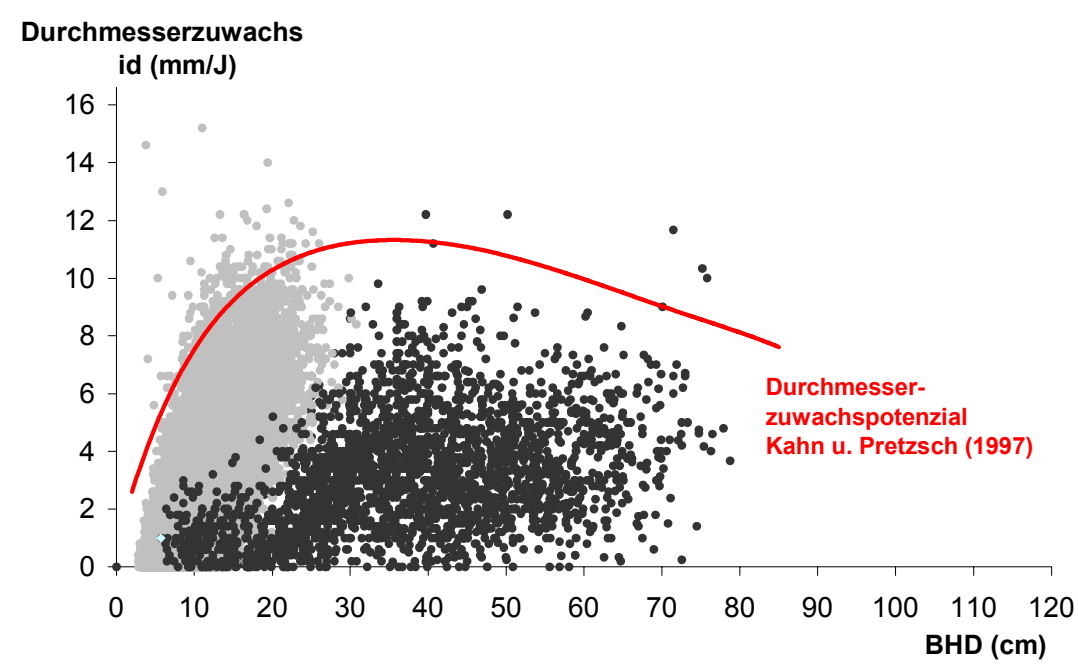

Abbildung 5: Beobachtete Durchmesserzuwächse über dem BHD für Buchen-Durchforstungsversuche der NW-FV A; grau: Einzelbaumzuwächse in den jüngeren Durchforstungsversuchen, schwar:: Einzelbaumzuwächse in den älteren Versuchsanlagen (nach GUERICKE 2002) Referenz: Potenzialfunktion für den Durchmesserzuwachs der Buche aus dem Einzelbaumsimulator „Silva“ (KAHN u. PRETZSCH 1997, UTSCHIG 2000)

\subsection{Reaktionsfähigkeit von Einzelbäumen auf Standraumerweiterungen}

In jungen bis mittelalten Beständen findet sich normalerweise ein enger linearer Zusammenhang zwischen dem Ausgangsdurchmesser und dem Durchmesserzuwachs (PRODAN 1965). Diese Zuwachsgeraden sind in der Jugend auf höherem Niveau, verlaufen steiler und fallen mit dem Alter ab. Der Rückgang des Anstiegs des Durchmesserzuwachses mit dem Alter ist bei der Buche nicht so stark ausgeprägt wie bei Lichtbaumarten. Die Zuwachsgeraden für eine Parzelle des Versuchs Herborn 1333 mit schwacher Hochdurchforstung zu drei unterschiedlichen Zeitpunkten verdeutlichen diesen Zusammenhang (s. Abb. 6). 


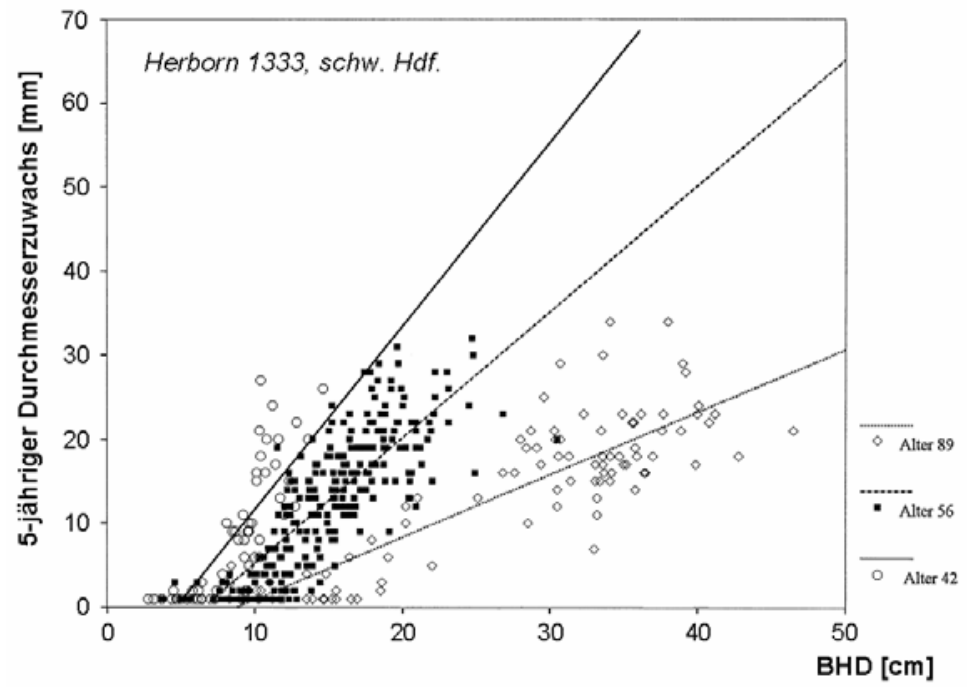

Abbildung 6: Durchmesserzuwachsgeraden für einen Buchenbestand in verschiedenen Altern; BuchenDurchforstungsversuch Herborn 1333, schw. Hochdurchforstung

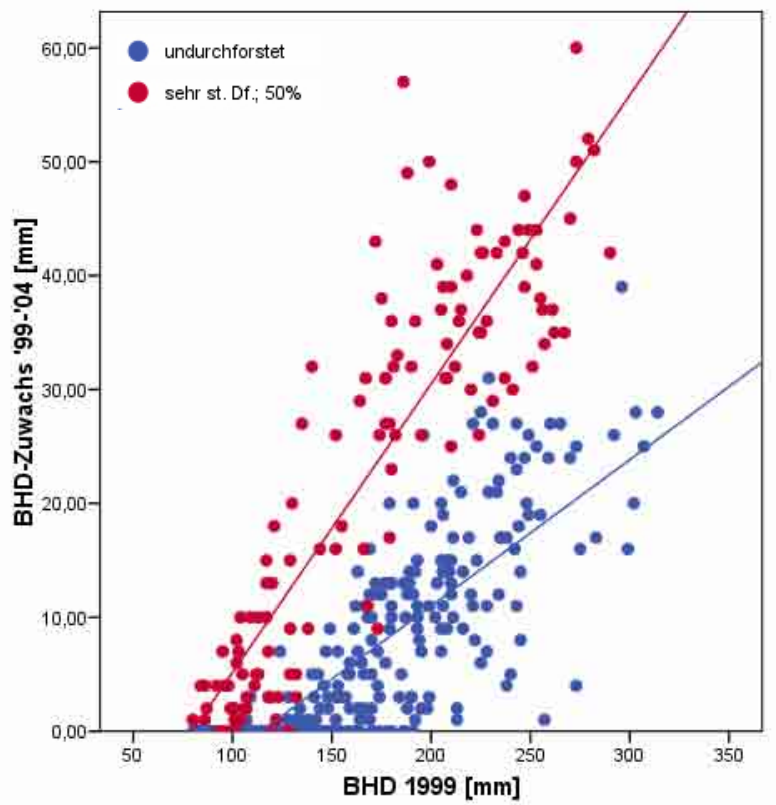

Abbildung 7: Durchmesserzuwachsgeraden für einen Buchenbestand bei unterschiedlicher Behandlung; Buchen-Durchforstungsversuch Münden 2028, Nulfläche und sehr starke Hochdurchforstung 
Die Lage und der Anstieg der Durchforstungsgeraden werden darüber hinaus auch durch die Eingriffsstärke wesentlich beeinflusst. Stärkere Eingriffe führen wie in dem Buchen-Durchforstungsversuch Münden 2028 zu einem steileren Anstieg, also zu einem höheren Durchmesserzuwachs bei gleichem Ausgangsdurchmesser (s. Abb. 7).

Aus diesen Zusammenhängen lässt sich folgern, dass auch bei der Buche, die sich durch eine lang anhaltende Reaktionsfähigkeit und ein dauerhaft hohes Zuwachsniveau auszeichnet, starke Eingriffe zu einem umso deutlicheren Durchmesserzuwachseffekt führen, je früher sie erfolgen. Allerdings muss zuvor die angestrebte astfreie Schaftlänge erreicht sein.

\section{Einfluss der Bestandesbehandlung auf Struktur, Qualitäts- entwicklung und Ertrag von Buchenbeständen}

\subsection{Durchmesserdifferenzierung bei unterschiedlichen Eingriffsstärken}

Viele Gründe sprechen dafür, den Strukturreichtum in unseren Wäldern zu erhöhen. Sie reichen von der Verbesserung der Stabilität und Elastizität der Bestände, der Erleichterung von Naturverjüngungen, über eine Erhöhung der Artendiversität bis hin zu ökonomischen Vorteilen hinsichtlich der Sortenstruktur und der Angebotsflexibilität. Es gibt jedoch mit Vertretern des Prozessschutzes eine kontroverse Diskussion darüber, inwieweit durch gezielte Pflegekonzepte die Strukturbildung gefördert werden oder der eigendynamischen Strukturentwicklung überlassen bleiben soll (SPELLMANN et al. 2004).

Durchforstungseffekte auf die strukturelle Diversität von Buchenbeständen wurden an der NW-FVA eingehend untersucht (MEYER 2000, SPELLMANN et al. 2004). Die Datenbasis für den Versuch Münden 2028 wurde für diesen Beitrag um die Daten einer weiteren ertragskundlichen Aufnahme im Jahre 2004 erweitert. In die Untersuchung wurden die Parzellen 2 (mäßige Hochdurchforstung), 4 (starke Hochdurchforstung), 6 (Nullfläche) und 8 (sehr starke Hochdurchforstung) des Buchen-Durchforstungsversuches Münden 2028 einbezogen. Nähere Angaben zu den standörtlichen Gegebenheiten der Versuchsfläche finden sich in Tabelle 2. Bei den Behandlungsvarianten handelt es sich, wie bereits näher erläutert (s. Kap. 4.1), um Z-Baum-orientierte Hochdurchforstungen verschiedener Eingriffsstärke und eine unbehandelte Nullfläche. Die Zahl der bei Versuchsanlage markierten ZBäume betrug 300 je ha bei mäßiger und starker Hochdurchforstung und 160 bei sehr starker Hochdurchforstung.

Die Datengrundlage der Diversitätsanalyse bildeten die BHD-Vollaufnahmen des nummerierten Bestandes (über $7 \mathrm{~cm}$ BHD) in den Bestandesaltern 44 Jahre (Durchforstungsbeginn) sowie 48, 53 und 58 Jahre. Die natürliche Mortalität wurde im Zuge der ertragskundlichen Aufnahmen in allen Parzellen erfasst. Es 
handelt sich dabei um Eingriffsinventuren, d. h. in den behandelten Parzellen wurde im Zuge der Aufnahmen der ausscheidende Bestand ausgezeichnet, erfasst und kurz darauf entnommen bzw. die abgestorbenen Bäume getrennt festgehalten.

Die strukturelle Diversität von Wäldern ist sehr komplex, da sie von einer Vielzahl von Merkmalen bestimmt wird. Die vorliegende Analyse beschränkt sich auf die strukturelle Diversität der Brusthöhendurchmesser (BHD) des lebenden Derbholzbestandes. Viele andere Kenngrößen sind eng mit dieser Schlüsselgröße korreliert. Aus der Vielzahl der Diversitätsindizes wird der Shannon-Index (SHANNON u. WEAVER 1949) verwendet. In seine Berechnung gehen die Anzahl vorhandener absoluter Durchmesserklassen mit $4 \mathrm{~cm}$ Klassenbreite ab Derbholzgrenze und die Besetzung dieser Klassen ein. Als Bezugsgröße maximaler Durchmesserdiversität von Wäldern diente der Plenterwald. In Übereinstimmung mit diesem Maßstab wurden die BHD-Klassen mit der Grundfläche gewichtet. Die Vorgehensweise ist bei MEYER (2000) ausführlich beschrieben.

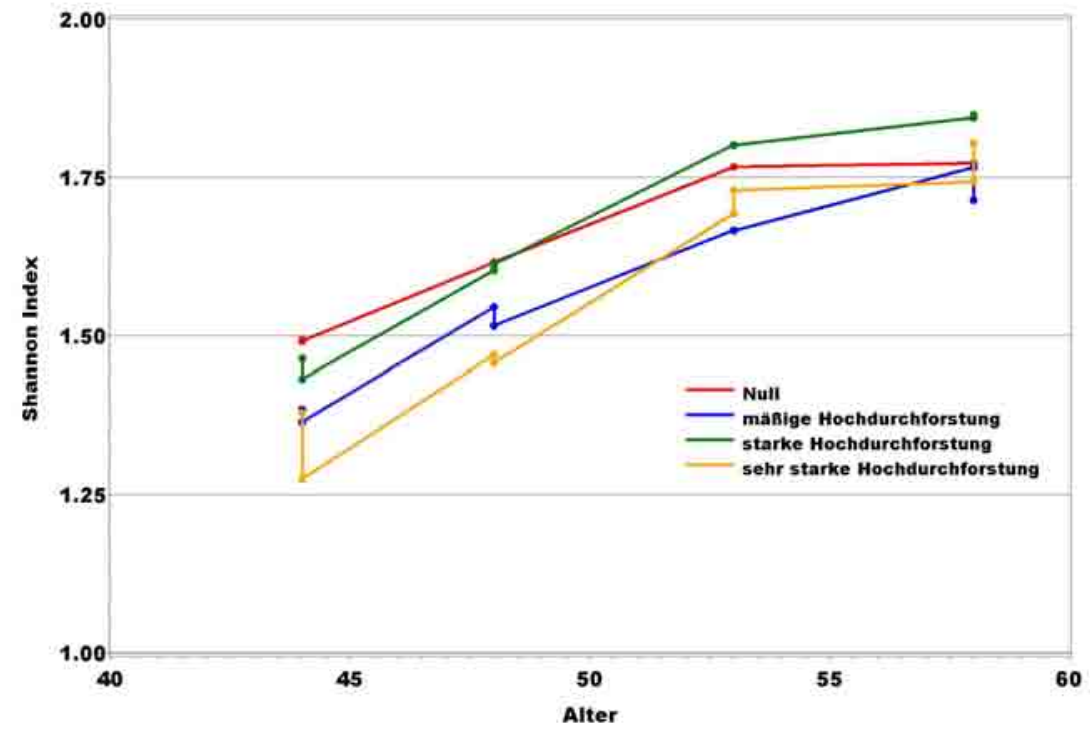

Abbildung 8: Entwicklung des Shannon-Index als Maß für die Durchmesserdiversität über dem Alter in Abhängigkeit von der Bestandesbehandlung (Buchen-Durchforstungsversuch Münden 2028; Nullfläche, mäßige, starke und sehr starke Hochdurchforstung)

Die durch den Shannon-Index ausgedrückte BHD-Diversität steigt für alle Eingriffsstärken bis zum Alter 53 Jahre deutlich an (s. Abb. 8). Die auftretenden „Sprünge“ zu den Aufnahmezeitpunkten sind unmittelbar auf die jeweils ausgezeichneten Durchforstungen zurückzuführen. Insgesamt fällt auf, dass sich die Durchmesserdiversität der einzelnen Parzellen während des Beobachtungszeitraumes nicht sehr stark voneinander unterscheidet. Der beträchtliche Rückgang des Shannon-Index bei der sehr starken Hochdurchforstung im Zuge des ersten 
Eingriffs ist auf größere Hiebsschäden am Unterstand zurückzuführen. In der Folge zeigt die Diversitätsentwicklung der Behandlungsvarianten jedoch unterschiedliche Trends. So nimmt die Diversität in der stark und der sehr stark durchforsteten Variante am deutlichsten zu, während die anfangs führende Nullfläche zurückfällt und zuletzt stagniert. Dies lässt sich wie folgt erklären:

Der Shannon-Index hängt stark von der Durchmessersspreitung ab (MEYER 2000, SPELLmanN et al. 2004) und erhöht sich mit steigender Anzahl der Durchmesserklassen. Eine Streckung der Durchmesserspanne ergibt sich zum einen aus der Erhöhung des BHD der dicksten Bäume und aus dem „Verharren“ dünner Bäume im unteren Durchmesserbereich. Eine maximale Differenzierung wird somit erreicht, wenn der Durchmesserzuwachs der herrschenden Bäume im Verhältnis zum unterständigen Baumkollektiv überproportional stark angeregt wird und zugleich ein Überleben der unterständigen Bäume gesichert ist. Ausdruck der unterschiedlichen Zuwachsverteilung bei unterschiedlicher Behandlung in Abhängigkeit vom Ausgangsdurchmesser sind die Lage und der Anstieg der Zuwachsgeraden (s. Abb. 7, Kap. 4.5). Daneben ist die Mortalität entscheidend für den Grad der Durchmesserdifferenzierung. Der stagnierende Shannon-Index der Nullfläche im Versuch Münden 2028 ist einerseits das Ergebnis des geringeren Durchmesserzuwachses der herrschenden Bäume, andererseits die Folge des zuletzt verstärkt einsetzenden Absterbens des Unterstandes. Ein Vergleich der ertragskundlichen Kennwerte des Unterstandes beider Versuchsparzellen verdeutlicht dies (s. Tab. 3).

Die Hochdurchforstung, die neben der Förderung des Durchmesserzuwachses der starken Bäume auch die Erhaltung eines vitalen Unterstandes gewährleistet, trägt danach am ehesten zur Differenzierung und Strukturierung in Buchenbeständen bei.

Tabelle 3: $\quad$ Einfluss der Behandlung auf den Unterstand nach vier Durchforstungen, Alter 58 Jahre, Durchforstungsbeginn mit 44 Jahren; Buchen-Durchforstungsversuch Münden 2028

\begin{tabular}{lcc}
\hline Unterstand & Nullfläche & starke Hdf. \\
\hline Grundfläche, lebend [m²/ha] & 4,7 & 5,4 \\
Stammzahl, lebend [N/ha] & 440 & 862 \\
$\mathrm{~d}_{\mathrm{g}}$ verbleibend [cm] & 11,7 & 8,9 \\
$\mathrm{~d}_{\mathrm{g}}$ ausscheidend [cm] (Mortalität) & 9,2 & 6,9 \\
\hline
\end{tabular}

\subsection{Möglichkeiten und Grenzen der positiven Beeinflussung der Qualitäts- entwicklung}

Die Optimierung der flächenbezogenen Wertleistung wurde bereits einleitend als eines der vorrangigen Ziele der Buchenwirtschaft herausgestellt. Diese Zielsetzung 
leitet sich aus der starken Gütedifferenzierung des Buchenholzes und den bedeutenden Preisunterschieden zwischen den Güteklassen ab. Bei den besseren Qualitäten ist zumindest bis zur Stärkeklasse L 5 auch die Stärkeklasse des Stammholzes ein wichtiges wertbestimmendes Merkmal (s. Tab. 4).

Tabelle 4: Buchen-Stammbolzerlöse in Abhängigkeit von Stärkeklasse und Güteklasse (durchschnittliche Erlöse in den Niedersächsischen Landesforsten im Jabre 2006)

stärkeklassenabhäng. Erlösentwicklung güteklassenabhäng. Erlösentwicklung

\begin{tabular}{cc|cc}
\hline Stärkeklasse & $\begin{array}{c}\text { Erlös für } \\
\text { Buchenstammholz } \\
\text { der Güte B } \\
{[€ / \text { Fm }]}\end{array}$ & Güteklasse & $\begin{array}{c}\text { Erlös für } \\
\text { Buchenstammholz } \\
\text { der Stärkekl. L5 } \\
{[€ / \text { Fm] }}\end{array}$ \\
\hline L 2a/b & $39,-$ & A & $205,-$ \\
L 3a & $50,-$ & B & $114,-$ \\
L 3b & $62,-$ & BK & $82,-$ \\
L4 & $93,-$ & C & $57,-$ \\
L5 & $114,-$ & & \\
L6 & $116,-$ & & \\
\hline
\end{tabular}

Betrachtet man die Anteile der Güteklassen am gesamten Buchen-Stammholzeinschlag in den Niedersächsischen Landesforsten für den Zeitraum von 1995 bis 2004 (SPELLMANN 2006) ${ }^{1}$, so wird die vergleichsweise starke Gütedifferenzierung des Buchenstammholzes ebenso deutlich wie der bescheidene Anteil des Stammholzes besserer Güte (s. Abb. 9). In der zehnjährigen Periode, die auch den Boom des Chinageschäftes zum Ende des letzten Jahrhunderts mit einschließt, wurden nur $6 \%$ des Buchen-Stammholzes als Wertholz und $40 \%$ als Stammholz der Güteklasse B sortiert, wobei dieses in Niedersachsen auch Stammholz der Güte „BK“ (Buchenstammholz mit Rotkernbildung von mehr als der Hälfte des Stammdurchmessers am stärkeren oder schwächeren Ende) umfasst. Demgegenüber liegt bei den Nadelhölzern der Anteil des Stammholzes der Güteklasse B und besser bei mindestens zwei Dritteln.

Angesichts der bereits seit über 100 Jahre andauernden Bemühungen um eine Verbesserung der Wertleistung von Buchenbeständen ist dieser Befund äußerst unbefriedigend. Er ist nicht nur im Zusammenhang mit den Behandlungskonzepten der Praxis, sondern auch mit den in der Vergangenheit oft weniger gelungenen Buchen-Naturverjüngungen, der starken Selbstdifferenzierung und der Plastizität

1 Vergleichbare Güteklassenverteilungen weisen auch die Holzeinschlagsstatistiken anderer Bundesländer aus. 
der Buchenkronen zu sehen. Darüber hinaus weisen u. a. KLEINSCHMIT u. SVOLBA (1996) und V. WÜHLISCH (2005) auf deutliche Herkunftsunterschiede in der Qualitätsentwicklung hin.

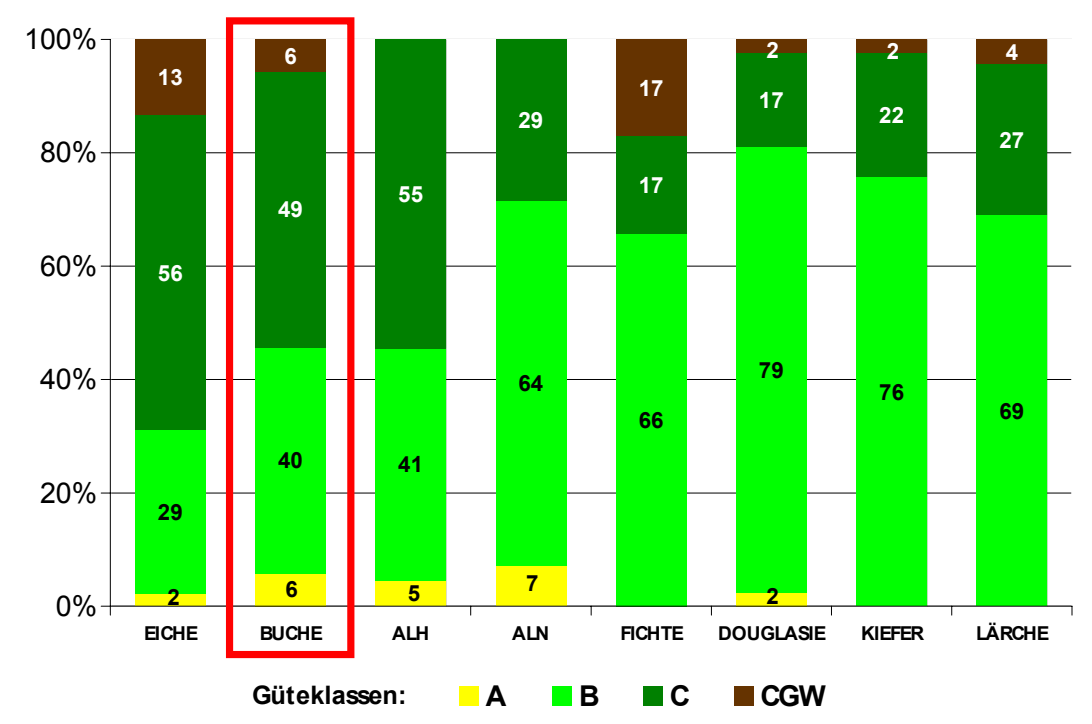

Abbildung 9: Güteklassen für den Gesamteinschlag Stammbolz der Niedersächsischen Landesforstverwaltung im Durchschnitt der Jahre 1995 bis 2004 (Quelle: SPELLMANN 2006)

Dass Durchforstungen dennoch ein sehr wichtiges waldbauliches Mittel zur Beeinflussung der Qualitätsentwicklung von Buchenbeständen sind, soll am Beispiel der periodisch durchgeführten Schaft- und Kronenansprachen im Buchen-Durchforstungsversuch Paderborn 616/618 verdeutlicht werden (s. Abb. 10). Verglichen werden die Behandlungsvarianten schwache Niederdurchforstung, schwache Hochdurchforstung (ohne Z-Baum-Markierung) und starke Hochdurchforstung (mit Z-Baum-Markierung). Die Schaftmerkmale gerade, bogig, Drehwuchs, Zwiesel, Klebäste, Wasserreiser und Ästigkeit mit ihren verschiedenen Ausprägungen wurden dabei zu den Schaftgüteklassen gut, befriedigend und schlecht zusammengefasst und hinsichtlich der Kronenausbildung nach gut, einseitig, geklemmt und gering unterschieden.

Die Ergebnisse zeigen, dass die Hochdurchforstungen im Laufe der Zeit zu besseren Kronen- und Schaftqualitäten bei den Bäumen der herrschenden Schicht führen als die Selbstdifferenzierung bei der schwachen Niederdurchforstung. Betriebswirtschaftlich erhalten die Qualitätsunterschiede im Zusammenhang mit der deutlich besseren Durchmesserentwicklung $\left(\mathrm{d}_{\mathrm{g}}\right)$ der Bäume der herrschenden Schicht ein noch größeres Gewicht (vgl. Tab. 4). 


\section{Schaftqualität}

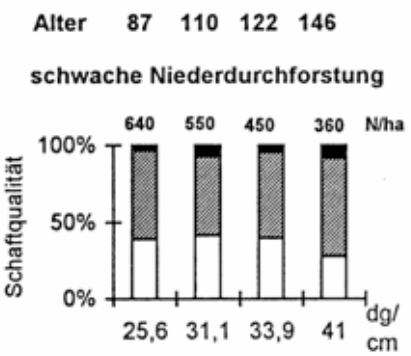

schwache Hochdurchforstung

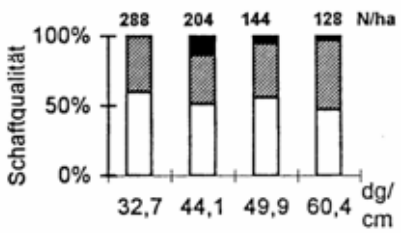

starke Hochdurchforstung

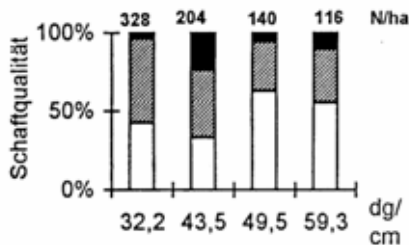

schlecht

$\mathbb{Z}$ befriedigend

gut

\section{Kronenqualität}

Alter $\quad 87 \quad 110 \quad 122 \quad 146$

schwache Niederdurchforstung

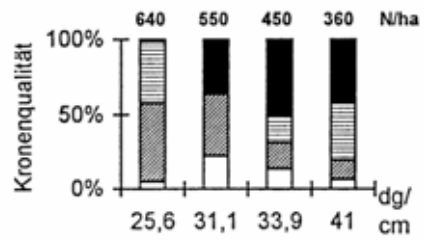

schwache Hochdurchforstung

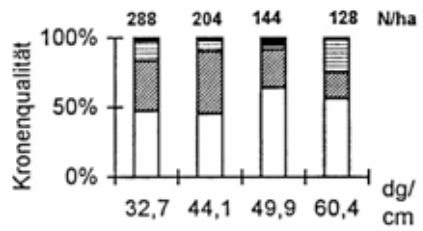

starke Hochdurchforstung

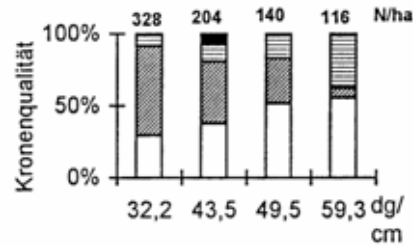

gering

豆 geklemmt

पIA einseitig

$\square$ gut

Abbildung 10: Entwicklung der Schaft- und Kronenqualitäten bei unterschiedlicher Durchforstung im Buchen-Durchforstungsversuch Paderborn 616/618 (aus SPELLMANN u. NAGEL 1996)

Der Wertertrag wird schließlich zu großen Anteilen von der Nutzung der hiebsreifen Bäume bestimmt. Die ökonomisch optimale Zielstärke lässt sich nach verschiedenen Verfahren herleiten (vgl. SPELLMANN 1995). Dabei gilt allgemein, je geringer der unterstellte Zinssatz, je höher der Zuwachs, je besser die Qualität und je geringer das Risiko, desto höher die Zielstärke und desto länger der Produktionszeitraum. Diese Zusammenhänge führen zu variablen Zielstärken, die insbesondere auch Qualitätsaspekte zu berücksichtigen haben. 
Betrachtet man die Wertentwicklung eines $8 \mathrm{~m}$ langen Buchen-Erdstammstückes mit zunehmender Zielstärke von $61 \mathrm{~cm}$ über $67 \mathrm{~cm}$ auf $74 \mathrm{~cm}$, so steigt der Wert bei B-Qualität von 226,- $€$ über 292,- $€$ auf 353,- $€$. Findet zwischenzeitlich eine Entwertung von B nach BK und schließlich durch Spritzkern nach $\mathrm{C}$ statt, so sinkt der Wert mit Dauer der Produktionszeit um 27,- $€$ bzw. 59,- $€$ (s. Abb. 11).

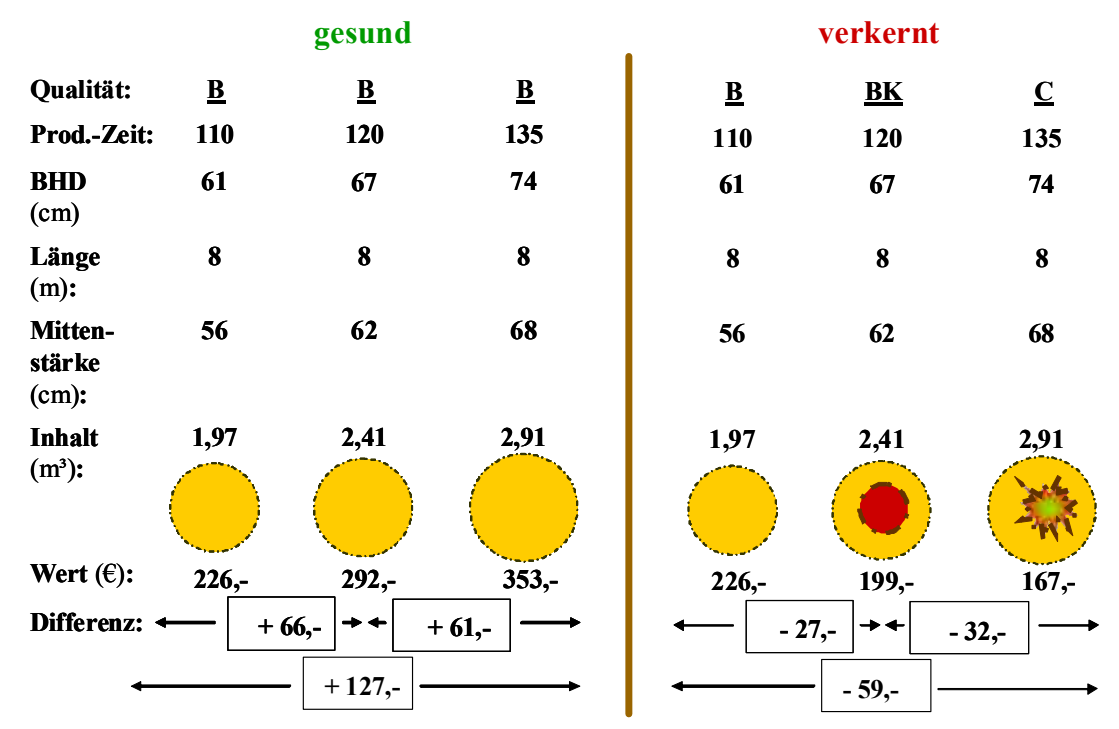

Abbildung 11: Auswirkungen von Zielstärke und Verkernung auf die Wertentwicklung bei Buche (aus SPELLMANN 2005)

\subsection{Durchmesserentwicklung von Z-Baumkollektiven und die Frage nach der Z-Baum-Zahl}

Die Auswahl von Z-Bäumen, ihre Markierung sowie der Zeitpunkt und die Stärke ihrer Freistellung sind wichtige Fragen einer wertertragsorientierten Steuerung von Buchenbeständen. Bereits im Kapitel 4.5 wurde der Einfluss des Alters und der Behandlung auf die Durchmesserentwicklung mit Hilfe von Zuwachsgeraden erläutert. Deren Anstieg ist umso steiler je früher die Durchforstungen einsetzen bzw. umso stärker sie geführt werden. Ähnlich sind die Versuchsergebnisse der vier jungen Buchen-Durchforstungsversuche Ahlhorn 106, Unterlüß 138 und Münden 2027 und 2028 zu interpretieren, wenn man den Einfluss der Durchforstungsstärke auf den Durchmesserzuwachs der jeweils 100 stärksten Z-Bäume je ha in den bisherigen drei Beobachtungsperioden betrachtet (s. Abb. 12). In der dritten Beobachtungsperiode nimmt jedoch der anfangs deutliche Vorsprung des Durchmesserzuwachses der sehr starken Durchforstung gegenüber demjenigen der starken Durchforstung wieder ab, was auf einen abklingenden Wuchsbeschleunigungseffekt schließen lässt. 

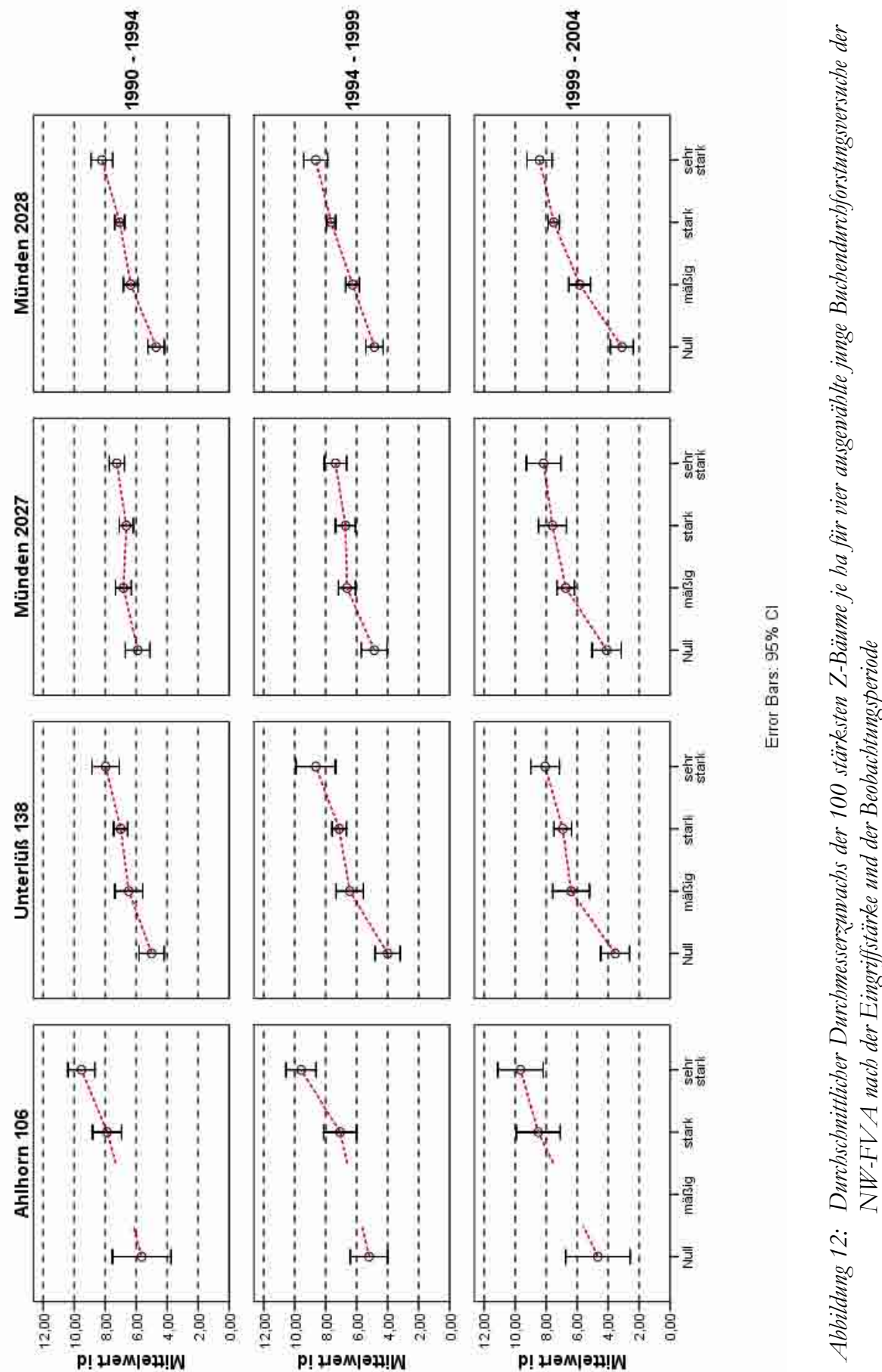

Beiträge aus der NW-FVA, Band 3, 2008 
Aufschlussreich sind auch die absoluten Beträge der durchschnittlichen jährlichen Durchmesserzuwächse der Z-Baumkollektive. Bei sehr starker Durchforstung und ab der zweiten Periode auch bei starker Durchforstung werden jährlich ca. $8 \mathrm{~mm}$ Dickenzuwachs erreicht, in dem besonders wüchsigen Versuch Ahlhorn 106 fast $10 \mathrm{~mm}$. Der Durchmesserzuwachs der nicht freigestellten Z-Bäume auf den Nullflächen geht von 4-6 $\mathrm{mm}$ in der ersten Periode auf ca. 3-4,5 $\mathrm{mm}$ in der dritten Periode zurück.

$\mathrm{Zu}$ Durchforstungsbeginn hatten die Z-Bäume in den hier vorgestellten Versuchen astfreie Schaftlängen von durchschnittlich $8 \mathrm{~m}$, in Münden 2027 von ca. 6,5 m. Während der 14-jährigen Beobachtungsdauer haben sich die Kronenansätze der Z-Bäume auf den undurchforsteten Nullparzellen um ca. 2,5 $\mathrm{m}$ nach oben verschoben. Daraus lässt sich ableiten, dass die Forderung nach einer astfreien Schaftlänge von ca. $10 \mathrm{~m}$ selbst in sehr wüchsigen Beständen eine Verzögerung der Erstdurchforstung um ca. 10 Jahre zur Folge hätte und den Verzicht auf $4-5 \mathrm{~cm}$ Dickenzuwachs der Z-Bäume bedeuten würde.

Versteht man unter Z-Bäumen im Anhalt an SCHOBER (1988b) die möglichen „Elitebäume“ für den Endbestand, die nur einmalig ausgewählt, laufend überprüft und dann kontinuierlich freigestellt und gefördert werden, so spielt für die Bemessung ihrer Anzahl neben der möglicherweise zu berücksichtigenden Reserve, der benötigte Standraum bei Erreichen des Produktionsziels eine wichtige Rolle. Die hier wiedergegebenen Ergebnisse zum Standraumbedarf von Buchen beruhen auf den Auswertungen der Buchen-Durchforstungsversuche der NW-FVA durch GUERICKE (2002). Danach haben Altbuchen mit Brusthöhendurchmessern zwischen 60 und $70 \mathrm{~cm}$ Kronenradien zwischen durchschnittlich $5 \mathrm{~m}$ und maximal $9 \mathrm{~m}$. Analog zur Beziehung zwischen BHD und Kronenbreite nimmt mit zunehmendem BHD auch die Kronenschirmfläche bis auf maximal $200 \mathrm{~m}^{2}$ bei einer $90 \mathrm{~cm}$ starken Buche zu (s. Abb. 13). Die am oberen Rand des Datenfeldes verlaufende Funktion beschreibt die Entwicklung der maximalen Kronenschirmfläche in Abhängigkeit vom BHD. Diese Funktion wurde aus Untersuchungen in BuchenLärchen-Mischbeständen übernommen (GUERICKE 2001) und damit an unabhängigem Datenmaterial parametrisiert. Mit wenigen Ausnahmen umschließt die Funktion den Wertebereich der Kronenschirmflächen über den gesamten beobachteten Durchmesserbereich. Auf Grundlage dieser Funktion wurde in einem zweiten Schritt aus den maximalen Kronenschirmflächen die dazu gehörige „theoretische" Stammzahlhaltung je ha Buchenreinbestand abgeleitet und über dem BHD aufgetragen. Ungeachtet der oft vorkommenden Kronenüberlappungen wurde vereinfachend ein Überschirmungsgrad von 1,0 unterstellt. Danach sinken die "potenziellen" Stammzahlen von rd. 220 Buchen je ha bei einem BHD von $25 \mathrm{~cm}$ auf rd. 70 je ha bei einem BHD von $65 \mathrm{~cm}$. Bei einer „finalen“ Betrachtung entsprechen die 70 Buchen je ha der nach dem Standraumbedarf theoretisch möglichen Z-Baumzahl unter der Annahme, dass alle Buchen zur selben Zeit einen 
BHD von $65 \mathrm{~cm}$ aufweisen, ihre Kronen rund sind und es keine Kronenüberlappung gibt.

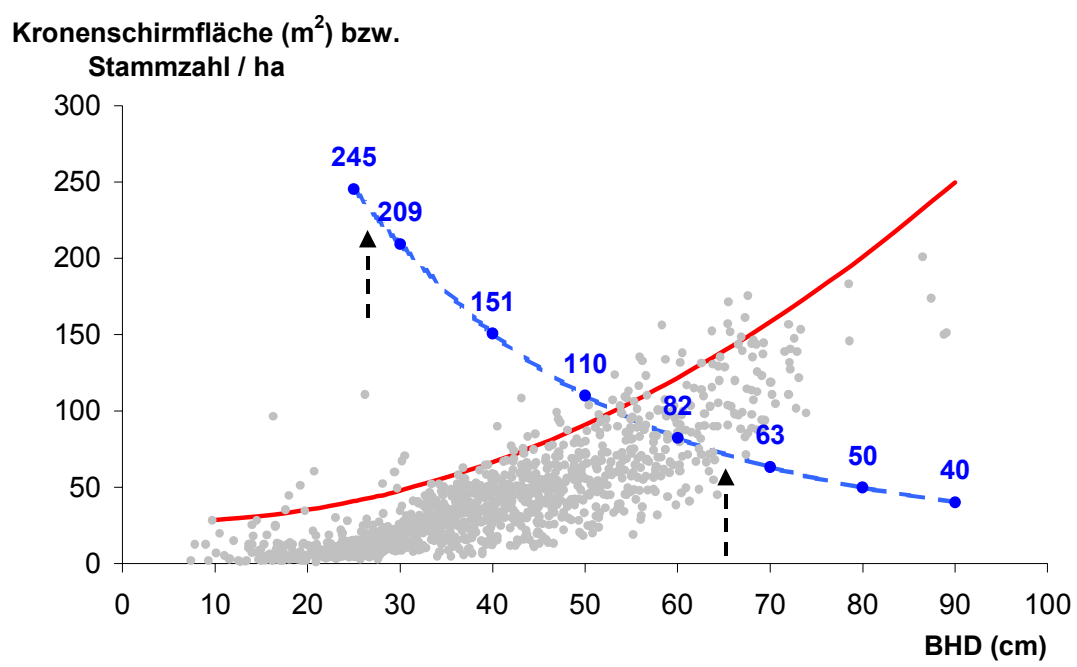

Abbildung 13: Beziehungen zwischen BHD, maximaler Kronenschirmfläche sowie der für vollständige, einfache Überschirmung abgeleiteten ,theoretischen" Stammzablentwicklung (aus GUERICKE 2002)

Diese theoretisch hergeleitete Z-Baumzahl kann aber nur eine minimale Forderung für die Anzahl Z-Bäume zu Beginn der Durchforstungsphase darstellen, denn es sind während des langen Produktionszeitraumes auch Risiken für die Z-Bäume und soziale und qualitative Umsetzungsprozesse zu berücksichtigen. Betrachtet man die Durchmesserentwicklung der Z-Bäume auf der Versuchsparzelle Saarforst 1606, starke Hochdurchforstung, getrennt für drei nach ihrem anfänglichen Brusthöhendurchmesser stratifizierte Z-Baum-Kollektive, so ist zunächst ihre mit der Zeit zunehmende Durchmesserdifferenzierung auffallend (s. Abb. 14). Sie ist die Grundlage für die Umsetzung einer zeitlich gestreckten Zielstärkennutzung, welche anerkanntermaßen bei der Buche zahlreiche ökonomische und ökologische Vorteile bietet (HOLM 1974, BACHMANN 1990, MOOG 1990, SPELLMANN 1999, KNOKE 2003). Von den anfänglich 236 Z-Bäumen je ha im Alter 49 Jahre sind im Alter 159 Jahre nach den ersten Zielstärkennutzungen noch 68 Z-Bäume je ha vorhanden. Während der Bestandesentwicklung hat es ein positives und negatives Umsetzen zwischen den drei Z-Baum-Kollektiven gegeben. Es ist daher bei der Festlegung der anfänglichen Anzahl Z-Bäume die statische finale Standraumbetrachtung durch eine dynamische zu ersetzen, da deutlich mehr als 60-80 Buchen innerhalb eines 30-50-jährigen Nutzungszeitraums die Zielstärke erreichen können. 


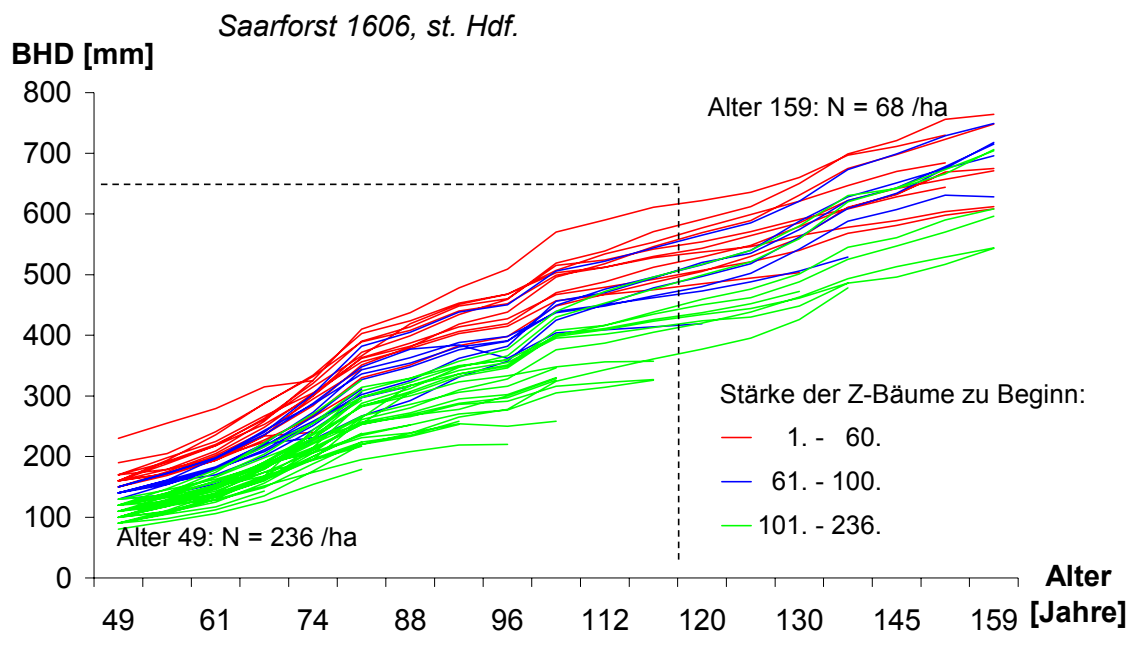

Abbildung 14: Durchmesserentwicklung eines zu Durchforstungsbeginn ausgewählten Z-Baumkollektivs (Buchen-Durchforstungsversuch Saarforst 1606, starke Hochdurchforstung)

Wie viele Z-Bäume bei laufender kritischer Überprüfung des anfänglichen ZBaum-Kollektives auszuwählen sind, hängt letztendlich auch von der Ausgangsqualität des jeweiligen Bestandes ab. In Beständen schlechterer Qualität dürfen dabei aber die Anforderungen an einen Z-Baum nicht zu hoch angesetzt werden. Auszuwählen sind die relativ besten Bäume in einer Anzahl, die auch unter diesen Umständen die höchstmögliche flächenbezogene Wertleistung sichert. Gerade in solchen Beständen mit geringerer oder sehr stark variierender Gesamtqualität ist die Entnahme besonders schlechter, konkurrenzstarker Bestandesglieder ab Beginn der Pflegephase auf der gesamten Bestandesfläche notwendig. Geschieht dies nicht und erfolgt die Pflege ausschließlich punktuell zur Förderung einer extrem geringen Z-Baumzahl (WILHELM et al. 1999a-d), bleiben dagegen in einer entscheidenden Entwicklungsphase größere Bestandespartien für eine längere Zeit ungepflegt und die Protzen setzen sich endgültig durch (s. Abb. 15).

Vergleichsweise niedrige Z-Baumzahlen zu Beginn der Durchforstungsphase im Anhalt an den erwarteten Endbestand oder noch darunter schränken auch die Möglichkeiten stark ein, Ausfälle vorgepflegter Wertträger z. B. durch Sturmwurf oder biotische Schäden zu kompensieren. Dies gilt erst recht, wenn der Füllbestand bis dahin völlig ungepflegt war. In den jüngeren Durchforstungsversuchen der NW-FVA traten in dem bisherigen Beobachtungszeitraum von 14 Jahren maximal Ausfälle in Höhe von $25 \%$ auf (Münden 2028, sehr starke Durchforstung, 40 von 155 Z-Bäumen, meist durch Schleimfluss). 


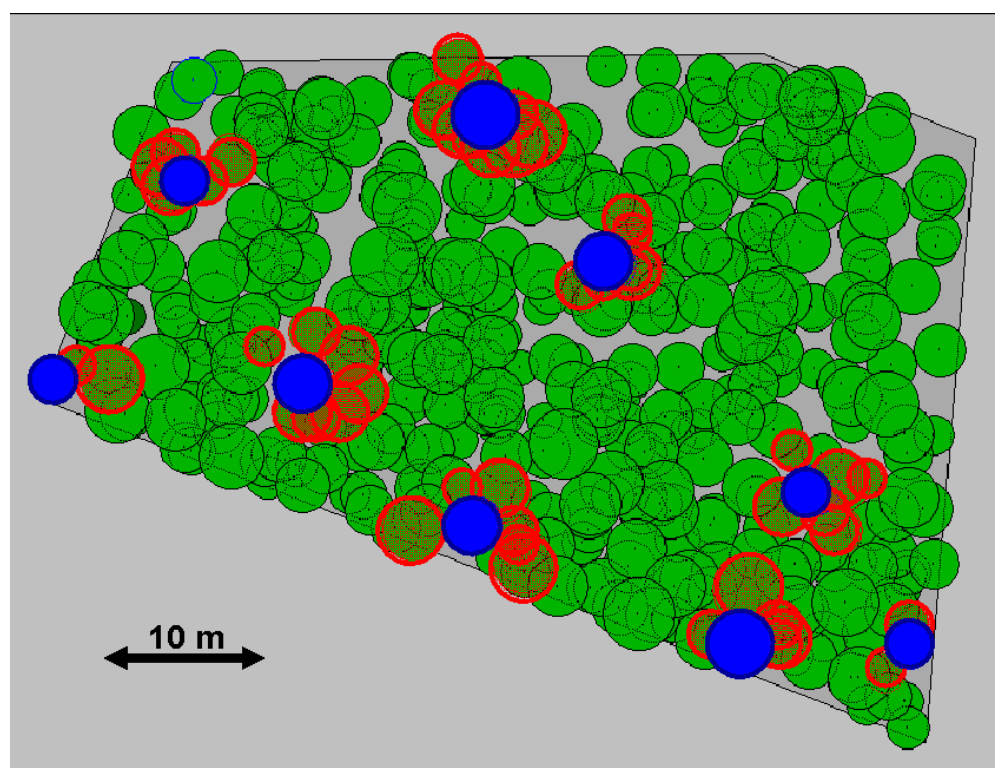

Abbildung 15: Erstdurchforstung in der Parzelle 12 des Buchen-Durchforstungsversuchs Labnstein 9a, Durchforstungsvariante „Dimensionierung"; blau: Z-Bäume (55 je ha), rot: ausscheidender Bestand, grün: verbleibender Füllbestand

\subsection{Auswirkungen unterschiedlicher Durchforstungsstärken auf den Massenzuwachs von Buchenbeständen}

Der Zusammenhang zwischen mittlerer Grundflächenhaltung und Volumenzuwachs bzw. Gesamtwuchsleistung von Buchenbeständen ist schon sehr lange Gegenstand der ertragskundlichen Forschung (u. a. WIEDEMANN 1932, ASSMANN 1950, 1953, 1956, 1965, SCHOBER 1972, BRYNDUM 1987). Die Untersuchungen zeigen, dass es in Buchenbeständen eine zuwachsoptimale Grundflächenhaltung gibt, die über längere Beobachtungszeiträume am ehesten der mittleren Grundflächenhaltung einer mäßigen Durchforstung entspricht. Der Einfluss des Standorts und des Bestandesalters auf die Ausprägung und die Lage der Optimumbeziehung wurde ebenfalls in mehreren der genannten Veröffentlichungen deskriptiv herausgearbeitet. PRETZSCH (2004) entwickelte auf der breiten Datenbasis zahlreicher Versuchsflächen, die auch drei Buchen-Durchforstungsreihen der NWFVA einschloss, ein Modell der Dichte-Zuwachs-Beziehung, das die bisherigen empirischen Befunde und vor allem den zeitlichen Aspekt der Beziehung mit dem sogenannten Wuchsbeschleunigungseffekt gut abbildet. Alle bisherigen Untersuchungen zeigen für die Buche eine höhere Gipfelung und eine breitere Wölbung der Dichte-Zuwachs-Beziehung als beispielsweise für die Fichte. Das spiegelt die 
besondere Fähigkeit von Buchenbeständen wider, aufgrund ihrer Plastizität Zuwachsminderungen bei Dichteabsenkungen abzupuffern.

Auf der Grundlage der Daten einiger Versuchsflächen der Nordwestdeutschen Forstlichen Versuchsanstalt wurde diesen Erkenntnissen erneut nachgegangen. Die jungen Buchendurchforstungsversuche verfügen ausnahmslos über eine Nullfläche, die die standörtlich mögliche maximale Grundflächenhaltung repräsentiert und über die die Grundflächensteuerung der durchforsteten Parzellen erfolgt. Grundflächenabsenkungen auf $80 \%$ (mäß. Df.), $65 \%$ (starke Df.) bzw. $50 \%$ (sehr starke Df.) der Oberstandsgrundfläche der Nullfläche ermöglichen einen systematischen Vergleich der Varianten innerhalb der Versuche, aber auch zwischen gleichen Varianten über die verschiedenen Versuche hinweg.

Tabelle 5 zeigt die laufenden Volumenzuwächse einiger junger Buchendurchforstungsversuche in Abhängigkeit von der Eingriffsstärke und der daran geknüpften relativen Grundflächenhaltung. Aufgeführt sind die Einzelwerte der bisherigen drei Beobachtungsperioden und der durchschnittliche laufende Volumenzuwachs für den gesamten 14-jährigen Beobachtungszeitraum. Bemerkenswert ist zunächst die absolute Höhe des laufenden Volumenzuwachses der beobachteten Versuche. Verglichen mit der Ertragstafel für den entsprechenden Altersbereich liegen die Zuwächse ca. $17 \%$ bis $90 \%$ über den Zuwachsangaben der Buchen-Ertragstafel von SCHOBER (1967) für die I. Ertragsklasse, mäßige Durchforstung. Trotz der deutlich überdurchschnittlichen Bonitäten der Versuche ist dieses Zuwachsniveau sehr hoch. Im Mittel aller Parzellen und aller Beobachtungen der jungen Durchforstungsversuche (auch derjenigen, die in Tab. 5 fehlen) beträgt der durchschnittliche laufende Volumenzuwachs $14,5 \mathrm{Vfm} \mathrm{ha}^{-1} \mathrm{a}^{-1}$, wodurch die zuvor getroffene Feststellung noch einmal unterstrichen wird.

Vergleicht man die laufenden Zuwächse der Varianten über den gesamten Beobachtungszeitraum, so leistet die mäßige Durchforstung in zwei Versuchen den höchsten Volumenzuwachs. Im Versuch Ahlhorn 106 wurde die mäßige Durchforstung aufgrund ihres 5 Jahre geringeren Bestandesalters nicht in den Vergleich einbezogen. Hier zeigt die Nullfläche von den verglichenen Varianten den höchsten Zuwachs. Im Versuch Münden 2027, wo die Durchforstung vergleichsweise früh bei einer Oberhöhe unter $14 \mathrm{~m}$ einsetzte, weist die starke Durchforstung den höchsten Volumenzuwachs auf. Die über den Gesamtzeitraum zuwachsschwächste Variante ist in allen Versuchen die sehr starke Durchforstung. Die Unterschiede zwischen der Variante mit dem höchsten laufenden Zuwachs und der sehr starken Durchforstung mit dem geringsten Zuwachs liegen für Ahlhorn 106 bei ca. $15 \%$, für die anderen Versuche zwischen 20 und $35 \%$. Die Werte für die erste und die zweite Beobachtungsperiode zeigen in mehreren Fällen noch ein besseres Abschneiden der starken und der sehr starken Durchforstung. Dies kann als Ausdruck eines temporären Wuchsbeschleunigungseffektes gewertet werden. 
Tabelle 5: $\quad$ Einfluss der Eingriffsstärke auf den laufenden Volumenzuwachs junger Buchenbestände (ausgewählte Parzellen der Buchen-Durchforstungsversuche Ablhorn 106, Unterlüß 138, Münden 2027 u. Münden 2028)

\begin{tabular}{|c|c|c|c|c|c|c|c|}
\hline \multirow[t]{2}{*}{ Versuch } & \multirow[t]{2}{*}{ Parz. } & \multirow[t]{2}{*}{ Df. } & \multirow{2}{*}{$\begin{array}{l}\text { Alter } \\
1990\end{array}$} & \multicolumn{4}{|c|}{ laufender Volumenzuwachs [Vfm ha-1 $\left.\mathrm{a}^{-1}\right]$} \\
\hline & & & & 1990-1994 & 1994-1999 & 1999-2004 & $1990-2004$ \\
\hline \multirow{4}{*}{$\begin{array}{c}\text { Ahlhorn } \\
106\end{array}$} & $2 \mathrm{~b}$ & ohne & 31 & 18,5 & 21,0 & 22,2 & 20,7 \\
\hline & - & mäßig & & - & - & - & - \\
\hline & 3 & stark & 31 & 16,0 & 21,6 & 20,0 & 19.4 \\
\hline & 4 & sehr stark & 31 & 16,7 & 17,3 & 19,7 & 18,0 \\
\hline \multirow{4}{*}{$\begin{array}{c}\text { Unterlüß } \\
138\end{array}$} & 2 & ohne & 35 & 13,9 & 12,7 & 13,7 & 13,4 \\
\hline & 3 & mäßig & 35 & 13,0 & 17,7 & 14,1 & 15,1 \\
\hline & 1 & stark & 35 & 13,6 & 12,5 & 13,3 & 13,1 \\
\hline & 5 & sehr stark & 35 & 11,0 & 14,6 & 9,7 & 11,8 \\
\hline \multirow{4}{*}{$\begin{array}{c}\text { Münden } \\
2027\end{array}$} & 2 & ohne & 36 & 12,3 & 14,9 & 16,0 & 15,1 \\
\hline & 1 & mäßig & 36 & 14,2 & 15,3 & 16,3 & 15,4 \\
\hline & 4 & stark & 36 & 11,2 & 14,5 & 19,6 & 16,0 \\
\hline & 3 & sehr stark & 36 & 13,5 & 11,7 & 12,6 & 11,9 \\
\hline \multirow{4}{*}{$\begin{array}{c}\text { Münden } \\
2028\end{array}$} & 6 & ohne & 44 & 14,3 & 17,9 & 9,4 & 13,8 \\
\hline & 2 & mäßig & 44 & 12,0 & 16,8 & 14,4 & 14,6 \\
\hline & 1 & stark & 44 & 11,1 & 14,8 & 14,9 & 13,8 \\
\hline & 8 & sehr stark & 44 & 12,1 & 13,3 & 11,2 & 12,2 \\
\hline
\end{tabular}

Am Beispiel von zwei Buchendurchforstungsversuchen in unterschiedlichen Altersphasen lässt sich die Altersabhängigkeit des Zusammenhangs zwischen relativer Grundflächenhaltung (Nullfläche $=100 \%$ ) und Zuwachs verdeutlichen (s. Abb. 16). Die Darstellungen sind allerdings aufgrund ihres Einzelbeobachtungscharakters vorsichtig zu interpretieren. Als Zuwachsgröße wird hier nicht der von der Höhen- und Formzahlentwicklung beeinflusste Volumenzuwachs, sondern der relative Grundflächenzuwachs betrachtet. Auffallend ist zunächst das hohe Niveau der positiven Zuwachsreaktion auf die Grundflächenabsenkung in dem jüngeren Bestand nach der hier in blau dargestellten dritten Durchforstung (Alter 53-58). Untereinander unterscheiden sich die drei durchforsteten Parzellen dabei relativ wenig. Unmittelbar nach der Erstdurchforstung (Alter 44-49) hatte es in dem betrachteten Versuch noch keinen klar erkennbaren Trend des Grundflächenzuwachses in Abhängigkeit von der Grundflächenhaltung gegeben. Erklärungsursachen dafür sind möglicherweise eine „Umstellungsphase“ in den durchforste- 
ten Parzellen und die mit 29,6 $\mathrm{m}^{2}$ je ha absolut gesehen noch nicht sehr hohe Grundflächenhaltung der Nullfläche. Für den Versuch Paderborn 616/618 sind in Bestandesaltern von 91 bzw. 136 Jahren bei Dichteabsenkungen auf unter $80 \%$ der Nullfläche nur noch geringe positive bis negative Reaktionen im Grundflächenzuwachs gegenüber der Nullfläche zu verzeichnen. Damit scheint sich die Annahme eines Nachlassens der an sich plastischen Zuwachsreaktion der Buche auf Grundflächenabsenkungen im höheren Alter auch an den hier gemachten Beobachtungen zu bestätigen (vgl. SCHOBER 1972).
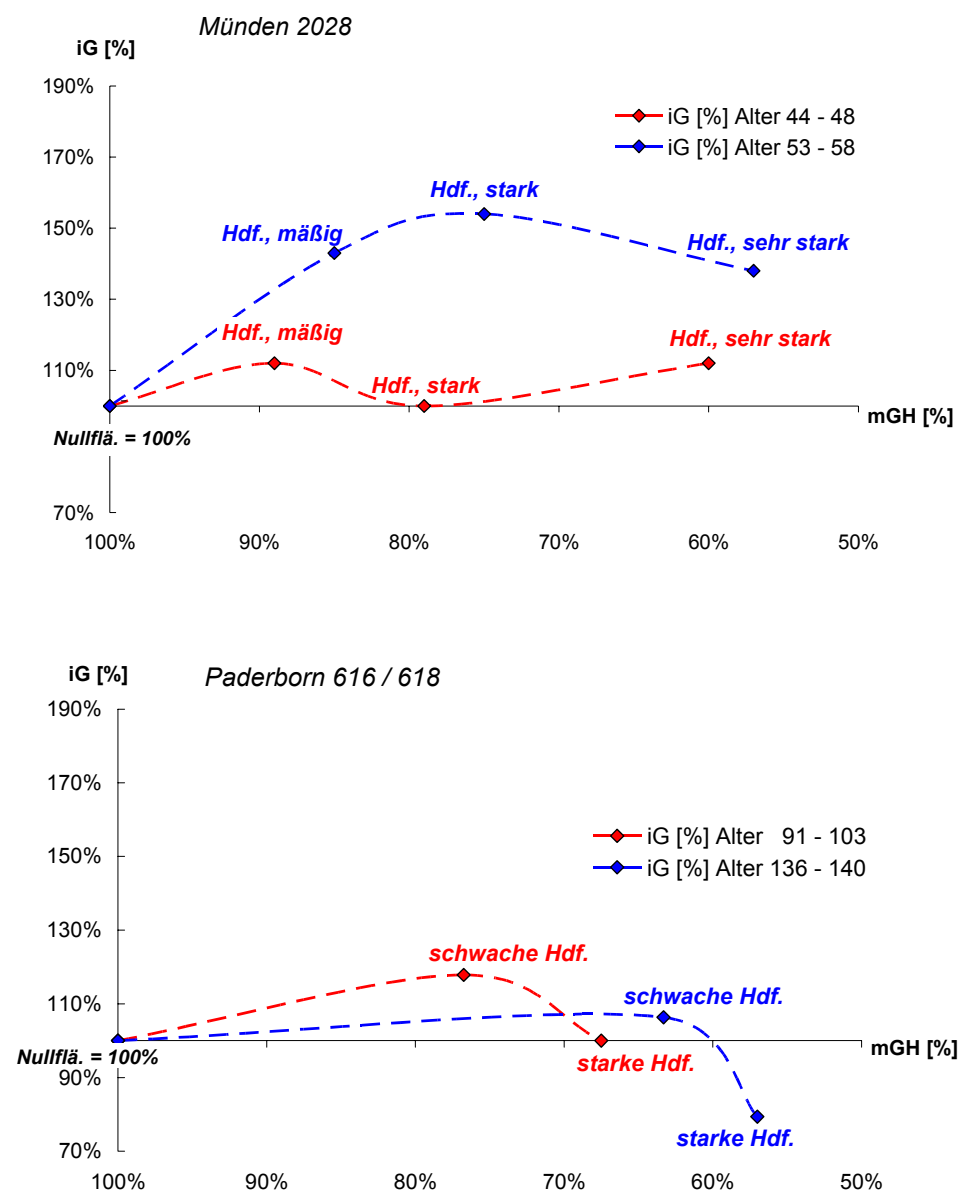

Abbildung 16: Relativer periodischer Grundflächenzuwachs von Buchenbeständen in Abhängigkeit von der mittleren Grundflächenhaltung in verschiedenen Bestandesaltern (Buchen-Durchforstungsversuche Münden 2028 (oben) und Paderborn 616/618 (unten)) 


\subsection{Modellkalkulationen zur Überprüfung von Handlungsalternativen}

Über die Frage der Bedeutung von Einzelbaum- bzw. Flächenproduktivität bei der Durchforstung von Buchenbeständen wird in jüngerer Zeit wieder kontrovers diskutiert. Einvernehmen herrscht darüber, dass Durchforstungen erst dann einsetzen sollen, sobald sie erste Deckungsbeiträge abwerfen. Umstritten sind hingegen die Zahl der Z-Bäume, der Grad ihrer Freistellung und die Behandlung der Zwischenfelder. Vereinfachend lassen sich zwei Gruppen unterscheiden. Auf der einen Seite werden niedrige Z-Baumzahlen und eine extreme Freistellung supervitaler Z-Bäume ohne Eingriffe in den übrigen Bestand befürwortet. Auf der anderen Seite werden anfänglich höhere Z-Baumzahlen, eine konsequente Hochdurchforstung und eine frühzeitige Entnahme schlecht veranlagter herrschender Bäume auf der gesamten Fläche empfohlen.

Die Auswirkungen beider Behandlungskonzepte auf den Massen-, Sorten- und Wertertrag von Buchenreinbeständen wurden mit Hilfe des Wachstumssimulators BWINPro 6.2 der Nordwestdeutschen Forstlichen Versuchsanstalt (NAGEL 1999, NAGEL et al. 2002, DÖBBELER et al. 2003) im Rahmen einer Vergleichssimulation überprüft. Die Datengrundlage bildete der sehr wüchsige Versuchsbestand (Buchen-Durchforstungsversuch Münden 2028, Parzelle 8) mit einer 0,8. Ertragsklasse, der jedoch, wie eingangs gezeigt, das Leistungsvermögen jüngerer Buchenbestände auf besseren Standorten gut repräsentiert. Die astfreie Schaftlänge lag zum Zeitpunkt der Erstdurchforstung im Alter von 44 Jahren bei 8 bis $9 \mathrm{~m}$. Der mittlere Brusthöhendurchmesser des Oberstandes $\left(\mathrm{d}_{\mathrm{g}}\right)$ betrug 13,2 cm, die Oberhöhe $\left(\mathrm{h}_{100}\right)$ 17,6 m, die Grundfläche $30,0 \mathrm{~m}^{2} / \mathrm{ha}$ und das mittlere Kronenprozent $44 \%$.

Die Z-Baumauswahl für beide Simulationsvarianten wurde auf der Grundlage der in der Versuchsparzelle tatsächlich ausgewählten 155 Z-Bäume je ha zu Durchforstungsbeginn vorgenommen. Für die Variante „Einzelbaum“ wurden aus diesen die stärksten 50 je ha ausgewählt, die Variante „Flächenproduktivität“ bezog 120 Z-Bäume je ha des tatsächlich ausgewählten Z-Baumkollektivs mit ein. Auffällig ist, dass auch in der z-baum-reichen Variante die Zukunftsbäume aufgrund der inhomogenen Bestandesqualität nicht gleichmäßig verteilt sind (s. Abb. 17 oben rechts).

Die 50 Z-Bäume bei der auf die Einzelbaumleistung ausgerichteten Durchforstungsvariante wurden mithilfe des A-Wertes nach JOHANN (1982) von Beginn an sehr stark freigestellt $(A-W e r t=3)$. Der A-Wert ist eine Verhältniszahl, die die Freistellungsstärke von Z-Bäumen ausdrückt. Der niedrige Wert wurde aufgrund der großen Kronenplastizität der Buche gewählt. Nach 35 Jahren wurde der AWert im Sinne einer „Ausreifungsphase“ (WILHELM 1999c) allmählich auf 4 angehoben. Die 120 Z-Bäume in der auf die flächenbezogene Wertleistung abzielenden Variante wurden nach einem ersten Eingriff, der die Z-Bäume mäßig freistellte (AWert 5,5) und vor allem der Entnahme starker, schlechter Bestandesglieder diente, 30 Jahre lang stark freigestellt $(\mathrm{A}$-Wert $=4,5)$. Danach ging die Freistellungsstärke 
allmählich auf mäßig zurück (A-Wert $=5$ bzw. 5,5). Das Vorgehen entspricht in etwa dem Pflegemodell für Buche nach SPELLMANN et al. (1999). Die Simulation erstreckte sich für beide Varianten über 70 Jahre bis zum Bestandesalter 114 Jahre. $\mathrm{Zu}$ diesem Zeitpunkt erreichen in beiden Varianten die ersten Bäume die Zielstärke.
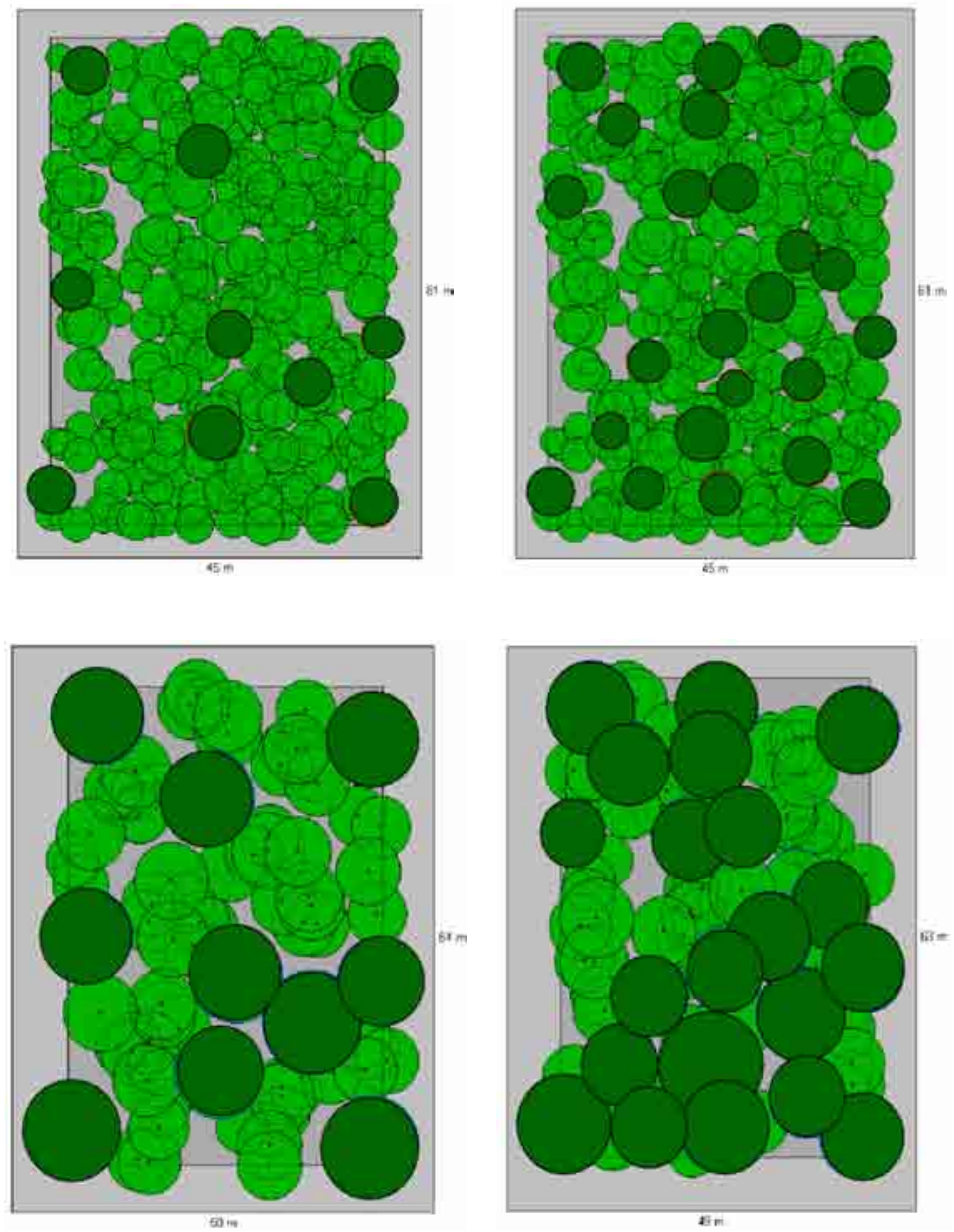

Abbildung 17: Stammverteilungspläne mit Kronenprojektionen, linke Seite: Variante 50 Z-Bäume je ha, rechte Seite: 120 Z-Bäume je ha; oben: Ausgangszustand zu Durchforstungsbeginn (Alter 44 Jahre), unten: nach 70-jäbriger Simulation (Alter 114 Jahre)

Die anfallenden Vornutzungen und der Endbestand beider Varianten wurden mithilfe eines in BWINPro 6.2 integrierten Sortiertools nach folgenden Vorgaben sortiert: 


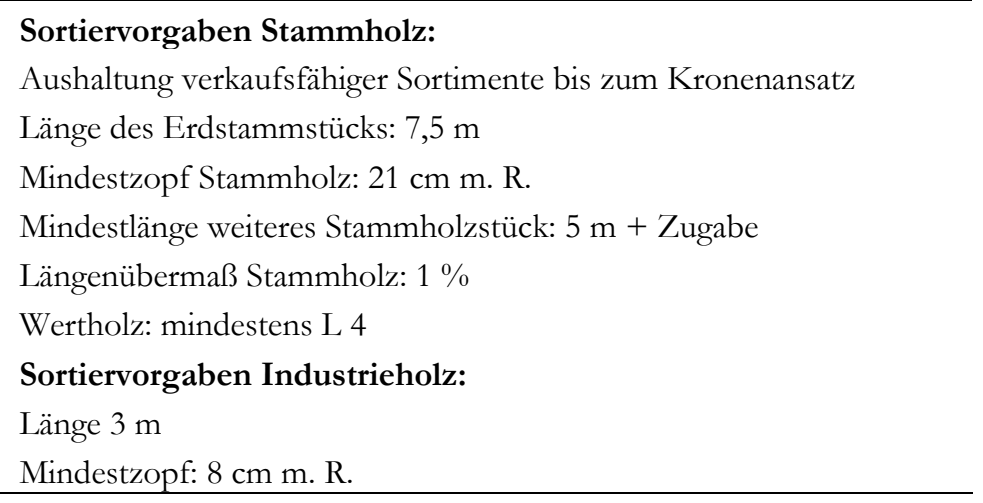

Auf der Grundlage der durchschnittlichen Güteklassenanteile des Buchen-Stammholzeinschlages der Niedersächsischen Landesforsten (Zeitraum 1995-2004, s. Kap. 5.2) wurden die Holzmengen über die Stärkeklassen den Güteklassen zugeordnet und mit den durchschnittlichen, im Jahre 2006 erzielten Holzerlösen für die einzelnen Güte- und Stärkeklassen bewertet. Bei den mitgeteilten Erlösen handelt es sich somit um Bruttoerlöse ohne den Abzug von Holzerntekosten.

Die Varianten unterscheiden sich in ihrer Wirkung auf die Bestandesstruktur (s. Abb. 17 unten), die Massenleistung, die Höhe der Vornutzungen, den Vorrat der Z-Bäume und die Sortimentsstruktur (s. Tab. 6).

Tabelle 6: Auswirkungen einer extremen Auslesedurchforstung (V ariante „Einzelbaum “) und einer starken Hochdurchforstung (Variante „Flächenproduktivität") auf den Massenertrag und den Gesamterlös

\begin{tabular}{|c|c|c|}
\hline $\begin{array}{l}\text { Ertragskundliche Daten im } \\
\text { Alter } 114 \text { Jahre }\end{array}$ & $\begin{array}{c}\text { Variante } \\
\text { „Einzelbaum“ } \\
\text { (50 Z-Bäume/ha) }\end{array}$ & $\begin{array}{c}\text { Variante } \\
\text { „Flächenproduktivität“" } \\
\text { (120 Z-Bäume/ha) }\end{array}$ \\
\hline Gesamtwuchsleistung $\left[\mathrm{m}^{3} / \mathrm{ha}\right]$ & 1.138 & 1.207 \\
\hline davon Restderbholz $\left[\mathrm{m}^{3} / \mathrm{ha}\right]$ & 170 & 208 \\
\hline Vornutzungen $\left[\mathrm{m}^{3} / \mathrm{ha}\right]$ & 283 & 356 \\
\hline Vorrat verbleib. Bestand $\left[\mathrm{m}^{3} / \mathrm{ha}\right]$ & 685 & 643 \\
\hline Vorrat Z-Bäume $\left[\mathrm{m}^{3} / \mathrm{ha}\right]$ & 185 & 350 \\
\hline Gesamterlös [€/ha] & 37.800 & 39.600 \\
\hline
\end{tabular}

In der Gesamtwuchsleistung, im Vorrat der Z-Bäume und im Gesamterlös gibt es einen Vorteil der Variante „Flächenproduktivität“, der jedoch bis zum Alter 114 Jahre mit $6 \%$ bzw. $5 \%$ nicht hoch ausfällt. Diese Variante hat im Alter 114 einen 
leicht geringeren Bestandesvorrat, erbringt jedoch ca. $25 \%$ höhere Vornutzungen, die auch höhere Stammholzanteile enthalten (s. Abb. 18).

\section{[Efm/ha] Vornutzungen, Variante 50 Z-Bäume /ha}
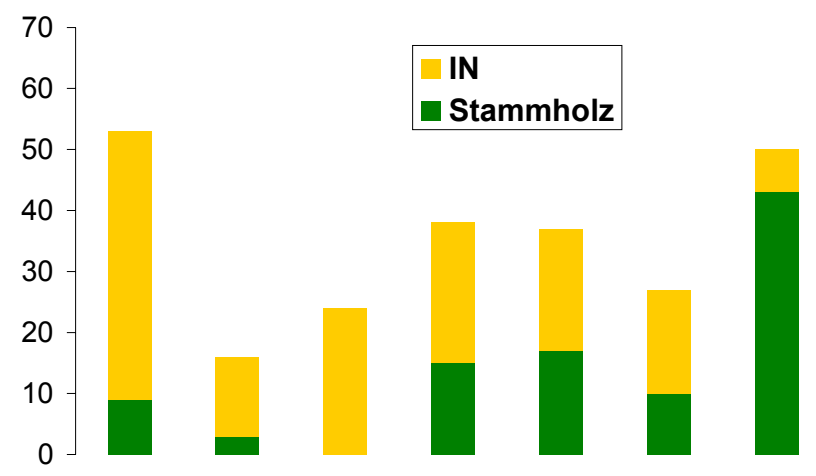

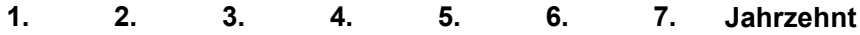

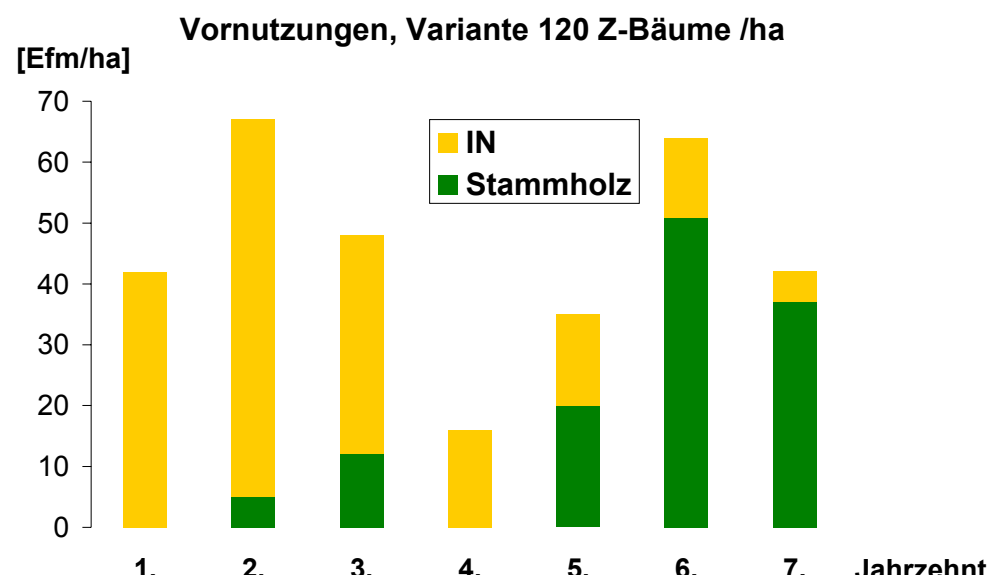

Abbildung 18: Auswirkungen einer extremen Auslesedurchforstung (Variante „Einzelbaum", oben) und einer starken Hochdurchforstung (V ariante „Flächenproduktivität“, unten) auf die Sortenstruktur und die zeitliche Verteilung der Vornutzungen

Deutlichere Vorteile der Variante „Flächenproduktivität“ sind mit dem Einsetzen der Zielstärkennutzung zu erwarten. Wie Abbildung 19 zeigt, ist die Stärkeklassenverteilung der wenigen Z-Bäume der „Einzelbaum“-Variante sehr eng. Fast der gesamte Z-Baumvorrat befindet sich in den Stärkeklassen L4 und L5. Damit wachsen diese Bäume aufgrund ihrer geringen Durchmesserdifferenzierung relativ zeitgleich in die Zielstärke und werden genutzt. Dagegen weist der größere ZBaumvorrat der Variante „Flächenproduktivität“ eine deutlich größere Durchmesserdifferenzierung auf. Im Zuge der Zielstärkennutzung werden bei dieser 
Variante mehr Bäume die Zielstärke erreichen, was angesichts des Bestandesalters noch ohne stark erhöhte Entwertungsgefahr möglich sein sollte.

Nicht berücksichtigt ist in diesen Modellkalkulationen das Ertragsrisiko, dass sich aus dem Ausfall bzw. dem qualitativen Umsetzen einzelner Z-Bäume ergeben kann. Dieses Risiko würde sich in der Variante mit 50 Z-Bäumen je ha deutlich stärker negativ bemerkbar machen. Auch zugunsten dieser Variante wurde bei der Sortierung des Holzes die gleichen Güteklassenanteile wie bei der Variante „Flächenproduktivität“ unterstellt, obwohl zu erwarten ist, dass sich in den ungepflegten Zwischenfeldern die Protzen durchsetzen und die sehr stark freigestellten Z-Bäume oberhalb des Kronenansatzes sehr schlechte, grobastige Stammholzabschnitte und einen höheren Industrieholzanteil aufweisen werden.
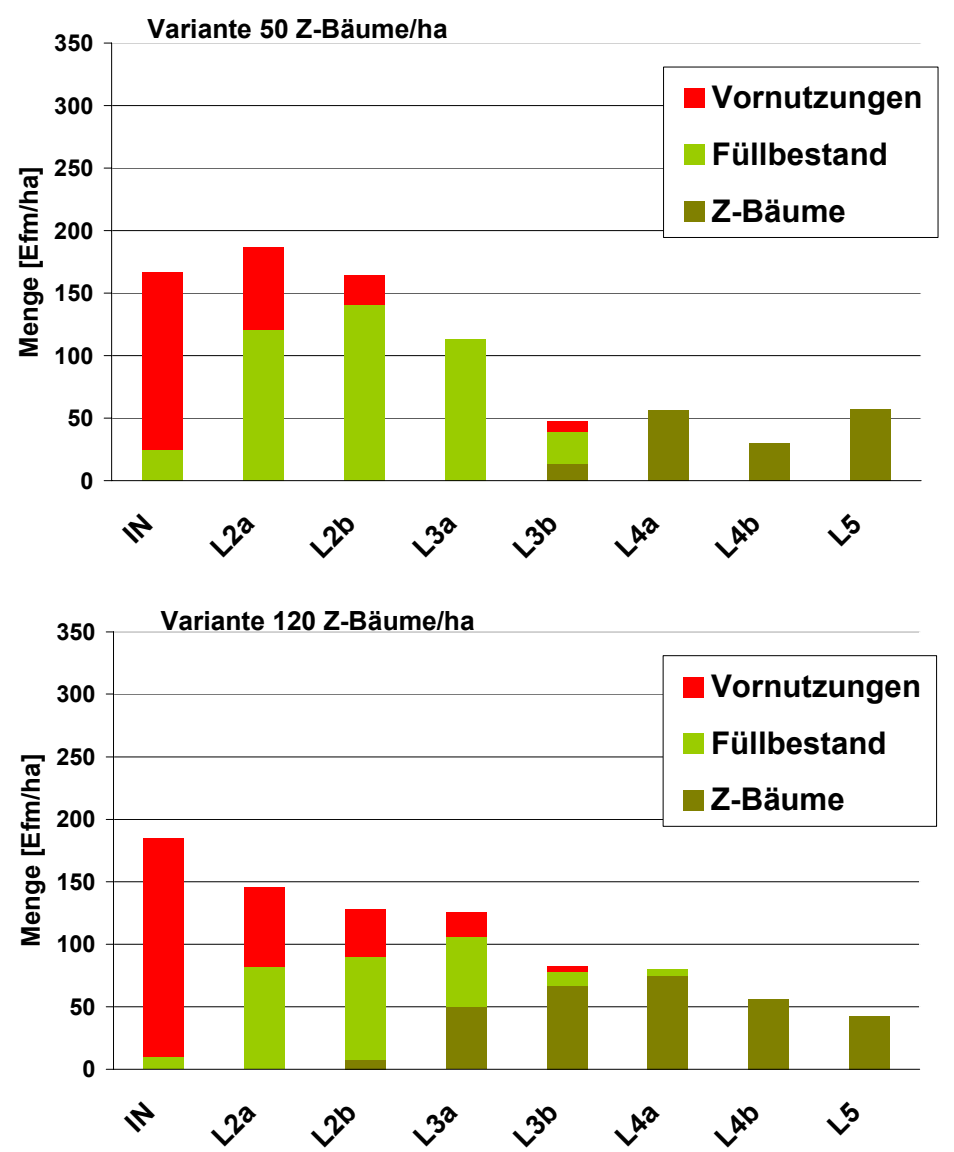

Abbildung 19: Auswirkungen einer extremen Auslesedurchforstung (,Einzelbaumvariante", oben) und einer starken Hochdurchforstung (Variante „Flächenproduktivität", unten) auf die Sortenstruktur 


\section{Diskussion}

Die Untersuchung von Wachstum, Behandlung und Ertrag der Rotbuche im Reinbestand zählt seit den Anfängen des forstlichen Versuchswesens zu den Kernaufgaben der ertragskundlichen Forschung. Während die abgesicherten Untersuchungsergebnisse Bestand haben, unterliegen ihre Bewertungen immer auch den Einflüssen, die sich aus dem Wandel der forstlichen Grundanschauungen, der wirtschaftlichen Zielsetzungen und der Ansprüche der Gesellschaft an den Wald ergeben. Vor dem Hintergrund der Verknappung fossiler Rohstoffe, steigender Holznachfrage und zunehmender Konkurrenz zwischen stofflicher und energetischer Nutzung einerseits (SPELLMANN et al. 2008) und der „Entdeckung“ des Buchenwaldes als europäisches Naturerbe durch den Naturschutz (BFN 2008) wird der Bewirtschaftung der Buchenwälder derzeit ein besonderes Augenmerk geschenkt.

Wie bei kaum einer anderen Baumart in Deutschland stehen bei der Buche die ökologischen Ansprüche und Eigenschaften im Einklang mit den Anbauschwerpunkten und den Behandlungskonzepten. Angesichts der großen Standortsamplitude ist die überwiegende Mehrzahl der Buchenwälder standortsgemäß und stabil (OtTO 1994, ElLENBERG 1996, HeinKen 1996, LeusChNer 1998), aber nicht immer ertragreich (MÖHRING 2004). Die Ökologie der Buche war in den achtziger und neunziger Jahren des letzten Jahrhunderts die Grundlage für die Entwicklung der Grundsätze eines naturnahen Waldbaus (z. B. ОтTO 1991). Mit dem Klimawandel sind jedoch gravierende Veränderungen zu erwarten, die eine Neubewertung der standörtlichen Zuordnung der Buchenwälder und eine Anpassung ihrer Bewirtschaftung notwendig machen werden (V LÜPKE 2004, RENNENBERG et al. 2004, BOLTE 2005, BrÈDA et al. 2006, SPELLMANN et al. 2007, BOLTE u. IBISCH 2007, KÖLLING u. ZIMMERMANN 2007, MANTHEY et al. 2007). Ungeachtet dessen wird man auch in Zukunft darauf angewiesen sein, die hier aufgezeigten ökologischen Eigenschaften der Buche im Sinne einer biologischen Rationalisierung zu nutzen.

Für die Analyse von Wachstumstrends bilden echte Zeitreihen aus langfristig beobachteten Versuchsflächen die wichtigste Auswertungsgrundlage. Mit Dauerversuchsflächen lassen sich am besten Waldentwicklungen verfolgen und kurzfristige Effekte von langfristigen Trends trennen. Die für die jüngeren BuchenDurchforstungsversuche festgestellte bessere Höhenwuchsleistung ist im Zusammenhang mit den erhöhten Stickstoffeinträgen seit Anfang der sechziger Jahre zu sehen (vgl. SPIECKER et al. 1996, GAUGER et al. 2002). Für die Forsteinrichtung ist die Aussage wichtig, dass die jungen Versuche in Nordwestdeutschland im Wachstumsgang aber auch dem extrapolierten Bonitätsfächer der Schober`schen Ertragstafeln weitgehend entsprechen.

Anders verhält es sich bei den Grundflächenentwicklungen. Die Schober`schen Ertragstafen geben für die älteren Versuchsbestände über 60 Jahre in der Jugend deutlich zu hohe und im Alter zu geringe Grundflächenhaltungen vor, die 
nicht den Entwicklungen der heute noch in Beobachtung befindlichen BuchenDurchforstungsversuchsflächen entsprechen. Diese Abweichungen lassen sich u. U. dadurch erklären, dass SCHOBER (1972) auf ein wesentlich größeres und räumlich breiter gestreutes Buchen-Versuchsflächennetz zurückgreifen konnte, als es für diese Auswertungen noch zur Verfügung stand. ASSMANN (1976) forderte allerdings schon früher höhere Grundflächenhaltungen für ältere Buchenbestände, während die DDR-Buchenertragstafel (DITTMAR et al. 1986) sich weitgehend an die Schober`sche Ertragstafel für mäßige Durchforstung anlehnt.

Gänzlich unterschätzt werden die Grundflächenhaltepotenziale in den jüngeren Versuchen unter 60 Jahren (vgl. KLÄDKE 1997, UTSCHIG 2000, V. TEUFFEL 2002). Demgegenüber entsprechen die am natürlichen Bestockungsgrad (DÖBBELER 2004) orientierten Grundflächenhaltungen für eine summarische Nutzungsplanung von SPELLMANN et al. (1999) weitgehend der Grundflächenentwicklung auf den jüngeren Versuchsflächen. Sie heben in höheren Altern die Bestandesgrundflächen wieder an, um das Zuwachspotenzial der Buche voll auszuschöpfen. Diese gestaffelte Hochdurchforstung entspricht in etwa dem Durchforstungsmodell von ALTHERR (1971), während der Lichtwuchsbetrieb von FREIST (1962) zwar in der Jugend mit höheren Grundflächen als die Schober`schen Ertragstafeln für mäßige und starke Durchforstung arbeitet, sich aber ab dem Alter 60 Jahre in Erwartung keiner größeren Minderleistungen der Schober`schen Ertragstafel für starke Durchforstung weitgehend annähert.

Die in den letzten Jahrzehnten verbesserten Wachstumsbedingungen für Buchenbestände spiegeln sich auch in dem Durchmesserwachstums der jüngeren Buchen-Durchforstungsversuche wider. Die Durchmesserentwicklung verläuft schneller als in den älteren Versuchen. Wie an den Versuchen Herborn 1333 und Münden 2028 verdeutlicht, müssen zur wirksamen positiven Beeinflussung des Durchmesserzuwachses die ersten Durchforstungen früh einsetzen und zunächst stark geführt werden. Der dabei festzustellende, aber nur eine begrenzte Zeit wirkende Wuchsbeschleunigungseffekt kann dazu genutzt werden, den Zuwachs auf die bestveranlagten Bäume zu konzentrieren. Für die Praxis bedeutet dies, dass die Erstdurchforstung nach dem Erreichen einer astfreien Schaftlänge von einem Viertel der erwarteten Endhöhe, je nach Ertragsklasse sind das ca. 6-10 m, erfolgen sollte. Bei Beständen I. Ertragsklasse entspricht dies einer Oberhöhe von ca. 16 m und einem Alter von 40 bis 45 Jahren. Forderungen nach einer längeren astfreien Schaftlänge (z. B. FLEDER 1987) stehen der beschleunigten Durchmesserentwicklung der Z-Bäume entgegen. Dies kann durch den lang anhaltenden Durchmesserzuwachs der Buche, speziell den Lichtungszuwachs im höheren Alter, zwar teilweise kompensiert werden (ASSMANN 1965), führt aber zur Verlängerung der Produktionszeit und erhöht damit gleichzeitig das Entwertungsrisiko (SEELING 1998, KNOKE u. SCHulZ Wenderoth 2001, KNOKE 2003, KOHNLE 2003).

Die starke Hochdurchforstung fördert auf Dauer die Durchmesserdifferenzierung in mehr oder weniger gleich alten Buchenbeständen, indem sie einen 
lebensfähigen Unterstand erhält und das Durchmesserwachstum der am besten veranlagten Bäume steigert (ALBERT 1999, MEYER 2000, SPELLMANN et al. 2004). Demgegenüber führt der alleinige Selbstdifferenzierungsprozesses bei der Buche zu einer Homogenisierung der Durchmesserverteilungen und langfristig zu Hallenbeständen mit einer relativ geringen Artenvielfalt (WEINREBE 2004, ZERBE u. SCHMIDT 2006). Aus waldbaulicher Sicht kommt der Erhaltung eines vitalen Unterstandes eine besondere Bedeutung zu, weil er in Altbeständen die Steuerung der Naturverjüngung ermöglicht, die Schäfte der Altbuchen vor Sonnenbrand schützt und die Ausbildung einer starken Bodenvegetation mit hoher Verdunstung verhindert, wodurch die Wasserbilanz verbessert wird.

Als vorrangiges wirtschaftliches Ziel der Buchenwirtschaft muss die positive Beeinflussung der flächenbezogenen Wertleistung herausgestellt werden. Gerade weil die waldbaulichen Möglichkeiten der Qualitätsverbesserung aufgrund der genetischen Eigenschaften (KLEINSCHMIT u. SVOlBA 1996, Hosius et al. 2003), der Selbstdifferenzierung und des ungleichmäßigen Auflaufens der Naturverjüngung begrenzt sind, ist es umso wichtiger, die Möglichkeiten der Bestandespflege voll auszuschöpfen. In der Dickungsphase soll der Dichtschluss zur Förderung der Astreinigung und Streckung der Schäfte möglichst wenig unterbrochen werden (V. LÜPKE 1986). Je nach Gesamtqualität sind in der Stangenholzphase Läuterungen unterschiedlicher Intensität und Wiederkehr erforderlich, die sich aber in jedem Fall auf die Entnahme schlechter Vorwüchse (Protzen) konzentrieren und ansonsten den Dichtschluss erhalten sollen (u. a. RÖHRIG 1968, V. LÜPKE 1986). Das entscheidende waldbauliche Mittel zur Qualitätsverbesserung der Bestände sind die Durchforstungen. Angesichts der starken Gütedifferenzierung der Buche wird hierfür im Sinne der Empfehlungen von SCHOBER (1987, 1988a, b) die „frühe Auswahl, Markierung und Förderung einer größeren Anzahl von Auslesebaum-Anwärtern, als sie der geschätzten Endbaumzahl entspricht“ vorgeschlagen. SCHOBER gibt als groben Anhalt für die im Zuge der Erstdurchforstung auszuwählenden Z-Bäume im Laubholz etwa die doppelte Anzahl der zu erwartenden Endbaumzahl an, die nach den örtlichen Verhältnissen zu modifizieren ist. Dies entspräche nach den hier bestätigten Beziehungen zwischen BHD, maximaler Kronenschirmfläche und Stammzahlentwicklung von GUERICKE (2002) einer anfänglich auszuwählenden Z-Baumzahl in Buchenbeständen von 140-160 Stück je ha. Diese Zahlen liegen im oberen Bereich des Z-Baum-Rahmens für die Niedersächsischen Landesforsten (NDS. LANDESFORSTVERWALTUNG 2000), aber erheblich über den Angaben extrem einzelbaumorientierter Behandlungsprogramme anderer Bundesländer (LANDESFORSTEN RHEINLAND-PFALZ 2001, 2003, MLU BRANDENBURG 2004, SAARFORST 2004, AG „BUCHE“ LANDESBETRIEB WALD UND HOLZ NRW 2006). Die anfänglich höheren Z-Baumzahlen geben aber die Möglichkeit zu einer stufenweisen Auslese und berücksichtigen damit die Gefahren eines Umsetzens bzw. des Verlustes von Z-Bäumen. Gegenüber einer freien Auslesedurchforstung (SCHÄDELIN 1942) werden die Vorteile einer Markierung der Z-Bäume in einer dadurch konsequenteren Pflege und Frei- 
stellung dieser Hauptwertträger und in ihrem besseren Schutz vor Fällungs- und Rückeschäden gesehen (vgl. BORT et al. 1993). Die bisher in der Forstpraxis oft fehlende konsequente Markierung der Z-Bäume in Buchenbeständen mag auch ein Grund dafür sein, dass der Anteil des Stammholzes besserer Güte bisher so gering ist (vgl. Abb. 9).

Ausgehend von der Beobachtung der ungleichmäßigen Verteilung guter Bäume entwickelten KATÓ u. MÜLdER (KATÓ 1973, KATÓ u. MÜLDER 1979, 1983, 1988, 1992) ihr Konzept der qualitativen Gruppendurchforstung mit dem Ziel der Ertragsverbesserung von Buchenbeständen mit einem knappen und unregelmäßig verteilten Vorrat guter Stämme. SPELLMANN u. NAGEL (1996) empfehlen in qualitativ unbefriedigenden Buchenbeständen ebenfalls die Förderung einzelner Gruppen guter Bäume im Rahmen einer starken Hochdurchforstung, allerdings mit der Einschränkung, dass dieses Prinzip genauso wenig schematisch gehandhabt werden darf, wie das Streben nach möglichst regelmäßigen Standräumen der Z-Bäume.

In der späteren Nutzungsphase gilt es, die Zielstärkennutzung so zu führen, dass größere Verluste durch Rot- oder Spritzkern vermieden werden. Auf die einschlägigen Arbeiten von MOOG (1990), MOOG u. KARBERG (1992) sowie KNOKE (2003) sei ebenso verwiesen wie insbesondere auf die umfangreichen Auswertungen von SCHMIDT et al. in diesem Band der Schriftenreihe.

Die auf den jüngeren Versuchsflächen festgestellten hohen Massenzuwächse der Buche decken sich mit ähnlichen Befunden auf süddeutschen Versuchsflächen (UTSCHIG 2000, KENK 2002, NICKEL et al. 2007). Der anfängliche Wuchsbeschleunigungseffekt nach sehr starken bzw. starken Eingriffen klingt nach der dritten Durchforstung deutlich ab und die zuwachsoptimale Grundflächenhaltung nähert sich anschließend der mäßigen Durchforstung. PRETZSCH (2006) führt den Wuchsbeschleunigungseffekt nicht nur auf die unmittelbare Durchforstungswirkung zurück, sondern auch auf die schnelle ontogenetische Entwicklung der Einzelbäume im Zuge der Durchforstung, die eine Vorverlegung des Zeitpunkts ihrer höchsten Zuwachseffizienz bewirkt. Für die praktische Umsetzung empfiehlt sich hiernach eine gestaffelte Hochduchforstung mit starken Eingriffen in der Jugend und einem Übergang zur mäßigen Hochdurchforstung nach dem dritten starken Eingriff. Dies stimmt auch mit den grundlegenden Untersuchungen von ASSMANN (1956) und SCHOBER (1972) überein, die die „kritische Grundflächenhaltung“ (Grundfläche bei der noch $95 \%$ des maximal möglichen Zuwachses geleistet wird) bei einem natürlichen Bestockungsgrad von 0,60 bis 0,70 sehen.

Die Modellkalkulationen zur Überprüfung der Handlungsalternativen Einzelbaum- und Flächenproduktivität zeigen, dass die starke Hochdurchforstung mit einer höheren Z-Baumzahl der extremen Auslesedurchforstung mit einer deutlich geringeren Z-Baumzahl nach 70 Jahren Simulation im Massen-, Sorten- und Wertertrag überlegen ist. Es ist davon auszugehen, dass sich dieser Vorsprung mit dem Einsetzen der Zielstärkennutzung weiter vergrößern wird. Bei dem „Q/D“- 
Konzept werden die Möglichkeiten, den Durchmesserzuwachs durch sehr starke Freistellungen zu fördern, offensichtlich überschätzt, die naturale und finanzielle Bedeutung der Vornutzungen wird vernachlässigt, die geringen Z-Baum-Zahlen tragen den Risiken in den langen Produktionszeiträumen nicht angemessen Rechnung und die Forstbetriebe werden in ihren Möglichkeiten eingeschränkt, auf veränderte Rahmenbedingungen flexibel zu reagieren

Als Fazit dieses Beitrages lassen sich die vorgestellten Untersuchungsergebnisse zur Skizzierung eines Behandlungskonzepts für Buchenbestände zusammenfassen (s. Tab. 7).

\section{Tabelle 7: $\quad$ Skizze eines Behandlungskonzepts für Buchenbestände}

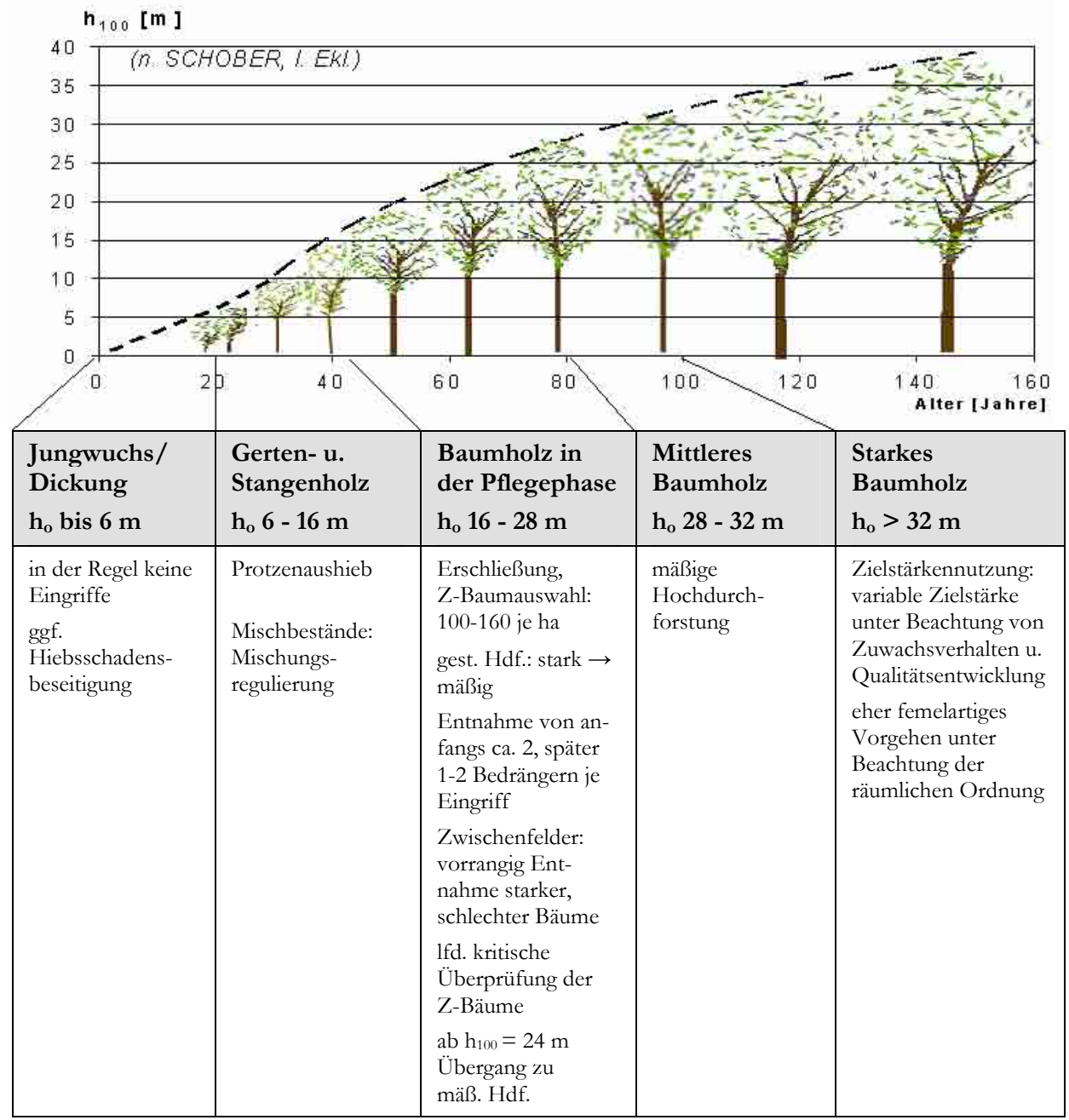




\section{Literatur}

AG „BuchE“ LANDEsbetrieb Wald und Holz Nordrhein-WestFalen (2006): Empfehlungen für eine naturnahe Bewirtschaftung von Buchenrein- und Mischbeständen in Nordrhein-Westfalen. $50 \mathrm{~S}$.

AlberT, M. (1999): Analyse der eingriffsbedingten Strukturveränderung und Durchforstungsmodellierung in Mischbeständen. Dissertation Universität Göttingen, Hainholz Verlag. Göttingen, $131 \mathrm{~S}$.

AltherR, E. (1971): Wege zur Buchen-Starkholzproduktion. Bericht zur 15. Hauptversammlung des Baden-Württembergischen Forstvereins, 123-127

ANONYMus (1902): Anleitung zur Durchführung von Durchforstungs- und Lichtungsversuchen des Vereins Deutscher Forstlicher Versuchsanstalten.

Arend, J.-P.; Eisenbarth, E. U. u. Petercord, R. (2006): Buchenkomplexkrankheit in Luxemburg und Rheinland-Pfalz - Schadsymptome, Ausmaß und Entwicklung der Schäden. Mitteilungen aus der Forschungsanstalt für Waldökologie und Forstwirtschaft Rheinland-Pfalz, Nr. 59, 11-22

AssmanN, E. (1950): Grundflächen- und Volumenzuwachs der Rotbuche bei verschiedenen Durchforstungsgraden. Forstw. Cbl. 69, 257-286

Assmann, E. (1953): Bestockungsdichte und Holzerzeugung. Forstw. Cbl. 72, 69-101

Assmann, E. (1956): Bestockungsgrad und Zuwachs. Forstw. Cbl. 75, 257-265

Assmann, E. (1965): Buchenlichtwuchsbetrieb. Forstw. Cbl. 84, 329-346

Assmann, E. (1976): Behandlung und Produktionsmöglichkeiten der Rotbuche nach dem jetzigen ertragskundlichen Wissensstand. Forstw. Cbl. 95, 245-251

BaChmann, P. (1990): Produktionssteigerung im Wald durch vermehrte Berücksichtigung des Wertzuwachses. Ber. D. Eidgenöss. Forsch. Anst. F. Wald, Schnee u. Landschaft 327, 73 S.

BFN (Bundesamt FÜr NATURSCHUTZ) (2008): Bonner Thesen zum „Naturerbe Buchenwälder“. Download unter http://www.bfn.de/6.html?\&cHash=8affa1489b\&tx_ttnews \%5Btt_news \%5D $=1115$

BMVEL (BUNDESMINISTERIUM FÜR VERBRAUCHERSCHUTZ, ERNÄHRUNG UND LANDWIRTSCHAFT) (2004): Die zweite Bundeswaldinventur - BWI2. Das Wichtigste in Kürze. Berlin

Bolte, A. (2005): Die klimatische Anpassungsfähigkeit der „Mutter des Waldes“. Zur Zukunft der Buche in Mitteleuropa. AFZ/Der Wald 60, 1077-1078

Bolte, A. u. IBISCH, P. L. (2007): Neun Thesen zu Klimawandel, Waldbau und Waldnaturschutz. AFZ/Der Wald 62, 572-576

Bort, U.; Mahler, G. u. Pfeil, C. (1993): Mechanisierte Holzernte - Wechselwirkungen von Erschließungsdichte, Pfleglichkeit und Betriebserfolg. Forsttechn. Informationen, 121-124

Brèda, N., Huc, R., Granier, A. u. Dreyer, E. (2006): Temperate forest trees and stands under severe draught: a review of ecophysiological responses, adaptation processes and long-term consequences. Ann. For. Sci. 63, 625-644

BRYNDUM, H. (1987): Buchendurchforstungsversuche in Dänemark. AFJZ 158, 115-121

Dieter, M.; ENGLERT, H. u. KLEIN, M. (2001): Abschätzung des Rohholzpotenzials für die energetische Nutzung in der Bundesrepublik Deutschland, Hamburg: Institut für Ökonomie der BFH, Arbeitsbericht 2001/11, $40 \mathrm{~S}$.

DitTmar, O.; KNApP, E. u. LembCKE, G. (1986): DDR- Buchenertragstafel 1983. In: IFE-Berichte aus Forschung und Entwicklung 4/1986. Eberswalde

DÖBBELER, H. (2004): Simulation und Bewertung von Nutzungsstrategien unter heutigen und veränderten Klimabedingungen mit dem Wuchsmodell SILVA 2.2. Dissertation an der GeorgAugust-Universität. Göttingen

Döbbeler, H.; Albert, M.; Schmidt, M. u. NAgel, J. (2003): BWINPro. Programm zur Bestandesanalyse und Prognose. Handbuch zur Version 6.2. Göttingen 
Dounavi, K. (2000): Familienstrukturen in Buchenbeständen (Fagus sylvatica L.). Dissertation an der Georg-August-Universität. Göttingen

EllenberG, H. (1996): Vegetation Mitteleuropas mit den Alpen. 5. Aufl., Ulmer-Vlg., Stuttgart

FLEDER, W. (1987): Erziehungsgrundsätze für Buchenbestände. Forst u. Holz 42, 107-111

FREIST, H. (1962): Untersuchungen über den Lichtwuchszuwachs der Rotbuche und seine Ausnutzung im Forstbetrieb. Forstwiss. Forschungen, Beihefte z. Forstw. Cbl., Heft 17, 78 S.

Gauger, Th.; Anshelm, F.; Schuster, H.; Erisman, J.W.; Vermeulen, A.T.; Draajers, G.P.J.; BleeCKer, A. u. NAgEL, H.-D. (2002): Mapping of ecosytem specific long-term trends in deposition loads and concentrations of air pollutants in Germany and their comparison with critical Loads and Critical Levels. Teil 1 Depositon Loads 1990-1999. Institut für Navigation der Universität Stuttgart und Umweltbundesamt (Final Repot 29942 210), 207 S.

Gerstengarbe, F.-W (2008): Der Klimawandel - Ursachen und Veränderungen. Gartower Gespräche. Eigenverlag, 12-22

GUERICKE, M. (2001): Untersuchungen zur Wuchsdynamik von Mischbeständen aus Buche und Europ. Lärche (Larix decidua, Mill.) als Grundlage für ein abstandsabhängiges Einzelbaumwachstumsmodell. Dissertation an der Georg-August-Universität. Cuvillier-Vlg.. Göttingen

GuERICKE, M. (2002): Untersuchungen zur Wuchsdynamik der Buche. Forst u. Holz 57, 331-337

HeIN, S.; LENK, E.; KLÄDKE, J. u. KoHNLE, U. (2007): Z-Baum orientierte Auslesedurchforstung in Buche [Fagus sylvatica L.]: Auswirkungen auf Qualität, Sortenstruktur und Wertleistung. AFJZ $178,8-20$

HeINKEN, T. (1996): Die naturnahe Waldvegetation grundwasserferner Standorte im niedersächsischen Tiefland - ein aktueller Überblick. Forst u. Holz 51, 429-435

HoLM, M. (1974): Untersuchungen zur einzelstammweisen Nutzung nach Zieldurchmessern. Diss. an der Albert-Ludwigs-Universität, Freiburg

HoLm, M. (1995): Naturnahe Buchenwirtschaft. AFZ/DerWald 50, 408-410

Hosius, B.; Leinemann, L.; Bergmann, F.; Maurer, W. D. u. TABel, U. (2003): Genetische Untersuchungen zu Familienstrukturen und zur Zwieselbildung in Buchenbeständen. Forst u. Holz $58,51-54$

Johann, K. (1982): Der „A-Wert“ - ein objektiver Parameter zur Bestimmung der Freistellungsstärke von Zentralbäumen. Berichte von der Jahrestagung der Sektion Ertragskunde im DVFFA in Weibersbrunn, 146-158

Kahn, M. u. Pretzsch, H. (1997): Das Wuchsmodell SILVA - Parametrisierung der Version 2.1 für Rein- und Mischbestände aus Fichte und Buche. AFJZ 168, 115-123

KATÓ, F. (1973): Begründung der qualitativen Gruppendurchforstung. Habilitationsschrift an der Georg-August-Universität, Göttingen

KATÓ, F. u. MÜLDER, D. (1979): Qualitative Gruppendurchforstung der Buche. Grundsätze, Wertentwicklung nach 10 Jahren, praktische Anleitung. AFJZ 150, 105-111

KATÓ, F. u. MÜLDER, D. (1983): Qualitative Gruppendurchforstung der Buche - Wertentwicklung nach 15 Jahren. AFJZ 154, 139-145

KATÓ, F. u. MÜLDER, D. (1988): Qualitative Gruppendurchforstung der Buche - Wertentwicklung nach 20 Jahren. AFJZ 159, 4-9

KATÓ, F. u. MÜLDER, D. (1992): Qualitative Gruppendurchforstung der Buche - Wertentwicklung nach 25 Jahren. AFJZ 163, 197-203

KENK, G. (2002): Variation der periodischen Zuwächse von Fichten und Buchen. AFZ/Der Wald $57,300-302$

KLÄDTKE, J. (1997): Seit 25 Jahren nach Altherr: Buchen-Lichtwuchsdurchforstung. AFZ/Der Wald $52,1019-1023$

KLÄDTKE, J. (2001): Konzepte zur Buchen-Lichtwuchsdurchforstung. AFZ/Der Wald 56, 1047-1050

KLÄDTKE, J. (2002): Wachstum großkroniger Buchen und waldbauliche Konsequenzen. Forstarchiv $73,211-217$ 
Kleinschmit, J. u. SvolBA, J. (1996): Ergebnisse der Buchenherkunftsversuche von Krahl-Urban. AFZ/Der Wald 51, 779-782

KNOKE, T. (2003): Eine Bewertung von Nutzungsstrategien für Buchenbestände (Fagus sylvatica L.) vor dem Hindergrund des Risikos der Farbkernbildung- eine waldbaulich-forstökonomische Studie. Forstliche Forschungsberichte. München, $200 \mathrm{~S}$.

Knoke, T. u. Schulz Wenderoth, S. (2001): Ein Ansatz zur Beschreibung von Wahrscheinlichkeit und Ausmaß der Farbkernbildung bei Buche (Fagus sylvatica L.). Forstw. Cbl. 120, 154-172

Kramer, H. (1988): Waldwachstumslehre. Vlg. Paul Parey, Hamburg u. Berlin, S. 188

Kölling, C.; Walentowski, H. u. Borchert, H. (2005): Die Buche in Mitteleuropa. Eine Waldbaumart mit grandioser Vergangenheit und sicherer Zukunft. AFZ/Der Wald, 696-701

Kölling, C. u. Zimmermann, L. (2007): Die Anfälligkeit der Wälder Deutschlands gegenüber dem Klimawandel. Gefahrstoffe - Reinhaltung der Luft 67, 259-268

KoHnLE, U. (2003): Sortierungsrelevante Kernmerkmale bei Buche. AFZ/Der Wald 58, 210-213

LANDESFOrSTVERWALtung RHEINLAND-PFalz (2001): Richtlinie zur naturnahen Erzeugung von Wertholz bei den Baumarten Buche, Berg-, Feld-, Spitzahorn und Esche. Aktuelle waldbauliche Richtlinien u. Hinweise 9/2001, Az. 10524-5001, Mainz

Landesforstverwaltung Rheinland-Pfalz (2003): Z-Baum-orientierte Pflegeeingriffe in der Dimensionierungsphase. Aktuelle waldbauliche Richtlinien u. Hinweise 4/2003, Az. 10524-5001, Mainz

LEONHARDT, B. u. WAGNER, S (2006): Qualitative Entwicklung von Buchen-Voranbauten unter Fichtenschirm. Forst u. Holz 61, 454-457

LeusChNeR, C. (1998): Mechanismen der Konkurrenzüberlegenheit der Rotbuche. Ber. Reinh. Tüxen-Ges., 10, 5-18

LÜPKE, B. V. (1986): Die Durchforstung, insbesondere Jungdurchforstung, von Buchenreinbeständen. Forst u. Holz 41, 54-61

LÜPKE, B. v. (2004): Risikominderung durch Mischwälder und naturnaher Waldbau: ein Spannungsfeld. Forstarchiv 75, 43-50

Mantau, U.; Sögel, C. u. Wimar, H. (2007): Holzrohstoffbilanz Deutschland, Szenarien des Holzaufkommens und der Holzverwendung bis 2010. Hamburg

Manthey, M.; Leuschner, C. u. HÄrdtle, W. (2007): Buchenwälder und Klimawandel. Natur und Landschaft 82, 441-445

MeYER, P. (2000): Strukturelle Diversität und waldbauliche Eingriffe. Entwicklung der DurchmesserDiversität von Buchen- und Fichtenbeständen in Abhängigkeit von der Eingriffsstärke. Tagungsband der Jahrestagung 2000 der Sektion Waldbau im DVFFA in Sachsen-Anhalt, 3-48

MitsCherLiCH, G. (1953/54) : Einzelstamm- oder Bestandeswirtschaft ? AFJZ 125, 179 -185

MLU (MINISTERIUM FÜR LANDWIRTSCHAFT, UMWELTSCHUTZ UND RAUMORDNUNG DES LANDES) BRANDENBURG (HRSG.) (2004): Waldbau-Richtlinie 2004 „Grüner Ordner“ der Landesforstverwaltung Brandenburg. Potsdam

MÖHRING, B. (2004): Betriebswirtschaftliche Analyse des Waldumbaus. Forst u. Holz 59, 523-530

Moog, M. (1990): Überlegungen zur optimalen Zielstärke der Buche. AFZ 45, 1168-1160

Moog, M. u. Karberg, B. (1992): Ökonomische Gesichtspunkte zur Zielstärke von Kiefern und Buchen. AFZ 47, 85-90

Nabuurs, G. J.; De Goede, D. M.; Michie, B.; SchelhaAs, M. J. u. Wesseling, J.G. (2002): Longterm international impacts of nature-oriented forest management on European forests - an assessment with the EFISCEN model. Journal of World Forest Resource Management, Bd.9, 101-129

NAGEL, J. (1999): Konzeptionelle Überlegungen zum schrittweisen Aufbau eines waldwachstumskundlichen Simulationssystems für Nordwestdeutschland. Schriftenreihe der Forstlichen Fakultät der Universität Göttingen, Band 128 
Nagel, J.; Albert, M. u. Schmidt, M. (2002): Das waldbauliche Prognose und Entscheidungsmodell BWINPro 6.1. Forst u. Holz 57, 486-493.

NDS. LANDESFORSTVERWALTUNG (2000): Z-Baumzahlen im Landeswald. RdErl. des ML, VORIS 79100000060061

Nickel, M.; KLemmt, H.-J. u. H. Pretzsch (2007): Unter optimalen Wuchsbedingungen in Bayern: Durchforstungsreaktionen der Buche. AFZ/Der Wald 62, 676-679

NiESAR, M. (2007): Symptom- und Ursachenanalyse der aktuellen Buchenerkrankungen in höheren Lagen von Nordrhein-Westfalen. Diss. Universität Göttingen, 115 S.

Отто, H.-J. (1991): Langfristige ökologische Waldentwicklung in den Landesforsten. Programm der Landesregierung Niedersachsen v. 23.07.1991. Sonderdruck

OтTо, H.-J. (1994): Waldökologie. Ulmer-Vlg., Stuttgart

Petercord, R.; DelB, H. u. SCHRÖTER, H. (2007): Vermeintliche Dürreschäden an Buche entpuppen sich als Käferbefall: Schwere Schäden durch den Buchen-Prachtkäfer in Baden-Württemberg. AFZ/Der Wald 62, 686-690

PRETzsCH, H. (2004): Gesetzmäßigkeiten zwischen Bestandesdichte und Zuwachs. Lösungsansatz am Beispiel von Reinbeständen aus Fichte (Picea abies [L.] Karst.) und Buche (Fagus sylvatica L.). AFJZ 175, 225-234

PretzsCH, H. (2006): Von der Standflächeneffizienz der Bäume zur Dichte-Zuwachs-Beziehung des Bestandes. Beitrag zur Integration von Baum- und Bestandesebene. AFJZ 177, 188-199

Prodan, M. (1965): Holzmesslehre. J D. Sauerländer's Vlg., Frankfurt a. M.

RennenberG, H.; Seiler W.; Matyssek R.; Gessler A. u. Kreuzwieser, J. (2004): Die Buche (Fagus sylvatica L.) - ein Waldbaum ohne Zukunft im südlichen Mitteleuropa? AFJZ 175, 210224

RÖHE, P. (2003): Naturnahe Buchenwirtschaft im Landeswald Mecklenburg-Vorpommern. Forst u. Holz 58, 440-445

RÖHRIG, E. (1968): Probleme der Jungbestandspflege im Laubholz. Forst u. Holz 23, 409-412

SAARFORST LANDESBETRIEB (2004): Richtlinie für die Bewirtschaftung des Staatswaldes im Saarland (WaldbauRL Saarland). Saarbrücken

SCHÄDELIN, W. (1942): Die Auslesedurchforstung als Erziehungsbetrieb höchster Wertleistung. 3. Aufl.. Bern

SCHOBER, R. (1967): Ertragstafeln für Rotbuche, mäßige und starke Durchforstung. In: Ertragstafeln wichtiger Baumarten, 3., neu bearbeitete u. erw. Auflage. Sauerländer's Vlg.. Frankfurt a. M.

SCHOBER, R. (1972): Die Rotbuche. Schriftenreihe der Forstlichen Fakultät der Universität Göttingen, Band $43 / 44$

SCHOBER, R., (1987): Durchforstung nach Zahlen? AFJZ 158, 174-183

SCHOBER, R., (1988a): Von der Niederdurchforstung zu Auslesedurchforstungen im Herrschenden. AFJZ 159, 208-213

SCHOBER, R. (1988b): Von Zukunfts- und Elitebäumen. AFJZ 159, 239-2249

SCHWAPPACH, A. (1911): Die Rotbuche. Neudamm Vlg.

SEELING, U. (1998): Kerntypen im Holz - Konsequenzen für die Verwertung am Beispiel der Buche (Fagus sylvatica L.). Schweiz. Z. Forstwes. 149, 991-1004

SHANNON, C. E. u. WEAVER, W. (1949): The mathematical theory of communication. University of Illinois Press, Urbana

SpeKat, A.; EnKE, W. u. KreienKAmP, F. (2007): Neuentwicklung von regional hoch aufgelösten Wetterlagen für Deutschland und Bereitstellung regionaler Klimaszenarien auf der Basis mit dem Regionalisierungsmodell WETTREG auf der Basis von globalen Klimasimulationen mit ECHAM/MPI-OM T63L31 2010 bis 2100 für die SRES-Szenarios B1, A1B und A2; FuEVorhaben Förderkennzeichen 20441 138, Publikationen des Umweltbundesamtes

SPELlmanN, H. (1995): Vom strukturarmen zum strukturreichen Wald. Forst u. Holz 50, 35-44 
SpellmanN, H. (1999): Überführung als betriebliche Aufgabe. Berichte Freiburger Forstliche Forschung, Heft 8, 194-217

SpellmanN, H. (2005): Produziert der Waldbau am Markt vorbei? AFZ/Der Wald 63, 454-459

SpellmanN, H. (2006): Bestandespflege - Mittel zum Zweck - Entscheidungshilfen auf der Grundlage waldwachstumskundlicher Forschungsergebnisse. Deutscher Forstverein e. V., 62. Jahrestagung in Weimar, 126-136

Spellmann, H. u. NAgel, J., (1996): Zur Durchforstung von Fichte und Buche. AFJZ 167, 6-15

SpellmanN, H.; NAGEL, J. u. BÖCKMAnN, T., (1999): Summarische Nutzungsplanung auf der Basis von Betriebsinventuren. AFJZ 170, 122-128

Spellmann, H.; MeYer, P. u. Albert, M. (2004): Strukturbildung durch gezielte Pflegestrategien im Vergleich zur natürlichen Strukturentwicklung in Naturwäldern. In: Forschungszentrum Waldökosysteme (Hrsg.): Indikatoren und Strategien für eine nachhaltige, multifunktionelle Waldnutzung - Fallstudie Waldlandschaft Solling. Abschlussbericht 1999-2003 zum BMBF-Verbundforschungsvorhaben. Teil 2: Ausführliche Teilvorhabenberichte, B 71/2004, 339-374

Spellmann, H.; Sutmöller, J. u. MeesenburG, H. (2007): Risikovorsorge im Zeichen des Klimawandels. AFZ/Der Wald 52, 1246-1249

Spellmann, H.; Mantau, U. u. Polley, H. (2008): Nachhaltige Rohholzversorgung aus deutschen Wäldern. Positionspapier der Plattform Forst \& Holz von DFWR und DHWR vom 05.06.2008. http://www.dfwr.de/download/, $10 \mathrm{~S}$.

Spiecker, H.; Mielikäinen, K.; KöHL, M. u. SkovsgaArd, J. (1996): Growth Trends in European Forests: Studies from 12 Countries. Springer-Vlg.. Berlin Heidelberg. 372 S.

STERBA, H. (1975): Assmanns Theorie der Grundflächenhaltung und die „Competition-DensityRule“ der Japaner Kira, Ando und Tadaki. Cbl. f. d. ges. Forstwesen 92, 46-62

STERBA, H. (1981): Natürlicher Bestockungsgrad und Reinekes SDI. Cbl. F. d. ges. Forstwesen 98, $101-116$

Sterba, H. (1987): Estimating Potential Density from Thinning Experiment and Inventory Data. Forest Science 33, 1022-1034

Teuffel, K. V. (2002): Aspekte zur Behandlung der Buche. AFZ/Der Wald 57, 851-853

UTSCHIG, H. (2000): Wachstum vorherrschender Buchen in Abhängigkeit von Standort und Behandlung. Forst u. Holz 55, 44-50

WAGNER, S. (1999): Ökologische Untersuchungen zur Initialphase der Naturverjüngung in EschenBuchen-Mischbeständen. Schriftenreihe der Forstlichen Fakultät der Uni Göttingen und der Niedersächsischen Forstlichen Versuchsanstalt Göttingen, Sauerländer's Vlg., Frankfurt a. M.

WeINREBE, H. (2004): Entwurf und Erprobung eines transektbasierten Methodensets zur Waldstrukturkartierung. Ergebnisse aus den Naturwäldern und Spechtkartierungsgebieten Limker Strang und Winterlieth im Solling. Diplomarbeit an der Universität Karlsruhe. 142 S.

Wiedemann, E. (1932): Die Rotbuche 1931. Mitt. a. Forstwirt. u. Forstwiss. 3, 96-201

Wilhelm, G. F.; LetTer, H. A. u. EDER, W. (1999a): Zielsetzungen und waldbauliche Prinzipien. Konzeption einer naturnahen Erzeugung von starkem Wertholz. AFZ/Der Wald 54, 232-233

Wilhelm, G. F.; LetTer, H. A. u. EDER, W. (1999b): Die Phase der Qualifizierung. AFZ/Der Wald $54,234-236$

Wilhelm, G. F.; LetTer, H. A. u. EdeR, W. (1999c): Die Phase der Dimensionierung. AFZ/Der Wald 54, 236-238

Wilhelm, G. F.; LeTter, H. A. u. Eder, W. (1999d): Die Phase der Reife. AFZ/Der Wald 54, 239240

Willmann, U.; Mahler, G. u. Wurster, M. (2001): Güteansprache am stehenden Stamm im Rahmen der Bundeswaldinventur II. AFZ/Der Wald 56, 1024-1026

WÜHLISCH, G. VON (2005): Herkunft und wirtschaftlicher Ertrag bei Rotbuche. AFZ / Der Wald 60 1074-1076 
ZERBE, S. u. SCHMiDT, I. (2006): Auswirkungen des Waldumbaus auf die biologische Vielfalt. In: Abschlussbericht 2003-2006 zum BMBF-Forschungsvorhaben „Biologische Vielfalt und deren Bewertung am Beispiel des ökologischen Waldumbaus in den Regionen Solling und Lüneburger Heide." Göttingen, S. 41-60

Korrespondierender Autor:

Ralf-Volker Nagel

Nordwestdeutsche Forstliche Versuchsanstalt

Grätzelstr. 2

37079 Göttingen

E-Mail: Ralf.Nagel@nw-fva.de

URL: www.nw-fva.de

Prof. Dr. Hermann Spellmann

Nordwestdeutsche Forstliche Versuchsanstalt 



\section{Methodische Ansätze und Ergebnisse zur Quantifizierung des Buchen-Rotkerns in Hessen}

\section{Methods and results of quantifying beech red heartwood in Hesse}

Matthias Schmidt, Stefan Nowack und Rüdiger Riebeling

\section{Zusammenfassung}

Im Zeitraum von 1993 - 1999 erfolgte im Rahmen des regulären Bucheneinschlages in ausgewählten hessischen Forstämtern eine umfangreiche Rotkernerhebung. Die in fast allen Landesteilen durchgeführte Erhebung umfasst über 80.000 angesprochene Erdstämme ${ }^{1}$. Eine adäquate Auswertung dieser Daten stand bisher aus. Der vorliegende Beitrag präsentiert ein Modell, das Zusammenhänge zwischen unabhängigen Variablen und der Wahrscheinlichkeit, eine bestimmte Verkernung aufzuweisen, auf der Grundlage dieser Daten quantifiziert. Dabei kann neben den Einflüssen des BHDs und des Bestandesalters u. a. auch der Einfluss von Standortsvariablen wie dem Ausgangsgestein und der Wasserhaushaltsstufe beschrieben werden. Das entwickelte Modell ist die Grundlage für ein umfassendes Entscheidungsunterstützungssysstem zur Rotkernproblematik für die forstliche Praxis.

\footnotetext{
${ }^{1}$ Die Autoren danken Herrn Wolfgang Raschka und Herrn Volker Graumann (Servicestelle Forsteinrichtung und Naturschutz - FENA) für die Verschneidung der Rotkerndaten mit Forsteinrichtungsund Geometriedaten.
} 
Zukünftig wird es somit möglich sein, optimale Zielstärken in Abhängigkeit des Standortes, des Bestandesalters und des aktuellen Einzelbaum-BHDs zu definieren

Stichworte: Rotbuche, Rotkern, non-proportional odds Modell, Standortssensivität

\begin{abstract}
Between 1993 - 1999, an extensive survey of red heartwood was conducted as part of a scheduled beech harvest in selected forest districts in Hesse. The survey, carried out in almost all regions of the state, investigated more than 80.000 butt logs. Until now, an adequate analysis of these data had not been undertaken. This paper presents a model that defines relationships between independent variables and the probability of a particular heartwood formation occurring, and then quantifies them using these data. In addition to the influences of DBH and stand age, the influence of site variables such as the parent rock material and hydrology class also can be described. The model developed forms the basis of a comprehensive decision support system for addressing problems associated with red heartwood in forestry practice. In future this model will facilitate definitions of optimum target diameters in relation to site, stand age and actual single-tree diameters.
\end{abstract}

Keywords: Common beech, red heartwood, non-proportional odds model, sitesensivity

\title{
1 Einleitung
}

Die Rotbuche ist sowohl vom Einschlagsvolumen (ZMP 2007) als auch von der Waldfläche her die mit Abstand wichtigste Laubholzart Deutschlands (BMVEL 2004). Trotz der starken Nachfrage- und Preisschwankungen für Buchenwertholz in den letzten Jahren wird das Produktionsziel aufgrund der großen dimensionsund qualitätsbedingten Preisstaffelung (Wertholzbaumart) auch zukünftig starkes, wertholzhaltiges Stammholz sein. Einer optimalen Ausnutzung des spät kulminierenden und auf vielen Standorten hohen durchschnittlichen Zuwachses dieser Baumart (KRAMER 1988: S. 103 und 220 ff.) steht jedoch häufig die Entwertung durch eine fakultative Verkernung entgegen (SEELING 1991, SACHSSE 1991, SEELING u. SACHSSE 1992). Immer wieder initiierte Werbekampagnen (z. B. WAGEMANN 2001) haben - mit Ausnahmen bei geringfügigen Sonderverkäufen nichts an den deutlichen Preisabschlägen für rotkerniges, ansonsten aber hochwertiges Buchenstammholz geändert. Die besondere Bedeutung des Buchenrotkernes, der häufig zu mehr als $50 \%$ das für eine Abwertung in die Güteklasse C (BK) ausschlaggebende Kriterium ist (HAPLA u. STEINFATT 2001), wird sowohl in der Handelsklassensortierung für Rohholz (HKS 1969) als auch in der Gütesor- 
tierung nach der EU-Norm EN 1316-1 durch die Ausweisung von Unterklassen berücksichtigt (DIN EN 1997). Andere Kerntypen wie der Spritzkern werden noch negativer beurteilt, da hier eine besonders starke Zellverthyllung (SACHSSE 1991) oder sogar beginnende Holzzersetzung (NECESANY 1958) auftritt. Die statistische Beschreibung des Auftretens und Ausmaßes der unterschiedlichen fakultativen Kerntypen in Abhängigkeit von Einzelbaum-, Bestandes- und Standortsvariablen ist somit von besonderem wirtschaftlichem Interesse. Erkenntnisse über Zusammenhänge zum Brusthöhendurchmesser (BHD), Einzelbaumalter oder Standort könnten dafür genutzt werden, optimale Produktionszeiträume und Zielstärken auf unterschiedlichen Standorten für die Rotbuche zu bestimmen. Daher sind schon früh Untersuchungen zum Buchenrotkern durchgeführt worden (RACZ 1961, ZYCHA 1948), wobei das Thema in den letzten Jahren noch einmal an Aktualität gewonnen hat und zahlreiche Ergebnisse auch aus neueren Untersuchungen vorliegen (SCHMIDT u. HEIN 2005, SCHMIDT et al. 2005, KNOKE 2003, BÖRNER 2002, BÜREN 2002, KNOKE u. SCHULZ WENDEROTH 2001, REDDE 1998, BÜREN 1998, HÖWECKE 1998). Allerdings dienen die Erkenntnisse trotz eines relativ hohen Forschungsaufwandes bisher kaum der Entscheidungsunterstützung in der Forstwirtschaft. Als Ursachen sind die uneinheitlichen Ergebnisse, die hohe nicht erklärte Reststreuung bei der Modellierung des Auftretens (Kerntyp) und Ausmaßes (Durchmesser) der Verkernung sowie die fehlende Einbindung in Planungssoftware zu vermuten. Gleichzeitig muss jedoch darauf hingewiesen werden, dass die verwendeten Methoden das Muster der fakultativen Buchenverkernung in vielen Fällen nur unzureichend erfassen. Im Folgenden wird ein Ansatz vorgestellt, der eine adäquate Beschreibung dieser Erscheinungsmuster und damit die Entwicklung eines umfassenden Entscheidungsstützungssystems ermöglicht.

\section{Datengrundlage}

Die uneinheitlichen Ergebnisse vieler Untersuchungen können dahingehend interpretiert werden, dass die Zusammenhänge zwischen Variablen wie dem Alter, dem BHD sowie Standortsvariablen und der Ausbildung des Buchenkerns durch zufällige, d. h. nicht beobachtete bzw. erfasste Einflüsse überlagert werden. Als Beispiele für derartige Einflüsse können das Auftreten von Totästen (ZYCHA 1948) und Rindenbrand (RACZ et al. 1961) oder aber auch nicht erfasste Bodeneigenschaften wie ein Stauhorizont genannt werden. Für eine statistisch saubere Quantifizierung der Effekte der beobachteten Einflussfaktoren werden daher große Datenmengen benötigt. Nur dann kann davon ausgegangen werden, dass alle Kombinationen von berücksichtigten Einflussfaktoren in einem ähnlichen Ausmaß von zufälligen Einflüssen überlagert werden. Große Datenmengen sind auch notwendig, wenn der Einfluss von kategorischen Standortsvariablen wie dem geologischen Ausgangsgestein oder der Wasserhaushaltsstufe zusätzlich zu den Effekten von (Bestandes)alter und BHD auf überregionaler Ebene quantifiziert 
werden soll. Die neueren Untersuchungen zum Buchenrotkern (s. Einleitung) können somit eher als exemplarische Pilotstudien denn als Grundlage für den Aufbau eines umfassenden Rotkerninformationssystems betrachtet werden, da ihre Datenbasis nur einige hundert bis einige tausend Datensätze umfasst. Die vorliegende Untersuchung basiert dagegen auf 80.155 Erdstämmen, an denen die Verkernung am Fuß- und Zopfende in kategorialer Form angesprochen wurde (s. Tab. 1). Die Daten wurden im Rahmen des regulären Bucheneinschlages in hessischen Forstämtern im Zeitraum von 1993 - 1999 mittels mobiler Datenerfassungsgeräte erhoben. Damit stehen auch der Mittendurchmesser und die Stammlänge als Informationen für Auswertungen zur Verfügung. Die Teilnahme an der Erhebung war freiwillig, und es wurden nur Einschläge im Staatswald erfasst. An der Erhebung der Buchenverkernung beteiligten sich im Einschlagsjahr 1993 insgesamt 12 Forstämter des Lahn-Dill-Berglandes, des Östlichen Hinter-Taunus, des Vorderen Vogelsberges und des Amöneburg-Neustädter Gebietes. Ab dem Jahr 1994 kamen Forstämter aus dem westlichen Taunus, der Hessischen Rheinebene, dem Odenwald, dem östlichen Vogelsberg, dem Nordost- sowie Nordwesthessischen Bergland und aus dem Weserbergland hinzu. Dadurch erhöhte sich die Zahl zunächst auf 19, später zeitweise auf 29 Forstämter.

Übergeordnete Motivation für die Erhebung war es, Informationen über die Auftretenswahrscheinlichkeit und das Ausmaß der Kernbildung in älteren Buchenbeständen auf unterschiedlichen Standorten in verschiedenen Regionen Hessens zu gewinnen und für Entscheidungsprozesse zu nutzen. Die Erhebung sollte zusätzlich dazu dienen, den örtlichen Umfang der Verkernung in den Forstämtern selbst zu dokumentieren. Darüber hinaus wollte man aus längeren Zeitreihen Entscheidungshilfen für die mittelfristige Planung und für die laufende Bewirtschaftung einzelner Waldteile ableiten. Nach einigen Erhebungsjahren erwartete man umfangreiche Informationen für die Entwicklung von Strategien (Zielstärken) zur Nutzung älterer Buchenbeständen auf unterschiedlichen Standorten. Das übergeordnete Ziel der Entwicklung eines umfassenden Rotkerninformationssystems ist bisher aber nicht erreicht worden.

Die Erhebung der Kernparameter geschah in praxisüblicher Weise durch okulare Einschätzung, die durch gelegentliche Kontrollmessungen ergänzt wurde. Es erfolgte keine Unterscheidung nach unterschiedlichen Kerntypen wie Rot- und Spritzkern. Die Ergebnisse der Rotkernansprache wurden abschließend mit Forsteinrichtungs- und Standortsinformationen verschnitten, um deren Zusammenhänge zur Verkernung prüfen zu können. Eine zusätzliche Erfassung von Merkmalen wie Totästen und die Quantifizierung ihres potenziellen Einflusses erscheinen vor allem aus wissenschaftlicher Sicht interessant. Gegen ihre Verwendung spricht jedoch, dass diese Informationen in der Praxis für die Anwendung eines zukünftigen Rotkerninformationssystems im Allgemeinen nicht zur Verfügung stehen. Das heißt ein derartiges System sollte nur auf relativ einfach zu erhebenden Daten basieren, wie sie von der Forsteinrichtung und Standortskartierung zur 
Verfügung gestellt werden. Gleichzeitig zeigen viele Untersuchungen, dass Kenngrößen wie Totäste oder Zwiesel im Vergleich zum (Bestandes)alter oder dem BHD nur eine nachrangige oder keine signifikante Bedeutung für die Verkernung haben (z. B. KNOKE u. SCHULZ WENDEROTH 2001).

Tabelle 1: Definition der Kategorien, die bei der Ansprache des Buchenrotkerns an Erdstämmen verwendet wurden

\begin{tabular}{cc|cc}
\hline $\begin{array}{c}\text { Rotkern- } \\
\text { kategorie }\end{array}$ & Definition & $\begin{array}{c}\text { Rotkern- } \\
\text { kategorie }\end{array}$ & Definition \\
\hline 0 & kein Rotkern & 3 & $\begin{array}{c}1 / 3-1 / 2 \text { des } \\
\text { Stammdurchmessers } \\
1\end{array}$ \\
bis 12 cm Kerndurchmesser & 4 & $\begin{array}{c}1 / 2-2 / 3 \text { des } \\
\text { Stammdurchmessers } \\
>2 / 3 \text { des }\end{array}$ \\
\hline & 12 cm Kerndurchmesser - & 5 & Stammdurchmessers \\
\hline
\end{tabular}

Einen ersten Überblick über die Datenstruktur erhält man durch die Stratifizierung nach Kenngrößen, die als relevant für die Verkernung eingestuft werden. Im Folgenden werden dabei nur die Variablen verwendet, für die bei der späteren Modellbildung ein signifikanter Effekt identifiziert wurde. Diese Variablen sind das geologische Ausgangsgestein, die Wasserhaushaltsstufe, die Trophiestufe, die Seehöhe, das Bestandesalter, der BHD und die Erdstammlänge.

Die Datenbelegung der geologischen Ausgangsgesteine ist dabei sehr unterschiedlich (s. Abb. 1 links). Auf Basalt, Buntsandstein und die Gruppe der Tonschiefer und Grauwacken entfallen $71 \%$ der Datensätze. Die Bezeichnung Buntsandstein umfasst hier den mittleren und unteren Buntsandstein. Ebenso wurden die Tonschiefer und Grauwacken sowie deren Mischformen zusammengefasst. Die Zusammenfassung der Ausgangsgesteine erfolgte, da aus bodenkundlicher Sicht große Ähnlichkeiten bezüglich der Zusammenhänge zur Verkernung angenommen wurden. Die Annahmen bestätigten sich während des Modellbildungsprozesses auch aus statistischer Sicht, da für die entsprechenden Ausgangsgesteine keine signifikanten Unterschiede auftraten. Neben den 3 dominierenden Ausgangsgesteinsgruppen weisen Diabas, Quarzit, die Gruppe Gneis und Granit sowie Löß noch jeweils mehrere tausend Datensätze auf. Die Zusammenfassung von Gneis und Granit zu einer Gruppe erfolgte unter den bereits erwähnten bodenkundlichen und statistischen Gesichtspunkten. Die übrigen Ausgangsgesteine umfassen in der Summe nur noch knapp $5 \%$ der Datensätze, was bei der späteren Quantifizierung und Interpretation ihrer Effekte auf die Verkernung berücksichtigt werden muss.

Die Verteilung nach Wasserhaushaltsstufen wird von den Ausprägungen 'frisch' und 'mäßig frisch' dominiert ( $85 \%$ der Datensätze). Die Ausprägungen 'feucht' und 'trocken' wurden aufgrund ihrer extrem geringen Belegung den Aus- 
prägungen 'betont frisch' bzw. 'mäßig trocken' zugeordnet. Die Ausprägungen 'mäß. trocken - trocken' und 'wechselfeucht' umfassen dabei relativ geringe Anzahlen an Datensätzen (s. Abb. 1 rechts).

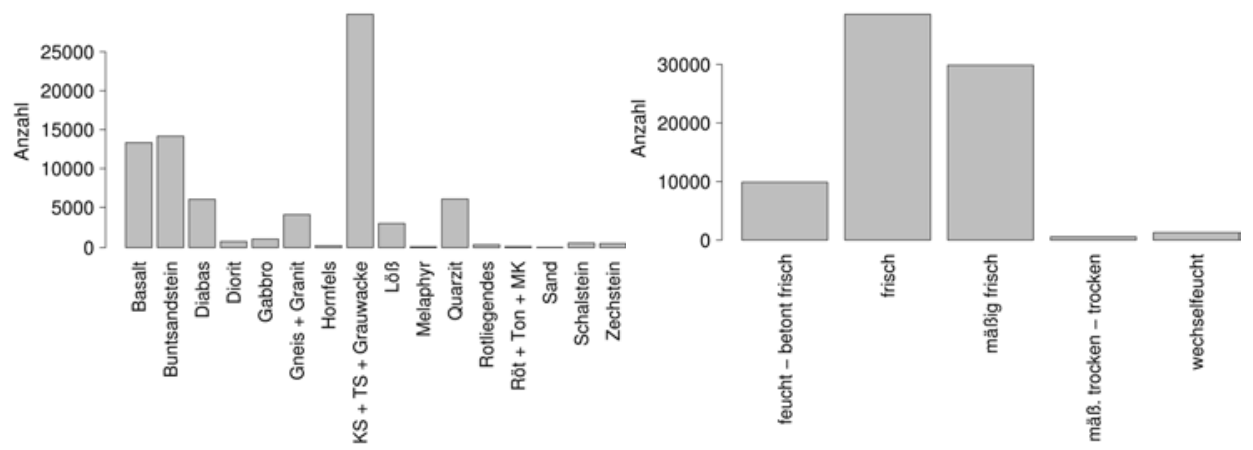

Abbildung 1: Häufigkeitsverteilungen der erfassten Erdstämme nach geologischem Ausgangsgestein (links) und Wasserhaushaltsstufe (rechts). Die Gruppe 'KS + TS + Grawwacke' umfasst Tonschiefer, Grawwacke sowie den seltenen Kieselschiefer, die Gruppe 'Buntsandstein' den mittleren und unteren Buntsandstein sowie den seltenen Glimmersandstein, die Gruppe 'Röt + Ton + MK' kombiniert die seltenen Ausgangsgesteine Oberer Buntsandstein (Röt), Ton und Muschelkalk.

Die Trophiestufen oligo- und mesotroph wurden zusammengefasst, da die Trophiestufe oligotroph nur selten vorkommt. Damit werden nur die Trophiestufen eutroph (36\% der Datensätze) bzw. meso - oligotroph (64\% der Datensätze) unterschieden. Neben der Seehöhe als weiterer Standortsvariablen wurden auch das Bestandesalter, der BHD sowie die Erdstammlänge als Kenngrößen mit signifikantem Effekt identifiziert (s. Tab. 2). Sowohl die Alters- als auch die BHDHäufigkeitsverteilung weisen eine breite Spreitung mit einem mittleren Alter von 142 Jahren bzw. einem mittleren BHD von 48,6 cm auf (s. Abb. 2 / Tab. 2).

Tabelle 2: $\quad$ Kennwerte der Häufigkeitsverteilungen und Korrelationskoeffizienten der metrischen Variablen, für die ein signifikanter Einfluss auf die Verkernung identifiziert wurde

\begin{tabular}{lcccccc}
\hline Variable & Min. & Mittel & Max. & \multicolumn{3}{c}{ Korrelationskoeffizienten } \\
& & & & $\begin{array}{c}\text { Alter } \\
\text { [J] }\end{array}$ & $\begin{array}{c}\text { Stammlänge } \\
{[\mathrm{m}]}\end{array}$ & $\begin{array}{c}\text { Seehöhe } \\
{[\mathrm{m}]}\end{array}$ \\
\hline BHD [cm] & 21,5 & 48,6 & 123,0 & 0,32 & 0,21 & $-0,20$ \\
Alter [J] & 42 & 142 & 227 & & 0,05 & 0,18 \\
Stammlänge [m] & 2,1 & 7,6 & 24,0 & & & 0,01 \\
Seehöhe [m] & 150 & 369 & 670 & & & \\
\hline
\end{tabular}



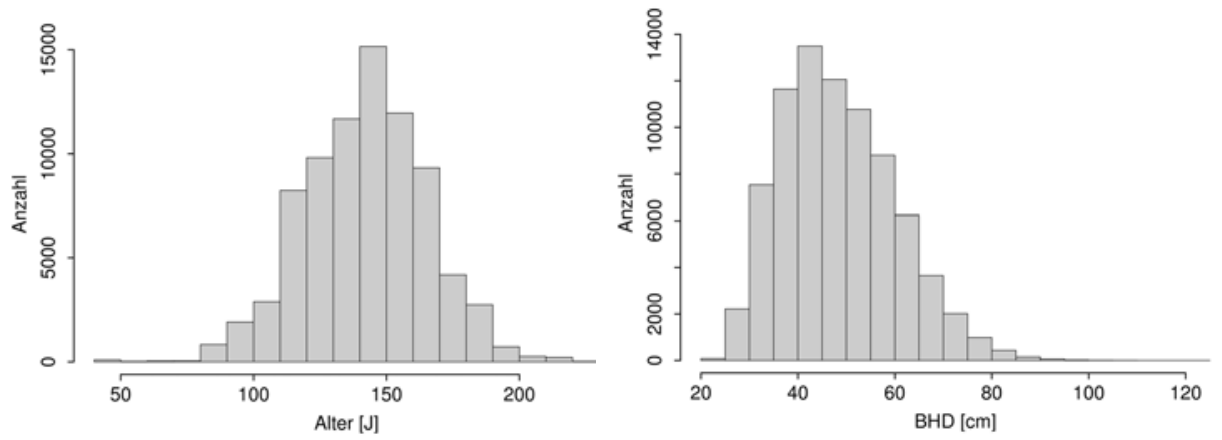

Abbildung 2: Häufigkeitsverteilungen der erfassten Erdstämme nach Alters- $[10 \mathrm{~J}]$ (links) und Durchmesserstufen $[5 \mathrm{~cm}]$ (rechts)

Die Korrelationskoeffizienten zwischen den metrischen Variablen sind gering, was vorteilhaft für die spätere unverzerrte Quantifizierung der einzelnen Modelleffekte ist. Insbesondere die geringe Korrelation zwischen dem BHD und dem Bestandesalter spiegelt wider, dass auch die Bäume mit geringerer Dimension aus älteren Beständen stammen.

Eine spezielle Problematik resultiert daraus, dass der BHD im Gegensatz zu den übrigen Variablen nicht gemessen bzw. direkt erfasst wurde, sondern aus dem Mittendurchmesser und der Erdstammlänge hergeleitet werden musste. Eine Verwendung des BHDs als Eingangsgröße ist aber unbedingt erforderlich, da die Ernteentscheidung am BHD (Zielstärke) und nicht am Mittendurchmesser orientiert ist. Aufgrund der großen Variation der Erdstammlängen (s. Tab. 2) und Mittendurchmesser, erscheint es jedoch unzulässig, den BHD unter Annahme einer einheitlichen Abholzigkeit von z. B. $1 \mathrm{~cm}$ pro laufendem Meter direkt aus dem Mittendurchmesser herzuleiten. Stattdessen wurde mit Hilfe eines über dem Bestandesalter funktionalisierten Höhen-Durchmesser-Modells für jeden Bestand eine altersspezifische Höhenkurve geschätzt. Bonitätsunterschiede konnten dabei nicht berücksichtigt werden, da nicht auf Höhenmessungen oder Bonitätsschätzungen zurückgegriffen werden konnte. Anschließend wurden iterativ BHDHöhen-Paare aus den Bestandeshöhen abgegriffen und zur Initialisierung des Schaftformmodells BDATPro (KUBLIN 2003) verwendet. Der BHD wurde dabei ausgehend vom Wert des zugehörigen Mittendurchmessers schrittweise um $0,5 \mathrm{~cm}$ erhöht. Für jedes der resultierenden BHD-Höhen-Paare wurde unter Vorgabe einer Stockhöhe von $30 \mathrm{~cm}$ der Stammdurchmesser auf der Hälfte der Erdstammlänge bestimmt und mit dem Wert aus der Holzaufnahme verglichen. Dem jeweiligen Erdstamm wurde dann der BHD zugeordnet, für den die zugehörige Abweichung zwischen erfasstem und berechnetem Mittendurchmesser minimal ist. 


\section{Methodik}

In Untersuchungen zum Rotkern wird häufig sowohl der Kerntyp angesprochen als auch der maximale Kerndurchmesser erfasst. Bezüglich des Kerntyps werden entweder nur die Ausprägungen 'kein Kern' und '(Rot)kern' unterschieden (KNOKE u. SCHULZ WENDEROTH 2001) oder es erfolgt eine weitere Differenzierung nach Rot- und Spritzkern (SCHMIDT et al. 2005) und sogar Wundkern sowie abnormem Kern (SACHSSE 1991) oder Wolkenkern sowie unregelmäßigem Kern (HÖWECKE 1998).

Bei den in dieser Arbeit verwendeten Daten wurde bei einer vorhandenen Verkernung nicht nach Rot- und Spritzkern unterschieden. Der Kerntyp wurde immer als Rotkern angesprochen. Weiterhin wurde der maximale Kerndurchmesser nicht exakt vermessen, sondern in Form einer ordinalen (rangskalierten) Variablen beschrieben (s. Tab. 1).

Jede der verschiedenen Erfassungsarten erfordert spezifische Modellansätze, um eine adäquate Auswertung der Daten zu gewährleisten. Modelle sind die Voraussetzung für die Überprüfung und Quantifizierung von Variablen-Effekten auf die Verkernung und damit die Grundlage für die Entwicklung eines Rotkerninformationssystems. Liegt eine exakte Vermessung des Kerndurchmessers vor, werden typischerweise 2-stufige Modellansätze verwendet. Dabei werden erst die Auftretenswahrscheinlichkeiten der Kerntypen in Abhängigkeit von Kovariablen und anschließend der Kerndurchmesser als Funktion des Kerntyps und gegebenenfalls weiterer Kovariablen geschätzt. Werden bei der Datenerhebung nur die zwei Ausprägungen 'kein Kern' und '(Rot)kern' angesprochen, können die Auftretenswahrscheinlichkeiten mit Hilfe eines verallgemeinerten linearen (additiven) Modells mit binomial verteilter Zielvariable geschätzt werden (KNOKE 2003). Liegt eine Unterscheidung nach mehr als 2 Kerntypen vor, sollte ein multinomiales Regressionsmodell verwendet werden (SCHMIDT et al. 2005).

Da in der vorliegenden Untersuchung nur die Typen 'kein Kern' und 'Rotkern' unterschieden wurden und der Kerndurchmesser in ordinaler Form erfasst wurde, kann ein 1-stufiger Modellansatz verwendet werden. Dabei werden die Auftretenswahrscheinlichkeiten für die verschiedenen Kerndurchmesserstufen direkt über ein multinomiales Regressionsmodell mit ordinaler Zielvariable geschätzt. Zielvariable ist die Auftretenswahrscheinlichkeit für die stärkere (sortierrelevante) Verkernung am Zopf oder Stammfuß eines Erdstammes. Allerdings werden in der Methodik der hier verwendeten logistischen Regressionsmodelle anstelle der Wahrscheinlichkeiten transformierte Erwartungswerte geschätzt (Formel 1a). Die transformierten Erwartungswerte werden durch den sogenannten linearen Prädiktor $\mathrm{X}_{i} \beta$ mit der Designmatrix $\mathrm{X}_{i}$ und dem Parametervektor $\beta$ bestimmt. Die Transformation erfolgt mit Hilfe der Logit-Funktion g. Die zugehörigen (kumulierten) Wahrscheinlichkeiten lassen sich durch eine Rücktransformation mit Hilfe der Responsefunktion (inverse Logit-Funktion) berechnen (Formel 1b) (FAHRMEIR et al. 1996). 
Die Ebene des linearen Prädiktors ist vor allem für den Bereich der Modellselektion und -interpretation von Bedeutung. Dagegen bilden die geschätzten Auftretenswahrscheinlichkeiten die eigentliche Basis für die Entscheidungsunterstützung der forstlichen Praxis.

Die einfachste Form multinomialer Regressionsmodelle für ordinale Zielvariablen ist das sogenannte 'proportional odds model' (MCCULLAGH 1980; ANDERSON u. PHILIPS 1981), das ein Spezialfall des kumulativen logistischen Regressionsmodells ist. Das Modell unterstellt bezogen auf die Rotkernproblematik für alle Kovariablen jeweils einheitliche und konstante Modelleffekte auf die Wahrscheinlichkeiten der Kerndurchmesserstufen (Formel 1a / 1b). Der Modellansatz unterstellt somit, dass die Verhältnisse der kumulierten Chancen konstant und nicht von den Kovariablen anhängig sind.

$$
\begin{aligned}
& \mathrm{y}_{i r} \sim \text { multinomiale rangskalierte Zielvariable }\left(1, \pi_{i r}\right) \quad \begin{array}{l}
\text { mit } \pi_{i r}=\mathrm{E}\left(\mathrm{y}_{i r}\right) \text { und } \\
\operatorname{cov}\left(\mathrm{y}_{i r} \mathrm{y}_{i s}\right)=\mathrm{y}_{i r}\left(1-\mathrm{y}_{i s}\right) / n_{i}, r \leq s
\end{array} \\
& \mathrm{~g}\left(\mathrm{y}_{i r}\right)=\ln \frac{\sum_{j=1}^{r} \pi_{i j}}{1-\sum_{j=1}^{r} \pi_{i j}}=x_{i}^{\prime} \beta_{(r)}, \text { mit } \mathrm{y}_{i r}=\pi_{i 1}+\ldots+\pi_{i r} \text { und } \pi_{i \mathrm{R}}=1 . \\
& \mathrm{P}\left(y \leq r \mid x_{i}\right)=\frac{\exp \left(\beta_{0 r}-\sum_{k=1}^{p} x_{i k} \beta_{k}\right)}{1+\exp \left(\beta_{0 r}-\sum_{k=1}^{p} x_{i k} \beta_{k}\right)}
\end{aligned}
$$

mit:

: Erwartungswert der transformierten kumulierten Wahrscheinlichkeiten aller Rotkernklassen $\leq r$ für die $i$-te Kovariablenkombination (unabhängige Variablen/Regressoren). Als Link-Funktion wird die logistische Funktion verwendet. Die Rotkernklasse resultiert aus dem jeweils höheren Verkernungsprozent am Zopf oder Stammfuß des Erdstammes.

$\mathrm{P}\left(y \leq r \mid x_{i}\right) \quad: \begin{aligned} & \text { Kumulierte Wahrscheinlichkeit aller Rotkernklassen } \leq r \text { für die } i \text {-te } \\ & \text { Kovariablenkombination }\end{aligned}$

$\mathrm{y}_{\text {ir }} \quad$ : Empirischer Anteil der Rotkernklasse $r$ für die $i$-te Kovariablenkombination, wobei eine multinomiale Verteilung mit $\sim$ multinomial mit geordneten Kategorien $\left(1, \pi_{i r}\right)$ unterstellt wird 


$$
\begin{aligned}
& \pi_{i r}=\mathrm{E}\left(\mathrm{y}_{i r}\right) \quad: \text { Erwartungswert der (bedingten) Wahrscheinlichkeit der Rotkern- } \\
& \text { klasse } r \text { für die } i \text {-te Kovariablenkombination } \\
& \mathrm{x}_{i}^{\prime} \quad: \quad i \text {-ter Vektor von Kovariablen } \\
& r \\
& \text { : } \quad r \text {-te Rotkernklasse der } 1 \text { bis R-1 Rotkernklassen, wobei } R \text { gleich der } \\
& \text { Anzahl Rotkernklassen (hier 6) ist } \\
& \text { P : Anzahl der unabhängigen Variablen } \\
& \beta \quad \text { : Vektor der Regressionskoeffizienten }
\end{aligned}
$$

Anhand von Modellvergleichen mit Hilfe der Prüfstatistik 'Bayesian Information Criterion' (BIC, BURNHAM u. Anderson 2004) wurde deutlich, dass das 'proportional odds modell' eine unzulässige Vereinfachung des zugrunde liegenden Datenmusters darstellt. Der weiterführende Prozess der Modellentwicklung resultierte schließlich in einem verallgemeinerten kumulativen logistischen Regressionsmodell mit teilweise kategorie-spezifischen Regressionskoeffizienten und nicht-linearen Modelleffekten zur Beschreibung des Verkernungsmusters. Die gesamte Datenanalyse und Modellentwicklung wurde mit dem Statistikpaket $R$ ( R DEVELOPMENT CORE TEAM 2007) unter Verwendung der Zusatzbibliothek VGAM (YEE 2005) durchgeführt.

\section{Ergebnisse}

Als Ergebnis der Modellauswahl wurden lineare kategorie-spezifische Effekte für die Variable BHD und zusätzlich nicht-lineare Effekte für die Variablen Bestandesalter und Erdstammlänge spezifiziert. Die übrigen unabhängigen Variablen gehen weiterhin nur über einen linearen kategorie-unspezifischen Effekt in das Modell ein. In der Schreibweise von MCCULLAGH (1980) erhalten alle Regressionskoeffizienten außer dem Interzept ein negatives Vorzeichen, womit sich das resultierende Modell wie folgt beschreiben lässt (Formel 2):

$P\left(y \leq r \mid x_{i}\right)=\frac{\exp \left(\alpha_{0 r}-f_{1}\left(\mathrm{Alter}_{i}\right)-\beta_{2 r} \mathrm{BHD}_{i j}-f_{3} \mathrm{SL}_{i j}-\beta_{4} \mathrm{AG}_{\mathrm{i}}-\beta_{5} \mathrm{WH}_{\mathrm{i}}-\beta_{5} \text { Trophie }_{\mathrm{i}}-\beta_{6} H N N_{i}\right)}{1+\exp \left(\alpha_{0 r}-f_{1}\left(\mathrm{Alter}_{i}\right)-\beta_{2 r} \mathrm{BHD}_{i j}-f_{3} \mathrm{SL}_{i j}-\beta_{4} \mathrm{AG}_{\mathrm{i}}-\beta_{5} \mathrm{WH}_{\mathrm{i}}-\beta_{5} \text { Trophie }_{\mathrm{i}}-\beta_{6} H N N_{i}\right)}$ [2]

mit:

Alter $_{i} \quad$ : Bestandesalter des $i$-ten Bestandes [J]

$\mathrm{BHD}_{i j} \quad$ : Brusthöhendurchmesser des $j$-ten Baumes im $i$-ten Bestand [cm]

$\mathrm{SL}_{i j} \quad$ : Erdstammlänge des $j$-ten Baumes im $i$-ten Bestand $[\mathrm{m}]$

AG $_{i} \quad:$ Geologisches Ausgangsgestein des $i$-ten Bestandes (kategorial)

$\mathrm{WH}_{i} \quad$ : Wasserhaushaltsstufe des $i$-ten Bestandes (kategorial) 
Trophie $_{i} \quad$ : Trophiestufe des $i$-ten Bestandes (kategorial)

$\mathrm{HNN}_{i} \quad$ : Seehöhe des $i$-ten Bestandes [m]

$f_{1}, f_{3} \quad:$ kategorie-unspezifische glättende Spline-Funktionen zur Beschreibung des nicht-linearen Alterseffektes bzw. des nicht-linearen Effektes der Erdstammlänge

$\alpha_{0 r} \quad:$ kategorie-spezifisches Interzept

$\beta_{2 r} \quad:$ kategorie-spezifischer Regressionskoeffizient des BHD

$\beta_{4}, \beta_{5}, \beta_{6}$ : kategorie-unspezifische Regressionskoeffizienten der übrigen Regressoren

Die Ergebnisse werden zuerst für die Ebene des linearen Prädiktors dargestellt, da hier die statistische Beurteilung des Modells erfolgen muss. Allerdings erlaubt auch diese Ebene eine inhaltliche Interpretation. Anschließend werden exemplarisch Ergebnisse auf der Wahrscheinlichkeitsebene dargestellt, da diese die letztlich für die forstliche Praxis notwendigen Informationen bereitstellt. Die Integration der kategorialen Variablen Ausgangsgestein, Wasserhaushalts- und Trophiestufe führte zu signifikanten Verbesserungen der Modellgüte, wobei als Kriterium das BIC verwendet wurde. Neben dem Aspekt einer Verbesserung des Gesamtmodells ist für kategoriale Variablen von besonderem Interesse, wie sich ihre verschiedenen Ausprägungen unterscheiden. Dabei weisen die verschiedenen geologischen Ausgangsgesteine teilweise sehr unterschiedliche Koeffizienten bzw. Effekte auf die Verkernung auf (s. Abb. 3 links). Zur Abschätzung der Signifikanz der Unterschiede zwischen den Effekten sind 2-fache Standardfehler der Koeffizienten dargestellt. Allerdings geben diese Konfidenzintervalle nur einen Anhalt, da sie aufgrund der hierarchischen Datenstruktur (mehrere Beobachtungen aus einem Bestand) bzw. der Verletzung der Unabhängigkeitsannahme tendenziell zu niedrig geschätzt werden. Trotz der Einschränkung, insbesondere die Unterschiede für seltene Ausgangsgesteine vorsichtig zu interpretieren, können viele der Unterschiede als gesichert angesehen werden. Die Effekte sind dabei so zu interpretieren, dass die Verkernungsmuster umso ungünstiger sind, je kleiner der Wert des Effektes ist. Innerhalb der 3 dominierenden Ausgangsgesteine weist Basalt somit ein deutlich ungünstigeres Verkernungsmuster auf als die Buntsandstein- und die Tonschiefer-Grauwacken-Gruppe. Dabei unterscheiden sich die Buntsandsteinund die Tonschiefer-Grauwacken-Gruppe kaum von einander. Von den 4 weiteren Ausgangsgesteinsgruppen, die noch mit mehreren tausend Erdstämmen vertreten sind, weist die Gneis-Granit-Gruppe den ungünstigsten Einfluss auf die Verkernung auf. Diabas ähnelt der Buntsandstein- und der Tonschiefer-GrauwackenGruppe während Löß und Quarzit etwas ungünstiger zu beurteilen sind. Die ungünstigsten Eigenschaften weisen Diorit (768 Datensätze) und Hornfels (235 
Datensätze), die günstigsten weist Zechstein auf (509 Datensätze), wobei die geringen Datenumfänge beachtet werden müssen.
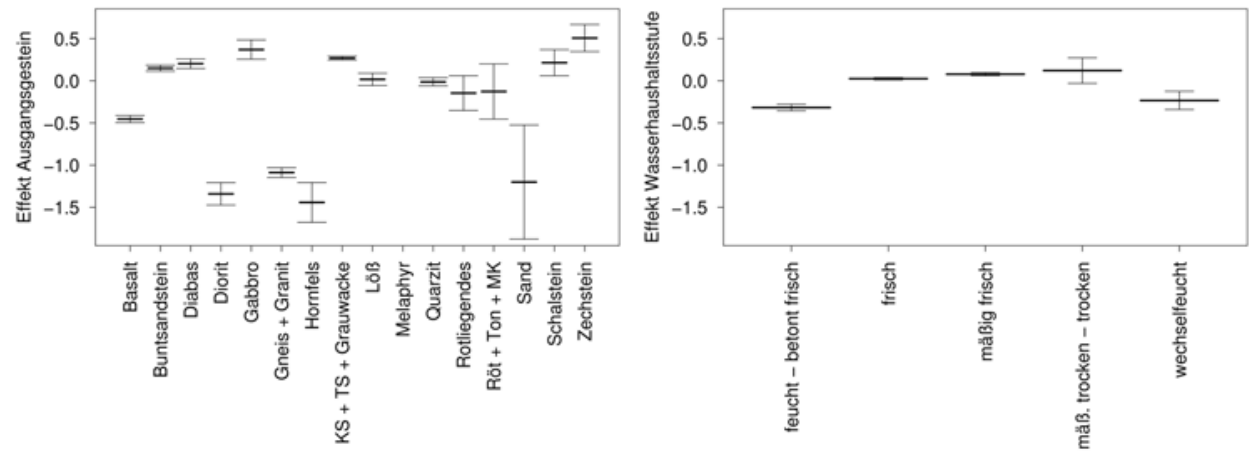

Abbildung 3: Modelleffekte der verschiedenen Ausprägungen der Variablen 'Ausgangsgestein' (links) und 'W asserhaushaltsstufe' (rechts) mit Angabe des zugehörigen 2-fachen Standardfeblers

Die Unterschiede zwischen den Wasserhaushaltsstufen sind insgesamt weniger stark ausgeprägt als zwischen den Ausgangsgesteinen (s. Abb. 3 rechts). Dabei sind die Unterschiede zwischen den Stufen 'frisch', 'mäßig frisch' und 'mäßig trocken trocken' bei einem leicht positiven Trend nur geringfügig. Deutlich ungünstiger wirken sich die Stufen 'feucht - betont frisch' und 'wechselfeucht' aus. Die Differenz der Effekte zwischen den beiden Trophiestufen 'eutroph' und 'meso - oligotroph' ist mit 0,34 noch etwas geringer (s. Tab. 3) als die Differenz zwischen der ungünstigsten und günstigsten Ausprägung der Wasserhaushaltsstufe (die Differenz von 'feucht - betont frisch' zu 'mäßig trocken - trocken' beträgt 0,44).

Tabelle 3: $\quad$ Regressionskoeffizienten der linearen Effekte für die metrischen Variablen BHD und Seeböhe sowie für die 2 Ausprägungen der Trophiestufe

\begin{tabular}{lcc}
\hline & Koeffizient & $\begin{array}{c}\text { 2-facher } \\
\text { Standardfehler }\end{array}$ \\
\hline BHD (Rotkernkategorie 1) & $-0,0785$ & 0,00212 \\
BHD (Rotkernkategorie 2) & $-0,0646$ & 0,00162 \\
BHD (Rotkernkategorie 3) & $-0,0457$ & 0,00153 \\
BHD (Rotkernkategorien 4 und 5) * & $-0,0558$ & 0,00343 \\
HNN (Seehöhe) & $-0,0027$ & 0,00023 \\
Trophiestufe eutroph (Interzeptabweichung) & $-0,2188$ & 0,03143 \\
Trophiestufe meso-oligotroph (Interzeptabweichung) & 0,1227 & 0,01762 \\
\hline
\end{tabular}

* Für die Rotkernkategorien 4 und 5 wurde ein einheitlicher BHD-Effekt spezifiziert, da hier kein signifikanter Unterschied auftritt 
Für die metrischen Variablen BHD und Seehöhe wurden lineare Effekte spezifiziert, wobei für den BHD kategorie-spezifische Koeffizienten geschätzt wurden (s. Tab. 3). Die negativen Vorzeichen der Effekte von BHD und Seehöhe sind dahin zu interpretieren, dass das Verkernungsmuster mit steigendem BHD und steigender Seehöhe ungünstiger wird.

Der nicht-lineare Alterseffekt zeigt ebenfalls eine deutlich abnehmende Tendenz mit einem annähernd linearen Verlauf bis zu einem Alter von ca. 150 und einer anschließend degressiven Abnahme, bis ab ca. 190 Jahren kein Alterseffekt mehr zu beobachten ist (s. Abb. 4 links). Somit beschreibt das Modell bis zu einem Alter von ca. 190 Jahren ein immer ungünstiger werdendes Verkernungsmuster, wobei sich diese Entwicklung ab einem Alter von ca. 150 verlangsamt. Der Effekt der Erdstammlänge weist bis ca. $14 \mathrm{~m}$ eine positive Steigung auf und nimmt ab einem deutlichen Maximum wieder ab (s. Abb. 4 rechts). Oberhalb von $14 \mathrm{~m}$ Stammlänge nimmt die statistische Unsicherheit jedoch aufgrund der immer geringeren Datengrundlage schnell zu. Die Effekte der Erdstammlänge und der Seehöhe auf die Verkernung sind schwächer als die Effekte der übrigen metrischen Variablen Alter und BHD.
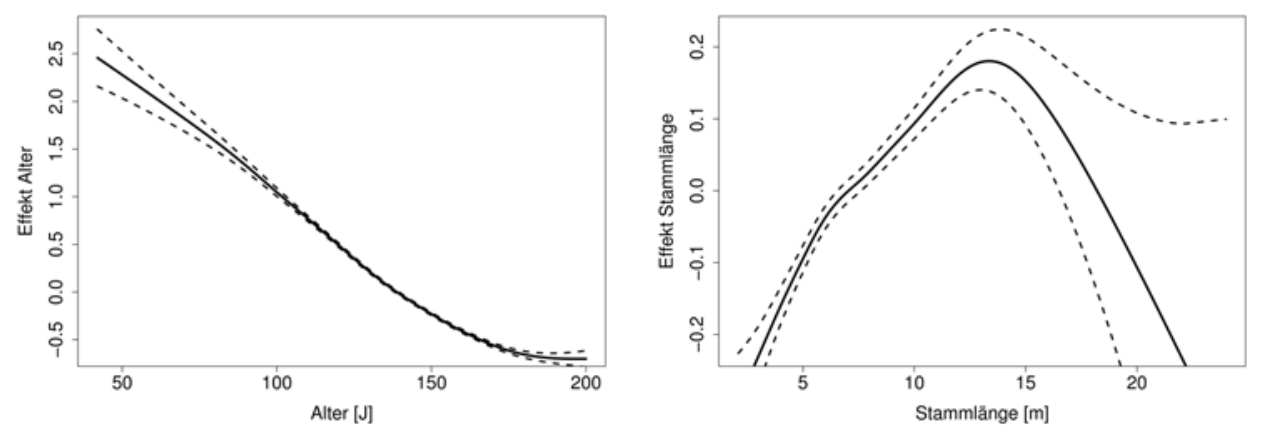

Abbildung 4: Nicht-lineare Modelleffekte für das Alter (links) und die Erdstammlänge (rechts) mit Angabe des 2-fachen punktweisen Standardfehlers (gestrichelte Linien)

Die bisher dargestellten Modelleffekte und zugehörigen Standardfehler dienen sowohl der Beurteilung des Modells aus statistischer Sicht, als auch der Einschätzung, wie sich die verschiedenen Ausprägungen der kategorialen Variablen bzw. Veränderungen der metrischen Variablen auf die Verkernung auswirken. Für die metrischen Variablen mit nicht-linearen Effekten können Bereiche unterschieden werden, die eine größere oder geringere Dynamik der Verkernung aufweisen (s. Abb. 4). Für die kategorialen Variablen können die verschiedenen Ausprägungen miteinander verglichen werden und dabei Ähnlichkeiten und Unterschiede identifiziert werden (s. Abb. 3). Für eine umfassende Modelldarstellung unter spezieller Berücksichtigung der von der forstlichen Praxis benötigten Informationen ist es jedoch notwendig, neben der Ebene des linearen Prädiktors auch die Schätzungen auf der Wahrscheinlichkeitsebene zu betrachten. Im Folgenden sollen daher die 
durch das Modell prognostizierten Wahrscheinlichkeiten für ausgewählte Kombinationen von Kovariablen dargestellt werden (s. Tab 4 / Abb. 5).

Tabelle 4: Kombinationen von unabhängigen Variablen, für die der Vektor der Auftretenswabrscheinlichkeiten der Kerndurchmesserstufen dargestellt wird. Die konstanten Werte der Variablen Erdstammlänge $(7,6 \mathrm{~m})$ und Seehöhe $(370 \mathrm{~m})$ entsprechen den Mittelwerten in der Datenbasis

\begin{tabular}{|c|c|c|c|c|c|c|c|}
\hline & Alter & {$[\mathrm{cm}]$} & $\begin{array}{c}\text { Erd- } \\
\text { stamm- } \\
\text { länge } \\
{[\mathrm{m}]}\end{array}$ & $\begin{array}{l}\text { Geolog. } \\
\text { Aus- } \\
\text { gangs- } \\
\text { gestein }\end{array}$ & $\begin{array}{l}\text { Wasser- } \\
\text { haushalts- } \\
\text { stufe }\end{array}$ & $\begin{array}{l}\text { Trophie- } \\
\text { stufe }\end{array}$ & $\begin{array}{l}\text { See- } \\
\text { höhe }\end{array}$ \\
\hline Kombination 1 & 80 & $20-90$ & 7,6 & Basalt & frisch & $\begin{array}{c}\text { meso - } \\
\text { oligotroph }\end{array}$ & 370 \\
\hline Kombination 2 & 80 & $20-90$ & 7,6 & Basalt & $\begin{array}{l}\text { feucht - } \\
\text { betont frisch }\end{array}$ & $\begin{array}{c}\text { meso - } \\
\text { oligotroph }\end{array}$ & 370 \\
\hline Kombination 3 & 80 & $20-90$ & 7,6 & $\begin{array}{c}\text { Bunt- } \\
\text { sandstein }\end{array}$ & frisch & $\begin{array}{c}\text { meso - } \\
\text { oligotroph }\end{array}$ & 370 \\
\hline Kombination 4 & 80 & $20-90$ & 7,6 & $\begin{array}{l}\text { Bunt- } \\
\text { sandstein }\end{array}$ & $\begin{array}{c}\text { feucht - } \\
\text { betont frisch }\end{array}$ & $\begin{array}{c}\text { meso - } \\
\text { oligotroph }\end{array}$ & 370 \\
\hline Kombination 5 & 140 & $20-90$ & 7,6 & Basalt & frisch & $\begin{array}{c}\text { meso - } \\
\text { oligotroph }\end{array}$ & 370 \\
\hline Kombination 6 & 140 & $20-90$ & 7,6 & Basalt & $\begin{array}{c}\text { feucht - } \\
\text { betont frisch }\end{array}$ & $\begin{array}{c}\text { meso - } \\
\text { oligotroph }\end{array}$ & 370 \\
\hline Kombination 7 & 140 & $20-90$ & 7,6 & $\begin{array}{l}\text { Bunt- } \\
\text { sandstein }\end{array}$ & frisch & $\begin{array}{c}\text { meso - } \\
\text { oligotroph }\end{array}$ & 370 \\
\hline Kombination 8 & 140 & $20-90$ & 7,6 & $\begin{array}{l}\text { Bunt- } \\
\text { sandstein }\end{array}$ & $\begin{array}{l}\text { feucht - } \\
\text { betont frisch }\end{array}$ & $\begin{array}{c}\text { meso - } \\
\text { oligotroph }\end{array}$ & 370 \\
\hline
\end{tabular}

Folgende Seite:

Abbildung 5: Über das kumulierte logistische Regressionsmodell (Formel 2) geschätz̨te Vektoren der Auftretenswabrscheinlichkeiten der Kerndurchmesserstufen für unterschiedliche in Tab. 4 definierte Kombinationen von Kovariablen. Die gestrichelte Linie markiert eine Zielstärke von $65 \mathrm{~cm} B H D$ bzw. den zugebörigen Wabrscheinlichkeitsvektor. 


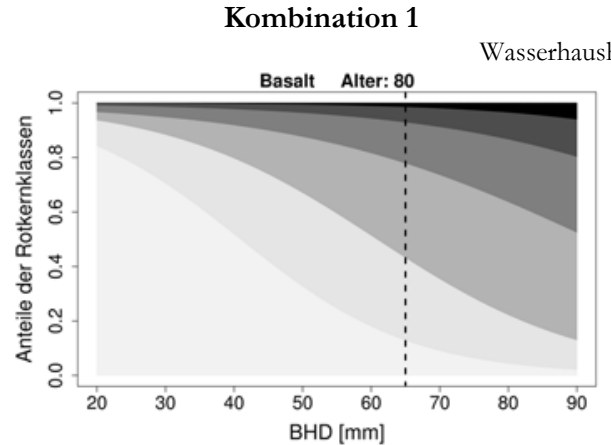

Kombination 2

\section{Kombination 3}

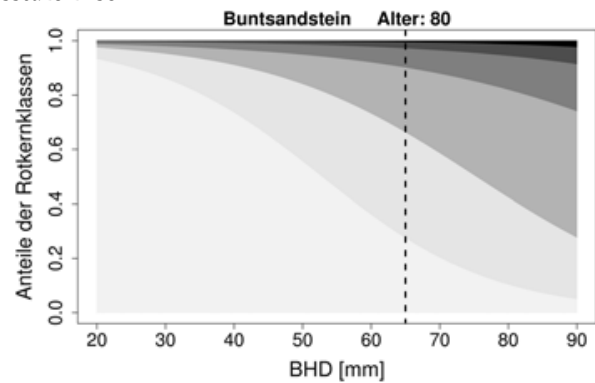

Kombination 4

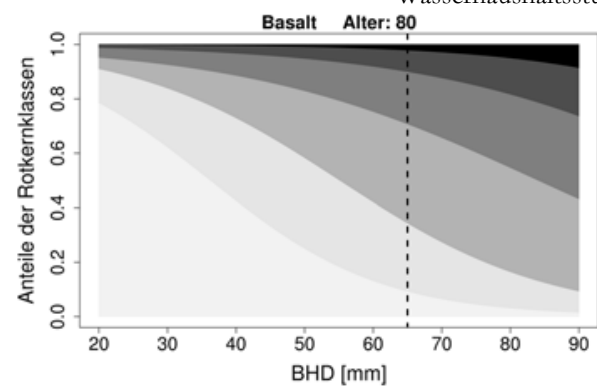

Kombination 5

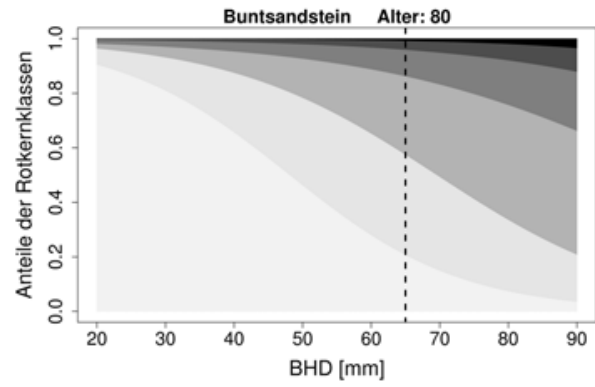

Kombination 7

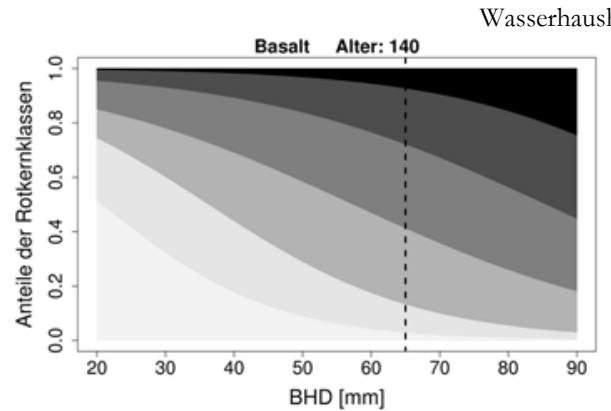

Kombination 6

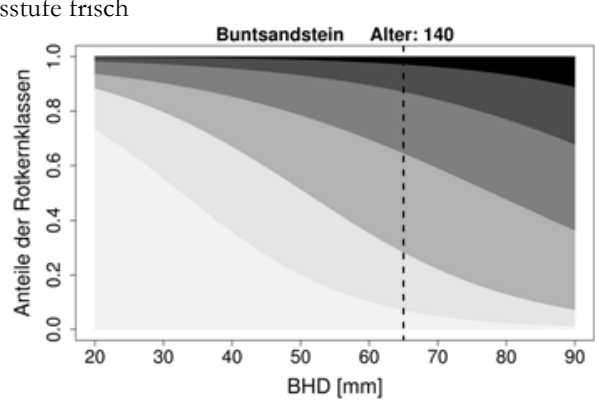

Kombination 8
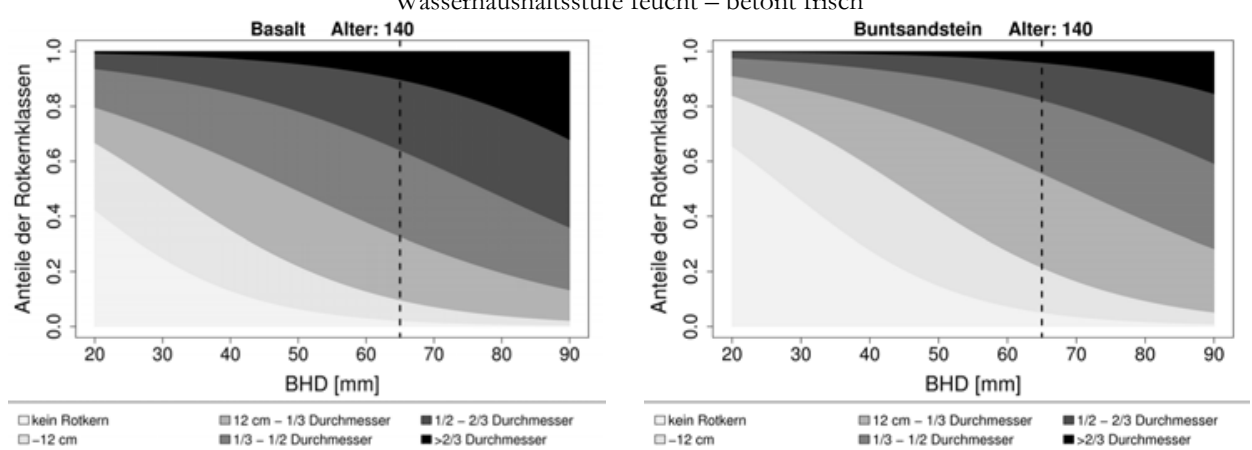

Beiträge aus der NW-FVA, Band 3, 2008 
Der Vektor der Wahrscheinlichkeiten für die verschiedenen Rotkernstufen lässt sich, wie hier exemplarisch dargestellt, für beliebige Kombinationen der unabhängigen Variablen schätzen (s. Abb. 5). Die bereits auf der Ebene des linearen Prädiktors beschriebenen Unterschiede werden auch auf der Wahrscheinlichkeitsebene deutlich. So weist Basalt bei sonst gleichen Bedingungen ein deutlich ungünstigeres Verkernungsmuster als Buntsandstein auf (jeweils zeilenweiser Vergleich in Abb. 5). Die Wasserhaushaltsstufe 'frisch' ist günstiger als die Stufe 'feucht betont frisch' zu beurteilen (Vergleiche Kombination 1 versus 2, Kombination 3 versus 4, Kombination 5 versus 6, Kombination 7 versus 8). Auch der negative Effekt des Bestandesalters (Vergleiche Kombination 1 versus 5, Kombination 2 versus 6, Kombination 3 versus 7, Kombination 4 versus 8) und des BHDs (jeweils innerhalb einer Graphik) sind deutlich zu erkennen.

Die Unterschiede der Kombinationen in der Verkernung sind für Bäume im Bereich der Zielstärke besonders interessant und beispielsweise bedeutungsvoller als für unterständige Bäume schwächerer Dimension. Daher werden die Wahrscheinlichkeiten für die Kernausprägung für eine potenzielle Zielstärke von $65 \mathrm{~cm}$ BHD noch einmal gesondert betrachtet. Die Wahrscheinlichkeiten können auch als Schätzung des Stammzahlprozents interpretiert werden, das bei einer bestimmten Kombination von unabhängigen Variablen einer bestimmten Verkernungsstufe zuzurechnen ist (s. Tab. 5). Graphisch ist der zugehörige Wahrscheinlichkeitsvektor in Abbildung 5 durch eine gestrichelte Linie markiert. Dabei setzt das Erreichen einer Zielstärke von $65 \mathrm{~cm}$ innerhalb von 80 Jahren optimale Wuchsbedingungen für den Einzelbaum voraus. Dagegen kann das Erreichen dieser Zielstärke in einem Alter von 140 Jahren eher als typisch bei der Buche betrachtet werden.

Vergleicht man exemplarisch zu beiden Altern die ungünstigste und günstigste Kombination (Kombination 2 versus 3 und Kombination 6 versus 7) so fallen die teilweise beträchtlichen Unterschiede auf (s. Tab. 5). Die Kombination 3 übertrifft die Kombination 2 in der Rotkernkategorie 0 um ca. $15 \%$ und in der Kategorie 1 um ca. $8 \%$. Die Kombination 2 weist ab der Kategorie 2 höhere Werte auf, wobei die beiden ungünstigsten Kategorien im Alter 80 in beiden Kombinationen mit zusammen nur ca. 7 bzw. $3 \%$ vertreten sind. In den mittleren Kategorien 2 und 3 weist die Kombination 2 ca. 11 und $8 \%$ mehr auf als die Kombination 3.

Im Alter 140 übertrifft die günstigste Kombination 7 die ungünstigste Kombination 6 in den Kategorien 0,1 und 2 um ca. 4, 11 und 8\%. Ab der Kategorie 3 weist dann die Kombination 6 höhere Prozente auf. 
Tabelle 5: $\quad$ Stammzahlprozente, die auf die verschiedenen Rotkernausprägungen entfallen, für einen Baum mit $65 \mathrm{~cm}$ BHD und zusätzlichen Kovariablenkombinationen, wie sie in Tabelle 4 definiert sind

\begin{tabular}{ccccccc}
\hline Kombination & \multicolumn{7}{c}{ Rotkernkategorie } \\
& 0 & 1 & 2 & 3 & 4 & 5 \\
& $\begin{array}{c}\text { kein } \\
\text { Rotkern }\end{array}$ & $\begin{array}{c}\text { bis } 12 \mathrm{~cm} \\
\text { des } \varnothing\end{array}$ & $\begin{array}{c}12 \mathrm{~cm}- \\
1 / 3 \text { des } \varnothing\end{array}$ & $\begin{array}{c}1 / 3-1 / 2 \\
\text { des } \varnothing\end{array}$ & $\begin{array}{c}1 / 2-2 / 3 \\
\text { des } \varnothing\end{array}$ & $\begin{array}{c}>2 / 3 \\
\text { des } \varnothing\end{array}$ \\
\hline $\mathbf{1}$ & 17,2 & 34,6 & 31,4 & 11,6 & 4,1 & 1,1 \\
$\mathbf{2}$ & 12,8 & 30,4 & 34,5 & 15 & 5,6 & 1,6 \\
$\mathbf{3}$ & 27,5 & 38,7 & 23,8 & 7,1 & 2,3 & 0,6 \\
$\mathbf{4}$ & 21,2 & 37 & 28,3 & 9,4 & 3,2 & 0,9 \\
\hline $\mathbf{5}$ & 4 & 13,7 & 32 & 28,8 & 16,2 & 5,4 \\
$\mathbf{6}$ & 2,9 & 10,3 & 27,9 & 30,9 & 20,5 & 7,4 \\
$\mathbf{7}$ & 7 & 21,1 & 36,1 & 22,6 & 10,1 & 3 \\
$\mathbf{8}$ & 5,1 & 16,7 & 34,4 & 26,4 & 13,3 & 4,2 \\
\hline
\end{tabular}

\section{Diskussion}

Im Folgenden soll ein Vergleich mit anderen Untersuchungen bezüglich der selektierten Einflussgrößen und der zugehörigen Effekte erfolgen und der vorgestellte Ansatz abschließend bewertet werden. Dabei muss noch einmal darauf hingewiesen werden, dass sich die verschiedenen Arbeiten sowohl methodisch als auch bezüglich der Datenumfänge deutlich unterscheiden. So sind die 2-stufigen Ansätze, in denen erst das Auftreten der Verkernung und anschließend der Kerndurchmesser geschätzt wird, nur eingeschränkt mit dem hier vorgestellten 1-stufigen Ansatz vergleichbar. Deshalb muss der Modellvergleich auf die Variablenselektion und eine qualitative Beurteilung ihrer Effekte beschränkt bleiben. Bei den 2-stufigen Ansätzen werden die Kovariableneffekte beider Modellebenen für den Vergleich mit der vorliegenden Arbeit herangezogen. Ein weiterer Unterschied besteht darin, dass in den meisten Arbeiten alle Schnittflächen eines Stammes (in der Regel 2) in die Untersuchung mit einbezogen werden. Die Verkernungen am Fuß- und Zopfende werden dabei unzulässigerweise fast immer als 2 unabhängige Beobachtungen betrachtet und nur in Ausnahmefällen wurde die Korrelation zwischen Beobachtungen an ein und demselben Stamm erfasst (SCHMIDT et al. 2005). In der vorliegenden Arbeit wird nur die jeweils stärkere (sortierrelevante) Verkernung eines Stammes berücksichtigt, so dass an dieser Stelle keine Verletzung 
der Unabhängigkeitsannahme auftritt. Daher ist der Effekt der Erdstammlänge (s. Abb. 4 rechts) aber nur indirekt mit den Aussagen anderer Autoren zum Einfluss der Stammhöhe auf die Verkernung zu vergleichen. Die Abhängigkeit von der absoluten oder relativen Höhe am Stamm wird fast ausnahmslos beobachtet. Sowohl bei KNOKE und SCHUlZ WeNDEROTH (2001; 195 Stämme), BÖRNER (2002; 146 Stämme) als auch KREMPL u. MARK (1962; 187 Stämme) werden die identifizierten Verläufe eher der häufig beschriebenen (asymmetrischen) Spindelform zugeordnet (SEELING u. SACHSSE 1992). Die auf einer sehr viel breiteren Datengrundlage basierende Untersuchung von RACZ et al. (1961; 41291 Stämme) in Niedersachsen weist als weitaus häufigste Kernmuster den Spindel- und den Kegel-Typ zu etwa gleichen Anteilen auf. HÖWECKE (1998: 3961 Stämme) beschreibt den Effekt der Stammhöhe nur bezüglich verschiedener Kerntypenanteile nicht aber bezüglich des Kerndurchmesseranteils. Aber auch er beobachtet einen deutlich geringeren Anteil von Erdstämmen, die am Stammfuß verkernt sind. Die höchsten Anteile verkernter Schnittflächen treten bei ca. $5 \mathrm{~m}$ Stammhöhe auf, danach nehmen die Anteile wieder langsam ab. Als methodisch suboptimal muss in denjenigen dieser Arbeiten, die Regressionsmodelle entwickeln, beurteilt werden, dass der Verlauf durch relativ starre Modellterme beschrieben wird. In der vorliegenden Arbeit wird durch die Verwendung eines glättenden Splineterms das eigentliche empirische Muster stärker berücksichtigt (s. Abb. 4 rechts). Der hier identifizierte Verlauf lässt sich gut mit der asymmetrischen Spindelform in Einklang bringen. Bei Vorliegen der Kegelform würde die stärkere Verkernung immer am Stammfuß und damit in Stockhöhe auftreten und wäre somit unabhängig von der Stammlänge. Bei Vorliegen der Spindelform jedoch, die nach den Erkenntnissen von RACZ et al. (1961) im Mittel ein Maximum bei ca. $4 \mathrm{~m}$ aufweist, würde die stärkere Verkernung über einen weiten Bereich von Stammlängen am Zopf auftreten und bei kurzen Stammlängen um $4 \mathrm{~m}$ die höchsten Werte aufweisen. Mit zunehmender Stammlänge erfolgt der Zopfschnitt im sich langsam verjüngenden oberen Spindelteil. Damit würde die Verkernung um so mehr abnehmen, je weiter man sich vom Maximum nach oben entfernt. Die stärkere Verkernung würde aber immer noch am Zopfende auftreten. Bei 13-15 m Stammlänge schwächt sich der Trend deutlich ab (s. Abb. 4 rechts), bevor bei noch größeren Längen keine statistisch abgesicherten Aussagen mehr möglich sind. Der schwache Effekt der Stammlänge auf die Verkernung im Bereich von 13-15 m könnte so interpretiert werden, dass sich der obere Teil der Kernspindel so stark verjüngt hat, dass die stärkere Verkernung jetzt überwiegend am Stammfuß auf Stockhöhe auftritt und damit unabhängig von der Stammlänge ist.

Einschränkend wird noch einmal darauf verwiesen, dass in den meisten Arbeiten eine Korrelation zwischen der Kronenansatzhöhe und der ausgehaltenen Stammlänge auftritt und die ermittelten Effekte somit durch weitere Zusammenhänge überlagert sein dürften. Die Korrelation tritt auf, da überwiegend auf Daten aus regulären Nutzungen zurückgegriffen wird, in denen die Aushaltung durch äußere Holzmerkmale bestimmt wird. Abweichend wurde in der Untersuchung 
von BÖRNER (2002) einheitlich bei $7 \mathrm{~m}$ und in der Untersuchung von KREMPL u. MARK (1962) teilweise auch nach versuchstechnischen Gesichtspunkten abgelängt.

In allen Rotkernuntersuchungen finden sich Aussagen zum Einfluss des Bestandesalters und des BHD. Allerdings konnten BÖRNER (2002) und SCHMIDT et al. (2005: 1252 Stämme) keinen bzw. keinen biologisch plausiblen Alterseffekt zusätzlich zum BHD-Effekt identifizieren. SCHMIDT u. HEIN (2005: 1728 Stämme) konnten einen biologisch plausiblen Alterseffekt nur über zusätzliche Modellbeschränkungen integrieren. Wie zu erwarten, führt in diesen Untersuchungen ein steigender BHD zu einer Zunahme der Verkernung. KREMPL u. MARK (1962) betrachten den BHD-Effekt nicht gesondert, stellen aber einen Alterseffekt fest, der nach einem sprunghaften Anstieg im Bereich von 100-120 Jahren ein anschließend langsameres Fortschreiten der Verkernung beschreibt. RACZ et al. (1961) betrachten den BHD und das Alter und stellen dabei einen deutlich stärkeren BHD- als Alterseffekt fest. KNOKE u. SCHULZ WENDEROTH (2001) beschreiben einen BHD-Effekt und über den durchschnittlichen Durchmesserzuwachs indirekt auch einen Alterseffekt. KNOKE (2003) verwendet das Alter und den durchschnittlichen Durchmesserzuwachs eines Baumes als unabhängige Variablen. In beiden Untersuchungen führt ein höheres Alter bzw. ein höherer BHD zu einer stärkeren Verkernung. Dabei hängt es von der jeweiligen Modellformulierung ab, ob der Alters- oder der BHD-Effekt einen degressiven Trend aufweist. Auch BÜREN (1998: 2402 Stämme) stellt eine steigende Kernwahrscheinlichkeit mit steigendem Alter und BHD fest. Allerdings werden beide Effekte getrennt betrachtet und es werden keine Aussagen bezüglich ihrer Einflussrelation gemacht. Auch HÖWECKE (1998) betrachtet das Alter und den BHD getrennt. Der BHDEffekt ist in Übereinstimmung mit der hier dargestellten Untersuchung stärker, wobei der Alterstrend nur undeutlich ausgeprägt ist. In einigen der Untersuchungen werden Einzelbaumalter verwendet. Abweichend wird in Untersuchungen, die auf regulären Einschlagsdaten basieren, die nicht bei jeder einzelnen Aufnahme direkt wissenschaftlich begleitet wurden, das Bestandesalter verwendet. Das betrifft die Untersuchung von SCHMIDT u. HEIN (2005), SCHMIDT et al. (2005), HÖWECKE (1998), RACZ et al. (1961) und die hier dargestellte Untersuchung.

In der vorliegenden Untersuchung weisen sowohl der BHD als auch das Alter einen deutlichen Modelleffekt auf. Insofern besteht eine grundlegende Übereinstimmung mit den übrigen Untersuchungen. Die Verwendung von Splinetermen macht es allerdings möglich, objektiver zu erfassen, welche Dynamik die Verkernung in Abhängigkeit vom BHD und Alter aufweist. Danach konnte für den BHD ein linearer Trend (s. Tab. 3) und für das Alter ein erst linearer und später degressiv nachlassender Trend identifiziert werden (s. Abb. 4 links). Allerdings treten durch die spezifische Formulierung des Modells mehrere kategorie-spezifische BHDEffekte auf (s. Formel 2 und Tab. 3). In der Zusammenschau aller Untersuchungen muss unter Berücksichtigung der extrem unterschiedlichen Datenumfänge sowie methodischen Unterschiede davon ausgegangen werden, dass der BHD 
einen stärkeren Einfluss auf die Verkernung hat als das Alter und dass der Alterseffekt im hohen Alter ab ca. 150 Jahren immer stärker nachlässt. Nichtsdestotrotz ist der Alterseffekt sehr deutlich. Ein durch die waldbauliche Behandlung gefördertes Dickenwachstum bzw. ein schnelleres Erreichen der Zielstärke senkt damit das Entwertungsrisiko durch die Rotkernbildung.

Für die Quantifizierung der Effekte von Bestandesvariablen, d. h. Variablen, die für alle Bäume eines Bestandes den gleichen Wert aufweisen, sind große Datenmengen zwingend notwendig. Die hierarchischen Datenstrukturen mit mehreren Beobachtungen aus demselben Bestand verstärken diese Notwendigkeit noch. Modellansätze zur Rotkernproblematik mit geringer Datenbasis sind deshalb häufig nicht standortssensitiv. Entweder ist die Datengrundlage bei gleichzeitig hoher Variabilität der Verkernung so begrenzt, dass keine signifikanten Effekte auftreten (BÖRNER 2002). Nicht signifikante Zusammenhänge können auch in einer geringen Standortsvariabilität der Datenbasis begründet sein (REDDE 1998). Falls eine Einbeziehung dennoch direkt über Standortsvariablen (KNOKE u. SCHULZ WeNDEROTH 2001) oder indirekt über die Bonität (KREMPL u. MARK 1962) erfolgt, ist dies kritisch zu beurteilen. Die daraus gewonnenen Ergebnisse sollten auf keinen Fall verallgemeinert werden sondern sind als exemplarische Auswertungen zu betrachten. Ungeachtet dessen stimmen die Ergebnisse von KNOKE u. SCHULZ WENDEROTH (2001) aber tendenziell mit den Ergebnissen der vorliegenden Untersuchung überein. So weisen in ihrer Arbeit die Bäume, die auf eher trockeneren und ärmeren, sandigen Standorten stocken, bei sonst gleichen Eigenschaften im Schnitt eine geringere Verkernung auf.

Eine Zwischenstellung nehmen die Arbeiten von SCHMIDT u. HeIN (2005), BÜREN (1998) und Höwecke (1998) ein, die bei deutlich größeren Datenumfängen bereits besser abgesicherte Aussagen bezüglich ausgewählter Standortgruppen oder Ökoserien zulassen. Sollen die Effekte einer Vielzahl von geologischen Ausgangsgesteinen, Wasserhaushalts- und Trophiestufen quantifiziert werden, so sollten pro Ausprägungskombination mindestens tausend Stämme aus möglichst vielen verschiedenen Beständen vorliegen, da die Effekte sonst nicht mit einer ausreichenden Genauigkeit geschätzt werden können (s. Abb. 3). Trotz der umfangreichen Datenbasis der Untersuchung von RACZ et al. (1961), werden in dieser Arbeit nur wenige Zusammenhänge zu Standortsvariablen identifiziert. Wichtigste Erkenntnis, die auch in einer neueren Untersuchung zumindest für die Relation zum Buntsandstein bestätigt wird (SCHMIDT u. HEIN 2005), ist eine stärkere Verkernung auf Muschelkalk- als auf Buntsandstein- und Geschiebelehmstandorten. Muschelkalk kommt in Hessen nur selten vor und spielt daher in der vorliegenden Untersuchung keine Rolle. BÜREN (1998) betrachtet nicht direkt das geologische Ausgangsgestein, sondern stärker differenziert den Bodentyp, die Humusform, die pflanzensoziologische Gesellschaft und die Wuchsregion. Gewisse Übereinstimmungen mit der vorliegenden und anderen Untersuchungen treten dennoch auch hier auf. So wird die stark erhöhte Wahrscheinlichkeit für das Auftreten von 
Rotkern auf Karbonat-Gesteinsböden bestätigt, wobei es sich hierbei überwiegend um Böden auf Jurakalken handelt. Abgesehen vom negativen Einfluss der Kalkböden treten in der Untersuchung von BÜREN (1998) höhere Verkernungsprozente eher auf schwächeren Standorten auf. Damit würde die hier beobachtete Relation von Granit zu Basalt, nicht aber die von Basalt zu Grauwacke bestätigt (s. Abb. 3 links). Übereinstimmend tritt ein negativer Effekt der Seehöhe auf. Letztlich aber erscheint die Datenbasis von BÜREN (1998) für die Vielzahl gleichzeitig abgeleiteter und untereinander korrelierter Zusammenhänge insgesamt zu gering.

In der Untersuchung von HÖWECKE (1998) wird vor allem für Buntsandstein ein gegenüber anderen Bodentypengruppen geringeres Verkernungsrisiko beobachtet.

Die in dieser Arbeit verwendete sehr umfangreiche Datenbasis erfasst eine große Bandbreite von Ausgangsgesteinen mit einer ausreichenden bis hohen Repräsentativität und übertrifft diesbezüglich alle übrigen überwiegend exemplarischen Studien bei weitem. Das auf dieser Basis entwickelte Modell ist den meisten der genannten Ansätze auch methodisch überlegen. Damit wird erstmals eine geeignete Grundlage für die Entwicklung eines überregional gültigen Entscheidungsunterstützungssystems für die Rotkernproblematik bereitgestellt. Prinzipiell sind zukünftig weitere Modellverbesserungen wie die Modellierung der Korrelation zwischen der Verkernung am Zopf und Stammfuß und damit die Verwendung beider Schnittflächen denkbar. Auch eine Quantifizierung zufälliger bestandesspezifischer Abweichungen vom hier vorgestellten Modell sowie der zeitlichen Korrelation innerhalb von Beständen ist denkbar. Letztlich ist der Ansatz in der jetzigen Form aber bereits voll einsetzbar. Die relativ einfache Datenstruktur würde auch eine weitere Ergänzung der Datenbasis im Rahmen von regulären Nutzungen insbesondere für bisher unzureichend belegte Variablenkombinationen und unzureichend berücksichtigte geographische Gebiete zulassen. Auch ließen sich relativ umfangreiche Datenquellen mit höherer Informationsdichte, wie sie für südniedersächsische Buntsandstein- vor allem aber Röt- und Muschelkalkstandorte vorliegen, umkodieren und bei der Parametrisierung des Modells berücksichtigen.

Aus dem deutlichen Einfluss der verschiedenen Standortsvariablen auf die Verkernung resultiert, dass eine einheitliche Zielstärke das Ertragspotential der Baumart Buche nur unzureichend ausschöpft und Zielstärken standortsabhängig definiert werden müssen. Durch die Abhängigkeit von der veränderlichen Einflussgröße Alter variiert die optimale Zielstärke in ein und demselben Bestand zusätzlich mit dem Alter. Schlussendlich variiert die optimale Zielstärke sogar zu einem Zeitpunkt innerhalb eines Bestandes in Abhängigkeit vom BHD. Die optimale Zielstärke ist bei einer Erntemaßnahme somit für jeden Baum individuell zu bestimmen. Ein auf dem eigentlichen Rotkernmodell, einem Durchmesserzuwachs- und einem Schaftformmodell basierendes Entscheidungsunterstützungssystem könnte vor jeder Erntemaßnahme mit den notwendigen Informationen ini- 
tialisiert werden. Neben den unabhängigen Variablen des Rotkernmodells müssten Preise für die verschiedenen kernabhängigen Güteklassen hinterlegt sein. Bis auf den Einzelbaumdurchmesser ließen sich alle Variablen einschließlich einer mittleren Erdstammlänge vor der Erntemaßnahme bereits ermitteln oder einschätzen. Ausgehend von diesen Informationen würde für einen beliebigen Durchmesserbereich, der optimale durchmesserspezifische Erntezeitpunkt berechnet und auf eine elektronische Kluppe übertragen. Durch die BHD-Messung im Bestand würde die letzte fehlende Einflussgröße ermittelt und das optimale Erntealter für den Einzelbaum bestimmt. Selbstverständlich müsste in die Entscheidungsfindung noch die äußere Holzqualität gutachtlich miteinfließen. So macht es keinen Sinn, Bäume, die bereits aufgrund äußerer Holzmerkmale in die Güteklasse C fallen, vor einem Absinken in die Güteklasse $\mathrm{C}$ aufgrund von Verkernung zu nutzen.

Neben der skizzierten Herleitung von einzelbaumspezifischen Zielstärken und Erntezeitpunkten kann das vorgestellte Modell auch zur Erhöhung der Aussagefähigkeit von waldbaulichen Szenariosimulationen eingesetzt werden. Waldbauliche Szenariosimulationen mit Hilfe von Wachstumssimulatoren dienen der Herleitung optimaler waldbaulicher Eingriffsfolgen. Um unterschiedliche Szenarien adäquat miteinander vergleichen zu können, müssen Werterträge ermittelt werden. Diese werden üblicherweise aus Stärkeklassenverteilungen abgeleitet. Für die Buche könnte die Herleitung der Werterträge zukünftig unter Einbeziehung des Verkernungsrisikos erfolgen und damit zu realistischeren Ergebnissen und verbesserten Empfehlungen für die forstliche Praxis führen.

\section{Literatur}

Anderson, J. u. P. Philips (1981): Regression, discrimination and measurement models for ordered categorical variables. Applied Statistics 30: 22-31

BÖRNER, M. (2002): Zieldurchmesser und Rotkern bei der Buche. Forst und Holz, 57 (5): 123-128

BUNDESMINISTERIUM FÜr VERBRAUCHERSCHUTZ, ERNÄHRUNG UND LANDWIRTSCHAFT (BMVEL) (2004): Die zweite Bundeswaldinventur - BWI ${ }^{2}$. Das Wichtigste in Kürze. Referat Öffentlichkeitsarbeit (Berlin): $87 \mathrm{~S}$.

BÜREN, S. (1998): Buchenrotkern: Erkennung, Verbreitung und wirtschaftliche Bedeutung. Schweiz. Z. Forstwes. 149 (12): 955-970

Büren, S. (2002): Der Farbkern der Buche (Fagus sylvatica L.) in der Schweiz nördlich der Alpen. Beiheft zur Schweizerischen Zeitschrift für Forstwesen Nr. 86 (Dissertation, Professur für Holzwissenschaften, ETH Zürich): $137 \mathrm{~S}$.

Burnham, K. P. u. Anderson, D. R. (2004): Multimodel Inference: Understanding AIC and BIC in Model Selection. Sociological Methods and Research, Vol. 33: 261-304

DIN EN (1997): Laub-Rundholz-Qualitäts-Sortierung - Teil 1: Eiche und Buche; DIN EN 13161:1997; Beuth Verlag, Berlin

Fahrmeir, L.; Hamerle, A. u. Tutz G. (1996): Multivariate statistische Verfahren. 2. überarbeit. Auflage, Walter de Gruyter, Berlin, New York: $902 \mathrm{~S}$.

Hapla, F. u. STEINFATT, H.-C. (2001): Kritische Analyse der EN 1316-1 bei Sortierung von BuchenStarkholz im Vergleich zur HKS. Allg. Forst- u. J.-Ztg., 173 (5): 86- 94 
HKS (1969): Gesetz über gesetzliche Handelsklassen für Rohholz vom 25.02.1968, Verordnung über gesetzliche Handelsklassen für Rohholz mit Anlage zu \1 vom 31.07.1969. Die Rundholzsortierung in Deutschland mit Zusatzbestimmungen der Bundesländer.

HÖWECKE, B. (1998): Untersuchungen zum Farbkern der Buche (Fagus sylvatica L.) in Baden-Württemberg. Schweiz. Z. Forstwes. 149 (12): 971-990

KNOKE, TH. (2003): Predicting red heartwood formation in beech trees (Fagus sylvatica L.). Ecological Modelling, 169 (2-3): 295-312

Knoke, Th. u. Schulz Wenderoth, S. (2001): Ein Ansatz zur Beschreibung von Wahrscheinlichkeit und Ausmaß der Farbkernbildung bei Buche (Fagus sylvatica L.) Forstw. Cbl. 120: 154-172

Kramer, H. (1988): Waldwachstumskunde. Verlag Paul Parey, Hamburg und Berlin: 374 S.

Krempl, H. u. MARK, E. (1962): Untersuchungen über den Kern der Rotbuche. Allgemeine Forstzeitung (Wien), 73: 186-191

Kublin, E. (2003): Einheitliche Beschreibung der Schaftform - Methoden und Programme BDATpro. Forstw. Cbl. 122, 183-200

McCullagh, P. (1980): Regression models for ordinal data (with discussion). J. R. Statist. Soc. B, 42: 109-142

NeCESANY, V. (1958): Die Vitalitätsveränderung parenchymatischer Zellen und die physiologische Grundlage der Kernbildung bei der Buche. Drevarsky Vyskum 3 (1): 13-26

RaCZ, J.; SCHUlZ, H. u. KNIGGE, W. (1961): Untersuchungen über das Auftreten des Buchenrotkerns. Der Forst- und Holzwirt 16 (19): 413-417

R Development Core TeAm (2007): R: A language and environment for statistical computing. R Foundation for Statistical Computing, Vienna, Austria. ISBN 3-900051-07-0, URL http://www.R-project.org.

REDDE, N. (1998): Fakultative Farbkernbildung an wertholzhaltigen Starkbuchen. Diplomarbeit, Fakultät für Forstwiss. u. Waldökologie, Universität Göttingen: 104 S.

SACHSSE, H. (1991): Kerntypen der Rotbuche. Forstarchiv 62: 238-242

SCHMidT, M.; GADOW, K. V. u. HÖFLE, H., (2005): Vorkommen und Ausprägung von fakultativen Kerntypen bei Rotbuche auf südniedersächsischen Kalk- und Rötstandorten. AFJZ 176 (11/12): 200-212

SCHMidT, M. u. HeIN, S. (2005): Modelling different core types in European beech [Fagus sylvatica L.]. Proceedings of the 5th Workshop on Wood Quality Modelling, IUFRO Working Party 5.01.04 (im Druck)

SEELING, U. (1991): Abnorme Kernbildung bei Rotbuche und ihr Einfluss auf holzbiologische und holztechnologische Kenngrößen. Dissertation, Forstwissenschaftlicher Fachber., Univ. Göttingen: 167 S.

SEELING, U. u. SACHSSE, H. (1992): Abnorme Kernbildung bei Rotbuche und ihr Einfluss auf biologische und holztechnologische Kenngrößen. Forst und Holz 47 (8): 210-217

Wagemann, M. (2001): Vermarktungsoffensive Rotkernbuche. AFZ/Der Wald (26): 1406-1407

YEE, T. W. (2005): VGAM: Vector Generalized Linear and Additive Models. R package version 0.63. http://www.stat.auckland.ac.nz/ yee/VGAM/

ZMP (2007): Zentrale Markt- und Preisberichtstelle GmbH Bonn. Marktbilanz Forst und Holz 2007

ZYCHA, H. (1948): Über die Kernbildung und verwandte Vorgänge im Holz der Rotbuche. Forstw. Cbl. (67): 80-109 
Korrespondierender Autor:

Dr. Matthias Schmidt

Nordwestdeutsche Forstliche Versuchsanstalt

Grätzelstr. 2

37079 Göttingen

E-Mail: Matthias.Schmidt@nw-fva.de

URL: www.nw-fva.de

Stefan Nowack

Landesbetriebsleitung Hessen-Forst

Rüdiger Riebeling

ehemals: Hessische Landesanstalt für Forsteinrichtung, Waldforschung u. Waldökologie (HLFWW) 


\section{Das mittelfristige Buchenholzaufkommen in Niedersachsen und Deutschland}

\section{Medium-term predictions of beech timber stocks in Lower Saxony and Germany}

Jan Hansen, Jürgen Nagel, Matthias Schmidt und Hermann Spellmann

\section{Zusammenfassung}

Vor dem Hintergrund der steigenden Holznachfrage werden zur Entwicklung von wettbewerbsfähigen, nachhaltigen waldbaulichen Nutzungsstrategien zuverlässige Informationen über das künftige Holzaufkommen der in Deutschland wichtigsten Laubbaumart Buche benötigt. Die Bundeswaldinventur (BWI) erlaubt Aussagen auch für nachgeordnete Befundeinheiten (Bundesländer, Regionen etc.) und stellt für Holzaufkommensprognosen eine ideale Datenbasis dar. Im Rahmen dieses Beitrages werden, basierend auf der zweiten Bundeswaldinventur (BWI 2), für die Buche der Status-Quo (2002) und Szenariorechnungen zum Holzaufkommen bis 2022 für Niedersachsen und Gesamtdeutschland vorgestellt. Die Ergebnisse werden dem Basisszenario der Holzaufkommensmodellierung HAM (BMVEL 2004) gegenübergestellt. Die betrachteten Szenarien weisen ein durchschnittliches Nutzungspotenzial bei der Buche zwischen 14 Mio. und $20 \mathrm{Mio.} \mathrm{Efm} \mathrm{a}^{-1}$ für Deutschland aus, welches deutlich über dem Holzeinschlag von 2006 $\left(10\right.$ Mio. Efm a $\left.^{-1}\right)$ liegt. Dabei führen die Szenarien teilweise zu einem Buchen- 
Vorratsaufbau. Für Niedersachsen ergibt sich ein ähnliches Bild, wobei hier Potenziale zwischen 1,5 Mio. und 2 Mio. Efm a ${ }^{-1}$ erzielt werden.

Die beobachteten Zuwächse im Zeitraum zwischen BWI 1 und BWI 2 werden zur Beurteilung der räumlichen Gültigkeit einer für denselben Zeitraum durchgeführten Prognose herangezogen. Dabei wurden für die Buche sowohl der Effekt der geographischen Lage als auch der Seehöhe als signifikant identifiziert. Dieses Ergebnis bildet die Grundlage für eine Modellkalibrierung zur Erhöhung der Wuchsmodellgenauigkeit und somit zur Verbesserung von Holzaufkommensprognosen.

Stichworte: Buche, Holzaufkommensprognose, Bundeswaldinventur, Zuwachs, Nutzung, Vorrat

\begin{abstract}
Given the increasing demand for wood, reliable information about future stocks of timber for beech, the most important broadleaved tree species in Germany, is needed to develop competitive, sustainable silvicultural management strategies. The national forest inventory (BWI) provides an ideal database for the prediction of future timber stocks at different levels (national, state, regional, etc.). Based on the second national forest inventory (BWI 2), this paper presents the status quo (2002) and scenario calculations for beech timber stock up to 2022 for Lower Saxony and Germany. The results then are compared to the base scenario from the timber stock prediction model HAM (BMVEL 2004). The scenarios considered predict an average utilisation potential for beech between 14 Mio. and 20 Mio. $\mathrm{m}^{3} \mathrm{a}^{-1}$ for Germany, which is well above the wood volume harvested in 2006 (10 Mio. $\left.\mathrm{m}^{3} \mathrm{a}^{-1}\right)$. These scenarios predict in part an increase in beech growing stock. A similar situation was found for Lower Saxony, whereby here a utilisation potential of between 1.5 Mio. and 2 Mio. $\mathrm{m}^{3} \mathrm{a}^{-1}$ is targeted.

The growth observed in the period between BWI 1 and BWI 2 was used to assess the spatial validity of a prognosis conducted for the same period. Both the effect of geographical location and elevation were found to be significant for beech. This outcome was used then to calibrate the growth model to increase its accuracy, and hence improve the timber stock prognoses.
\end{abstract}

Keywords: beech, timber stock prediction, national forest inventory, growth, utilisation, growing stock 


\section{Einführung}

Die Globalisierung der Rohstoff- und Warenmärkte und die Verknappung fossiler Rohstoffe haben in Deutschland zu einer steigenden Holznachfrage und einer zunehmenden Konkurrenz zwischen stofflicher und energetischer Holznutzung geführt. Zur Entwicklung von Nutzungsstrategien, die die Grundsätze der Nachhaltigkeit wahren, die Wettbewerbsfähigkeit der deutschen Forst- und Holzwirtschaft sichern, Investitionen lenken und die Entwicklung der ländlichen Räume stärken, werden zuverlässige Informationen über das künftige Holzaufkommen benötigt. Dies gilt in besonderem Maße auch für die wichtigste Laubbaumart in Deutschland, die Buche.

Buchenwälder bedecken in Deutschland rund 15\% der Waldfläche. Nach den Ergebnissen der zweiten Bundeswaldinventur (BWI 2) betrug der Vorrat der Buche zum Stichtag 01.10.2002 ca. 583 Mio. $\mathrm{m}^{3}$, was einem Anteil von ca. $17 \%$ am Gesamtvorrat (3,38 Mrd. $\mathrm{m}^{3}$ ) entspricht (BMVEL 2004). Der Buchenholzeinschlag erreichte im Jahr 2006 ca. 10 Mio. $\mathrm{m}^{3}$ o. R. bzw. ca. $16 \%$ des gesamten deutschen Holzeinschlages (ZMP 2007). Drei Viertel des Buchen-Einschlages bzw. 7,4 Mio. $\mathrm{m}^{3}$ o. R. wurden als Industrieholz inkl. Brennholz verkauft.

Die Bundeswaldinventur erlaubt Aussagen auch für nachgeordnete Befundeinheiten (Bundesländer, Regionen, thematische Einheiten), deren Genauigkeit allerdings mit abnehmender Stichprobenzahl sinkt. Für Niedersachsen wird der Stichprobenfehler des mittleren Buchenvorrates $\left(\sim 54\right.$ Mio. $\left.\mathrm{m}^{3}\right)$ mit $\pm 7,8 \%$ und für die Buchenfläche ( 149.000 ha) mit $\pm 7,3 \%$ angegeben (BMVEL 2004). Der Fehler des Vorrates getrennt nach $10 \mathrm{~cm}$ breiten Durchmesserstufen beträgt im Brusthöhendurchmesser-Bereich von 10 bis $70 \mathrm{~cm}$ zwischen \pm 10 und $\pm 12,5 \%$. Somit können für die Buche in Niedersachsen Hochrechnungen, die den Vorrat und die Fläche betreffen, auch bei weiterer Differenzierung nach Durchmesserstufen [10 cm] und Altersklassen [20 Jahre] aus statistischer Sicht als aussagekräftig beurteilt werden. Allerdings sind dabei die Interpretationsmöglichkeiten an den Rändern der Durchmesserstufen- und Altersklassenverteilung eingeschränkt.

Für Holzaufkommensprognosen stellen derartige Großrauminventuren eine hervorragende Datenbasis dar. Ihr großer Vorteil gegenüber den Informationen der klassischen Forsteinrichtung besteht darin, dass repräsentativ erfasste dendrometrische Größen wie der Brusthöhendurchmesser (BHD) und die Baumhöhe als Startwerte für Simulationen für große Gebiete zu einem Stichtag zur Verfügung stehen (POLLEY et al. 1996, KÄNDLER et al. 2005, LWF 2005). Für regionale Prognosen, vor allem im Bereich der mittelfristigen Forstplanung, können auch Betriebsinventuren verwendet werden (PRETZSCH et al. 1998, ĎUSKŶ 2000, SCHMIDT et al. 2005). Der Einsatz von Waldwachstumssimulatoren zur Durchführung von Holzaufkommensprognosen hat in den letzten Jahren immer mehr an Bedeutung gewonnen (BÖSCH 1995, STERBA 1996, POLLEY et al. 1996, SODTKE 2003, KÄNDLER et al. 2005, LWF 2005). Sie sind mittlerweile als optimale Werk- 
zeuge für waldbauliche Simulationen anerkannt. Zu ihren Vorteilen zählt, dass hoch aufgelöste Einzelbauminformationen genutzt werden können, ohne dass Informationen durch eine Aggregierung zu Bestandeswerten verloren gehen (PRETZSCH 2001). Außerdem werden die Wachstumssimulatoren in regelmäßigen Abständen unter Einbeziehung aktuell erhobener Versuchsflächendaten reparametrisiert, wodurch sich ändernde Umwelt- bzw. Standortsbedingungen in den Wachstums- und Mortalitätsmodellen Berücksichtigung finden.

Im Rahmen dieses Beitrages werden Holzaufkommensprognosen bis zum Jahre 2022 für die Baumart Buche für Niedersachsen und Gesamtdeutschland vorgestellt, die ausgehend von den Ergebnissen der BWI 2 im Jahre 2002 an der Nordwestdeutschen Forstlichen Versuchsanstalt berechnet wurden und die Vergleichsmöglichkeiten zum Basisszenario der Holzaufkommensmodellierung (HAM) des Bundesministeriums für Ernährung, Landwirtschaft und Verbraucherschutz (BMVEL 2004) bieten. Die Holzaufkommensprognosen umfassen sowohl eine differenzierte Abschätzung der zukünftigen Vorräte als auch der periodisch möglichen Nutzungspotenziale. Darüber hinaus werden Wege einer Berücksichtigung regionaler Wachstumsunterschiede bei künftigen Holzaufkommensprognosen skizziert.

\section{Methodik der Szenariosimulation}

Die Holzaufkommensprognosen wurden mit Hilfe des für Nordwestdeutschland gültigen Wachstumssimulators BWINPro bzw. des auf seiner Basis entwickelten Entscheidungsunterstützungssystems WaldPlaner (HANSEN 2006, ALBERT u. HANSEN 2007) simuliert. Der Wachstumssimulator wurde an der ehemaligen Niedersächsischen Forstlichen Versuchsanstalt entwickelt (NAGEL 1996, 1999, NAGEL et al. 2002, NAGEL et al. 2006). Der biometrische Modellansatz ist ein statistisches, positionsunabhängiges Einzelbaumwachstumsmodell. Das baumartenspezifische Wachstum bzw. die Veränderung von BHD, Höhe, Kronenansatz und Kronenbreite über der Zeit wird als Funktion des Alters, der Kronenmantelfläche sowie des Kronenkonkurrenzfaktors $\mathrm{C}_{66}$ und dessen Veränderung aufgrund von Eingriffen beschrieben. Mit diesem Ansatz lassen sich Zuwachsreaktionen als Folge von waldbaulichen Eingriffen (Freistellungseffekt) abbilden. Die auf der Dimension der Baumkrone basierenden unabhängigen Variablen werden über einfache Funktionen aus dem BHD und der Höhe zum Beginn der Simulation geschätzt. Der Vorteil kronenbasierter Konkurrenzindices besteht darin, dass sich mit ihnen zumindest teilweise die unterschiedliche Konkurrenzkraft der Baumarten berücksichtigen lässt. In einer Untersuchung von BIGING u. DOBBERTIN (1992) haben sie sich gegenüber durchmesserbasierten Indizes als überlegen erwiesen.

Der Waldwachstumssimulator wurde in den letzten Jahren mehrmals reparametrisiert und mit zusätzlichen Funktionen, u. a. einem Sortierungsmodul, versehen (ALBERT 2000, NAGEL et al. 2002). Auf der Grundlage von Schaftform- 
modellen (SCHMIDT 2001) kann der Effekt unterschiedlicher Aushaltungsvarianten auf die Stärkeklassenverteilung für beliebige regionale Einheiten (Bestand, Betrieb, Bundesland) simuliert werden. Die Stärkeklassenverteilungen können für ökonomische Analysen mit Preisen hinterlegt werden und ermöglichen so eine Abschätzung der Wertleistung (SPELLMANN u. SCHMIDT 2003). Neben diesen und anderen Routinen verfügt der Simulator auch über eine Durchforstungs- und Nutzungsroutine, mit der waldbauliche Zielvorgaben unter Berücksichtigung von Beschränkungen automatisiert und regelbasiert umgesetzt werden können (SPELLMANN et al. 1999, AlBERT u. GUERICKE 2003, DudA 2006). Der Simulator wird von der Nordwestdeutschen Forstlichen Versuchsanstalt über das Internet bereitgestellt und steht privaten und öffentlichen Forstbetrieben zur Verfügung. Für die hier vorgestellten Auswertungen des Waldzustandes 2002 und der Holzaufkommensprognosen wurde das Entscheidungsunterstützungssystem WaldPlaner verwendet, das die Funktionalitäten des Wachstumssimulators mit der Möglichkeit zur Stapelverarbeitung sowie zusätzlichen GIS- und Analyse-Funktionalitäten kombiniert. Nur über das automatisierte Einlesen, Aufbereiten und Fortschreiben einer Vielzahl von Stichprobenpunkten sind Holzaufkommensprognosen auf der Grundlage der Bundeswaldinventur überhaupt möglich. Der W aldPlaner unterstützt verschiedene Datenbanksysteme und kann Daten unterschiedlicher Inventurverfahren (z. B. Winkelzählprobe, konzentrische Probekreise) verarbeiten.

Die Justierung der Waldbehandlungsroutinen über Steuergrößen wie die Zielstärke erlaubt es, den Einfluss waldbaulicher Strategien auf das Holzaufkommen abzubilden. Über die Berücksichtigung von Freistellungseffekten erfolgt dabei auch eine Rückkopplung auf das Wachstum. Einen größeren Einfluss auf das Holzaufkommen haben allerdings die gewählten Ausprägungen der Steuergrößen, die die unterschiedlichen waldbaulichen Szenarien charakterisieren. Für die Holzaufkommensprognose dieser Studie wurden die zwei Szenarien „naturnaher Waldbau“ und „,ertragsorientierter Waldbau“ gewählt, die durch die in Tabelle 1 aufgeführten Steuergrößen definiert sind.

Das Szenario „naturnaher Waldbau“ orientiert sich an dem für den niedersächsischen Landeswald entwickelten Konzept der Langfristigen Ökologischen Waldentwicklung (LÖWE - NIEDERSÄCHSISCHE LANDESFORSTVERWALTUNG 1991). Es fördert durch die Vorgaben der Steuerungsgrößen Schutz seltener Baumarten, Habitatbaumauswahl und Totholzmanagement die biologische Diversität stärker als die Variante „ertragsorientierter Waldbau“, die lediglich ein Totholzmanagement vorsieht. Das Szenario "ertragsorientierter Waldbau" unterstellt bei den Zielstärken der Baumarten gegenüber dem „naturnahen Waldbau“ jeweils um $5 \mathrm{~cm}$ geringere Werte. Eine detaillierte Beschreibung des verwendeten Systems zur modellhaften Umsetzung waldbaulicher Szenarien findet sich bei DUDA (2006). 
Tabelle 1: Szenariorahmenwerte

\begin{tabular}{|c|c|c|}
\hline Steuergrößen & naturnah & ertragsorientiert \\
\hline Schutz seltener Baumarten & ja & nein \\
\hline Habitatbäume (n ha ${ }^{-1}$ ) & 3 & 0 \\
\hline Totholz $\left(\mathrm{m}^{3} \mathrm{ha}^{-1}\right)$ & 20 & bis 10 \\
\hline Zielstärke (cm) & $\begin{array}{l}\mathrm{Ei} 70, \mathrm{Bu} 60 \\
\mathrm{Fi} 45, \mathrm{Ki} 45\end{array}$ & jeweils $5 \mathrm{~cm}$ geringer \\
\hline Endnutzungsmasse pro Hieb $\left(\mathrm{m}^{3} \mathrm{ha}^{-1} 5 \mathrm{a}^{-1}\right)$ & $\begin{array}{l}\text { Min. } 10-15 \\
\text { Max. } 70-115\end{array}$ & $\begin{array}{c}\text { Min. } 10-15 \\
\text { Max. } 70-115\end{array}$ \\
\hline $\begin{array}{l}\text { Durchforstungsbeginn } \\
\text { Bestandesoberhöhe (m) }\end{array}$ & $10-16$ & $12-18$ \\
\hline Durchforstungsmasse pro Hieb $\left(\mathrm{m}^{3} \mathrm{ha}^{-1} 5 \mathrm{a}^{-1}\right)$ & $25-100$ & $25-110$ \\
\hline $\begin{array}{l}\text { Freistellungsgrad der Z-Bäume (nach DUDA } \\
\text { 2006) }\end{array}$ & stark & sehr stark \\
\hline
\end{tabular}

Bei der Sortimentierung, d.h. der rechnerischen Zerlegung der modellhaft genutzten Buchenstämme in einzelne Sortimente wurde sowohl lang als auch in Abschnitten ausgehalten. Ein einzelner Stamm kann somit ausschließlich in Langholz, in Abschnitte oder in eine Kombination aus beiden Sorten aufgeteilt werden. Für das Langholz (mind. 5 Meter lang) betrug der Mindestzopfdurchmesser $21 \mathrm{~cm}$ und der Mindestmittendurchmesser $25 \mathrm{~cm}$. Für die Abschnitte wurden ein Mindestzopf von $12 \mathrm{~cm}$ und eine Fixlänge von $3 \mathrm{~m}$ festgelegt. Die unterstellte Stockhöhe betrug $30 \mathrm{~cm}$.

Den Szenarien „naturnaher Waldbau“ und „ertragsorientierter Waldbau“ werden Ergebnisse des Basisszenarios der Holzaufkommensmodellierung (HAM) des BMELV ${ }^{1}$ gegenübergestellt. Das Basisszenario schätzt das potenziell nutzbare Rohholzaufkommen mit einer als konstant unterstellten, bundeslandspezifischen Waldbehandlung für einen 40-jährigen Zeitraum, wobei hier die Resultate der ersten 20 Jahre verwendet werden. Die wesentlichen Steuergrößen des zugehörigen Nutzungsmodells sind Tabelle 2 zu entnehmen. Die wichtigsten Steuerparameter sind länder- und baumartenspezifische Definitionen der Durchforstungsart, Durchforstungsintensität (Turnus und Zielgrundfläche), Umtriebszeit und des Zieldurchmessers.

\footnotetext{
1 Auf der Internetplattform zur zweiten Bundeswaldinventur besteht die Möglichkeit, die Inventurergebnisse und die Ergebnisse der Rechnung eines Basisszenarios (HAM) abzurufen: www.bundeswaldinventur.de (20.07.07). Dieses Szenario wird den, im Rahmen dieses Projektes durchgeführten Szenariorechnungen („,naturnaher Waldbau“ u. „ertragsorientierter Waldbau“) vergleichend gegenübergestellt.
} 
Die Ergebnisse umfassen neben Angaben zum Gesamtvorrat auch eine Vielzahl an detaillierten Auswertungen wie Vorratsverteilungen nach Mittenstärken. Das Durchmesserzuwachsmodell dieser Prognose wurde direkt auf der Basis der BWIDaten parametrisiert und die Höhenschätzung erfolgte über eine Tariffunktion aus dem BHD. Die Bestandesdichte hat in der HAM-Prognose keinen Einfluss auf das Durchmesserwachstum der Einzelbäume. Auch können Freistellungseffekte nicht beschrieben werden, da die periodische Bundeswaldinventur zur Parametrisierung verwendet wurde und die Nutzungen hier zeitlich nicht eingeordnet werden können.

Tabelle 2: $\quad$ Steuergößßen des Nutzungsmodells der Holzaufkommensmodellierung (HAM). Die Verteilungsparameter spiegeln die unterschiedlichen Ländervorgaben wider.

\begin{tabular}{|c|c|c|c|c|c|c|c|c|c|c|c|c|c|c|c|}
\hline \multirow[t]{2}{*}{ Steuerparameter } & \multicolumn{3}{|c|}{ Buche } & \multicolumn{3}{|c|}{ Eiche } & \multicolumn{3}{|c|}{ Fichte } & \multicolumn{3}{|c|}{ Kiefer } & \multicolumn{3}{|c|}{ Lärche } \\
\hline & Mi & $\mathrm{Me}$ & $\mathrm{Ma}$ & Mi & $\mathrm{Me}$ & $\mathrm{Ma}$ & Mi & $\mathrm{Me}$ & $\mathrm{Ma}$ & Mi & $\mathrm{Me}$ & $\mathrm{Ma}$ & Mi & $\mathrm{Me}$ & $\mathrm{Ma}$ \\
\hline $\begin{array}{l}\text { Durchforstungs- } \\
\text { beginn im Alter }\end{array}$ & 15 & 40 & 45 & 15 & 30 & 35 & 15 & 30 & 30 & 10 & 10 & 30 & 10 & 20 & 20 \\
\hline $\begin{array}{l}\text { Durchforstungs- } \\
\text { beginn bei } \\
\text { Mittelhöhe [m] }\end{array}$ & 0 & 14 & 16 & 7 & 10 & 14 & 10 & 10 & 14 & 7 & 10 & 12 & 7 & 10 & 14 \\
\hline $\begin{array}{l}\text { Endnutzungs- } \\
\text { beginn im Alter }\end{array}$ & 110 & 130 & 150 & 120 & 165 & 179 & 60 & 100 & 130 & 100 & 120 & 140 & 70 & 110 & 130 \\
\hline $\begin{array}{l}\text { Endnutzung } \\
\text { abgeschlossen im } \\
\text { Alter }\end{array}$ & 160 & 190 & 200 & 180 & 219 & 240 & 100 & 155 & 190 & 150 & 170 & 200 & 130 & 160 & 190 \\
\hline Max. Zielstärke & 55 & 65 & 70 & 60 & 70 & 80 & 40 & 45 & 60 & 45 & 45 & 60 & 50 & 60 & 65 \\
\hline $\begin{array}{l}\text { Max. Zielstärken- } \\
\text { Entnahmeprozent }\end{array}$ & 10 & 50 & 60 & 20 & 50 & 50 & 25 & 50 & 60 & 10 & 50 & 60 & 25 & 50 & 80 \\
\hline
\end{tabular}

$\mathrm{Mi}=$ Minimum, $\mathrm{Me}=$ Median, $\mathrm{Ma}=$ Maximum

Quelle: BMELV (2004)

(ohne Pappel, Erle, Weide Ahorn, Esche, Tanne und Douglasie).

\section{Ausgangszustand, Zuwachs und Nutzungen in der Inventurperiode 1987 - 2002}

Zum Stichtag der zweiten Bundeswaldinventur (2002) lag der bundesweite Vorrat der Buche bei ca. 583 Mio. Vfm. Dies entspricht ca. $17 \%$ des Gesamtholzvorrates. In Niedersachsen stockten 2002 ca. 54 Mio. Vfm Buche, was einem Anteil am niedersächsischen Gesamtvorrat von ca. $18 \%$ entspricht. Die Verteilung des Vorrats über Brusthöhendurchmesserstufen ist in Abbildung 1 dargestellt, wobei 
der Schwerpunkt sowohl in Niedersachsen (43\%) als auch im Bundesgebiet (42\%) im Bereich von 30 bis 49,9 cm BHD liegt.

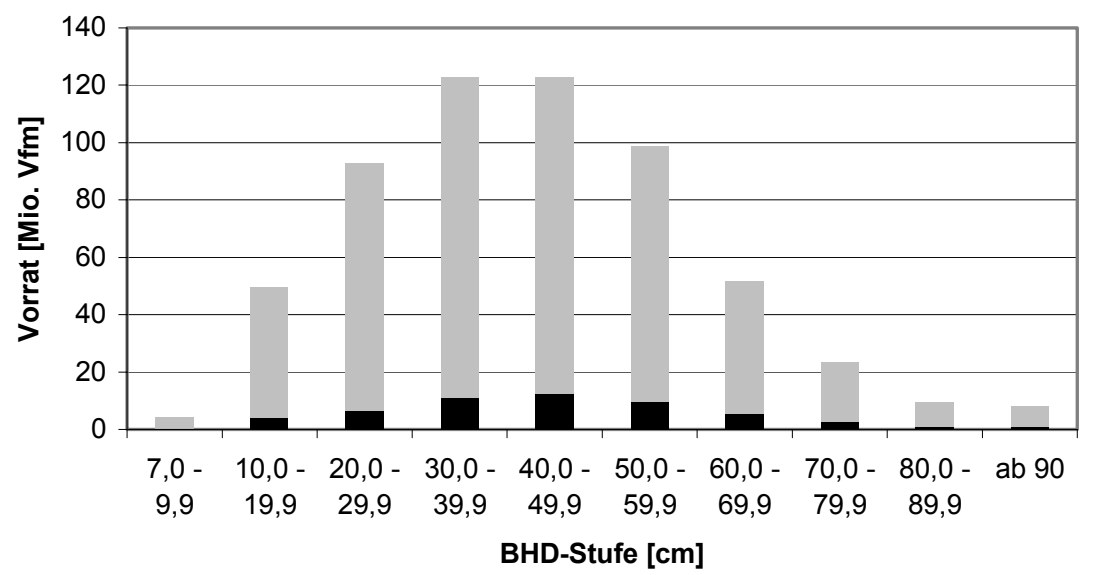

- Niedersachsen $\square$ Deutschland

Abbildung 1: Vorratsverteilung nach BHD-Stufen der Baumart Buche in Deutschland und Niedersachsen auf Basis der zweiten Bundeswaldinventur

Die bundesweite Flächenverteilung nach Altersklassen weist für die Buche eine weitgehend gleiche Ausstattung der Altersklassen aus. Unterrepräsentiert sind die bis 40-jährigen Bestände. Die Bestände über 140 Jahre nehmen ca. 250.000 ha ein (s. Abb. 2). In Niedersachsen liegt der Schwerpunkt der Altersklassenverteilung im Bereich von 81-120 Jahren. In den Jahren zwischen 1987 und 2002 wurden in den alten Bundesländern jährlich durchschnittlich ca. 7,5 Mio. Efm Buchenholz genutzt. (BMVEL 2004). Die Holzeinschlagsstatistiken weisen demgegenüber für den gleichen Zeitraum nur einen durchschnittlichen jährlichen Einschlag von ca. 5,9 Mio. Efm Buchenholz aus (ZMP 2007). Diese Diskrepanz erklärt sich in erster Linie durch nicht verbuchte Einschläge für den Eigenbedarf (z. B. Brennholz im Privatwald). Auf die Unterschiede zwischen den Ergebnissen der BWI 2 und den Einschlagsstatistiken weisen DIETER u. ENGLERT (2005) hin. Hinsichtlich des Zuwachses der Buche im Vergleich der alten Bundesländer liegt Niedersachsen mit 11,24 $\mathrm{m}^{3} \mathrm{ha}^{-1} \mathrm{a}^{-1}$ knapp unter dem Durchschnitt von 11,74 $\mathrm{m}^{3} \mathrm{ha}^{-1} \mathrm{a}^{-1}$. Die höchsten Zuwächse wurden in Bayern festgestellt $\left(13,25 \mathrm{~m}^{3} \mathrm{ha}^{-1} \mathrm{a}^{-1}\right)$. Der geringste durchschnittliche periodische Zuwachs der Buche wurde in Hessen beobachtet $\left(10,37 \mathrm{~m}^{3} \mathrm{ha}^{-1} \mathrm{a}^{-1}\right)$. Der jährliche Holzeinschlag für die Buche in Niedersachsen betrug zwischen den Jahren 1987 und 2002 0,92 Mio. Efm a-1 bzw. 0,9 Efm ha-1 a-1. 


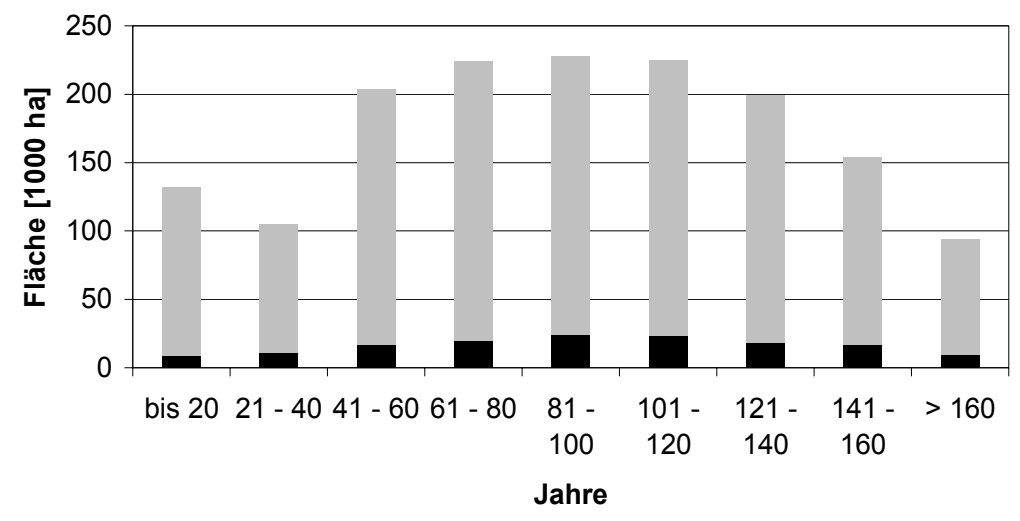

- Niedersachsen Deutschland

Abbildung 2: Flächenverteilung nach Altersklassen der Baumart Buche in Deutschland und Niedersachsen auf Basis der zweiten Bundeswaldinventur

\section{Buchenholzpotenziale Niedersachsen}

Beim Vergleich und der Bewertung der Szenarien, die mit dem WaldPlaner durchgeführt wurden, und der Variante HAM sind Unterschiede vor allem auf die unterschiedlichen Steuergrößen zurückführen (s. Tab. 1). Geringfügigere Unterschiede können aber auch aufgrund unterschiedlicher biometrischer Modelle bei der Wachstumsprognose oder der Volumenberechnung auftreten. Weiterhin ist ein geringfügig unterschiedlicher Flächenbezug in den Szenarien zu beachten. So beziehen sich alle Prognosen (zukünftiger Vorrat und Nutzung) des HAM-Szenarios nur auf den Hauptbestand (Plenterbestände werden voll berücksichtigt) solcher Flächen, die laut Definition begehbarer Holzboden einschließlich Lücken im Bestand sind, welche aber keinem Nutzungsverbot unterliegen. Der Zustand 2002 (s. Abb. 1 und 2) und alle Prognosen mit dem WaldPlaner (Szenarien „,naturnaher“" und „,ertragsorientierter Waldbau“") berücksichtigen auch Flächen mit einem Nutzungsverbot (bundesweit 92.366 ha, ca. 1\%) und alle Bestandesschichten, so dass bei einem Vergleich hier generell von etwas höheren Gesamtwerten ausgegangen werden muss. Weiterhin ist bei der Interpretation zu beachten, dass ein eigentümerspezifisches Verhalten in keinem der WaldPlaner-Szenarien berücksichtigt wird. Es wird aufgezeigt, welche Holzmengen theoretisch bei vollflächiger Umsetzung der definierten Szenarien zur Verfügung stehen würden.

Betrachtet man die Vorratsentwicklung der Buche in Niedersachsen, so weist das Basisszenario (HAM) einen fallenden Trend auf (s. Abb. 3). 


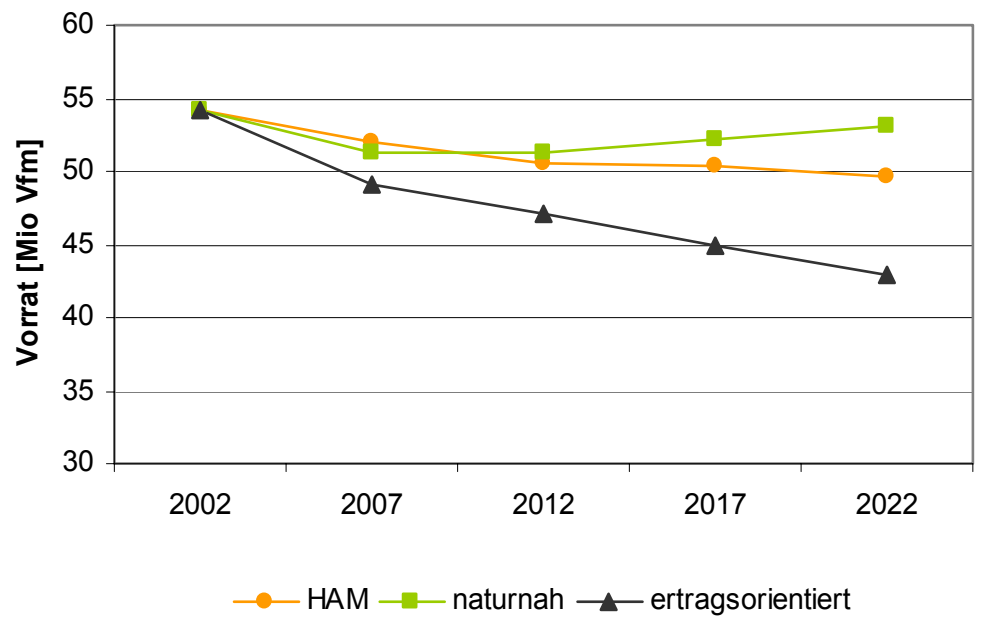

Abbildung 3: Prognostizierte Entwicklung der Buchenholzvorräte in Niedersachsen von 2002 bis 2022

Von ca. 54 Mio. Vfm im Jahre 2002 geht der Buchenholzvorrat auf ca. 49,7 Mio. Vfm im Jahre 2022 zurück. Demgegenüber ist bei der ertragsorientierten Variante eine noch stärkere Vorratsabsenkung zu verzeichnen. Im Jahr 2022 liegt der prognostizierte Buchenvorrat nur noch bei ca. 43 Mio. Vfm. Das Szenario „naturnaher Waldbau“ zeigt insgesamt eine ausgeglichene Entwicklung des Buchenvorrates. Bis 2007 ist ein leichter Abfall auf ca. 51 Mio. Vfm zu verzeichnen. Danach steigt der Vorrat auf ca. 53 Mio. Vfm an und erreicht somit fast das Ausgangsniveau des Jahres 2002.

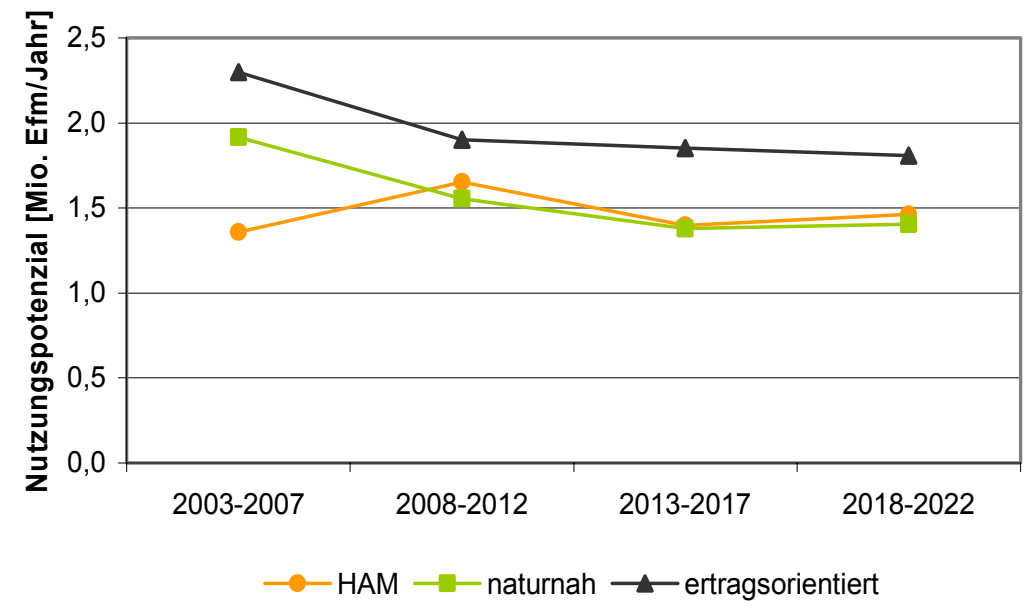

Abbildung 4: Prognostizierte Entwicklung des mittleren periodischen (5 Jahre) Nutzungspoteñials bei Buche in Niedersachsen 
Die Entwicklungen der Nutzungspotenziale verlaufen bei den Szenarien „naturnaher Waldbau“ und „ertragsorientierter Waldbau“ annährend parallel (s. Abb. 4). Die Nutzungen liegen in den ersten 5 Jahren bis zum Jahr 2007 im Mittel bei jährlich 2,3 bzw. 1,9 Mio. Efm, wobei es sich bei allen dargestellten Werten um mittlere periodische Nutzungen innerhalb von 5-jährigen Perioden handelt. Der abnehmende Trend führt in der Periode 2018 - 2022 bei der naturnahen Variante zu einem Nutzungspotenzial von ca. 1,4 Mio. Efm a ${ }^{-1}$ und bei der ertragsorientierten Variante von 1,8 Mio. Efm a ${ }^{-1}$. Durchschnittlich beläuft sich das Buchenholzpotenzial für den 20-jährigen Simulationszeitraum in Niedersachsen unter den Bedingungen eines ,naturnahen Waldbaus“ auf ca. 1,6 Mio. Efm a ${ }^{-1}$. Die Variante „ertragsorientierter Waldbau“ übertrifft diesen Wert mit durchschnittlich ca. 2,0 Mio. Efm a ${ }^{-1}$. Mit durchschnittlich 1,5 Mio. Efm a ${ }^{-1}$ liegt das Potenzial, das durch die HAM-Prognose ausgewiesen wird, knapp unter dem Wert der naturnahen Variante.

Die aus den Nutzungspotenzialen abgeleiteten Sortimente (s. Tab. 3 u. Tab. 4) weisen bei der Variante „naturnaher Waldbau“ einen Schwerpunkt in der Stärkeklasse $^{2} 5$ auf. Auf diese entfallen im Mittel der 20 Jahre jährlich 446.500 Efm. Bei der Variante „ertragsorientierter Waldbau“ verlagert sich der Schwerpunkt entsprechend der geringeren Zielstärke ab der Periode 2008 - 2012 auf die Stärkeklasse 4. In diese fallen dann durchschnittlich 530.200 Efm. In beiden Szenarien steigen die Potenziale in der Stärkeklasse 4 im Laufe der Simulation an. In allen anderen Stärkeklassen gehen die prognostizierten Mengen zurück, wobei das Szenario „naturnaher Waldbau“ in der letzten Simulationsperiode teilweise steigende Tendenzen aufweist (Stärkeklassen 2a, 2b).

Tabelle 3: $\quad$ Das mittlere jährliche Nutzungspotenzial der Buche [1000 Efm $\left.a^{-1}\right]$ nach Stärkeklassen für das Szenario „naturnaber Waldbau“ in Niedersachsen

\begin{tabular}{lccccccccc}
\hline \multicolumn{1}{l}{ naturnah, Niedersachsen } & \multicolumn{8}{c}{ Stärkeklasse } \\
Jahr & $1 \mathrm{a}$ & $1 \mathrm{~b}$ & $2 \mathrm{a}$ & $2 \mathrm{~b}$ & $3 \mathrm{a}$ & $3 \mathrm{~b}$ & 4 & 5 & $\geq 6$ \\
\hline $2003-2007$ & 34,47 & 73,18 & 57,66 & 105,12 & 89,05 & 73,55 & 120,53 & 526,84 & 390,10 \\
\hline $2008-2012$ & 31,68 & 87,45 & 63,86 & 115,86 & 71,69 & 58,25 & 216,36 & 453,26 & 126,57 \\
\hline $2013-2017$ & 27,44 & 65,34 & 41,56 & 71,14 & 74,67 & 69,07 & 260,53 & 411,26 & 59,54 \\
\hline $2018-2022$ & 21,73 & 63,62 & 54,64 & 76,01 & 73,37 & 56,65 & 304,67 & 394,55 & 58,49 \\
\hline
\end{tabular}

\footnotetext{
2 Die Stärkeklasse bezieht sich auf den Mittendurchmesser ohne Rinde der ausgehaltenen Sortimente. 1a: 10 bis $14 \mathrm{~cm}, 1 \mathrm{~b}: 15$ bis $19 \mathrm{~cm}, 2 \mathrm{a}: 20$ bis $24 \mathrm{~cm}, 2 \mathrm{~b}: 25$ bis $29 \mathrm{~cm}, 3 \mathrm{a}: 30$ bis $34 \mathrm{~cm}, 3 \mathrm{~b}: 35$ bis $39 \mathrm{~cm}, 4: 40$ bis $49 \mathrm{~cm}, 5: 50$ bis $59 \mathrm{~cm}, 6: 60 \mathrm{~cm}$ und stärker.
} 
Tabelle 4: $\quad$ Das mittlere jährliche Nutzungspotenzial der Buche[1000 Efm $\left.a^{-1}\right]$ nach Stärkeklassen für das Szenario ,ertragsorientierter W aldbau" in Niedersachsen

\begin{tabular}{lccccccccc}
\hline \multicolumn{1}{l}{ ertragsorientiert, Niedersachsen } & \multicolumn{7}{c}{ Stärkeklasse } \\
Jahr & $1 \mathrm{a}$ & $1 \mathrm{~b}$ & $2 \mathrm{a}$ & $2 \mathrm{~b}$ & $3 \mathrm{a}$ & $3 \mathrm{~b}$ & 4 & 5 & $\geq 6$ \\
\hline $2003-2007$ & 38,73 & 93,52 & 75,67 & 134,15 & 126,70 & 90,74 & 308,41 & 512,88 & 409,62 \\
\hline $2008-2012$ & 41,70 & 107,83 & 70,88 & 137,55 & 99,96 & 63,98 & 467,04 & 399,62 & 119,31 \\
\hline $2013-2017$ & 38,58 & 94,94 & 73,04 & 117,36 & 99,30 & 82,66 & 616,06 & 273,09 & 74,05 \\
\hline $2018-2022$ & 26,12 & 84,22 & 54,56 & 90,44 & 85,53 & 63,95 & 729,41 & 234,12 & 66,24 \\
\hline
\end{tabular}

\section{Buchenholzpotenziale Deutschland}

Für ganz Deutschland zeigen alle drei Szenarien bis zum Jahr 2007 einen fallenden Trend der Buchenholzvorräte (s. Abb. 5). Von ca. 583 Mio. Vfm wird der Vorrat auf Werte zwischen ca. 530 Mio. und 550 Mio. Vfm absinken. Ab dem Jahr 2007 wird der Buchenvorrat beim Szenario „naturnaher Waldbau“ wieder aufgebaut. Dieser erreicht im Jahr 2022 mit 579 Mio. Vfm fast das Ausgangsniveau von 2002. Die Szenarien HAM und ,ertragsorientierter Waldbau“ senken die Vorräte ab dem Jahr 2007 weiter ab und führen 2022 zu einem geschätzten Vorrat von ca. 516 Mio. Vfm (HAM) bzw. 483 Mio. Vfm („,ertragsorientiert“). Dies ist vor allem durch die gegenüber dem „naturnahen Waldbau“ höheren maximal zulässigen Nutzungsmassen und niedrigeren Zielstärken bedingt.

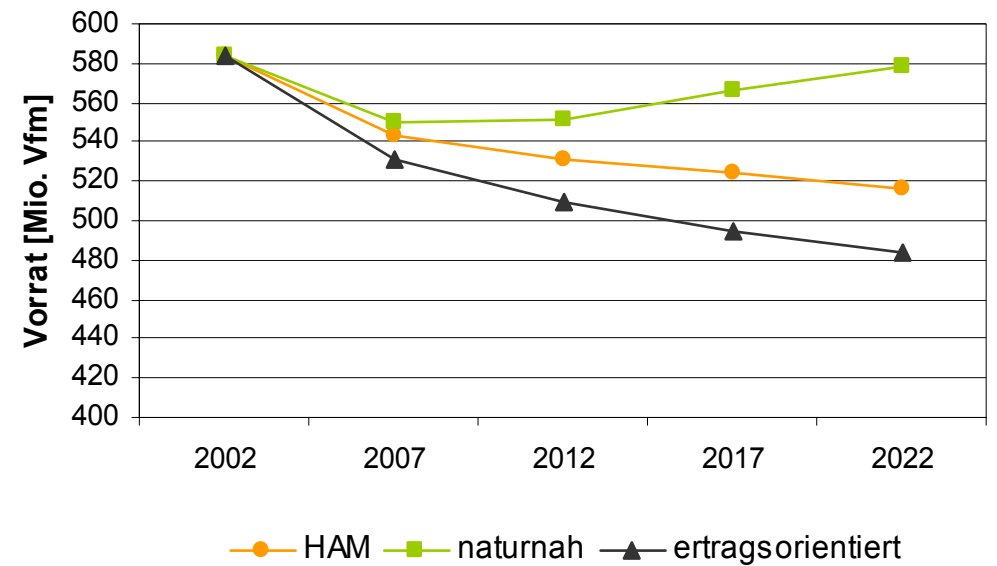

Abbildung 5: Prognostizierte Entwicklung der Buchenholzvorräte in Deutschland von 2002 bis 2022 
Die resultierenden Vorratsverteilungen werden deutlich von den gewählten Zielstärken bestimmt. In beiden Szenarien (,naturnah“/,,ertragsorientiert") ergibt sich sowohl für Deutschland als auch für Niedersachsen eine linksschiefe Verteilung der Vorräte über dem Brusthöhendurchmesser (s. Abb. 6 u. Abb. 7). Ab der Durchmesserstufe 60-69,9 cm sind bei dem Szenario „naturnaher Waldbau“ die Anteile am Gesamtvorrat sehr gering. Da bei der ,ertragsorientierten“ Variante die Zielstärke bei $55 \mathrm{~cm}$ liegt, ist hier auch die Stufe 50-59,9 cm deutlich schwächer besetzt. Die Vorratsstruktur für Niedersachsen entspricht grundsätzlich für beide Varianten dem bundesweiten Muster.

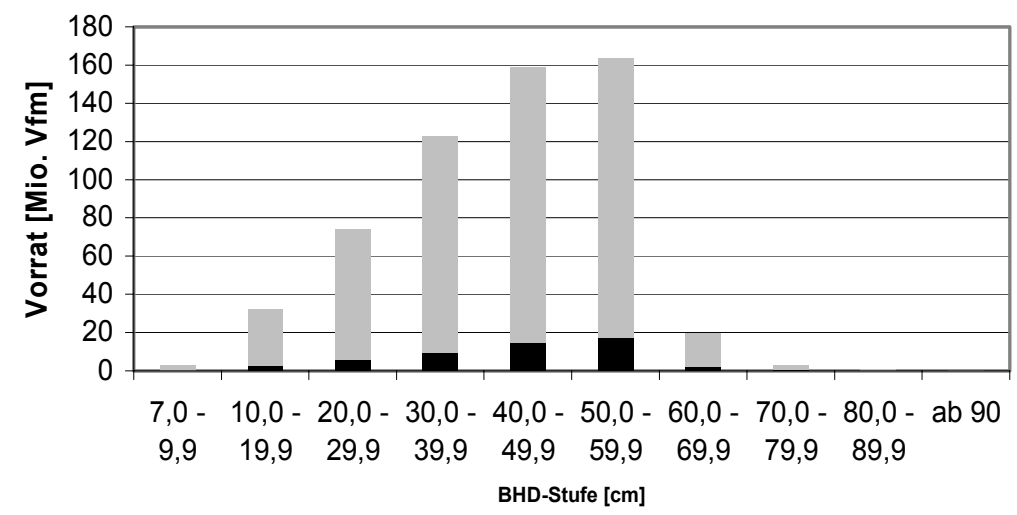

- Niedersachsen Deutschland

Abbildung 6: Prognostizierte Vorratsverteilung nach BHD-Stufen der Baumart Buche im Jahr 2022 in Deutschland und Niedersachsen für das Szenario "naturnaher Waldbau"

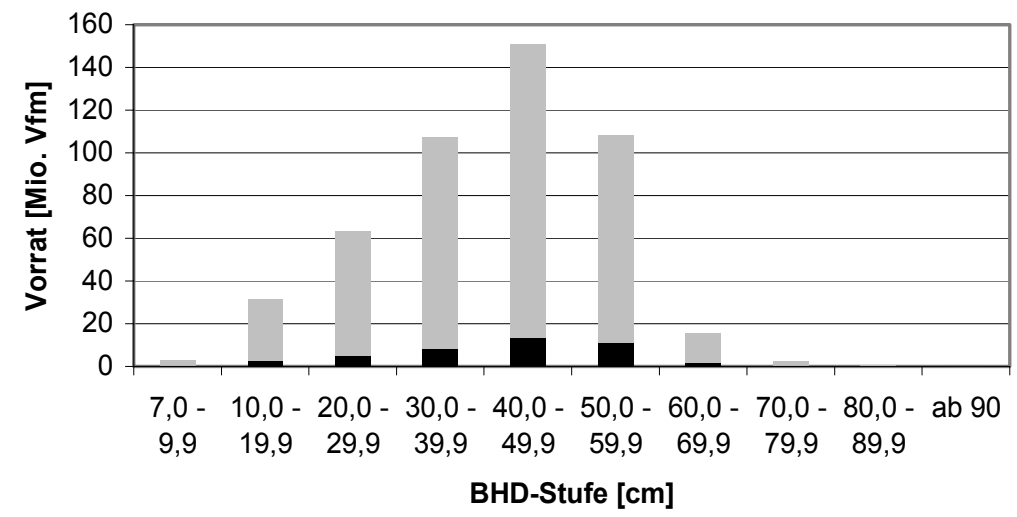

- Niedersachsen $\square$ Deutschland

Abbildung 7: Prognostizierte Vorratsverteilung nach BHD-Stufen der Baumart Buche im Jahr 2022 in Deutschland und Niedersachsen für das Szenario „ertragsorientierter Waldbau“" 
Die prognostizierten Nutzungspotenziale für Deutschland verlaufen wie in Niedersachsen für die Szenarien „naturnaher“ und „ertragorientierter Waldbau“ annähernd parallel und weisen einen abnehmenden Trend auf (s. Abb. 8). Ab der Periode 2013 - 2017 wird in beiden Szenarien ein gleichbleibendes Nutzungsniveau von 14,4 Mio. bzw. 18,5 Mio. Efm a ${ }^{-1}$ erreicht. Die Variante HAM weist wie für Niedersachsen von Beginn an einen deutlich ausgeglicheneren Verlauf mit durchschnittlich 13,9 Mio. Efm a ${ }^{-1}$ auf. Im Durchschnitt der simulierten 20 Jahre zeigt das ertragsorientierte Szenario das höchste jährliche Nutzungspotenzial (19,8 Mio. Efm). Auf einem deutlich tieferen Niveau liegt das Nutzungspotenzial des Szenarios „,naturnaher Waldbau“ (15,9 Mio. Efm). Hier steigt in den letzten 5 Simulationsjahren in Übereinstimmung mit der Simulation für Niedersachsen das Potenzial wieder leicht an.

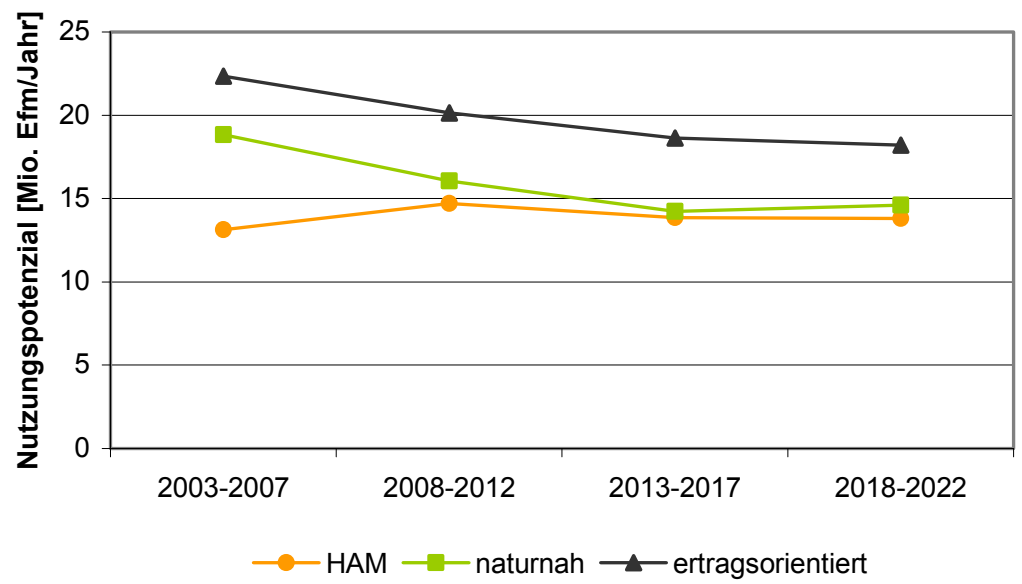

Abbildung 8: Entwicklung des mittleren periodischen (5 Jahre) Nutzungspotenzials bei Buche in Deutschland von 2002 bis 2022

Auffällig ist, dass die naturnahe Variante bei durchschnittlich etwas höheren Nutzungen einen deutlich höheren Vorrat im Vergleich zur HAM-Variante aufbaut. Dies ist auf mehrere Faktoren zurückzuführen, wie den unterschiedlichen Bestandes- und Flächenbezug. Die Vorratsangaben des HAM-Szenarios beziehen sich auf Bestände ohne Nutzungsverbot und nur auf den Hauptbestand bzw. den Gesamtbestand bei plenterartigen Bestandesstrukturen, so dass der Vorrat insgesamt geringer berechnet wird. Im Jahr 2002 beträgt der Buchenvorrat über alle Bestandesschichten ca. 583 Mio. Vfm. Der Vorrat nur für den Hauptbestand (oder Plenterwald) ohne Nutzungsverbot liegt 2002 hingegen bei ca. 544 Mio. Vfm und ist somit um $7 \%$ geringer als der Vorrat bei Berücksichtigung aller Bestandesschichten. 
Weiterhin könnten unterschiedliche Verjüngungsmechanismen und Zuwachsmodelle bei der WaldPlaner-Simulation und der HAM-Prognose zu den beobachteten Unterschieden führen.

Die Verteilung des Nutzungspotenzials auf Durchmesserstufen verdeutlicht den Einfluss der alternativen waldbaulichen Szenarien (s. Abb. 9). Zwar weisen die Szenarien „ertragsorientierter" und „naturnaher Waldbau“ aufgrund ihrer spezifischen Steuergrößen für Grundflächenhaltung und Zielstärke beide eine 'Hiebsruhe' zwischen Vor- und Endnutzung auf. Die obere Grenze dieses Bereiches schwächerer Nutzungsintensität wird aber vor allem durch die Zielstärke bestimmt (s. Tab. 1). So tritt dieser Bereich in der ,naturnahen“ Variante bei 50-59 cm, in der „ertragsorientierten“ Variante aber bereits bei 40-49 cm auf. Umgekehrt fallen die Endnutzungsmassen im „ertragsorientierten Waldbau“ ausgeglichener sowohl in der Stufe $50-59 \mathrm{~cm}$ als auch in der Stufe $\geq 60 \mathrm{~cm}$ mit beträchtlichen Mengen an (5,7 Mio. und 7,3 Mio. Efm a ${ }^{-1}$. Dagegen führt die höhere Zielstärke im „naturnahen Waldbau“ erst ab der Durchmesserstufe $>60 \mathrm{~cm}$ zu einem Vorratsabbau bzw. hohen Nutzungen (9,5 Mio. Efm a-1).

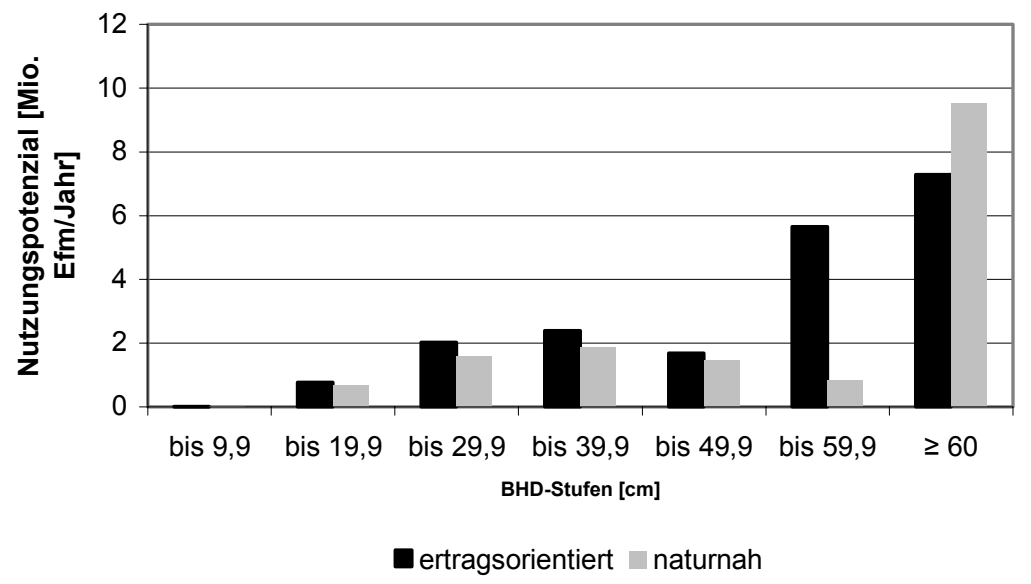

Abbildung 9: Verteilung der mittleren jäbrlichen Nutzungspotenziale (2003 bis 2022) nach Durchmesserstufen der Szenarien ,ertragsorientierter" und ,naturnaber W aldbau" für Buche in Deutschland

Die Verteilung des Nutzungspotenzials auf die Stärkeklassen zeigt ein ähnliches Muster (s. Tab. 5 u. Tab. 6) wie für Niedersachsen. Die Mengen in den einzelnen Stärkeklassen sind für beide Szenarien mit Ausnahme der Stärkeklasse 4 rückläufig.

Bei der Variante „naturnaher Waldbau“ treten die größten Mengen dennoch durchgängig in der Stärkeklasse 5, bei der Variante „ertragsorientierter Waldbau“ ab der Periode 2008 - 2012 durchgängig in der Stärkeklasse 4 auf. Der stärkste Rückgang ist in beiden Szenarien für die Stärkeklasse $6 \mathrm{zu}$ verzeichnen. Die auftre- 
tenden Muster lassen sich wie bei der Verteilung nach Durchmesserstufen vor allem über die unterschiedlich gewählten Zielstärken erklären. Die maximalen Eingriffsstärken für Buchenbestände liegen bei $100 \mathrm{~m}^{3} \mathrm{ha}^{-1}$ pro Eingriff (1 Eingriff in 5 Jahren), so dass zielstarke Bäume im Allgemeinen innerhalb von 1-2 Eingriffen entnommen werden. Eine konsequent umgesetzte Zielstärkennutzung führt daher in beiden Szenarien dazu, dass nach ca. 10 Jahren nur noch geringe Anteile der Stärkeklasse 6 anfallen. Bei der naturnahen Variante kommt hinzu, dass Habitatbäume mit großen Brusthöhendurchmessern geschützt und somit der Nutzung entzogen werden.

Tabelle 5: $\quad$ Das mittlere jährliche Nutzungspotenzial der Buche [1000 Efm $\left.a^{-1}\right]$ nach Stärkeklassen für das Szenario „naturnaber Waldbau" in Deutschland

naturnah, Deutschland
\begin{tabular}{lccccccccc} 
Jahr & $1 \mathrm{a}$ & $1 \mathrm{~b}$ & $2 \mathrm{a}$ & $2 \mathrm{~b}$ & $3 \mathrm{a}$ & $3 \mathrm{~b}$ & 4 & 5 & $\geq 6$ \\
\hline $2003-2007$ & 467,2 & $1.181,9$ & 856,6 & $1.126,6$ & 934,6 & 765,1 & $1.434,6$ & $4.769,5$ & $3.428,5$ \\
\hline $2008-2012$ & 370,2 & $1.212,5$ & 916,9 & $1.220,8$ & 964,8 & 783,5 & $2.247,9$ & $4.071,2$ & $1.182,9$ \\
\hline $2013-2017$ & 287,7 & 814,0 & 687,0 & 987,6 & 859,1 & 729,4 & $2.699,6$ & $3.655,6$ & 615,4 \\
\hline $2018-2022$ & 280,4 & 745,9 & 622,7 & 934,9 & 834,0 & 683,2 & $3.224,5$ & $3.844,5$ & 419,1 \\
\hline
\end{tabular}

Tabelle 6: $\quad$ Das mittlere jährliche Nutzungspotenzial der Buche [1000 Efm $\left.a^{-1}\right]$ nach Stärkeklassen für das Szenario ,ertragsorientierter Waldbau“ in Deutschland

\begin{tabular}{l}
\multicolumn{1}{l}{ ertragsorientiert, Deutschland } \\
\begin{tabular}{lccccccccc} 
Jahr & $1 \mathrm{a}$ & $1 \mathrm{~b}$ & $2 \mathrm{a}$ & $2 \mathrm{~b}$ & $3 \mathrm{a}$ & $3 \mathrm{~b}$ & 4 & 5 & $\geq 6$ \\
\hline $2003-2007$ & 499,2 & $1.447,8$ & $1.072,9$ & $1.429,8$ & $1.132,7$ & 778,2 & $3.313,5$ & $4.764,7$ & $3.474,6$ \\
\hline $2008-2012$ & 482,7 & $1.501,6$ & $1.157,4$ & $1.549,4$ & $1.232,8$ & 864,9 & $4.969,9$ & $3.457,7$ & $1.208,9$ \\
\hline $2013-2017$ & 382,8 & $1.158,1$ & 965,7 & $1.395,9$ & $1.233,2$ & 906,9 & $5.914,9$ & $2.493,6$ & 656,1 \\
\hline $2018-2022$ & 337,1 & 952,2 & 783,4 & $1.199,5$ & $1.152,6$ & 749,6 & $7.099,6$ & $1.994,7$ & 416,7 \\
\hline
\end{tabular}
\end{tabular}




\section{Regionale Validierung und Kalibrierung der Durchmesser- zuwachsprognose}

Abschließend soll skizziert werden, wie Großrauminventuren zukünftig dazu verwendet werden können, Wachstums- und damit Holzaufkommensprognosen $\mathrm{zu}$ überprüfen und gegebenenfalls deren Genauigkeit zu erhöhen. Großrauminventuren sind durch ihre Flächenrepräsentativität insbesondere für eine Überprüfung der regionalen Gültigkeit von Wachstumssimulatoren geeignet. Auf Grundlage der beobachteten Zuwächse im Zeitraum zwischen BWI 1 (1987) und BWI 2 (2002) erfolgte die Beurteilung einer Prognose, die mit dem WaldPlaner für denselben Zeitraum durchgeführt ${ }^{3}$ wurde. Dabei steht nach der ersten Folgeinventur (BWI 2) erstmals für den gesamten Bereich der alten Bundesländer eine Vergleichsdatenbasis zur Verfügung, die eine Überprüfung der Prognose unter dem speziellen Aspekt ihrer räumlichen Gültigkeit ermöglicht. Die Validierung wurde mit Hilfe eines speziellen verallgemeinerten additiven Regressionsmodells (WOOD 2006) durchgeführt. Der verwendete Ansatz ermöglicht es, potenzielle Verzerrungen der Prognose auch in Abhängigkeit von der geographischen Lage zu quantifizieren. Als Verzerrung wird eine systematische Unter- und Überschätzung der beobachteten Zuwachswerte bezeichnet. Dabei wurden für die Buche u. a. sowohl der Effekt der geographischen Lage als auch der Seehöhe als signifikant identifiziert. Eine Verzerrung in Abhängigkeit von der geographischen Lage und der Seehöhe kann dahingehend interpretiert werden, dass die derzeitige Parametrisierung des Durchmesserzuwachsmodells auf Basis von ertragskundlichen Versuchsflächen keine Berücksichtigung von räumlichen Wachstumstrends oder gar einer Wechselwirkung derartiger Trends mit der Seehöhe ermöglicht. Dies war auch zu erwarten, da das Durchmesserzuwachsmodell an ertragskundlichen Versuchsflächendaten parametrisiert wurde, die eine Beschreibung von regionalen und durch die Höhenlage beeinflussten Wachstumsunterschieden nur bedingt zulassen. Durch die Identifizierung der unterschiedlichen Einflussgrößen und die Quantifizierung ihrer Effekte auf die Verzerrung, bietet das verwendete Regressionsmodell jedoch die Möglichkeit für eine Verzerrungskorrektur. Anhand der Zielgröße „summarischer periodischer Grundflächenzuwachs 1987 - 2002“ wird die starke Verbesserung der Prognose durch die Verzerrungskorrektur dargestellt. Bei einer stratenweisen Betrachtung der prozentualen Abweichungen von Beobachtung und Prognose nach räumlichen Einheiten (Bundesländer, ausgewählte Wuchsgebiete) wird die Erhöhung der Prognosegüte für die meisten der Bezugsgebiete deutlich (s. Abb. 10).

Zukünftig werden durch die sich ständig erweiternde Datenbasis sowohl der ertragskundlichen Versuchsflächen als auch vor allem der Bundeswald- und

3 Die ausführliche Beschreibung der Kalibrierung ist dem Beitrag „Validierung der Durchmesserzuwachsprognose des Wachstumssimulators BWINPro 7.0 für Fichte und Buche für den Bereich der alten Bundesländer“" (SCHMIDT u. HANSEN, 2007) zu entnehmen. 
Betriebsinventuren biometrische Modelle für das Durchmesser- und Höhenwachstum entwickelt werden können, die regionale Wachstumsunterschiede direkt berücksichtigen, so dass nachgeschaltete Verzerrungskorrekturen nicht mehr notwendig sein werden. Bis dahin bietet das skizzierte Vorgehen einen pragmatischen Ansatz, um die Prognosegüte in der Holzaufkommensmodellierung speziell bei regional differenzierten Auswertungen zu erhöhen.

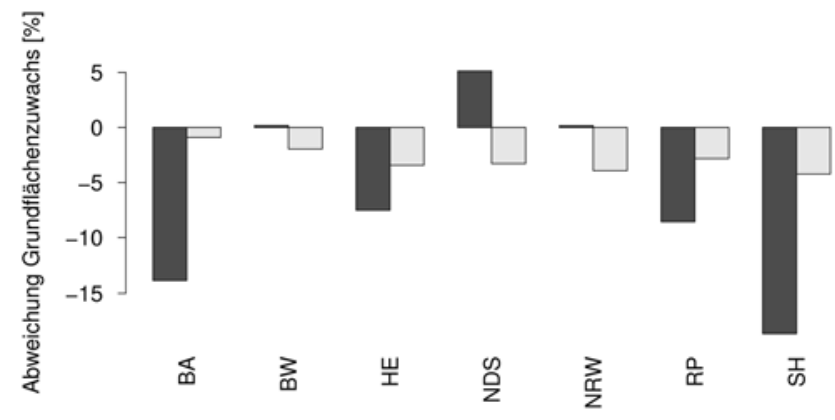

Bundesland

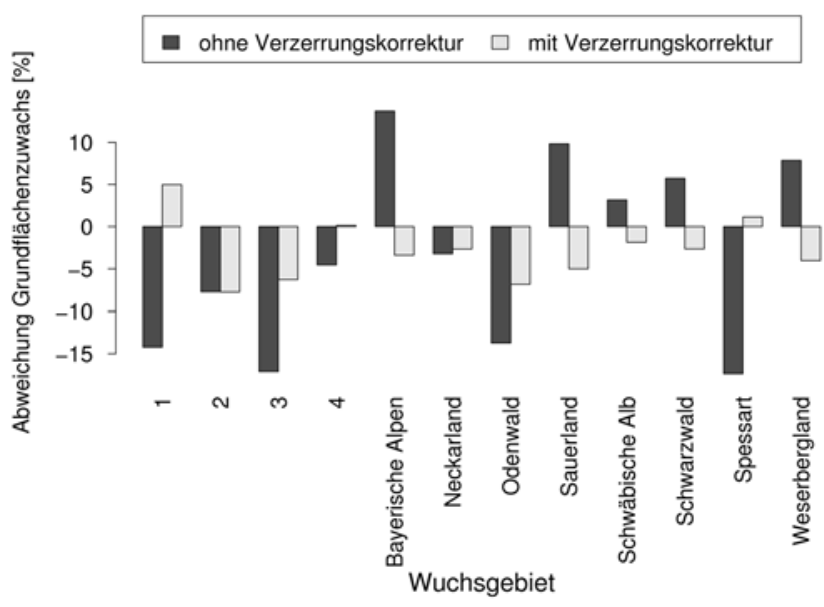

Abbildung 10: Abweichungen des prognostizierten periodischen Grundflächenzuwachses (1987 - 2002) in Prozent der beobachteten Werte der BWI mit und obne nachgeschaltete Verzerrungskorrektur für die Westdeutschen Bundesländer (oben) und ausgewählte Wuchsgebiete (unten) 1: Frankenalb und Oberpfälzer Jura, 2: Oberrbeinisches Tiefland und RheinMain-Ebene, 3: Schleswig-Holstein Ost, 4: Südwestdeutsches Alpenvorland) 


\section{Literatur}

AlBERT, M. (2000): Ein funktionaler Höhenergänzungsalgorithmus für Einzelbaumwachstumsmodelle. Tagungsbericht der Sektion Ertragskunde DVFFA (Kaiserslautern), 32-43

Albert, M. u. GuericKe, M. (2003): Zur Prognose waldbaulicher Behandlungsvarianten in Mischbeständen mit einzelbaumbasierten Durchforstungsmodellen. Forstarchiv 74, 79-89

Albert, M. u. HANSEN, J. (2007): Ein Entscheidungsunterstützungssystem für die multifunktionale Forstplanung auf Landschaftsebene. Forst und Holz 62, 14-18

Biging, G.S. u. DOBBERTin, M. (1992): A comparison of distance-dependent competition measures for height and basal area growth of individual conifer trees. Forest Sciences 38, 360-377

BÖsCH, B. (1995): Ein Informationssystem zur Prognose des künftigen Nutzungspotenzials. Forst und Holz 50, 19

BUNDESMINISTERIUM FÜr ERNÄHRUNG, LANDWIRTSCHAFT UND VERBRAUCHERSCHUTZ (BMELV) (2004): HAM - Das Wichtigste in Kürze - www.bundeswaldinventur.de/enid/ 0522e2b28521f1 f349eafd7173633096,0/9l.html

BUNDESMINISTERIUM FÜR VERBRAUCHERSCHUTZ, ERNÄHRUNG UND LANDWIRTSCHAFT (BMVEL) (2004): Die zweite Bundeswaldinventur. Das Wichtigste in Kürze. BMVEL Bonn, 87 S.

DieTER, M. u. ENGLERT, H. (2005): Gegenüberstellung und forstpolitische Diskussion unterschiedlicher Holzeinschlagsschätzungen für die Bundesrepublik Deutschland. Institut für Ökonomie der Bundesforschungsanstalt für Forst- und Holzwirtschaft, Hamburg, Arbeitsbericht 2005/2, 10 S., http://www.bfafh.de/bibl/pdf/iii_05_02.pdf

DuDA, H.A.A. (2006): Vergleich forstlicher Managementstrategien. Umsetzung verschiedener Waldbaukonzepte in einem Waldwachstumssimulator. Dissertation, Georg-August-Universität Göttingen, Books on Demand GmbH, Norderstedt, $182 \mathrm{~S}$.

DúRSKŶ, J. (2000): Einsatz von Waldwachstumssimulatoren für Bestand, Betrieb und Großregion. Habilitationsschrift an der Forstwissenschaftlichen Fakultät der Technischen Univ. München, Freising-Weihenstephan, $223 \mathrm{~S}$.

HANSEN, J. (2006): Der WaldPlaner - Ein System zur Entscheidungsunterstützung in einer nachhaltigen Forstwirtschaft. In: Degenhardt, A. u. Wunn., U. (Hrsg.): Sammlung der Beiträge von der 18. Jahrestagung der Sektion Forstliche Biometrie und Informatik des DVFFA vom 25.27.09.2006 in Trippstadt. Die Grüne Reihe, 112-119

KÄNDLER, G.; BÖsCH, B. u. SCHMIDT, M. (2005): Wesentliche Ergebnisse der zweiten Bundeswaldinventur in Baden-Württemberg - Rückblick und Ausblick. Forst und Holz 60, 45-49

LWF (2005): Holzaufkommensprognose für Bayern. Bayrische Landesanstalt für Wald und Forstwirtschaft (LWF), LFW Wissen 50, Freising, $73 \mathrm{~S}$.

NAGEL, J. (1996): Anwendungsprogramm zur Bestandesbewertung und zur Prognose der Bestandesentwicklung. In: Hempel, G. (Hrsg.): Deutscher Verband Forstlicher Forschungsanstalten. Sektion Forstliche Biometrie und Informatik. Tagung (8; 1995; Tharandt) - Ljubljana. Biotechnische Fakultät, Abteilung für die Forstwirtschaft, 133-141

NAGEL, J. (1999): Konzeptionelle Überlegungen zum schrittweisen Aufbau eines waldwachstumskundlichen Simulationssystems für Nordwestdeutschland. Schriften aus der Forstlichen Fakultät der Universität Göttingen und der Nieders. Forstl. Versuchsanstalt, Band 128, J.D. Sauerländer's Verlag, Frankfurt a.M., $122 \mathrm{~S}$.

Nagel, J.; Albert, M. u. Schmidt, M. (2002): Das waldbauliche Prognose- und Entscheidungsmodell BWINPro 6.1 - Neu-Parametrisierung und Modellerweiterungen. Forst und Holz 57, 486-493

NAgEL, J.; DudA, H. u. HANSEN, J. (2006): Forest Simulator BWINPro7. Forst und Holz 61, 427-429

NiEDERSÄCHSISCHE LANDESFORSTVERWALTUNG (1991): Langfristige Ökologische Waldentwicklung in den Landesforsten. Programm der Landesregierung, Niedersächsische Landesregierung Hannover, $49 \mathrm{~S}$. 
POlLey, H.; SAsSe, V. u. ENGLERT, H. (1996): Entwicklung des potentiellen Rohholzaufkommens bis zum Jahr 2020 für das Gebiet der Bundesrepublik Deutschland. Mitteilungen der Bundesforschungsanstalt für Forst- und Holzwirtschaft NR. 183, Wiedebusch, Hamburg: 266 S.

Pretzsch, H. (2001): Waldwachstumsforschung. Ihre Bedeutung für die Nutzung und den Schutz von Waldökosystemen. Beitr. Forstwirtsch. u. Landsch.ökol. 35 (3): 138-144

PreTZSCH, H.; KAHN, M. u. ĎURSKÝ, J. (1998): Stichprobendaten für die Entwicklungsprognose und die Nutzungsplanung. AFZ/Der Wald 25: 1552-1558

SCHMiDT, M. (2001): Prognosemodelle für ausgewählte Holzqualitätsmerkmale wichtiger Baumarten. Dissertation, Fakultät für Forstwissenschaften und Waldökologie der Universität Göttingen (Selbstverlag): $309 \mathrm{~S}$.

SChMidt, M. u. HANSEN, J. (2007): Validierung der Durchmesserzuwachsprognose des Wachstumssimulators BWINPro für Fichte und Buche für den Bereich der alten Bundesländer. Tagungsband der Jahrestagung der Sektion Ertragskunde im DVFFA vom 21.-23.05.2007 in AlsfeldEudorf, 164-179

Schmidt, M.; NAgel, J. u. BöCKmann, Th. (2005): The Use of Tree Models for Silvicultural Decision Making. In: Hasenauer, H. (ed.). (2005): Sustainable Forest Management, Growth Models for Europe. Springer, Berlin, Heidelberg, New York: 389 S.

SodtKe, R.; Schmidt, M.; FAbrika, M.; NAgel, J.; ĎurskÝ, J. u. Pretzsch, H. (2003): Anwendung und Einsatz von Einzelbaummodellen als Komponenten von entscheidungsunterstützenden Systemen für die strategische Forstbetriebsplanung. Forstarchiv 75, 51-64

SpellmanN, H. u. SCHMidT, M. (2003): Massen-, Sorten- und Wertertrag der Fichte in Abhängigkeit von der Bestandesbehandlung. Forst und Holz 58, 412-419

SpellmanN, H.; NAgEL; J. u. BöCKMAnN, T. (1999): Summarische Nutzungsplanung auf der Basis von Betriebsinventurdaten. Allgemeine Forst- und Jagdzeitung 170, 122-128

STERBA, H. (1996): Holzaufkommen in Österreich. Die Sägeindustrie Österreichs (CD und Begleittext), Wirtschaftskammer Österreich, Wien

Wood, S.N. (2006): Generalized additive models: an introduction with R. Chapman \& Hall/CRC, Boca Raton, $391 \mathrm{~S}$.

Zentrale MarkT- und Preisberichtsstelle GmbH (ZMP) (2007): ZMP-Marktbilanz Forst und Holz 2007, Bonn

Korrespondierender Autor:

Jan Hansen

Nordwestdeutsche Forstliche Versuchsanstalt

Grätzelstr. 2

37079 Göttingen

E-Mail: Jan.Hansen@nw-fva.de

URL: www.nw-fva.de

Prof. Dr. Jürgen Nagel

Dr. Matthias Schmidt

Prof. Dr. Hermann Spellmann

Nordwestdeutsche Forstliche Versuchsanstalt 


\title{
Verwertung und Verwendung von Buchenholz
}

\author{
Utilisation of beech wood
}

František Hapla und Holger Militz.

\section{Zusammenfassung}

Sowohl die ökologisch ausgerichteten waldbaulichen Konzepte als auch die Ergebnisse der zweiten Bundeswaldinventur machen deutlich, dass die deutsche Forstund Holzwirtschaft zukünftig mit einem steigenden Aufkommen an Buchenholz konfrontiert sein wird. Zumindest beim Verkauf von Buchenwertholz dürfte sich eine holzproduktorientierte Rundholzsortierung durchsetzen. Die Kenntnis der Holzeigenschaften des weißen sowie des rotkernigen Buchenholzes ist eine wichtige Voraussetzung für die Weiterverarbeitung von Buchenstammholz. Vor dem Hintergrund der qualitätsorientierten Ausbeute an Furnier- und Schnittholzprodukten aus rotkernigen Buchen spielt die optimale Zielstärke bei der Bewirtschaftung der Buche eine wichtige Rolle. Einige Forschungsergebnisse ergaben diesbezüglich für die Produktion von Furnier und Schnittholz die höchste Wertschöpfung bei Buchenstammholz der Stärkeklasse 5 nach der noch gültigen Handelsklassensortierung. Befragungen verschiedener buchenholzverarbeitender Furnier- und Sägewerke bestätigen diese Forschungsergebnisse. Heutzutage werden aus dem von Natur aus nicht dauerhaften Buchenholz zahlreiche Produkte erzeugt, die allerdings fast ausschließlich im Innenbereich ihre Verwendung finden. Ein wesentlicher Aspekt der zukünftigen Buchenholzverwendung betrifft die Verbesserung der Qualität und des Absatzes von Buchenholzprodukten. Dies 
schließt vor allem neuartige innovative Verfahren der Holzmodifizierung ein, die es ermöglichen, Buchenholzprodukte verstärkt im Außenbereich einzusetzen.

Stichworte: Holzqualität und Holzeigenschaften, Rotkern, Ausbeute an Furnier und Schnittholz, innovative Holzprodukte, Holzmodifizierung, Verwendungsbereiche

\begin{abstract}
The ecologically based silvicultural objectives of forest management as well as the results of the second national forest inventory clearly show that, in future, the supply of beech timber to the forest and wood industries in Germany will increase. Certainly, for the sale of high-grade beech a wood products based log grading system will assert itself. Knowledge of the wood properties of the white and red beech heartwood will be essential for the processing of beech logs. Target diameters play an important role in the management of beech to optimise the yield of high quality wood from red heartwood for the production of veneer and sawn timber. Some research shows that, for the production of veneer and sawn timber, the greatest value is derived from the utilisation of beech in diameter class 5 under the current log grading system. A survey of various veneer mills and sawmills processing beech confirms these results. Today, numerous products are made from beech, a non-durable wood by nature, yet these are designed almost exclusively for interior use. For the utilisation of beech in the future, an improvement in wood quality and in the marketing of beech products is important. This involves new, innovative methods of wood modification to enhance the suitability of beech for outdoor use.
\end{abstract}

Keywords: wood quality and properties, red heartwood, veneer and sawn timber yield, innovative products, wood modification, wood utilisation

\title{
1 Einleitung
}

Da zwischen den beiden Begriffen Holzverwendung und Holzverwertung nicht nur sprachlich, sondern auch in Fachpublikationen häufig nicht unterschieden wird, ist es sinnvoll an dieser Stelle eine Begriffsabgrenzung vorzunehmen. Die Holzverwertung wird als jede sinnvolle Nutzung von Holz gleich welcher Art, Form oder welchen Veredelungsgrades definiert. Dies schließt Wald-Industrieholz und Industrierestholz sowie Holz für Energiegewinnung mit ein (MOMBÄCHER (Hrsg.) 2003). Aus Sicht der forstwirtschaftlichen Betriebe umfasst die Verwertung des Holzes jedoch verschiedene Arbeitsgänge der Holzwerbung sowie die Vermarktung von Rohholz. Erst dann steht das Rundholz vor seiner handwerklichen oder industriellen Bearbeitung und Verwendung in Form verschiedener Produkte in unterschiedlichen Bereichen. 
Der Einschlag von Buche erreichte im Jahr 2006 in ganz Deutschland ein Volumen von ca. 10 Mio. $\mathrm{m}^{3}$ ohne Rinde (o. R.), was einen Anteil von ca. $16 \%$ am gesamten Einschlag ausmachte (ZMP 2007). Dem Einschlag von Buchen-Stammholz von ca. 2,5 Mio. $\mathrm{m}^{3}$ o. R. im Jahr 2006 stehen fast 7,5 Mio. $\mathrm{m}^{3} \mathrm{o}$. R. geernteten Buchen-Waldindustrieholzes gegenüber. Die forstwirtschaftliche Bedeutung dieser Baumart spiegelt sich außerdem in der Anbaufläche von rund 1,565 Mio. ha wider. Damit stockt die Buche auf etwa 14,8 \% der gesamten Anbaufläche der Wirtschaftswälder (BMVEL 2004). In den letzten Jahren nahm der internationale Handel mit Buchenstammholz deutlich zu. Abgesehen vom turbulenten Asienmarkt der letzten Jahre ist auch ein starker Anstieg der Buchen-Stammholzexporte in Länder der Europäischen Union zu verzeichnen.

\section{Rundholzsortierung}

Vor dem Hintergrund dieser Entwicklung war es von großem Interesse, die Europäische Sortiernorm DIN EN 1316-1 (1997) für Buchenstammholz einem kritischen Vergleich mit der inländischen Handelsklassensortierung für Rohholz zu unterziehen. Insgesamt wurden 42 Starkbuchen der Stärkeklassen 5 bis 7 nach Forst-HKS (Anlage zu \1 der Verordnung über gesetzliche Handelsklassen für Rohholz, 1969), die eine fakultative Verkernung unterschiedlichen Ausmaßes aufwiesen, für die Untersuchung herangezogen.

Die Untersuchungsergebnisse zeigten deutlich, dass die DIN EN 1316-1 zu einer wesentlich schlechteren Qualitätsklassenverteilung der sortierten Buchenstämme führte als die im Vergleich angewandte Forst-HKS mit Zusatzbestimmungen des Landes Niedersachsen. Im Rahmen der vergleichenden Sortierung wurde unter anderem die Frage nach der Praktikabilität der EU-Norm für Buchenstammholz gestellt. Anhand der erzielten Ergebnisse erwies sich die EU-Norm als nicht praxistauglich. Bezüglich der Auswirkung der einzelnen Qualitätsmerkmale auf die Güte- bzw. Qualitätsklassenverteilung der sortierten Buchenstämme kann auf die Veröffentlichung von HAPLA u. STEINFATT (2002) verwiesen werden.

Für einen monetären Vergleich wurden bei der Holzerlöskalkulation die nach Forst-HKS sortierten Güteklassen und Stärkeklassen erzielten Holzerlöse auch für die jeweiligen Qualitätsklassen nach der EU-Norm zugrunde gelegt. Demnach lagen die rein rechnerisch erzielten Erlöse für das Stammholz der Versuchsbuchen nach der EU-Norm ausgehalten um rund $20 \%$ niedriger, da der Anteil an den Qualitätsklassen A und B im Vergleich zur Forst-HKS deutlich kleiner war. Die Untersuchungsergebnisse führten zu folgenden Schlussfolgerungen: Die DIN EN 1316-1 für Buchenrundholz in der vorliegenden Fassung wird sich in der Praxis kaum durchsetzen können. Ein monetärer Kalkulationsvergleich auf der Basis der gleichen aktuellen Holzpreise für das sortierte Buchenstammholz führte auf der Forst-HKS-Seite zu einer höheren Wertschöpfung für den Forstbetrieb. 
Dennoch verliert die Handelsklassensortierung (Forst-HKS) immer mehr an Akzeptanz unter den mit Rundholz handelnden Beteiligten. Gerade für den Buchenstammholzmarkt mit seiner turbulenten Preisentwicklung in den letzten Jahren erwies sich die Sortierung nach der HKS als nicht mehr brauchbar. Inzwischen verwies der Deutsche Holzwirtschaftsrat auf die geplante Aufhebung der Richtlinie 68/89/EWG für die Sortierung von Rohholz auf Grund eines Vorschlags der EU-Kommission vom 29. September 2006. Deutschland ist offensichtlich das einzige Land innerhalb der Europäischen Union, das diese Richtlinie umgesetzt hat. Die Aufhebung der Richtlinie 68/89/EWG führt zur Aufhebung der Forst-HKS. Die Anwendung der Forst-HKS war jedoch nur fakultativ und somit nicht zwingend vorgeschrieben. Dennoch sollte dem Rundholzhandel in Deutschland ein einheitlicher Rahmen hinsichtlich der Dimensionsmessung und Gütesortierung gegeben werden. Die diesbezügliche Diskussion ist bei den betroffenen Akteuren der Forst- und Holzwirtschaft im Gange. Derzeit wird eine neue „Rahmenvereinbarung für den Rohholzhandel in Deutschland“ erarbeitet. Der Arbeitskreis wird durch die Forstliche Versuchs- und Forschungsanstalt BadenWürttemberg koordiniert.

Zukünftig dürfte sich zumindest beim Buchenwertholzverkauf eine holzprodukt- bzw. kundenorientierte Rundholzsortierung, die eine immer größere Akzeptanz seitens der Forstwirtschaft und der Holzindustrie erfährt, durchsetzen. Wie die Buchen-Submissionsverkäufe der jüngsten Zeit zeigten, wurden von internationalen Holzkäufern im Einvernehmen mit den Anbietern eigene Sortierregeln verwendet. Weiterhin muss festgehalten werden, dass bei den Submissionen teilweise hohe Erlöse und Deckungsbeiträge für das hochwertige Buchenstammholz erzielt werden. Allerdings sind die Forstbetriebe gut beraten, wenn sie vor Durchführung von Submissionen genau prüfen, ob der erwartete Mehrerlös auch den verursachten Mehraufwand decken kann. Andernfalls könnte der Freihandverkauf von starkem Buchenstammholz zu einem günstigeren Betriebsergebnis führen (LUDWIG et al. 2006).

\section{Holzeigenschaften}

Für den Buchenstammholzmarkt stellt die fakultative Verkernung der Buche nach wie vor noch einen wesentlichen wertmindernden Faktor dar. Über die Ursachen der Rotkernbildung bei der Buche enthält die Arbeit von HANSKÖTTER (2004) ein solides Literaturkompendium. Im Zuge des ökologisch orientierten Waldbaus wird die Buche in relativ hohen Umtriebszeiten bewirtschaftet. Mit zunehmendem Alter der Bäume nimmt nachweislich auch der Anteil an rotkernigem Buchenholz zu. Dieser Farbkern wird in der Fachliteratur häufig als Rotkern bezeichnet und variiert von Stamm zu Stamm bezüglich seiner Ausprägung, Größe und Form teilweise beachtlich. Auf Submissionen wurden für unverkernte Einzelstämme Spitzenerlöse erzielt. Für rotkernige Sortimente werden häufig deutlich geringere 
Preise bezahlt. Die Absatzkonditionen von rotkernigem Buchenholz haben sich jedoch in den letzten fünf Jahren etwas verbessert. Infolge einer gezielten Marketingstrategie insbesondere bei Möbel- und Fußbodenprodukten, wird eine gestiegene Nachfrage nach Buchen-Rotkern mit seinen teils lebhaften Farbtonausprägungen verzeichnet.

Eine weitere Ursache für den noch moderaten Absatz rotkerniger Produkte ist darin begründet, dass rotkerniges Buchenholz als individuelle Gestaltungsmöglichkeit dem Endverbraucher unlängst noch fast völlig unbekannt war. Weiterhin spielen Vorurteile im Hinblick auf die Holzeigenschaften rotkernigen Buchenholzes eine ernstzunehmende Rolle. Trotz Untersuchungen von WOBST (1969) und MOLNÁR et al. (2001) werden im Vergleich zu unverkerntem Buchenholz dem Buchen-Rotkern immer noch schlechtere physikalische und mechanische Holzeigenschaften nachgesagt. Im Rahmen einer vergleichenden Untersuchung der physikalischen und mechanischen Eigenschaften an unverkerntem und rotkernigem Buchenholz sollten diese Wissenslücken geschlossen werden (KRAL 2004).

In Tabelle 1 sind die Untersuchungsergebnisse zusammengefasst. Die unwesentlich höheren Dichtewerte im Rotkernbereich können mit hoher Wahrscheinlichkeit auf holzanatomische Strukturen zurückgeführt werden. Hieraus resultiert eine etwas höhere Härte im Unterschied zum Splintholz. Sowohl die Quellung als auch die Schwindung in tangentialer Richtung wiesen bei dem Rotkern günstigere Werte auf. Bei der Bruchschlagarbeit wurden höhere Werte im Splintholz festgestellt. Ingesamt jedoch zeigten beide Xylemzonen Splint und gesunder Rotkern ebenbürtige für die Holzverarbeitung und Holzverwendung relevante Eigenschaften. Tatsache ist aber auch, dass die Erfahrungen mit unzureichender UVLichtbeständigkeit sowie die starken Form- und Farbvariationen des rotkernigen Buchenholzes eine schlechtere Nachfrage begünstigten. Hinzu kommt, dass aufgrund der Verthyllung der Gefäße im Rotkernbereich eine schlechtere Imprägnierbarkeit zu erwarten ist. 
Tabelle 1: $\quad$ Mittlere physikealische und mechanische Kennwerte von weißem und rotkernigem Buchenbolz.

\begin{tabular}{|c|c|c|c|c|c|c|}
\hline \multirow{2}{*}{\multicolumn{2}{|c|}{ Holzeigenschaften }} & \multicolumn{2}{|c|}{ Splint } & \multicolumn{2}{|c|}{ Rotkern } & \multirow{3}{*}{$\begin{array}{c}\mathbf{P} \\
0,000\end{array}$} \\
\hline & & \multirow{2}{*}{$\frac{\bar{x}}{0,694}$} & \multirow{2}{*}{$\begin{array}{l}n \\
75\end{array}$} & \multirow{2}{*}{$\frac{\bar{x}}{0,706}$} & \multirow{2}{*}{$\begin{array}{l}n \\
75\end{array}$} & \\
\hline Rohdichte $\left[\rho_{u=12 \%}\right]$ & {$\left[\mathrm{g} / \mathrm{cm}^{3}\right]$} & & & & & \\
\hline Darrdichte $\left[\rho_{u=0 \%}\right]$ & {$\left[\mathrm{g} / \mathrm{cm}^{3}\right]$} & 0,664 & 75 & 0,674 & 75 & 0,000 \\
\hline Schwindung $\left[\beta_{\max }\right]$ & & & 120 & & 120 & \\
\hline$\left[\beta_{\text {radial }}\right]$ & {$[\%]$} & 6,0 & & 6,2 & & \\
\hline$\left[\beta_{\tan \text { gential }}\right]$ & {$[\%]$} & 13,2 & & 11,9 & & \\
\hline$\left[\beta_{\text {longitudinal }}\right]$ & [\%] & 0,3 & & 0,3 & & \\
\hline$\left[\beta_{\text {Volumen }}\right]$ & [\%] & 19,4 & & 18,3 & & \\
\hline Quellung $\left[\alpha_{\max }\right]$ & & & 120 & & 120 & \\
\hline$\left[\alpha_{\text {radial }}\right]$ & {$[\%]$} & 6,0 & & 6,6 & & \\
\hline$\left[\alpha_{\text {tan gential }}\right]$ & {$[\%]$} & 15,3 & & 14,4 & & \\
\hline$\left[\alpha_{\text {longitudinal }}\right]$ & {$[\%]$} & 0,5 & & 0,5 & & \\
\hline$\left[\alpha_{\text {Volumen }}\right]$ & {$[\%]$} & 22,5 & & 22,6 & & \\
\hline Janka Härte $[J H]$ nach Is & & & 24 & & 26 & \\
\hline$J H_{\text {parallel }}$ & {$\left[\mathrm{N} / \mathrm{mm}^{2}\right]$} & 66,2 & & 74,3 & & 0,000 \\
\hline$J H_{\text {senkerecht }}$ & {$\left[\mathrm{N} / \mathrm{mm}^{2}\right]$} & 49,7 & & 55,3 & & 0,001 \\
\hline Druckfestigkeit $\left[\delta_{\text {parallel }}\right]$ & {$\left[\mathrm{N} / \mathrm{mm}^{2}\right]$} & 64,4 & 129 & 63,6 & 108 & \\
\hline Biegefestigkeit [MOR] & {$\left[\mathrm{N} / \mathrm{mm}^{2}\right]$} & 96,6 & 160 & 97,3 & 73 & \\
\hline \multicolumn{2}{|l|}{ Elastizitätsmodul [MOE] } & & & & & \\
\hline$M O E_{\text {dynamisch }}$ & {$\left[\mathrm{N} / \mathrm{mm}^{2}\right]$} & 13.337 & 160 & 13.660 & 73 & 0,031 \\
\hline$M O E_{\text {statisch }}$ & {$\left[\mathrm{N} / \mathrm{mm}^{2}\right]$} & 12.027 & 160 & 12.135 & 73 & \\
\hline Bruchschlagarbeit $[\omega]$ & {$\left[\mathrm{kJ} / \mathrm{m}^{2}\right]$} & 77,9 & 91 & 68,3 & 58 & 0,016 \\
\hline
\end{tabular}

$\bar{x}$ : arithmetisches Mittel; n: Stichprobenumfang der DIN-Proben; P: signifikante Differenzen, Quelle: KRAL (2004) 


\section{Furnierausbeute von rotkernigen Buchen in Zielstärke}

Die wichtigste einheimische Laubholzart der deutschen Furnierindustrie ist die Buche mit einem Anteil von $28 \%$ gefolgt von der Eiche mit $19 \%$ und dem Ahorn mit $17 \%$ (ANONYMUS 2006). Das helle, gut zu bearbeitende Holz der Buche erfreut sich bei der Holzindustrie, insbesondere bei der Möbelindustrie einer hohen Nachfrage. Dank der modernen Furniertechnik sind die Furnierverarbeiter heute in der Lage, Produkte mit farblich homogenen Buchen-Furnieroberflächen auf den Markt zu bringen. Daher erobert die Buche stetig Marktanteile als Oberflächenholzart in der Holztürproduktion. Da die Diskussion über die Auswirkung der Zielstärkennutzung der Buche sowie des Rotkerns auf die Wertschöpfung der Buchen-Holzprodukte innerhalb der Forst- und Holzwirtschaft weiterhin anhält, wurde eine Untersuchung durchgeführt, mit dem Ziel den Produktionsablauf vom Buchenrundholz in Zielstärke bis zum verkaufsfähigen Messerfurnier-Produkt aufzuzeigen. Unter Erfassung aller die Ausbeute reduzierenden Produktionsschritte wurde die Messerfurnier-Ausbeute von Starkbuchen ermittelt. Die erzeugten Messerfurnierpakete wurden schließlich nach verwendungsorientierten Qualitätsklassen sortiert und preislich bewertet. Bezogen auf das Untersuchungsmaterial ergaben die Buchen der Stärkeklasse 5 sowohl für die anbietende Forstwirtschaft als auch für die abnehmende Furnierindustrie eine deutlich höhere Wertschöpfung als die dimensionsstärkeren Buchen der Stärkeklasse 6 (HAPLA et al. 2002).

Das Produktionsziel des Furnierwerkes war, eine höchstmögliche Ausbeute an Messerfurnier aus dem eingesetzten Rundholz zu erzeugen. Während die Holzverluste nach dem Kappen 1,4\% betrugen, lagen diese nach den Verarbeitungsschritten Entrinden, Zuricbten und Hobeln bei 16,7\%. Weitere Holzverluste wurden infolge der Produktionsschritte Messern, Trocknen und Formatieren mit insgesamt $32,1 \%$ kalkuliert. Für das im Werk vermessene Rundholzvolumen wurde damit ein Gesamtverlust von 50,2\% errechnet. Das bei der Furnierproduktion angefallene Restholz (Kappstücke, Rinde, Schwarten und Späne) wurde werksintern energetisch verwertet. Die Restbretter aus dem Produktionsschritt Messern wurden als Verpackungsmaterial zur Verstärkung der Versandpaletten eingesetzt. Aus den insgesamt 29,027 $\mathrm{m}^{3}$ Buchen-Rundholz m. R. (alle Stämme wiesen Rotkern auf) wurden im Rahmen dieser Untersuchung $23.562 \mathrm{~m}^{2}$ Messerfurnierfläche hergestellt, was einer Messerfurnier-Ausbeute von 49,8\% entspricht. Bezogen auf das gesamte Untersuchungskollektiv der Wertholzbuchen konnten aus einem Kubikmeter Buchen-Rundholz im Durchschnitt $841 \mathrm{~m}^{2}$ Messerfurnier mit einer Dicke von $0,55 \mathrm{~mm}$ erzeugt werden. Die Messerfurnierpakete der Buchen-Versuchsstämme wurden schließlich nach produktionsorientierten Qualitätsklassen sortiert und preislich bewertet. Die Qualitätsfurniere wurden dabei nach Verwendungsbereichen wie Innenausbau, Möbel, Türen, Paneele und Kantenmaterial (Streifer) eingeteilt, wobei diese Furnier-Qualitätsklassen weiter untergliedert wurden. Etwa $10 \%$ der Messerfurniere wurden als Blindfurniere und sog. Nullpakete von geringerer Qualität ausgewiesen. Der größte Sortieranteil mit 24,2 \% an der Gesamt-Messer- 
furnierfläche entfiel auf die preislich hoch bewertete Furnier-Qualitätsklasse Schlafzimmer, die dem Innenausbau zugeordnet ist (HAPLA et al. 2002).

Die technisch bedingten Holzverluste betragen beim jetzigen Stand der Messerfurnier-Technik etwa ein Drittel. Die über dieses Maß hinausgehenden Verluste werden durch „Wuchsmerkmale“ verursacht. Zu den letzteren zählt insbesondere der fakultative Buchen-Farbkern, der bei allen Buchen-Versuchsstämmen auftrat. Ein wesentlicher Rotkern-Volumenanteil fällt mit dem nach dem Messern entstehenden Restbrett aus der Produktion heraus. Somit trug der Rotkern zur Reduzierung der Messerfurnier-Ausbeute bei, jedoch in einem geringeren Ausmaß als ursprünglich angenommen wurde. Die Festlegung der Zielstärke bei der Buche wird vor allem von der vermuteten Wert- und der RotkernEntwicklung des Einzelbaumes beeinflusst. Im Rahmen der eigenen Untersuchungen ergaben die sechs Versuchsstämme der Stärkeklasse 6 im Durchschnitt $825 \mathrm{~m}^{2} / \mathrm{m}^{3}$ Messerfurnier-Ausbeute. Im Vergleich dazu lag die Ausbeute an Messerfurnier bei den anderen sechs Versuchsstämmen der Stärkeklasse 5 im Durchschnitt bei $914 \mathrm{~m}^{2} / \mathrm{m}^{3}$ und damit um $10 \%$ höher als bei den deutlich stärkeren Buchen. Der errechnete mittlere Rotkern-Volumenanteil lag bei den stärkeren Buchen (Stärkeklasse 6) deutlich höher. Beim Verkauf der Versuchsstämme an das Furnierwerk erzielte die Forstwirtschaft für die Buchen der Stärkeklasse 5 einen deutlich höheren Durchschnittserlös als für die Buchen der Stärkeklasse 6. Bezogen auf das Untersuchungsmaterial ergaben die Buchen der Stärkeklasse 5 sowohl für die anbietende Forstwirtschaft als auch für die abnehmende Furnierindustrie eine deutlich höhere Wertschöpfung als die dimensionsstärkeren Buchen der Stärkeklasse 6.

Die Buche ist die wichtigste Holzart für die Herstellung von Schälfurnieren. Genaue Angaben über Produktionsmengen an Buchen-Sperrholz sind nicht verfügbar. Die Sperrholzindustrie war ein Kernsegment der Verarbeitung von stärkerem Buchenstammholz. In den letzten Jahren jedoch wurden mehrere Sperrholzwerke geschlossen. Derzeit werden in Deutschland jährlich zwar ca. 1 Mio. $\mathrm{m}^{3}$ Sperrholz verbraucht, es werden jedoch weniger als $20 \%$ davon im Lande produziert (ANONYMUS 2007). Die wenigen verbliebenen Sperrholzhersteller konzentrieren sich auf spezielle Produkte wie z. B. Formsperrholz. Vor dem Hintergrund der Ergebnisse der 2. Bundeswaldinventur wird ein Verbundprojekt in Zusammenarbeit mit drei deutschen Sperrholzherstellern bearbeitet. Das Ziel dabei ist eine verstärkte Nutzung des Buchenstarkholzes. Des Weiteren soll der Einfluss der altersbedingten fakultativen Verkernung des Buchenstarkholzes auf die Ausbeute und Qualität der Schälfurnierprodukte untersucht werden (ADERHOLD et al. 2007). Insgesamt betrachtet, sank die Furnierproduktion in Deutschland in den letzten Jahren unter die Marke von $200 \mathrm{Tsd} . \mathrm{m}^{3}$ pro Jahr und zugleich erfolgte eine Kapazitätsverlagerung nach Osteuropa. 


\section{Schnittholzausbeute von rotkernigen Buchen in Zielstärke}

Im Jahre 2006 haben deutsche Sägewerke mit einem Jahreseinschnitt von mehr als fünftausend Kubikmeter Rundholz ca. 1,2 Mio. $\mathrm{m}^{3}$ Laubschnittholz produziert (ZMP 2007). Die deutsche Sägeindustrie, hauptsächlich die reinen Laubholzsägewerke, sind die größten Abnehmer des Buchenstammholzes. Die Auswirkungen des Rotkerns auf die Ausbeute von hochwertigem Buchen-Schnittholz wurden von HAPLA u. OHNESORGE (2005) untersucht. Aufbauend auf Erfahrungen von Buchenholz-Sägewerken wurde ein Kalkulationsmodell entwickelt, mit dem die Simulation der Schnittholzausbeute in Abhängigkeit vom Rotkernanteil am Zopfdurchmesser bei drei definierten Einschnittarten (Rund-, Tangential- und Modelschnitt) erfolgte. Der Vergleich der Einschnittarten zeigte, dass bei einem kleineren Rotkernanteil der Rundschnitt unter den definierten Sortierungsvorgaben im Vorteil ist. Liegt der Rotkernanteil jedoch über $20 \%$, erzielen die Einschnittarten Tangential- und Modelschnitt eine höhere kernfreie Schnittholzausbeute und damit einen höheren Schnittholzerlös. Die für den Tangentialschnitt durchgeführte Deckungsbeitragskalkulation ergab einen maximal tolerierbaren Kernanteil am Zopfdurchmesser des Einzelstammes, der zwischen $65 \%$ und $75 \%$ liegt. In diesem Kernteilbereich decken die Schnittholzerlöse nicht mehr die Kosten, die durch Produktherstellung anfallen. Das Wissen um den Einfluss des Rotkerns auf die Schnittholzausbeute stellt eine wichtige Argumentationsgrundlage für Verkaufsverhandlungen dar und ermöglicht es kundenspezifisch Buchenstammholz anzubieten. Für die Produktion von hochwertigem Schnittholz werden unter ökonomischen Gesichtspunkten ein qualitätsorientierter Einschnitt sowie eine differenzierte und kundenorientierte Schnittholzsortierung immer wichtiger. Ein namhafter Buchen-Schnittholzhersteller verarbeitet mit großem Erfolg vor allem rotkernige Buche. Wesentlich ist dabei das große Sortimentsangebot an verschiedenen Qualitäten und Abmessungen.

\section{Innovative Holzprodukte}

Unbehandelt verliert das rotkernige Buchenholz im Laufe der Zeit an Farbintensität und bleicht aus. Als Auslöser werden photochemische Reaktionen im Holz vermutet, die durch das UV-Licht hervorgerufen werden. Da das Holz der Buche bislang ausschließlich im Innenbereich Verwendung findet, liegt im Bereich der Holzfarbe ein wichtiger Schwerpunkt, den es abzusichern gilt, um das rotkernige Holz auf dem Markt besser zu positionieren. Auch die Verklebbarkeit von rotkernigem Buchenholz stellt einen sehr wichtigen Aspekt in der Weiterverarbeitung und Verwendung von Buchen-Rotkern dar. Zu diesen holztechnologischen Fragen erfolgten bereits orientierende Untersuchungen (HAPLA u. MILITZ 2004, OHNESORGE et al. 2007a). 
Da sowohl das helle als auch das rotkernige Buchenholz nur eine geringe Pilzresistenz aufweisen und unter Feuchtigkeitseinfluss wenig dimensionsstabil sind, erfolgte bislang eine Verwendung unbehandelter Buchenholzprodukte ausschließlich im Innenbereich. Neue innovative Modifizierungsprozesse sind jedoch in Entwicklung, wodurch sich das Einsatzspektrum der Buche im Außenbereich erweitern lässt.

Im Rahmen einer neueren Untersuchung konnte gezeigt werden, dass sich mittels eines entwickelten Vernetzungsverfahrens Massivholz, Furniere und Sperrholzplatten aus Buchenholz mit einer deutlich verbesserten Witterungsstabilität und biologischer Dauerhaftigkeit herstellen lassen. Das vernetzte Buchensperrholz wies im Rahmen der Bewitterungsversuche eine sehr gute Formstabilität, eine deutlich verringerte Rissbildung und Oberflächenrauhigkeit auf (WEPNER $2005 \mathrm{u}$. 2006, KRAUSE et al. 2008). In weiterführenden Untersuchungen an Buchenholz wurden vernetzte Produkte hergestellt, die eine sehr gute Dimensionsstabilität sowie eine biologische Resistenzklasse 1 bis 2 gemäß DIN EN 350-1 (1994) aufwiesen. Während die Härte der modifizierten Buchenholzprodukte um das Zweibis Dreifache verbessert werden konnte, blieb die Optik des modifizierten Holzes gegenüber dem unbehandelten Buchenholz unverändert (KRAUSE 2006). Diese für Buchenholz vollkommen neuartigen Eigenschaften führen zu neuen Produkten und Absatzmöglichkeiten, die sich die Holzindustrie bereits zunutze macht (www.becker-kg.de; http://belmadur.de).

Als Produktalternative wurde die Verwendung rotkernigen Buchenholzes für die thermische Modifizierung untersucht. Je nach Behandlungsstufe erhöhten sich die Dimensionsstabilität und die Pilzresistenz deutlich, jedoch begleitet von einer Reduzierung der Holzdichte sowie der Festigkeitseigenschaften (OHNESORGE et al. 2007b, MiLITZ 2008). Verschiedene Eigenschaften wie Quellung und Schwindung, kapillare Wasseraufnahme und $\mathrm{pH}$-Wert haben auch Einfluss auf die Verklebung von thermisch behandeltem Holz. SCHNIDER et al. (2008) untersuchten Festigkeitseigenschaften von mit verschiedenen Klebstoffen verklebten thermisch vergüteten Buchenproben.

Die möglichen Einsatzbereiche des vergüteten Buchenholzes sind vielfältig. Aufgrund der besseren biologischen Dauerhaftigkeit ist eine Verwendung im Außenbereich (Lärmschutzwand, Terrassendeck oder Gartenmöbel) möglich. Die sehr gute Dimensionsstabilität sowie der ansprechende Farbton von thermisch behandeltem Buchenholz machen eine Verwendung als Design-Möbel oder im Fußbodenbereich interessant (OHNESORGE et al. 2007b). Im Rahmen eines abgeschlossenen EU-Projektes wurden das Farbverhalten von Buchen-Rotkern nach Dämpfung, Trocknung und UV-Lichtbehandlung sowie Keilzinkung, Verklebbarkeit und Festigkeit von Buchen-Brettschichtholz untersucht. Die Studie beinhaltet auch Versuche zur thermischen Behandlung von Buchen-Kernholz (SEELING et al. 2007). 


\section{Verwendungsbereiche}

In Tabelle 2 sind die wesentlichen Verwendungsbereiche des Buchenholzes in Abhängigkeit von der Stammdimension zusammengestellt.

Tabelle 2: $\quad$ Verwendung von Buchenholz in Abhängigkeit von Stammdimension und Holzqualität

\begin{tabular}{|c|c|c|}
\hline Dimensionen & Qualität & Mögliche Produkte \\
\hline \multirow{2}{*}{$\begin{array}{l}\text { Starkholz } \\
\text { Stkl. } \geq 5\end{array}$} & Hochwertig & $\begin{array}{l}\text { Messerfurnier (Deckfurnier) } \\
\text { Schälfurnier, Sperrholz, Formteile, } \\
\text { Multiplexplatte, Panzerholz } \\
\text { Möbel und Innenausbau, } \\
\text { Musikinstrumente }\end{array}$ \\
\hline & Mittlere Qualität & $\begin{array}{c}\text { Treppen, Gestellware, Wagenbau } \\
\text { Massivholzplatten, Innenausbau, } \\
\text { Blindholz } \\
\text { Marknahes Holz (Palette, Brennholz) }\end{array}$ \\
\hline \multirow[t]{2}{*}{$\begin{array}{l}\text { Mittelstarkes Holz } \\
\text { Stkl. 2b - } 4\end{array}$} & Hochwertig & $\begin{array}{c}\text { Möbel und Innenausbau, } \\
\text { Massivholzplatten } \\
\text { Parkett und Fußböden } \\
\text { Gestellware, Haushalts- und Spielwaren } \\
\text { Turn- und Sportgeräte } \\
\text { Treppen, Bugholz, Werkzeugstiele } \\
\text { Eisenbahnschwellen }\end{array}$ \\
\hline & Mittlere Qualität & $\begin{array}{l}\text { Parkett, Fußböden, Holzpflaster } \\
\text { Palette, Verpackungen, Brennholz }\end{array}$ \\
\hline $\begin{array}{l}\text { Schwachholz } \\
\text { Stkl. } \leq 2 \mathrm{a}\end{array}$ & $\begin{array}{c}\text { Wald- } \\
\text { Industrieholz }\end{array}$ & $\begin{array}{c}\text { MDF (10\% Anteil) } \\
\text { Spanplatte (10\% Anteil), OSB } \\
\text { Zellstoff und Papier } \\
\text { Holzkohle, Brennholz, Hackschnitzel, } \\
\text { Pellets }\end{array}$ \\
\hline
\end{tabular}

Stkl.: Mittenstärkensortierung nach HKS; MDF: Mitteldichte Faserplatte; OSB: Oriented Strand Board 
Noch im 19. Jahrhundert wurden große Mengen von Buchenholz für Pottaschegewinnung (für Glas- und Seifenherstellung) benötigt. Bis in die Mitte des 20. Jahrhunderts galt die Buche in Europa als nicht wertvolle Holzart und fand hauptsächlich als Brennholz und in Form von imprägnierten Bahnschwellen ihre Verwendung. Doch dann machte die Buche eine steile Karriere. Heutzutage zählt die Buche zu den am vielseitigsten verwendeten Holzarten unter den einheimischen Nutzhölzern. Die gute Rohstoffverfügbarkeit sowie die günstigen Bearbeitungseigenschaften erschlossen der Buche viele Verwendungsmöglichkeiten (vgl. Tab. 2).

Die Möbelproduktion stellt den mengenmäßig größten Verwendungsbereich für stärker dimensioniertes Buchenholz. Derzeit laufen verschiedene Untersuchungen über Möglichkeiten des Einsatzes von Brettschichtholz aus Buche als Leimbinder für tragende Zwecke. Hier wäre der Einsatz von rotkernigem Schnittholz grundsätzlich möglich. Mit einer Zulassung von Buchen-Brettschichtholz für die Nutzungsklasse I (nach DIN 1052 (2004): z. B. Verwendung in allseitig geschlossenen und beheizten Gebäuden) ist $2008 \mathrm{zu}$ rechnen. Wie im Möbelbau könnte die Verwendung von Brettschichtholz aus rotkerniger Buche dann hoffentlich architektonische Akzente setzen (OHNESORGE et al. 2007 a).

Für die Holzwerkstoffindustrie ist die Buche die wichtigste Laubholzart. Wobei der Anteil des Wald-Industrieholzes am gesamten Buchenholzeinschlag bereits $75 \%$ erreichte (ZMP 2007). Dieses begehrte Sortiment wird vorwiegend von der Spanplatten- und Faserplattenindustrie aber auch in der Zellstoff- und Papierindustrie verarbeitet. Im Rahmen des BMBF-Förderschwerpunktes „Nachhaltige Waldwirtschaft" werden unter anderem Möglichkeiten untersucht, aus Buchen- und Küstentannenholz kombinierte zukunftsfähige Holzwerkstoffe zu entwickeln.

Von der Preisentwicklung bei Buchen-Brennholz sind nicht nur die Parkettund Palettenhersteller betroffen, sondern alle Branchen der Holzwerkstoffindustrie sowie der Papier- und Zellstoffindustrie. Es hat sich eine echte Konkurrenz zur stofflichen Nutzung von Holz entwickelt. Die derzeit anhaltende Diskussion hat den Gedanken der sog. „Kaskadennutzung“ ins Spiel gebracht. Dies bedeutet eine zuerst stoffliche Nutzung wegen der langfristigen Bindung von Kohlenstoff in Holzprodukten, gefolgt von der Wiederverwendung sowie Weiterverwendung der Holzprodukte. Erst in der letzten Nutzungsphase sollte auch unter den Klimaschutzgesichtspunkten die energetische Verwertung von Holz erfolgen. Ob das Prinzip einer ressourcenschonenden Kaskadennutzung von Holz in der Säge- und Holzwerkstoffindustrie etabliert werden kann, wird letztendlich die zukünftige Preisentwicklung für Brennholzsortimente mitentscheiden. Mit gutem Beispiel gehen die niedersächsischen Landesforsten voran, in dem sie für 2008 Verträge mit der Holzwerkstoffindustrie bezüglich der Lieferung von Buchenindustrieholz zu einem beidseitig akzeptablen garantierten Preis schlossen. Im Jahr 2006 wurden allein in Niedersachsen ca. 1,93 Mio. Fm Holz (in Form von Scheitholz, Hack- 
schnitzeln und Pellets) zur Wärmeerzeugung verwertet. Der Scheitholzanteil betrug ca. $90 \%$, wobei die Buche dabei eine wesentliche Rolle spielte (RÜTHER et al. 2007). Inzwischen kommt es in einigen Forstämtern zu einer Konkurrenzsituation zwischen den Selbstwerbern des Brennscheitholzes und den Produzenten von Holzhackschnitzeln. Bedingt durch diese Konkurrenzsituation wurden in letzter Zeit Versuche zur Gewinnung alternativer Holzsortimente wie Schlagabraum und Landschaftspflegeholz durchgeführt (NEFF u. HAPLA 2007, NEFF et al. 2007).

\section{Fazit}

Vor dem Hintergrund der ökologisch orientierten waldbaulichen Konzepte sowie der Entwicklungen in der Holzindustrie ist es sehr wichtig, die Möglichkeiten und Grenzen der Produktion von Buchenwertholz aufzuzeigen. Dabei müssen die verwendungsorientierten Belange der holzbe- und holzverarbeitenden Industrie ihre Berücksichtigung finden. Die niedersächsische Landesforstverwaltung strebt eine Erhöhung des Laubbaumartenanteils bis auf $65 \%$ an. Dabei soll die wichtigste heimische Baumart Buche (Fagus sylvatica L.) mit weiteren Begleitbaumarten wie Douglasie, Fichte und Küstentanne zu stabilen, wertholzreichen zukunftsfähigen Mischbeständen erzogen werden. Infolge des angestrebten ökologischen Waldbaus wird das Angebot an Laubhölzern - vor allem Buche mit und ohne Rotkern - am Holzmarkt zukünftig stark zunehmen.

Zukünftig ist mit einer verstärkten Nutzung von Buche im Holzleimbau zu rechnen. Jedoch sind noch weiterführende Forschungsarbeiten auf den Gebieten: Haltbarkeit der Verleimungen, Delaminierungsversuche, Prüfung der elastomechanischen Eigenschaften von Leimbindern sowie maschinelle Sortierung von Buchen-Schnittholz notwendig. Auf Grund der sehr geringen Feuchte- und Pilzresistenz des Buchenholzes war bislang dessen Einsatz nur im Innenbereich möglich. Neuartige Verfahren der Holzmodifizierung lassen einen neuen feuchteresistenten Werkstoff entstehen, der im Außenbereich einsetzbar ist. Aus diesem Grund besteht bis auf Weiteres ein Forschungsbedarf im Bereich der Weiterverarbeitung von Buchenholz mit und ohne Rotkern. Dies schließt insbesondere die Dämpfung und Trocknung, Farberhaltung, Verklebung sowie die Modifizierungsprozesse von Buchenholz ein. 


\section{Literatur}

Aderhold, J.; Plinke, B.; Duschl, C. u. GAFKA, G. (2007): Starkholzfurnier. Furnier-Magazin 2007, 91-96

ANONYMus (2006): Furnier-Magazin, 2006. Ein Supplement von Holz-Zentralblatt und HK.

ANONYMus (2007): Furnier-Magazin, 2007. Ein Supplement von Holz-Zentralblatt und HK.

BMVEL (2004): Die zweite Bundeswaldinventur - BWI 2. Das Wichtigste in Kürze. Bonn, 87 S.

DIN EN 350-1 (1994): Dauerhaftigkeit von Holz und Holzprodukten - Natürliche Dauerhaftigkeit von Vollholz - Teil 1: Grundsätze für die Prüfung und Klassifikation der natürlichen Dauerhaftigkeit von Holz. Deutsche Fassung, Ausgabe 1994

DIN EN 1316-1 (1997): Laub-Rundholz - Qualitäts-Sortierung - Teil 1: Eiche und Buche; Deutsche Fassung EN 1316-1: 1997

DIN 1052 (2004): Bemessung von Holzbaukonstruktionen. Deutsche Fassung, Ausgabe 2004

HANSKÖTTER, B. (2004): Diagnose fakultativer Farbkerne an stehender Rotbuche (Fagus sylvatica L.) mittels „Elektrischer Widerstandstomographie“. Dissertation, Institut für Holzbiologie und Holztechnologie, Georg-August-Universität Göttingen, Cuvillier Verlag, ISBN 3-86537-123-X, $133 \mathrm{~S}$.

Hapla, F. u. Steinfatt, H.-C. (2002): Kritische Analyse der EN 1316-1 bei Sortierung von BuchenStarkholz im Vergleich zur HKS. Allg. Forst- und Jagdzeitung, 173. Jg., 86-94

Hapla, F.; Meggers, L.; MilitZ, H. u. MAI, C. (2002): Investigation on the yield and quality of sliced veneer produced from beech trees (Fagus sylvatica L.) containing red heartwood. Holz als Rohund Werkstoff, 60. Jg., 440-442

Hapla, F. u. MiLiTZ, H. (2004): Colour measurements and gluability investigation on red heart beech wood (Fagus sylvatica L.). Wood Research, 49 (4): 1-12

Hapla, F. u. OhNesorge, D. (2005): Qualitätsorientierte Schnittholzausbeute in Abhängigkeit von Durchmesser und Rotkernanteil bei Buchenstammholz. - Ein Modellansatz mit unterschiedlichen Einschnittarten -. holztechnologie, (4), 5-9

HKS (1969): Gesetz über gesetzliche Handelsklassen für Rohholz vom 25.02.1968, Verordnung über gesetzliche Handelsklassen für Rohholz mit Anlage zu \$1 vom 31.07.1969, Die Rundholzsortierung in Deutschland mit Zusatzbestimmungen der Bundesländer.

KRAL, S. (2004): Vergleichende Untersuchung der physikalischen und mechanischen Eigenschaften an unverkernter und rotkerniger Buche (Fagus sylvatica L.). Masterarbeit, Institut für Holzbiologie und Holztechnologie, Georg-August-Universität Göttingen, 80 S.

Krause, A. (2006): Holzmodifizierung mit N-Methylol-Vernetzern. Dissertation, Institut für Holzbiologie und Holztechnologie, Georg-August-Universität Göttingen, Sierke Verlag Göttingen, $229 \mathrm{~S}$.

Krause, A.; Wepner, F.; Xie, Y. u. Militz, H. (2008): Wood Protection with Dimethylol-dihydroxyEthyleneurea and Its Derivates. In: Schultz, T., Nicholas, D., Militz, H.; FreEman, M.H. u. Goodell, B. (Hrsg.): Development of Commercial Wood Preservatives - Efficacy, Environmental, and Health Issues. 356-371

Ludwig, K.; Hapla, F. u. MÖHRing, B. (2006): Submissionen von Buche gerechtfertigt? HolzZentralblatt, (2), 34-35

Militz, H. (2008): Processes and Properties of Thermally Modified Wood Manufactured in Europe. In: Schultz, T., Nicholas, D., Militz, H.; Freeman, M.H. u. Goodell, B. (Hrsg.): Development of Commercial Wood Preservatives - Efficacy, Environmental, and Health Issues. 372-389

MolnÁr, S.; Németh, R.; Fehér, S.; Tolvaj, L.; PApp, G.; VArga, F. u. Apostol, T. (2001): Technical and technological properties of Hungarian beech wood consider the red heart. Wood research - Drevársky Výskum 46 (1), 21-30

Mombächer, R. (Hrsg.) (2003): Holz-Lexikon. 4. Auflage, DRW-Verlag, Band 1, 734 S. und Band 2, 689 S., ISBN 3-87181-355-9 
NefF, A. u. Hapla, F. (2007): Aufbereitungsversuche von Holzhackschnitzeln zur energetischen Nutzung - Erfahrungsberichte aus Norddeutschland. holztechnologie 48, (2), 5-10

NefF, A., Nelles, M., Loewen, A. u. Hapla, F. (2007): Perspektiven für die energetische Verwertung von Landschaftspflegeholz. Jahrbuch der Baumpflege 2007-09-26, S. 23-36, Hrsg.: Dirk Dujesiefken \& Petra Kockerbeck, Haymarket Media GmbH \& Co. KG, Braunschweig, 11. Ausgabe, ISBN 978-3-87815-222-4, ISSN 1432-5020

Ohnesorge, D.; Aicher, S. u. BeCKer, G. (2007a): Brettschichtholz aus Buche. Fachbeitrag in KernHol₹, Magazin für Architekten und Planer, Ausgabe 4, 3-5

Ohnesorge, D.; Seeling, U. u. Becker, G. (2007b): Ganzheitliches Konzept für die rotkernige Buche. Holz-Zentralblatt, (32), 865-866

Rüther, B.; Hansen, J.; Ludwig, A.; Spellmann, H.; Nagel, J.; Möhring, B. u. Dieter, M. (2007): Clusterstudie Forst und Holz Niedersachsen. Beiträge aus der Nordwestdeutschen Forstlichen Versuchsanstalt, Band 1, Universitätsverlag Göttingen, ISSN 1865-6994, ISBN-13 978-3940344-07-6

SCHNider, T.; NiemZ, P. u. HuRst, A. (2008): Verklebung von thermisch vergüteter Buche. HolzZentralblatt, (4), 100-101

Seeling, U.; Ohnesorge, D.; Helzle, C.; Burgbacher, C.; Nemeth, R.; Tolvaj, L.; Teischinger, A.; Hansmann, C.; Mitteramskogler, H.; Huber, H.; Oliver, J.V.; Abian, M.A.; Pons, L. u. Custodio, R. (2007): Red Heartwood Handbook. Integrated concepts for processing European Beech (Fagus sylvatica L.) containing red heartwood. Project No. COOP-CT-2004-508137, Printed by Sorell Impresores, 48 S., ISBN 84-95077-26-4

WePNER, F. (2006): Entwicklung eines Modifizierungsverfahrens für Buchenfurniere (Fagus sylvatica L.) auf Basis von zyklischen N-Methylol-Verbindungen. Dissertation, Institut für Holzbiologie und Holztechnologie, Georg-August-Universität Göttingen, Sierke Verlag Göttingen, ISBN 10 3-933893-57-7, $258 \mathrm{~S}$.

WePNER, F. (2005): Produktinnovationen durch Holzmodifizierung. Tagungsband: Holzmärkte der Zukunft - wo liegen die Chancen? Anlässlich der 62. Jahrestagung des Deutschen Forstvereins e.V., 15.-18. September 2005 in Weimar, 330-344

WOBST, H. (1969): Auswirkungen der Rotkernigkeit auf kennzeichnende Eigenschaften und industrielle Verwendung von Buchenstammholz. Der Forst- und Holzwirt, 27, 80-83

ZMP (2007): Forst und Holz - Marktbilanz. Zentrale Markt- und Preisberichtstelle GmbH, Bonn, $147 \mathrm{~S}$.

Korrespondierender Autor:

Prof. Dr. Dr. h.c. František Hapla

Burckhardt-Institut der Georg-August-Universität Göttingen

Abteilung Holzbiologie und Holzprodukte

Büsgenweg 4

37077 Göttingen

E-Mail: fhapla@gwdg.de

URL: www.wood.uni-goettingen.de

Prof. Dr. Holger Militz

Burckhardt-Institut der Georg-August-Universität Göttingen 



\title{
Betriebswirtschaftliche Bewertung von Buchenwäldern
}

\author{
Economic valuation of beech forests
}

Bernhard Möhring, Georg Leefken und Claudia Gutsche

\section{Zusammenfassung}

Die Baumart Buche ist beim Naturschutz hoch geschätzt, da sie auf vielen Standorten in Deutschland die potenziell natürliche Waldgesellschaft prägt. Die Forstwirtschaft verbindet mit ihr auch betriebswirtschaftliche Erwartungen, insbesondere im Zusammenhang mit einer Minderung der Bestandesrisiken, aber auch durch die Erweiterung der Sortimentspalette.

Eine kritische Analyse der betriebswirtschaftlichen Ergebnisse sowohl bei Reinertragskalkulationen als auch bei Ergebnissen eines forstlichen Betriebsvergleiches zeigt im Baumartenvergleich jedoch, dass trotz der in der jüngeren Vergangenheit vergleichsweise günstigen Marktbedingungen für Buchenstammholz die Buche bis heute nachhaltig eine geringere Rentabilität - einen geringeren Reinertrag - erzielt hat als beispielsweise die Fichte und sich gerade in den letzten 2-3 Jahren die Marktverhältnisse zuungunsten der Buche entwickelt haben.

Auch die deutlich größeren Risiken der Fichtenwirtschaft führen nicht zu einer Umkehr dieser Verhältnisse. Die Buche wird jedoch insbesondere auf den besser nährstoffversorgten kollinen und submontanen Standorten auch in Zukunft sehr verbreitet bleiben, nicht zuletzt wegen der Möglichkeiten sie einfach natürlich zu 
verjüngen und vielfältig mit anderen Baumarten zu mischen, der relativ geringen Risikoanfälligkeit und der durch das Buchenholz verbreiterten Sortimentspalette, die von den Forstbetrieben angeboten werden kann.

Ein großflächiger Waldumbau in Richtung Buche ist nicht zuletzt auch vor dem Hintergrund der damit einhergehenden langfristigen Liquiditätsprobleme kritisch zu sehen. Ob und inwieweit die laufenden Klimaänderungen hier zu einer Neubewertung Anlass geben, ist an anderer Stelle zu diskutieren.

Stichworte: Buchenwirtschaft, Rentabilität, Risiko, Liquidität

\begin{abstract}
As a characteristic tree species of potential natural forest communities on many sites in Germany, beech is accorded a high priority in nature conservation. In forestry, it also has an economic value: in particular it is managed with the aim of reducing risks in stand management, but also of expanding the range of timber assortments.

However, a critical analysis of economic results of net yield calculations and economic comparisons of different enterprises for different tree species shows that, despite the recent comparatively favourable market conditions for beech stemwood, even today beech continues to realise a lower profitability - a lower net yield - than Norway spruce, for example. Furthermore, in the last two to three years the market developments have been unfavourable for beech.

Not even the much higher risks in the management of Norway spruce have brought about a reversal of this trend. Yet beech will continue to have a broad distribution in the future, especially on colline and sub-montane sites with better nutrient status, primarily because beech can be regenerated naturally with ease and grown in mixture with a diversity of species, because of its relatively low risk of susceptibility and the range of beech wood assortments that forest enterprises can supply.

A critical appraisal of the large-scale conversion of forests to beech is necessary, not only in view of the long-term liquidity problems associated with it. A discussion of whether and to what extent the ongoing climate change presents an occasion for a renewed evaluation also is required.
\end{abstract}

Keywords: beech management, profitability, risk, liquidity 


\section{Einleitung}

Mit der Baumart Buche verbinden sich spätestens seit den in den 1980er Jahren insbesondere in den Fichtenbeständen der deutschen Mittelgebirge deutlich gewordenen großflächigen Immissionsschäden sowohl die Hoffnung des Naturschutzes auf einen zukünftig naturnäheren Waldaufbau als auch der Wunsch vieler Forstbetriebe nach einer risikoärmeren und gleichzeitig ertragreicheren Forstwirtschaft. Vor diesem Hintergrund wurde in der Vergangenheit vielerorts, z. B. in Niedersachsen u. a. im Rahmen des Walderneuerungsprogramms im Harz (s. OTTO 1989) und später im Rahmen der langfristigen ökologischen Waldentwicklung („LÖWE“, s. NDS. ML 1991) der Waldumbau in Richtung Buchenwälder durch großflächige Voranbauten der Buche unter der Fichte vorbereitet bzw. bereits umgesetzt.

In den letzten Jahren wird die Diskussion um eine weitere Erhöhung der Buchenwaldfläche in Deutschland vor allem durch die naturschutzfachlichen Vorgaben für die Natura 2000- bzw. die FFH-Gebiete sowie durch die Diskussion um den Erhalt der biologischen Vielfalt gestützt. Die daraus abgeleiteten Forderungen, naturnahe, d. h. weitgehend reine Buchenwälder als solche zu erhalten (d. h. eine Anreicherung mit Nadelbaumarten zu unterlassen) und weitere Nadelbaumbestände in Buchenbestände umzuwandeln, werden augenscheinlich auch durch die "Jahrhundert"-Stürme der letzten 20 Jahre „Vivian und Wiebke" (1990), „Lothar“ (1999) und „Kyrill“ (2007), denen insbesondere eine große Anzahl von Fichtenbeständen zum Opfer fielen, gestützt.

Die naturalen Risiken einer Baumart sind jedoch nur ein Teil der Faktoren, die Forstbetriebe in ihren Dispositionen bei der Baumartenwahl berücksichtigen müssen. Denn Forstbetriebe sind es, die in Mitteleuropa die Wälder bewirtschaften und dabei zwingend nachhaltigen Erfolg benötigen, mithin die Deckung ihrer Ausgaben durch die Einnahmen aus ihrer betrieblichen Tätigkeit erreichen müssen. Mindestens genauso bedeutsam sind in dieser Hinsicht die naturale Produktivität einer Baumart, die Produktionsdauer, die Kosten der Begründung und Ernte und nicht zuletzt die technologischen Holzeigenschaften und die damit verbundene Nachfrage nach bestimmten Holzsortimenten. Die betriebswirtschaftliche Bewertung einer Baumart muss wegen der Langfristigkeit der forstlichen Produktion neben der Rentabilität und Stabilität (Risikoarmut) auch betriebliche Liquiditätsaspekte berücksichtigen, die für Forstbetriebe gerade in der Umbauphase besonders relevant sind.

Der nachfolgende Beitrag zeigt diese verschiedenen Aspekte der betriebswirtschaftlichen Bewertung von Buchenwäldern im Vergleich zu waldbaulichen Alternativen, hier exemplarisch insbesondere der Fichte, wobei die Aussagen in ähnlicher Form für die vergleichsweise schnellwachsenden Nadelbaumarten wie Lärche, Douglasie, Küstentanne etc. gelten. 


\section{Reinerträge im Wandel der Zeit}

Eine erste Annäherung an die wirtschaftliche Vorteilhaftigkeit einer bestimmten Baumart gegenüber einer oder mehrerer Alternativen erfolgt „traditionell“ durch den statischen Vergleich der Wald-Reinerträge, zinsfrei berechnet für normale Betriebsklassen (s. u. a. AlbERT u. WOMELSDORF (1987), JUNACK (1925), Keuffel (1980), ManN (1990), Mixdorf (1997), Möhring (1986), MÖHring (2004), MÖHRING (2007), RIPKEN (1989), RIPKEN u. SPELLMANN (1979), STUHR (1985a)). Das diesen Kalkulationen zugrunde gelegte Normalwaldmodell verkörpert das idealtypische Modell eines nachhaltigen Forstbetriebes (s. MÖHRING 1994). Hier sind alle Altersstufen - von der Bestandesbegründung bis hin zum hiebsreifen Endbestand - mit dem gleichen Flächenanteil vertreten. Jährlich werden die planmäßigen Durchforstungen durchgeführt und jeder geerntete Altbestand wird wieder durch einen Jungbestand ersetzt. Insgesamt halten sich Zuwachs und Nutzung stets die Waage. Naturale Risiken allerdings, die einen vorzeitigen Verlust von Beständen und mithin eine Verschiebung der Altersklassenflächen in Richtung der jüngeren Bestände bewirken, werden dabei jedoch meist nicht angemessen berücksichtigt.

\section{Fichte $(€)$}

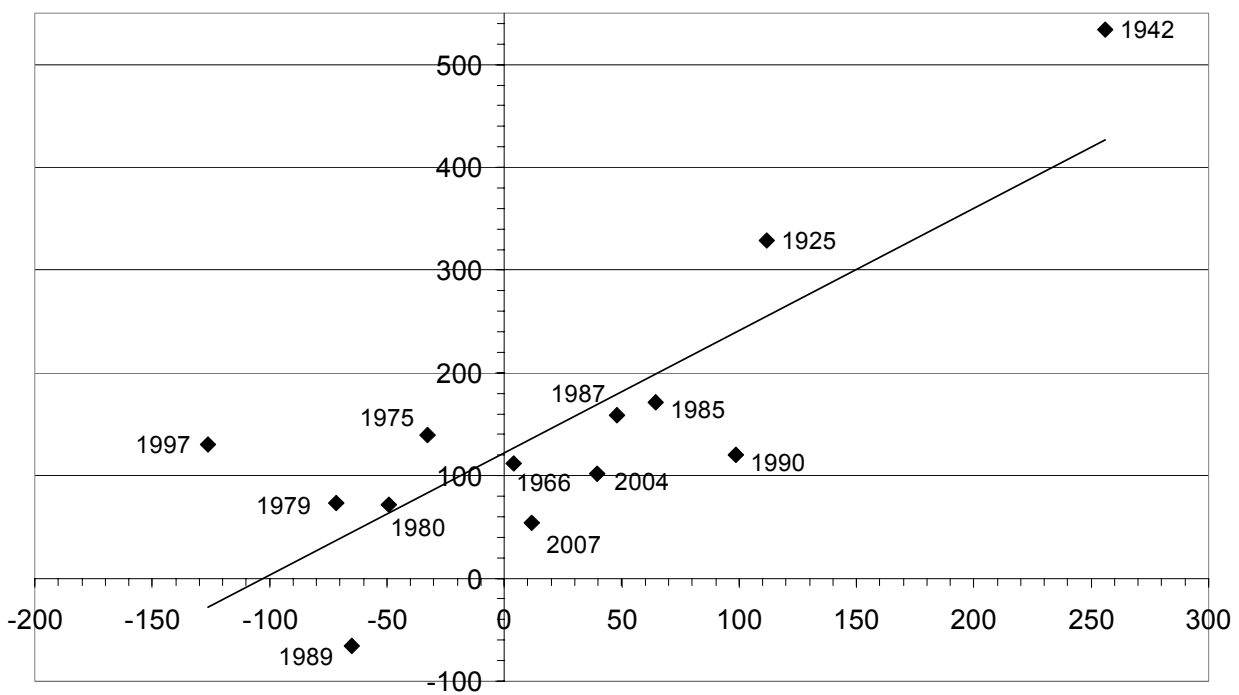

Buche $(€)$

Abbildung 1: Gegenüberstellung ausgewählter Ergebnisse von Reinertragskalkulationen normaler Buchen- und Fichtenbetriebsklassen für den Zeitraum von 1925 - 2007

Trotz seiner bekannten Nachteile ist das Normalwaldmodell ein etabliertes und akzeptiertes Modell für die Beschreibung der nachhaltigen ökonomischen Leistungsfähigkeit einer Baumart, das in der Literatur vielfach verwendet wird. Die 
Abbildung 1 zeigt anhand einer größeren Zahl in der Literatur veröffentlichter Reinertragskalkulationen exemplarisch das Verhältnis der Waldreinerträge je Hektar Waldfläche für Buche und Fichte im Paarvergleich für den Zeitraum von 1925 bis 2007, wobei die historischen Werte einheitlich in Euro-Beträge umgerechnet wurden. Dargestellt sind in der Regel die Reinerträge, die für mittlere Wachstumsverhältnisse, d. h. für die Bonität der II. Ertragsklasse und Umtriebszeiten für die Buche zwischen 120 und 140 Jahren sowie für die Fichte zwischen 80 und 100 Jahren hergeleitet wurden.

Insgesamt lässt sich für den über 80-jährigen Zeitraum feststellen, dass zu allen Bewertungszeitpunkten die nachhaltigen Reinerträge der Buche je Hektar unter denen der Fichte liegen. Die mittlere Reinertragsdifferenz liegt bei gut $100 € /$ ha. Ein Grund hierfür ist unter anderem die auf gleichen Standorten deutlich geringere naturale Produktivität der Buche gegenüber der Fichte. Untersuchungen im Solling (s. MÖHRING 2004) ergaben für den Paarvergleich von Buche und Fichte auf gleichem Standort eine im mittleren Bereich im Vergleich zur Fichte um vier Vorratsfestmeter je Hektar (Vfm/ha) geringere jährliche Produktivität der Buche (s. Abb. 2). Aus dieser geringeren naturalen Produktivität (geringerer Massenzuwachs je Hektar und Jahr) ergeben sich entsprechend niedrigere Einschlagsmengen je ha, was unmittelbaren Einfluss auf den nachhaltigen Reinertrag hat.

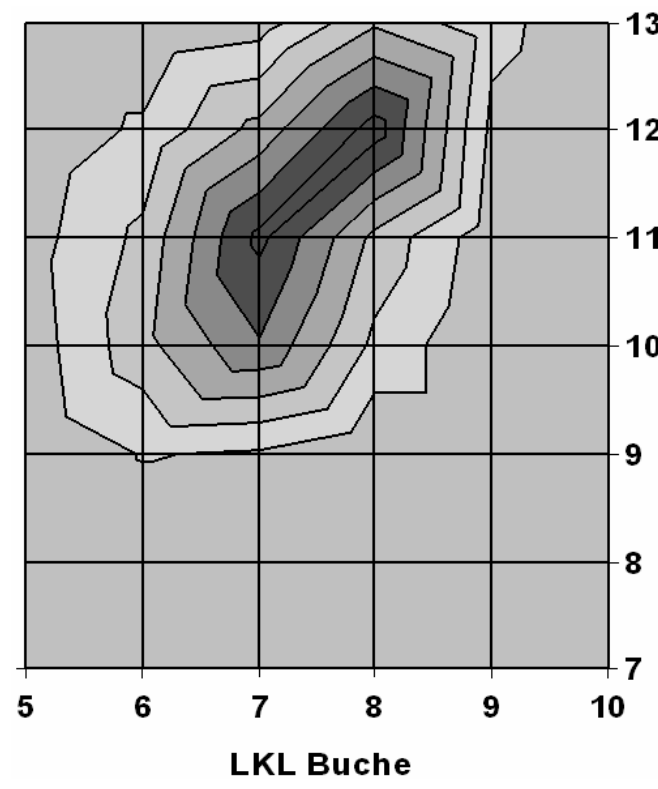

\section{Fläche (ha)}

\begin{tabular}{|l|}
$\square 1.200-1.400$ \\
$\square 1.000-1.200$ \\
$\square 800-1.000$ \\
$\square 600-800$ \\
$\square 400-600$ \\
$\square 200-400$ \\
$\square 0-200$ \\
\hline
\end{tabular}

Abbildung 2: Gegenüberstellung der Produktivitätsverbältnisse in Miscbbeständen des Sollings (LKL = Leistungsklasse = dGrmax in Vfm je ha und Jahr; Quelle: MÖHRING (2004)) 
Neben den Nutzungsmassen spielen auch die Holzerlöse bzw. Deckungsbeiträge je Erntefestmeter (Efm) eine zentrale Rolle für den wirtschaftlichen Erfolg der Baumarten, darauf wird an späterer Stelle in diesem Beitrag noch eingegangen. Der durch die Buchenwirtschaft erzielbare Reinertrag wird gegenüber dem der Fichte weiterhin durch die deutlich höheren Kulturkosten der Buche belastet, da die meisten Kalkulationen von einer Pflanzung der Buche ausgehen. Die Pflanzung einer Fichten-Kultur kann beispielsweise mit $2000 € /$ ha, die der Buche mit gut $5000 € /$ ha kalkuliert werden. Zumindest im Fall eines Waldumbaus in Richtung Buche sind die Kulturkosten auch nicht zu vermeiden und damit plausibel. Unterstellt man jedoch - durchaus realistisch - für vorhandene Buchenbetriebsklassen eine natürliche Verjüngung, die in der Regel deutlich geringere Kosten verursacht, dann können sich Reinerträge ergeben, die mit denen der Fichte durchaus vergleichbar sind, zumal, wenn man zusätzlich die Kosten des in der Kultur gebundenen Kapitals berücksichtigt (vgl. MÖHRING u. RÜPING 2006). Dies zeigt, dass die Aufrechterhaltung der Buchenwirtschaft auf gegebenem Standort, so wie es verbreitet in der Praxis vorzufinden ist, unter Rentabilitätsgesichtspunkten betriebswirtschaftlich durchaus sinnvoll sein kann, also wenn entsprechend alte Buchenbestände bereits vorhanden sind und die Naturverjüngung problemfrei möglich ist. Eine Umwandlung von Fichten- in Buchenbestände lässt sich aber unter der Prämisse des Ziels der Reinertragsmaximierung - danach nicht begründen, wenn der Waldbesitzer die Kulturkosten der Buche tragen muss.

\section{Risiko und Diversifikation}

Unter Risiken der Forstwirtschaft werden in der Regel zuerst naturale Risiken wie Sturmwurf, Käferbefall und Waldbrand verstanden. Hier gilt die Buche als vergleichsweise weniger gefährdet, während die Fichte diesen Einflüssen bekanntermaßen deutlich stärker unterworfen und Gegenstand vieler diesbezüglicher Forschungen ist. An dieser Stelle sei erneut auf die Untersuchung im Solling hingewiesen (s. MÖHRING 2004), welche mit Hilfe einer Sensitivitätsanalyse unter anderem der Frage nachgegangen ist, wie groß die Risikobelastung der Fichte maximal sein darf, bis sich der oben beschriebene Reinertragsvorteil der Fichte gegenüber der Buche aufzehrt. In einer Risikosituation, bei der die mittlere Lebenserwartung (das mittlere Nutzungsalter) der Fichte auf 90 Jahre begrenzt ist, sind die Reinerträge von risikobelasteter Fichte und risikofreier Buche gleich. Das tatsächliche mittlere Nutzungsalter liegt im Solling jedoch deutlich oberhalb dieses Wertes. Insofern kann man zumindest unter den vergleichsweise stabilen Verhältnissen des Sollings auch bei Einbeziehung des Bestandesrisikos von der Fichtenbetriebsklasse einen höheren Reinertrag erwarten als von der Buche. Eine Risikosenkung durch die Reduzierung des Fichtenanteils wäre demnach tendenziell mit einer Minderung der Deckungsbeiträge bzw. Reinerträge je ha verbunden. Hier zeigt sich der klassische ökonomische Zusammenhang zwischen Rendite und 
Risiko. Geringeres Risiko ist regelmäßig mit geringerer Rendite (geringeren Reinerträgen) verbunden (s. MÖHRING 2004). Zu einem ähnlichen Ergebnis kommt auch DIETER (2001) - mit Hilfe von Monte-Carlo-Simulationen konnte er zeigen, dass durch eine Erhöhung des Buchenanteils zu Lasten der Fichtenbestandesfläche das Risiko von Bestandesschäden zwar gesenkt werden kann, dies aber zu Rentabilitätsverlusten führt.

Im Rahmen der ökonomischen Bewertung stellt sich dennoch die Frage, mit welchen Risiken auch in der Buchenwirtschaft tatsächlich gerechnet werden muss. Für die Baumart Buche typische naturale Risiken sind insbesondere der Schleimfluss und die im Zusammenhang mit der „Waldsterbens-Diskussion“ mehrfach beschriebene Komplexkrankheit, die bevorzugt zum Absterben von Altbuchen führt, aber auch die Stürme verschonen die Buche bekanntermaßen nicht. KNOKE u. WURM (2006) gehen davon aus, dass $20 \%$ der Buchenbestände vor Ende des planmäßigen Nutzungszeitpunktes kalamitätsbedingt ausscheiden.

Weitere typische Risiken der Buchenwirtschaft liegen in der Entwertung des vergleichsweise „empfindlichen“ Rohholzes. Hier sind insbesondere der Rotkern und Spritzkern zu nennen. Aber auch Sonnenbrand, der Holzeinschlag während der „Saftzeit“ sowie eine verzögerte Holzabfuhr können zu einer starken Entwertung des Holzes führen.

Weiterhin ist zu berücksichtigen, dass das forstbetriebliche Gesamtrisiko nicht nur durch naturale oder technische, sondern insbesondere auch durch Marktrisiken bestimmt wird. Hier besteht grundsätzlich die Möglichkeit, durch Diversifikation des Produktangebotes, also die Ausweitung des betrieblichen Sortiments bzw. die Aufgabe einer Monostruktur, die normalen marktbedingten Risiken zu mindern. Ein methodisches Konzept, wie Diversifikation die Wirkung von unsystematischen Marktpreisschwankungen zu mindern vermag, bietet die sogenannte PortfolioTheorie. KNOKE u. HAHN (2007) konnten zeigen, dass die Baumartenwahl vor dem Hintergrund der Schwankungen der Holzpreise (unsystematischen Marktrisiken) mit der Risikostrukturentscheidung für ein Aktien-Portfolio vergleichbar ist. Durch die Kombination von verschiedenen Baumarten mit möglichst negativ korrelierten Preisschwankungen können sich auch für Forstbetriebe positive Diversifikationseffekte ergeben, die das Gesamtrisiko mindern. In Bezug auf eine großflächige Mischung von Buche und Fichte konnten KNOKE u. HAHN (2007) zeigen, dass eine Kombination von $60 \%$ Fichte und $40 \%$ Buche für den risikomeidenden Waldbesitzer optimal ist. Weiterhin zeigten sie, dass bei einer Erhöhung des Fichtenanteils zwar die Rendite gesteigert werden kann, aber eben auch das Risiko. Von der individuellen Risikopräferenz ist es abhängig, inwieweit ein Waldbesitzer (Investor) zusätzliche Risiken eingeht, um diese zusätzlichen Rendite zu realisieren.

Ein Grundproblem bei der Anwendung der Portfolio-Theorie besteht jedoch darin, dass man für die Berechnung der Marktrisiken auf Daten der Vergangenheit zurückgreifen muss und mithin nur die in der Vergangenheit bereits manifest 
gewordenen nicht systematischen Risiken berücksichtigt werden können. Die verbleibenden systematischen Risiken können nicht „weg-diversifiziert“ werden.

Ein solches systematisches Marktrisiko kann beispielsweise in der Änderung der Wertschätzung für bestimmte Güter liegen. Für den Bereich der Holzerlöse verdeutlicht die Abbildung 3, dass es offensichtlich systematische, nicht nur zufällige Preisentwicklungen auf den Holzmärkten gibt.

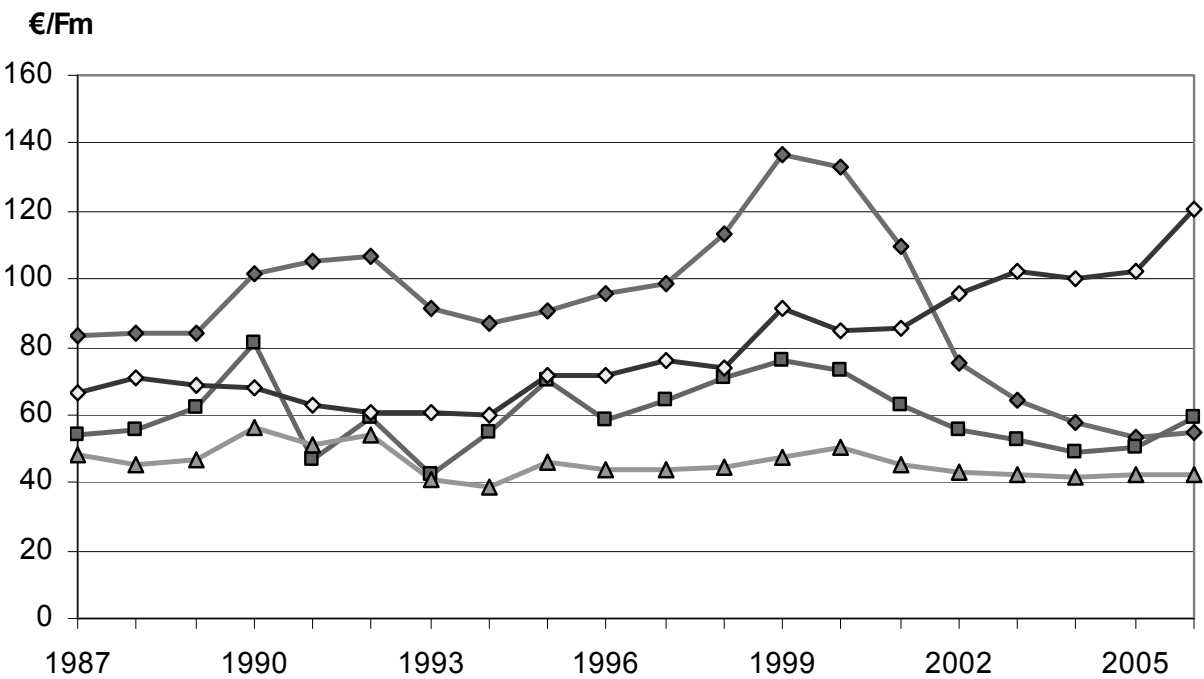

$\leadsto$ Bu-Sth A-C $\rightarrow$ Fi-Sth B-C $\triangle$ Ki-Sth B-C $\leadsto$ Ei-Sth A-C

Abbildung 3: Entwicklung der von 1987 bis 2006 durch die Niedersächsische Landesforstverwaltung/ Niedersächsische Landesforsten A.ö.R. realisierten Stammbolzppreise für die Hauptbaumarten Buche, Fichte, Kiefer und Eiche (A, B, C = Güteklassen nach HKS). Datenquelle: ZMP 2007

Die Abbildung 3 zeigt für die letzten 20 Jahre interessante Entwicklungen der durchschnittlichen Stammholzpreise der vier Hauptbaumarten: Das KiefernStammholz lag beständig auf dem geringsten Niveau ohne nennenswerte Schwankungen. Das Fichtenstammholz hatte eine positive Erlösentwicklung vor 1990, die durch die Stürme Wiebke und Vivian abrupt zunichte gemacht wurde; nach einem Auf und Ab folgten Jahre steigender Stammholzpreise, bis der Sturm Lothar 1999 erneut für starken Preisverfall sorgte; 2004 wurde die Talsohle durchschritten und seither zeigt sich wieder ein deutlicher Preisanstieg für das Fichtenstammholz, was insbesondere auch mit dem Aufbau der entsprechenden Holzverarbeitungskapazitäten im Zusammenhang steht. Das Eichenstammholz zeigte nach einer Stagnation bis Mitte der 1990er Jahre einen bemerkenswert stetigen Preisanstieg, der sich durch die neue Wertschätzung des Eichenholzes im Möbelbau erklären lässt. Das 
Buchenstammholz hat sich hingegen recht anders entwickelt. Ausgehend von einem Durchschnittserlös (Güteklassen A-C, alle Stärkeklassen) von gut $80 € / \mathrm{Efm}$ im Jahr 1987 stiegen die Buchenstammholzpreise mit einem Zwischenhoch 1993 auf das „Allzeithoch“ von $133 € /$ Efm im Jahr 1999, das in der Branche allgemein als „China-Boom“ bezeichnet wird und eine Phase beschreibt, in der Buchenholz als wertvolle Möbeloberfläche oder als Raumausstattungsholz weltweit gefragt war. Seither ist es mit den Buchenstammholzerlösen kontinuierlich bergab gegangen bis auf $54 € /$ Efm im Jahr 2005. Im Jahr 2006 lag erstmals der Durchschnittserlös des Fichtenstammholzes oberhalb des durchschnittlichen Buchenstammholzerlöses. Die traditionelle Sichtweise, Fichtenstammholz als standardisierte BauholzMassenware und Buchenstammholz als Möbel-Wertholz anzusehen, erscheint unter den aktuellen Marktentwicklungen nicht mehr angebracht.

Die skizzierten Entwicklungen verdeutlichen, dass es offensichtlich systematische Veränderungen in der Wertschätzung des Holzes der verschiedenen Baumarten gegeben hat und dass trotz der vergleichsweise geringeren naturalen Risiken ${ }^{1}$ die Buchenwirtschaft augenscheinlich mit nicht unerheblichen Marktrisiken beim Zielprodukt belastet ist.

\section{Berücksichtigung der betrieblichen Liquidität}

Ein Wechsel der Wirtschaftsbaumarten ist wegen der langfristigen Ausrichtung der forstlichen Produktion in nachhaltig wirtschaftenden Betrieben kurzfristig nicht möglich, sondern ist in der Regel ein Projekt, das sich über viele Jahrzehnte erstrecken muss. Dies erfordert im Rahmen der betriebswirtschaftlichen Bewertung auch die Berücksichtigung der Folgen für die betriebliche Liquidität, welche durch die Dynamik des Baumartenwechsels in der Umbauphase beeinflusst wird. Eine komparativ-statische Betrachtung der Reinerträge normaler Betriebsklassen reicht hier nicht mehr aus, da ein solcher Vergleich letztlich unterstellt, dass die entsprechenden, ideal aufgebauten Modellwälder bereits vorhanden sind. Sowohl der Aufbau mit der Zielbaumart als auch der Abbau der Ausgangsbaumart werden von statischen Reinertragskalkulationen monetär nicht angemessen erfasst.

Man kann sich diesem Problem nähern, indem man durch eine dynamische Simulation beispielsweise den Wechsel von einer nachhaltigen Fichten- in eine Buchenbetriebsklasse nachbildet und aus den so berechneten naturalen Größen die jährlichen Überschüsse zum jeweiligen Zeitpunkt herleitet. Nach MÖHRING (2004) ergeben sich beim Umbau einer bisher im Kahlschlag bewirtschafteten Fichtenbetriebsklasse (mit einer Umtriebszeit von 100 Jahren und einer Bonität in Höhe

${ }^{1}$ Natürlich ist auch die Buche nennenswerten Bestandesrisiken, wie z. B. Einwirkungen durch Luftschadstoffe (s. DAmmanN et al. 2007) ausgesetzt. Diese führen jedoch im Gegensatz zur Fichte i. d. R. nicht zu einer vorzeitigen Nutzung der Buche. 
einer Leistungsklasse 12) ${ }^{2}$ durch Voranbau von Buchen in einen Buchennaturverjüngungsbetrieb (mit einer Umtriebszeit von 150 Jahren und einer Bonität in Höhe einer Leistungsklasse 8) hinsichtlich der Überschüsse drei sehr unterschiedliche Phasen (s. Abb. 4).

1. Entnahmephase: Zu Beginn des Waldumbaus wird durch die Zielstärkennutzung in Verbindung mit dem Voranbau der Buche verstärkt in die Fichtenaltbestände eingegriffen, mithin werden deutlich höhere Überschüsse als in der Ausgangssituation realisiert.

2. Abbauphase: Auf diese erste Phase verstärkter Nutzungen folgt eine Phase der Einschlagsreduktion, da der Fichtenschirm zum Schutz des Buchennachwuchses über die bisherige Umtriebszeit hinaus erhalten werden muss. Dies erfordert Einschlags- und damit Einnahmeverzichte. Hinzu kommen die erhöhten Bestandesbegründungskosten für den künstlichen Voranbau der Buche. Da noch in nennenswertem Umfang Fichtenaltbestände, die jedoch zunehmend abgebaut werden, zur Ernte anstehen, können noch positive Überschüsse erzielt werden. Die aus der Buche erzielbaren Durchforstungserlöse können den Ausfall bei der Fichte jedoch nicht wettmachen.

3. „Ausfallphase“: Nachdem der Umbau der letzten Fichtenbestände in Angriff genommen ist, wird deutlich, dass die Buche wegen des späteren Erreichens ihrer Zielstärke die Ausfälle der Fichte nicht kompensieren kann. Die anfangs vorhandene Fichte ist bereits geerntet, die Buche aber hat noch nicht die erntefähigen Dimensionen erreicht. Daraus ergibt sich eine lange Periode deutlich negativer Überschüsse erheblicher Größenordnung, die selbst zum Ende des hier dargestellten Zeitraumes von 160 Jahren noch nicht beendet ist.

Demnach schafft die geringere Produktivität der Buche in Verbindung mit der längeren Produktionsdauer beim Waldumbau langfristig massive Liquiditätsprobleme - Defizite über Jahrzehnte hinweg sind programmiert. Von einem beschleunigten Umbau vorhandener wüchsiger Fichtenbestände ist also auch unter dem Liquiditätsaspekt betrieblich dringend abzuraten, zumindest wenn die damit verbundenen betriebswirtschaftlichen Nachteile nicht anderweitig (z. B. durch Ausgleichszahlungen im Zusammenhang mit Vertragsnaturschutz, Kompensationsmaßnahmen etc.) kompensiert werden.

\footnotetext{
2 Die Leistungsklasse entspricht dem maximalen durchschnittlichen Gesamtzuwachs (DGZmax), angegeben in Vfm je ha und Jahr.
} 


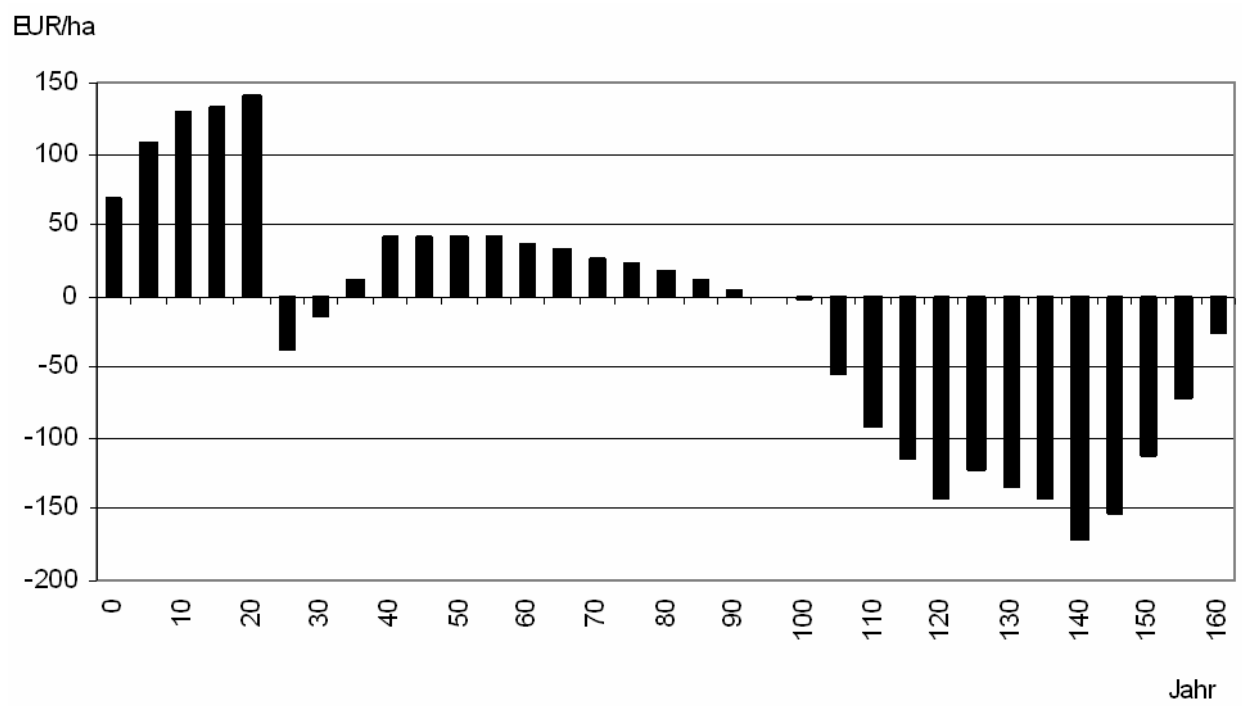

Abbildung 4: Entwicklung der Überschüsse in Euro/ ha für die Simulation des Wechsels vom FichtenKablschlagbetrieb in Richtung naturnaber Buchenwirtschaft. Quelle: MÖHRING (2004)

\section{Forstwirtschaftliche Realität: Betriebsvergleichsergebnisse}

Die bisherigen betriebswirtschaftlichen Betrachtungen bauten ganz überwiegend auf Modellkalkulationen auf. Abschließend soll daher der Frage nachgegangen werden, wie die betriebswirtschaftlichen Ergebnisse in der forstbetrieblichen Realität wirklich aussehen bzw. ausgesehen haben.

Denn die Zusammenhänge zwischen Baumart und den damit verbundenen betriebswirtschaftlichen Erfolgen müssen sich letztlich auch in den tatsächlichen forstbetrieblichen Ergebnissen manifestieren. Solche Betriebsergebnisse werden beispielsweise für den Privatwald durch den Forstlichen Betriebsvergleich Westfalen-Lippe seit 1969 erhoben. Er ist damit der älteste Betriebsvergleich seiner Art in Deutschland. Von Anfang an wurde dieser Betriebsvergleich wissenschaftlich begleitet, seit 1974 durch Forstökonomen der Universität Göttingen. Durchschnittlich nahmen in den letzten Jahren an diesem Betriebsvergleich rund 35 private Forstbetriebe mit einer mittleren Größe von rund 1.700 ha teil, so dass der Betriebsvergleich demnach Forstbetriebsflächen von insgesamt rund 60.000 ha repräsentiert. Die Betriebe werden innerhalb des Betriebsvergleichs entsprechend ihrem Baumartenschwerpunkt einem von drei „Beratungsringen“ (Fichte, Laubholz/Buche oder Kiefer) zugeordnet, wobei dies auch gleichzeitig einer räumlichen Gliederung entspricht; Fichtenbetriebe liegen überwiegend im Sauerland, Laubholz/Buchenbetriebe im Weserbergland und Kiefernbetriebe im Münsterland. 
Für diese drei Beratungsringe wird jeweils eine große Vielzahl betriebswirtschaftlicher Kennziffern berechnet. Für eine korrekte Deutung der Zahlen ist es wichtig $\mathrm{zu}$ wissen, dass die Forstbetriebe in den jeweiligen Beratungsringen nicht jeweils reine Fichten-, Buchen- und Kiefernbetriebe sind, vielmehr weisen praktisch alle Betriebe neben der namengebenden Hauptbaumart eine mehr oder minder breite Mischung der gängigen Wirtschaftsbaumarten auf (s. KAUL u. LEEFKEN 2001). Dennoch lassen sich daraus belastbare Hinweise auf die wirtschaftliche Situation der Buche im Vergleich zu den anderen Baumarten (Fichte, Kiefer) ableiten.

Die Zeitreihe der Holzeinschläge in Efm/ha in Abbildung 5 zeigt, dass die Fichtenbetriebe regelmäßig die höchsten Holzeinschläge realisiert haben - im Durchschnitt über den betrachteten Zeitraum 8,8 Efm/ha -, wobei in dieser Betriebsgruppe auch die stärksten Schwankungen im Zusammenhang mit den Kalamitäten und Kalamitätsfolgejahren auftraten. Die Laubholz/Buchenbetriebe lagen mit ihrem Holzeinschlag im Schnitt um 1,7 Efm/ha unter dem der Fichtenbetriebe, die Kiefernbetriebe lagen mit durchschnittlich 2,6 Efm/ha nochmals deutlich unter den Laubholz/Buchenbetrieben.

\section{Efm/ha}

Einschlag (inkl. X-Holz) Efm/ha

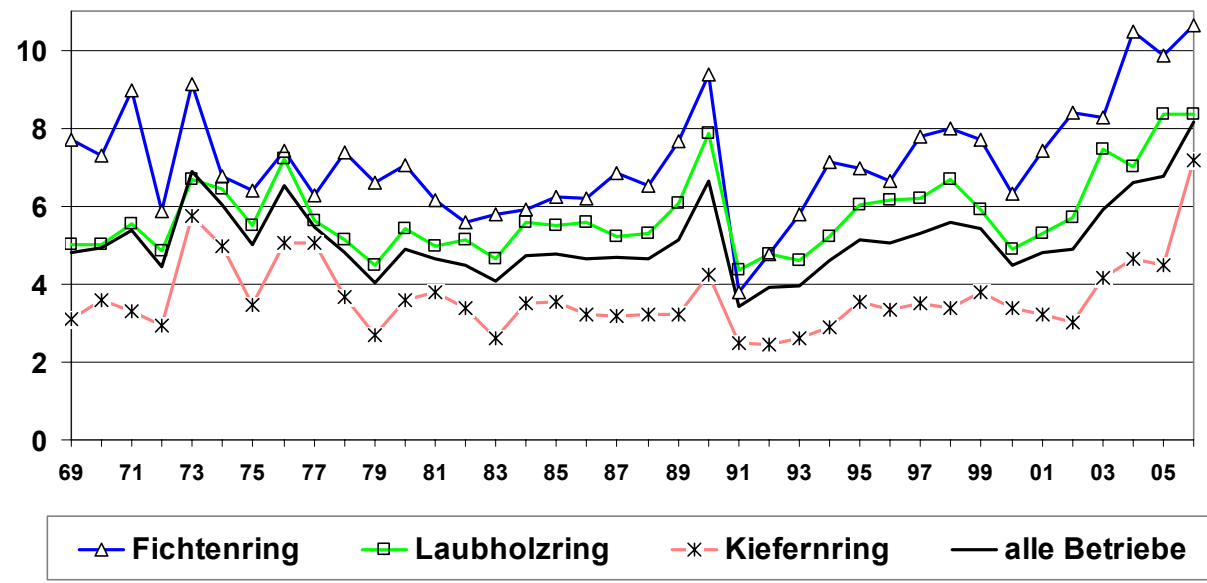

Abbildung 5: Holzeinschlag in Erntefestmeter je ha und Jahr des Forstlichen Betriebsvergleichs Westfalen-Lippe von 1969 bis 2006. Quelle: LEEFKEN u. MÖHRING (2007)

Eine ebenfalls sehr aussagekräftige Kennziffer ist der in Abbildung 6 dargestellte sogenannte Nettoholzerlös, auch erntekostenfreier Holzerlös genannt. Es ist der Deckungsbeitrag, der dem Forstbetrieb aus der Holzernte nach Abzug der Holzerntekosten je Efm verbleibt. Es ist erkennbar, dass die Fichten- und Laubholz/Buchenbetriebe das Niveau, das von Mitte der 70er Jahre bis Ende der 90er Jahre bei rund 35 ,- $€ /$ Efm lag, nach der Talfahrt der letzten fünf Jahre im Jahr 2006 noch nicht wieder erreicht haben, die Tendenz aber eindeutig positiv ist. Es zeigt sich aber auch, dass die Laubholz/Buchenbetriebe, anders als seit Mitte der 
70er Jahre, in der jüngsten Vergangenheit geringere Nettoholzerlöse als die Fichtenbetriebe erzielt haben.

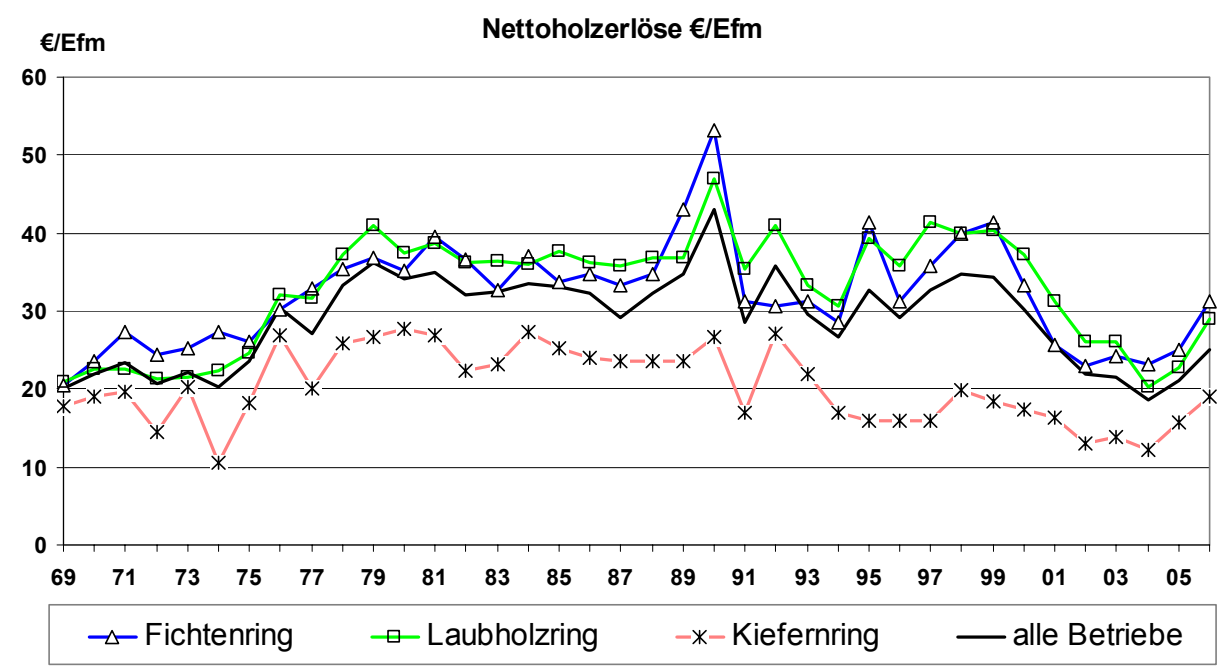

Abbildung 6: Nettoholzerlöse (erntekostenfreie Holzerlöse, $(€ / E f m)$ ) des Forstlichen Betriebsvergleichs Westfalen-Lippe von 1969 bis 2006. Quelle: LEEFKEN u. MÖHRING (2007)

Das Ergebnis der wirtschaftlichen Tätigkeit läuft in der Kennziffer des betrieblichen Reinertrages zusammen. Betrachtet man die Entwicklung dieser Größe über den vom Betriebsvergleich Westfalen-Lippe erfassten Zeitraum, so ist festzuhalten, dass die Reinerträge der Laubholz/Buchenbetriebe, bis auf wenige Ausnahmen, regelmäßig unter denen der Fichtenbetriebe liegen (s. Abb. 7). Die Unterschiede der Reinerträge sind dabei aber bei weitem nicht so gravierend, wie die oben dargestellten baumartenbezogenen Modellberechnungen vermuten lassen. Dabei muss jedoch berücksichtigt werden, dass die am forstlichen Betriebsvergleich teilnehmenden Laubholz/Buchenbetriebe in der Regel auch über nennenswerte Fichtenanteile und umgekehrt auch die Fichtenbetriebe über Buchenanteile verfügen, so dass die Abweichungen zwischen beiden Betriebsgruppen geringer sein müssen, als für die Reinbestände berechnet. Auch erzielen die Laubholz/Buchenbetriebe regelmäßig höhere Einnahmen aus sonstigen Erträgen wie Jagd, forstlichen Nebennutzungen (Saat- und Pflanzgut), Vertragsnaturschutz etc. als die Fichtenbetriebe; zu berücksichtigen ist auch, dass die Fichtenbetriebe im Sauerland nennenswert ungünstigere standörtliche Bedingungen bezüglich Höhenlage, Nährstoffarmut, Winddisposition etc. aufweisen, als die besser nährstoffversorgten, oft mit Lösslehm überlagerten Standorte der Laubholz/Buchenbetriebe im Weserbergland. Es mag schon manchen überraschen, dass die Fichtenbetriebe, trotz der nennenswert ungünstigeren Produktionsbedingungen und größeren Kalamitätsbelastungen langfristig bessere Betriebsergebnisse erzielt haben als die 
standörtlich begünstigten Laubholz/Buchenbetriebe. Insofern wird der Trend, welcher schon anhand der Modellkalkulationen aufgezeigt wurde, durch die Ergebnisse des Betriebsvergleichs durchaus bestätigt.

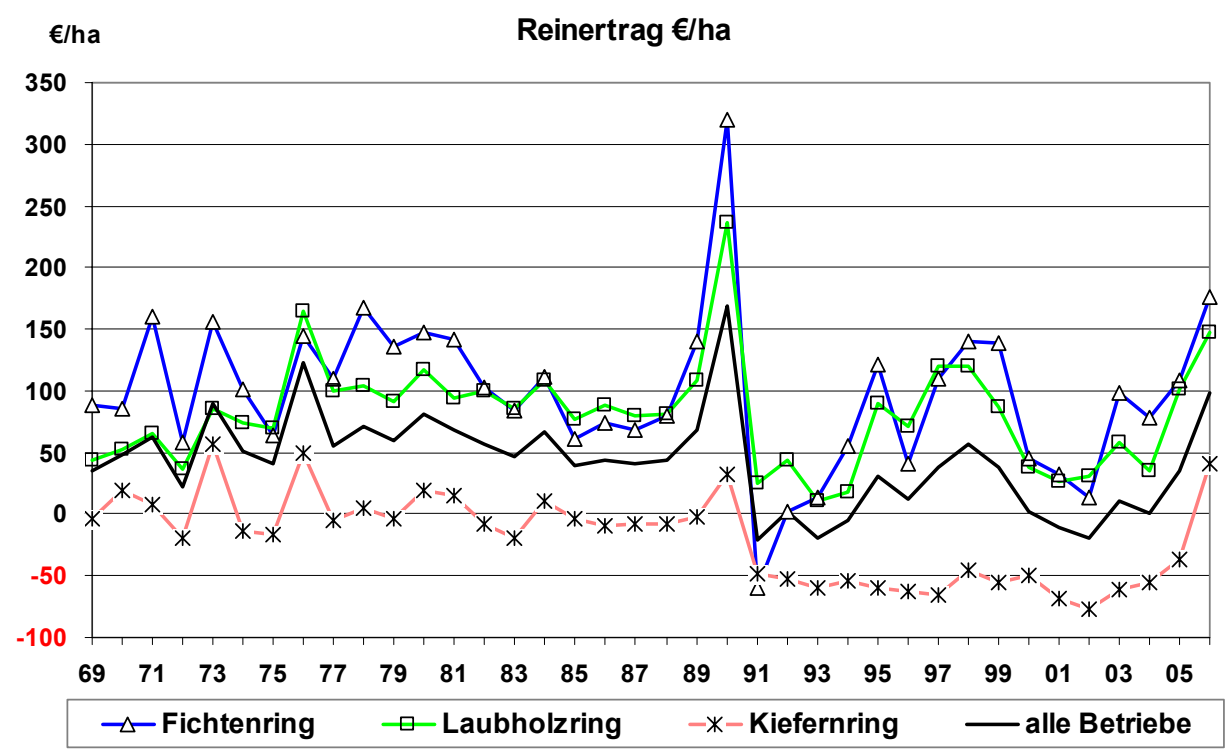

Abbildung 7: Reinerträge des Forstlichen Betriebsvergleichs Westfalen-Lippe von 1969 bis 2006. Quelle: LEEFKEN u. MÖHRING (2007)

\section{Schlussfolgerungen}

Aus den vorgestellten, sicher eher desillusionierenden Ergebnissen sind verschiedene Schlussfolgerungen in Bezug auf die betriebswirtschaftliche Bewertung von Buchenwäldern und den Waldumbau in Richtung Buche zu ziehen.

Die Buche ist die Schlüsselbaumart der potenziellen natürlichen Vegetation in Deutschland. Sie ist insbesondere auf den besser nährstoffversorgten kollinen und submontanen Standorten - auch im Privatwald - recht verbreitet und ist dort das Objekt nachhaltiger Bewirtschaftung. Es spricht vieles dafür, dass dies auch in Zukunft so bleiben wird, nicht zuletzt wegen der Möglichkeiten, sie einfach natürlich $\mathrm{zu}$ verjüngen und vielfältig mit anderen Baumarten $\mathrm{zu}$ mischen, der relativ geringen Risikoanfälligkeit und der durch das Buchenholz verbreiterten Sortimentspalette, die von den Forstbetrieben angeboten werden kann. In ihrer betriebswirtschaftlichen Leistungsfähigkeit bleibt die Buche jedoch deutlich hinter dem Nadelholz zurück, wobei auch die ohne Zweifel höhere Risikoanfälligkeit namentlich der Fichte die Verhältnisse nicht umkehrt. Erschwerend kommt hinzu, 
dass sich in den letzten Jahren die Verhältnisse auf den Holzmärkten zuungunsten der Buche entwickelt haben.

Ein großflächiger Waldumbau von Nadelholz in Richtung Buche, so wie er vielfach gefordert und zzt. auch in der Praxis umgesetzt wird (die Nadelholz-Nachwuchs-Flächen sind dramatisch zurückgegangen), wird deshalb auch keinen Betrag zur Lösung betriebswirtschaftlicher Probleme der Forstwirtschaft leisten. Es ist vielmehr umgekehrt, sowohl unter dem Aspekt der Rentabilität als auch der Liquidität schneidet die Buche schlechter als das Nadelholz ab. Dabei ist auch zu berücksichtigen, dass eine Ausdehnung der Buchenanbaufläche auf Standorten erfolgen muss, deren Leistungskraft tendenziell geringer und deren Risikobelastung höher ist als im derzeitigen Gebiet des Buchenanbaus.

Es gibt starke betriebswirtschaftliche Gründe, den Waldumbau in Richtung einer naturnahen Buchenwirtschaft nur auf Teilflächen vorzusehen und nur langsam (generationsübergreifend) umzusetzen, um die ökonomische Nachhaltigkeit auf Betriebsebene nicht zu stark zu schwächen. Parallel sollten Konzepte umgesetzt werden, die die zu erwartenden Ertragseinbußen durch andere Baumarten (z. B. die Douglasie, Küstentanne etc.) ausgleichen. Zudem gilt es, die waldbaulichen Maßnahmen zur Stabilisierung der Nadelholzbestände konsequenter als bisher anzuwenden.

Ist der Waldumbau in Richtung Buche aufgrund gesellschaftlicher Ziele erwünscht, so bedarf er des finanziellen Ausgleiches durch öffentliche Mittel. Die Gesellschaft als „Kunde“ muss lernen, dass sie für derartige gesellschaftliche Leistungen der Forstbetriebe zahlen muss. Erste erfolgversprechende Ansatzpunkte können im Bereich des Vertragsnaturschutzes und bei Ausgleichs- und Ersatzmaßnahmen im Wald, beim Wasserpfennig und bei der Zertifizierung gesehen werden.

Neue Bewertungen der hier dargestellten betriebswirtschaftlichen Zusammenhänge werden sich gegebenenfalls wegen der zu erwartenden Klimaänderungen ergeben, wobei hier jedoch die Rahmenbedingungen sowohl für die Fichte als auch für die Buche nicht besonders günstig aussehen (s. V. LÜPKE 2007).

\section{Literatur}

Albert, G. u. WomelsDorf, G. (1987): Reinerträge der Hauptwirtschaftsbaumarten in RheinlandPfalz. Modellkalkulationen 1987. Mitteilungen der Landesforstverwaltung Rheinland-Pfalz Nr. 7, $52 \mathrm{~S}$.

Dammann, I.; Paar, U.; Gawehn, P.; Wendland, J. u. Eichhorn, J. (2007): Waldzustandsbericht 2007. Nordwestdeutsche Forstliche Versuchsanstalt (Hrsg.), 31 S. Veröffentlicht auch im Internet unter: http://www.nw-fva.de/fileadmin/user_upload/ Sachgebiet/Waldzustand_ Boden/WZB2007Niedersachsen.pdf

DieTeR, M. (2001): Land expectation values for spruce and beech calculated with Monte Carlo modelling techniques. In: Forest Policy and Economics, Heft 2, 157-166. Shannon: Elsevier 
JUNACK (1925): Reinertragstafeln für die Berechnung von Ertragswerten der Waldbestände. Der deutsche Forstwirt, 2. Auflage, Berlin, $87 \mathrm{~S}$.

Kaul, R. u. LeefKen, G. (2001): Der Forstliche Betriebsvergleich Westfalen-Lippe, Schriftenreihe der Landesforstverwaltung NRW, Sonderheft 30 Jahre Forstlicher Betriebsvergleich, 5-14. Druck- und Verlagshaus Bitter GmbH, Recklinghausen, Bezug über Forstliche Dokumentationsstelle der Landesforstverwaltung NRW

Keuffel, W. (1980): Programmierte Deckungsbeitragskalkulation als betriebswirtschaftliche Entscheidungshilfe. Dissertation. Albert-Ludwig-Universität Freiburg, 216 S.

KNOKE, T. u. HAHN, A. (2007): Baumartenvielfalt und Produktionsrisiken: Ein Forschungseinblick und -ausblick. Schweiz Z. Forstwesen 10, 312-322

KNOKE, T. u. Wurm, J. (2006): Mixed forests and a flexible harvest policy: A problem for conventional risk analysis? European Journal of Forest Research 125 (3): 303-315

LeEfKen, G. u. MÖHRING, B. (2007): Privatwald-Betriebsvergleich Westfalen-Lippe 2006. AFZ/Der Wald, 62. Jg., 1192-1194

LÜPKE, B. V. (2004): Risikominderung durch Mischwälder und naturnaher Waldbau: ein Spannungsfeld. Forstarchiv, 45. Jg., 43-50

ManN, H.-J. (1990): Reinertrag im kleineren Privatwald Niedersachsens. Forst und Holz, 45. Jg., 627630

MiXDORF, U. (1997): Reinertragskalkulationen als Maßstab der ökonomischen Leistungsfähigkeit von Bestandeszieltypen - Modellkalkulationen. Sächsisches Staatsministerium für Landwirtschaft, Ernährung und Forsten (SML), Dresden, $83 \mathrm{~S}$.

MÖHRING, B. (1986): Dynamische Betriebsklassensimulation. Ein Hilfsmittel zur Bewertung ökonomischer Folgen neuartiger Waldschäden. Dissertation. Georg-August Universität Göttingen, 268 $\mathrm{S}$.

MÖHRING, B. (1994): Über ökonomische Kalküle bei forstlichen Nutzungsentscheidungen. J.D. Sauerländers Verlag, Frankfurt/M., 267 S.

MÖHRING, B. (2004): Betriebswirtschaftliche Analyse des Waldumbaus. Forst und Holz, 59. Jg., 523530

MÖHRING, B. (2007): Möglichkeiten/Vorschläge für ein typisierendes Verfahren zur Bewertung forstlicher Betriebe im Zusammenhang mit der Erbschaft- und Schenkungssteuer. Arbeitspapier. Institut für Forstökonomie. Georg-August Universität Göttingen, 37 S.

MÖHRING, B. u. RüPING, U. (2006): Bewertungskonzept für forstliche Nutzungsbeschränkungen. Schriften zur Forstökonomie, Band 32. J.D. Sauerländers Verlag, Frankfurt/M., 48 S.

NDS. ML (1991): Niedersächsisches Programm zur langfristigen ökologischen Waldentwicklung in den Landesforsten (LÖWE), Beschluß der Niedersächsischen Landesregierung vom 23.07.91, als RdErl. d. ML in Kraft getreten am 05.05.94

Отто, H.-J. (1989): Das Walderneuerungsprogramm Harz im Rahmen der langfristigen, regionalen Waldbauplanung. Allgemeine Forst Zeitschrift für Waldwirtschaft und Umweltvorsorge (AFZ), 468-470

RIPKEN, H. (1989): Ertrag und Aufwand im Forstbetrieb - Stand und Tendenzen. Forst und Holz, 44. Jg., 51-57

Ripken, H. u. SpelLmanN. H. (1979): Modell-Berechnungen der Reinerträge der wichtigsten Baumarten sowie der gesamten Holzproduktion in den Niedersächsischen Landesforsten. In: Aus dem Walde. Mitteilungen der Niedersächsischen Landesforstverwaltung, Heft 30, 346-403

STUHR, G. (1985a): Reinertrag einer Buchenbetriebsklasse im Jungdiluvium Schleswig-Holsteins. Der Forst- und Holzwirt, 40. Jg., 545-546

ZMP (2007): Forst und Holz Marktbilanz 2007. Zentrale Markt- und Preisberichtsstelle GmbH. CD$\mathrm{ROM}$ 


\section{Weiterführende Literatur}

Dummel, K. (1975): Analyse und Gestaltung der Intensität im Forstbetrieb. Dissertation. AlbertLudwig-Universität Freiburg, $331 \mathrm{~S}$.

Kennel, H. (1942): Vom forstlichen Zinsfuß, von der Umtriebszeit und andern statischen Fragen. Forstw. Cbl. 9, 193-201

MAtThies, F. (1966): Die Kostenträgerrechnung als Teilgebiet der forstlichen Kostenrechnung. Aus dem Walde. Mitteilungen der Niedersächsischen Landesforstverwaltung, Heft 9, 178 S.

Michaelis, C. (1934): Wie bringt Durchforsten die größere Stärke- und Wertzunahme des Holzes? Neudammer forstliche Belehrungshefte 2, 6. Auflage, $43 \mathrm{~S}$.

Möhring, B.; Glißmann, S.; KrÜCKe, B.; Leefken, G.; Wippermann, Ch. u. Ziegeler, M. (2004): Ökonomisch/ökologisch optimierte Nutzungsverfahren für Mischbestände. Kurzfassung eines Forschungsberichtes. In: FORSCHUNGSZENTRUM WALDÖKOSYSTEME DER UNIVERSITÄT GÖTTINGEN (Hrsg.): Indikatoren für eine nachhaltige, multifunktionelle Waldnutzung - Fallstudie Waldlandschaft Solling. Abschlußbericht zum BMBF-Forschungsvorhaben. Selbstverlag

STUHR, G. (1985b): Auswirkungen früherer Zuwachsrückgänge und geminderter Starkholzpreise auf die Fichtenumtriebszeiten. AFZ 11, S. 231

Korrespondierender Autor:

Prof. Dr. Bernhard Möhring

Burckhardt-Institut der Georg-August-Universität Göttingen

Abteilung Forstökonomie und Forsteinrichtung

Büsgenweg 5

37077 Göttingen

E-Mail: forecon@uni-forst.gwdg.de

URL: http://ufobiu.forst.uni-goettingen.de/ufbl/

Dr. Georg Leefken

Claudia Gutsche M.Sc.

Burckhardt-Institut der Georg-August-Universität Göttingen 
Buchenwälder bedecken in der Bundesrepublik Deutschland derzeit knapp 1,6 Millionen Hektar, dies entspricht einem Anteil von 14,8 \% an der Gesamtwaldfläche. Rund ein Viertel des natürlichen Verbreitungsgebietes der Rotbuche (Fagus sylvatica L.) liegt in Deutschland. Damit kommt dieser Baumart sowohl ökonomisch, ökologisch als auch kulturell eine besondere Bedeutung zu. In diesem Band werden Ergebnisse aus der angewandten Forschung zur Buche in 14 Beiträgen vorgestellt. Diese decken ein breites Themenspektrum ab: vom Wasser- und Nährstoffhaushalt von Buchenwäldern, der genetischen Vielfalt, den Auswirkungen des Klimawandels auf Buchenwälder über Aspekte des Waldschutzes, der Biodiversität, des Waldumbaus und der Bestandesbehandlung bis hin zur Verwendung von Buchenholz und der betriebswirtschaftlichen Bewertung von Buchenwäldern. 